\title{
Lessons in Modeling, Preventing, and Treating Neural Injury: Improving Bench-to- Bedside Translation
}

\author{
Ryan Coddington Turner \\ West Virginia University
}

Follow this and additional works at: https://researchrepository.wvu.edu/etd

\section{Recommended Citation}

Turner, Ryan Coddington, "Lessons in Modeling, Preventing, and Treating Neural Injury: Improving Benchto- Bedside Translation" (2013). Graduate Theses, Dissertations, and Problem Reports. 3665.

https://researchrepository.wvu.edu/etd/3665

This Dissertation is protected by copyright and/or related rights. It has been brought to you by the The Research Repository @ WVU with permission from the rights-holder(s). You are free to use this Dissertation in any way that is permitted by the copyright and related rights legislation that applies to your use. For other uses you must obtain permission from the rights-holder(s) directly, unless additional rights are indicated by a Creative Commons license in the record and/ or on the work itself. This Dissertation has been accepted for inclusion in WVU Graduate Theses, Dissertations, and Problem Reports collection by an authorized administrator of The Research Repository @ WVU.

For more information, please contact researchrepository@mail.wvu.edu. 


\title{
Lessons in Modeling, Preventing, and Treating Neural Injury: Improving Bench-to-
} Bedside Translation

\author{
Ryan Coddington Turner \\ Dissertation submitted to the \\ School of Medicine \\ at West Virginia University \\ in partial fulfillment of the requirements \\ for the degree of \\ Doctor of Philosophy \\ in \\ Neuroscience \\ Charles L. Rosen, M.D., Ph.D., Chair \\ Jason D. Huber, Ph.D. \\ James P. O'Callaghan, Ph.D. \\ Rae R. Matsumoto, Ph.D. \\ Taura L. Barr, Ph.D. \\ Department of Neurosurgery \\ The Center for Neuroscience \\ Morgantown, West Virginia \\ 2013
}

Keywords: aging, stroke, traumatic brain injury, blast injury, signal transduction 


\section{Abstract \\ Lessons in Modeling, Preventing, and Treating Neural Injury: Improving Bench-to- Bedside Translation}

\section{Ryan Coddington Turner}

The translation of therapeutic agents for ischemic stroke and traumatic brain injury from the preclinical laboratory to clinical trials has been fraught with failure as evident by the $1,000+$ preclinical studies that have failed to translate to a proven therapeutic clinically. Numerous potential reasons for these shortcomings have been identified and include the use of animal models that fail to capture clinically relevant risk factors or injury parameters, targeting of pathological events occurring rapidly after injury but before the likely time of patient presentation for treatment, emphasis on acute rather than chronic outcome measures, and mismatch in outcome measures used between preclinical and clinical studies. To address these shortcomings, we describe the use of aged animals in a clinically relevant model of ischemic stroke produced through thrombus insertion and reperfusion mediated via administration of tissue plasminogen activator (tPA), the only FDA-approved pharmacologic agent for treatment of ischemic stroke. Using this ischemic stroke model with tPA administered at 2 hours post-stroke, we demonstrate the neuroprotective properties of bryostatin-1, a protein kinase $c$ (PKC) modulator. Specifically, bryostatin-1 treated animals displayed an improved survival rate, reduced infarct volume, and diminished functional impairment following ischemic stroke. As tPA administration is limited clinically by the time of presentation, extending the therapeutic window will be therapeutically advantageous. We show, in our aged model of ischemic stroke, that administration of a recombinant human apyrase (APT 102), a novel form of the naturally occurring human apyrase/ADPase, improves the safety and efficacy of tPA administration at 6 hours post-stroke based on functional and histological endpoints. Furthermore, we characterized behavioral and functional deficits following ischemia at numerous time points in order to more closely replicate clinical studies that emphasize behavioral outcomes rather than the use of infarct volume or other histological measures as an experimental endpoint. These studies demonstrate a significant correlation between infarct volume and functional ability, particularly with regards to striatal infarcts. Similar paradigms were applied to a diffuse axonal injury model and revealed no substantive deficits between injured and non-injured animals within one week of trauma. This indicates the challenge of identifying injury severity in preclinical trauma models as the lack of deficits is more representative of subconcussive injury rather than concussion or more substantive brain injury. Using this diffuse axonal injury model, we also explore preventative measures drawing on biologically inspired discovery. We show for the first time, the ability to reduce intracranial injury through manipulation of the vasculature through an externally applied device. Data from these studies demonstrate potential advances addressing the shortcomings associated with neural injury research, namely the development and use of clinically relevant animal models, selection of appropriate experimental endpoints, and varied approaches to both improving neural injury prevention and treatment. 
This work is dedicated to my loving and supportive family and the many others that have helped and encouraged me along the way. 


\section{ACKNOWLEDGEMENTS}

The work presented within this dissertation would not have been possible without the support, assistance, and guidance of many individuals both inside and outside of the workplace. I would like to thank the many members of the Department of Neurosurgery for support of our work, particularly those that kept the lab supplied (Mrs. Penny Humberson) and me reimbursed (Mrs. Mary Ann Meleady). I'd also like to thank the many other members of the Huber/Rosen lab, both past and present. Xinlan Li and Zhenjun Tan for assistance with experimental preparations and perhaps most importantly, never ending entertainment. Aric Logsdon for contributing to many different experiments and papers, particularly those requiring skills with Chem Bio Draw, Western blotting, and polymerase chain reaction (PCR). You also taught me a thing or two about patience, a lesson that while not enjoyable at the time will serve me well long-term. Ken DiPasquale for your extensive efforts in the laboratory, particularly those related to experiments requiring extensive knowledge of Windows 98, and endless source of laughter, often at Aric's expense. Alisa Elliott for assistance in navigating the bureaucracy of the National Institute of Occupational Safety and Health (NIOSH) and acquisition of supplies from our neighbors. Brandon Lucke-Wold for your relentless dedication to the task-at-hand and assistance in completing the numerous review papers on which we've worked together. l'd also like to thank former lab members for assistance with experimental techniques and guidance in navigating lab dynamics, namely Dr. Rachel Leon, Dr. Kimberly Kelly, and Dr. Reyna Vangilder.

I owe an immense amount of gratitude to Zach Naser for your contributions to many experiments and papers, as well as a lasting friendship. Without your assistance and dedication to carrying out many of the experiments I started, I never would have been able to complete them. You were instrumental in nearly all of the immunohistochemistry work and microscopy performed by the laboratory during my time here and for that I am forever grateful. When I first met you during my early time in the lab we both had next to no idea what we were doing but with time, persistence, and lots of reading and discussion with collaborators, it's amazing how far we've come.

l'd also like to thank members of the Matsumoto lab that have been not only good friends but great collaborators, specifically Mike Seminerio and Matt Robson. Mike was instrumental in getting me involved with the Biomedical and Behavioral Science training grant and introducing me to many of the behavioral techniques that were utilized throughout my work. Matt, you've been an endless source of help in learning various techniques and debating aspects of science, all the while forming a productive collaboration and close friendship. It's been a pleasure to work with you and I look forward to following your career and the potential for working together again in the future.

Dr. Charles Rosen, my mentor and advisor, has been instrumental in my development as not only a scientist but also a hopeful clinician-scientist and neurosurgeon upon completion of my training. You've served as a prime example illustrating both the challenges and successes inherent to the career I'm pursuing, something that most in my position probably do not experience and if so, not with the proximity with which I have. This experience alone has probably shaped my career more than any other throughout my training as I have gained a far greater appreciation for the elements and atmosphere required to be successful integrating the realms of basic science and clinical practice. You've allowed me the freedom to pursue topics and projects of interest to me while helping ensure the resources required were available. No matter how little time you have available, you've always found time to guide and assist when needed, something for which I'm extremely grateful. Furthermore, you've taught me to question 
the way science is conducted and not to be afraid to 'swim upstream', against conventional wisdom, in pursuit of improving science and the betterment of patients in the future.

Dr. Jason Huber, l'd like to thank you for your role in my experience within the lab and helping to demonstrate and teach me how to write grants, an invaluable experience and one that will hopefully serve me well throughout my career. You also significantly furthered my understanding of experimental design and instilled an appreciation of how various techniques are conducted and how to troubleshoot problems, all skills that will be of assistance going forward in my scientific career.

l'd also like to thank the other members of my committee, Dr. Rae Matsumoto, Dr. James O'Callaghan, and Dr. Taura Barr. In both our formal and informal meetings, each of you provided encouragement, recognition, and suggestions for improving my science without fail. I owe a special thanks to Dr. Matsumoto and Dr. O'Callaghan for providing resources for my work and equally important, many insightful conversations about science, funding, and the politics of universities and government agencies.

My acknowledgments wouldn't be complete without recognizing and thanking other influential figures and collaborators that l've had the experience of working with at various points in my training. Dr. Diane Miller has always provided assistance in utilizing the resources available at $\mathrm{NIOSH}$ and more importantly, guidance and suggestions for a number of experiments, particularly those assaying some form of behavior. Dr. Julian Bailes was instrumental in me getting involved in traumatic brain injury research and provided me much of my early experience in animal models of traumatic brain injury. You've also continued to keep in touch and provided mutually beneficial opportunities for me despite having switched institutions, and for that I'm grateful. You also introduced me to others in the field that contributed to my work such as Dr. David Smith. Dr. Smith, you have provided many interesting ideas to consider and always a different perspective for improving science for the betterment of patients. Dr. Robert Gettens of Western New England University was essential in the design and production of the blast model utilized within this work and for that I am extremely grateful.

Finally, and most importantly, I want to thank my wife, Julie. You have been a continual source of support behind my efforts and tolerated the many hours I've spent working, both in the lab and at home. You've been a source of empathy and encouragement through the many challenges encountered, both in the lab and more importantly, in general life. Your belief in me, often exceeding my own, is greatly appreciated and a source of motivation and pride. You've also given me the greatest gift imaginable in our daughter Olivia. I owe Olivia thanks for training me for the many nights and years of sleep deprivation required for a successful career in academic neurosurgery. 


\section{TABLE OF CONTENTS}

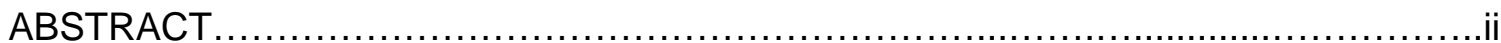

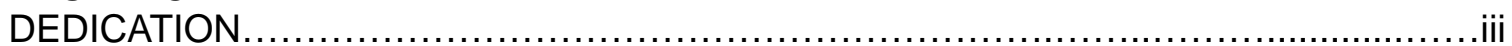

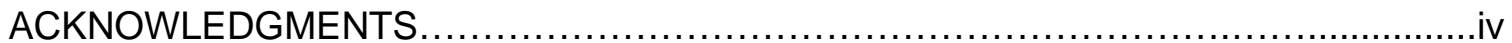

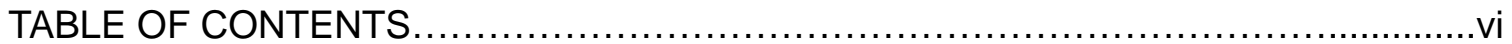

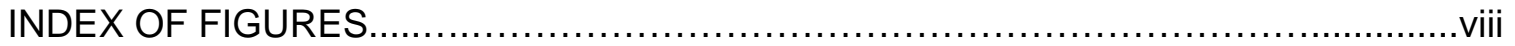

INDEX OF TABLES.

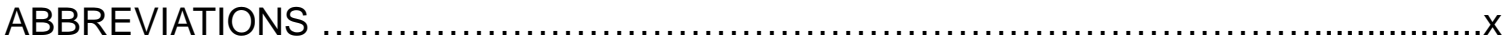

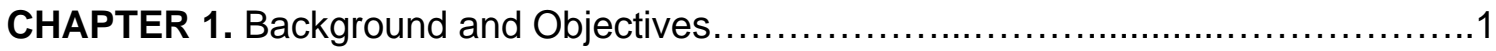

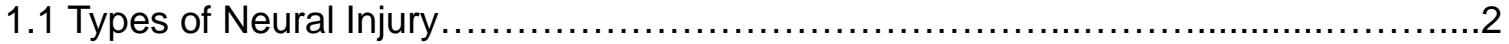

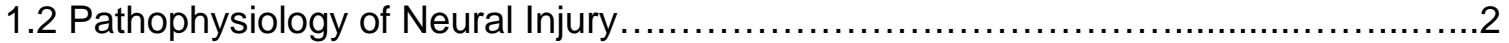

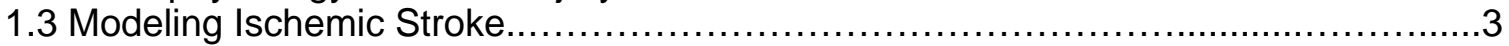

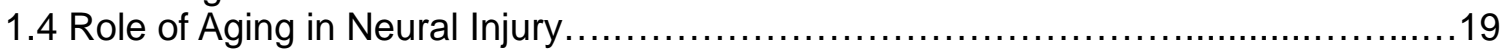

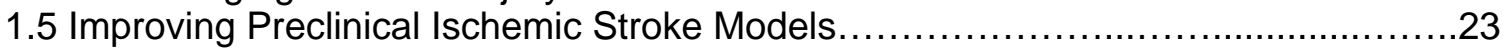

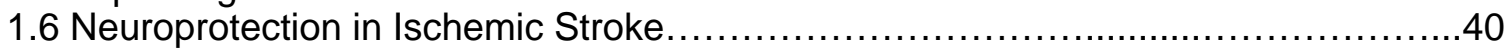

1.7 Jak2/Stat3-mediatd Signaling in Ischemic Stroke.....................................55

1.8 Protein Kinase $C$ as a Therapeutic Target for Ischemic Stroke..........................69

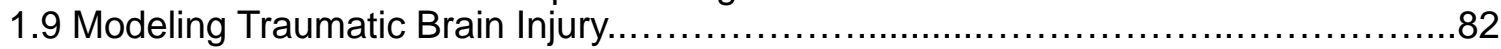

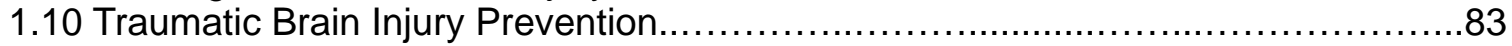

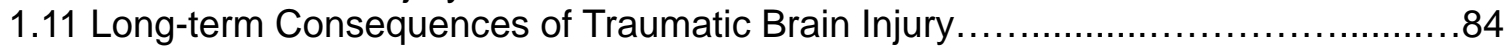

CHAPTER 2. Bryostatin Improves Stroke Outcome in a Clinically Relevant Model of Ischemic Stroke via Protein Kinase C Activation...................................................98

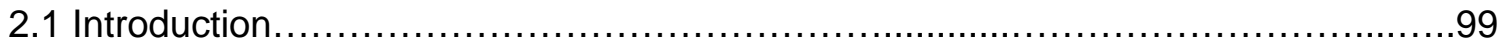

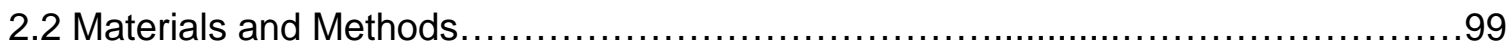

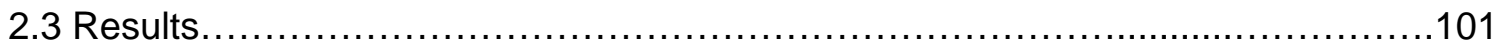

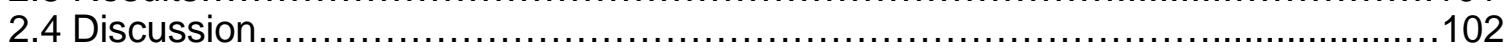

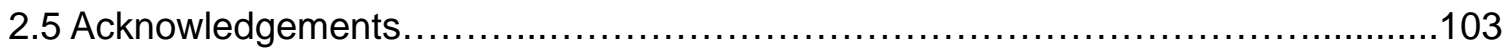

CHAPTER 3. Combination Treatment of $r$-tPA and Apyrase Reduces Mortality Rate and Hemorrhagic Transformation in Acute Ischemic Stroke in Aged Female Rats.............110

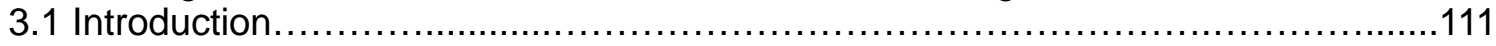

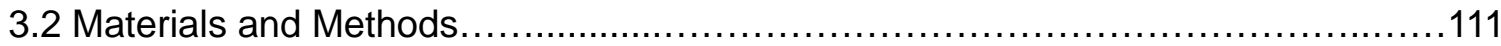

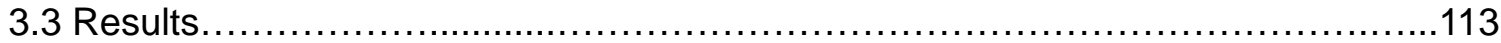

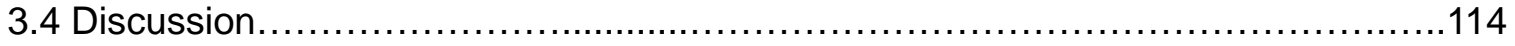

CHAPTER 4. Effects of Aging on Behavioral Assessment Performance: Implications for Clinically Relevant Models of Neurological disease ...............................................121

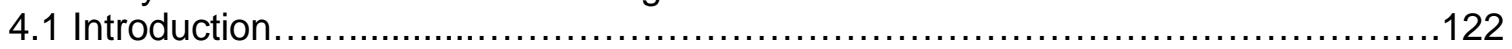

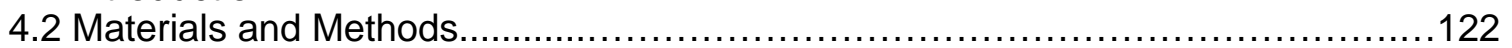

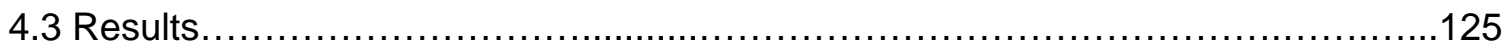

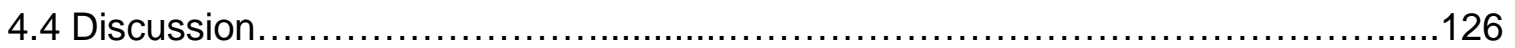

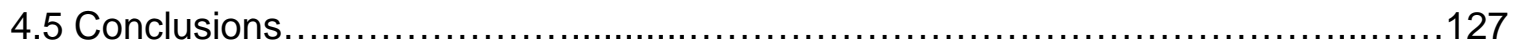

CHAPTER 5. The Role for Infarct Volume as a Surrogate Measures of Functional Outcome Following Ischemic Stroke: Reconciling Preclinical and Clinical Studies......137

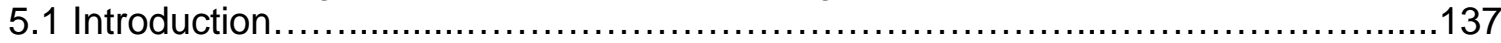

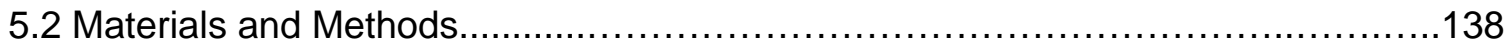

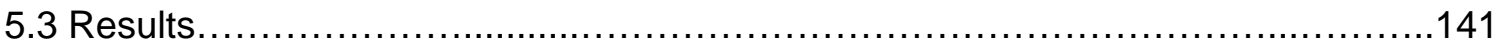

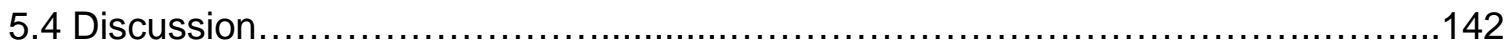


CHAPTER 6. Anabolic Steroids and Head Injury..............................................148

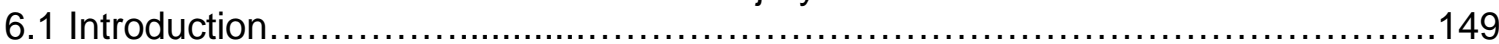

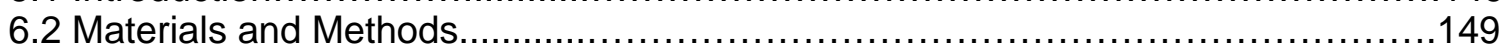

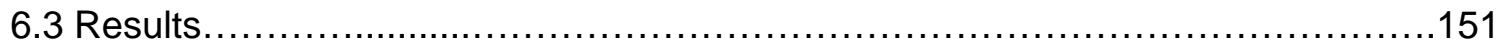

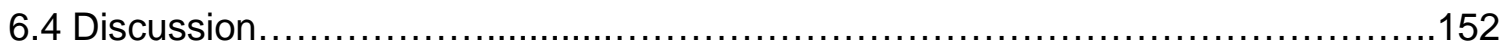

CHAPTER 7. Internal Jugular Vein Compression Mitigates Traumatic Axonal Injury in a Rat Model by Reducing the Intracranial Slosh Effect...............................................157

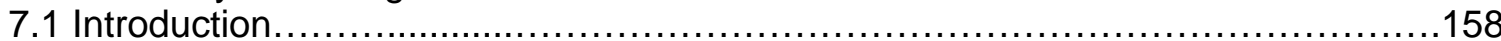

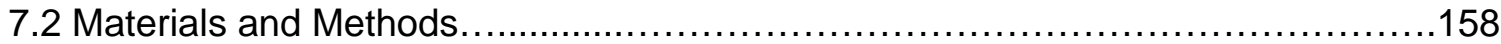

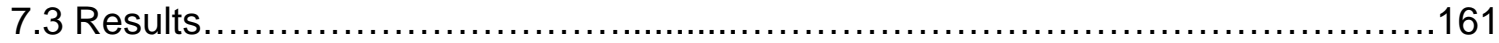

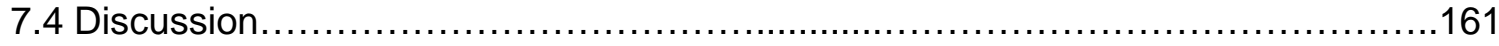

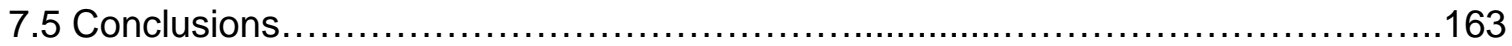

CHAPTER 8. Effect of Slosh Mitigation on Histologic Markers of Traumatic Brain

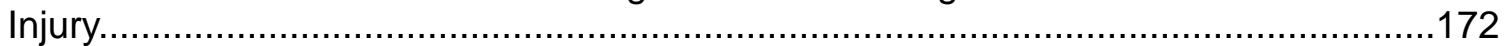

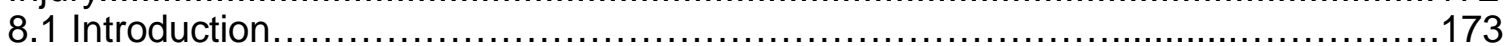

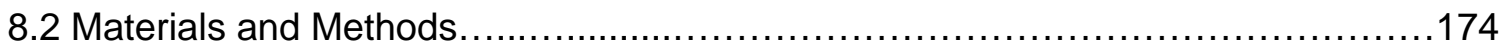

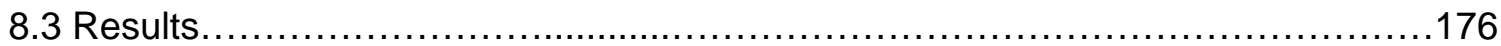

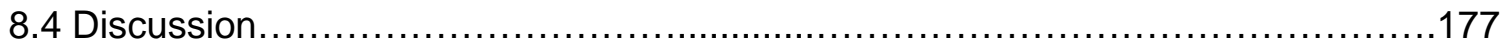

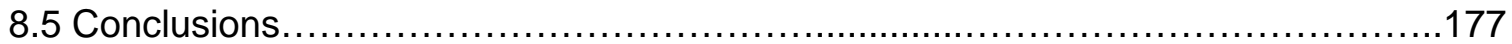

CHAPTER 9. Modeling Clinically Relevant Blast Parameters based on Scaling Principles Produces Functional \& Histological Deficits in Rats................................................187

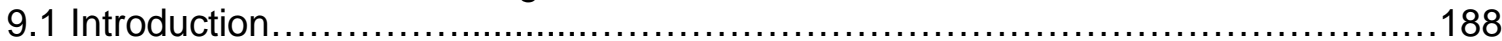

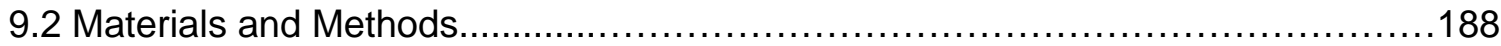

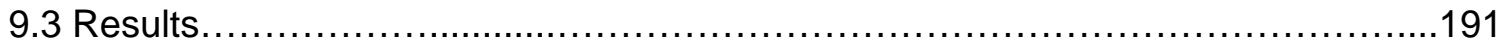

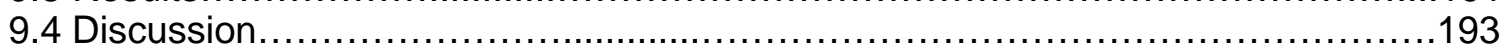

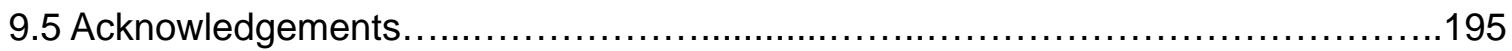

CHAPTER 10. Elucidating the Severity of Preclinical Traumatic Brain Injury Models:

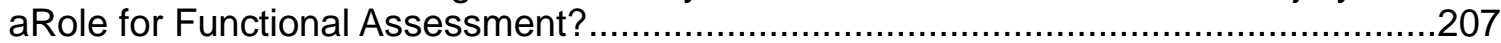

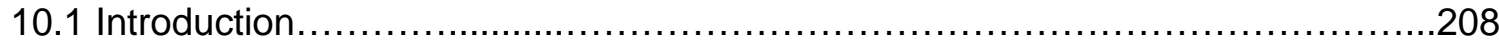

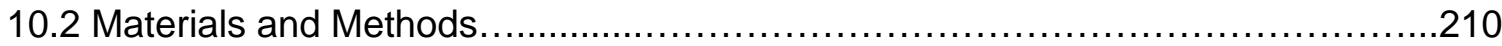

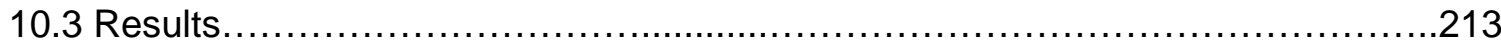

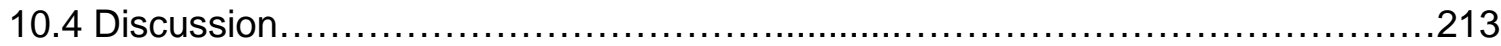

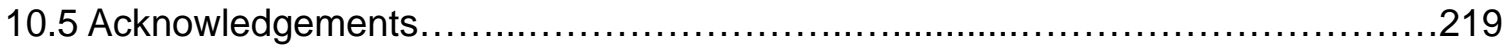

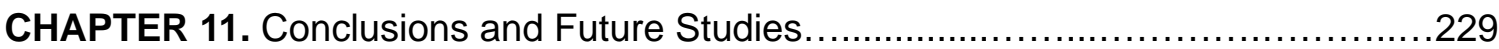

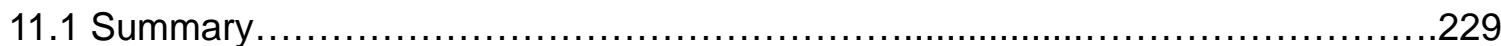

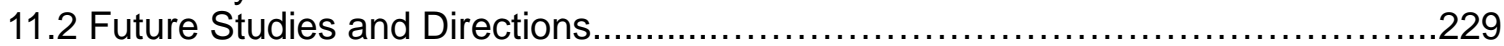

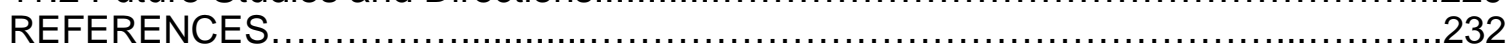

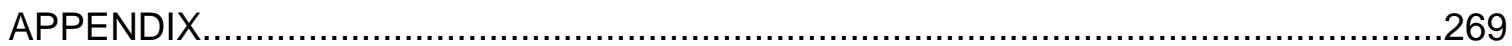

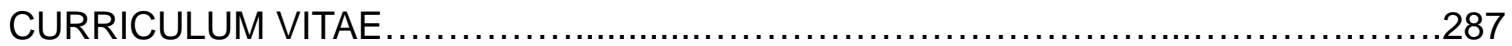




\section{INDEX OF FIGURES}

Chapter One

$\begin{array}{lll}\text { 1.1. } & \text { Femoral artery dissection } & 7\end{array}$

1.2. Burr hole preparation 10

1.3. Vessel preparation for thrombus insertion 11

1.4. Diagrammatic representation of vasculature 13

1.5. Laser Doppler recording of CBF during MCAO 14

1.6. Aging and Stroke 26

1.7. Obesity and Stroke 31

1.8. Rat Models of Diabetes Type II 34

1.9. Hypertension and Stroke 36

1.10. Ischemic injury cascade 42

1.11. Targets for preventing neuronal excitotoxicity 43

1.12. Ischemia produces increased intracellular calcium 46

1.13. Inflammation as a therapeutic target 49

1.14. Neuroprotection versus cerebroprotection 51

1.15. Receptor subunits for neuropoietic cytokine signaling 57

1.16. Complexity of the STAT3 pathway 59

1.17. Schematic of PKC activation 70

1.18. PKC isozyme activation and function 81

1.19. Imaging and biomarker study outline for CTE 97

Chapter Two

2.1. Survival rate and functional status post-MCAO 104

2.2. Infarct volume is reduced by bryostatin-1 105

2.3. TUNEL staining is diminished with bryostatin-1 106

2.4. Astrocyte activation and glial scarring is reduced 107

2.5. Colocalization of PKC isozymes in neurons post-MCAO 108

2.6. Colocalization of PKC isozymes in endothelium post-MCAO 109 Chapter Three

3.1. Mortality rate is reduced with APT 102 administration 116

3.2. Infarct size is diminished following APT 102 administration 117

3.3. Functional status post-MCAO with and without APT $102 \quad 118$

$\begin{array}{ll}\text { 3.4. Hemoglobin as a marker of hemorrhagic transformation } & 119\end{array}$

3.5. Edema post-MCAO is not altered by APT $102 \quad 120$

Chapter Four

4.1. Effect of age on sensorimotor assessment 130

4.2. Effect of age on motor coordination 131

4.3. Impaired balance beam performance with age 132

4.4. Effect of age on anxiety-like behavior 133

4.5. Effect of age on depressive-like behavior 134

4.6. Role of age in spatial acquisition 135

4.7. Effect of age on reference memory 136

Chapter Six

6.1. Micrographs of corticospinal tracts post-TBI 154

6.2. APP positive axons receiving treatment pre-injury 155 Chapter Seven

6.3. APP positive axons receiving post-injury treatment 156

7.1. ICP with and without compression 165

7.2. IOP with and without compression 166

$\begin{array}{lll}\text { 7.3. } & \text { Representative physiologic tracing with manipulation } & 167\end{array}$

$\begin{array}{lll}\text { 7.4. } & \text { Corticospinal tract APP staining } & 168\end{array}$

7.5. Effect of IJV compression on axonal injury 169

$\begin{array}{ll}\text { 7.6. Newton's Cradle } & 170\end{array}$ 
7.7. IJV compression device in action

Chapter Eight

$\begin{array}{ll}\text { 8.1. Cresyl violet staining } & 179\end{array}$

$\begin{array}{ll}\text { 8.2. Hematoxylin and eosin staining } & 180\end{array}$

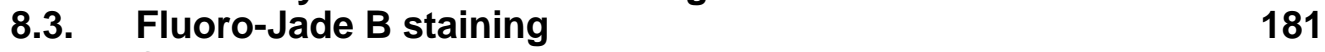

8.4. Stereologic quantification of Fluoro-Jade B 182

8.5. Glial fibrillary acidic protein staining 183

8.6. Quantification of glial fibrillary acidic protein staining 184

8.7. Ionized calcium binder adapter molecule 1 staining 185

8.8. Quantification of ionized calcium binder adapter molecule 1186 Chapter Nine

9.1. Experimental setup for blast modeling 196

$\begin{array}{ll}\text { 9.2. } & \text { Shock tube recordings } \\ & 197\end{array}$

$\begin{array}{ll}\text { 9.3. } & \text { Gross morphology post-blast } \\ & 198\end{array}$

$\begin{array}{ll}\text { 9.4. Hematoxylin and eosin staining } & 199\end{array}$

$\begin{array}{ll}\text { 9.5. Neural degeneration quantification } & 200\end{array}$

9.6. Astrocyte reactivity post-blast 201

9.7. Microglial activation with blast exposure 203

9.8. Gene expression with blast exposure 205

9.9. Effect of blast exposure on activity 206

$\begin{array}{cc}\text { Chapter Ten } & \\ 10.1 . & \text { Neural degeneration post-DAl }\end{array}$

10.2. Astrocyte activation post-DAI 222

10.3. Microglial activation post-DAl 223

10.4. DAl does not produce sensorimotor deficits acutely 224

10.5. Lack of retrograde amnesia acutely with DAl 225

10.6. DAl does not cause changes in anxiety- or depressive-like behaviors acutely 


\section{INDEX OF TABLES}

Chapter One

1.1. Proposed neuroprotectants targeting excitotoxicity

1.2. Proposed neuroprotectants targeting inflammation 50

1.3. Sites for neuropoietic cytokine binding 56

1.4. Diversity of pathological findings in CTE 90

1.5. Imaging modalities for CTE 92

Chapter Four

4.1. Modified Neurologic Severity Score (mNSS) 129

Chapter Five

5.1. Description of the mNSS scoring system for neural injury 144

5.2. Spearman correlation coefficients (d1 volume, d1 function) 145

5.3. Spearman correlation coefficients (d21 volume, d21 function) 146

5.4. Spearman correlation coefficients (d21 volume, d1 function) 147 Chapter Ten

10.1. Performance on fixed speed rotarod on Day 2 post-injury 227

10.2. Performance on fixed speed rotarod on Day 6 post-injury 228 


\section{CHAPTER 1}

BACKGROUND AND OBJECTIVES 
The overall goal of this work is to better understand neural injury in an effort to develop improved treatment for patients suffering neural injury. Neural injury takes numerous forms ranging from ischemic stroke to hemorrhagic stroke to traumatic brain injury to drug abuse. Consequently, numerous tools exist for studying neural injury and include animal models of ischemic stroke and traumatic brain injury, both of which are explored in greater detail throughout this work as these animal models are utilized as tools for the study of neural injury.

\subsection{Types of Neural Injury}

Neural injury represents a leading cause of death and disability in the developed world, likely due to the diverse and highly prevalent nature of neural injury. While neural injury has historically been categorized as a product of ischemic or hemorrhagic stroke and trauma, further sub-classifications and other forms of neural injury require consideration. Traumatic brain injury, for example, can be classified as open or closed, linear or rotational, and can occur from a traditional impact or from blast exposure. Other forms of neural injury include toxicant exposure and drug abuse, each of which may result in not only acute pathological changes but also persistent neuroinflammation, not unlike more traditionally considered forms of neural injury such as stroke and trauma.

\subsection{Pathophysiology of Neural Injury}

While the diversity of conditions and causes associated with neural injury leads to various inciting injurious elements, the pathophysiology of neural injury often shares many similar events. Using ischemia as an example, the cessation of blood flow is associated with a rapid decoupling of oxidative phosphorylation and hypoglycemia that leads to decreased ATP production and failure of ionic pumps. Ionic imbalance leads to membrane depolarization and subsequent glutamate release from the pre-synaptic neuron ${ }^{1}$. Excess glutamate can lead to prolonged opening of $\mathrm{Ca}^{2+}$ channels and a number of damaging events ranging from free radical production to activation of proteases, lipases, and endonucleases ${ }^{2}$. Ultimately, these events may lead to irreversible cell damage and death. While these events occur rapidly following injury, others demonstrate a delayed onset or persist for prolonged periods. Perhaps the best example of a delayed but persistent event following injury is that of inflammation ${ }^{3}$. Induction of the inflammatory process remains under investigation but emerging evidence indicates a role for not only signaling molecules released from the damaged tissue directly (proteases, DAMPs, ATP, etc) but also a disruption in communication between neurons and glial cells essential for homeostasis as well as the response to injury, namely microglia and astrocytes. How exactly these glial cells contribute to injury as well as repair is not entirely clear and consequently remains a topic of investigation ${ }^{3}$.

Other forms of neural injury exhibit similar pathophysiological processes as ischemic stroke. For example, traumatic brain injury, particularly open head injury, results in vascular disruption and therefore produces similar pathological events to ischemic stroke acutely. Traumatic brain injury, particularly closed head injury, also produces diffuse axonal injury (DAI). DAI leads to microstructural changes in connectivity and excess glutamate release, a finding that frequently correlates with injury severity ${ }^{4}$, and initiates a pathological cascade similar to that observed in ischemic stroke ${ }^{5}$. Furthermore, trauma induces significant glial activation as evident by astrocyte reactivity, microglial activation, and corresponding events such as cytokine release and formation of the glial scar ${ }^{6,7}$. Glial activation, a hallmark of neuroinflammatory processes, is shared amongst 
most, if not all, forms of neural injury and represents a critical pathological process in drug abuse and neurotoxicant exposure as well.

\subsection{Modeling Ischemic Stroke}

One of the tools developed and honed by the Rosen lab at West Virginia University for the study of neural injury is that of ischemic stroke in a clinically-relevant animal model utilizing aged rodents and a thromboembolic mechanism of ischemia. This model, surgical technique, and some of the finer points required for experimental success are detailed in the following section.

This work was published in TRP Channels in Drug Discovery: Volume II, Methods in Pharmacology and Toxicology.

Citation: Turner RC, Elliott AS, Huber JD, Rosen CL (2012) A Clinically Relevant Thromboembolic Stroke Model in the Aged Rat. Edited by Arpad Szallasi and Tamás Bíró. TRP Channels in Drug Discovery: Volume II. Methods in Pharmacology and Toxicology.

\section{ABSTRACT}

Animal models have been an integral component of scientific discovery concerning ischemic stroke pathophysiology and the accompanying therapeutic targets. Unfortunately, the knowledge gleaned from these studies has failed to translate to enhanced therapeutics in the clinical environment ${ }^{8}$. We argue that this failed translation is a direct result of inadequate animal models that fail to address the greatest risk factor for stroke, age ${ }^{9}$. Herein we provide comprehensive step-by-step instructions for a clinically-relevant thromboembolic stroke model in aged rats ${ }^{10}$. This technique, utilizing the only FDA approved therapeutic agent for the treatment of stroke, tissue plasminogen activator (tPA), allows for pathophysiologic studies in addition to testing potential therapeutic agents in combination with the currently approved treatment regime.

\subsubsection{INTRODUCTION}

The desire to model stroke in the preclinical environment remains relevant due to the immense societal impact of stroke. Stroke remains the world's second leading cause of mortality, resulting in $\sim 6,000,000$ deaths annually ${ }^{11}$. Worse yet, survivors often experience significant morbidity. These factors necessitate the development of improved therapeutic agents that can be applied to a greater percentage of those afflicted with stroke.

In spite of more than 100 agents that have progressed from what appeared to be successful preclinical studies to clinical trials, tissue plasminogen activator remains the only FDA approved treatment and can be used in a relatively small number of patients due to contraindications and a limited window of opportunity ${ }^{8}$. The question of why so many agents have failed to translate successfully remains largely unanswered. Assessing this failed translation, it is clear that one key difference exists between the animal models and the clinical realm - the consideration of age.

The average stroke patient is elderly - approximately $72 \%$ of patients are over the age of 65 yet the vast majority of preclinical studies are completed in 3 month old rats which equates to essentially a young-adult ${ }^{9}$. Additionally, $52 \%$ of stroke victims are female but most preclinical studies utilize male animals. Furthermore, $87 \%$ of strokes are ischemic 
and caused by a thrombus or embolus occluding a vessel, most often being the middle cerebral artery. Despite this fact, many preclinical studies utilize other methods of occlusion ranging from a suture to endothelin-1 to photothrombosis.

The stroke model described in this work seeks to model the clinical scenario as closely as possible by utilizing aged, female rats and a thrombus to occlude the middle cerebral artery ${ }^{10}$. While this is but one model of ischemia in terms of technique and animal type used, we believe it is the most clinically relevant for studying the pathophysiology of stroke and assessing various proposed pharmacologic agents.

\subsubsection{MATERIALS}

\subsubsection{Equipment}

Equipment described below is used in our laboratory. Alternatives are available and may be substituted where appropriate.

1. VetEquip $\mathrm{RC}^{2}$ (Rodent Circuit Controller) anesthesia machine (VetEquip; Pleasanton, California)

2. Harvard Apparatus Homeothermic Blanket Control Unit with Probe(Harvard Apparatus; Holliston, Massachusetts)

3. Harvard Apparatus Homeothermic Blanket (Harvard Apparatus; Holliston, Massachusetts)

4. Oster GOLDEN A5 with \#10 clip for hair removal (Oster; McMinnville, Tennessee)

5. Leica M400-E Operative Microscope (Leica Microsystems Inc; Buffalo Grove, IL)

6. Moor Instruments DRT4 Laser Doppler Perfusion Monitor (Moor Instruments Inc; Wilmingtown, Delware)

7. Aitecs SEP-215 Plus syringe pump for administering saline (Aitecs; Vilnius, Lithuania)

8. Harvard Apparatus Pump 11 for administering tissue plasminogen activator (Harvard Apparatus; Holliston, Massachusetts)

9. Integra $2.7 \mathrm{~mm}$ twist drill bit with adjustable safety stop (Integra; Plainsboro, New Jersey)

10. Integra Camino Bolt (cranial bolt for insertion of Doppler probe) (Integra; Plainsboro, New Jersey)

11. ISMATEC Reglo Peristaltic Pump for cardiac perfusion of the animal (ISMATEC; Wertheim-Mondfeld, Germany)

12. $-80^{\circ} \mathrm{C}$ freezer

13. Autoclave (for sterilizing surgical equipment)

14. Incubator (for thrombus preparation)

\subsubsection{Supplies for Thrombus Preparation}

1. INTRAMEDIC CLAY ADAMS Brand NON-RADIOPAQUE POLYETHYLENE TUBING (BD; Franklin Lakes, New Jersey)

2. Petri dish

a. PE $50-$ I.D. $0.58 \mathrm{~mm}$ and O.D. $0.965 \mathrm{~mm}$

b. PE $10-$ I.D. $0.28 \mathrm{~mm}$ and O.D. $0.61 \mathrm{~mm}$

3. $1 \mathrm{ml}$ syringe

4. Bunsen Burner

5. $0.9 \%$ Physiologic Saline 


\subsubsection{Surgical Instruments and Supplies}

1. Kendall Curity Gauze Sponges (for making gauze pillow) (Covidien; Mansfield, Massachusetts)

2. VWR Laboratory Labeling Tape (for making gauze pillow) (VWR International; Radnor, Pennsylvania)

3. Puritan Cotton Tipped Applicators (VWR International; Radnor, Pennsylvania)

4. Kendall MonoJect $20 \mathrm{ml}$ syringe (Covidien; Mansfield, Massachusetts)

5. Ethicon 3-0 Coated Vicryl Suture (Ethicon; Somerville, New Jersey)

6. Syneture 5-0 Sofsilk Suture (Covidien; Mansfield, Massachusetts)

7. Forceps (sharp \& dull; different sizes)

8. Surgical scissors (large and small sizes)

9. Surgical scalpel

10. Bard-Parker Stainless Steel Surgical Blade \#15 (BD; Franklin Lakes, New Jersey)

11. Micro-scissors

12. Micro-vascular clips (x 2)

13. Micro-vascular clip holder

14. Hemostat

15. Needle Driver

16. Bone Rongeur for craniotomy

17. $2 \mathrm{~mm}$ brain block

18. Razor blades

19. Sterile surgical gowns

20. Sterile surgical gloves

21. Sterile surgical drapes

22. Instrument pouch for autoclave

23. Micro-syringe for thrombus injection

\subsubsection{Drugs/Chemicals}

1. Baxter $0.9 \%$ Sodium Chloride Injection USP $1000 \mathrm{~mL}$ (Baxter; Deerfield, Illinois)

2. 2,3,5 - Triphenyl tetrazolium chloride (Sigma-Aldrich; St. Louis, Missouri)

3. Activase (tissue plasminogen activator) (Genentech; San Francisco, California)

4. Phosphate-buffered Saline (PBS)

5. Scrub Care ${ }^{\circledR}$ Providone lodine Cleansing Solution (Cardinal Health; Dublin, Ohio)

6. Dechra VETROPOLYCIN veterinary ophthalmic ointment (Dechra Veterinary Products; Overland Park, Kansas)

7. Isoflurane

\subsubsection{METHODS}

The following sections describe all steps of the thromboembolic stroke model beginning with chemical and surgical preparation through achieving reperfusion.

\subsubsection{Chemical Preparation}

1. Activase (tissue plasminogen activator)

a. Add $100 \mathrm{~mL}$ of physiologic saline to $100 \mathrm{mg}$ bottle of Activase, creating a concentration of $1 \mathrm{mg} / \mathrm{ml}$. 
b. Dissolve the mixture by gentle shaking.

c. Aliquot $1.5 \mathrm{~mL}$ in $2 \mathrm{~mL}$ Eppendorf tubes and store at $-80^{\circ} \mathrm{C}$ until needed. To achieve the final dose of $5 \mathrm{mg} / \mathrm{kg}, 5 \mathrm{ml} / \mathrm{kg}$ is given. For example, a 300 gram rat receives $1.5 \mathrm{ml}$ of the $1 \mathrm{mg} / \mathrm{ml}$ mixture.

\section{2. $2 \%$ Triphenyl tetrazolium chloride}

a. Weight 2 grams of 2,3,5 - Triphenyl tetrazolium chloride.

b. Place in a $100 \mathrm{~mL}$ Pyrex round media storage bottle with screw cap.

c. Fill to $100 \mathrm{~mL}$ with phosphate-buffered saline.

\subsubsection{Surgical Preparation}

\section{Preparation of Sterile Surgical Instruments}

a. Place clean instruments in autoclave pouch. Instruments should be in the open position when applicable to ensure proper sterilization.

b. Place autoclave pouch containing instruments in the autoclave.

c. Ensure autoclave is filled with deionized water.

d. Run autoclave (most cycles are at least 20 minutes).

e. After cycle is complete and pressure reduced, crack the autoclave door slightly (Note 1 ).

f. Run drying cycle (per manufacturer instructions).

g. Allow temperature to normalize with room temperature.

h. Remove pack.

\section{Anesthesia Induction}

a. Place rat in the anesthesia induction chamber.

b. Induce anesthesia for 10 minutes with the gas valve set at $4.0 \%$.

c. Remove rat from induction chamber and place the nose of the rat in the nose cone.

d. Maintain anesthesia via nose cone with the gas valve set at $2.0 \%$ for remainder of procedure.

\section{Aseptic Surgical Preparation}

a. Shave the hair of the rat in the proper position (see below).

b. Apply sterile surgical drapes around the surgical site.

c. Ready these areas for surgery using a surgical preparation solution such as betadine.

d. Place a drop of eye ointment in each eye to prevent post-surgical ophthalmic infections.

\section{Preparation of Gauze Pillow}

a. Roll gauze pads together creating a $2.5 \mathrm{~cm}$ diameter by $5 \mathrm{~cm}$ long cylinder.

b. Secure with laboratory labeling tape. 


\subsubsection{Creation of Thrombus}

1. Position rat on the homeothermic heating pad in a supine position while placing the lower limbs in the down position, exposing the groin region (Note

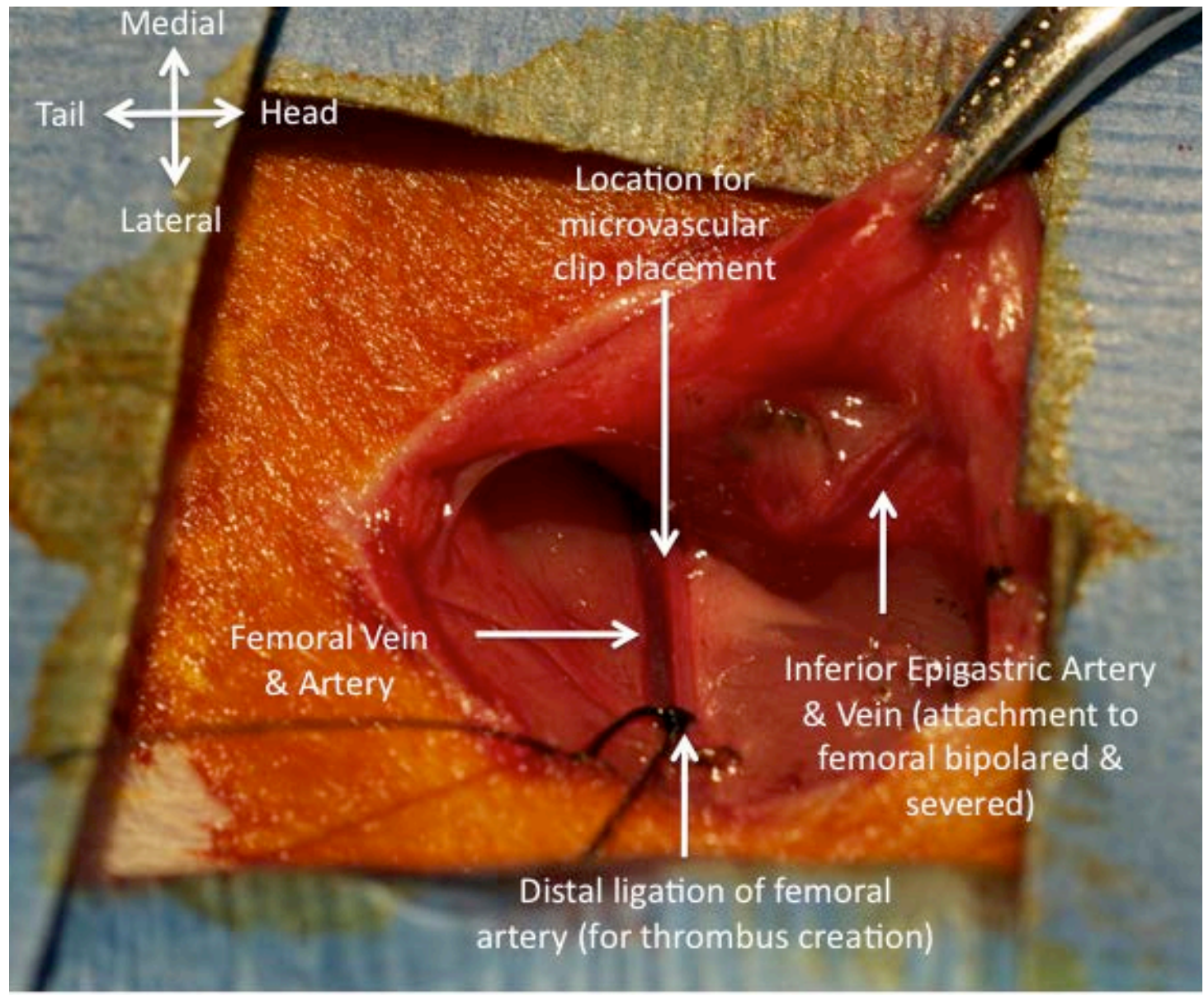

Figure 1.1. Femoral artery dissection in preparation for blood draw to create thrombus. Note space between suture and microvascular clip - this is where femoral artery lumen will be exposed to insert PE50 tubing.

8).

2. Prepare the right groin area for aseptic surgery (see above).

3. Using a surgical scalpel, make a $4 \mathrm{~cm}$ incision parallel to the midline in the groin.

4. Using blunt dissection techniques, dissect down to the femoral artery and vein. Passing through the layers of fat and connective tissue, the inferior epigastric artery will become visible, arising from the femoral artery and coursing towards the superficial layers of the lower abdomen.

5. Using a 3-0 suture, retract the external oblique muscle overlying the femoral artery using a hemostat in order to widen the field of view.

6. Isolate the femoral artery from the surrounding femoral vein and nerve.

7. Using a 5-0 suture, ligate distal portion of femoral artery.

8. Place microvascular clip (aneurysm clip) proximally to ligated portion of the femoral artery. Leave sufficient space (approximately $2 \mathrm{~cm}$ ) between suture and clip. This process is visualized in Figure 1. 
9. Once microvascular clip and suture are in place, effectively ceasing blood flow through this portion of the femoral artery, use microscissors to make a small cut in the top portion of the femoral artery. Use caution to not sever the entire vessel so that the base of the artery remains intact despite an opening in the top portion.

10. Cut a $15 \mathrm{~cm}$ long segment of PE50 tubing. Bevel one end of the cut segment at approximately $45^{\circ}$.

11. Insert beveled end of PE50 tubing into opening of the femoral artery. With application of light pressure, artery circumference will stretch to accommodate the tubing.

12. Once beveled end of PE50 tubing is entirely inside the artery lumen $(0.5 \mathrm{~cm}$ inside), release the microvascular clip briefly.

13. PE50 tubing should rapidly fill with blood. Upon filling, the microvascular clip is replaced.

14. Remove blood-filled PE50 tubing and bipolar coagulate the femoral artery proximal to incision, in order to prevent bleeding.

15. Remove microvascular clip and remove traction on overlying muscles.

16. Suture wound with 3-0 suture.

17. Place blood-filled PE50 tubing in an incubator at $37^{\circ} \mathrm{C}$ for 120 minutes.

18. Store thrombus in refrigerator overnight.

\subsubsection{Preparation of Thrombus for MCAO}

1. Remove thrombus from refrigerator.

2. Add saline to petri dish to wash thrombus.

3. Affix a $20 \mathrm{~cm}$ segment of PE10 tubing to a $1 \mathrm{ml}$ syringe via a sharp 27 gauge needle.

4. Insert free end of PE10 tubing into lumen of thrombus-filled PE50 tubing.

5. With slow but constant pressure, eject thrombus into saline-filled petri dish.

6. Visually assess thrombus diameter and appearance for uniformity using the microscope. A well-formed thrombus should appear consistent throughout (a smooth exterior with no missing pieces).

7. Wash clot by drawing into PE10 tubing attached to a syringe and expelling repeatedly back into saline. Upon initial expulsion, red cells will become detached from thrombus resulting in a cloudy appearance within saline. When clot is washed thoroughly it should appear $\sim 25 \%$ narrower and no additional cloudiness produced.

8. Prepare modified PE50 tubing for injection of the thrombus. This is done by heating the PE50 tubing over a bunsen burner, removing from the heat, and then stretching the heated portion quickly, reducing the diameter.

9. You should now have a piece of PE50 tubing that is regular in diameter on the left and right end and much thinner in the middle. Cut tubing in the middle of the thin portion creating two pieces of the modified PE50 tubing.

10. Set a micrometer to $0.3 \mathrm{~mm}$. Pull the modified PE tube between the jaw blades, starting at the thinnest portion and progressing to the thicker segment. Where blades 'catch' the tubing is where the tubing is then cut. This creates a modified PE50 segment of tubing with an outer diameter of 0.3 $\mathrm{mm}$.

11. When ready to inject thrombus, draw clot into modified PE50 tubing either directly or indirectly (via PE10 tubing).

\subsubsection{Measurement of Cerebral Blood Flow}


When performing middle cerebral artery occlusion, confirmation of proper thrombus (or suture in other models) placement is essential for verifying the induction of ischemia as well as for monitoring tissue plasminogen activator-induced reperfusion (Note 2). Briefly, a cranial bolt with accompanying laser Doppler cerebral blood flow (CBF) probe is inserted into the cranium above the area corresponding to the vascular region supplied by the middle cerebral artery (MCA). This provides continual readings to document CBF at baseline as well as during and after insertion of thrombus and during reperfusion.

1. Position rat on homeothermic heating pad such that it is on its left side, with the right side exposed.

2. Prepare region between ear and eye for aseptic surgery (see above).

3. Using a surgical scalpel, make an incision that is $2 \mathrm{~cm}$ medial and cephalic to right ear to $2 \mathrm{~cm}$ lateral and cephalic to right ear.

4. Temporalis muscle should now be exposed. Using scalpel, make a $1 \mathrm{~cm}$ incision along right edge at the point where temporalis muscle inserts into skull. Make another $1 \mathrm{~cm}$ incision parallel to the first incision in cephalic direction. Now to complete temporalis flap, cut along medial insertion. Use the bipolar at low setting (i.e. 15-20 Malis units) to control bleeding.

5. Reflect temporalis flap using 3-0 suture and a hemostat to expose Bregma.

6. After removing excess tissue to clearly expose sutures, use tip of scalpel blade to start a small burr hole approximately $3 \mathrm{~mm}$ caudal to Bregma and 3 $\mathrm{mm}$ medial to the linea temporalis (twirl the scalpel with attached blade in your fingers, similar to a drill bit).

7. Once a starting groove has been made in skull with scalpel blade, switch to drill bit (approximately 7/64 of an inch) and continue making burr hole. Do this part slowly and remove bone fragments frequently, particularly once dural surface is exposed (Note 3 ).

8. When the burr hole is finished, it should be a perfect circle and dura mater should be exposed such that you can see vasculature (Figure 2). 


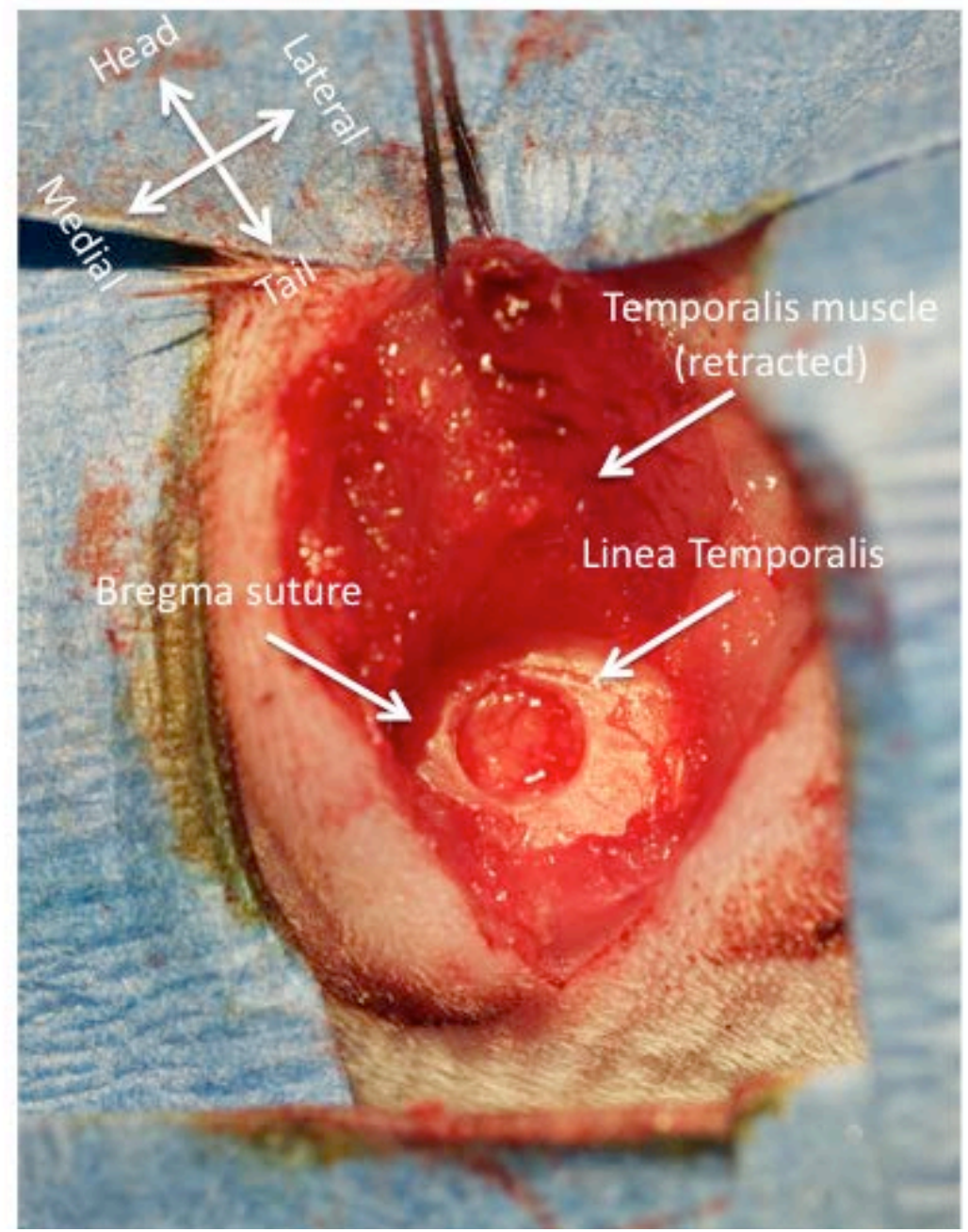

Figure 1.2. Burr hole ready for insertion of cranial bolt and laser Doppler

9. Affix cranial bolt over middle cerebral artery by carefully screwing it into parietal bone surrounding burr hole (Note 4).

10. Insert laser Doppler probe into cranial bolt opening for monitoring cerebral blood flow (Note 5).

11. Remove hemostat providing traction and withdraw suture from temporalis muscle.

12. Rat is now ready to be positioned for middle cerebral artery occlusion procedure.

\subsubsection{Middle Cerebral Artery Occlusion}

The thromboembolic model described herein is advantageous in that it most closely mimics the clinical scenario. This is accomplished by administering a thrombus created from a donor rat into the middle cerebral artery, the vessel most frequently occluded in the human population. Additionally, proper placement can be verified through the use of the laser Doppler cerebral blood flow recording discussed previously. 
1. Place rat in supine position on homeothermic heating pad being careful not to disrupt laser Doppler probe that has been inserted previously for monitoring purposes.

2. Place a small pillow (see above) under head of rat such that anterior portion of head and neck are parallel to operating table. This is important as it brings anatomical structures of interest (carotid artery and its branches) more

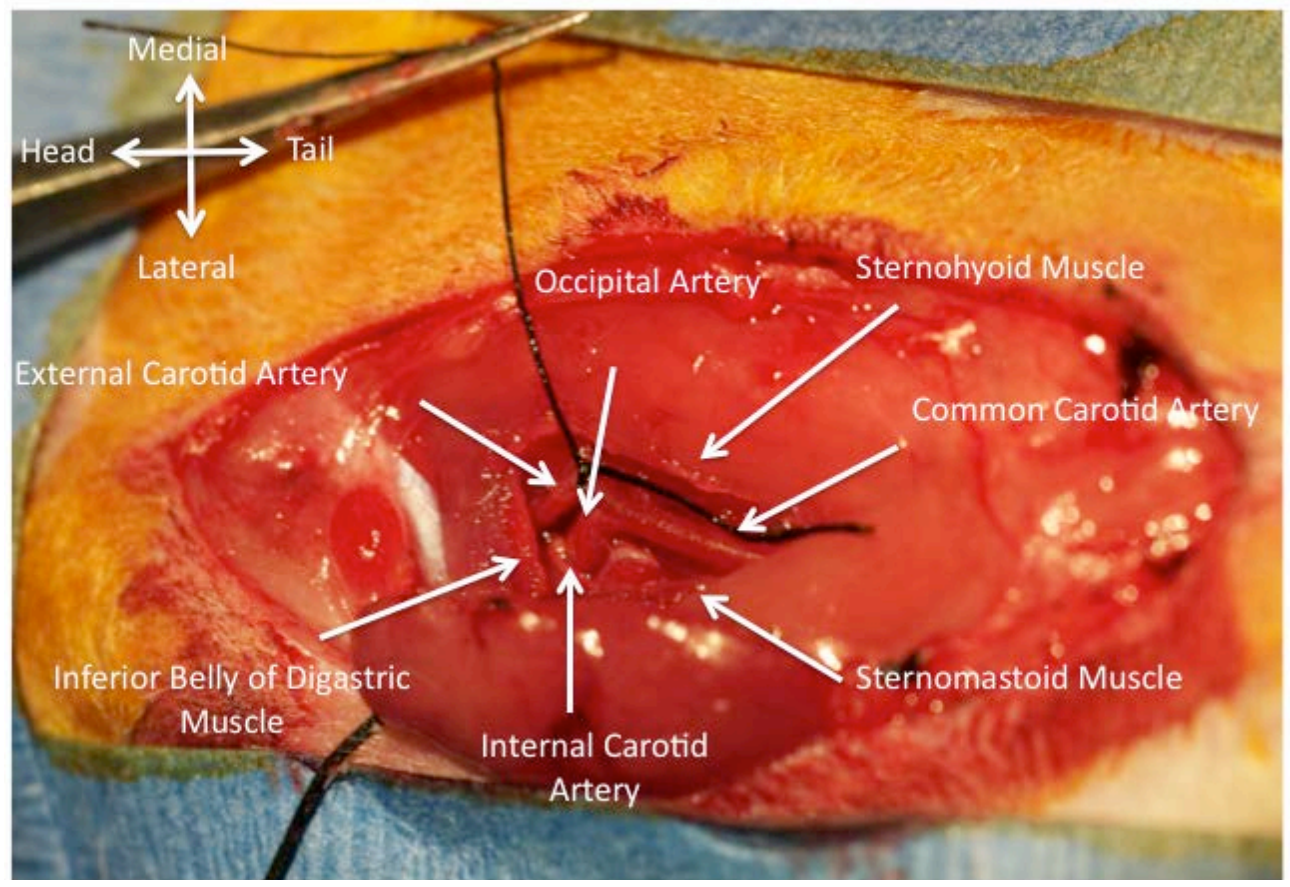

Figure 1.3. Vessels prepared for insertion of thrombus. The external carotid artery stump, seen ligated by the suture, is the site of insertion. This stump can be manipulated such that it is in line with the internal carotid artery, allowing for a straight shot from the ECA stump into the ICA and subsequently the MCA.

superficial. This allows increased access to the bifurcation of the carotid artery and visualization of the internal carotid artery.

3. Place forelimbs of rat in traction such that chest is fully exposed, enhancing visualization during dissection.

4. Using surgical scalpel, make a midline cervical incision running from near mandible to the sternum.

5. Dissect through superficial connective tissue to visualize mandibular glands and muscles overlying the trachea.

6. Focusing to right (the rat's right side) of the midline, a triangle formed by three different muscles should be apparent (Figure 3). The medial border of this triangle is created by the sternohyoid, the inferior lateral border by the sternomastoid, and the superior lateral by the inferior portion of the digastric.

7. Separate these muscles by dissecting through connective tissue between the muscles. Use care to avoid damaging muscles as this can result in unnecessary trauma and excessive bleeding.

8. Retract superior lateral border of triangle (inferior portion of digastric) by encircling muscle with 3-0 suture and providing traction using a hemostat. Common carotid artery should now be visible in center of the triangle.

9. Carefully isolate common carotid artery from surrounding connective tissue. 
10. Follow common carotid artery superiorly to its bifurcation into external and internal carotid arteries.

11. Carefully isolate external carotid artery, the more superficial of the branches from the common carotid artery. The other branch from bifurcation is the internal carotid artery.

12. Following external carotid artery cephalically until the superior thyroid artery (first anterior-oriented branch off of external carotid) comes into view (Figure 4). The superior thyroid artery may be attached underneath the superior angle of the triangle, between the digastric muscle and sternohyoid muscle.

13. Bipolar coagulate superior thyroid artery and cut in the center of bipolared portion, separating superior thyroid artery from external carotid artery.

14. Ligate external carotid artery near origin from common carotid artery. Use a single tie that can be untied easily as this is a temporary ligation.

15. Bipolar coagulate external carotid artery in the most distal region possible, above where the superior thyroid artery branched off.

16. Cut external carotid artery in middle of the bipolared segment, creating the external carotid artery stump at the bifurcation of the common carotid artery. This stump should move freely while attached to the common carotid artery at this point - if not, dissect more thoroughly such that the stump can be moved such that it is inline with the internal carotid artery.

17. Isolate internal carotid artery. While isolating, a branch originating near the ECA-ICA bifurcation becomes apparent called the occipital artery.

18. Isolate the portion of the occipital artery nearest the internal carotid artery and bipolar this vessel. Once the vessel is bipolared sufficiently, cut this vessel in the bipolared segment. The external carotid artery stump can be seen in Figure 3. Also visible in this figure is the common carotid artery and the internal carotid artery.

19. Finish isolating the internal carotid artery down to the next branch (pterygopalatine artery). Ensure that both the ICA and the pterygopalatine artery can be seen at this bifurcation (Figure 4) as it is essential that the tubing can be visualized in the proper vessel when inserted in subsequent steps.

20. Apply two microvascular clips - one on the common carotid artery and one on the internal carotid artery. These are to occlude blood flow temporarily while inserting the thrombus. A drop in CBF in the MCA territory, as measured with the laser Doppler monitoring system, should be observed at this point (Figure 5).

21. Mark the modified PE50 tubing prepared previously with a permanent marker at $18 \mathrm{~mm}$ from the modified end. This mark will allow for visualization through the artery as the clot is inserted in subsequent steps.

22. Draw the clot into the modified PE50 tubing using the micro-syringe. If the clot cannot be drawn into the modified tubing, wash the clot more in the saline until small enough to be drawn into the modified tubing (Note 6 \& 7).

23. Position the micro-syringe with attached modified PE50 tubing filled with the clot such that the modified tubing is in line with the external carotid artery stump. To do this, use modeling clay positioned in the groin of the rat as the mounting point for the micro-syringe and adjust this superiorly or inferiorly such that the modified tubing and clot are in the proper location. 


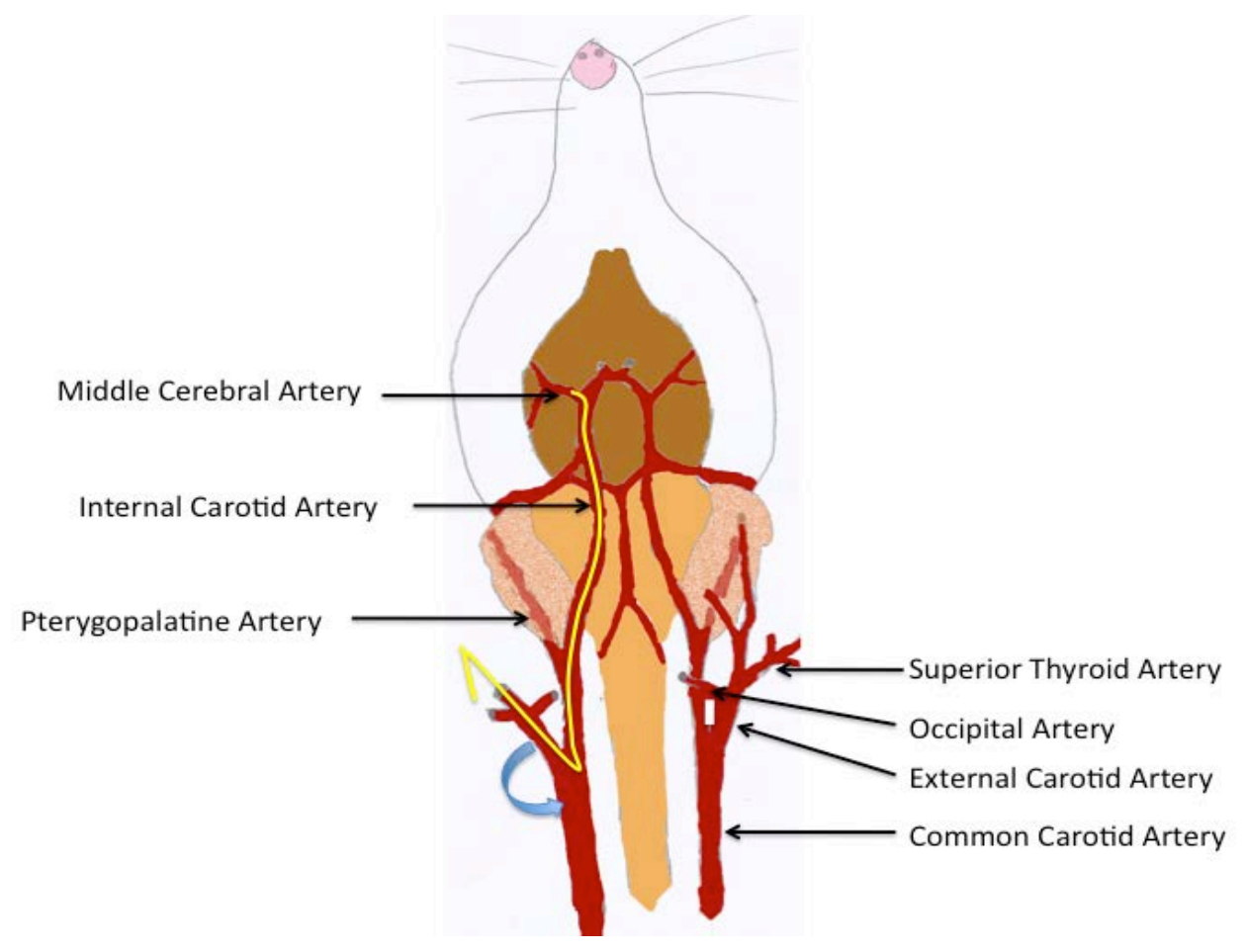

Figure 1.4. Diagramatic representation of vasculature in the neck and brain relevant for middle cerebral artery occlusion (MCAO). It is important to note the location of the superior thyroid artery and the occipital artery as these must be dissected and bipolared prior to thrombus insertion. When inserting the thrombus, ensure the modified tubing is placed from the external carotid artery stump into the internal carotid and then the middle

24. Cut the bipolared segment off of the external carotid artery stump so that the lumen can be visualized.

25. Loosen the suture placed around the external carotid artery and begin to insert the modified tubing containing the clot while bending the external carotid artery stump towards the syringe, inline with the internal carotid artery. Once the tubing is inserted beyond the suture, tighten the suture once again around the tubing.

26. Remove the micro-vascular clip from the internal carotid artery but leave the other micro-vascular clip on the common carotid artery at this time.

27. Continue inserting the modified tubing containing the clot into the internal carotid artery. Ensure that the tubing remains in the internal carotid artery and does not enter the pterygopalatine artery by observing the tubing inside the vessel.

28. When the $18 \mathrm{~mm}$ mark approaches the bifurcation, slow the insertion process and watch the laser Doppler monitor for changes in cerebral blood flow.

29. When the laser Doppler number drops, indicating blood flow to the MCA is disrupted, the tubing is in the correct place (Figure 5). Pull back approximately $1 \mathrm{~mm}$ on the modified tubing and watch the cerebral blood flow be restored, as indicated by the laser Doppler recording (Figure 5). This is a confirmatory step for ensuring no hemorrhage was caused.

30 . When the cerebral blood flow is restored, inject $5 \mu$ of saline to eject the blood clot from the modified tubing. 


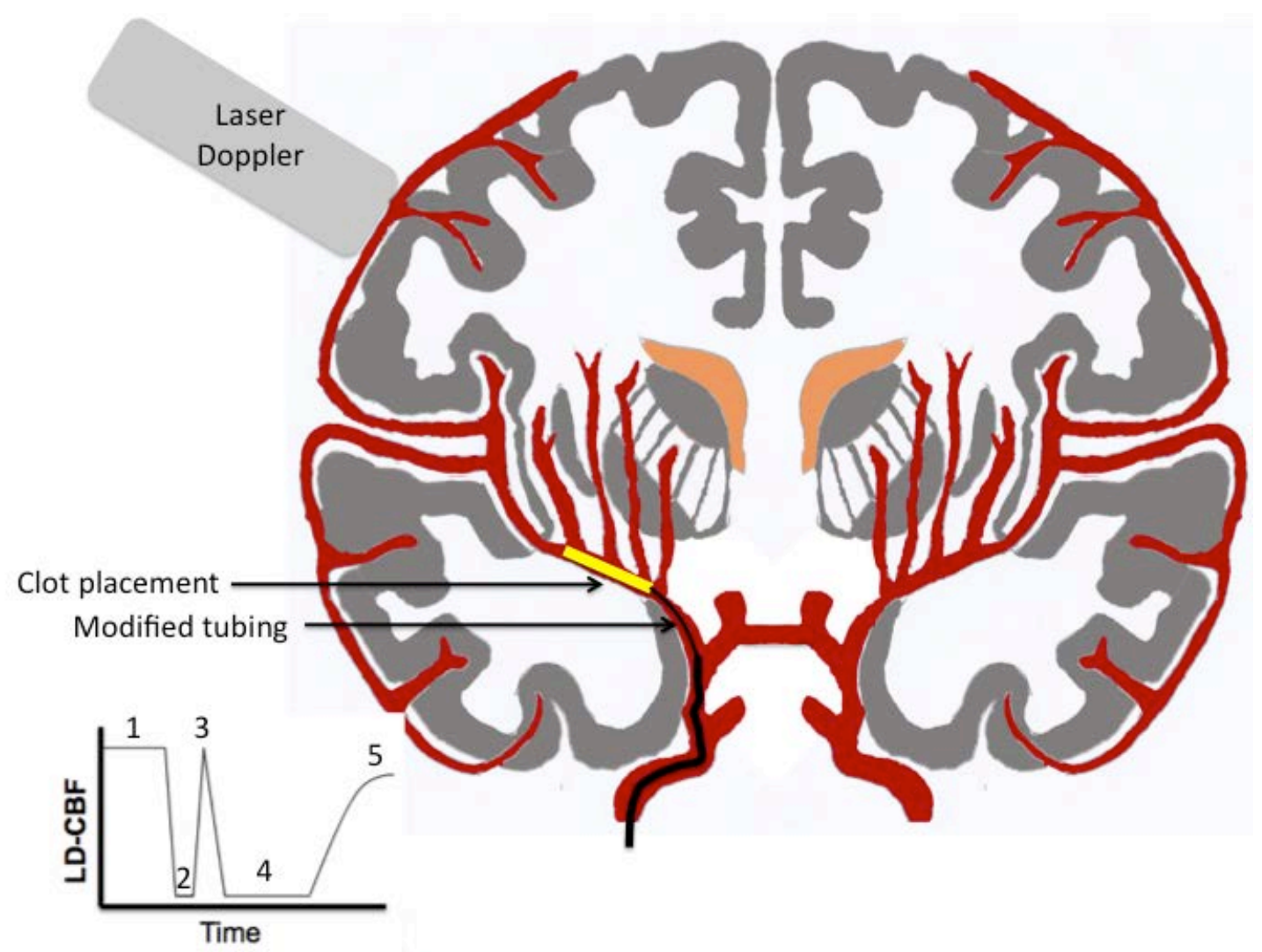

Figure 1.5. Laser Doppler recording of CBF during MCAO procedure. 1) Baseline CBF recording, 2) Occlusion with modified tubing prior to clot injection, 3) Verification by withdrawing catheter approximately $1 \mathrm{~mm}, 4$ ) Injection of clot and CBF reduction to $20 \%$ or less of baseline, 5) Reperfusion from tPA administration.

31. Once the clot is injected, the cerebral blood flow should drop again to a value similar to that seen previously when occluding with the tubing only (Figure 5).

32. After injecting the clot, wait 5 minutes to ensure clot stability prior to slowly removing the modified tubing.

33. When removing the modified tubing, a micro-vascular clip will need to be placed on the internal carotid artery prior to withdrawing the modified tubing entirely.

34. Once the clip is placed, remove the modified tubing the rest of the way and use the remaining suture around the external carotid artery stump to ligate the stump permanently. The stump can also be bipolar coagulated to ensure proper closure and prevent bleeding.

35. Remove both micro-vascular clips at this time. The CBF in the MCA territory should remain decreased at $20 \%$ or less of baseline (Figure 5 ).

36. Cover the exposed tissues with a saline-soaked gauze pad while the animal remains under anesthesia prior to closing the wound using a 3-0 suture.

\subsubsection{Achieving Reperfusion}


In this thromboembolic model, reperfusion is obtained via administration of tissue plasminogen activator, the same compound that is utilized clinically. This allows for the study of new therapeutics in conjunction with the presently approved agent - a combination therapy approach. Briefly, tissue plasminogen activator can be given intravenously via the same catheter used to administer saline in the femoral vein.

1. Follow the procedure for 'Creation of Thrombus', found above.

2. Isolate the femoral vein from the artery and nerve.

3. Using a 5-0 suture, ligate the distal end of the exposed femoral vein.

4. Using a 5-0 suture, prepare a loose ligation more proximally. This will be to hold the intravenous catheter in place but cannot be tightened until the catheter is inserted.

5. Insert the 22-gauge catheter proximal to the total ligation of the femoral vein but distal to the loose suture.

6. Secure the intravenous catheter with the previously prepared 5-0 suture.

7. Connect the intravenous catheter to a syringe pump loaded with saline.

8. Administer saline at a rate of $1 \mathrm{ml} /$ hour to maintain proper fluid balance.

9. At 2 hours after induction of ischemia by injecting the clot, administer tissue plasminogen activator via a syringe pump at a dose of $5 \mathrm{mg} / \mathrm{kg}$. A $30 \%$ bolus should be given and the remaining administered over a period of 30 minutes. This is done via the same intravenous catheter used for saline administration and is done simultaneously.

10. While administering tissue plasminogen activator, closely observe the rat for bleeding from open wounds and stop via bipolar.

11. Observe the cerebral blood flow measurement, as blood flow should be restored following tissue plasminogen activator administration (Figure 5).

12. Once complete, carefully remove the intravenous catheter and either suture or bipolar this open segment of the femoral vein.

13. Remove the laser Doppler probe and cranial bolt.

14. Suture all wounds using a 3-0 suture.

\subsubsection{Post-stroke Assessment}

Assessment of animals post-stroke in the past has largely focused on volumetric measurements and the ability of the compound being tested to alter infarct volume. In recent years the focus has begun to shift towards behavioral outcomes to increase clinical relevancy ${ }^{12}$. This shift has been largely predicated on the failure of compounds to translate from preclinical models to clinical trials. Despite the hundreds of clinical trials initiated for pharmacologic agents to treat ischemic stroke, tissue plasminogen activator remains the only FDA approved compound.

As such, we will discuss briefly both aspects of assessment post-stroke: functional and volumetric.

\section{Functional Assessment}

\subsection{Modified Neurological Severity Score (MNSS)}

The MNSS has long been utilized as a functional assessment for stroke as it is proposed to assess both motor and sensory deficits after ischemia ${ }^{13}$. This scale, while simple when applied to the young animal, is fraught with challenges when applied to the aged animal. The aged animal is fundamentally unique in that motor activity, even prior to injury, is greatly reduced as well as balance and sensory perception. Therefore, the 
utility of this scale in the aged population may simply be more of a qualifier for whether or not stroke occurred rather than an accurate assessment of stroke severity.

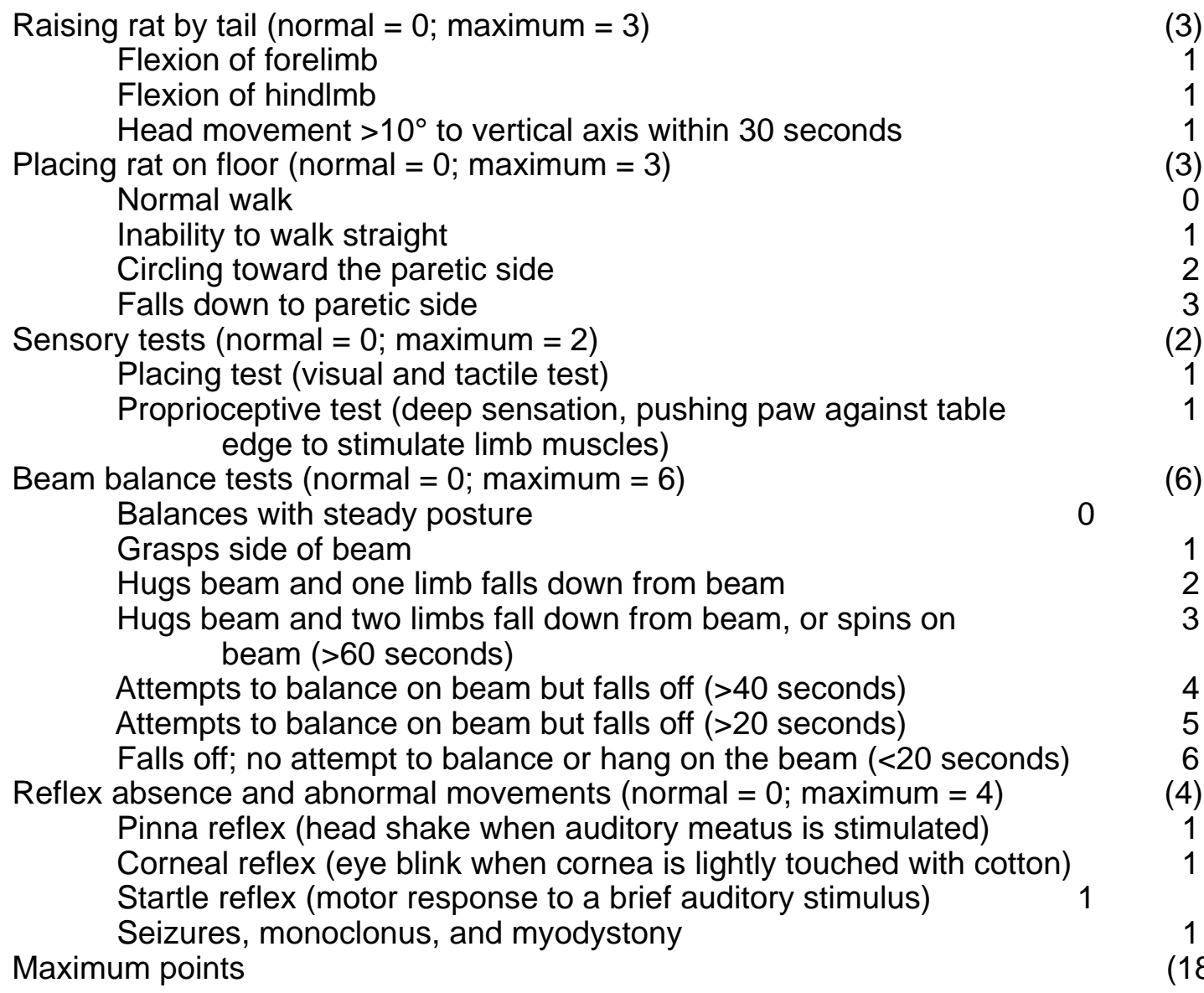

*Points are assessed for inability to perform specified task or for absence of reflex, resulting in more severely impaired animals receiving a higher score. The following ranges have been used to categorize injury severity: 1-6 mild injury, 7-12 moderate injury, and 13-18 severe injury.

\subsection{Sensorimotor Assessment}

Assessment of sensorimotor activity can be done as part of scoring systems such as the aforementioned MNSS but can also be done using other techniques such as open-field/ locomotor activity, rotarod, staircase test, the cylinder test, grip-strength, adhesive label test, and others ${ }^{14}$. While the intent of this work is not to review all functional tests, it is important to consider the variety of factors that impact the assessment of motor ability. Perhaps most important are the inherent differences seen from animal to animal at baseline. Therefore, it is important to either normalize to baseline or provide adequate training to meet a preset criterion. Additionally, age must be considered. Young adult animals behave differently than aged animals and have significantly more mobility before and after ischemia. Furthermore, alterations in the methodology used to induce MCAO produces highly variable results depending on occlusion type, location, duration, and strain of animals used.

\subsection{Cognitive Assessment}


Cognitive assessment of rodents post-stroke remains a widely debated topic, similar to other behavioral assessments. There is little agreement as to the most appropriate measures or expected results as past work has been highly variable. Some commonly utilized techniques include the Morris Water Maze, radial maze, passive avoidance, and active avoidance ${ }^{15}$. Each of these tests has been designed to assess some aspect of learning or memory but requires significant motor ability and baseline ability may vary from strain to strain and with age.

\section{Volumetric Assessment}

1. At time of sacrifice, anesthetize the rat as described previously.

2. Once anesthetized, place the rat in a supine position.

3. Make a horizontal incision, approximately 2 inches wide, in the upper abdomen of the rat.

4. Cut vertically at each end of this incision, cutting through the diaphragm and rib cage.

5. A flap has now been created consisting of the sternum and the anterior portion of the rib cage that can be retracted using a large hemostat.

6. Quickly remove any fascia remaining around the heart and aorta so that both structures can be visualized clearly.

7. Insert the peristaltic pump needle into the left ventricle and into the aorta. Visualize the needle in the aorta to confirm proper placement (Note 9).

8. Start the pump and set to a rate of approximately $10 \mathrm{~mL} / \mathrm{min}$ for 10 minutes using physiologic saline to perfuse the animal.

9. Cut the right atrium to allow for the blood to drain out of the animal. Over time the fluid should become clear and the tissues/organs should become pale (particularly liver and eyes), indicating a successful perfusion.

10. After 10 minutes (and $100 \mathrm{~mL}$ of saline), turn off the pump and remove the needle from the aorta.

11. Decapitate the animal.

12. Extract the brain, being careful not to damage the cortex.

13. Place the brain in the $2 \mathrm{~mm}$ brain block.

14. Place in $-80^{\circ} \mathrm{C}$ freezer for approximately 5 minutes.

15. Remove from freezer and slice using razor blades.

16. Place each slice in a separate well in a 12 well plate.

17. Add $2 \mathrm{~mL}$ of $2 \%$ TTC in PBS to each slice.

18. Place in incubator for 20 minutes.

19. Remove plate from incubator and scan into an image file (jpg, tif, etc).

20. Calculate lesion volume using previously described techniques (Photoshop, Image J, etc) ${ }^{16}$.

\subsubsection{NOTES}

1. If the instrument pouch is wet upon removal from the autoclave, it is likely that the door was not opened/cracked appropriately and/or the drying cycle did not run properly. Do NOT open the door to the maximum extent immediately as this allows colder room air to rush in and create condensation on the instruments.

2. We strongly recommend relying on CBF measurements throughout the procedure. CBF changes can be seen with each step, ranging from the initial brief reduction seen when placing microvascular clip on the CCA to those corresponding to duration of ischemic episode. 
3. Creating a high quality burr hole without damaging the brain is vital for confirming ischemia and reperfusion but is often challenging, even for the experienced surgeon. After initiating the hole with the scalpel and switching to the drill bit, be sure to frequently remove bone fragments to prevent forcing them into the brain. Also, try applying enough downward force to engage the drill bit in the cranium but then apply slight upward force to prevent the bit from entering the dura and brain. This upward force also assists in directing bone fragments out of the cranium rather than inside.

4. Our group, as well as others in the field, has experimented with different methods of recording cerebral blood flow such as extracranial measurements from the cranium surface. While it is possible to obtain readings from this position, we have not found consistent success at ensuring proper occlusion and reperfusion. One of the primary obstacles encountered is the ability to achieve preset criteria for assessing ischemia/reperfusion. We define ischemia as a drop of $80 \%$ or more from baseline laser Doppler recording and define reperfusion as a return to $80 \%$ or greater of the baseline value or visual absence of thrombus upon craniotomy.

5. Prior to inserting the cranial bolt, verify the position of the Doppler probe within the bolt. Depending on the supplies/equipment used, the probe may be longer than the bolt. In this case, it may be helpful to apply a moldable but somewhat rigid material (such as bone wax) to the probe to prevent the probe from extending beyond the bolt.

6. Washing the clot properly is one of the most critical steps of the process. Wash too little and reperfusion becomes unreliable, wash too much and spontaneous reperfusion occurs. The proper amount of washing requires practice and a trained eye - this can be acquired with attention to detail and some basic experience.

7. We have found significant variance in the PE 50 tubing used to form the clot when purchased from various manufacturers. In addition to slight variances in size, lumen texture is also variable with some appearing smoother while others are slightly ribbed. For this reason, we utilize the INTRAMEDIC listed in the materials section. We have found the most success with this tubing as it conforms to rigid tolerances and as such is consistent from reel to reel.

8. We have also found that the method of anesthesia used influences volume of the clot. Isoflurane may result in smaller diameter clots than using Ketaject. For this reason, it may be advantageous to utilize Ketaject for blood-draws from the donor animal.

9. When perfusing the animal, it may be desirable to use a dulled needle. Often a sharp needle may penetrate the aorta leading to poor quality perfusions. If you experience this problem, file the tip of the needle to create a dulled tip. 


\subsection{Role of Aging in Neural Injury}

One of the greatest shortcomings of prior work in neural injury is the failure to consider the effect of aging on pathophysiology and subsequent therapeutic targets. This is particularly alarming when considering subsets of neural injury such as ischemic stroke, a disease predominately affecting the elderly population. The following work explores the importance of aging as an experimental parameter in neural injury research using ischemic stroke as a fundamental example.

This work was published in the Journal of Aging Research.

Citation: Popa-Wagner A, Buga AM, Turner RC, Rosen CL, Toescu E (2012) Cerebrovascular Disorders: Role of Aging. Journal of Aging Research. Article ID 128146. doi: $10.1155 / 2012 / 128146$.

Therapeutic development for neurological diseases, whether cerebrovascular disease, vascular dementia, or Alzheimer's disease (AD), has been largely unsuccessful. Despite the apparent success of numerous pharmacologic agents in preclinical animal models, few have translated successfully from bench to bedside. As such, a vast need remains unmet and additional investigation is required.

Speculation concerning the reasons for this failed translation from bench-to-bedside often highlights either the lack of reproducibility or a poor choice of the outcome measure parameters. However, in most of the literature, the lack of clinical relevance of the animal model used often goes unnoticed. The role of age in neurologic disease development is well known, in fact, age is the greatest risk factor for development of $A D$ as well as stroke. In sharp contrast to this clinical reality, the vast majority of preclinical studies utilize young or young-adult animal models of neurologic disease, despite the suggestion of collaborative advisory groups such as the Stroke Therapy Academic and Industry Roundtable (STAIR).

The aging process affects various biological axes, from the status of the cardiovascular system to the status of the inflammatory responsiveness and finally to the changes in the ability of the neural systems to handle pathological insults and a significant reduction in the recovery capability. At one end of this spectrum of changes in cerebral cardiovascular status are the morphological changes in vasculature, manifested as large increases in the vascular path due to increased tortuosity of arterioles in the deep white matter ${ }^{17}$ and an age-associated decrease in capillary number and length, leading to a significant increase (25\%) of the inter-capillary distance in both the hippocampus and cortex ${ }^{18}$ and white matter ${ }^{19}$. Such processes would lead to tissular hypoxia that in young or adult brain would trigger an angiogenesis response, mediated by HIF-1 as a transcription factor for VEGF. However, in the aged brain, there is a reduction in the angiogenesis response, due to decreased responsiveness to HIF-1. Such ageassociated changes in the blood vessels architecture are even more relevant to the brain, because of the specifics of vascularization: the system of feeding arteries is situated near the surface of the brain, from where end arteries are penetrating through the gray and white matter in a network that terminates in a capillary bed but with very few shunts ${ }^{20}$.

As a result of such anatomical changes, there is little surprise that in the aged brain there is a significant reduction in the cerebral blood flow (CBF), affecting mainly the cortex, and more sparingly the subcortical regions, as revealed by a variety of imaging techniques, from positron-emission tomography (PET) and single-photon emission 
computed tomography (SPECT) to high-resolution, contrast-enhanced MRI and arterialspin labeling (ASL) ${ }^{21}$. However, as it has been pointed out ${ }^{22}$, CBF is aff ected not only by morphological changes, but it is also modulated by a variety of functional parameters such as perivascular innervation ${ }^{23}$, astroglial control of arteriolar constriction ${ }^{24}$, and autocrine endothelial signaling in response to rheostatic forces and neural environment 25

For the clinical category of cerebrovascular diseases, the stroke is, by a distance, the most significant entity, both in terms of prevalence and consequences. The stroke can result from either an occlusion of the vessel (ischemia), which can be either transient (e.g., the transient ischemic attack, TIA) or of longer duration; or from the rupture of a vessel, leading to regional hemorrhage, either within the cortical matter or in the dural cavities. While the biological and medical consequences of a stroke are significant at any age, the incidence and the severity of a stroke are significantly increased with age. The paper from Russo et al. provides a systematic review, looking at the first stroke incident and shows that the trend continues for the very old (older than 80 years old) ${ }^{26}$.

Several factors are likely contributors to this increased severity of stroke with age. One such factor is a metabolic decrease in the capacity of neural cells to counteract extreme stressors and/or neurotoxic challenges (decreased metabolic reserve) ${ }^{27}$ that appear in the penumbra region of a stroke, in which a combination of hypoxia and resulting decrease in ATP provisions will lead to the generation of a hyperexcitable environment, posing significant metabolic challenges.

Stroke in the very old is a very frequent condition, with an unfavorable outcome, and makes a relevant contribution to the social burden of stroke and may require more efficient dedicated stroke services ${ }^{26}$. Aged individuals recover less well from stroke, and rehabilitation aims at improving the physical and cognitive impairments and disabilities of patients with stroke. Therefore, studies on behavioral recuperation after stroke in aged animals are necessary. Various experimental settings have been used to assess the recovery of sensorimotor functions, spontaneous activity, and memory after ischemia in aged rats. Overall, the results indicate that aged rats have the capacity to recover behaviorally after cortical infarcts, albeit to a lesser extent than their young counterparts ${ }^{28,29}$. It should be kept in mind, however, that before stroke aged rats are already impaired as compared to young animals and show significantly decreased performance in some tests like spontaneous activity ${ }^{30}$. Accelerated glial reactivity to stroke in aged rats correlates with reduced functional recovery ${ }^{30}$ including Morris water maze ${ }^{31}$. Behavioral and histological effects of chronic antipsychotic and antidepressant drug treatment in aged rats with focal ischemic brain injury are discussed in the work by Zhao and colleagues ${ }^{31}$.

Another crucial component of the brain tissue response to stroke is the inflammatory response. As reviewed in the article by Shah and Di Napoli 32 the age-associated changes in the immune function determine that in the aged and individuals, the response to an acute stroke involves a more intense inflammatory reaction in the first phase of acute ischemia, involving cytokine activation and chemokine expression that lead to an early scar formation and fibrosis. In agreement with previous observations ${ }^{33}$, the article also points out the fact that the biology of the aged brain is different from that of the young brain, and this has significant implications for the current translational efforts to define effective therapies, since a large majority of interventional studies are performed on young and young-adult animals. 
Another important perspective in asserting new potential therapeutical interventions for stroke is to assess if the differences in responsiveness between young adult and old brains are due only to functional changes or if they involve more elaborate changes in protein expression. Recent detailed analyses of the genomics data from adult and aged animals indicate changes in the balance of the various systems and regulatory processes. Using categorized DNA arrays, we found inappropriate gene regulation in response to stroke both in the ipsilateral and the contralateral hemisphere of aged rats. The gene expression profile in the brains of post-stroke aged rats is indicative of increased cell death due to DNA damage and apoptosis, especially in the first week after stroke. Similarly, we reported persistent down-regulation of genes that are required for neurogenesis after stroke in aged rats ${ }^{34}$.

However, the category of cerebrovascular diseases is not restricted to the pathologies involving the large vessels. Cerebral small vessels disease (cSVD) is a group of pathological processes associated with established vascular risk factor such as hypertension, atherosclerosis, diabetes mellitus, and atrial fibrillation. The consequences of small vessel disease on the brain parenchyma are mainly lesions located in the subcortical structures such as lacunar infarcts, white matter lesions, large hemorrhages, and microbleeds. This compendium of tissue lesions has been compiled in the last decade or so by the ever-expanding use of more powerful and sensitive brain imaging technologies, rather than by a better understanding or direct assessment of actual small vessels territories. As a result, the cerebral small vessels diseases are now invoked in explaining a variety of clinical syndromes, and van Uden and collaborators discuss in their paper the possibility that such vascular pathology is underlining the clinical manifestations of the late onset depression in the aged people ${ }^{35}$. Even more daring, Teggi et al. propose that cSVD might also explain some forms of the Meniere disease ${ }^{36}$.

Another area of neuropathology in which small vessel disease has a well-established role that gathers ever increasing recognition in both the clinical and basic research world is that of being a leading cause of cognitive decline and functional loss in the elderly. Vascular dementia is a broad concept that has evolved slowly. While general anecdotal references linking putative cerebral vascular events with reductions in cognitive performance have existed for long time, it is the articles of Binswanger and Alzheimer around the turn of the 20th century describing in detail the various types of vascular lesions that could be encountered in human brains that set the field on a strong morphopathological footing, and it took another 70 years until Hachinski suggested that dementia of vascular origin was a consequence of multiple strokes ${ }^{37}$. Subsequently, the concept continued to evolve to include multiple mechanisms related to deficiencies in cerebral blood supply, including large vessel disease, small vessel pathology, consequences of cerebral hypoperfusion and hemorrhage.

While the risk factors for vascular diseases are relatively well established, much less consensus exists on the specific risk factors for vascular dementia, that is, apart, from the acknowledged role of increased age. Ongoing interest in cerebrovascular diseases research has provided data showing that Alzheimer's proteins and other factors may be involved in the pathogenesis of gradual ischemic brain injury. Thus, both focal and global brain ischemia in rodents produce a stereotyped pattern of selective neuronal degeneration, which is just the same as in Alzheimer's type dementia. As hypothesized in the article by Enciu et al. ${ }^{38}$, there is an overlap of events between chronic hypoxia and $A D$ on several levels, such as hypoxic-triggered cellular pathways, inflammatory environment, growth factor signaling, and calcium homeostasis. A reduction of CBF and a series of molecular events precede the major ischemic events in vascular cognitive 
impairment. Based on these subtle changes, intervention at early stages could prevent the full-blown development of dementia, which might represent a "point of no return" for the neurovascular units and neuronal networks with few chances for efficient treatment. In their extensive review, Enciu and her colleagues also discuss some of the molecular mechanisms that are triggered by the cerebrovascular diseases and that lead to overcognitive dysfunction and argue convincingly the case for an early intervention at the point at which the dysfunction of the neurovascular units is still potentially reversible 38 .

However, it is important to keep in mind that the genetic makeup is a hugely important factor, from the subtle alterations in the function of various proteins (e.g., ApoE4) to the overt cerebrovascular diseases with a genetic control, as the cerebral autosomal arteriopathy with subcortical infarcts and leukoencephalopathy (CADASIL) that involves mutations in Notch-3 protein. Equally important is the discovery that fundamental genetic changes may occur with aging, such as the CLOCK genes, as discussed by Thome et al. ${ }^{39}$. Taken together, aging may result in fundamental changes throughout the body, beginning at a gene level and progressing to protein expression or posttranslational modification level.

It is clear that the quest for improved therapeutics will require increased understanding of disease pathophysiology and, in particular, the changes induced by the aging process. Similarly, neurologic disease research has often maintained a "neurocentric" focus, in which the role of the neuron was emphasized. This is unlikely to result in successful development of therapeutics due to the intimate relationship evident between neurons, surrounding glia, and the neurovascular unit-in both health and disease. The effect of aging on astrocyte and microglial response to injury and how this process can be manipulated successfully for therapeutic development must be considered.

Similarly, preclinical studies of neurologic disease utilizing aged animals must incorporate functional outcome measures in addition to histological measures. Clinically, the primary assessment remains functional outcome and presence/absence of significant disability yet preclinical studies often emphasize histological outcomes such as infarct volume. The difficulty of identifying high fidelity functional studies in aged animals remains a vexing problem. 


\title{
1.5. Improving Preclinical Models of Ischemic Stroke
}

Research in neural injury, particularly ischemic stroke, has largely failed to advance proposed therapeutic agents successfully from bench-to-bedside. Reasons for this lack of success are primarily speculative in nature at this point but have frequently focused on the use of inadequate animal models, failure to address the role of age and comorbidities in disease pathophysiology, and the pursuit of hyperacute injury mechanisms rather than those more likely present at the time of patient presentation. In these works, we explore each of these topics to varying degrees in an effort to shed light upon what may be more promising directions for future investigations.

This work was published in part in Journal of Neurosurgery and the Yale Journal of Biology and Medicine.

\section{Citations:}

Turner RC, Dodson SC, Rosen CL, Huber JD (2013) The science of cerebral ischemia and neuroprotection: navigating past failure to future success. Journal of Neurosurgery 118(5): 1072-1085.

Lucke-Wold BP, Turner RC, Lucke-Wold AN, Rosen CL, Huber JD (2012) Age and the Metabolic Syndrome as Risk Factors for Ischemic Stroke: Improving Preclinical Models of Ischemic Stroke. Yale Journal of Biology and Medicine 85: 523-539.

\begin{abstract}
Ischemic stroke represents a leading cause of morbidity and mortality in the developed world. This disabling and sometimes fatal event puts an ever-increasing burden on the family members and medical professionals who care for stroke victims. Preclinical ischemic stroke research has predominantly utilized young adult, health animals, a clear discrepancy when considering the clinical population affected by stroke. A broad spectrum of risk factors such as age, obesity, diabetes, and hypertension has been associated with an increased stroke risk. The effect of these comorbidities on both stroke pathophysiology and outcome has not been emphasized and has been recognized as a shortcoming of preclinical studies. By addressing these conditions in experimental models of ischemic stroke, it may be possible to more accurately represent the clinical scenario and improve therapeutic translation from bench-tobedside. In this work, we review many of the risk factors associated with increased stroke risk, particularly as each risk factor relates to inflammation. Additionally, we explore potential animal models that could be utilized in identifying the contribution of these risk factors to stroke outcome. By investigating the risk factors for stroke and how these may alter stroke pathophysiology, the present discrepancies between preclinical studies and the clinical reality can be reconciled in an effort to improve therapeutic development and translation from bench-to-bedside.
\end{abstract}

\subsubsection{INTRODUCTION}

Stroke is the second largest contributor to mortality worldwide and the primary cause of disability among the elderly in Western Europe and the United States ${ }^{40,41}$. Among the various types of stroke, ischemic stroke is the most prominent and accounts for the most long-term disability ${ }^{42}$. This review will deal only with preclinical models of ischemic stroke. It is widely accepted that age is the greatest risk factor for stroke. The process of aging results in a large number of inflammatory changes. It is predicted that the number of 
people in the United States past the age of 65 will double within the next 30 years ${ }^{43}$. Thus, the need for new treatments and therapeutic options for stroke is a pressing concern as the population ages. Current available therapeutics are limited to thrombolytics such as recombinant tissue plasminogen activator ( $r$-tPA) and mechanical means of thrombolysis 44. Although r-tPA drastically improves patient outcome when used within the suggested time period, only a small percentage of presenting patients are candidates to receive $r$ tPA due to an extensive list of contraindications. As such, a large medical need remains unmet for the vast majority of patients afflicted with ischemic stroke.

As more than 100 potential therapeutic agents have progressed from pre-clinical studies with young animals to unsuccessful clinical trials, the need for improved pre- clinical models is clear ${ }^{45}$. These failures call into question the validity of the models being used to represent ischemic stroke. An area of specific concern is how the age and health of the animals used in these experiments replicates disease pathogenesis and pathophysiology observed in the clinic. Previously, stroke work was done principally with young adult, healthy animals despite the fact that the people most susceptible to stroke are older and are often overweight, diabetic, and hypertensive ${ }^{46}$. Recent recommendations from the Stroke Therapy Academic and Industry Roundtable (STAIR) encourage research to be performed with healthy, young adult, male animals first and then proceed to aged, diabetic, hypertensive, and female animals ${ }^{47}$. Currently, these recommendations have not been broadly implemented. In the following sections, physiologic changes associated with aging, obesity, diabetes, and hypertension will be examined for their roles in predisposition to stroke. It will be argued that more accurate models are necessary to improve the applicability of future research endeavors and to adhere to the suggestions made by STAIR. We identify potential models allowing for investigation of ischemic stroke in relation to a given comorbid condition and, in particular, identify pathophysiologic changes associated with each respective disease state that may represent therapeutic targets. By better understanding pathologic alterations induced by common comorbid conditions as related to ischemic stroke, it is our hope that therapeutics may more successfully move from preclinical studies to accepted treatments clinically.

We specifically focus on molecules associated with inflammatory processes and cascades, as not only does inflammation represent a potentially promising therapeutic target but is also heightened or altered in aging and various comorbid conditions associated with ischemic stroke. Pharmacologic agents targeting inflammation have been developed and, in the case of minocycline, appear promising for the treatment of ischemic stroke. Inflammation involves interplay between cellular and molecular components that results in brain tissue loss. Leukocytes, microglia, astrocytes, and neurons are but a few cell types involved in inflammation related to stroke. On the molecular side, a broad range of chemokines, cytokines, and adhesion molecules play a role in infarct development and subsequent deficits. The activity of these components can be altered by the presence of various disease states, aging, and lifestyle factors ${ }^{48}$. These factors have not been emphasized in most preclinical models of ischemic stroke and may represent a potential reason for the lack of successful therapeutic translation from bench-to-bedside. This review addresses the importance of considering other comorbid processes and how they relate to stroke.

\subsubsection{Aging and Stroke}

Age: The Greatest Risk Factor 
Age is the greatest risk factor for stroke yet is rarely considered in preclinical models 9 , 49. The aged brain is fundamentally different and responds to pathologic insults in a detrimental manner, resulting in increased infarct volume and worsened functional outcome, when compared to young-adult animals $9,50-52$. This is particularly evident when considering inflammatory processes and astrocyte activation $30,53-56$.

The aged brain is believed to exist in a state of chronic, low-grade inflammation that diminishes the ability of the aged brain to respond appropriately to pathologic stimuli, such as ischemia. Throughout normal aging, microglia, the primary immune cell of the brain, are primed following peripheral inflammation. This priming, characterized by morphologic changes and by cell-surface protein expression, leads to increased proinflammatory molecule release following additional pathologic insult 53 . While the release of pro-inflammatory results in a wide array of downstream effects beyond the scope of this review, the effect of aging on pro-inflammatory molecule expression has been well-documented 56 . Furthermore, work in an aged animal model has shown differences, when compared to young-adult animals, in regulation of the JAK2/STAT3 pathway ${ }^{51}$. Notably, young-adult and aged animals differ in inflammatory response across a variety of time-points post-stroke. Badan, et al showed a gradual activation of microglia and astrocytes in the young-adult animal, peaking 14 to 28 days after ischemia. Alternatively, aged animals exhibit rapid activation of these glial cells, peaking within the first week post-ischemia ${ }^{30}$. This is consistent with the findings of DiNapoli, et al in which morphologic differences in astrocytes and microglia were seen when comparing young-adult and aged animals after stroke ${ }^{51}$.

The mitigation of oxidative stress using free radical scavengers has proven successful in preclinical studies but failed to translate in clinical trials 57,58 . This may be related to methodology differences such as time of administration after stroke, type of stroke, delivery method, and others, or differences in downstream effects associated with age. An example of age-related effects was seen with apocynin, an inhibitor of the NOX2 isoform of NADPH oxidase, which has contrasting effects when used in young-adult or aged animals. Apocynin was shown to be neuroprotective in young-adult animals but worsened stroke outcome in aged animals 59 .

While the precise downstream consequences of these differences remain to be elucidated, it is clear that age and the aging process fundamentally alter the response to pathologic stimuli. To truly address the lack of translation from bench-to-bedside, and increase the clinical relevance of preclinical models, utilizing aged animals may prove fruitful. In the following sections, we attempt to briefly discuss implications of aging, how aging changes the response to an injurious event, and the benefits and limitations of aged animal models.

\section{The Aged Brain: Fundamentally Different}

Aging has been well documented to be associated with an overall dysregulation of the immune system hallmarked by a shift to- ward a pro-inflammatory condition 60-63. Age causes an increased secretion of tissue necrosis factor-alpha (TNF-a), a proinflammatory cytokine, and decreased inter- leukin-10 (IL-10), an anti-inflammatory cytokine ${ }^{61}$. These cytokines act on specific cells in the central nervous system. Numerous cell types throughout the body can release cytokines, and these cells can change with age. The concept of "inflamm-aging" (inflammation increasing with age) has been attributed to chronic macrophage stimulation ${ }^{60}$. Despite the mechanistic details 
being unclear, aging clearly results in inflammatory variations that are especially obvious in the brain. Some of these variations are due to genetics, lifestyle, and the envi ronment as seen in Figure 1. These alterations in inflammation are common to agerelated diseases and serve to prime the central nervous system. This often leads to an exaggerated response and worsened outcome following a systemic challenge ${ }^{61,62}$. Consistent with the link between age-related diseases and inflammatory changes are recent findings in humans and animals alike in which inflammation has been linked to cognitive impairment and altered brain structure and metabolism ${ }^{64}$. In fact, high levels of interleukin-6 (IL-6), a pro-inflammatory cytokine, have been described as exhibiting a strong correlation with morbidity and mortality in the aged population. Some discrepancy does exist as even "healthy" centenarians possess a highly elevated IL-6 level ${ }^{60}$. Cell-specific changes in the CNS mediate how the brain responds to and alters the immune system following injury. These cell-specific changes and the brain's global response to injury will be discussed below.

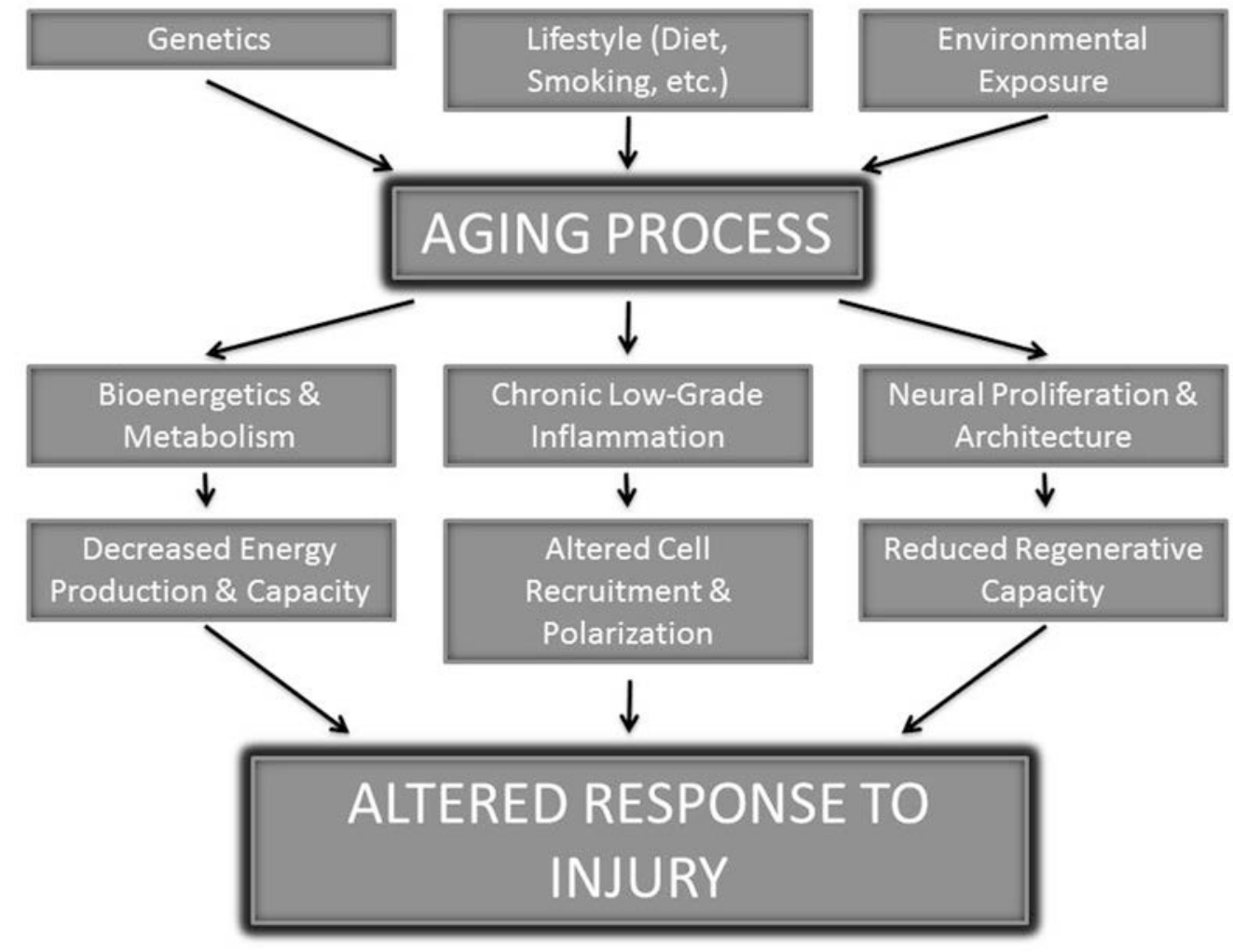

Figure 1.6. Aging and Stroke. A schematic diagram of the factors contributing to the aging process and how aging alters both the function and structure of the brain. These changes contribute to an altered and generally more deleterious response to injury when compared to the young adult brain.

Age and Cell-Specific Changes

Astrocytes 
With increased awareness of the role of glia in neurologic injury and the neurovascular unit, modulation of astrocytes and their associated functions have been identified as a potential target for ischemic stroke. Astrocytes are implicated in many functions associated with injury pathophysiology such as buffering of potassium ions and maintaining the integrity of the blood-brain barrier (BBB) ${ }^{65}$. Despite increased understanding of astrocyte functions pre- and post-injury, the effect of age on astrocyte function is not entirely clear. Inflammatory cytokines do, however, alter astrocyte ability to respond to injury. Recent studies have shown that astrocytes do express markers consistent with senescence in both aged humans and rodents. These markers include increases in glial fibrillary acidic protein (GFAP), cytokine release, and protein aggregates thought to induce cellular death ${ }^{66}$. Therefore, it is evident that astrocyte function changes with aging and may influence both normal homeostatic mechanisms as well as the response to injury. It will be important for future work to elucidate how agerelated inflammatory cytokines modify astrocytes.

\section{Endothelial Cells: A Source of Inflammatory Regulators}

Since stroke is a vascular disease, it is important to understand the effect of aging on the vasculature system. One of the primary components of the vasculature system is endothelial cells. Studies in aged rats that were designed to correspond to a 70- to 75year-old human have revealed a marked increase in pro-inflammatory cytokine expression. This increase in cytokines from endothelial cells may be responsible for altering vascular function and increasing permeability in the CNS. Key cytokines

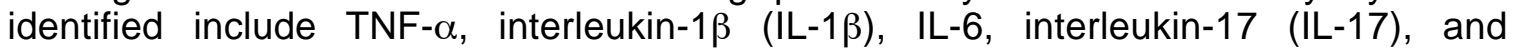
interleukin-6R $\alpha(\mathrm{IL}-6 \mathrm{R} \alpha)$. Aging was also associated with decreased expression of endothelial nitric oxide synthase (eNOS) and increased production of inducible nitric oxide synthase (iNOS), factors that clearly alter vascular function ${ }^{67}$.

\section{Microglial Cells}

Microglia, the resident immune cells of the central nervous system (CNS), undergo significant functional changes with aging ranging from altered iron storage to cytokine production and accumulation of lipofuscin 68. These age-related altercations are exemplified by morphologic changes and are likely involved in the transition of microglia from a neuroprotective phenotype in the young brain to a neurotoxic and destructive form in the aged brain. The destructive form of microglia is known to secrete increased amounts of IL-6 and TNF- $\alpha$ in the aged brain ${ }^{69,70}$. Interestingly, microglial senescence occurring during the aging process may mediate the transition from protective to deleterious effects. This transition is consistent with findings of senescent microglia in close proximity to degenerating neural cells ${ }^{63-69}$. Furthermore, recent work by Baker and colleagues demonstrated that increasing removal of senescent cells results in delayed acquisition of age-related disorders. Microglial senescence may perhaps be a promising therapeutic target for many neurodegenerative conditions ${ }^{71}$. Microglia in aged subjects possess altered surface markers such as major histocompatibility complex II (MHCII) and ED1. How exactly age-associated changes are induced in microglia remains to be elucidated, but it is clear that microglia in the aged brain are basally activated and respond differently to stimuli such as lipopolysaccharide (LPS) and Nogo B 63, 72-74.

Neuron-Microglia Interaction

One method of regulating microglial activation in the healthy, young adult brain is through neuron-microglia communication. Disruptions in this signaling mechanism may 
explain the age-associated shift of microglia from a protective to a destructive phenotype 75. Neurons can communicate and regulate microglia in multiple ways with ligandreceptor binding via the CD200-CD200R and CX3CL1-CX3CR1 pathways. CD 200, when bound to its receptor (CD200R), is commonly expressed on cells of the myeloid lineage such as microglia. This binding results in microglia being maintained in the quiescent state. As previous works have demonstrated, targeting CD200-CD200R interaction represents a potential therapeutic target to modulate microglial activation with age. In fact, Cox and colleagues have demonstrated that administration of CD200 fusion protein (CD200fc) restores microglia from a quiescent state and results in improved long-term potentiation (LTP) in aged animals ${ }^{76}$. Fractalkine (CX3CL1), a protein expressed by neurons, has been identified as playing a role in neuroimmune modulation by binding to the corresponding receptor, CX3CR1, on microglia. Consistent with the shift to a proinflammatory state seen in the aged brain, fractalkine levels are reduced in the aged rat hippocampus as early as 12 months of age. By correcting this fractalkine deficiency exogenously, hippocampal progenitor cell proliferation and neurogenesis is largely restored. This further illustrates the ability to modulate inflammation for neurologic benefit ${ }^{77}$.

\section{Global Changes in Neural Proliferation and Architecture}

The aged brain exhibits decreased neurogenesis in the subventricular zone (SVZ) and subgranular layer of the hippocampus in comparison to young adult animals. Proteins such as ubiquitin and GFAP are altered in the aged brain and may play a role in ageassociated effects 78,79 . It is currently unknown whether diminished regenerative capabilities are the product of stem cell impairment or, rather, changes in the surrounding environment due to age ${ }^{29}$. Besides changes in neurogenesis, the structure of neuron spines is altered as well. The aged brain is characterized by a loss up to nearly 50 percent of thin spines. No change in mushroom or stubby spine quantity has been noted ${ }^{80}$. Experiments assessing changes in the hypothalamus have gone as far as implicating the G protein-coupled receptor and cytoskeletal-associated protein, GIT2, as a critical regulator of the aging process ${ }^{81}$.

Responding to Injury: Role of Brain Aging

Age not only affects risk, but also has a profound impact on recovery ${ }^{29}$. Following ischemic injury, for example, aged animals exhibit a rapid development of the glial scar and an increased release of associated signaling molecules. The rapid progression demonstrates a dysregulated cellular and inflammatory response ${ }^{33}$. This altered response is likely a product of physiologic differences prestrike and also an associated increase in oxidative stress. The inflammation predisposes to a more deleterious response after injury $77,82,83$. The contribution of each of these factors and the role aging plays in the pathophysiologic differences is expanded upon below.

\section{Disruption of the Blood-Brain Barrier}

Aging has been associated with diminished BBB integrity following ischemic stroke ${ }^{84,85}$. The precise mechanism leading to this altered permeability is unclear, but an increase in matrix metalloproteinase-9 (MMP-9) has been associated with increased permeability. Similarly, claudin-5, a protein integral in BBB structure, is decreased in the aged brain following injury ${ }^{85}$. A disrupted BBB allows an influx of inflammatory cells that are responsible for most of the damage seen following stroke. Modulating BBB integrity 
therefore represents a promising therapeutic target because BBB disruption precedes neuronal damage and often correlates with the extent of injury ${ }^{84}$.

\section{Cell Survival and Degradation}

The altered response to injury exhibited by the aged brain results in diminished cell survival and the potential for changes in other key cellular processes such as autophagy. Work in various neural injury models utilizing aged rodents has demonstrated an increase in apoptotic cell death attributed to inflammatory processes and heightened oxidative stress ${ }^{86,87}$. Besides apoptosis, cellular autophagy represents another pathway for degradation. The pathway remains under investigation with respect to aging and deleterious injuries. It might represent yet another potential therapeutic target ${ }^{88}$.

\section{Inflammation}

Following injury, inflammatory responses differ between young adult and aged subjects. In ischemic stroke, suppressor of cytokine signaling 3 (SOCS3) is elevated in aged animals in the subacute period. It mitigates the effects of an elevated phosphorylated signal transducer and activator of transcription 3 (pSTAT3) ${ }^{51}$. This is consistent with other reports in aged animals. On the other hand, microglia are activated more quickly following ischemic stroke and contribute to the release of deleterious cytokines and reactive oxygen species ${ }^{33}$. Other studies of inflammatory diseases utilized young and aged animals to investigate microglial phenotype and demonstrated age-dependent differences. Specifically, microglia expressed a pro-inflammatory phenotype characterized by ED1 and IL-1 $\beta$ in older rats but not young adult rats in an adjuvant arthritis model ${ }^{89}$. IL-1 $\beta$ is involved in cell proliferation, differentiation, and apoptosis.

\section{Oxidative Stress}

Oxidative stress has long been recognized as a potential contribution to the aging process and associated pathologies, but the precise origin of oxidative stress has not been entirely clear. In addition to the changes in metabolism and energy production discussed previously, recent studies demonstrate an association between aging and NADPH oxidase (NOX). NOX is a key enzyme in producing reactive oxygen species. NOXs and their associated subunits appear to vary with the aging process. Similarly, lifestyle choices such as diet may influence the production of reactive oxygen species in the aged population ${ }^{90}$. A proposed neuroprotective agent apocynin, a NOX2 inhibitor, demonstrates contrasting effects in young adult and aged animals. While protective in young adult animals, apocynin worsened outcome from ischemic stroke in aged animals. This further proves the importance of utilizing aged animals in preclinical studies ${ }^{59}$.

\section{Preclinical Models of Aging}

Assessing the role of aging in preclinical models of ischemic stroke has been accomplished using both in vitro and in vivo models. While neither model system has resulted in the successful translation of therapeutics from bench-to-bedside, preclinical studies using aged rats have demonstrated findings in opposition to similar studies in young adult animals. Aging is one of many components that may lead to improved models.

The primary in vivo models for studying the effects of the aging process on ischemic stroke employ aged rats. These studies generally report the use of animals as disparate 
as 12 to 28 months old. However, female rats typically undergo reproductive senescence around 9 months of age. Therefore, 9 months of age can been equated to a period similar to the late 40 s or early 50 s in humans. Studying animals around 18 months of age may equate to a similar period in humans of $75+$ years old. In humans, this is the period of greatest stroke risk. Utilizing aged animals, 18 to 20 months of age, typically requires the establishment of an aging colony either by the investigator or the animal supplier.

An alternative to aging animals in the traditional fashion described above is made possible by the development and selection of Senescence-Accelerated Mouse (SAM) strains. These mice originate from inbred strains suffering from early onset of agerelated diseases and demonstrate other pathologic features consistent with aging such as dysregulation of the immune system. The SAMP10 strain in particular experiences both histologic (loss of spines, synapses, and neurons) and functional (impaired learning and memory, depressive-like behavior) changes. Notably, this strain exhibits many of the pro-inflammatory characteristics observed in the aged brain of both humans and rodents.

\section{Limitations}

Despite the potential for clear benefit not only in investigation of disease pathophysiology but also therapeutic development, models of aging have some drawbacks that must be considered. For example, due to per diem costs for housing and caring for animals, aging can be prohibitively expensive for many groups. Despite the expense, research with this model has been conducted successfully by groups in both North America and Europe. Conversely, SAMs may represent a quicker and more costefficient method of studying the aged brain, but the question of how well SAMs correlate with normal aging remains to be answered. It will be important for future work to investigate how closely SAMs replicate the process of aging.

\subsubsection{THE METABOLIC SYNDROME AND STROKE}

\section{Obesity: Inflammatory Mechanisms Driving the Epidemic}

The number of overweight and obese people in the United States has doubled within the past 30 years ${ }^{91}$. Obesity dramatically increases the risk for stroke, and several groups have proposed mechanisms to explain this phenomenon. The first mechanism is a decrease of the cytokine called adiponectin. Low adiponectin levels can cause an increase in inflammation, insulin resistance, and vascular degradation ${ }^{92}$. Savopoulos and colleagues showed that the cytokine resistin is altered with obesity. Resistin causes endothelial dysfunction by augmenting the release of endothelin-1 93 . Endothelin-1 constricts blood vessels and links obesity to hypertension. Still further, the Hishinuma 


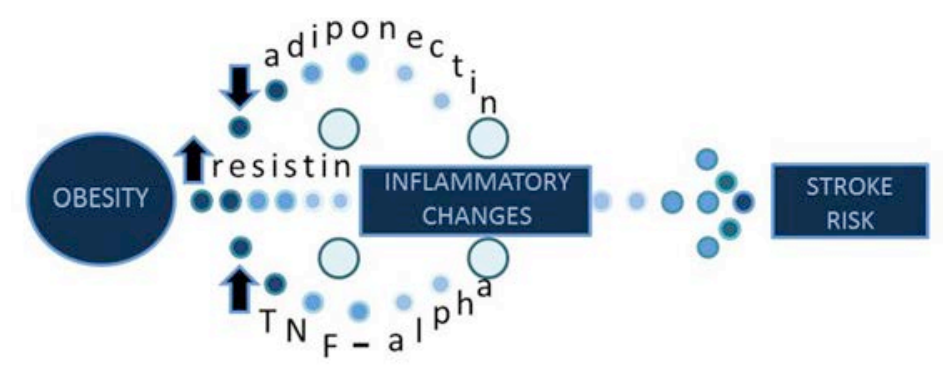

Figure 1.7. Obesity and Stroke. Obesity causes a decrease in adiponectin and an increase in resistin and TNF-alpha. These molecular changes predispose the body to increased inflammation. The inflammation leads to increased stroke risk. has been reported during vascular surgery. Obesity decreases the risk of post-operative stroke compared to the risk seen in non-obese individuals ${ }^{95}$. Further work has shown that even though obesity elevates the risk for stroke in the general population, it increases survival rates following stroke. Two prevailing theories about this paradox are an excess nutrient reserve available in obese and overweight individuals following stroke as well as an upregulation of TNF- $\alpha$ receptors in adipose tissue following infarct ${ }^{96}$. The activation of TNF- $\alpha$ receptors might increase the likelihood of stroke in obese individuals but it also provides greater neuroprotection following stroke. This occurs because TNF- $\alpha$ is known to activate apoptotic pathways through tissue necrosis factor receptor 1 (TNFR1) and neuroprotective pathways through tissue necrosis factor receptor 2 (TNFR2) ${ }^{97}$.

\section{Animal Models of Obesity}

In order to elucidate the role of obesity in stroke infarct damage, researchers have sought to develop appropriate models of obesity. Two primary forms of obesity exist in the general public. The first is uncontrollable genetic disorders such as leptin insensitivity and the second is obesity caused by poor diets. A model of the genetic form of obesity is the obese Zucker rat. Zucker rats are insensitive to satiety signals relayed to the hypothalamus ventromedial nucleus and also have deficient leptin receptors ${ }^{98}$. The model has been used to gather important data regarding the release of vasodilator and vasoconstrictor hormones associated with stroke development ${ }^{99}$. A model for the dietinduced obesity uses high fat diets in rats. Rats are fed these high-fat diets from 3 weeks of development onward. After these animals become obese, transient middle cerebral artery occlusion (tMCAO) is induced through a fibrin clot. Infarct damage is increased in these animals compared with non-obese controls ${ }^{100}$. This elevated level of damage is due to neurovascular matrix degradation and BBB disruption mediated by increases in MMP-9 101. Furthermore, matrix metalloproteinase 2 (MMP-2) is upregulated and causes degradation of type IV collagen, which is then replaced by inappropriate collagen type I deposits ${ }^{102}$. Since obesity is associated with many vascular disorders, it will be important in the future to investigate how other stroke risk factors such as age, diabetes, and hypertension interact with obesity.

Diabetes: A Growing International Problem 
Diabetes mellitus type 2 has become the most common serious metabolic disorder not only in the United States but across the world ${ }^{103}$. One research group estimates that 171 million people across the globe were inflicted with diabetes mellitus in the year 2000 and that number is projected to double by $2030{ }^{104}$. The most recognizable aspect of type 2 diabetes mellitus is the uncontrolled levels of hyperglycemia. Hyperglycemia can stimulate the formation of advanced glycosylated end-products (AGEs). In the clinical setting, diabetes mellitus is diagnosed as a fasting plasma-glucose of $110 \mathrm{mg} / \mathrm{dL}$ or more, or a random plasma-glucose of $200 \mathrm{mg} / \mathrm{dL}$ or more ${ }^{105}$. The pathophysiology of diabetes mellitus, however, is more complex than a simple elevation in serum glucose levels. Clinical manifestations also include glycosuria, polydipsia, polyuria, and renal failure from AGEs. Perhaps the most interesting aspect of the disease is the starvation of body tissues despite the fact that glucose remains abundant in the blood stream. This phenomenon is due to peripheral cells being either completely or partially resistant to the effects of insulin ${ }^{105}$. The body shifts from primarily receiving energy from glucose to receiving energy from lipids. Consequentially, this leads to ketone buildup in the body and a lowered $\mathrm{pH}$ level ${ }^{103}$.

\section{Causes of Diabetes: An Unknown Conglomeration of Many Factors}

A few theories have been proposed regarding the causes of diabetes. In reality, little is known about the causes, and like many other diseases, it is likely multifactorial. Many clinicians point to certain habits, such as sedentary lifestyle and high calorie and fat intake, as the main culprit of diabetes ${ }^{103}$. Interventions focusing on weight loss, diet modifications, and regular physical exercise of at least 150 minutes per week reduce the incidence of diabetes in at-risk patients. These activities also reduce hemoglobin A1C levels in type 2 diabetics ${ }^{106}$. Genetic links have likewise been recognized. Researchers discovered at least 36 genes associated with diabetes ${ }^{107}$. A high concordance level among identical twinsshows that type II diabetes is heritable ${ }^{108}$.

\section{Diabetes: Role in Chronic Inflammation and Ischemic Stroke Risk/Outcome}

Additionally, many groups began to focus on the influence of inflammation and innate immunity in diabetes. It is uncertain at this point if inflammation occurs prior to or after the onset of diabetes. Specific cytokines such as TNF- $\alpha$, IL-6, and C-reactive protein (CRP) are elevated in type 2 diabetic patients in comparison to non-diabetic controls ${ }^{109}$. TNF- $\alpha$ and IL- 6 cause generalized systemic responses such as fever and increased vascular permeability while CRP is a key player in complement-mediated immunity. $\mathrm{CRP}$ is the foundational block of the classical pathway. CQ binds to the pathogen, and $\mathrm{CR}$ and CS cleave C4 and C2 to make the convertase C4bC2a. The convertase then marks the pathogen for phagocytosis by cleaving $\mathrm{C} 4$ to $\mathrm{C} 4 \mathrm{~b}$ and $\mathrm{C} 4 \mathrm{a}$, which allows $\mathrm{C} 4 \mathrm{~b}$ to become membrane bound. Another innate immune response is driven by toll-like receptors (TLR). TLRs are activated by LPS or lipoteichoic acid on cell membranes or by DNA or RNA in endosomes. TLRs trigger nucleus specific changes that often lead to increased release of acute phase reactants ${ }^{110}$. These acute phase reactants are beneficial in situations in which the body's immune system is compromised with an infection or after an acute trauma. Over a longer period of time, these reactants become destructive to the systemic vascular system and cause damage to tissues throughout the body ${ }^{109}$. This destruction of the vascular system contributes to other diseases and injuries such as hypertension, cerebrovascular diseases, renal disease, and ischemic stroke 111,112 . The damage is mediated through numerous pro-inflammatory pathways. 
The signaling produces enhanced oxidative stress and the toxic build-up of degraded cells, proteins, and molecules ${ }^{112}$.

Although the role of inflammation in diabetes is still under investigation, it is important to consider which pathways may be targeted therapeutically. For example, TNF- $\alpha$ induces pathways that lead to increased insulin resistance throughout the body ${ }^{113}$. Insulin resistance is a strong risk factor for ischemic stroke ${ }^{112}$. Targeting TNF- $\alpha$ pathways may therefore be useful for preventing strokes in diabetic patients. Ultimately, any inflammatory trauma to the vascular system will have a large effect on the vasculature of the brain. If the inflammatory changes of diabetes can be controlled, it might greatly reduce the incidence of stroke in this population.

\section{Diabetes in Animal Models}

Many well-established rat models are used in type 2 diabetes research. This section will address three of the most prominent models as mentioned in Figure 1.8. The Israeli sand rat model focuses on the dietary factors of diabetes mellitus. As mentioned previously, an increased caloric intake can intensify the occurrence of diabetes mellitus type 2 . When the normal vegetarian diet is changed to a specific high-calorie laboratory diet, the rats eventually develop obesity, glucose intolerance, and hyperinsulinemia ${ }^{114}$. With time, the rat's intact pancreatic islet cells begin to degrade and die, thus leading to overt diabetes 115, 116. Essentially, this model relies on nutritional means to develop diabetes mellitus type 2 in the animals. The model is beneficial because it mimics the excessive calorie and glucose levels found in the human population. One limitation is that it does not address the genetic factors of diabetes. Many genetic factors have been identified in the human population and lead to activation of different pathways. These pathways present different therapeutic intervention opportunities. Therefore, genetic factors of diabetes are essential in the development of a proper diabetic model. 


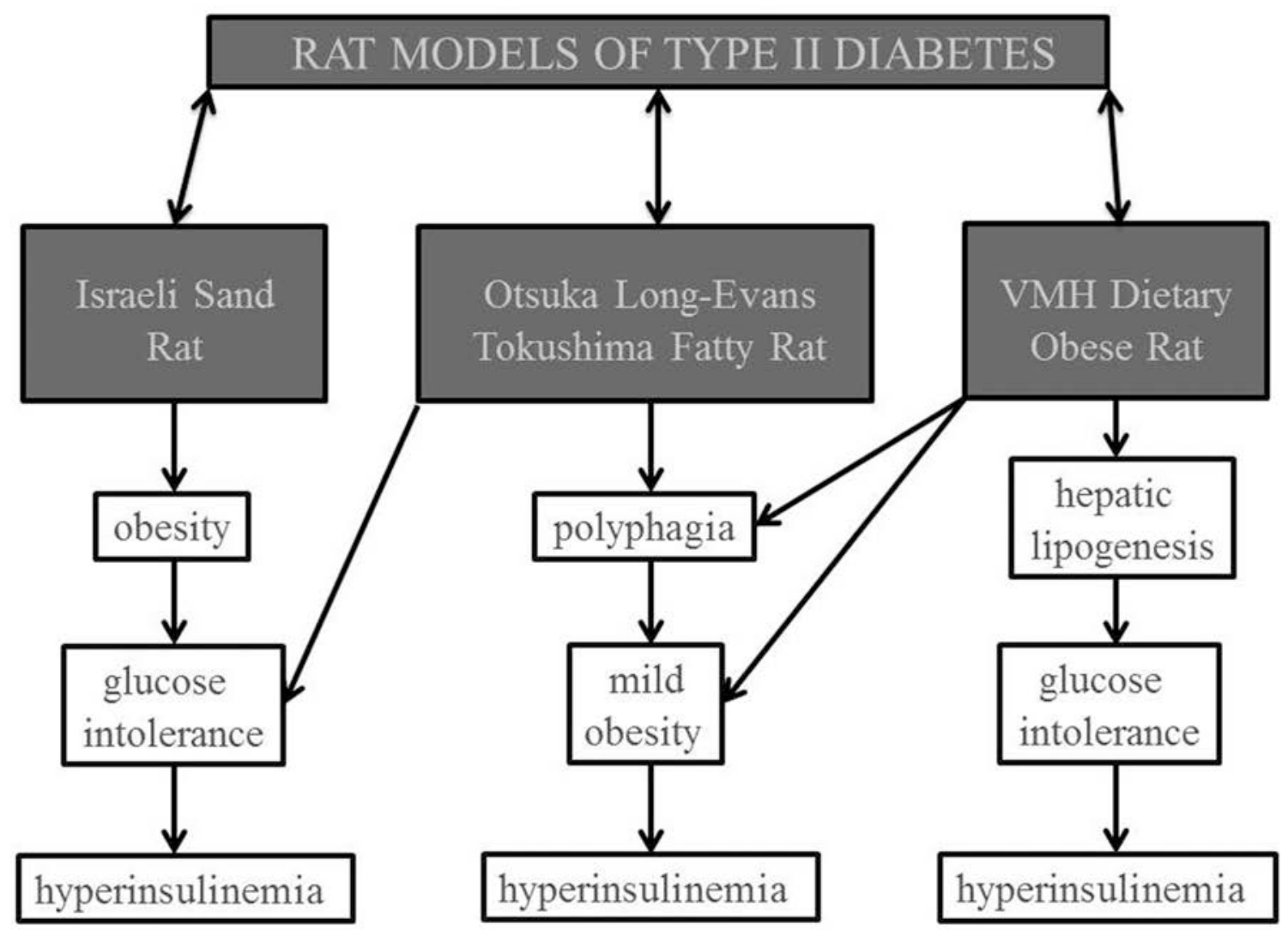

Figure 1.8. Rat Models of Diabetes Type II. Three well-known models of diabetes include the Israeli Sand Rat, Otsuka Long-Evans Tokushima Fatty Rat, and Ventromedial Hypothalamus Lesion (VMH) Dietary Obese Rat. Each of these models represents different characteristics of diabetes such as obesity, glucose intolerance, hyperinsulinemia, polyphagia, and hepatic lipogenesis.

The Otsuka Long-Evans Tokushima fatty (OLETF) rat is one example of a diabetic model that incorporates genetic factors. This rat model is developed by selective breeding from an outbred rat colony of Long-Evan rats. Although selective breeding is used, it is important to note that this is not a transgenic population. At 16 weeks of age, the OLETF rats' differentiating characteristics are thoroughly developed. This rat model demonstrates the following physical manifestations: glucose intolerance, hyperinsulinemia, mild obesity, and polyphagia ${ }^{115}$. The combination of polyphagia, obesity, and impaired glucose intolerance is a more accurate syndrome of the Western diabetic population. The polyphagia is assumed to be partially explained by a null allele coding for the cholecystokinin A receptor ${ }^{114}$. A problem with this model is that the diabetic characteristics are induced by breeding instead of the actual pathophysiology of diabetes mellitus. Using such models explains why current treatments of diabetes mellitus focus only on eliminating physical manifestations of diabetes instead of treating the underlying cause of the disease.

Another interesting model combines the use of a lesion in the ventromedial hypothalamus region of the brain and a high-fat/highsucrose diet. This is known as the ventromedial hypothalamus lesion $(\mathrm{VMH})$ dietary obese rat model ${ }^{117}$. The lesion itself is thought to cause the development of hyperphagia, obesity, decreased glucose 
metabolism, defective regulation of insulin, and increased hepatic lipogenesis ${ }^{115}$. As already discussed, the high-fat and high-sucrose diet is a key factor in exacerbating the lesion's effects. Within 3 weeks, these rats are found to have fasting hyperinsulinemia, hypertriglyceridemia, and impaired glucose tolerance ${ }^{117}$. Although this model is beneficial in studying the effects of the lesion and the diet on diabetes, it again does not accurately portray the entire pathophysiology of diabetes. Some diabetic pathways are highlighted while others are completely ignored. It is clear that these models do not accurately represent the pathophysiology of diabetes. A more comprehensive model will be necessary to elucidate the mechanisms of diabetes and how these altered pathways increase risk for stroke. Fan and colleagues studied stroke in a streptozotocin diabetic rat model. The model they used is limited because it represents type I diabetes mellitus and hemorrhagic stroke instead of the more common type II diabetes and ischemic stroke. Furthermore, this study focused only on tPA activity instead of investigating other mechanisms and pathways ${ }^{118}$.

Hypertension and Stroke: Increasing Prevalence in the United States

Two broad classifications of hypertension include primary hypertension and secondary hypertension. Primary hypertension is not caused by an independent disease, such as athersclerosis, but it can lead to higher risk for vascular and heart problems and predispose individuals to disease. The prevalence of primary hypertension is increasing in the United States due to the rise of obesity rates ${ }^{119}$. Three subcategories for primary hypertension are systolic and diastolic hypertension (SDH), isolated systolic hypertension (ISH), and isolated diastolic hypertension (IDH). SDH and ISH are risk factors for ischemic stroke as shown in Figure $1.9^{120}$.

\section{Hypertension as a Contributing Factor in Stroke Outcome: An Inflammatory Process}

Hypertension causes an increase in cell adhesion molecules, vascular adhesion molecules, and selectins. Subsequently, these molecules induce inflammatory responses by increasing the affinity of binding between immune cells and damaged tissue. The molecules also increase the build-up of atherosclerotic plaques in blood vessels through similar binding mechanisms. If a cerebral vessel becomes completely occluded, an ischemic event ensues leading to a cascade of inflammatory responses and still further immune cell binding ${ }^{121}$. Many of these responses are time-specific and depend on precise pathway activation. For example, Interleukin- 6 (IL-6) and MMP-9 can paradoxically lead to either neuroprotection or neurotoxicity depending on the specific period of observation ${ }^{122}$. IL-6 also causes a systemic fever useful for disrupting pathogen viability. It is therefore necessary to use models that relate well with human pathology and to observe changes at multiple time points post-ischemia.

\section{Hypertension in Animal Models}

Since hypertension is the largest factor in predicting stroke severity, it is essential that models of stroke incorporate the issue of hypertension ${ }^{123}$. It was proposed that 9 percent to 16 percent of ischemic strokes could be avoided if hypertension were kept under control in the general population 124. A model that addresses the issue of hypertension and stroke is known as the spontaneous hypertensive rats-stroke prone (SHR-SP). These rats have spontaneous increases in blood pressure, and upward of 80 percent develop stroke when placed on normal diets ${ }^{125}$. Not surprisingly, some groups have found that diets high in sodium chloride accelerate the time to first infarct in these animals ${ }^{126}$. A primary reason why this model works well is that it allows researchers to 
investigate how inflammation changes from a baseline state of hypertension to a postischemic state. Inflammation triggered by the sheer on an atherosclerotic vessel facilitates an increase in oxidative stress and the activation of the ubiquitin-proteasome system. Initially this leads to small vessel remodeling, but as long as the pressure remains elevated, these changes eventually result in occlusion and infarct ${ }^{127}$. Since this

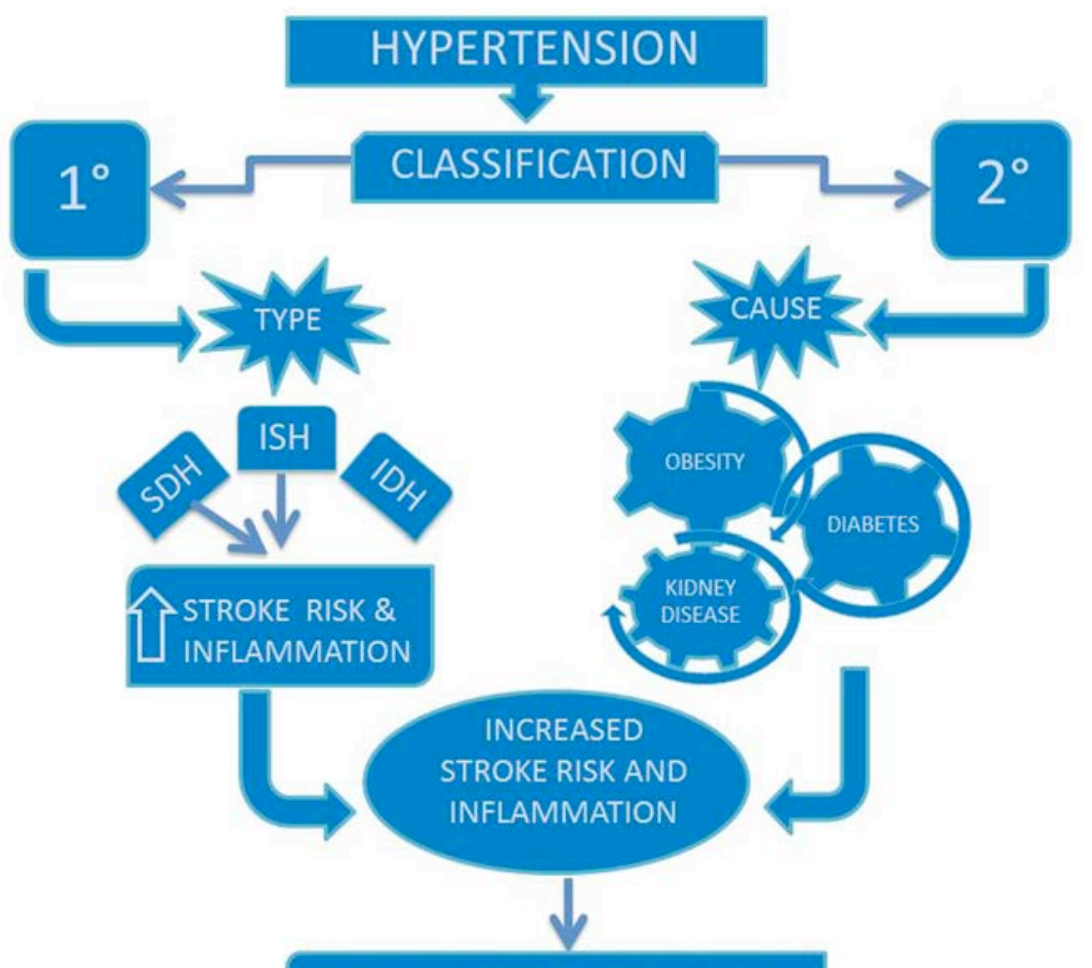

NEED FOR IMPROVED TREATMENTS

Figure 1.9. Hypertension and Stroke. Primary hypertension, specifically systolic and diastolic (SDH) and isolated systolic (ISH), increases inflammation and the risk for stroke. Improved treatments for all classifications of hypertension are necessary to manage the increasing rate in the population.

process is progressive, it also allows for measurement of time-specific changes. Unfortunately this model also has some limitations since the animals die before 1 year of age ${ }^{128}$. Since age is the biggest risk factor for stroke, it is important to consider how hypertension changes with age. The majority of people past the age of 65 will eventually develop hypertension due to age-related arterial changes ${ }^{129}$. It will therefore be beneficial for future models to use animals that develop hypertension later in their lives similar to when most humans develop it.

\subsubsection{OTHER CONSIDERATIONS}

In addition to use of clinically relevant animal models employing aged animals and those with comorbidities, other considerations need to be taken into account to improve the likelihood of translation from bench-to-bedside. This includes but is certainly not limited to the role of gender in outcome following ischemic stroke, occlusion methodology, combination treatment, time of treatment administration, and outcome measures utilized. Each of these topics is explored briefly below. 


\section{Role of Gender}

Another risk factor for stroke rarely considered is the role of gender. Females are more likely to be afflicted with ischemic stroke yet the vast majority of preclinical studies are conducted in young-adult, male animals ${ }^{9}$. This fails to account for the role genderrelated hormones may play in stroke outcome ${ }^{49}$. While numerous studies have illustrated a neuroprotective effect of gender-associated hormones, such as estrogen, on stroke outcome, these studies have been conducted in young-adult animals 130, 131. There remains no indication that these same hormones, when administered to the aged animal, will be neuroprotective. In fact, clinical experience from hormone replacement therapy (HRT) would indicate an increased stroke risk with estrogen replacement in the aged individual ${ }^{132}$.

\section{Occlusion Methodology \& Combination Treatment}

Methodology for inducing ischemia has been discussed at length in the literature, with each technique having distinct advantages and disadvantages 133-136. While suture occlusion remains the prevalent technique for inducing ischemia and is advantageous in ease and reproducibility, the $100 \%$ reperfusion that occurs immediately upon suture withdrawal lacks clinical relevance. Additionally, reperfusion in clinical stroke occurs with administration of tissue plasminogen activator (tPA) and this is a gradual reperfusion as the thrombus is broken down. This reperfusion profile seen clinically is replicated in preclinical models utilizing a thrombus and subsequent tPA administration. By failing to utilize one of the only approved treatments for stroke in the vast majority of preclinical models, it is difficult to study combination therapies. While combination therapies result in potentially more intricate experimental paradigms, it seems unlikely that any potential neuroprotectant can be entirely successful without some degree of reperfusion. Similarly, the ischemic cascade, as described above, is a complex process and may require multiple steps to be targeted simultaneously. Therefore, it seems plausible that future studies need to investigate combinatorial therapy, particularly with administration of tPA to achieve reperfusion ${ }^{137}$.

\section{Treatment Administration}

To increase the translation from bench-to-bedside, potential therapeutic agents need to be administered at extended time points in preclinical studies, time points more consistent with patient presentation. In the past decade since regulatory approval of tPA, less than $5 \%$ of patients receive intravenous tPA. The lack of use is related primarily to time-of-presentation, in that only $46 \%$ of patients present within 3 hours, and an extensive list of contraindications ${ }^{138}$. This reality necessitates that therapeutics capable of extending the thrombolytic window that can be administered safely, perhaps by first responders, need to be developed.

\section{Selecting Appropriate Outcome Measures}

Preclinical models of stroke emphasize volumetric assessments of stroke outcome whereas clinical assessment is based almost entirely on functional outcome. This discrepancy may play a role in the failed translation of therapeutics from bench-tobedside. Furthermore, functional assessment may prove to be a more sensitive indicator of recovery in preclinical models, raising the possibility that compounds previously thought to be a failure based on volumetric assessment, may be 
advantageous functionally. Commonly used functional evaluations in preclinical work, such as the modified Neurological Severity Score (mNSS), were developed in youngadult animals. If aged animals are to be utilized to increase clinical relevance, it will be important to obtain baseline assessments and determine the most appropriate functional and behavioral outcome measures for aged animals, as aged animals may require a unique battery of tests for detailed functional outcome assessment.

\subsubsection{CONCLUSION AND FUTURE DIRECTIONS}

Although it has been challenging to find therapeutics that translate successfully from bench-to-bedside, animal models are still a valuable and promising tool for therapeutic development. Animals experience several of the same physiological changes that humans encounter. With the use of transgenic animals and careful breeding, it is possible to simulate the majority of risk factors seen in complex human diseases ${ }^{139}$. Age, obesity, diabetes, and hypertension were examined in this review as they relate to stroke risk and inflammation. The models that represent these individual risk factors have been used successfully in relation to other disease states and furthermore have provided helpful insights into important components of stroke. The question that remains is how all of these risk factors fit together. In order to answer this question, it will be necessary to design a more comprehensive model that incorporates most of these risk factors.

In human diagnosis, the factors of obesity, dyslipidemia, glucose intolerance, and hypertension have all been linked into a single metabolic syndrome. Until recently, these factors were viewed as separate components and risk elements. Since they often occur together in individual patients, researchers and clinicians were encouraged to propose the idea of the metabolic syndrome ${ }^{140}$. Some cardiovascular researchers have adopted the idea of the metabolic syndrome and now use a transgenic rat model SHR/NDcp, which spontaneously develops obesity, hypertension, hypderlipidemia, and insulin resistance ${ }^{141}$. If such a model could be adapted to stroke research, it may offer a more comprehensive approach necessary to isolate the important pathways. Another possibility is to use the high-fat diet mentioned in the obesity section but initiate it at a later time point in development. This high-fat diet has been deemed the western diet in animal research and has consistently resulted in the development of the metabolic syndrome ${ }^{100}$. The results from this comprehensive model can then be compared with previous work in the other models. Other models have been used to isolate key inflammatory pathways important in stroke morbidity and mortality. A good starting point for using the more comprehensive model would be to look at common pathways consistently showing changes in most of the previous less-sophisticated models.

When looking through the literature, a few inflammatory pathways appear to be activated consistently post-ischemia and would be worth investigating with this comprehensive model. For example, some of the cytokines released post-ischemia such as IL-6, oncostatin $\mathrm{M}$, and ciliary neurotrophic factor bind to the gp130 receptor ${ }^{142}$. These cytokines cause increased inflammation and the recruitment of immune cells. Not surprisingly, these same cytokines are altered by age and the factors of the metabolic syndrome 51,143 . Once the gp130 receptor is activated, it induces downstream Janus kinase 2 (JAK2) phosphorylation of the tyrosine residue on STAT3. STAT3 is unique in its capacity to activate different genes in different cell types ${ }^{144}$. Another pathway that looks promising is TNF-a's activation of secretory phospholipase A2 IIA (sPLA2 IIA). SPLA2 IIA activation leads to a destruction of phosphatidylcholine in membranes and also to an increase in inflammation-dependent infarct size ${ }^{145}$. The cytokine TNF- $\alpha$ 
specifically causes endothelial widening and diapedesis of macrophages. As mentioned previously, TNF- $\alpha$ levels are altered by obesity, age, and other factors of the metabolic syndrome. Finally, protein kinase $\mathrm{C}$ (PKC) is a well-known modulator of inflammatory pathways. PKC has 12 different isoforms, many of which are altered by the metabolic syndrome and age 146,147. These responses can sometimes work against each other depending on which isoform of PKC is active. PKC $\delta$ is associated with neurotoxicity where PKC $\varepsilon$ initiates neuroprotection ${ }^{148}$.

Although much remains to be discovered about stroke, the use of a comprehensive animal model has been a useful tool for translational research in other disease states and may prove useful for ischemic stroke research as well. It will allow investigators to highlight neurotoxic and neuroprotective pathways and discover how they function with consideration to age and the metabolic syndrome. With increased therapeutic options, it may be possible to increase survival and recovery following an ischemic event. 


\title{
1.6. Neuroprotection in Ischemic Stroke
}

In this work we explore the concept of neuroprotection and potential therapeutic targets for future research and development.

This work was published in the Journal of Neurosurgery.

\section{Citation:}

Turner RC, Dodson SC, Rosen CL, Huber JD (2012) The science of cerebral ischemia and neuroprotection: navigating past failure to future success. Journal of Neurosurgery 118(5):1072-1085.

\begin{abstract}
:
Ischemic stroke remains a leading cause of morbidity and mortality for which few therapeutic options are available. The development of neuroprotective agents, a once promising field of investigation, has failed to translate from bench-to-bedside successfully. This work reviews the ischemic cascade, agents targeting steps within the cascade, and potential reasons for lack of translation. Additional therapeutic targets are highlighted and areas requiring further investigation discussed. It is clear alternative targets need to be pursued such as the role glia play in neurologic injury and recovery, particularly the interactions between neurons, astrocytes, microglia, and the vasculature. Similarly, the biphasic nature of many signaling molecules such as matrix metalloproteinases (MMPs) and high mobility group box 1 (HMGB1) protein must be further investigated to elucidate periods of detrimental versus beneficial activity.
\end{abstract}

\subsubsection{INTRODUCTION}

Decades of research on ischemic stroke have produced a heightened understanding of the pathophysiology, providing an extensive array of potential therapeutic targets. While many of these proposed targets have been manipulated through various pharmacologic compounds, many of which have progressed to clinical trials, there remains an unmet need for alternative, or supplemental, treatments besides tissue plasminogen activator (tPA) for stroke. In addition to the complex pathophysiology of stroke, the lack of successful translation from bench-to-bedside has resulted in controversy concerning the most appropriate preclinical models of ischemic stroke and assessment of potential therapeutics. This work highlights the pathophysiology of stroke with an emphasis of the time-dependent nature of the ischemic cascade and pharmacologic agents tested preclinically and/or clinically targeting events within the cascade. Preclinical modeling and alternative therapeutic approaches/targets are discussed at length with the goal of improving the likelihood of successful translation from bench-to-bedside.

\subsubsection{Significance of Stroke}

Stroke remains a leading cause of morbidity and mortality in the developed world with the only approved pharmacologic treatment being tPA. Due to many risks associated with tPA use, stringent guidelines must be followed, most specifically, treatment within three hours of symptom onset resulting in less than $5 \%$ of patients receiving tPA treatment ${ }^{149}$. As such, the development of alternative treatments is highly desirable.

\subsubsection{Concept of Neuroprotection}


The notion that neuroprotection may be possible following neurologic injury, and more specifically ischemia, is based largely on the findings of Astrup, et al 150. The onset of ischemia produces a 'core' region in which flow is severely reduced, by almost $90 \%$. This sharp reduction in blood flow results in deficient ATP levels and subsequent ionic disruption and metabolic failure, progressing to cell death via necrosis within minutes. Surrounding this 'core' region lies the 'penumbra' in which flow is less severely reduced, typically in the range of $35 \%$ of baseline 150 . This territory is characterized by the loss of action potential firing but maintenance of proper resting membrane potential. Upon restoration of blood flow to the penumbra, normal function returns. If flow is not restored acutely, tissue originally in the penumbral region dies and the core infarct region evolves to encompass what was previously penumbral tissue ${ }^{151}$. While the general consensus remains that the infarct core is not salvageable, it is believed that penumbral tissue can be salvaged through flow restoration and/or restoration of cellular homeostasis through manipulation with pharmacologic compounds. Potential neuroprotective agents target a variety of aspects of cellular physiolology ranging from membrane integrity to mitochondrial function to the regulation of apoptosis.

\subsubsection{Current Treatment Options}

This concept resulted in the development of intravenous tissue plasminogen activator (IV-tPA), the most widely used therapeutic option for ischemic stroke treatment. Unfortunately, IV-tPA can only be applied to a relatively small subset of those afflicted with ischemic stroke, less than $5 \%$, as a consequence of numerous contraindications and the late presentation time after stroke onset of most patients ${ }^{146}$. In an attempt to provide thrombolytic therapy to a broader patient population, other therapeutic techniques have been developed. These include intra-arterial delivery of tPA, the Penumbra System, and the $\mathrm{MERCl}$ clot retrieval device. Each of these alternative techniques, while expanding the therapeutic window of opportunity, require specialists found primarily at advanced academic medical centers and are rarely available at smaller community hospitals, making them less than an ideal therapeutic option.

\subsubsection{Targeting the Ischemic Injury Cascade}

Pharmacologic agents selected to interrupt the ischemic injury cascade that can be administered safely to a larger patient population are therefore desirable. Numerous compounds have been investigated as potential neuroprotective agents. These compounds have targeted events primarily occurring rapidly after ischemic onset. After cerebral blood flow is reduced, energy failure occurs due to a decoupling of oxidative phosphorylation, resulting in a series of events including glutamate release and ionic imbalance which subsequently causes membrane depolarization ${ }^{1}$. Calcium rapidly enters the cell causing the induction of various enzymes and mitochondrial damage. Further downstream, apoptotic mediators are released, free radicals produced, and inflammatory mediators released 2. The events of the injury cascade, and the pharmacologic agents designed to prevent them, will be discussed in further detail in subsequent sections.

\subsubsection{Current Status of Neuroprotectant Development}

To date there have been over 1000 preclinical studies identifying neuroprotective compounds with over 100 progressing to clinical trials. Of those progressing to clinical trials, none have proved efficacious in the human population ${ }^{8}$. Consequently, funding for the field has declined from both pharmaceutical corporations as well as government agencies. 
The prospect of neuroprotection remains at the forefront of the stroke field, despite the aforementioned failures, with the vast majority of researchers still believing it has merit as a potential therapeutic option. There is simply too great a need for addtional treatments and development of

I neuroprotective agents due to the economic burden of stroke with an estimated $\$ 28.3$ billion in direct costs and $\$ 25.6$ billion in indirect costs in the United States in the year 2010 152 .

The failed translation from bench-tobedside of therapeutic agents has resulted in much speculation about the requirements for achieving success. This work seeks to summarize the ischemic cascade

I (Figure 1.10), agents that have targeted a given step yet failed in clinical trials, and potential reasons for past failures. Additional discussion highlights potentially promising therapeutic targets and areas or topics in need of further investigation.

I. 1.6.2. PATHOPHYSIOLOGY OF
STROKE \& CORRESPONDING
THERAPEUTICS - ACUTE PERIOD

\subsubsection{Glutamate-mediated Excitotoxicity}

Blood flow cessation as a result of ischemia onset induces a shortage of oxygen and glucose delivery. As the main sources of energy production via oxidative phosphorylation, diminished oxygen and glucose supply results in a decoupling of oxidative

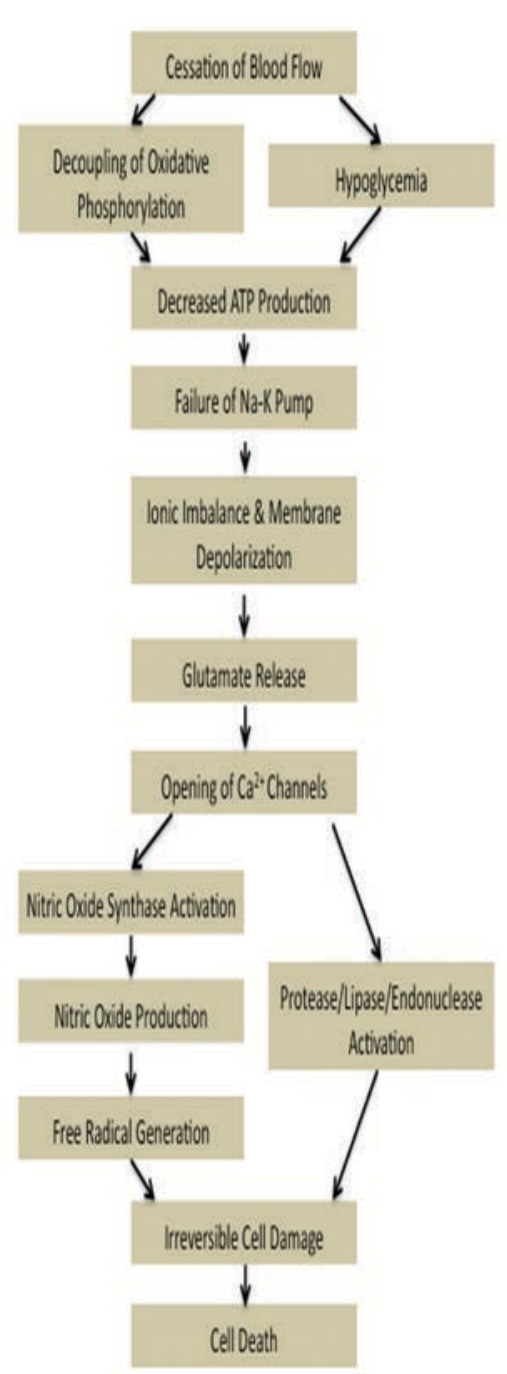

Figure 1.10. A diagrammatic representation of the ischemic injury cascade from the onset of ischemia to cell death.

phosphorylation and subsequently, reduced ATP production and disruption of ionic pump function. These events occur rapidly after onset of ischemia; in fact, ATP levels are decreased substantially within 2 minutes ${ }^{153}$. Diminished levels of ATP impedes the functioning of Na-K ATPase, preventing the maintenance of normal ionic gradients. As such, elevations in cytosolic $\mathrm{Na}^{+}$and a decrease in cytosolic $\mathrm{K}^{+}$is seen, leading to a depolarization of the neuronal membrane ${ }^{153}$. Voltage-gated calcium channels are activated by depolarization, allowing for rapid influx of $\mathrm{Ca}^{2+}$ which results in release of excitotoxic amino acids such as as glutamate. Under normal homeostatic conditions, glutamate is rapidly cleared from the synapse via presynaptic and astrocyte uptake but this response is altered in ischemia. In ischemia, the excess glutamate within synaptic cleft binds ionotropic N-methyl-D- 
aspartate (NMDA) and $\alpha-a m$ ino-3hydroxy-5-methyl-4isoazole propionic acid (AMPA) receptors on the postsynaptic membrane, p $r$ o m p t i n g depolarization of the post-synaptic neuron and propagating the wave of depolarization ${ }^{1}$.

\subsubsection{Blocking Excitotoxic Events}

Attempts to mitigate excitotoxicity have encompassed a wide array of targets, ranging from prevention of glutamate release to blocking glutamate receptors to facilitating the opening of ion channels that counteract the excitotoxicity (Figure 1.11). One of the most widely investigated targets is antagonism of the NMDA receptor on the post-synaptic membrane. The NMDA receptor is activated by the binding of both glutamate and glycine to their respective sites and is further modulated by an allosteric site ${ }^{154}$. Once activated, the NMDA receptor acts as a non-specific cation channel, allowing the passage of $\mathrm{Ca}^{2+}, \mathrm{Na}^{+}$, and $\mathrm{K}^{+}$into the cell ${ }^{155}$. By preventing activation of the NMDA receptor, cation flux through the ion channel is reduced, preventing depolarization. Numerous agents have been investigated for this purpose and include non-competitive antagonists such as Aptiganel Hydrochloride (Cerestat; CNS-1102) 154, 156, 157, Dextrorphan Hydrochloride 154, 158-160, and Magnesium 161-163; competitive antagonists such as Selfotel (CGS-19755) 154, 164, 165; glycine-site antagonists like Gavestinel (GV-150,526) 154, 166-168; and indirect blockers exemplified by Lubeluzole (Prosynap) 154, 169, 170.

The AMPA receptor, an ionotropic receptor for glutamate, is another well-documented target in the search for neuroprotection via reducing excitotoxicity. The AMPA receptor has multiple glutamate binding sites that when bound activate the receptor and open the cation channel, allowing passage of cations non-specifically ${ }^{171}$. Logic in targeting the AMPA receptor is similar to that of the NMDA receptor in that antagonizing the AMPA receptor should prevent cation influx and subsequent depolarization. One widely investigated therapeutic antagonizing the AMPA receptor is Zonampanel (YM872) 154, 172 .

In addition to targeting glutamate-binding receptors and preventing substrate-receptor binding, another alternative is to counteract the increase in cation influx. One way of doing this is to increase the influx of anions such as $\mathrm{Cl}^{-}$. Clomethiazole (Zendra ${ }^{\circledR}$ ), a $\mathrm{GABA}_{A}$ receptor agonist, was evaluated for this purpose ${ }^{173-175}$. Similarly, activation of the neuronal $5-\mathrm{HT}_{1 \mathrm{~A}}$ receptor has been shown to elicit hyperpolarization due to 
increasing inward $\mathrm{K}^{+}$current. A compound activating the $5-\mathrm{HT}_{1 \mathrm{~A}}$ receptor that has been evaluated in not only preclinical experimental stroke studies but also clinical trials is Repinotan (Bay $x$ 3702 ) 176-178. Maxipost (BMS-204352), a potassium channel opener, has also been evaluated for the treatment of ischemic stroke. Once again, the logic behind application of this compound was that an increase of potassium influx would hyperpolarize the membrane, preventing depolarization 154, 179, 180 .

A final target for preventing excitotoxicity is the opiate receptor, the kappa receptor in particular. This receptor results in the initiation of a G-protein signaling cascade when stimulated 181 . While the details of this cascade are not entirely elucidated, it is clear that the pathway is involved in the release of excitatory neurotransmitters, release of free fatty acids, and the presence of antioxidants. The agent Nalmefene (Cervene) was a kappa receptor antagonist investigated for the treatment of stroke $182,183$.

The target, preclinical development, clinical evaluation, and results of these agents is highlighted in Table 1.1. Notably, each of these agents failed when reaching clinical trials. The precise reason for each of these failures is unlikely to be identified but numerous weaknesses in preclinical design and the translation from bench-to-bedside are evident. For example, most preclinical studies utilize young-adult animals despite the role of age in the clinical stroke population. Furthermore, preclinical work often involves drug administration prior to, or shortly after, onset of ischemia yet clinical trials often have a much longer exclusion window in order to increase the number of eligible patients. Other potential shortcomings of studies are discussed in greater detail below. 


\subsubsection{Effects of Intracellular Calcium}

The excessive calcium influx results in activation of a multitude of enzymes including phospholipases and proteases, mediated through calcium serving as a messenger molecule for numerous downstream signaling pathways 184, 185. Activation of phospholipases and proteases results in membrane and protein degradation, decreasing cellular integrity and survival. Endonucleases are also activated, cleaving DNA and leading to further cellular changes and potentially cell death. Calcium influx also potentiates glutamate-mediated excitotoxicity as depolarization occurs and additional glutamate released. The release of excessive amounts of glutamate results in propagating waves of depolarization, termed peri-infarct depolarization, originating in the core and spreading to the peripheral penumbra ${ }^{186}$. Peri-infarct depolarization, at least in the initial 3 hours of ischemia, has been shown to correlate with final infarct volume in preclinical studies ${ }^{187}$. Other studies have shown wave propagation for at least 6-8 hours after ischemic onset, although the correlation with infarct volume at these later time points is unestablished ${ }^{188}$. Agents interrupting the waves of depolarization through targeted blockade of glutamate receptors (AMPA and NMDA) prevent progression of the penumbra to infarct in experimental models of ischemic stroke. In contrast, the creation of additional waves of depolarization through administration of potassium chloride causes expansion of the infarct core 188. While manipulation of peri-infarct depolarization has occurred in preclinical models, the propagating waves of depolarization have been shown to occur in humans afflicted with stroke and equally important, each wave has been shown to correlate with expansion of the infarct 188 . It is unclear as to whether the spreading depolarization may have some beneficial effects though as it may induce release of protective factors.

In addition to roles in protease, phospholipase, and endonuclease activation, and serving as an intermediary in downstream signaling, calcium influx is involved in mitochondrial damage and subsequent reactive oxygen species (ROS) generation 51. Following a rise in intracellular cytosolic calcium concentration, occurring following ischemia, calcium enters the mitochondria ${ }^{189}$. Calcium balance in the mitochondria is achieved via intake through the uniporter and output through $2 \mathrm{Na}^{+}-\mathrm{Ca}^{2+}$ exchange. This balance is disrupted in ischemia with calcium entering via the uniporter far more quickly than the $2 \mathrm{Na}^{+}-\mathrm{Ca}^{2+}$ exchanger can function ${ }^{185}$. Accumulation of excess calcium within the mitochondria most often results in opening of the permeability transition pore (PTP) found on the inner mitochondrial membrane. The PTP opening is associated with loss of membrane potential and subsequent osmotic swelling, leading to outer membrane rupture and release of cytochrome $c{ }^{190}$. While the mechanism has not been elucidated entirely, calcium influx and associated mitochondrial dysregulation may result in initiation of apoptosis and generation of reactive oxygen species. This process plays a role in not only initial ischemic injury but also subsequent reperfusion and reperfusion-associated injury (Figure 1.12).

\subsubsection{Blocking Actions of Excess Calcium}

Nimodipine, a calcium channel blocker frequently utilized in other forms of cardiovascular disease, was assessed as a potential therapeutic in the phase III trial Very Early Nimodipine Use in Stroke (VENUS) ${ }^{191}$. Preclinical studies, as well as previous clinical trials, reported mixed findings regarding the efficacy of Nimodipine 192-194. A subgroup analysis of a previous clinical trial employing Nimodipine suggested improvement in outcome when drug administration occurred within 18 hours of symptom 
onset, hence the VENUS trial was initiated. Unfortunately, the VENUS trial failed to show improved outcome, in both primary and secondary measures, at trial termination ${ }^{191}$.

\subsubsection{Oxidative Stress- induced Damage}

Oxidative stress has long been recognized as a potential target for therapeutic development following ischemia. Oxidative stress, induced by the production of reactive oxygen species (ROS), can be attributed largely to mitochondrial dysfunction ${ }^{189}$. In addition to PTP opening and release of cytochrome $c$, inhibition of normal respiration occurs, along with the release of nucleotides 189 . Furthermore, glutathione necessary for neutralization of reactive oxygen species is reduced. Free radical production leads to peroxidation of plasma membranes and intracellular organelle membranes ${ }^{187}$. Release of biologically active free fatty acids, such as arachidonic acid, is a direct consequence of free radical production. ROS production also leads to damage of intracellular organelles such as the endoplasmic reticulum, mitochondria, and induce DNA fragmentation. Disturbances to homeostasis induced by ROS contribute to the progression of downstream injury pathways and apoptosis, often similar to those induced by excess calcium influx ${ }^{187}$.

\subsubsection{Free Radical Scavenging}

Tirilazad mesylate, a compound inhibiting lipid peroxidation, was evaluated for treatment of stroke in a total of 6 trials (4 of which were published) ${ }^{57}$. Tirilazad failed to reduce morbidity or mortality in acute ischemic stroke. Reasons for failure remain unknown but preclinical evaluation of the drug produced mixed findings, often depending on time of drug administration 195, 196. When administered prior to ischemia, tirilazad appeared efficacious in preclinical studies but when administered post-stroke, no positive effect was found $195,196$.

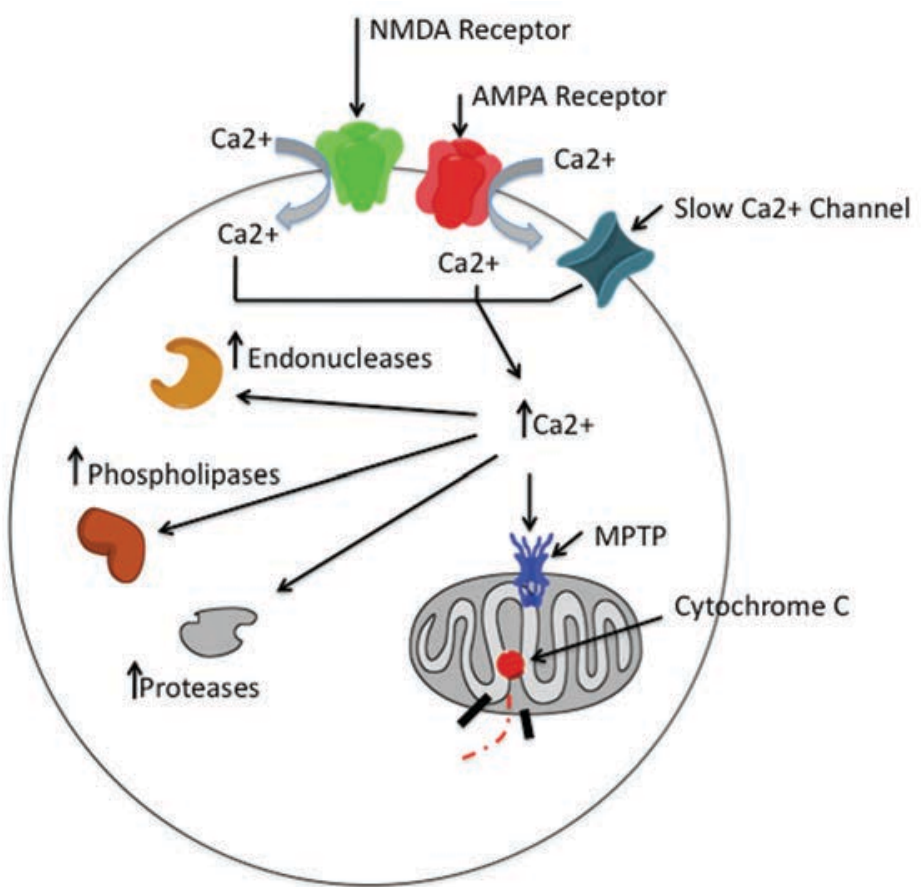

Figure 1.12. Schematic showing that ischemia results in increased intracellular calcium levels through not only the NMDA and AMPA receptors but also through a slow $\mathrm{Ca}^{++}$ of endonucleases and phospholipases as well as proteases. The MPTP allows calcium to enter the mitochondria, which causes production of ROS and further damage. Calcium in the mitochondria also causes swelling and eventual rupture, release cytochrome c, long known to play a role in apoptosis. 
The most recent well-documented failure of a proposed neuroprotective agent is that of Disufenton Sodium (Cerovive; NXY-059), a free-radical spin trapping agent. Believed by many to have undergone the most rigorous preclinical assessment of a potential therapeutic agent, Disufenton Sodium progressed to clinical trials (SAINT I \& II) 58 . In SAINT I, Disufenton Sodium appeared to be neuroprotective as disability, assessed with the modified Rankin scale, was reduced in the treatment group ${ }^{197 . ~ I n ~ t h e ~ c o n f i r m a t o r y ~}$ trial, SAINT II, Disufenton Sodium failed to reduce disability when administered within 6 hours of ischemic onset ${ }^{198}$. This lack of efficacy was also true for subgroup analysis and hemorrhage associated with tPA administration ${ }^{199}$. A critical review of not only preclinical studies but also design of the clinical trials reveals numerous discrepancies between studies, ranging from inconsistent time of administration to inadequate animal models and insufficient functional assessment 58.

\subsubsection{PATHOPHYSIOLOGY OF STROKE \& CORRESPONDING THERAPEUTICS - SUBACUTE PERIOD}

\subsubsection{Apoptosis}

Evolution of the infarct does not occur based on necrosis or apoptosis individually but is rather a complex interplay between the aforementioned forms of cell death 1, 200-202. In the infarct core, where ATP is severely depleted, necrosis may be the predominating form of cellular death whereas in the penumbra, apoptosis may be the predominant form of cell death 200 . The onset of ischemia induces apoptosis through two primary methods, one of which is through altered gene expression of apoptotic mediators such as Bax, $\mathrm{Bad}, \mathrm{Bid}, \mathrm{Bcl}-2, \mathrm{Bcl}-\mathrm{XL}$, etc and the second being the influx of calcium and subsequent mitochondrial damage, ultimately resulting in opening of the PTP. Mitochondrial damage and changes in mitochondrial membrane integrity can cause release of toxic mediators such as cytochrome $c$ and apoptosis inducing factor (AIF). This process, termed the intrinsic arm of the apoptosis program, leads to the activation of proteolytic caspases and ultimately, cell death. The extrinsic arm of the apoptosis program, in contrast, utilizes a series of interactions between cell-death factors, such as Fas ligand, and celldeath receptors, such as the Fas receptor. After binding of ligand and receptor, an adaptor protein (FADD) is recruited, resulting in the activation of the caspase cascade 200, 203 .

\subsubsection{Inhibiting Apoptosis}

As a result of the previously discussed cell stressors produced following ischemia, many of which are involved in mediating apoptosis, the pro- vs. anti-apoptotic protein balance is shifted towards the induction of apoptosis. One of the pro-apoptotic proteins, Bax, has been demonstrated to play an essential role in permeabilization of the outer mitochondrial membrane 204. By preventing the Bax-mediated disruption, the release of pro-apoptotic proteins can be avoided. When a Bax-inhibiting peptide was used in a neonatal model of ischemia, outcome was improved 204.

Inhibition of caspases is another alternative target in the attempt to reduce apoptosis and thus, improve outcome from cerebral ischemia 205 . Caspases are proteases, first synthesized in the pro-caspase form, that are rapidly activated in response to earlier events in the apoptotic cascade such as apoptosome formation or death receptor signaling 206. Caspase activation ultimately results in DNA fragmentation via cleavage and inactivation of inhibitor of caspase activated DNase (ICAD), producing the hallmark 
'DNA ladder' of apoptotic cells ${ }^{207}$. In experimental neonatal focal ischemia, application of a pan-caspase inhibitor failed to reduce infarct volume, despite cell death shifting towards necrosis 207.

While attempts to improve outcome after ischemia through the modulation of apoptosis have failed to progress to clinical trials and successful therapeutics, investigation of the apoptotic cascades in neonate, as well as young-adult animals, has revealed fundamental differences associated with age. For example, caspase-3 has been described as being highly involved in apoptosis in the immature brain but plays a minor role in adult brain 207. Similarly, the mitochondrial permeability often responsible for inducing apoptosis may be mediated via different age-related mechanisms. In neonatal hypoxic-ischemic injury, permeability is largely Bax-dependent but in adult brain, the cyclophilin D transition pore plays a prominent role 204 .

Besides appreciating age-related differences in apoptotic mechanisms, another challenge that must be overcome in the pursuit of pharmacologic apoptotic mediators is the variety of pathways resulting in the same end result - apoptotic cell death. Many studies focusing on one specific apoptotic pathway may delay cell death but not entirely prevent it. Rather, a pan-apoptotic inhibitor may be required 208.

\subsubsection{Inflammation}

Onset of ischemia results in a rapid activation of inflammatory cells residing in the brain, termed microglia 209 . This is followed by influx of circulating inflammatory cells such as granulocytes, leukocytes, T cells, monocytes, etc ${ }^{210}$. Similarly, over the first few hours, an array of pro-inflammatory mediators such as cytokines and chemokines are released from the damaged tissue, inducing adhesion molecule expression and subsequent transendothelial migration of circulating inflammatory cells ${ }^{210}$. Infiltration of leukocytes from the bloodstream into brain tissue results in release of additional cytokines and chemokines, ultimately leading to excessive oxidative stress and activation of matrix metalloproteinases (MMPs) ${ }^{209}$. MMP activation subsequently causes enhanced bloodbrain barrier (BBB) disruption, leading to further inflammatory cell recruitment and associated inflammation ${ }^{211}$. The inflammatory cascade is complicated by evidence that the initiating microglia can also produce neuroprotective and neurotrophic factors 212 .

\subsubsection{Mitigating Inflammation}

An extensive variety of targets exist in the effort to manipulate the inflammatory response to achieve neuroprotection (Figure 1.14). Pharmacologic agents modulating inflammation fall into a few general classes. This includes astrocyte modulators, blockade of inflammatory cell receptor complexes, inhibitors of microglia, and general immunosuppressive/anti-inflammatory agents.

The precise role of the astrocyte in ischemic stroke research has received much less emphasis than the neuron. This neuron-centered approach fails to consider the intimate relationship between neurons and supporting glia 213. Astrocytes have been credited with contributing to formation of the blood-brain barrier, modulating neurotransmitter levels in the synaptic cleft, regulating ion homeostasis, and also may alter inflammation via microglial influence. Furthermore, the neuron-astrocyte interaction is responsible for controlling the microcirculation of the brain 214, 215. The influence of astrocytes can be seen both in homeostasis as well as both the acute and chronic injury response via 
astrogliosis 216. Modulating astrocyte activity, via either fluorocitrate or Arundic Acid (ONO-2506), has produced mixed results 217-220. Arundic Acid, administered at up to 48 hours after stroke onset, appears promising based on early results yet fluorocitrate treatment at 5 days after ischemia resulted in decreased vascular remodeling and worsened functional status 217, 220. Arundic Acid has advanced to clinical trials and its development highlighted in Table 1.2 154, 218.

Following ischemia, peripheral inflammatory cells such as neutrophils are recruited to the region of infarction. Multiple attempts have been made to target adhesion molecules on neutrophils, as well as other inflammatory cells, to prevent infiltration following ischemic stroke. Examples include Hu23F2G (LeukArrest), an antibody recognizing the CD11/18 integrin, and Enlimomab (R6.5) which targets intercellular adhesion molecule-1 (ICAM-1) 221-224. More information on the preclinical development and results of clinical trials utilizing these compounds can be found in Table 1.2154.

Modulating general inflammation after stroke is another potential, and promising, therapeutic target. The effects of non-specific immunosuppressive agents, like

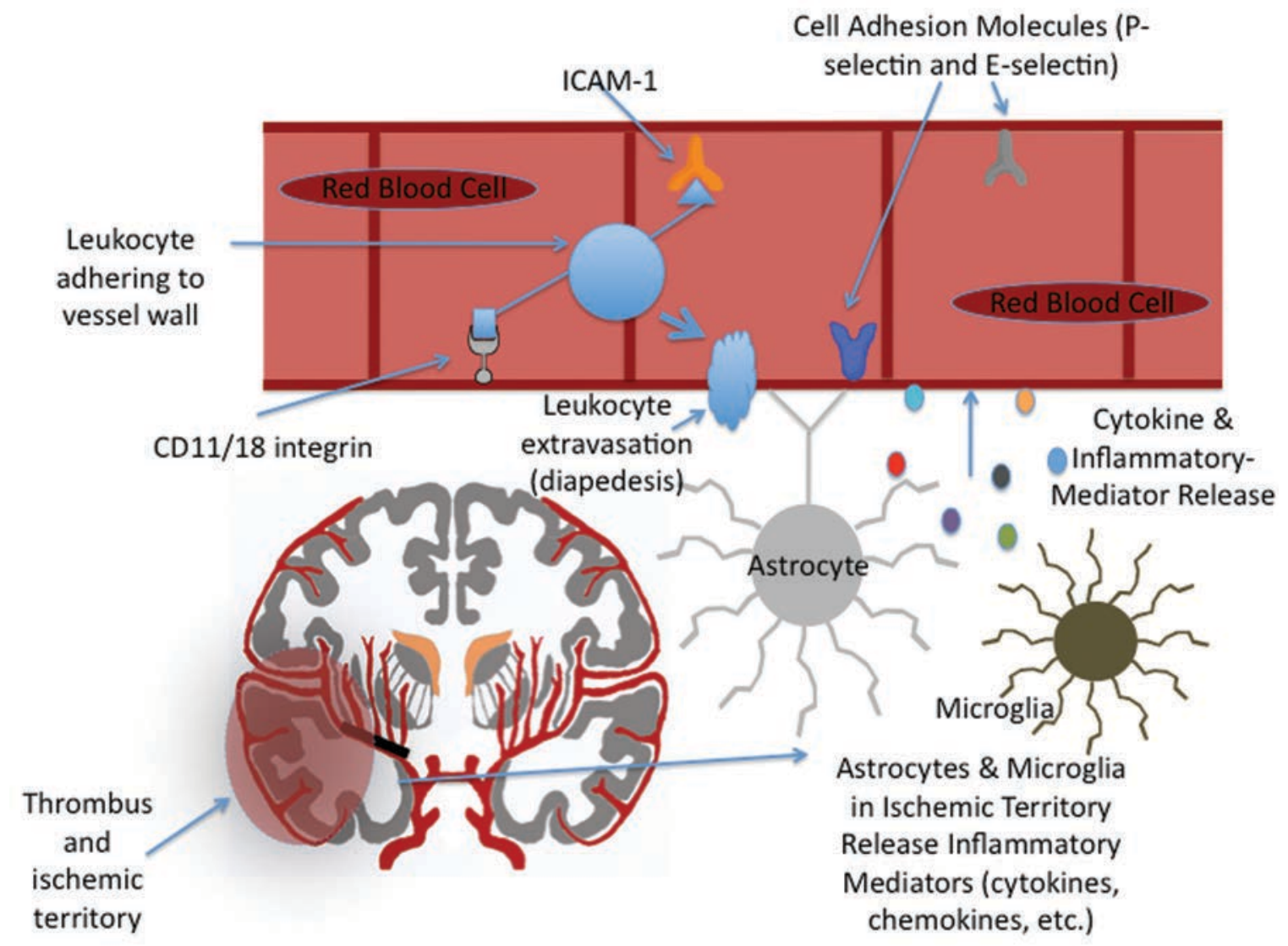

Figure 1.13. Inflammation has been identified as a promising avenue of therapeutic development. Multiple aspects can be inhibited, including release of inflammatory mediators from microglia, preventing adhesion, and blocking migration of inflammatory cells outside of the vasculature and into the parenchyma. 


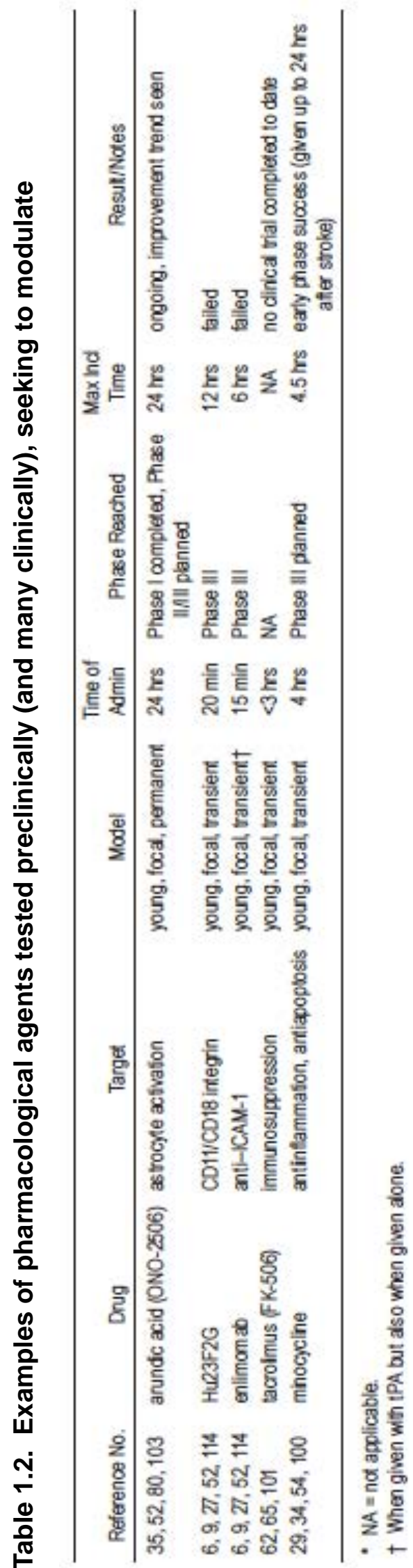

Tacrolimus (FK-506), as well as microgliaspecific inhibitors such as minocycline are highlighted in Table $1.2^{225-231}$.

\subsubsection{PATHOPHYSIOLOGY OF STROKE \& CORRESPONDING THERAPEUTICS - CHRONIC PERIOD}

\subsubsection{Inflammation}

While inflammation is initiated in the subacute period after onset of ischemia, inflammation has been shown to persist for an extended period, out to greater than 30 days ${ }^{3}$. Inflammation is generally thought of as a detrimental process, exerting further tissue damage but recent findings, particularly those associated with matrix metalloproteinases (MMPs), indicate a restorative role of inflammation in these extended time periods ${ }^{2}$.

As much of preclinical stroke models focus on the acute period, many questions remain unanswered regarding pathologic events occurring in the chronic period, particularly in terms of inflammation. Future work needs to elucidate the role of inflammation in repair and more specifically, how altering acute inflammation impacts chronic inflammation that may, in some cases, be required for recovery/ repair. Furthermore, determining the time course of expression of inflammatory mediators, and how this is altered with age and/or comorbidity, across both the acute and chronic periods may lead to additional insight into the role inflammation plays in not only injury but also recovery.

\subsubsection{Repair}

Recent work has focused on the development of stem cell therapies for post-stroke treatment. Early studies have appeared promising and shown effects ranging from inhibition of cell death, vasculature regrowth, modulation of inflammation, and induction of plasticity and neurogenesis. The use of stem cells, of which there are many subtypes, remains a promising future avenue of work with many questions remaining to be elucidated. Future studies will likely need to ascertain the precise cell types most likely to demonstrate success (exogenous, neural, embryonic, induced pluripotent, etc) and 
the optimal delivery of these cells. Furthermore, other questions remain such as how these cells integrate within the remaining intact neurologic structures and the prevalence of long-term side-effects such as tumor formation, etc. Additionally, how these implanted cells exert effects on the vasculature, surrounding neurons and glia, and the inflammatory process all remain to be elucidated 232 .

\subsubsection{FUTURE DIRECTIONS}

\subsubsection{A Shifting Approach - From Neurons to Neurovascular Unit}

For years, stroke research has emphasized neural survival and hence coined the term 'neuroprotection'. Perhaps 'neuroprotection' is simply not enough for clinical success and rather the focus needs to shift towards full 'cerebroprotection' in which glial cells and the vasculature are also considered (Figure 1.15) 137, 233. Not only has the neuro-centric approach failed to deliver clinically successful therapeutics but disregards the fact that glia outnumber neurons and play an integral role in maintaining homeostasis in the health brain and restoring neurologic function in the injured brain. Furthermore, it has been shown that glial cells play an integral role in protecting neurons from ischemia and can also lead to detrimental downstream consequences 216 . In fact, the question of whether neurons can even survive in the absence of supporting glia has been asked and remains to be addressed, further necessitating the need to consider and investigate the role of glia in injury more thoroughly.

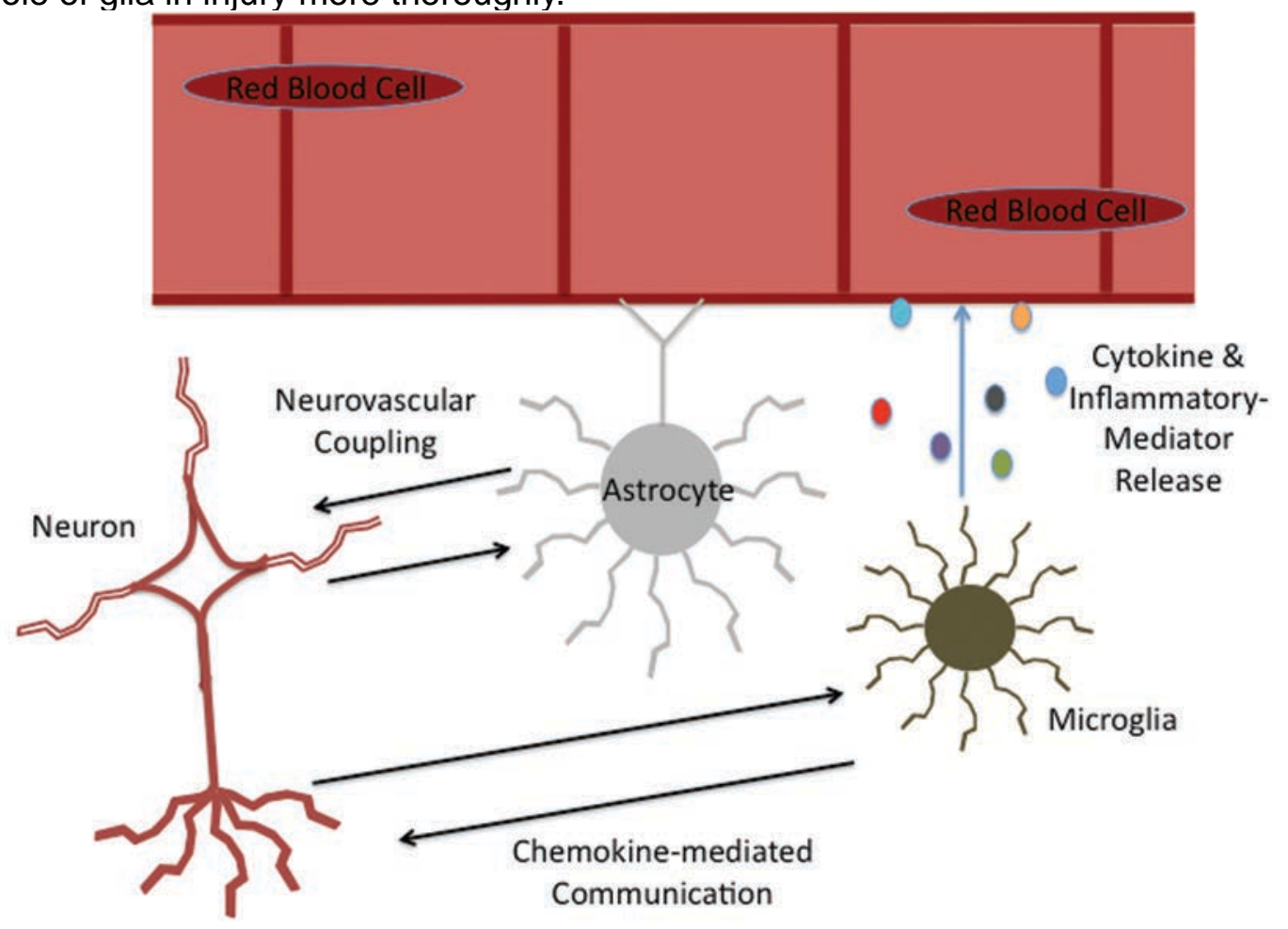

Figure 1.14. The concept of neuroprotection is perhaps misguided; the neurocentric approach has failed to produce effective therapeutic agents. Cerebroprotection is needed, and for that the entire neurovascular unit must be considered. Also, supporting glia play a crucial role in health and disease, necessitating inclusion in therapeutic development. 
Astrocytes, long recognized for having a role in neurologic homeostasis via actions on neurotransmitter and ionic balance, have also been implicated in control of the brains microcirculation through signaling with neurons 215 . Responding to synaptic release of glutamate from neurons, increases in intracellular calcium concentration within the astrocyte are observed, ultimately resulting in release of ATP, the primary signaling molecule through which astrocytes communicate with other cells of the CNS 213 . The release of ATP by astrocytes has been demonstrated following injury and results in microglial activation. Considering the proximity of astrocytic processes with neural synapses and cerebral arterioles, forming a possible relay from neuron to vasculature, it is easy to envision how astrocyte compromise as a consequence of ischemia may be deleterious neurologically. Similarly, neuronal dysfunction following ischemia results in excitotoxicity via glutamate release, which may then signal microglial activation through the astrocytic response.

The role astrocytes play in homeostasis and response to injury is likely increased in the aged animal due to an increased content of astrocytes, as well as pericytes, with age. Pilegaard, et al suggested an increase of $20 \%$ in astrocyte and pericyte population in the aged brain ${ }^{234}$. Besides an increase in number, astrocytes in the aged brain exhibit hypertrophy (associated with increase in glial fibrillary acidic protein expression) and altered expression of surface markers, intracellular antigens, and growth factors. Notably, these differences exist in the absence of neurologic injury, providing further indication that the aged brain is a fundamentally different entity in comparison to the young-adult brain, furthering the need to consider age in preclinical stroke models 50 .

Understanding the precise role of microglia, in both acute and chronic periods, after ischemia and how microglia influence not only neuronal function but that of the neurovascular unit is another area of investigation that may lead to successful therapeutic development. Microglia comprise approximately $12 \%$ of cells in the brain and potentially exert both detrimental and beneficial effects in the ischemic brain, necessitating further investigation and elucidation of these mechanisms ${ }^{235}$. While generally existing in an inactivated state in the young-adult brain, normal aging has been shown to produce increasing numbers of activated microglia as well as phagocytic microglial subtypes ${ }^{53}$. Similarly, microglial reactivity is increased in response to a numerous types of injury in the aged brain, ranging from neurotoxin (MPTP) exposure to traumatic brain injury (TBI) to ischemia. Microglial activation ultimately results in the production of many pro-inflammatory factors such as PGE2, IL-1 $\beta$, TNFa, NO, NOO-, $\mathrm{O}^{*}{ }^{*}$, and $\mathrm{H}_{2} \mathrm{O}_{2}$, furthering the damage initiated by initial pathologic stimuli 235 .

Besides release of inflammatory mediators, microglia likely play a role in oxidative stress-induced damage. As microglia are activated, cytosolic subunits of the NADPH oxidase enzyme translocate to the cell membrane and assemble the active form of the enzyme, resulting in superoxide production. In addition to the oxidative damage caused by extracellular ROS, such as superoxide, these molecules often can act intracellularly, serving to amplify the inflammatory response ${ }^{235}$.

The inflammatory response, mediated primarily through effects of astrocytes and microglia, represent a more logical target clinically as inflammation persists for an extended period and consists of beneficial and detrimental effects. Therefore, upregulation of the beneficial aspects of the inflammatory response may be a target as well as decreasing the detrimental components. Similarly, evidence indicates that events occurring later in the ischemic cascade such as apoptosis and neuroinflammation 
account for most of the penumbral tissue death, the region of interest in therapeutic development ${ }^{1}$.

Lack of therapeutic translation from bench-to-bedside may partially be explained by the neurocentric approach taken towards investigating and ischemia and subsequent cell death. It is clear there is continual communication between neurons-astrocytes, neurons-microglia, and neurons-vasculature. This communication, likely bi-directional in nature, is responsible for normal homeostasis as well as responding to injury. The previous focus on neuronal response alone fails to consider the complex relationships amongst the various elements of the neurologic system. Shortcomings of the field led to formation of the Stroke Progress Review Group by National Institutes of Neurological Disorders and Stroke (NINDS). One of the primary points of emphasis of this group is the need for cerebroprotection, in which the entire neurovascular unit and neurologic structure is considered, rather than solely neuroprotection. As such, future work needs to address the temporality and mechanistic details of signaling events within the neurovascular unit.

\subsubsection{Understanding Biphasic Signaling}

A significant challenge in therapeutic development is that of various messenger molecules having divergent actions temporally. For example, matrix metalloproteinases (MMPs), have been tied to hemorrhagic transformation in the acute period following ischemic injury yet also are essential for long-term recovery ${ }^{2}$. Similarly, high-mobility group box 1 (HMGB1) protein promotes necrosis and inflammatory cell infiltration immediately after ischemia yet is influential in the recovery phase ${ }^{236}$. Not only is it important to identify these biphasic pathways, but it is also important to elucidate how pharmacologic manipulation alters outcome. If inhibition is possible in the acute phase, preventing further damage, will the beneficial activity still occur later on?

\subsubsection{CONCLUSION}

The dearth of success in translation of proposed therapeutic agents for ischemic stroke from bench-to-bedside necessitates a reassessment of experimental methodology. Proposed therapeutics have too often targeted events occurring rapidly after the onset of ischemia such as glutamate excitotoxicity, calcium release, and oxidative stress. Investigating pathologic events occurring at more delayed periods, such as inflammation and apoptosis, may prove more realistic targets and consequently, translate more effectively from bench-to-bedside. Notably, early results of minocycline clinical studies appear promising. Minocycline acts via targeting inflammation which occurs later after the onset of ischemia than many of the previously unsuccessful pharmacologic agents. Additionally, protection afforded by minocycline is not neuro-centric in nature and rather acts via effects on surrounding glia. Modulating the response of glia to injury, much like targeting the neurovascular unit, is more likely to translate to effective therapeutic development due to the large role glia play in both health and disease. Ischemic stroke is a vascular disease impacting all cellular types within the nervous system, not just neurons. Similarly, the only approved therapeutic, tPA, exerts its effects through the vascular system rather than direct effects on neurons. This alone indicates the potential to improve outcome without a neuro-centric focus.

Future studies must more closely replicate the clinical environment to increase the likelihood of translational success. This includes incorporating aged animals in preclinical studies as well as animals with comorbidities typically seen in the clinical stroke population such as diabetes and hypertension. Furthermore, models can be 
improved by incorporating tPA for combination therapy. Agents extending the window of opportunity for tPA treatment would represent a significant improvement in stroke treatment.

Despite past failures, stroke research must press forward based on the immense need. By thoroughly reassessing reasons for the previous lack of success and pursuing new avenues and approaches to treatment, new advances are likely possible. 


\title{
1.7 Jak2/Stat3-mediated Signaling in Ischemic Stroke
}

One area identified as a weakness in preclinical neural injury research is the largely 'neurocentric' focus in which other cell types, such as glia, and how these cell types contribute to injury and/or repair, has not been a point of emphasis. In this work, we explore the Jak2/Stat3 signaling pathway that has clear implications in numerous cell types such as neurons and astrocytes. Furthermore, this pathway serves as a prime example of the potential for biphasic signaling in which a signaling pathway may contribute to both protective and detrimental effects depending on the duration and target of signal.

This work was published in Neurological Disorders: New Research.

Citation: Turner RC, Lucke-Wold B, Miller DB, O'Callaghan JP, Rosen CL, Huber JD (2012) Neuropoietic Cytokines and Neural Injury: Alterations in JAK2/STAT3 Signaling Associated with Aging. Edited by Chloe E. Thomas and Jayden R. Moore. Neurological Disorders: New Research. Chapter II. Nova Science Publishers, Inc.

\begin{abstract}
:
Neuropoietic cytokines have long been recognized as playing a role in neural injury with recent work implicating the janus-kinase 2/signal transducer and activator of transcription 3 (JAK2/STAT3) signal-transduction pathway. Released in response to neural injury, neuropoietic cytokines modulate a diverse array of processes ranging from apoptosis to vascular remodeling to inflammation. Neural injury, particularly in the case of ischemic stroke, often is associated with advanced age yet the aging process is rarely taken into account in preclinical models. Notably, aging has been linked with inflammation through the 'inflam-aging' hypothesis in which dysregulation of the immune system and an inability to successfully handle pathologic stimuli occurs with aging. Therefore, the ability to modulate the effects of inflammation-related cytokines and cytokine-associated signaling is an attractive therapeutic target and has resulted in promising therapeutics for non-neural disease processes such as arthritis and autoimmune conditions. Here, we highlight the role of neuropoietic cytokines in neural injury and more specifically, those that mediate their effects through the JAK2/STAT3 signaling pathway. The consequences of STAT3 signaling and phospho-STAT3 (pSTAT3) activation are empahsized as well as factors that modulate signaling via this pathway, in particular the age-dependent regulation of suppressor of cytokine signaling 3 (SOCS3), and potential therapeutic targets discussed.
\end{abstract}

\subsubsection{INTRODUCTION}

The role of inflammation in neural injury, regardless of whether the injurious event is a stroke or traumatic brain injury, has long been recognized and represents a possible option for therapeutic development. Neuropoietic cytokines, such as the IL-6 family, are produced in response to ischemia. Release of neuropoietic cytokines occurs in a variety of cell types ranging from endothelial cells to those more commonly associated with the inflammatory response such as macrophages and microglia. Regardless of origin, these cytokines mediate much of the neuronal, glial, and immune response to injury through signaling cascades such as the Janus-activated kinase-signal transducer activator of transcription (JAK-STAT) and the mitogen-activated protein kinase (MAPK) pathway initiated by the gp130 receptor complex ${ }^{237}$. This work focuses on the JAK-STAT pathway (specifically, the JAK2/STAT3 variation) - its activation, downstream effects, and regulatory processes. Furthermore, the age-dependent effects after injury 
associated with the pathway are discussed based on work conducted in the laboratory of the authors.

\subsubsection{Neuropoietic Cytokine Family}

Cytokines represent a varied group of small autocrine and paracrine signaling proteins, previously associated primarily with the immune response, but more recently a role in development, disease, and injury has been recognized 237,238 . Cytokines are produced by multiple cell types and often exert diverse effects that are dependent on not only the stimuli resulting in secretion but also the timing of stimulation ${ }^{238}$. As such, the study of cytokines and downstream signaling effects is inherently complex but provide extensive opportunities for intervention and modulation for possible therapeutic benefit.

In this work we focus on the neuropoietic cytokine family as these cytokines have been identified as playing a central role in the inflammatory response to stroke and central nervous system (CNS) trauma ${ }^{239}$. This cytokine family includes interleukin-6 (IL-6), interleukin-11 (IL-11), neuropoietin, ciliary neurotrophic factor (CNTF), leukemia inhibitory factor (LIF), oncostatin M (OSM), cardiotrophin-1 (CT-1), and cardiotrophin-like cytokine (CLC) $237,240,241$. Members of the neuropoietic cytokine family share a signaltransducing receptor, glycoprotein-130 (gp130), and consequently exhibit similar structures, particularly secondary, as well as overlapping activities ${ }^{239}$.

Structurally, cytokine ligands are composed of two pairs of anti-parallel $\alpha$-helices that are connected by polypeptide loops. This arrangement results in the presence of a shared protein fold termed the 'four-helix bundle'. Each family of cytokines is delineated primarily by the length, and in some cases number, of the $\alpha$-helices. For example, interleukins (IL)-2, -3 , and -4 have shorter $\alpha$-helices (8-10 residues long) in comparison to neuropoietic cytokines which exhibit $\alpha$-chains composed of 10 to 20 residues. Lastly, the number of $\alpha$-helices can vary which is demonstrated by the presence of eight $\alpha$ helices in IL-5 and interferon $\gamma^{242}$.

\subsubsection{Role of the gp130 Cytokine Receptor Complex}

Signal transduction involving members of the neuropoietic cytokine family involves the initial formation of a ligand-receptor complex at the cell surface. These receptors often consist of multiple subunits, creating a receptor complex, with the subunit composition dictating the affinity for various extracellular ligands, in this case, the binding potential of different members of the neuropoietic cytokine family. Furthermore, neuropoietic cytokine family receptors are transmembrane in nature with ligand binding resulting in formation of a protein-signaling complex on the intracellular surface of the membrane. Based on the multi-subunit composition of the gp130 cytokine receptor complex and transmembrane nature of the overall signaling complex, an extensive number of signals can be integrated in terms of both initiating molecules binding to the various receptor complexes but also the induced intracellular signaling cascade. The numerous receptor complexes incorporating the gp130 receptor are highlighted in Table 1.3.

Table 1.3. Sites for neuropoietic cytokine binding for activation of JAK/STAT signaling

$\begin{array}{llll}\text { Ligand } & \text { Site I } & \text { Site II } & \text { Site III } \\ \text { LIF } & - & \text { gp130 } & \text { LIF-R } \\ \text { OSM } & - & \text { gp130 } & \text { LIF-R/OSM-R }\end{array}$




$\begin{array}{llll}\text { CNTF } & \text { CNTF-R } & \text { gp130 } & \text { LIF-R } \\ \text { CT-1 } & ? & \text { gp130 } & \text { LIF-R } \\ \text { IL-6 } & \text { IL-6R } & \text { gp130 } & \text { gp130 } \\ \text { IL-11 } & \text { IL-11R } & \text { gp130 } & \text { gp130 } \\ \text { CLC } & ? & \text { gp130 } & \text { LIF-R }\end{array}$

The multi-subunit nature of the receptor complex is required for intracellular signaling as only gp130, LIF-R, and OSM are capable of binding the corresponding ligand and inducing the intracellular signaling cascade. The other receptors are capable of binding the ligand but do not directly associate with intracellular signaling mediators due to either shortened cytoplasmic domains, in the case of IL-6R and IL-11R, or are bound to the extracellular membrane surface by lipid anchors, as is the case with CNTF-R. Therefore, these molecules must bind the ligand and then associate with gp130 for intracellular signaling to ensue (Figure 1.1.6) ${ }^{242}$.

gp130 - gp130 LIF-R - gp130

OSM-R - gp130

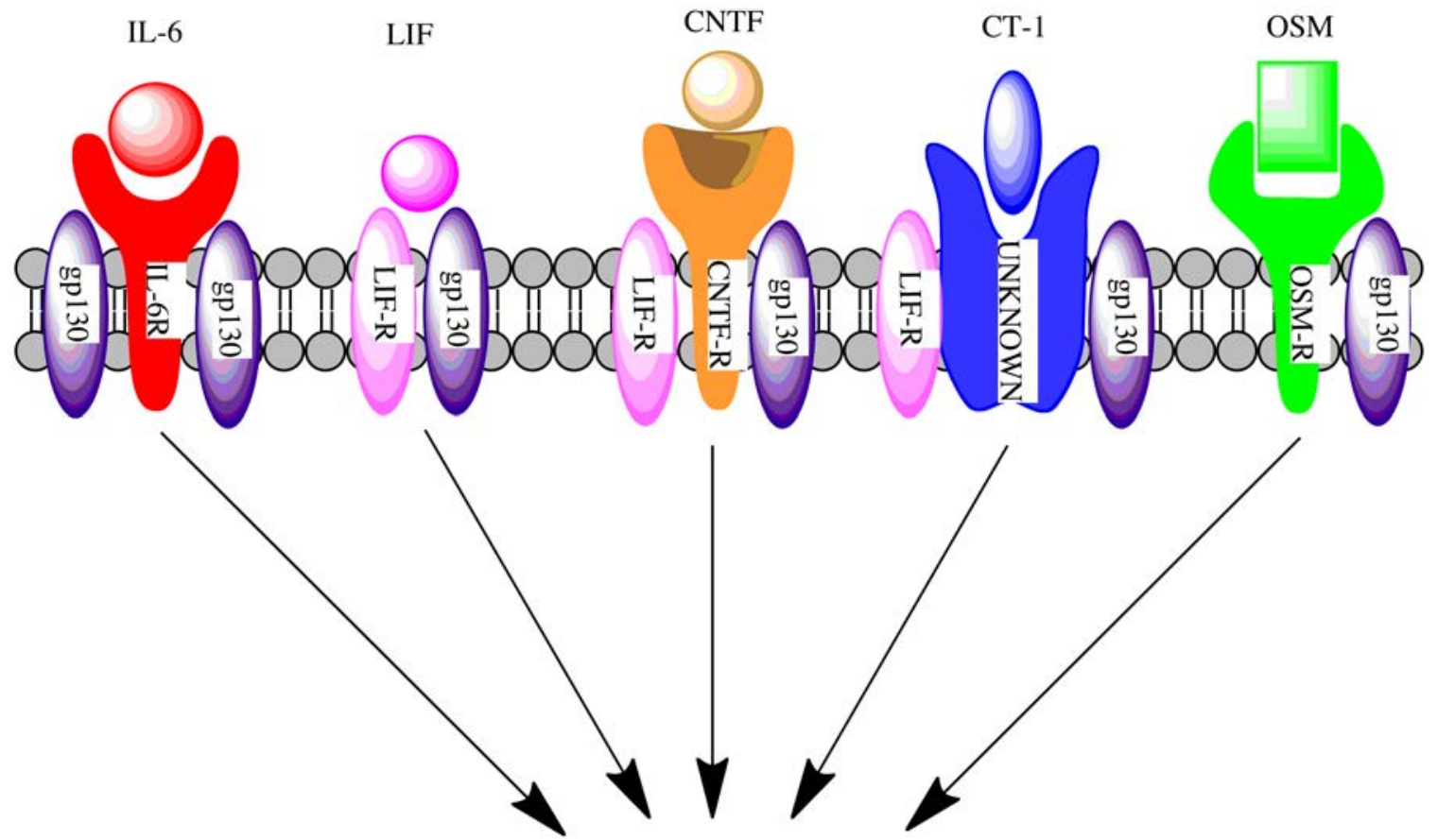

ACTIVATION OF JAK-STAT PATHWAY

Figure 1.15. Receptor subunits required for neuropoietic cytokine signaling. Each cytokine pictured requires a minimum of one gp130 receptor subunit to exert intracellular effects through this pathway.

Below we review Janus kinases and STAT3 as key downstream effectors of neuropoietic cytokines signaling through gp130 receptors. While beyond the scope of this brief review, it is important to note that other trophic signaling molecules can affect Jak/STAT3 signaling via crosstalk from other receptors and pathways (e.g MAPK, interferons, 
erythropoietin, etc.). These effects can occur at the level of Jak and/or STAT3 and can involve neuronal and glial responses to injury ${ }^{243}$.

\subsubsection{Role of Janus Kinases (Jaks)}

The ability of the various cytokine receptor families to exert biological effects via the induction of tyrosine phosphorylation following ligand binding has long been recognized 244. The Janus family of protein kinases (Jaks) have been identified as the primary means via which this initially extracellular signal results in intracellular effects. A variety of Jaks exist, many of which are expressed ubiquitously in mammals. These include Jak1, Jak2, and Tyk2. Others, such as Jak3, have also been identified but expression is not ubiquitous and in the case of Jak3 is localized primarily to hematopoietic cells. The specific Jak used for cytokine-based signaling depends upon the cytokine receptor utilized and more specifically, an interaction between the non-catalytic domain of the Jak with the membrane-associated region of the cytokine receptor chain ${ }^{245}$. The remainder of this work will focus specifically on JAK2.

Following ligand-receptor binding and associated conformational changes in the cytoplasmic portion of the receptor, Jak2 is rapidly phosphorylated, activating its kinase activity ${ }^{246}$. This initial phosphorylation has been attributed to ligand-receptor binding resulting in a transfer of $g$-phosphate of ATP to the tyrosine hydroxyl group on the receptor, which then causes an aggregation of the receptor and the associated Jaks, allowing for a transphosphorylation to occur amongst the Jaks 245, 247, 248. This transphosphorylation is necessary for kinase activity amplification as previous work has identified 10 major autophosphorylation sites with some being required for kinase activity. More specifically, the contribution of phosphorylation at the $Y^{1007}$ and $Y^{1008}$ have been studied in greater detail with mutation to $Y^{1008}$ revealing little effect on kinase activity while mutations in $Y^{1007}$ almost entirely eliminate kinase activity ${ }^{245}$. The varying kinase activity associated with mutated Jak phosphorylation sites illustrate the importance of protein phosphorylation as a regulatory mechanism in cell signaling and the role it likely plays in both homeostatic and pathologic processes.

\subsubsection{Activation of Signal Transducer and Activator of Transcription 3 (STAT3)}

Following activation, Jaks phosphorylate specific tyrosine residues of the receptor endodomains, allowing the binding of signal transducer and activator of transcription 3 (STAT3). The family of STAT proteins share structurally and functionally similar domains that include an amino-terminal domain (NH2), the coiled-coiled domain (CCD), the DNA binding domain (DBD), the linker domain, and the SH2/tyrosine activation domain. The key difference amongst individual members of the STAT protein family lies in the carboxy-terminal transcriptional activation domain (TAD) ${ }^{246}$. It is the $\mathrm{SH} 2$ domain, shared amongst the various STAT proteins, that allows binding to specific phosphotyrosine motifs. Consequently, the $\mathrm{SH} 2$ domain is described as being responsible for not only recruitment to the membrane-associated cytokine receptor but also for association with the activating Jak 246, 249, 250. Once bound to the Jak, STAT undergoes phosphorylation and subsequent activation. At this point, STAT dissociates from the receptor complex forming homo- or heterodimers. This process is once again dependent on the $\mathrm{SH} 2$ domain and interactions amongst phosphorylated tyrosine residues. The dimers then translocate to the nucleus where binding of response elements and gene transcription occurs ${ }^{247}$.

\subsubsection{Effects of phospho-STAT3 (pSTAT3)}


The effects mediated by PSTAT3 in the nucleus appear to be widespread and influenced by a number of factors ranging from the cell-type to the phosphorylation site. We will review some of the effects most commonly associated with the nervous and circulatory systems, particularly as they relate to neurologic disease. Some of these pathways are visualized in Figure 1.17.

\section{Cell Survival}

While initially identified as being involved in the acute-phase response associated with the inflammatory response, perhaps the most extensive research concerning STAT3 has been conducted in relation to cancer cell biology. Multiple works have illustrated the involvement of STAT3 in tumor transformation via regulation of suggested target genes such as c-myc, Bcl- $\mathrm{X}_{\mathrm{L}}$, and Fas ${ }^{251}$. As such, inhibition of STAT3 signaling has been suggested as a target for cancer therapy and appeared promising based on preclinical studies using malignant glioma cells both in vitro and in vivo. Application of a selective inhibitor of STAT3 phosphorylation suppressed additional tumor cell replication and promoted apoptosis through activation of Bax and suppression of c-myc, Bcl-X, and Mcl-1 ${ }^{252}$. Anti-apoptotic effects of STAT3 signaling, via effects on Bcl-2, have also been observed in cardiovascular studies. In a myocardial ischemia post-conditioning study, administration of AG490, a potent Jak2 inhibitor, resulted in reduced p-STAT3 and increased apoptosis following post-conditioning ${ }^{253}$. Furthermore, attempts have been made to generate Stat3 knockouts but this leads to early embryonic lethality ${ }^{254}$. Consequently, utilization of 'floxed' STAT3 alleles has been pursued generating tissue specific knockouts, allowing the role of STAT3 to be studied with regards to numerous processes $246,255,256$.

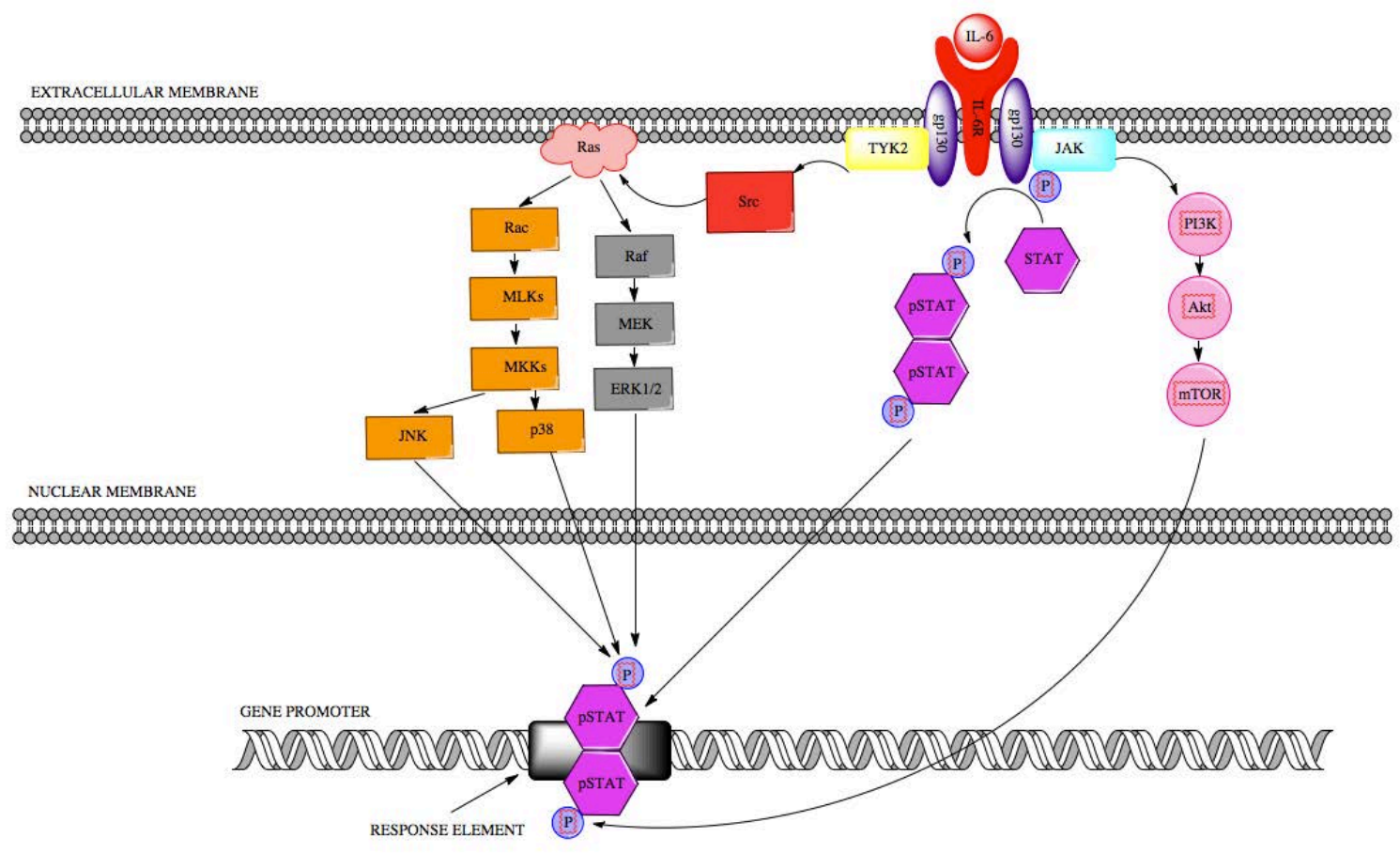


Figure 1.16. Complexity associated with the STAT3 pathway. Numerous pathways converge to act on STAT3 to modulate the cellular response. Notice the ability of different pathways to phosphorylate STAT3 at different sites. This change in structure induced by pohosphorylation may be responsible for the contrasting effects observed.

\section{Remodeling the Cellular Environment}

In addition to its possible oncogenic role in the regulation of cell survival, STAT3 activation may lead to a remodeling of the extracellular environment through effects on vascular endothelial growth factor (VEGF), basic fibroblast growth factor, and matrix metalloproteinase-2 (MMP-2) ${ }^{257}$. While this work was initially conducted in glioblastoma cell lines, it is likely applicable to other neurologic disease conditions due to some similarities in the cellular stress experienced. Glioblastoma, the most common and malignant primary brain tumor, is characterized by a relatively unique pseudopalisading necrosis in which a necrotic core is surrounded by a hypercellular zone. Notably, the pseudopalisading cells experience greater levels of hypoxia than surrounding regions and consequently, genes regulated by hypoxia are overexpressed in these areas ${ }^{257}$. These genes include hypoxic inducible factor-1 (HIF-1) and the aforementioned VEGF and MMP-2 $258-260$.

Besides vascular and extracellular matrix remodeling associated with oncogenic-induced changes in STAT3 signaling, vascular injury and associated neointima formation has been linked to STAT3 signaling as well. More specifically, following vascular injury, angiotensin II (Ang II) induces proliferation of vascular smooth muscle cells (VSMC). This process results in neointima formation and the deposition of extracellular matrix mediated through the rennin-angiotensin system. Seki and colleagues further investigated this work and the role of STAT3 signaling showing an induction of STAT3 phosphorylation through Ang II type 1 receptors. Inhibition of STAT3 signaling via AG490 administration resulted in reduced neointima formation in the injured artery ${ }^{261}$.

\section{Regulating Oxidative Stress}

A key element associated with inflammation and disease, as well as the aging process, is the regulation of oxidative stress. As such, Manea and colleagues investigated the role of the JAK/STAT pathway in the regulation of oxidative stress via NADPH oxidase in aortic smooth muscle cells. It was discovered that STAT3 is involved in controlling the transcription of p47phox and p67phox, two key subunits contributing to NADPH oxidaseassociated oxidative stress ${ }^{262}$. Regulation of NADPH oxidase function by STAT3 has also been demonstrated in a model of retinopathy of prematurity $(\mathrm{ROP})$ rat model and more specifically, the contribution of oxidative stress in the development of intravitreous neovascularization ${ }^{263}$. Furthermore, IL-6 and associated signaling via STAT3 controls the transcription of antioxidant enzyme promoters, mainly manganese-containing superoxide dismutase (Mn-SOD) ${ }^{264-266}$. This finding is consistent with others in the literature identifying increased oxidative stress after focal brain injury in IL-6-deficient mice ${ }^{267}$. Conversely, inhibiting IL- 6 has been identified as potentially therapeutic through a reduction in oxidative stress, specifically that generated by inducible nitric oxide synthase ${ }^{268}$.

Immune Tolerance 
The regulation and response of the immune system to both acute and chronic neurodegenerative diseases has garnered increasing attention in the scientific community in recent years. This is likely due to the promise offered by modulating these processes for potential therapeutic benefit. Pharmacologic agents such as minocycline, described as an anti-inflammatory and protease inhibitor, have reached clinical trials for the treatment of stroke yet little remains known about how precisely these agents work 269. In attempting to better understand regulatory processes of inflammation, STAT3 has been identified as a candidate pathway ${ }^{270}$. In fact, STAT3 has been shown to prevent macrophage activation in vitro, limiting the inflammatory response ${ }^{254,271-273}$. Inhibition of STAT3, in an attempt to reverse immunotolerance seen in many tumor studies, results in increased production of IL-12 by macrophages and a reversal of systemic immunotolerance. This principle of restoring immune tolerance was shown by Hussain, et al in patients afflicted with glioblastoma multiforme, illustrating the potential of such approaches not only for cancer patients but also other neurologic diseases involving a significant immune component ${ }^{270}$.

Besides playing a clear role in the immunoregulation and tolerance associated with CNS-based malignancies, STAT3 has been implicated in cardiovascular conditions. Inflammation induced via lipopolysaccharide (LPS) administration resulted in increased cardiomyocyte apoptosis in animals with cardiomyocte-restricted knockout of STAT3. Similarly, while young animals with the restricted knockout exhibited no outwardly visible signs of heart failure, aging of these animals resulted in spontaneously developing cardiac dysfunction ${ }^{274}$. As such, it is clear that STAT3 plays an essential role in modulating inflammation and cell survival, processes that may become even more important with aging based on the 'inflam-aging' hypothesis ${ }^{60}$.

\section{Role of the Phosphorylation Site}

STAT3 possesses multiple phosphorylation sites but the two primary sites implicated in modulating its functional activity are tyrosine $705\left(\mathrm{Y}^{705}\right)$ and serine $727\left(\mathrm{~S}^{727}\right)$. Thus, STAT3 DNA-binding activity appears to be regulated by the phosphorylation status of these key residues, once again illustrating the importance and regulatory control exhibited by site-specific protein phosphorylation state. For example, Wen et al reported serine phosphorylation controlled transcription but did not affect DNA-binding in both STAT1 and STAT3 ${ }^{275}$. The role of serine phosphorylation appears controversial though as others have shown a range of effects including potentiating tyrosine phosphorylation and DNA binding, inhibiting tyrosine phosphorylation, or having no effect on either activity. Therefore, it has been proposed that the effects of serine-727 phosphorylation likely vary across cell types and in response to various stimuli 276,277 . This site also is one source of interaction/overlap amongst various pathways with others identifying roles of $\mathrm{mTOR}$ and MAPK in regulating $\mathrm{S}^{727}$ phosphorylation ${ }^{278,279}$. Given that mulitple other phosphoryaltion sites exist on STAT3, their phosphorylation status likely could affect tyrosine $705\left(\mathrm{Y}^{705}\right)$ and serine $727\left(\mathrm{~S}^{727}\right)$ phosphorylation. Moreover, based on findings for other key signaling modules in the CNS, regulation of the phosphorylation of tyrosine $705\left(\mathrm{Y}^{705}\right)$ and serine $727\left(\mathrm{~S}^{727}\right)$ likely would be affected in a tissue and cell-type specific fashion ${ }^{280}$.

\subsubsection{Regulating pSTAT3: Role of Negative Regulators}

In addition to understanding the transcriptional events regulated by pSTAT3, much work has been completed regarding the regulation of Jak2/Stat3 signaling. Three classes of negative regulators exist. These include SOCS (suppressors of cytokine signaling), 
PIAS (protein inhibitors of activated stats), and PTPs (protein tyrosine phosphatases) 281. Each of these inhibitory pathways has been extensively reviewed elsewhere and details beyond those required for a basic understanding of the material are beyond the scope of this work ${ }^{282}$.

\section{Suppressors of Cytokine Signaling (SOCS)}

The SOCS family of regulatory proteins consists of eight plus members, each of which shares a common $\mathrm{SH} 2$ domain and in some cases, a small kinase inhibitory region. SOCS is somewhat different in comparison to the other regulatory proteins as it is a clear negative feedback loop in which cytokine stimulation results in increased transcription of SOCS, consequently downregulating cytokine-induced signaling ${ }^{281}$.

\section{Protein Inhibitors of Activated Stats (PIAS)}

The PIAS family of negative regulators remains somewhat of a mystery as only one conserved motif, a zinc-binding motif, has been identified. PIAS3, the STAT3-specific PIAS, appears to only interact with p-STAT3. The precise interaction is not clear but it may bind monomers, preventing dimmer formation or may facilitate the degradation of aforementioned dimers ${ }^{283}$.

\section{Protein Tyrosine Phosphatases (PTP)}

In addition to the activated gp130 receptor attracting cytoplasmic STAT, it also attracts PTP SHP2, a cytoplasmic PTP that binds the active site of the receptor and unfolds the protein. Numerous other PTPs are frequently available as a range of subtypes, some of which alter the kinetics of STAT3 activation whereas SHP2 is thought to regulate the total amount and amplitude of STAT3 activation ${ }^{284}$. This is notable in that modulation of the kinetics may partially be responsible for differences in downstream effects associated with other molecules, besides those in the neuropoietic cytokine family, that also signal through STAT3.

\subsubsection{DISCUSSION}

\subsubsection{Changes in Neural Injury}

While it is likely STAT3 signaling is altered in the vast majority of neurologic and neurodegenerative diseases due to the large number of potential signaling events relayed through this transducing element, most work focusing on STAT3 has been conducted with an emphasis on stroke and traumatic brain injury. For this reason, we will limit our discussion to these two conditions.

\section{Stroke}

In the past 10 years vast strides have been made in attempting to elucidate the role of STAT3 signaling on both the outcome from ischemia as well as the role played in modulating the benefit of ischemic preconditioning. We will first discuss findings in ischemia and then identify potential roles of STAT3 signaling in modulating the protective responses seen in ischemic preconditioning.

In the year 2000, one of the first papers related to STAT3 and ischemia was published by Justicia, et al. In this work, STAT3 was shown to be activated following ischemia, evidenced by translocation to the nucleus. This response was particularly pronounced in 
reactive astrocytes, identifying a possible role for regulation of astrocyte growth and expression of glial fibrillary acidic protein (GFAP). Importantly, changes in STAT3 regulation were seen in the neuron and microglial cells as well ${ }^{285}$. One substantial weakness of this preliminary work was the lack of STAT3 phosphorylation status data. This issue was remedied in the work of Wen and colleagues. In a mouse model of ischemia, phosphorylated STAT3 was found to be upregulated as early as 6 hours after ischemia. This increase though was found in peripheral regions of the ischemic territory and was constrained to neurons only - not astrocytes or microglia. Importantly, p-STAT3 positive neurons were identified as also being TdT-mediated biotinylated UTP nick end labeling (TUNEL)-positive, illustrating a potential role of p-STAT3 in modulating ischemia-induced neuron death ${ }^{286}$. Following up on these developments, Yamashita and colleagues assessed the effect of IL-6 inhibition on STAT3 activation and survival in a mouse model of ischemia. An IL-6 receptor antibody was administered immediately following ischemia and while successfully decreasing p-STAT3, IL-6 signaling blockade lead to increased apoptosis and subsequently an increased infarct size as well as deterioration in neurologic function ${ }^{287}$. This work illustrated the potential role of IL-6 as a neuroprotectant, particularly in the acute phase following injury. Similarly, more recent work identifying the contribution of p-STAT3 in regulating oxidative stress via the expression of antioxidants such as Mn-SOD, has shown IL-6 to be neuroprotective ${ }^{264}$, 266. Notably, both of these studies are in contrast to the findings of Satriomoto, et al in which inhibition of STAT3 signaling, whether it be via non-specific JAK2 phosphorylation inhibitor AG490 or siRNA, resulted in improved outcome as determined using infarct volume, functional assessments, and histologic studies. Recent work investigating the roles and regulation of p-STAT3 in a clinically relevant, thromboembolic rodent model of stroke has been conducted in our laboratory. This work will be described in greater detail in subsequent sections concerning age-related effects.

Preconditioning has been recognized in numerous injury modalities as a protective technique. Generally, the mechanisms through which preconditioning is believed to work are poorly elucidated. Common themes identified as potentially mediating the effects of preconditioning typically center around inflammation and a 'priming' of the immune response. Recent work by Yagi and colleagues sought to identify the role of STAT3 in a rodent model of global ischemic preconditioning. STAT3 phosphorylation at $S^{727}$ was shown to be protective and necessary for modulating the effects of ischemic preconditioning. Notably, phosphorylation at $S^{727}$ in preconditioning studies is associated with protein kinase C (PKC) $\varepsilon$-Raf-mitogen-activated protein kinase (MAPK)/ extracellular signal-regulated kinase kinase (MEK)-p44/42 MAPK signaling pathway ${ }^{288}$.

In addition to preclinical studies implicating the role of neuropoietic cytokines in the response to injury, Shakarishvili and colleagues assessed the correlation of numerous inflammatory cytokines with outcome from stroke in both young and aged patient populations. Elevated levels of IL-6 following the onset of ischemia, particularly in the acute period, were found to be a strong predictor of poor outcomes in the aged patient population ${ }^{289}$. Therefore, it appears likely that neuropoietic cytokines and associated STAT3 signaling may play a role in influencing clinical outcomes as well.

\section{Traumatic Brain Injury}

Less controversy appears to exist in the TBI literature concerning the role of p-STAT3 signaling. Numerous studies have been completed characterizing the expression of $p$ STAT3 following TBI as well as neuropoietic cytokines, such as IL-6, that would lead to p-STAT3 activation. The knock-out of IL-6 or application of a STAT inhibitor both result in 
worsened outcome following TBI $267,290-292$. One controversy does exist though in terms of the cell-type(s) in which p-STAT3 signaling is localized with astrocyte expression being more well-accepted and neuronal expression appearing slightly more controversial 291,292 . The conditional deletion of STAT3 in a mouse model of spinal cord injury results in enhanced inflammatory responses, increased lesion volume and reduced functional recovery, effects associated with astrocyte-specific responses related to scar formation 255

While clinical studies have not begun to elucidate the role of STAT3-associated signaling, the emphasis on biomarker discovery has led to identification of numerous cytokines following injury at an extensive list of time-points. These cytokines include those such as IL-6, which in fact, has been shown to correlate with intracranial pressure elevation following injury in the clinical population ${ }^{293}$.

\subsubsection{JAK2/STAT3 Regulation Altered With Aging}

The role of aging in neurologic disease has long been recognized, particularly as it relates to stroke, $A D$, and Parkinson's Disease (PD). Similarly, aged individuals generally fare far worse following neurologic injury, whether it be stroke or traumatic in origin, when compared to a younger population. As such, it has been hypothesized that the aged brain is fundamentally different than the young-adult brain. This hypothesis, in conjunction with our past work and reports of altered cytokine expression in the aged brain, is what led to our initial investigation of the Jak2/Stat3 pathway ${ }^{32,56,84}$.

In our investigation of the effect of age on STAT3-associated signaling, we confirmed previous reports demonstrating increased p-STAT3 as early as 6 hours and persisting to 72 hours in young-adult animals. When using aged animals, a scenario we believe far more representative of the aged patients afflicted with stroke, we found increased SOCS3 expression in comparison to young-adult animals. While the mechanism for this change in SOCS3 expression remains to be elucidated, it is clear that alterations pSTAT3 signaling may account for differences in outcome between young-adult and aged animals. One possibility is changes in STAT3 isoform expression associated with aging as the IL-6-mediated induction of SOCS3 is entirely dependent on STAT3 $\alpha^{144}$. A second possibility is alterations in an overlapping pathway or stimulus such as interferon-gamma (IFNgamma). IFNgamma has been shown to be capable of regulating SOCS3 and furthermore, is increased with age, raising the possibility that increased levels of IFNgamma are ultimately responsible for these age-related changes in SOCS3 expression and subsequent pSTAT3 signaling ${ }^{294,295 .}$

The notion that aging alters not only the initially inflammatory stimulus but also the response is consistent with findings by Liu and colleagues related to development and regeneration, furthering the concept that the response of the aged brain is altered in comparison to the young or young-adult brain ${ }^{296}$.

\subsubsection{Significance of Phosphorylation Site}

Studying STAT3 is significantly complicated by the multiple phosphorylation sites present on the molecule and the potential significance of each site in eliciting transcription following DNA binding (discussed above). As described previously, the $\mathrm{C}$ terminus contains the TAD segment responsible for transcriptional regulation. It remains unclear exactly how these domains regulate activity, numerous works have shown a promotion of transcription following serine phosphorylation $\left(S^{727}\right)^{297,298}$. Notably, the serine site is 
a mitogen-activated protein kinase (MAPK) phosphorylation motif, illustrating the complex nature of STAT3 signaling and the simultaneous interaction of multiple pathways 275, 299 . Nevertheless, at least for the case of neurotoxic injury of the CNS, STAT3 can translocate to the nucleus of astrocytes via phosphorylation of tyrosine 705 $\left(\mathrm{T}^{705}\right)$ in absence of a change in phoshorylation of serine $727\left(\mathrm{~S}^{727}\right){ }^{300}$. Importantly, the effects of phosphorylation at $S^{727}$ have not been widely considered in vivo and remain to be clearly elucidated, particularly in relationship to neural injury. Whether high-quality antibodies allowing such investigation are available remains to be clearly shown and may be a limiting factor currently.

\subsubsection{FUTURE DIRECTIONS}

\subsubsection{Elucidate the Role of STAT3 Across Cell Types}

Advances in therapeutics related to STAT3 signaling are likely to require an increased understanding of the response of various cell types to STAT3 signaling. More specifically, the timing and downstream effects must be elucidated in each cell type. The investigation into cell type specific responses has been recognized by others in the literature and is exemplified by the work of Haroon and colleagues. They showed, in an experimental autoiummune encephalomyelitis study, that loss of gp130 on astrocytes resulted in increased astrocyte apoptosis. Interestingly, the lack of gp130 expression in neurons did not alter the course of the disease in comparison to the control condition ${ }^{301}$.

In addition to possible discrepancies in the benefit/detriment of STAT3 signaling associated with different cell types, the timing of responses may also prove critical. This notion was reviewed by Dziennis and colleagues and notably, STAT3 phosphorylation tends to occur at earlier time points, namely 30 minutes to 24 hours, in neurons following injury but tends to arise later in astrocytes, microglia, and endothelial cells ${ }^{302}$. As such, perhaps there is some form of biphasic response in which STAT3 signaling and associated effects are beneficial in certain cell types during the acute period and detrimental in the chronic period. The opposite may be true for other cell types, therefore conveying both a cell-specific and time-dependent response.

This concept appears consistent with the multi-factorial role of STAT3 signaling and it's presence in development and disease. In the neuron, phosphorylated STAT3 is increased following ischemia and correlates with TUNEL-positive neurons ${ }^{286}$. On the other hand, STAT3 has been identified as playing a key role in synaptic plasticity, an important factor in not only normal development but also recovery from injury ${ }^{303}$. The complex role of astrocytes and implications of STAT3 signaling following neurologic injury provide yet another example of the cell- and time-dependent effects. Astrocyte activation and associated glial fibrillary acidic protein expression has been recognized as a consequence of neural injury and subsequent induction of gp130-related cytokines ${ }^{300}$. Activation of astrocytes results in the potentiation of astrogliogenesis and may also be involved in regulating other neurologic functions such as pain 304,305 . Perhaps most importantly, spinal cord injury studies have indicated the time-dependent effects of reactive astrocytes. In the acute phase, astrocytes have been implicated in tissue repair and functional restoration yet in the chronic phase, astrogliosis impairs axonal regeneration via both physical and chemical barriers ${ }^{256}$. Other studies in spinal cord and peripheral nerve injury identified a rapid induction of JAK2/STAT3 signaling, primarily in microglial cells, following injury. Blockade of this signal, via SOCS3, attenuates the development of mechanical allodynia, a common symptom associated with impactinduced neuropathic pain ${ }^{306}$. The prevention of STAT3 activation in microglia following 
electromagnetic field stimulation results in decreased release of nitric oxide (NO) and other inflammatory mediators such as TNF $\alpha$. Taken together, these studies indicate widespread effects of STAT3 signaling across cell types and the likely time-dependent response.

\subsubsection{Modulating Beneficial vs. Detrimental Effects}

One of the many challenges in understanding the role of STAT3 signaling, and potentially targeting the pathway therapeutically, is determining when, where, and to what extent STAT3 serves beneficial versus detrimental effects. Much like has been demonstrated in other inflammatory pathways associated with injury, such as matrix metalloproteinase activation, the initial response to injury may be damaging whereas the chronic response may be essential for recovery ${ }^{151}$. This phenomenon is certainly not unique to activation of matrix metalloproteinases and has been described in relation to astrocyte reactivity as well 256,307 . Interestingly, one of the primary regulators of STAT3 signaling, SOCS3, has been implicated in the modulation of Wallerian degeneration following peripheral nerve injury. In addition to regulating STAT3, SOCS3 may decrease expression of the associated cytokines such as IL-6 and in doing so may prevent continual high levels of expression associated with inflammation ${ }^{308}$. The timing of SOCS3 expression is important though as IL-6 and LIF play a role in both neuronal survival and regeneration following injury, further indicating a need to understand the precise timing of signaling events post-injury.

It is this dynamic between the potential beneficial results of neuroinflammation and the detrimental effects of sustained or excessive inflammation that must be more clearly elucidated. IL-6, and subsequent activation of STAT3, has been implicated in the acute phase response to inflammation, which may be beneficial, and also injury but the overall effects remain unclear 300,309 .

\subsubsection{Identifying Injury-Associated Changes}

Many neurologic disease conditions involve a vascular component and remodeling of the neurovascular unit, both during the aging process and as a consequence of injury. This is particularly true in stroke and traumatic brain injury and has implications for both injury and repair of the neurovascular unit and in particular, the blood-brain barrier. Proteases are commonly involved in this process with matrix metalloproteinases being the most well-described. STAT3 signaling has been implicated in this process in through work in ischemia as well as cancers such as glioblastoma through a link with microRNA-21 310-313. MicroRNAs have been recognized for their role in attenuation of protein expression via complementary binding to target mRNA and in doing so exert numerous potential effects both directly and indirectly. As such, the interaction of these various signaling pathways and intervening molecules likely requires greater investigation and understanding for proper therapeutic development.

\subsubsection{Understanding \& Manipulating Regulatory Processes for Therapeutic Benefit}

As discussed previously, the regulation of STAT3 signaling is a dynamic process involving not only ligand-receptor binding but also a series of downstream effectors such as SOCS, PIAS, and PTP. How these processes are altered with respect to various disease states, likely in a cell-specific and time-dependent nature, remains to be clearly elucidated. Furthermore, how these processes are altered by aging, which is the greatest risk factor for many neurologic diseases, remains to be seen. We have shown in our initial work that SOCS3 is upregulated following ischemia in the aged rodent 
producing a decrease in phosphorylated STAT3 not seen in the young-adult rodents ${ }^{51}$. Consequently, it seems not only plausible but likely that the response of the aged brain to injury is fundamentally unique and requires further investigation. Once again, the celltype and time-dependent nature of these processes, and how it impacts STAT3 signaling, may be fundamentally altered with aging and disease.

\subsubsection{The IL-6 \& IL-10 Signaling Dynamic}

Despite IL-6 and IL-10 both exerting effects primarily through modulation of STAT3, the response to each cytokine is notably unique. In macrophages, both cytokines have been recognized as anti-inflammatory in nature with IL-10 acting as the more potent antiinflammatory cytokine relative to IL-6. Three key elements emerged from this work in macrophages by Niemand, et al. First, pretreatment of macrophages with inflammationinducing substances such as LPS resulted in blockade of IL-6 induced STAT3 activation. This was not true of IL-10 signaling. Secondly, the order and timing of cytokine expression appears to dictate STAT3 signaling based upon pretreatment of macrophages with IL-10 impairing future IL-6-induced signaling via STAT3. Importantly, when IL-6 was administered first, IL-10 signaling was not affected. Lastly, the regulation of signaling via each cytokine differs. IL-6, and the associated gp130 receptor, attracts SOCS3, producing a negative feedback loop. This is in contrast to IL-10 and its associated receptor does not interact with SOCS3. 314 Importantly, some discrepancy does exist concerning the effect of inflammation induced via LPS administration on IL-6 and STAT3 levels as others have shown increases following inflammation. As such, it is important to consider the effect of priming or preconditioning on cellular response ${ }^{315}$.

Understanding the surely complex interaction between cytokines such as IL-6 and IL-10, particularly the similarities and differences present, may lead to breakthroughs in the development of therapeutics targeting this pathway. Furthermore, many questions remain concerning the relationship between $\mathrm{IL}-6$ and $\mathrm{IL}-10$ such as whether the signaling dynamic described above holds true for all cell types, does STAT3 undergo identical phosphorylation regardless of its inducing cytokine, what is the role of prior inflammation, and are these effects consistent across time points? Are acute phase IL-6 mediated increases in pSTAT3 distinct, perhaps in brain region and cell type from injury related increases in IL-6 and pSTAT3? Perhaps the most important question raised by studies concerning the effect of prior inflammation on IL-6 and IL-10 induced STAT3 signaling is how this may relate to models of disease involving an inflammatory component. For example, the aged brain is known to exist in a state of low-grade inflammation and how this reality alters STAT3 signaling is only beginning to be uncovered. Interestingly, our laboratory has shown an attenuation of STAT3 signaling associated with increased SOCS3 expression in the aged rodent following MCAO, a finding consistent with that described above in which prior inflammation leads to an attenuation of IL-6-induced STAT3 signaling 51,314. How this finding may apply to other models of neurologic disease commonly affecting the aged population remains to be seen.

Numerous challenges are likely to arise in attempting to address the questions stated previously. These included the complexity of phosphoprotein regulation, the presence of multiple STAT3 isoforms, isolation of effects in a given cell type in a biologicallymeaningful environment, and overlapping/intersecting pathways creating a complicated dynamic of both redundancy and abundance of effects. Phosphoprotein regulation, particularly when multiple sites are present, is inherently more difficult to study appropriately. Furthermore, depending on the inhibitor chosen, one or both phosphorylation sites may be blocked. For example, the use of AG490 has been shown 
to inhibit $\mathrm{Y}^{705}$ phosphorylation of STAT3 but not $\mathrm{S}^{727}$ in an in vitro astrocyte-stress model, possibly due to overlapping pathways ${ }^{316}$. The notion of intersecting pathways further complicating studies is expanded upon by Planas, et al. In this work, STAT1 and STAT3 are discussed highlighting the ability of anti-inflammatory cytokines like IL-10 to activated STAT3 but not STAT1 whereas pro-inflammatory cytokines like IFN-gamma induce STAT1 but not STAT3. IL- 6 on the other hand activates both pathways ${ }^{317}$. Lastly, work concerning STAT3 may need to focus on isoform-specific events. STAT3 is found in two isoforms: $\alpha$ and $\beta$. STAT3 $\beta$, long thought to be the dominant negative isoform, has been shown to exert a negative effect on the synthesis of inflammatory cytokines and may actually increase synthesis of anti-inflammatory cytokines. The $\alpha$ isoform on the other hand has been implicated in control of SOCS3 ${ }^{144}$. As such, it is clear that while current and past studies have greatly expanded our knowledge of STAT3 and associated signaling, many questions remain unanswered, particularly those related to the study of neurologic disease.

\subsubsection{Therapeutic Development: Challenges of Inhibition}

The challenges of developing therapeutics to modulate proinflammatory pathways, particularly for neurologic disease, are numerous and consist of course of timing, dosage, and delivery challenges. For example, IL-6 antibodies have been developed for the successful treatment of rheumatoid arthritis in preclinical models but are complicated by issues such as delivery across the BBB and immunogenicity challenges ${ }^{318}$. Other challenges include the use of inhibitors that can't penetrate the blood-brain barrier and inhibit aspects of the signaling cascade upstream of STAT3, such as AG490 ${ }^{319}$.

Perhaps the greatest challenge to the development of therapeutics targeting STAT3 signaling is when to administer them, for how long, and to what cells. It appears that STAT3-induced effects likely vary from cell-type to cell-type, making cell-specific inhibitors desirable. Furthermore, methods of inhibition utilizing SOCS3 are now available. These include genetic overexpression or downregulation as well as pharmacologic mimetics ${ }^{320}$.

Brain inflammatory responses signaling through STAT3 represent promising therapeutic targets. STAT3 signaling, while complex in nature, seems to have powerful effects on a variety of cell types important for the study of neurologic disease and allows for targeting of multiple aspects of pathophysiology ranging from ECM modification to angiogenesis to apoptosis. Similarly, STAT3 signaling plays a role in not only initial injury but also represents a promising therapeutic avenue for repair and recovery via effects on neural stem cells and the modulation of neuroplasticity 237,303 .

\subsubsection{CONCLUSION}

The effects of STAT3 signaling are diverse and likely play a significant role in modulating the body's response to neural injury. While STAT3 has been implicated in vascular remodeling, control of apoptosis, and regulation of oxidative stress, much remains unknown concerning its regulation in response to injury. Following stroke and traumatic brain injury, STAT3 signaling is altered across a range of cell types, with each undergoing time-dependent changes. The precise role STAT3 plays in the initial injury and subsequent recovery phase remains to be elucidated but represents a potentially promising therapeutic target. Similarly, aging is associated with the development of many neurologic diseases and alters the STAT3 signaling pathway in response to injury, once again demonstrating the need to consider age in the study of neurologic disease. 


\title{
1.8 Protein Kinase C as a Therapeutic Target for Ischemic Stroke
}

Another potential therapeutic target that appears promising in a variety of neural injury models (ischemic stroke, traumatic brain injury, and Alzheimer's disease) is regulation of protein kinase C. In this work, we explore the current status of protein kinase C research and promising future directions. Much of this work sets the stage for one of our publications discussed later in this dissertation relating to bryostatin-1 for ischemic stroke.

This work was published in Ischemic Stroke: Symptoms, Prevention and Recovery.

Citation: Turner RC, Lucke-Wold B, Tan Z, Rosen CL, Huber JD (2013) Modulation of Protein Kinase C Isoforms: A Potential Therapeutic for Ischemic Stroke? Chapter 6. Ischemic Stroke: Symptoms, Prevention and Recovery. Edited by Virag Lakatos and Balazs Somogyi. Nova Biomedical. Pgs. 171-190.

\begin{abstract}
Manipulation of the protein kinase C (PKC) signaling pathway has been recognized as a potential therapeutic target for numerous disease states, the most well-investigated being cardiovascular disease. Recent discoveries in preclinical models of neurologic disease ranging from Alzheimer's disease (AD) to traumatic brain injury (TBI) to stroke have identified changes in PKC expression and in some cases, successfully targeted this pathway therapeutically. The effects of altered PKC expression following injury remain under investigation with some studies implicating PKC in the regulation of oxidative stress, apoptosis, inflammation, and vascular remodeling. Additionally, various PKC isoforms may be involved in both beneficial and detrimental processes following injury as evident in the role of PKC mediating neuroprotection in hypoxic preconditioning but also being associated with blood-brain barrier (BBB) disruption in other studies. Furthermore, preclinical studies of neurologic injury and disease often fail to emphasize the effect of aging, despite the fact that age is the greatest risk factor for numerous neurologic diseases such as stroke and AD. This is of particular relevance in the regulation of $\mathrm{PKC}$ function as aging has been shown to alter enzyme translocation, thus altering function. This work reviews the location and pathways associated with various $\mathrm{PKC}$ isoforms, the role of PKC isoforms in the aging process, as well as implications of PKC signaling following neurologic or cerebrovascular injury. We provide insight into potential downstream pathways of interest and the potential role of PKC modulation as a therapeutic target for ischemic stroke based on findings in not only preclinical studies of stroke but also studies of other neurologic and cardiovascular diseases.
\end{abstract}

\subsubsection{INTRODUCTION}

\subsubsection{PKC Isoforms - A Lesson in Structure-Function Relationships}

The PKC family is a series of threonine/serine kinases. Up to twelve different isoforms exist each with their own actions and specific pathways of activation. All isoforms are present in some form or another within the brain ${ }^{321}$. These isoforms have been grouped into three classifications: conventional, novel, and atypical. The classification is based on the unique polypeptide chains and the cofactors associated with each group. The $\mathrm{NH} 2$-domain is specific for the membrane phospholipids that interact with PKC during translocation. The $\mathrm{COOH}$-domain is specific for the activating cofactors, and the $\mathrm{C} 2$ domain has binding capacity for receptors for activated $\mathrm{C}$ kinases (RACKs). Conventional isoforms (cPKC $\alpha,-\beta I,-\beta I I$, and $\gamma$ ) require calcium, phosphotidylserine (PS), 
and diacylglycerol (DAG) for activation. The novel isoforms (nPKC $\delta,-\varepsilon,-\eta,-\theta$, and $-\mu$ ) require PS and DAG but not calcium. The atypical isoforms $(\mathrm{aPKC} \zeta,-\lambda)$ are unresponsive to DAG, PS, and calcium (Figure 1.18). Both cPKCs and nPKCs activate the extracellular signal-regulated kinase (ERK) and mitogen-activated protein kinase (MAPK) cascade among other pathways ${ }^{322}$. These pathways will be discussed in detail in later sections. In investigating PKCs, it was discovered that a panel of nine genes express the phospholipid-kinases. These genes produce the PKCs in various tissues throughout the body and account for their broad distribution and functions. PKC $y$, $\mathrm{PKC} \delta, \mathrm{PKC} \alpha$, and $\mathrm{PKC} \varepsilon$ are the most predominant isoforms associated with neurologic diseases. Other isoforms play more predominant roles in other organ systems such as the heart.

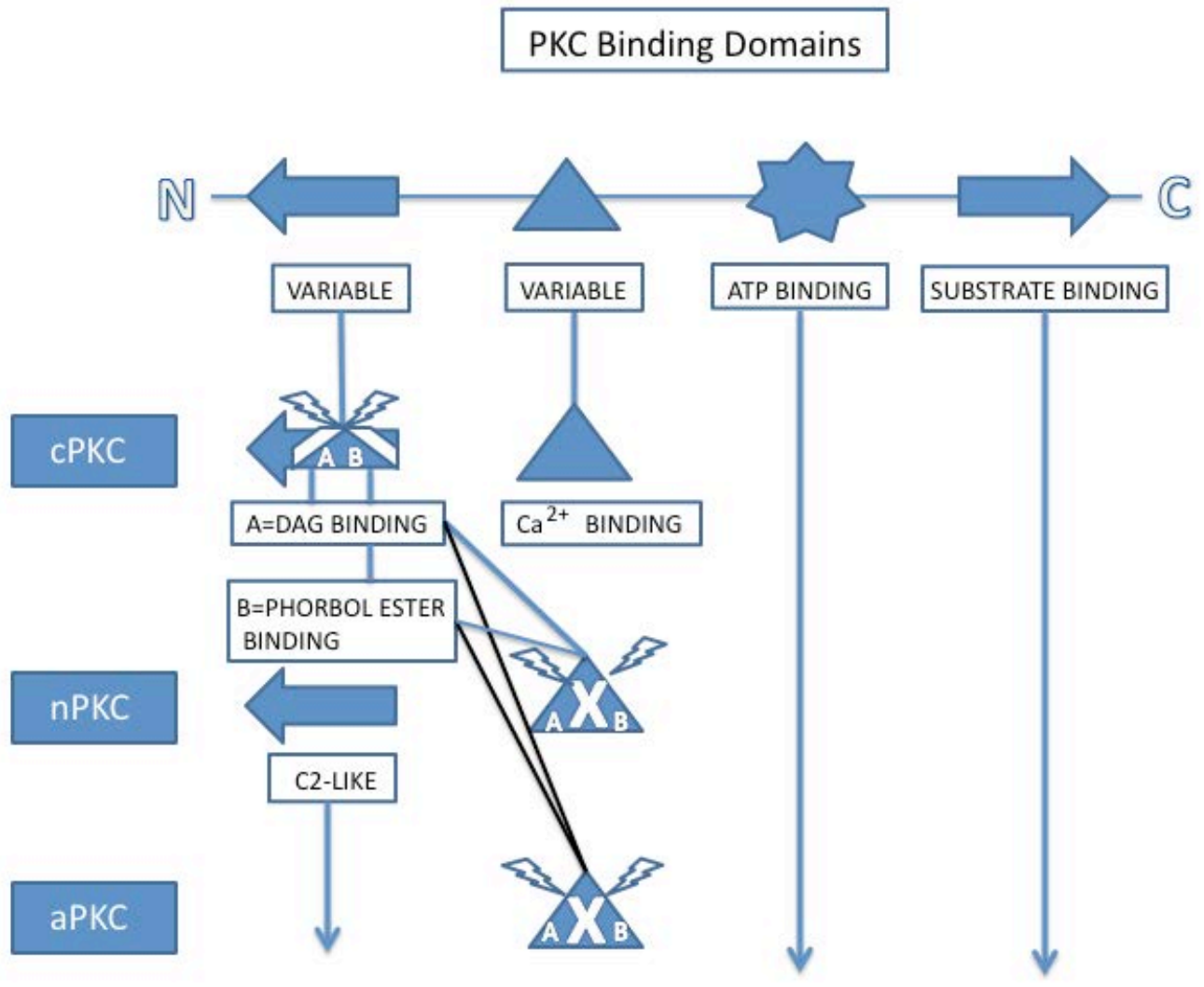

Figure 1.17. Schematic of typical protein sequence for PKC showing the variations between conventional, novel, and atypical subgroups. cPKC requires diacylglycerol, phorbolester, and calcium for activation. nPKC requires diacylglycerol and phorbolester but not calcium for activation. aPKC is not dependent on diacylglycerol or phorbolester but activates quicker in their presence which is represented by the black lines.

\subsubsection{Expression of PKC Isoforms - Systemic and Cellular Considerations}

PKCs have been found in a large number of organs such as the heart, brain, lungs, kidneys, and organs of the gastrointestinal tract as well as in cells of the hematopoietic system ${ }^{323-326}$. Different isoforms are located in a tissue-specific manner. PKCa is highly expressed in $\beta$ cells of the pancreas, but has also been linked to pathological tissue 
such as breast cancer and malignant gliomas ${ }^{327}$. PKCY is endogenous to the a cells in the pancreas. $\mathrm{PKC} \zeta$ has been linked to matrix metalloproteinase (MMP) regulation in smooth muscle cells ${ }^{328}$. PKCס is upregulated in endothelial cells following ischemia ${ }^{329}$. $\mathrm{PKC} \varepsilon$ is present in large quantities in the hippocampus and olfactory tubercle ${ }^{330}$. PKCßII is present in both normal and pathological B-cells ${ }^{331}$. Other isoforms such as PKCO and PKCa work together and have important roles in T-cell regulation and IL-2 activation ${ }^{332}$. All PKCs are originally localized in the cytosol of specific cells and must undergo a translocation to a membranous site. Depending on the isoform or tissue, this membranous site could be the nucleus, golgi apparatus, endoplasmic reticulum, mitochondria, or even the cell membrane ${ }^{333,334 .}$

\subsubsection{Activation of PKC Isoforms}

PKCs are completely phosphorylated following translation but are inactive due to pseudosubstrate interference with the binding domain. The pseudosubstrate domain keeps the PKC polypeptide in a folded configuration. PKCs are localized in the cytosol of specific cells and must be activated by removing the pseudosubstrate domain. After activation, PKCs must then be translocated to membranous sites in order to phosphorylate downstream pathways ${ }^{335}$. Lipopolysaccharide (LPS) in the presence of the aforementioned endogenous cofactors (DAG, $\mathrm{Ca}^{++}$, and PS) induces activation of PKC $\beta$, $-\varepsilon$, and $-\zeta^{336}$. Phorbolesters such as tissue plasminogen activator (tPA) and even the $\beta$-adrenergic system have also been shown to activate PKCs ${ }^{337}$. A variety of growth factor receptors can activate PKCs as well. The increase in growth factors during cancer is one of the reasons PKCs have become ideal targets for therapeutic interventions ${ }^{338}$. Once activated, PKCs can be translocated. The translocation mechanism is initiated through PKC binding with RACKs. RACKs are 30-36 kilo-Dalton proteins that are located in cytoskeletal compartments. They interact with a $\mathrm{C} 2$ domain on the PKC polypeptide and thus do not interfere with the substrates binding site. Each isoform of PKC has a specific RACK. RACKs also have other functions besides binding PKCs such as anchoring phosphodiesterase and integrin, and contributing to vesicle release and cell-to-cell communication 339,340 . After PKC binds to its isoform specific RACK, the entire unit will translocate from the cytoplasm to the membranous sites previously mentioned.

\subsubsection{Regulating PKC Activation}

In general, PKCs are self-regulated proteins. When PKCs are activated, the pseudosubstrate dissociates. Eventually, the PKC loses one of its three phosphate groups at a site known as the activation loop. When this occurs, the cofactor separates from the active site and the PKC is no longer functionally active. The pseudosubstrate reattaches and the PKC is in its inactive state. In order for the PKC to return to a state where it can activate again, it must regain the phosphate group that it has lost. A phosphoinositide-dependent kinase 1 (PDK-1) phosphorylates the PKC at the activation loop. This additional phosphorylation causes the other remaining phosphate groups to reconfigure and realign. In some PKCs, PDK-1 does not directly phosphorylate the activation loop but instead induces autophosphorylation. Once all phosphate groups have been restored and the other phosphate groups have been reconfigured, the PKC can undergo activation ${ }^{341}$.

\subsubsection{PKC - A Role in Disease Processes?}


PKCs typically regulate important functions such as cell growth and differentiation, secretion of hormones, and normal membrane functioning. They also play a key role in the regulation of the $\mathrm{Na} / \mathrm{K} / 2 \mathrm{Cl}$-cotransporter (NKCC) and neuronal sodium and potassium transport. Several of the PKC isoforms contribute to the pathology of neurologic and cardiovascular disease sometimes in direct contrast to one another. In stroke, the PKC isoforms $-\alpha,-\beta I$, and $-\varepsilon$ contribute to the activation of NKCC at the blood-brain-barrier (BBB). This leads to the protectant mechanism of increased $\mathrm{K}^{+}$ transport across endothelial cells and an increased $\mathrm{K}^{+}$flow back into the blood stream. 342 PKC $\beta$ I also acts as a neuroprotectant for astrocytes by inducing phosphorylation and increasing the activation of excitatory neurotransmitter transporters. Similarly, PKCa acts as a neuroprotectant for neurons by activating the adenosine signaling pathway ${ }^{343}$. In contrast to these neuroprotectant roles, PKC $\delta$ in conjunction with amplified $\mathrm{Ca}^{++}$influx has been shown to cause increased constriction of the middle cerebral artery (MCA) prior to and during ischemic stroke ${ }^{329,344}$. Following ischemia, PKCßII has been linked to increased endothelial permeability and inflammation ${ }^{345}$. Stroke is not the only neurologic disease where PKCs are active. The isoform PKC $\delta$ also regulates mitochondrial-induced apoptosis and has been linked to diseases such as Alzheimer's disease (AD), Parkinson's disease (PD), Amyotrophic Lateral Sclerosis (ALS), and Traumatic Brain Injury (TBI) ${ }^{346-349}$. PKC $\delta$ is cleaved by caspase-3 and translocates to specific cells via the appropriate RACK. It then enters the mitochondria of these target cells, and eventually phosphorylates a series of enzymes such as the GTPase Drp1. This signaling cascade ultimately leads to mitochondrial-mediated apoptosis ${ }^{350}$. PKCs also play a critical role in cardiovascular disease. PKC $\varepsilon$ provides cardioprotection through activation of mitochondrial aldehyde dehydrogenase 2 (ALDH2). PKCE is originally found in the cytosol and translocates to the cell particulate fraction following hypoxia preconditioning. In addition to an interaction with the appropriate RACK, PKCE associates with heat shock protein 90 (HSP90) before it translocates ${ }^{351}$. Similar to the brain, PKC $\delta$ leads to reperfusion injury following the initial onset of myocardial infarction. Importantly, cardiovascular-based studies have revealed an increase in PKC translocation by over $50 \%$ following injury. When inhibited, injury is significantly reduced through attenuation of the pro-apoptotic pathway in mitochondria ${ }^{352}$. If an animal is preconditioned to ischemia before injury, the protectant isoform $\mathrm{PKC} \varepsilon$ will be upregulated and the harmful isoform PKCס will be downregulated 353, 354. As more is discovered about PKCs, it is becoming increasingly apparent that they have important functions in a broad spectrum of diseases. This is particularly true when considering the greatest risk factor for the development of neurologic disease is aging. Since age in and of itself alters the dynamics of PKC signaling, it is important to realize that unique modifications occur in aged animals even before the onset of disease ${ }^{355}$.

\subsubsection{EFFECT OF NEURAL DISEASE \& INJURY ON PKC ACTIVITY}

Numerous disease states, both neurologic and non-neurologic in nature, have been associated with changes in both PKC isozyme activity and translocation. The following sections highlight the role of common neurologic disease states in modulation of PKC activity but it's important to consider that much of what is known concerning the role of PKC in pathophysiology is derived from work in other organ systems, namely cardiovascular. Many comprehensive reviews focusing on the general role and effects of PKC following injury, as well as specific isozyme-mediated effects, have been published by the Mochly-Rosen group and others, often in reference to cardiovascular disease, a disease process that likely shares many similarities with cerebral ischemia. As such, the cardiovascular literature and the aforementioned reviews provide insight 
into the role of PKC in neural injury and can serve as further reference for material not covered herein ${ }^{356}$.

The interest in PKC isozyme modulation following neurologic injury appears warranted due to the identification of deficits and abnormalities in PKC-mediated signaling cascades, particularly in relation to learning and memory deficits, in the brain that occur acutely in patients with Alzheimer's disease and/or ischemic stroke ${ }^{148}$. Of particular interest is the role of the epsilon isozyme as PKCE is expressed more highly in the brain than most other tissue, suggesting a critical role in nervous system homeostasis. This notion is consistent with the role of $\mathrm{PKC} \varepsilon$ in various functions including perhaps most notably, neurotransmitter release and ion channel function ${ }^{330}$. Furthermore, contrasting viewpoints concerning the effect of neural injury on PKC activity exist and therefore, additional investigation is required to understand both these effects and how PKC can be manipulated pharmacologically for therapeutic benefit ${ }^{326}$.

\subsubsection{Alzheimer's Disease (AD)}

Of the neurologic disease states in which PKC has been investigated in relationship to disease pathophysiology, AD comprises the vast majority of reported studies and importantly, deficient PKC isozyme function has been described as a key component of disease pathogenesis and consequently, raised the prospect of PKC as a promising therapeutic target ${ }^{357}$. Pathologic studies conducted on the brains of AD patients reveals a close correlation between dementia and synaptic loss. The precise mechanism leading to synaptic loss have been poorly elucidated until recent work of Hongpaisan, et al in which a reduction in synaptogenic PKC isozymes, particularly $\alpha$ and $\varepsilon$, and associated substrates was found to occur in conjunction with increased soluble beta amyloid but prior to both plaque development and corresponding neuronal loss. These findings were consistent with clinical autopsy results in which age-matched, nondemented control patients exhibited a higher level of PKC expression and activity in comparison to patients afflicted with AD ${ }^{358}$.

Mechanistic studies have revealed a role for PKC activation in activation of $\alpha$-secretase, an enzyme responsible for converting amyloid precursor protein (APP) to soluble sAPP $\alpha$, potentially preventing amyloid beta $(A \beta)$ production. Besides altering production of $A \beta$, PKC-mediated pathways have been reported to increase the degradation of $A \beta$, further altering the ratio of production and degradation in a beneficial manner ${ }^{359}$. In addition to providing a potential therapeutic approach to $A D$, these studies have also demonstrated clear roles for many PKC isozymes in learning and memory-associated tasks that are affected in many neurologic disease states such as ischemia and traumatic brain injury ${ }^{357}$.

\subsubsection{Ischemia}

Conflicting evidence exists concerning the effect of ischemia on PKC expression and activity $322,326,345,360,361$. This is thoroughly reviewed by Bright and colleagues and perhaps most importantly, only recently have the effects of various isozymes been recognized as frequently divergent, necessitating the consideration of disease-isozyme relationships rather than consideration of PKC activity/expression as a whole ${ }^{321}$. We attempt to identify these often different, if not opposing, roles of various isozymes as related to disease pathophysiology and therapeutic development in the remainder of this work. 
In addition to consideration of potentially contrasting izoyme effects, the impact of preclinical model and corresponding severity of ischemia has often gone unrecognized. Importantly, Sun and colleagues investigated this precise issue, demonstrating that while PKC activity is increased with mild ischemia, severe ischemia results in diminished PKC activity ${ }^{148}$. Besides severity of ischemia, the location of both ischemia and sample must be taken into account. Cortical and striatal tissue may respond differently to ischemia due to unique metabolic demands and cellular composition. Whether tissue samples analyzed are from infarct core or penumbral regions may further influence PKC-related findings ${ }^{362}$. Other factors to consider include the time of analysis post-onset of ischemia, the role of isozyme phosphorylation in activation and downstream-signaling, and discrepancies between in vitro and in vivo studies. Contrasting results between cellbased and animal-based studies, as reported by Fleegal and colleagues, indicate the potential for cell-specific effects, further complicating the study of PKC-mediated pathways ${ }^{345}$. Whether manipulation of PKC in response to injury has similar effects across the various cell-types found within the brain remains to be seen.

Regardless of the aforementioned issues in comparing results across studies, it is clear that PKC is involved in both the initial, acute response to ischemia as well as more delayed effects throughout the subacute and chronic periods. This is consistent with work showing PKC $\delta$ increased throughout both infarct core and penumbra regions ${ }^{148}$. Likewise, PKC $\varepsilon$ has been shown to be up-regulated rapidly following onset of ischemia and remains elevated in both in vitro and in vivo studies 322,363 . Most importantly, preclinical studies targeting specific PKC isozymes using either pharmacologic agents or genetic modifications have demonstrated the ability to reduce cerebral injury $321,364,365$.

\subsubsection{Ischemic Preconditioning}

In addition to the effect of ischemia on PKC and modulation of PKC for therapeutic benefit discussed above, PKC is also involved in the beneficial effects associated with ischemic preconditioning 148,330,366. Historically, the PKC $\varepsilon$ isozyme has been most frequently recognized as essential in ischemic preconditioning $148,330,367$. The protective effects associated with $\mathrm{PKC \varepsilon}$ activation that occurs with preconditioning have been ascribed to the role of $\mathrm{PKC \varepsilon}$ in the adenosine/NMDA-activated signal transduction pathway ${ }^{148}$. Recent work in mice undergoing hypoxic preconditioning (HPC) prior to middle cerebral artery occlusion (MCAO) has implicated PKC $\beta I$. This finding is consistent with observed increases in $\mathrm{PKC} \beta \|$ membrane translocation in the acute phase of HPC, but further work remains to clearly elucidate the mechanism of protection 366, 367. Other isozymes such as PKCס, a pro-apoptotic kinase, have been identified as being decreased with HPC in cardiac tissue, which may lead to the protective effects associated with HPC ${ }^{354}$.

\subsubsection{Traumatic Brain Injury}

Relatively little work has been done investigating the effect of TBI on PKC activity with the exception of that by Yang and colleagues and Zohar and colleagues. Yang, et al demonstrated significant changes in PKC activity at 1 and 3 hours after TBI but not at 5 minutes or 24 hours post-injury. At the 3 hour time point, PKC translocation to membrane fractions was elevated ${ }^{326}$. While Zohar, et al did not assess activity or translocation, administration of bryostatin-1, a potent PKC activator, resulted in diminished learning and memory deficits following TBI. The reduction in learning and 
memory deficits was consistent with improved maintenance of synapses as evident by pre-synaptic synaptophysin and post-synaptic spinophylin immunohistochemistry ${ }^{349}$.

\subsubsection{ROLE OF PKC IN NEURAL INJURY OUTCOME}

The effect of altered PKC activity observed in many of the aforementioned neurologic disease and injury states has not been entirely elucidated but many recent works have tied various PKC isozymes to oxidative stress, regulation of apoptosis, blood-brain barrier (BBB) breakdown, and processes associated with recovery/repair. In the following sections we expand upon work investigating the role of PKC in these diseaseassociated events by again linking work completed across multiple organ systems, mainly neurologic and cardiovascular.

\subsubsection{Modulation of Response to Oxidative Stress}

Oxidative stress has frequently been cited as a potentially precipitating factor in many pathologic states, particularly those associated with the aging process resulting in the theory of 'inflamaging' ${ }^{368}$. A reduction or loss of PKC activity has previously been correlated with injury severity but how PKC modulated this response was unclear. Emerging evidence implicates PKC in the modulation of oxidative stress-induced apoptosis, generation of oxidative stress through NADPH oxidase, and mitochondrial function.

Oxidative stress leads to PKC $\delta$ translocation to the mitochondria, termed mitochondrial targeting, ultimately causing loss of mitochondrial transmembrane potential and release of cytochrome $\mathrm{c}$ that leads to apoptosis 352,369 . PKC $\delta$ also appears to be somewhat unique in the response to oxidative stress in that it may also further amplify the apoptotic cascade via caspase activation and subsequent feedback ${ }^{370}$. Importantly, previous work has shown these processes can be inhibited using PKC $\delta$ inhibitors 369,371 . These inhibitors may also prevent upregulation of NOX1, part of the NADPH oxidase complex, via $\mathrm{PKC} \delta$ that contributes to oxidative stress and has been identified previously as a potential therapeutic target ${ }^{372-374}$.

\subsubsection{Effect of PKC on Cell Survival}

Consistent with divergent effects of PKC isozymes on other cellular functions, PKC isozymes have been characterized as pro- or anti-apoptotic in nature. Consequently, work utilizing non-specific PKC modulators has shown both protective and deleterious effects in response to injury, likely due to isozyme-specific effects 375 . PKC isozymes identified as suppressing apoptosis include $\alpha, \beta, \varepsilon$, and $\zeta$ while those acting as proapoptotic are $\delta$ and $\theta^{148}$. Most work investigating PKC-mediated effects on cell survival, namely apoptosis, has centered on the role of PKC $\delta$ and PKCE.

Inhibition of PKCD has been found to be universally protective regardless of organ system following onset of ischemia as evident by diminished pathologic features of apoptotic processes such as DNA laddering and fragmentation ${ }^{352,376}$. Similarly, proteins involved in apoptotic signaling cascades such as cytochrome c, caspase 3, and Bcl-2 assocaited death protein (BAD) were reduced following inhibition 352, 376-379. Perhaps most importantly, at least from a therapeutic development standpoint, is that administration of PKC $\delta$ inhibitors results in improved outcome following ischemic stroke, even when administered up to 6 hours after onset ${ }^{377}$. How exactly PKC $\delta$ mediates the 
aforementioned effects remains to be clearly elucidated but numerous pathways have been proposed in which glycogen synthase kinase-3B (GSK-3B) and Akt signaling pathways are altered 376,377 . Others have shown a role for Drp1, a mitochondrial fission protein to which PKC $\delta$ binds, that is phosphorylated upon binding resulting in enhanced mitochondrial fragmentation ${ }^{379}$. Furthermore, $\mathrm{PKC} \delta$ may be partially responsible for mediating the protective effects of hypothermia following ischemia ${ }^{360}$.

Investigation of $\mathrm{PKC \varepsilon}$ is more limited and the effects not as clearly elucidated as those associated with $\mathrm{PKC} \delta$ modulation. Bright and colleagues demonstrated a protective role of $\psi \varepsilon$ RACK and associated PKC $\varepsilon$-mediated effects when activator peptide is administered near the time of stroke onset, implicating PKC $\varepsilon$ in acute pathophysiologic events. When given 24 hours before ischemia or at the onset of reperfusion, $\psi \varepsilon$ RACKmediated activation did not improve nor worsen outcome ${ }^{363}$. In other work, activation of $\mathrm{PKC} \varepsilon$ restores the phosphorylation state of endothelial nitric oxide synthase (eNOS), increasing cell survival ${ }^{380}$.

\subsubsection{Regulating BBB Integrity through Matrix Remodeling}

Neural injury and degeneration often involves transient periods of blood-brain barrier (BBB) disruption. Improving the maintenance of BBB integrity has been identified as a promising therapeutic target for neurologic disease, particularly ischemic stroke. Much of the previous work into mechanism of BBB breakdown after injury has focused on the role matrix metalloproteinases (MMPs) play in both acute breakdown, associated with deleterious processes, as well as chronic regulation required for recovery/repair. While regulation of MMP activity has largely remained unclear and complicated, emerging evidence indicates a clear role for PKC signaling. Fleegal and colleagues have shown that PKC inhibition with chelerythrine chloride reduces BBB permeability, which lends credence to the idea that PKC may control expression and function of junctional proteins 345. This is consistent with the suggestion that PKC may regulate other junctional proteins associated with the epithelial barrier ${ }^{381}$. The attempt to identify the role of specific isozymes involved in BBB permeability has produced multiple candidates depending on the model used, time points, and severity of injury utilized. In a global ischemia model, analysis of cortical microvessels demonstrated changes in PKC distribution, particularly that of $\mathrm{PKC} \theta$ and $\mathrm{PKC} \zeta$, as well as phosphorylation activity of these isozymes. This suggests that these signaling molecules may further mediate tightjunction strength ${ }^{382}$. Others have suggested a role for PKC $\zeta$ in MMP regulation as well with Hussain and colleagues showing that $\mathrm{PKC} \xi$ activity is required for cytokine-induced activation of MMP-1, -3 , and $-9{ }^{328}$.

Other isozymes identified as contributing to BBB regulation include $\mathrm{PKC} \delta$ and $\mathrm{PKC} \varepsilon{ }^{381}$, 383, 384. Notably, some proposed neuroprotective agents, such as doxycycline, have reduced BBB breakdown and improved neurologic outcome while downregulating $\mathrm{PKC} \delta$, suggesting protective effects may be mediated via PKC isozyme modulation ${ }^{385}$.

How PKC activation and translocation leads to MMP activation is not well elucidated but indications are that it may involve downstream signaling cascades mediated through the NF-KB or MAP/ERK pathways ${ }^{386,387 . ~ W o r k ~ i n ~ o t h e r ~ o r g a n s ~ s u c h ~ a s ~ t h e ~ k i d n e y ~ h a s ~}$ shown other pathways and molecules may be involved in regulating MMP gene expression such as C-Fos and C-Jun, two cellular proto-oncogenes ${ }^{387}$. Other studies indicate potential modulation of cytokine-induced effects on MMP expression by PKC 
isozymes as well as identify a possible role of PKC-mediated phosphorylation of MMP-2 that then alters the activity of the protease ${ }^{388-390}$.

In addition to the role the BBB serves in regulating solute passage and preventing vascular dysfunction, it also is responsible for maintaining the immunoprivileged state. PKC $\delta$ activation in endothelial cells studied in vitro revealed diminished neutrophil migration following stimulation with leukotriene B4 (LTB4) or tissue necrosis factor- $\alpha$ (TNF- $\alpha$ ) without altering endothelial barrier integrity ${ }^{391}$. This would appear to be in contrast with previous findings in which PKC $\delta$ inhibition reduces endothelial dysfunction and improves BBB integrity $329,379,392$.

\subsubsection{Role in Recovery \& Repair following Neural Injury}

Understanding the mechanisms associated with recovery and repair from neural injury remains the subject of intense investigation due to the potential promise for therapeutic development for a number of neurologic disease states ranging from ischemic stroke to TBI. Recovery/repair involves a complex array of simultaneously occurring processes including neural migration and angiogenesis. Recent work highlights roles for PKCmediated signaling in each of these repair-associated components.

One of the greatest challenges in recovery/repair events is overcoming inhibitory activity attributed to the glial scar found at the lesion site. Much of the inhibition resulting from myelin and other molecules composing the glial scar is regulated by cPKC activity. By blocking PKC activity, neurite outgrowth has been stimulated both in vitro and in vivo despite the normally prohibitive presence of myelin ${ }^{148}$. This process likely requires a series of protease and metalloprotease-mediated steps, which may be regulated by PKC as discussed above. A prime example is that of semaphoring 3A (Sema3A), a guidance molecule that activates MMP-2 in a PKC $\alpha$-dependent fashion ${ }^{393}$. Besides involvement in axonal guidance, PKC activation using bryostatin has been shown to increase mushroom spine number on neurons in a memory-specific manner ${ }^{394}$.

Actual neurite outgrowth has been shown to be PKCe-dependent in nature with an increase in $\mathrm{PKC \varepsilon}$ promoting nerve growth factor-induced growth whereas a decrease inhibits the process ${ }^{330}$. This overall pro-repair event appears consistent with work completed in the cardiovascular system in which sustained PKCE inhibition following heart failure prolonged survival while reducing dysfunction through a variety of mechanisms, many of which are associated with the regulation of inflammatory pathways and inflammatory cell migration ${ }^{334}$.

PKCס, often thought of in a negative light with regards to impact on neural injury in the acute period, has been shown to modulate critical aspects of repair/regeneration such as angiogenesis and cell cycle modulators, making PKC $\delta$ activation potentially beneficial in the chronic period post-injury. Specifically, Kim and colleagues demonstrated that PKC $\delta$ activation promotes NADPH oxidase activity and increases HIF-1 $\alpha$ levels, leading to angiogenesis ${ }^{395}$. PKC $\delta$ is also involved in cell cycle events based on actions on Cdk5 via p35 and consequently regulates cortical radial migration of neurons ${ }^{396}$.

\subsubsection{Effect of PKC Modulation on Learning and Memory}

In addition to the role of PKC in neural migration and repair following injury, the effect of PKC on learning and memory has been well established in both healthy and injured 
animals. In Hermissenda, bryostatin was found to enhance memory acquisition at lowdoses consistent with up-regulation of specific PKC isozymes but at high doses, bryostatin resulted in down-regulation and no recall of the associative training task 397 . One of the major cellular mechanisms associated with learning and memory, long-term potentiation (LTP), seems to require PKC-mediated signaling based on previous findings assessing the effect of phorbol esters and PKC inhibitors on LTP. While PKC has been described as necessary, but not sufficient, to induce LTP, it is clear that PKC isozymes such as PKC $\varepsilon$ contribute to presynaptic control ${ }^{330}$. Furthermore, PKC modulators such as bryostatin have been shown to induce synthesis of proteins associated with memory consolidation, a fact that makes PKC modulators a potentially promising therapeutic target for conditions associated with memory impairment like Alzheimer's disease ${ }^{398}$.

\subsubsection{STUDYING PKC ISOFORM-SPECIFIC EVENTS: USING AVAILABLE TOOLS}

Work in other organ systems, such as the cardiovascular system, has illustrated the importance of elucidating isozyme-specific events for the development of therapeutic agents. For example, Chen and colleagues showed that various isozymes may have similar effects with regard to one cellular function and opposing effects on another function, explaining contrasting findings often reported due to use of non-isozyme selective pharmacologic agents. Furthermore, the isozyme-dependence of cellular functions is of particular interest considering the elevation of multiple isozymes following injury and the idea that it is the balance struck between various isozymes that determine the overall outcome post-injury ${ }^{353}$.

The elucidation of isozyme-specific events can be accomplished via genetic manipulations such as knockouts or through the use of pharmacologic inhibitors. Of the isozymes identified, at least four have been successfully mutated to produce null mice. This includes the $\gamma, \beta, \varepsilon$, and $\theta$ isozymes. While null mice may provide insight into neurologic effects associated with a respective isozyme, animals are affected systemically as evidenced by $\beta$ and $\theta$-null mice, which have shown phenotypes consistent with immunologic changes ${ }^{399}$. One of the advantages of null mice though is the ability to target a given isozyme specifically, a significant challenge observed in studies using pharmacologic inhibitors that often inhibit one isoform class rather than a specific isozyme. For example, Go6976 has been described as inhibiting classical $\mathrm{Ca}^{2+}$ dependent isozymes whereas Go6850 inhibits both conventional and novel isozymes ${ }^{381}$, 393. Other agents such as Ro 31-8220 have been shown to inhibit PKC $\alpha$ more selectively at low concentrations than high concentrations where additional PKC isozymes may be affected ${ }^{394}$. More recently, isozyme specific inhibitors have become available as well as the development of peptide-based specific inhibitors $321,358,376$. In addition to inhibitors of PKC, activators are also available and include promising therapeutic candidates, such as bryostatin, for a host of neurologic disorders ${ }^{349,358,364}$.

\subsubsection{FUTURE DIRECTIONS}

Modulation of PKC using isozyme-specific pharmacologic and peptide-based inhibitors has yielded positive results in many preclinical studies, particularly in models of neurologic diseases such as stroke, TBI, and AD. Despite the apparent success of these studies and progression to early phase clinical trials in some cases, further studies are required to elucidate effects of common disease comorbidities such as aging, hypertension, and diabetes that have not been addressed in early preclinical studies. Similarly, many of the early preclinical studies have been conducted in vitro on neurons and have not addressed the potential of cell-specific or time-dependent effects. 
Furthermore, investigating signaling mechanisms associated with beneficial and detrimental PKC-mediated effects may lead to optimal therapeutic development by regulating multiple isozymes.

\subsubsection{Alterations in PKC-mediated Signaling Associated with Aging}

The greatest risk factor for neurologic diseases such as ischemic stroke and AD is age. Most preclinical studies investigating PKC-mediated effects have not considered the role of age and the aging process. In one study that did address the effect of age on PKC in the brain demonstrated that cPKCs are substantially down-regulated in the cortex, striatum, and hippocampus of middle-aged animals compared to young animals. This down-regulation drastically changes the expression of substrates normally modulated by PKCs such as nitric oxide synthase (NOS) ${ }^{146,380}$. Ultimately, such age-related altercations might predispose the brain to Alzheimer's disease, dementia, or other memory dysfunctions. In addition to PKC downregulation, the availability of the remaining PKC isoforms is disrupted. Most isoforms are not present in membranous regions due to a deficit in translocation. RACKs have decreased expression, up to $50 \%$, in the aged brain. Without RACKs, PKCs are isolated to the cytosol of cells and cannot translocate even if activated. This leads to deficits in PKC-mediated neuronal plasticity and subsequent loss of LTP 333,400 . To further complicate matters, oxidative stress and aluminum-induced neurotoxicity can cause PKC to transfer from the plasma membranes to the cytosol in aged brains ${ }^{401}$. In other tissues such as the heart, some RACKs may be functional while others are not. When an aged heart is confronted with increased preload, the expression of most PKCs is up-regulated. The RACKs specific for PKC $\beta$ and $P K C \varepsilon$ are still functional and can therefore initiate translocation. Since PKC $\beta$ and $\mathrm{PKC} \varepsilon$ are overactive compared to the other regulatory cytosol-bound PKCs, the heart hypertrophies and becomes fibrotic ${ }^{356}$. Considering the potential effect of age on RACK functionality, the ideal therapeutic would not depend on RACK function to mediate therapeutic effects on the targeted isozyme. Several drugs have been designed for such targets, and one specific drug of interest is Bryostatin-1. Bryostatin-1 is a potent activator of PKCs and also aids in increased translocation of PKCa ${ }^{357,397 .}$

Kostyak and colleagues demonstrated that at baseline, $\mathrm{PKC} \delta$ levels in the mitochondria of the heart were increased by approximately $40 \%$ in comparison to the normal adult heart. Similarly, aged hearts contained a nearly 2 -fold increase in cytosolic PKC $\delta$ in comparison to adult heart. Perhaps most importantly, inhibition of PKC $\delta$ with KID1-1 reduced the increased infarct volume typically found in aged hearts to a level more consistent with the adult heart ${ }^{376}$.

\subsubsection{Elucidating Cell-Specific and Time-Dependent Events}

Investigating the pathophysiology of neurologic diseases such as ischemic stroke, as well as developing therapeutics, is complicated by the numerous cell-types required for both normal homeostasis as well as the response to injury and subsequent repair/ recovery. Being that each cell-type may respond differently to injury, or at least exhibit varying sensitivity to injury, may necessitate investigation of PKC-mediated processes independently for targeted therapeutic delivery. This idea is consistent with the varied findings across cell-types such as the role of PKC in modulating the inflammatory process and microglial adhesion, astrocytic control of MMP-9, and oxidative stress in neurons $371,386,391,402$. 
Besides cell-specific events, previous work has shown time-dependent changes in PKCmediated signaling in cerebrovascular studies. For example, PKC $\delta$ is activated early in cerebrovascular events and likely plays a greater role in the acute period whereas PKC $\alpha$ may play a larger role in the chronic period after injury. Therefore, studies more adequately addressing the timing of isozyme increases or decreases following an injurious event may lead to more appropriate therapeutic development ${ }^{403}$.

\subsubsection{Understanding PKC-mediated Pathways}

PKC-mediated signaling is involved in a wide array of homeostasis-associated pathways as well as those that occur in response to injury but how precisely many cellular effects are controlled through PKC activity remains unclear. Numerous pathways have been implicated based on work in the brain as well as cardiovascular systems but how these pathways all interact, and the role of various PKC isozymes in these pathways, remains to be seen. One signaling molecule of interest is glycogen synthase kinase 3 beta (GSK-3 $\beta$ ), an enzyme responsible for phosphorylating glycogen synthase kinase (GSK). Aging has been recognized as altering the ratio between phosphorylated GSK-3 $\beta$ and GSK-3 $\beta$, a difference that coincides with worsened cardiovascular injury in aged subjects. Reperfusion results in a worsening of this ratio in the mitochondria of aged hearts, an effect prevented by PKC $\delta$ inhibition ${ }^{376}$. Vascular endothelial growth factor (VEGF) appears to exert its mitogenic and permeability-associated effects via PKC $\beta$ demonstrating another potential pathway of investigation ${ }^{404}$. Lastly, the protein kinase Akt, which has been identified as regulating apoptotic events, has been shown to correlate with PKC $\delta$ activity in the acute period after ischemia but not after reperfusion 377. This indicates a potential link between the two pathways but also demonstrates the inherent complexity of signaling cascades, particularly those that overlap or display timedependent effects.

\subsubsection{CONCLUSION}

PKC clearly plays a role in neurologic injury and represents a promising therapeutic target based on work in numerous preclinical models of $A D$, ischemic stroke, and TBI. Activity of some PKC isozymes has been demonstrated in both clinical samples and preclinical studies at a variety of time points post-injury, likely in an isozyme-specific fashion. While the precise mechanism through which PKC isozymes influences outcome following injury is unclear, evidence indicates a role for PKC in regulating apoptosis, blood-brain barrier integrity, inflammatory cell adhesion/migration, and multiple aspects of recovery/repair (Figure 1.19). 


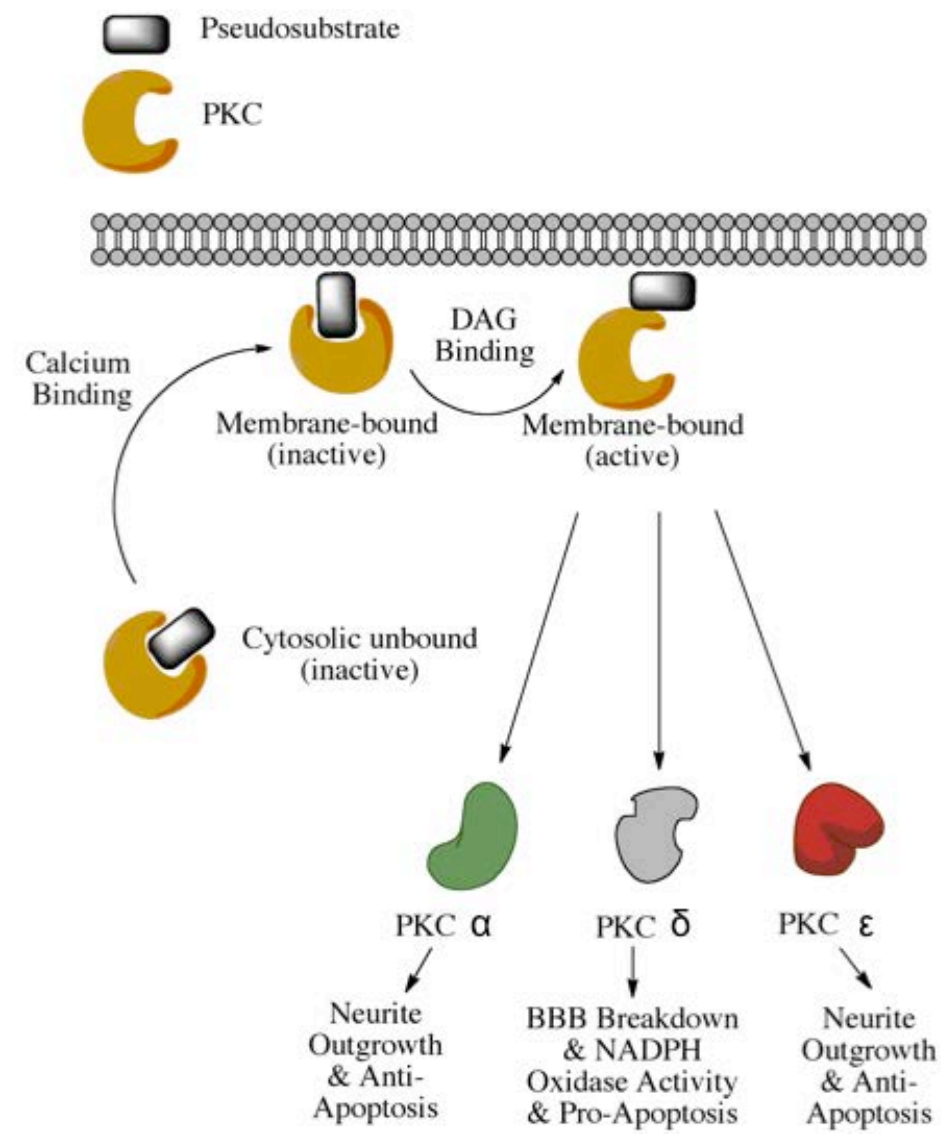

Figure 1.18. Schematic demonstrating generic PKC activation processes and subsequent isozymes with identified functions in neural injury/repair. Each isozyme likely has time-dependent and isozyme-specific actions that contribute to a range of cellular effects both during normal homeostassis as well as in response to injury.

Understanding isozyme-specific functions and how to most appropriately target these effects remains under investigation but recent improvements in peptide-based inhibitors provides promise in more clearly elucidating the role of a given isoform over time. As progress continues in this regard, successful therapeutics in preclinical studies that have progressed to clinical trials, such as Bryostatin-1, may potentially be used to carefully target individual isoforms and likewise increase functional recovery following a variety of neurologic injury types. 


\subsection{Modeling Traumatic Brain Injury}

Increased awareness of the diversity of events producing traumatic brain injury, whether it be on the athletic or even the battlefield, or the result of accidents (automotive and others), has led to a multitude of model systems. Models are generally classified as open or closed, focal or global, or blast-induced. Similarly, the mechanism of injury with regards to inciting event can also be used to classify models. The most common events include impacts from weight-drop, fluid percussion, a pneumatic cylinder, and blast. The advantages and disadvantages of these models have generally been well characterized with some appreciation of injury biomechanics and relevance to human trauma. Weightdrop models, in which a weight travels down a guide tube and impacts either the cranium or the brain directly, are generally well regarded for modeling biomechanics similar to those experienced by humans and are more simplistic in nature than other models. Disadvantages of these models include the potential for high mortality rates, some of which can be avoided through experimental modification (ventilation, etc), and issues with reproducibility. Variations of these models can be utilized to produce focal or diffuse injury with what is known as Feeney's model and the Shoami's model producing focal injury whereas Marmarou's and Maryland's result in diffuse injury ${ }^{5}$.

Fluid percussion injury models on the other hand are highly reproducible and allow nearly limitless adjustment of injury severity and can be applied to a broad range of species with relative ease and only minor modifications. Unfortunately, fluid percussion injury requires a craniotomy and uses an injury mechanism with unclear relevance to clinical studies ${ }^{405}$. Fluid percussion induces a mixed injury with focal and global components.

Another model commonly employed is that of controlled cortical impact. In controlled cortical impact the skull is fixed within a stereotactic frame and a craniotomy made (can be unilateral, bilateral, and of different locales depending on type of injury desired). The electromagnetic or pneumatic-driven cylinder is lined up with the craniotomy and the depth of impact desired can be set. While this model exhibits high reproducibility ${ }^{5}$, it does require craniotomy and produces a focal injury, which limits the models applicability to the more commonly sustained closed head injury in a clinical population. Variations of this model exist in which the impactor is fitted with a rubberized tip that is then used to impact the intact cranium, often in a repeated manner.

Perhaps the model technique of greatest interest due to wartime conflicts in Iraq and Afghanistan is blast injury. Blast exposure has been described as the signature injury of war and consequently has garnered increased attention by the lay and scientific communities alike. Many models of blast injury exist with little standardization ${ }^{406}$. Most commonly, for numerous practicality and regulatory reasons, compressed gas-driven shock tubes are utilized. While these models have successfully been used to produce a classical Friedlander curve (ideal blast wave), parameters of the wave and how these measurements are reported varies greatly from laboratory to laboratory. Specifically, the two main components of interest with regards to modeling blast include the peak overpressure and the duration of the wave. While others have demonstrated the ability to simulate a range of peak overpressures, measured in incident or reflected positions, little emphasis has been placed on exposure duration until recently 406,407 . Emerging evidence suggests that relevant exposures in rodents, based on scaling principles derived from pulmonary lethality work across species (including humans), are far shorter than the 4-10 ms durations commonly reported in the literature 406, 407. As such, it remains to be seen the effect of scaled blast on neural injury in a rodent model, a key 
question that needs to be addressed for elucidation of blast injury pathophysiology and the future development of therapeutics.

\subsection{Traumatic brain injury prevention}

The prevention of traumatic brain injury in potential contact scenarios, whether it is in the general public (bicycles, motorcycles, etc), athletes (football, lacrosse, baseball, hockey, etc), or battlefield, has always focused on the application of external protective equipment such as helmets. While helmets have dramatically reduced the incidence of skull fractures and have substantially improved in comfort due to improvements in materials and design, closed head injury, namely concussion, remains a prominent health issue in sports and on the battlefield. This is likely due to inherent shortcomings associated with the application of helmets, specifically an inability to address differential movement between the brain and the skull that occurs due to the floating nature of the brain in cerebrospinal fluid within the cranial vault. As such, alternative approaches for reducing traumatic brain injury and concussion are required going forward. 


\subsection{Long-Term Consequences of Traumatic Brain Injury}

Investigating neural injury using trauma as a tool has generally focused on acute injurious events. Only recently has evidence emerged that trauma, in the form of concussions, has lasting effects that often emerge years later. Perhaps the most wellknown of these is chronic traumatic encephalopathy, a neurodegenerative disease diagnosed in numerous athletes and soldiers at autopsy. The fact that chronic traumatic encephalopathy remains an autopsy-based diagnosis limits the ability to understand disease pathophysiology and progression, as well as the development of potential therapeutics. This work highlights the pathological findings of chronic traumatic encephalopathy and explores potential tools for improving detection prior to death, through the use of imaging modalities and biomarkers.

This work was published in Frontiers Neurology.

Citation: Turner RC, Lucke-Wold BP, Robson MJ, Omalu BI, Petraglia AL and Bailes JE (2013) Repetitive traumatic brain injury and development of chronic traumatic encephalopathy: a potential role for biomarkers in diagnosis, prognosis, and treatment? Front. Neur. 3: 186. doi: 10.3389/fneur.2012.00186.

\section{ABSTRACT}

The diagnosis of chronic traumatic encephalopathy (CTE) upon autopsy in a growing number of athletes and soldiers alike has resulted in increased awareness, by both the scientific/medical and lay communities, of the potential for lasting effects of repetitive traumatic brain injury. While the scientific community has come to better understand the clinical presentation and underlying pathophysiology of CTE, the diagnosis of CTE remains autopsy-based, which prevents adequate monitoring and tracking of the disease. The lack of established biomarkers or imaging modalities for diagnostic and prognostic purposes also prevents the development and implementation of therapeutic protocols. In this work the clinical history and pathologic findings associated with CTE are reviewed, as well as imaging modalities that have demonstrated some promise for future use in the diagnosis and/or tracking of CTE or repetitive brain injury. Biomarkers under investigation are also discussed with particular attention to the timing of release and potential utility in situations of repetitive traumatic brain injury. Further investigation into imaging modalities and biomarker elucidation for the diagnosis of CTE is clearly both needed and warranted.

\subsubsection{INTRODUCTION}

Increasing awareness by both medical professionals and the lay community concerning the potential long-term effects of repetitive traumatic brain injury, such as CTE and cognitive impairment $408-410$, has led to the identification of a need for improved diagnostic and prognostic tests. Investigators have focused primarily on the use of various imaging modalities and development of blood- or CSF-based biomarkers. In the following sections we attempt to briefly review findings associated with CTE diagnosis, proposed disease pathophysiology, and how these findings may potentially relate to imaging and/or biomarker discovery.

\subsubsection{Epidemiology \& Clinical Presentation}

Exposure to repetitive mild traumatic brain injury (mTBI) is a common occurrence in athletes on the playing field and soldiers on the battlefield. In fact, playing American 
football at higher levels results in documented exposure up to 1400 impacts per season for select positions such as linemen with some players involved in both offense and defense sustaining nearly 2000 impacts ${ }^{411}$. Similarly, mTBI has been identified as the most common combat-related injury in soldiers returning from present-day conflicts in Iraq and Afghanistan and consequently, has been described as the "signature injury of war" 412 . The diagnosis of mTBI remains particularly challenging due to the usual lack of abnormal findings on conventional CT and MR imaging ${ }^{412}$. Lack of a diagnostic test for $\mathrm{mTBI}$ is problematic considering the potential for both enduring cerebral effects (cognitive, neurophysiological, and clinical) and for identification of those at risk for development of CTE later in life.

CTE represents a progressive neurodegenerative disease currently diagnosed only upon autopsy and subsequent neuropathological examination ${ }^{413}$. Despite the lack of specific diagnostic criteria required for pre-mortem clinical diagnosis, patients afflicted with CTE diagnosed post-mortem are often described as suffering behavioral, cognitive, and emotional changes or impairments prior to death ${ }^{413-415}$. Notably, symptom development occurs following a prolonged latency in most cases, although exceptions do exist 415,416 . The tendency for a latent period creates a clear distinction between initial symptoms associated with TBI and the persistent, long-term degeneration, much like other neurodegenerative diseases such as Alzheimer's disease (AD).

CTE has been diagnosed in a broad spectrum of individuals with a history of head trauma, although the number of and severity of impacts is often unclear, ranging from athletes playing American football, soccer, hockey, boxers, and wrestlers to soldiers who have received battlefield injuries ${ }^{415,417-426}$. Due to the variety of individuals afflicted by CTE, emerging evidence indicates that CTE is likely more common than previously thought 411,417 . Based on the experience of one group of investigators, a conservative estimate of lifetime prevalence of CTE in American football players is at least $3.7 \% 413$. Estimates of CTE prevalence in retired professional boxers have been as high as $20 \%$ 419 . Considering the number of individuals actively engaged in contact sports such as football or exposed to explosive devices on the battlefield, it is clear that CTE represents a clear public-health risk. Much of the early work involving CTE diagnosis focused on concussion history but recent studies have documented a potential role of repetitive subconcussive blows as well 417,424 .

\subsubsection{Neuropathologic Findings of CTE}

\section{$\underline{\text { Gross }}$}

Gross neuropathologic examination of the brain in individuals afflicted with CTE may produce a range of findings. The brain may appear grossly normal or may show minimal lobar cortical atrophy for age without remote cortical contusions or lacerations ${ }^{423}$. There may be other non-specific gross pathologic changes like fenestrations of the septi pellucidi, communicating ventriculomegaly, subcortical ganglionic atrophy, cerebellar folial atrophy and pallor of the substantia nigra ${ }^{414}$. In general, however, the CTE brain of non-boxers is grossly normal without evidence of focal traumatic brain injury.

The frequent lack of gross neuroanatomical changes observed in CTE is in striking contrast to dementia pugilistica, believed to represent a more severe form of chronic traumatic encephalopathy observed in boxers ${ }^{427-430}$. Dementia pugilistica, described originally as 'punch drunk' by Martland ${ }^{431}$, was characterized neuropathologically by 
Corsellis and colleagues based on a tetrad of findings: 1) abnormalities of the septum pellucidum; 2) cerebellar and other scarring of the brain; 3) degeneration of the substantia nigra; and 4) the presence of neurofibrillary tangles in a regional manner ${ }^{429}$. While some controversy exists concerning identification of CTE, the work of Corsellis and colleagues is notable in that at no point is encephalopathy defined by the presence of a fenestrated or cavum septum pellucidum and equally important, the complete tetrad was not observed in a third of the cases characterized as dementia pugilistica ${ }^{428}$.

\section{Microscopic}

Microscopic investigation of CTE has focused primarily on several factors: the presence of tau, amyloid, and presence of TDP-43 proteinopathy as well as low grade diffuse white matter rarefaction, microglial activation and presence of reactive astrocytes ${ }^{414}$. The presence of a tauopathy, whether it be in the form of neurofibrillary tangles (NFTs), neuropil threads (NTs), or glial tangles (GTs), is a defining feature of CTE. While other neurodegenerative diseases such as $A D$ are also frequently defined and/or described by the presence of tau, CTE is clearly unique based on the topographic distribution of tauopathy ${ }^{414}$. AD is characterized by a relatively uniform distribution of tau NFTs in layers containing large projection neurons, such as layers III and V ${ }^{414}$. In contrast, CTE is exemplified by an irregular distribution of tau in more superficial cortical layers such as II and III. Similarly, the progressive topographic involvement of regions of the brain as seen in CTE differs from what is seen in other neurodegenerative diseases like AD. While neuritic amyloid plaques are seen in $A D$, neuritic amyloid plaques are not defining features of CTE, and are less frequently seen in CTE ${ }^{414}$.

Another delineating factor between $A D$ and $C T E$ is the presence of neuritic beta amyloid $(A \beta)$ plaques. Found extensively throughout the brains of those afflicted with AD, neuritic amyloid plaques are found in a minority of CTE sufferers. Diffuse and neuritic amyloid plaques are found in less than $40-45 \%$ of individuals with CTE $414,432$. Additionally, when found in CTE, amyloid plaques are more likely to be diffuse plaques and not the typical neuritic plaques that are diagnostic of $A D{ }^{414}$. The role $A \beta$ plays in CTE pathophysiology, and why it is present in some brains but not others, remains to be elucidated. Interestingly, amyloid precurosor protein (APP), which can undergo cleavage to form $A \beta$, accumulates following axonal injury and likely plays a role in plaque formation ${ }^{414}$.

CTE has been recently associated, in greater than $80 \%$ of cases, with accumulation of yet another phosphorylated protein aggregate, TDP-43 ${ }^{414}$. In some cases, TDP-43 has been found extending into the anterior horns of the spinal cord, particularly in patients exhibiting motor neuron disease symptoms similar to those of amyotrophic lateral sclerosis 411, 414. While TDP-43 proteinopathy occurs as a primary or secondary proteinopathy in a variety of neurodegenerative diseases, the significance of TDP-43 proteinopathy in CTE is presently not clear. The presence of another phosphorylated protein in aggregate form may indicate a shared process resulting in neurodegeneration following repetitive brain trauma ${ }^{414}$. Being that TDP-43 has been suggested to mediate the response of the neuronal cytoskeleton following injury, brain trauma and subsequent axonal injury may trigger a TDP-43-mediated process involved in neurodegeneration ${ }^{433}$.

The diversity of pathological findings associated with CTE, as represented by the varied findings presented above, has begun to be described with four distinct phenotypes I emerging (Table 1.4) ${ }^{426}$. The significance of these phenotypes with regards to clinical 
correlate remains to be elucidated but it is becoming increasingly clear that CTE represents a diverse spectrum of disease, a finding consistent with the heterogeneous nature of injury history, genetic predisposition, and a variety of other factors.

\subsubsection{CTE Pathogenesis \& Pathophysiology}

The pathogenesis and pathophysiology of CTE remain unclear, as with many neurodegenerative diseases, but is believed to be a multifactorial process initiated by brain trauma. The development of CTE begins with sub-concussive or concussive injury. The damage is progressive and often accelerated by the number of brain injuries that occur in an individual. Initially, mTBI causes diffuse axonal injury (DAI), which results in disruption of axonal transport and subsequent axonal swelling. The swelling causes a disconnection of the axons and later Wallerian degeneration ${ }^{434}$. This degenerative process, a portion of which is referred to in the literature as immunoexcitotoxicity, may lead to the development of CTE ${ }^{432}$. It is interesting to note that the abnormal tau and amyloid accumulations, which are seen in CTE are peptide derivatives of both membrane and cytoskeletal proteins, which are involved in traumatic axonal injury following concussive and subconcussive injury. This process is still relatively poorly understood clinically with post-mortem studies on young adults revealing that repetitive head injury is associated with the formation of NFTs and tau-based pathology surrounding vascular elements within the cortex ${ }^{435}$. Consequently, it is perhaps likely that microvascular damage plays a role in formation of the classical neuropathology associated with CTE. This is consistent with findings from classical literature exploring dementia pugilistica in which a large percentage of ex-boxers experienced perivascular hemorrhages with evidence of meningeal and/or subpial siderosis ${ }^{427}$. In addition to repetitive brain injury, there may be other identified factors that may contribute to or alter disease development, such as presence of certain genotypes ${ }^{424}$. Notably, anabolic steroid use had been previously suggested as a potential contributing factor to CTE development but the use of exogenous anabolic steroids has been shown experimentally to not worsen $\mathrm{mTBI}{ }^{436}$.

While the precise pathway or mechanism via which repetitive brain trauma predisposes to CTE development is poorly elucidated, Blaylock and Maroon posit a logical process via which immunoexcitotoxicity mediates the transition ${ }^{432}$. As part of this process, microglia are primed by initial impacts and with sustained trauma as well as aging, undergo phenotypic conversion from a non-destructive to destructive mode ${ }^{413,432}$. Once phenotype switching occurs, this pro-inflammatory state can be maintained for prolonged periods, consistent with neurodegenerative processes and emergence of hyperphosphorylated tau. Similarly, mild injury has been demonstrated to damage axons due to degenerative processes, resulting in a progressive deterioration, rather than rapidly occurring axonal shearing. This furthers the notion of CTE as a chronic neurodegenerative process, clearly distinct from the immediate sequelae often associated with TBI ${ }^{432}$.

\subsubsection{IMAGING MODALITIES FOR REPETITIVE BRAIN INJURY \& CHRONIC TRAUMATIC ENCEPHALOPATHY (CTE)}

A major concern for clinicians and researchers is how to detect small but important brain changes prior to the development of CTE symptoms so that preventative measures can be taken or treatments implemented, once available 413, 417. The obvious short term deficits seen in concussive injuries such as loss of consciousness, post-traumatic amnesia, and altered mental status have been historically hard to quantify using 
traditional imaging modalities such as computed tomography (CT) and magnetic resonance imaging (MRI) 437,438 . The difficulties and shortcomings of more commonly utilized imaging techniques have led researchers and clinicians to define concussion as a biomechanically induced brain injury with no gross anatomic lesions ${ }^{439}$. Despite the lack of gross lesions, it is still necessary to look at diffuse and microscopic alterations in the brain. Until recently, imaging techniques were not sophisticated enough to detect the subtle changes that are the hallmark of DAl. Clinicians have previously been forced to rely on post-mortem tissue analysis to discover mechanisms of CTE pathology ${ }^{419}$. With the invention and application of new imaging modalities, it is now possible to identify and investigate more of the pathological changes that are a consequence of mTBI. The following sections will highlight several of the new imaging modalities that are being used successfully to study various phenomena associated with $\mathrm{mTBI}$ and also promising modalities for further investigation of CTE. These modalities and potential strengths, as well as challenges, associated with each are summarized in Table 1.5.

\subsubsection{Diffusion Tensor Imaging (DTI)}

Diffusion tensor imaging (DTI) represents one of the more thoroughly investigated techniques for detecting axonal injury clinically ${ }^{412,440-442}$ and has even been shown to correlate with long-term outcome in preclinical rodents models of TBI ${ }^{443}$. Perhaps most notably, preliminary studies in retired professional athletes subjected to repeat head trauma demonstrate a correlation between history of concussion and DTI measures, particularly in the callosal white matter ${ }^{416}$. How DTI-based findings are altered with age and whether these findings correlate with neurodegenerative and behavioral changes associated with CTE remains to be seen ${ }^{444}$. Regardless, DTI appears to be a promising technique for detecting and tracking structural correlates of repetitive brain injury, including those believed to be specific to CTE.

\subsubsection{Functional Magnetic Resonance Imaging (fMRI)}

Functional magnetic resonance imaging (fMRI) is unique amongst the various imaging modalities discussed within this work due to the ability to provide insight into functional alterations, particularly working memory, and anatomic or structural changes simultaneously. The capability to measure brain-behavior relationships has proved useful in other neurodegenerative diseases and appears promising for further investigation of CTE-related changes ${ }^{416}$. Based on blood oxygen level dependent (BOLD) signal, fMRI is advantageous in that serial scans can be administered without exposure to harmful ionizing radiation ${ }^{445}$. This is particularly useful for fMRI-based studies as baseline measurements are required for comparison due to inter-individual variability ${ }^{446}$. Following exposure to head trauma, deficits on fMRI are apparent in both concussive and subconcussive injury groups with concussive injury producing a more severe deficit 447, 448. Importantly, studies utilizing both fMRI and ImPACT for neuropsychological testing have revealed deficits in working memory, even in the group sustaining only subconcussive impacts, and these deficits appear to be related to the number of impacts rather than the magnitude of any one impact ${ }^{449}$. Furthermore, in a preclinical model of $\mathrm{TBI}, \mathrm{fMRI}$ response was diminished acutely and correlated with functional deficits based on the forepaw placement test ${ }^{450}$. The use of $\mathrm{fMRI}$ for assessing the more subtle deficits produced by repetitive subconcussive impacts warrants further investigation, and in particular, longitudinal studies investigating the persistence of deficits on $\mathrm{FMRI}$ and the likelihood of further development for detecting, diagnosing and tracking CTE. 


\subsubsection{Magnetic Resonance Spectroscopy (MRS)}

Magnetic resonance spectroscopy (MRS) is a noninvasive method that measures brain chemistry associated with DAl and neuronal injury 412,416 . It is sensitive for changes occurring with age, mTBI, and mild cognitive impairment $(\mathrm{MCl}){ }^{451}$. As an imaging modality, ${ }^{1} \mathrm{H}$ MRS is used to detect changes in $\mathrm{N}$-acetylaspartate (NAA), myoinositol $(\mathrm{ml})$, choline, lactate, and adenosine triphosphate (ATP) production in specific ROI. Nacetylaspartate is a neuronal marker and is reported as a NAA:creatinine ratio. Other markers that may prove useful in neural injury include myoinositol, a glial marker, choline-based compounds, indicative of membrane integrity, and lactate, an indirect marker for ischemia ${ }^{452}$. The metabolite levels in an injured brain are compared to levels in a healthy brain for analysis ${ }^{453}$. A decrease in NAA and ATP is indicative of reversible neuronal and mitochondrial dysfunction ${ }^{454}$. An elevation of the NAA:creatinine ratio indicates persistent damage following mTBI ${ }^{439}, \mathrm{H}_{2} \mathrm{O}$ is often measured as an internal control in the patient during imaging ${ }^{444}$. By monitoring the metabolites and $\mathrm{H}_{2} \mathrm{O}$, the progress of a patient can be tracked longitudinally ${ }^{455}$. It is even possible to detect changes in the brain prior to onset of symptoms such as in subconcussive injuries ${ }^{456}$. Furthermore, metabolite levels remain altered long after symptoms subside, which gives credence to the idea of long-term degeneration occurring and presence of a latent period 457. As MRS utilizes current clinical MR scanners and does not administer ionizing radiation to the patient, MRS may represent an ideal imaging modality for long-term studies requiring repeated measurements. Furthermore, a preliminary study conducted by Lin and colleagues demonstrated persistent changes in Cho and Glx in 5 professional athletes, aged 31-54 years. Specifically, both Cho and Glx were significantly increased as compared to controls ${ }^{416,458,459}$. Additionally, studies have demonstrated changes in $\mathrm{ml}$ consistent with $\mathrm{MCl}$ literature that correlate with NFT count in AD brains upon pathological examination ${ }^{444}$. Consequently, understanding the effect of repetitive brain injuries on $\mathrm{ml}$ levels over time may be of interest for future investigation due to the strong correlation with episodic memory deficits observed in formerly concussed athletes 444. Based on this evidence, MRS appears promising for not only elucidation of CTE pathophysiology but also tracking the effects of repetitive brain trauma longitudinally. In this manner, MRS may also be useful for the diagnosis of CTE in effected individuals.

\subsubsection{Positron Emission Tomography (PET)}

Positron emission tomography (PET) can be used to detect metabolic rates in a variety of tissues of interest, including the brain ${ }^{460}$. While PET has traditionally been used in cases of more severe injury, recent evidence indicates a potential role in $\mathrm{mTBI}{ }^{461}$. In boxers, hypometabolism was observed in regions likely affected by impacts to the side of the head such as the portion of the frontal lobe anterior to Broca's area ${ }^{462}$. Similarly, hypometabolism was observed in the posterior cingulate cortex and posterior parietal lobes ${ }^{462}$. In other patients afflicted with TBI, hypometabolism may be seen commonly in the anterior temoporal lobe and orbitofrontal lobe due to rapid acceleration-deceleration mechanisms 462. Other promising developments in PET imaging include the development of ligands specific for disease states such as AD. For example, Pittsburgh compound B selectively binds beta-amyloid whereas others under development nonselectively bind both beta amyloid and tau ${ }^{416}$. While beta amyloid is not diagnostic for CTE, this technology appears potentially promising for the imaging of CTE should tauspecific compounds be identified. Furthermore, recent studies using $\mathrm{F}^{18}$ DDNP glucose- 


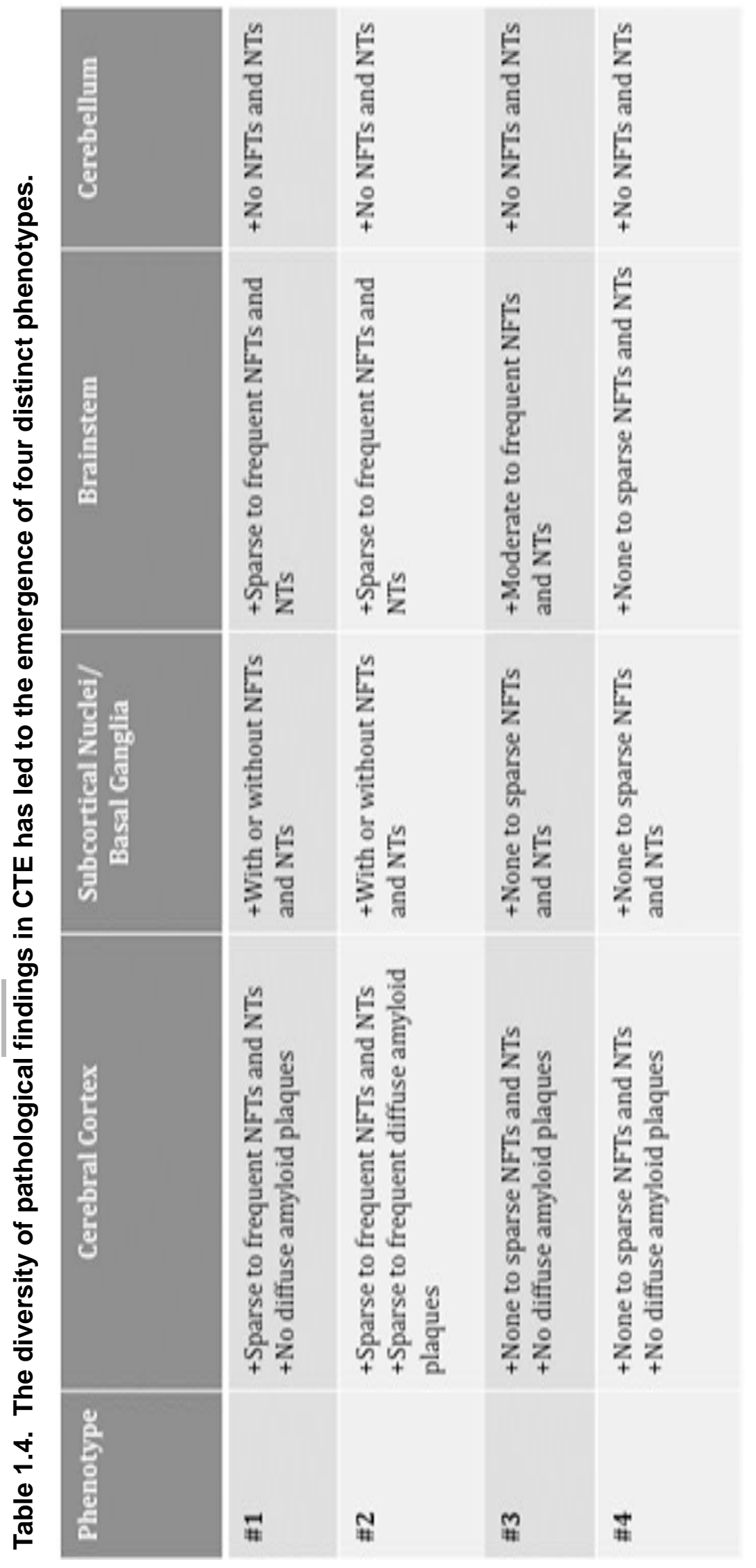

PET, conducted by Dr. Gary Small at UCLA, show promise for identifying tau protein deposition in the form of NFT's in subjects with potential CTE ${ }^{463}$. $F^{18}$ DDNP glucose-PET allows for labeling of both plaques and tangles, as has been shown in cases of mild cognitive impairment as well as $A D$, making this technique exceptionally promising in identifying c h a r a c t e ris t i c pathological features of CTE as they develop pre-mortem ${ }^{464}$. This effort is significant in that it could potentially allow, for the first-time ever, the diagnosis of CTE pre-mortem. Similarly, identification of NFT's following brain injury would provide substantial insight into the timing of development and pathophysiology of CTE.

1.11.2.5. Single Photon Emission Computer Tomography (SPECT)

Single photon emission computer tomography (SPECT), similar to PET in the use of radionuclides, can be used for measurement of cerebral perfusion and has been shown to detect abnormalities in $41 \%$ of amateur boxers in comparison to $14 \%$ of controls. Furthermore, abnormalities were generally singular in the control group yet 
multiple in the group of boxers subjected to repetitive brain trauma ${ }^{465}$. While SPECT has not been utilized in studies of CTE, CTE is associated with amyloid protein deposition in a significant number of cases and amyloid is notably largely perivascular in deposition, particularly in regions of abnormal vasculature, which could account for abnormal findings on SPECT ${ }^{465}$. It has also been correlated with functional deficits revealed on neuropsychological testing (NPT). Specifically, NPT strongly predicts diminished perfusion on SPECT but the inverse is not true ${ }^{458}$. The reasons for this mismatch are currently unknown but may be due to SPECT being obtained at a resting state while NPT requires neural activity and processing ${ }^{458}$. In spite of this lack of correlation and lack of specificity when considering other disease conditions, SPECT has clear value in recovery prognostication based on previously reported findings ${ }^{458}$.

\subsubsection{Susceptibility Weighted Imaging (SWI)}

Susceptibility weighted imaging is used to detect microhemorrhages, the intact structure of the venous system, and oxygen saturation following neurotrauma ${ }^{466}$. Microhemorrhages, venous thrombosis, and ischemia are common secondary injuries following $\mathrm{mTBI}{ }^{467}$. Furthermore, the extent of blood brain barrier (BBB) disruption and perivascular tau deposition can be mapped with SWI ${ }^{417}$. Tau accumulates in the brain during the progression of CTE ${ }^{411}$. The imaging technique works by taking phase data from a T2* weighted MRI and creating a second mapped image. This image allows both blood oxygen levels and the amount of deoxyhemoglobin to be measured ${ }^{468}$. It also localizes concentrations of paramagnetic iron and detects areas of iron translocation ${ }^{469}$. Iron deposition may play a role in CTE symptom manifestation ${ }^{470}$. The images produced with SWI are highly functional, velocity-compensated, and gradient echoed ${ }^{471}$. When used with DTI, SWI can accurately show which areas of white matter are damaged by microhemorrhages ${ }^{472}$. This modality offers a unique tool to detect areas of injury early, and provides clinicians with information on how to manage the care of the patient, particularly in children in which SWI has been shown to predict future outcomes following injury 416,473 . Unfortunately, the predictive ability is more limited in adults at present but necessitates further study ${ }^{416}$.

\subsubsection{Electroencephalography (EEG)}

Electroencephalography has been used to demonstrate mental processing impairment in 12 professional concussed boxers based on increased P300 latency and lessened amplitude. P300 has been identified as a cognitive event-related potential (ERP) with a novel amplitude and latency, making it a potentially suitable measure of brain processing ability. Similarly, investigators have demonstrated altered components of P300, such as $\mathrm{P} 3 \mathrm{a} / \mathrm{P} 3 \mathrm{~b}$, decades after concussion in older athletes with a history of past concussion compared to controls ${ }^{416,433}$. Based on these findings, EEG may have utility in assessing long-term cognitive effects associated with concussion and therefore, may be useful in identifying those predisposed to CTE development.

\subsubsection{Future Directions}

Advances in imaging technology and improved access provide nearly limitless opportunities for both understanding the pathophysiology and diagnosis of CTE premortem. As such, it may be possible to initiate both treatments and preventive measures prior to emergence of CTE. Many challenges remain such as clarifying the role of subconcussive injury in CTE development and how to most readily identify 


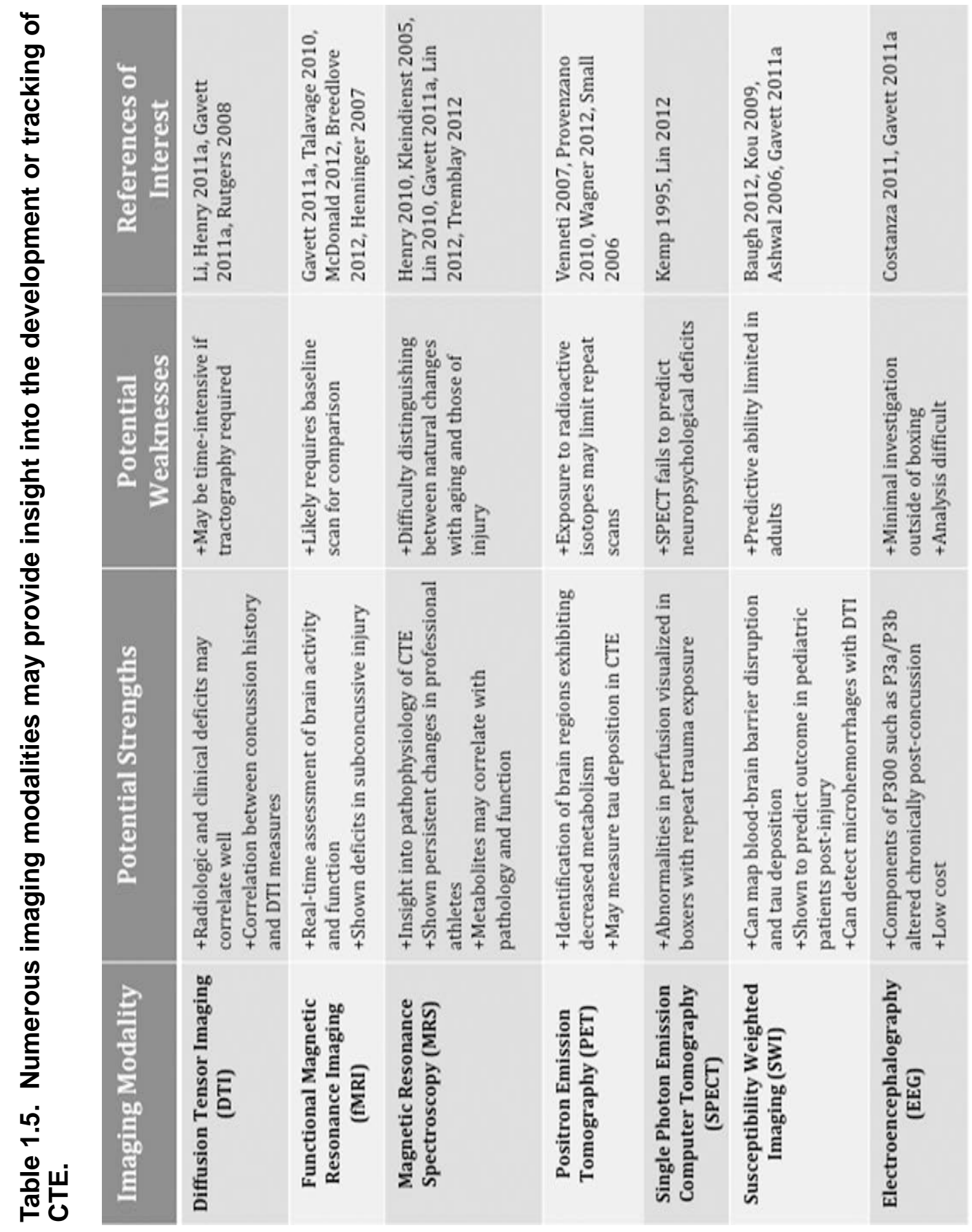

suspected

subconcussive injuries on the athletic or battlefield. By utilizing techniques such as MRS and $\mathrm{fMRI}$, pathology can be correlated with functional deficits and hopefully lead to more appropriate identification and monitoring of those with suspected injuries, regardless of severity. Other challenges such as the need for baseline measurements remain to be overcome as well but clearly warrant additional investigation.

\subsubsection{BIOMARKERS FOR CTE: A NEW FRONTIER}


As discussed in previous sections modern imaging techniques are one way by which diagnosing and tracking the progression of CTE may be feasible in the near future. Unfortunately, access and economic issues associated with imaging techniques may somewhat limit their ultimate usefulness in the diagnosis of CTE, especially during the acute phases of injury. With CTE potentially being a large public health issue, a simple and cost effective manner to diagnose and possibly track progression of the disease is crucial $^{417}$.

Biomarkers represent one method by which CTE may one day be diagnosed. Additionally, it may be possible to track disease severity and progression through the use of a biomarker(s). Ideally, a potential biomarker for CTE would be minimally invasive, have diagnostic potential and would correlate to disease severity, allowing care providers to track progression of the disease. It should be sensitive enough to detect the disease and when used in conjunction with clinical evidence of CTE symptoms and a past history of repeated head injuries, allow for a diagnosis. As described above, there is currently no accepted method of diagnosing CTE until post-mortem pathological analysis has been conducted ${ }^{417}$. A readily available biomarker with the aforementioned traits would give practitioners a useful tool for the diagnosis and tracking of patients suffering from CTE.

Currently there is a paucity of studies aimed at determining a biomarker(s) specifically for CTE. Many studies aimed at elucidating biomarkers for traumatic brain injury and other types of neurotrauma have been conducted. One difficult aspect of searching for a CTE biomarker is that CTE symptomology can be similar to a variety of other neurologic disorders. This may add a layer of complexity to conducting human clinical studies in an attempt to discover suitable biomarkers. The neuropathology of CTE, however, is not distinctly different, as hyperphosphorylated tau and TDP-43 deposition are found in a variety of neurodegenerative diseases outside CTE 413, 417, 474. Additionally, recent reports indicate that CTE may be associated with symptomology similar to ALS, further complicating the likelihood of clinical diagnosis without improved diagnostic tests ${ }^{474}$. These reports emphasize the need for a biomarker with high specificity that can delineate CTE from other neurologic disorders.

There are two primary body fluids where readily attainable biomarkers of CTE may be located. These include the cerebral spinal fluid (CSF) and the blood. Blood or plasma samples have the distinct advantage of being minimally invasive and easy to obtain. Cerebrospinal fluid samples, however, are in more direct contact with the CNS and may confer an advantage in this regard to the determination of suitable biomarkers for CTE. Routine use of CSF in the diagnosis or tracking of CTE presents several obvious problems associated with obtaining CSF samples. Therefore, ideally a biomarker aimed at detecting CTE in the periphery would be found in serum or plasma samples obtained via venipuncture. As discussed above, currently there is a lack of primary studies aimed at the elucidation of biomarkers in either one of these body fluids for CTE. However, several studies have been conducted in the realm of TBI and mTBI and these will be discussed herein.

It may be possible that biomarkers in development for TBI may be useful during the acute injury phase of patients at risk for CTE. The repeated concussive and subconcussive blows that result in CTE may alter levels of particular biomarkers studied for TBI immediately following these events. The tracking of these acute changes, although not specific to CTE, may give healthcare professionals insight into those patients at the greatest risk for developing CTE later in life. Equally important though, is 
the potential utility for biomarker surveillance in patients with known or suspected postconcussion syndrome.

Many studies have been conducted aimed at determining a biomarker for TBI or mTBI in the plasma or serum. A study conducted by Mondello et al. has determined that glial neuronal ratio (GNR), characterized as the ratio between GFAP and ubiquitin carboxyterminal hydrolase-L1 (UCH-L1) in the serum of TBI patients is characteristic of the type of TBI injury, focal vs. diffuse ${ }^{475}$. It was noted by Mondello et al. that their studies may have implications for diagnosis in TBI patients in the early acute phases of injury, however whether these results would have implications for CTE patients has yet to be determined ${ }^{475}$. It is possible that after multiple concussive or subconcussive blows to the head that the GNR may be altered and may offer insight into the extent of injury and potential for later development of CTE.

Several other studies aimed at elucidating valuable biomarkers for $\mathrm{TBI}$ in the serum have been conducted and may offer insight into the production of a biomarker(s) specific to CTE. It has been reported that plasma bilirubin levels in TBI patients are elevated on days subsequent to injury ${ }^{476}$. Unfortunately, this study only determined bilirubin levels up to 4 days post-injury. The determination as to whether the increases in plasma bilirubin remain elevated for a significant amount of time post-injury or whether it is specific to the acute injury phase has yet to be made. Additionally, the determination as to whether bilirubin concentrations in the plasma would be elevated in patients at risk for CTE has also yet to be made.

Another intriguing potential serum biomarker for CTE may be the protein neuron-specific enolase. Zetterberg et al. have shown that this protein is increased in the serum of boxers as compared to healthy controls after the boxers abstained from boxing for a period of 2 months ${ }^{477}$. Interestingly, elevated levels of neuron-specific enolase were detected two months after a nonparticipatory period of boxing, however other biomarker candidates for CTE such as S-100B, BDNF and heart-type fatty acid binding protein were found to be unchanged ${ }^{477}$. These results indicate that neuron-specific enolase may be one protein biomarker that has the potential for use as a diagnostic tool. It is possible that neuron-specific enolase remains elevated for an extended time period after injury and may be a useful tool for diagnostic purposes in athletes and patients who have suffered multiple concussive and subconcussive blows to the head.

An area of biomarker research that has received much attention is the field of microRNA. It is possible that potential biomarkers for CTE may not only be protein-based in nature. A recent report by Balakathiresan et al. has shown that levels of microRNA let-7i are elevated post-blast-induced TBI in both the CSF and the serum of rats exposed to a model of this type of $\mathrm{TBI}{ }^{478}$. Additionally, this group showed that the expression of 5 microRNA's were altered post-blast TBI in the serum of these animals ${ }^{478}$. These included let-7i, miR-122, miR-200b, miR-340-5p and miR-874 ${ }^{478}$. Further study is needed in both animal models, as well as humans, to validate the potential for using microRNA's as potential biomarkers for TBI and possibly CTE. Although this study was conducted using a blast-TBI animal model, a recent report has shown that this model produces pathology similar to that seen in CTE and models similar to this may be viable animal models for the study of CTE ${ }^{418}$. The production of validated animal models could greatly aid in the discovery of biomarkers aimed at detecting CTE and the production of potential clinical therapies for treating CTE. Other studies have also successfully identified RNA-based biomarkers for $\mathrm{mTBI}$ based on both micro- and snoRNA in peripheral blood mononuclear cells (PBMCs). Using a panel of 3 markers, veterans that 
experienced mTBI were detected with $89 \%$ accuracy, $82 \%$ selectivity, and $78 \%$ specificity ${ }^{479}$. Future studies utilizing both animal models as well as clinical samples may reveal additional RNA-based molecules that may be viable biomarkers for detecting and possibly tracking CTE.

One particularly interesting report recently published, studied a variety of biomarkers aimed at detecting TBI and mTBI in Olympic boxers with an extended history of many bouts ${ }^{480}$. This study determined extent of increases in neurofilament light protein (NFL), total tau (T-tau), tau phosphorylated at threonine 181 (P-tau $\left.{ }_{181}\right)$, heart-type fatty acid binding protein (H-FABP), glial fibrillary acidic protein (GFAP), S-100B, and the 42 amino acid form of amyloid $\beta$ (A $\beta 1-42)$ in olympic boxers both in the acute phase after a bout and an extended phase of two weeks post-bout and compared them to non-boxing controls. It was found that both NFL and GFAP levels within the CSF were significantly different from controls in both the acute and extended phase of testing ${ }^{480}$. The determination as to whether either of these correlated to injury severity was unable to be conclusively made in this study ${ }^{480}$.

However, a previous study has shown that there may be a potential correlation between NFL levels in the CSF and injury severity ${ }^{477}$. Zetterberg et al. showed that NFL, T-tau and GFAP were increased in the CSF of boxers and the levels appear to be correlated to injury severity, as boxers who received more hits in number or severity had increased levels of these markers ${ }^{477}$. Only NFL in these studies however, was increased for an extended time period (tested at 3 months) as compared to controls ${ }^{477}$. The extended increase in NFL levels within the CSF post-injury may make it a viable candidate for a biomarker for $\mathrm{mTBI}$ and CTE however whether levels of NFL within the CSF correlate to later disease severity in the case of CTE or mTBI has yet to be conclusively made. NFL levels however, have been shown to be altered in other types of neurologic trauma and disorders so this may ultimately decrease its viability as a tool for detecting, diagnosing and tracking CTE disease progression ${ }^{481,482}$.

Although levels of proteins such as NFL and tau may not be specific with regards to CTE, a battery of various biomarker proteins combined with patient history and imaging studies [discussed in previous sections] may result in the ability to make a pre-mortem diagnosis of CTE via a reference fractional laboratory index using a high throughput analytical system. In this respect, a combined battery of proteins such as tau, GFAP, NFL, S100-B and bilirubin may offer more accurate quantitative data with regards to a patients risk for developing CTE and when combined with other modalities of relevant clinical data, may be used as even more accurate diagnostic indicators at some point in the future.

\subsubsection{LINKING PATHOPHYSIOLOGY TO IMAGING \& BIOMARKER DISCOVERY}

Perhaps the greatest challenge concerning development of biomarkers or imaging modalities for prevention, diagnosis, and prognosis of CTE is the current lack of wellelucidated disease pathophysiology. Consequently, few clearly identifiable and specific disease markers have been identified. Without first understanding disease development more clearly, likely via both clinical research and utilization of animal models of repetitive brain trauma, logical discovery of CTE-associated markers appears unlikely. Recent work has demonstrated the presence of CTE-like neuropathology in wild-type mice following single-blast exposure and increased formation of neurofibrillary tangles in a transgenic mouse model following repetitive injury ${ }^{418,483}$. In a single-injury experiment, again using transgenic mice utilized in Alzheimer's disease models, tau and amyloid- $\beta$ 
accumulation was accelerated ${ }^{484}$. Similarly, studies have shown the presence of phosphorylated tau and amyloid- $\beta$ months after initial fluid percussion injury in the genetically unmodified rat ${ }^{485}$.

Despite the present lack of clear elucidation of disease pathophysiology, certain imaging techniques such as MRS, fMRI, and tau-specific labeling compounds in PET, have begun and will likely continue to provide insight into CTE development via longitudinal changes in neurologic function and health via a variety of markers. Similarly, work in other disease conditions that allows for imaging of activated microglia, consistent with the notion of immunoexcitotoxicity, may provide further insight into disease development and progression ${ }^{461}$.

In addition to assisting in the diagnosis and prognosis of patients suffering from CTE, continued improvements in imaging and biochemical assays associated with CTE identification may promote risk factor identification and provide insight into periods of increased risk. For example, the role of age at time of impact and associated brain trauma in disease development is unclear. Understanding this issue warrants further investigation as neuropathologic changes associated with CTE have been found in an asymptomatic 18-year old high school football player and consequently, age at time of first head injury may contribute to CTE incidence ${ }^{413}$.

\subsubsection{CONCLUSION}

CTE is an emerging public-health concern due to disease prevalence likely higher than previously recognized and continued popularity of contact sports as well as involvement in military combat that places soldiers at risk for explosive blasts and subsequent TBI. As CTE represents a devastating deterioration of neurologic function, a clear need for improved diagnostic and prognostic tests exists. To accommodate this need, as well as more clearly elucidate disease pathophysiology, imaging and/or biomarker-based tests are required. We propose a potential work-flow for implementation of both imaging and biomarker-based studies for improving the understanding of the role concussive and subconcussive impacts play in long-term disease development (Figure 1.20). While the specifics remain open to interpretation, obtaining pre-injury exposure studies as well as after a suspected or confirmed injury parameters is likely to provide further insight into the effects of TBI. It is only by more accurately identifying CTE pre-mortem and studying disease progression and mechanisms that we can establish improved therapeutic approaches. 
A.)

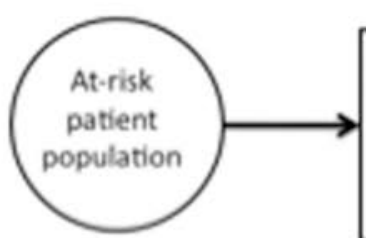

Prescreening/Baseline Measurements (Imaging, biomarker levels, neuropsychological testing)

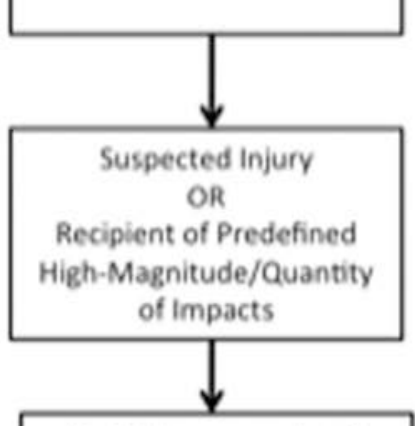

Post-injury screening \& tracking

(Imaging, biomarker levels, neuropsychological testing)
Ideal workflow for future clinical CTE studies aimed at studying both the psychological and physiological effects of the disease.

\section{B.)}
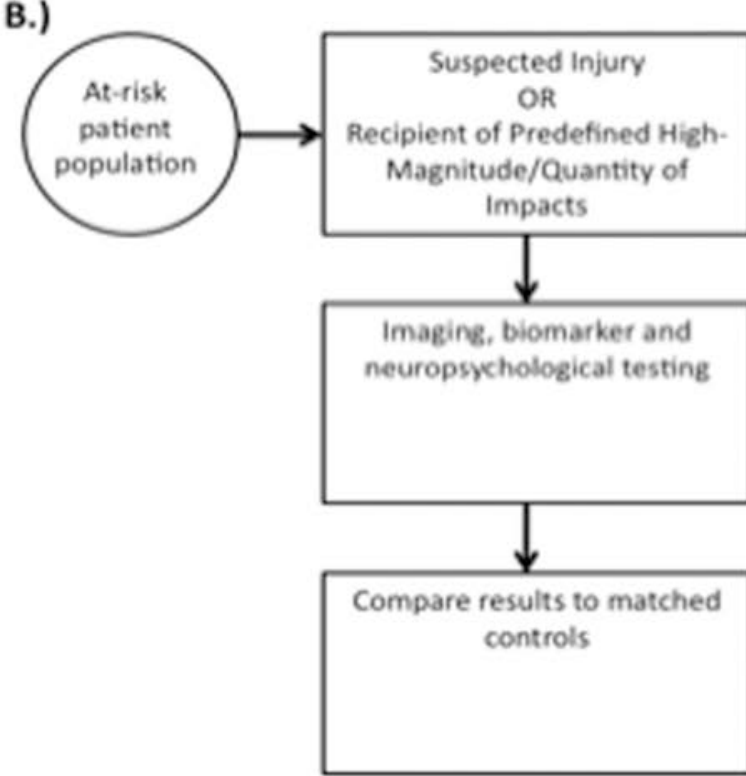

Likely clinical workflow for future CTE studies aimed at studying both the psychological and physiological effects of the disease.

Post-mortem histological studies

(Biomarker levels, presence

of tauopathies or other neuropathology)

Figure 1.20. Imaging and biomarker studies may be useful in improving understanding of the role of repetitive concussive and subconcussive injury in disease development. Two experimental paradigms are presented representing a potentially more ideal, but costly, scenario (A) and more cost-conscious approach (B). 


\section{Chapter Two}

The role of protein kinase $C$ modulation in neural injury outcome is not well elucidated but work completed by Sun and colleagues using a global model of ischemia, akin to cardiac failure, has indicated the potential benefit of protein kinase $\mathrm{C}$ modulation via bryostatin-1 administration. This chapter seeks to investigate this potential protective effect in a clinically relevant thromboembolic model of ischemic stroke utilizing aged animals and tissue plasminogen activator for reperfusion. Characterization of bryostatin-1-mediated effects on outcome was pursued through the use of both histological measures (infarct volume, edema, and atrophy) as well as functional assessments (mNSS) as outlined by the Stroke Therapy Academic Industry Roundtable (STAIR). Insight into the mechanism of bryostatin-1 was obtained through assessment of protein kinase $C$ expression, specifically $\alpha$ and $\varepsilon$ isozymes, post-stroke in both neurons, astrocytes, and also endothelial cells.

\section{Bryostatin-1 Improves Outcome in a Clinically Relevant Model of Ischemic Stroke via Protein Kinase C Activation}

Zhenjun Tan, Ryan C. Turner, Rachel L. Leon, Xinlan Li, Jarin Hongpaisan, Wen Zheng, Aric F. Logsdon, Zachary J. Naser, Daniel L. Alkon, Charles L. Rosen, Jason D. Huber

This work is currently under revision for publication in Stroke.

\section{ABSTRACT}

Background and Purpose. Bryostatin-1, a potent protein kinase $C$ (PKC) activator, has demonstrated therapeutic efficacy in preclinical models of associative memory, Alzheimer's disease, global ischemia, and traumatic brain injury. In this study, we tested the hypothesis that administration of bryostatin-1 provides a therapeutic benefit in reducing brain injury and improving stroke outcome using a clinically relevant model of cerebral ischemia with tissue plasminogen activator (tPA) reperfusion in aged rats.

Methods. Focal cerebral ischemia was produced by reversible occlusion of the right middle cerebral artery (MCAO) in 18-20 month old female Sprague-Dawley rats using an autologous blood clot with tPA-mediated reperfusion. Bryostatin-1 was administered at 6 $\mathrm{h}$ after MCAO then at 3, 6, 9, 12, 15, and $18 \mathrm{~d}$ after MCAO. Functional and assessment was conducted at 1, 7, 14, and $21 \mathrm{~d}$ after MCAO and histological assessment was performed at $21 \mathrm{~d}$ post-MCAO.

Results. Bryostatin-1 treated rats showed improved survival following MCAO, especially during the first $4 \mathrm{~d}$. Repeated administration of bryostatin- 1 following MCAO resulted in reduced infarct volume, atrophy formation, and improved neurological function at $21 \mathrm{~d}$ after MCAO. Changes in PKC alpha expression and PKC epsilon expression in neurons were noted in bryostatin-1 treated rats at $24 \mathrm{~h}$ following MCAO.

Conclusions. Repeated bryostatin-1 administration following MCAO protected the brain from severe neurological injury following MCAO. Bryostatin-1 treatment improved survival rate, reduced infarct volume, salvaged tissue in infarcted hemisphere by reducing necrosis and peri-infarct astrogliosis, and improved functional outcome following MCAO. 


\subsection{INTRODUCTION}

There is an urgent clinical need for improved treatment of acute ischemic stroke. Despite impressive gains in our knowledge of the mechanisms underlying ischemic brain injury, there has been a failure in translating these promising preclinical findings into therapeutics that improve stroke outcome. Thrombolytic treatment with recombinant tissue plasminogen activator (tPA) remains the only FDA approved drug for treatment of focal ischemic stroke. However, less than $2 \%$ of people suffering an ischemic stroke receive tPA due to increased risk of secondary cerebral hemorrhage and edema formation ${ }^{486}$. Based on the aging population and increased stroke burden, an unmet need exists to develop alternative approaches to treat acute ischemic stroke, either independently of tPA or in an effort to increase the number of patients eligible for tPA.

Preclinical studies of proposed therapeutics for ischemic stroke have largely failed to consider the greatest risk factor for stroke, age ${ }^{9}$. Previous studies suggest that aged rats represent a more clinically relevant model of ischemic stroke in comparison to the younger animals typically used, a difference characterized by an altered inflammatory response following brain injury ${ }^{84,487}$. These studies illustrate an increased severity of ischemic injury and altered neural injury progression ${ }^{30,51,84,487}$. Therefore, inclusion of aged animals may increase clinical relevance and the likelihood of bench-to-bedside therapeutic translation.

Protein kinase $\mathrm{C}(\mathrm{PKC})$ is critical for storage of associative memory and related synaptogenesis in normal animals ${ }^{394}$; reducing accumulation of A Beta, synaptic loss, cognitive deficits, and neurodegeneration in preclinical models of Alzheimer's disease 358, 488; and protection against ischemic pathology 364,365 and traumatic brain injury in rodents ${ }^{349}$. PKC isozymes alpha $(\alpha)$ and epsilon $(\varepsilon)$ regulate synaptogenic and antiapoptotic signaling pathways ${ }^{489}$, as well as critical functional pathways in the multicellular response to ischemia-reperfusion injury ${ }^{321}$. Individual PKC isozymes play differing, and sometimes opposing, roles in injury response that often depend on cell types and degree of pathophysiology ${ }^{362,490}$. Observations that PKC activation mediates both protective and harmful messages results from different PKC isoforms being activated by different signals that play concentration- and time- dependent roles in the development of neuronal damage and regeneration 375,394 .

Bryostatin-1, an ultrapotent PKC activator may provide substantial benefit in the treatment of focal ischemic stroke. Studied extensively as an anti-tumorogenic agent, recent studies demonstrated that administration of bryostatin-1 following ischemic insult resulted in curative neurogenesis, synaptogenesis, and cognitive enhancement ${ }^{362}$. The purpose of our current study was to investigate the pharmacological potential of repeated administration of bryostatin-1 to improve outcome following focal ischemic stroke in aged rats using a clinically relevant experimental stroke model.

\subsection{MATERIALS AND METHODS}

\subsubsection{Animals and Treatment Protocol}

Forty-eight female Sprague-Dawley rats (18-20 months old) were purchased from Harlan Laboratories (Indianapolis, IN) and housed under 12-h:12-h light-dark conditions with food and water ad libitum. All work involving rats was approved by the West Virginia University Animal Care and Use Committee. Rats were randomly divided into two treatment groups $(\mathrm{N}=38)$. Group $1(\mathrm{~N}=19)$ underwent a $2 \mathrm{~h}$ MCAO with tPA-mediated (5 
$\mathrm{mg} / \mathrm{kg}$; i.v.) reperfusion as previously described 9,10 , and at $6 \mathrm{~h}$ was administered bryostatin-1 (15 mg/m²; i.v.) with subsequent doses every $3 \mathrm{~d}$ for a total of 7 doses over 21 d. Group $2(\mathrm{~N}=19)$ served as the control group and underwent the same procedures except was administered $0.9 \%$ saline instead of bryostatin-1. A second set of rats ( $\mathrm{N}=10 ; 6$ saline and 4 bryostatin-1 treated rats) underwent MCAO with bryostatin-1/ saline treatment at $6 \mathrm{~h}$ and were then euthanized at $24 \mathrm{~h}$ to visualize changes in PKC isozyme ( $\alpha$ and $\varepsilon$ ) expression in neurons, endothelial cells, and astrocytes. Ischemia and reperfusion were defined as a minimum of $80 \%$ decrease in cerebral blood flow at onset of ischemia and return to $80 \%$ of baseline blood flow measurement, respectively. Rats not achieving these standards were excluded from the study ${ }^{9,10}$. Chemicals and antibodies were acquired from specified manufacturers (Supplemental Methods; please see http://stroke.ahajournals.org).

\subsubsection{Neurological Functional Assessment}

The modified Neurological Severity Scores (mNSS) was used to assess neurological function at 1, 7, 14, and $21 \mathrm{~d}$ after MCAO. The mNSS is a composite score of motor, sensory, balance and reflex measures ranging from scores of 1 to 17 , with higher scores indicating greater neurological injury ${ }^{493}$.

\subsubsection{TUNEL Staining, Immunohistochemistry, and Infarct Volume Measurement}

To identify cells undergoing DNA fragmentation, TUNEL staining was performed according to manufacturer's instructions (Roche Applied Science). After mounting and nuclear staining with 4',6-diamidino-2-phenylindole (DAPI), slices were visualized for apoptotic tissue using a Zeiss LSM 510 confocal microscope.

Using alternating slices from the same brain (Supplemental Methods; please see http:// stroke.ahajournals.org), immunohistochemistry using a modified ABC procedure (Benkovic et al., 2004) was performed with anti-GFAP (1:1000) for evaluation of astrocyte morphology ( $\mathrm{n}=7$ saline and 10 bryostatin-1 treated rats). Infarction volumes were determined using standard protocols for hematoxylin and eosin (H\&E) staining as well as cresyl violet staining. Degree of cerebral atrophy at $21 \mathrm{~d}$ following MCAO was calculated from $\mathrm{H}$ \& $\mathrm{E}$ stained tissue using the formula: cerebral atrophy $=(\mathrm{LV}-\mathrm{RV}) /$ LVx $100 \%$, where LV was volume of left hemisphere $\left(\mathrm{mm}^{3}\right)$ and RV was volume of right hemisphere $\left(\mathrm{mm}^{3}\right)$.

Tri-labeled confocal microscopy was performed to determine immunolocalization of aPKC (1:100) and EPKC (1:100) with neurons (1:150), endothelial cells (1:100), and astrocytes (1:100) at $24 \mathrm{~h}$ following MCAO ( $\mathrm{n}=4$ rats per treatment). Nuclear staining was performed on sections using DAPI and secondary antibodies were incubated at 1:200 dilution.

Expression levels of DNA damage (TUNEL), $\alpha \mathrm{PKC}$, and $\varepsilon P K C$ were evaluated based on immunofluorescence intensity. Confocal images were quantified using ImageJ as previously described ${ }^{358}$.

\subsubsection{Statistical Analysis}

All data were compiled and analyzed by an investigator blinded to treatment and presented as mean \pm SEM. Physiologic parameters, functional data, and immunofluorescence intensity were compared by Student's t-test grouped by treatment 
(saline vs bryostatin-1). Mortality data were compared using Fisher's exact test (2X2 contingency table). $\mathrm{P}<0.05$ was considered statistically significant.

\section{RESULTS}

\subsubsection{Administration of Bryostatin-1 Following MCAO in Aged Rats Improved Survival Rate \& Neurological Function}

A total of 48 rats were used in this study. Thirty-eight rats were used to evaluate the effects of bryostatin-1 administration on ischemic brain injury over $21 \mathrm{~d}$ following MCAO. Two rats from this cohort, both saline-treated, were excluded from further study for not meeting ischemia/reperfusion criteria. Survival rates were documented daily from 1 to 21 d (Figure 2.1A). Administration of bryostatin-1 following MCAO improved survival rates through $21 \mathrm{~d}(\mathrm{~N}=36 ; 17$ saline and 19 bryostatin-1 treated rats) with positive effects most evident during the first $4 \mathrm{~d}$ following MCAO and a significant increase in survival achieved from 2 to $17 \mathrm{~d}$ after MCAO. Although survival decreased in bryostatin-1 treated rats after day 4, improved survival was demonstrated at $7 \mathrm{~d}(68 \%$ bryostatin- $1, n=13$, versus $41 \%$ saline, $n=7$, treated rats), $14 d(59 \%$ bryostatin $-1, n=11$, versus $41 \%$ saline, $n=7$, treated rats), and 21 d (53\% bryostatin- $1, n=10$, versus $41 \%$ saline, $n=7$, treated rats) after MCAO.

Neurological functional assessment revealed significant improvement in mNSS scores at $21 \mathrm{~d}$ post-MCAO in bryostatin- 1 treated rats $(2.6 \pm 0.3 ; n=10)$ compared to saline-treated rats $(3.7 \pm 0.2 ; n=7)$ (Figure 2.1B). No difference $(p>0.05)$ in mNSS scores was observed at $1 \mathrm{~d}$ ( $\mathrm{n}=15$ saline and 18 bryostatin- 1 treated rats), $7 \mathrm{~d}(\mathrm{n}=7$ saline and 13 bryostatin- 1 treated rats), and $14 \mathrm{~d}$ ( $\mathrm{n}=7$ saline and 11 bryostatin-1 treated rats) following MCAO.

\subsubsection{Bryostatin-1 Results in Diminished Infarct Volume Post-MCAO}

Quantification of infarction volume for cortex, striatum and total cerebral hemisphere using H\&E-stained and cresyl violet-stained coronal sections was measured at $21 \mathrm{~d}$ with bryostatin-1 administration $(n=10)$ resulting in a significant $(p<0.05)$ decrease in infarct volume in cortex $(6 \pm 2 \%)$, striatum $(19 \pm 3 \%)$, and total hemisphere $(10 \pm 2 \%)$ as compared to saline-treated $(n=7)$ rats, which showed infarct volumes in cortex $(18 \pm 5 \%)$, striatum $(31 \pm 2 \%)$, and total $(23 \pm 3 \%)$ (Figure 2.2A). Measurements of cortical atrophy revealed a significant $(p<0.05)$ decrease in the infarcted hemisphere in bryostatin-1 treated rats $(5 \pm 1 \% ; n=10)$ compared to saline-treated rats $(14 \pm 2 \% ; n=7)$ (Figure 2.2B). Figure $2.2 \mathrm{C} \& \mathrm{D}$ shows representative photomicrographs of the infarcted hemisphere in saline control $(n=7)$ and bryostatin-1 $(n=10)$ treated aged rats at $21 \mathrm{~d}$ following MCAO. The infarcted hemisphere from saline-treated rats exhibited profound atrophy marked by necrosis and vacuolation. The peri-infact area showed intense astrogliosis throughout the hemisphere. The infarcted hemisphere of bryostatin-1 treated rats showed a smaller brain injury with astrogliosis confined to the MCA region.

\subsubsection{Administration of Bryostatin-1 Reduces Cellular Apoptosis and Decreases Astrogliosis in Aged Rats at 21 Days Following MCAO}

At $21 \mathrm{~d}$ after MCAO, a significant reduction $(\mathrm{p}<0.01)$ in TUNEL-positive cells, representing those undergoing apoptosis due to DNA fragmentation, were detected in the peri-infarct region surrounding the infarct in bryostatin-1 treated rats when compared to saline-treated rats ( $n=7$ saline and 10 bryostatin- 1 treated rats) (Figure 2.3). This finding was reinforced by a profound reduction in reactive astrocytes, identified by GFAP expression, observed in bryostatin-1 treated rats, in which not only was astrogliosis reduced but the degree of tissue destruction was markedly reduced (Figure 
2.4). Astrocytic hypertrophy with enlarged processes was evident in tissue surrounding the necrotic zone around the infarct in saline-treated aged rats (Figure $2.4 \mathrm{~A} \mathrm{\&} \mathrm{B}$ ), consistent with the astrocytic response. Bryostatin-1 attenuated this response and reduced tissue damage in the peri-infarct area (Figure $2.4 \mathrm{C} \& \mathrm{D}$ )

\subsection{4. $\alpha P K C$ Expression Was Reduced in Neurons Surrounding the Penumbral Region of Bryostatin-1 Treated Rats at $24 \mathrm{~h}$ Following MCAO}

Colocalization of aPKC with neurons (NeuN), endothelial cells (CD31), and astrocytes (GFAP) was investigated in penumbra surrounding the infarct in aged rats at $24 \mathrm{~h}$ after MCAO ( $n=4$ rats per treatment group). aPKC immunoreactivity was observed in neurons and endothelial cells (Figure 2.5A \& 2.6A). Expression of $\alpha \mathrm{PKC}$ was diffusely localized throughout the cytoplasm of the cell. No colocalization of aPKC was shown with GFAP-positive astrocytes (data not shown). Rats treated with bryostatin-1 showed a significant $(p<0.05)$ decrease in immunoreactivity of $\alpha P K C$ in neurons at $24 \mathrm{~h}$ following MCAO (Figure 2.5A \& 2.5C). No change ( $p>0.05)$ in aPKC expression was observed in endothelial cells at $24 \mathrm{~h}$ following MCAO (Figure 2.6A \& 2.6C).

\subsubsection{Bryostatin-1 Increased $\varepsilon P K C$ Within Neurons in the Penumbra at $24 \mathrm{~h}$ Following MCAO and TPA Reperfusion}

Colocalization of $\varepsilon P K C$ with NeuN and CD31 in the penumbral area at $24 \mathrm{~h}$ after MCAO was investigated ( $n=4$ rats per treatment group). $\varepsilon P K C$ immunoreactivity was observed in neurons and endothelial cells (Figure 2.5B \& 2.6B). Expression of $\varepsilon$ PKC was punctate along cytoplasmic edge of the plasma membrane of the cell. No colocalization of $\varepsilon P K C$ was shown with GFAP-positive astrocytes. Rats treated with bryostatin-1 showed a significant $(p<0.05)$ increase in immunoreactivity of PKC in neurons at $24 \mathrm{~h}$ following MCAO (Figure 2.5B \& 2.5C).

\subsection{DISCUSSION}

The primary finding of this study was that administration of bryostatin-1 after MCAO and tPA reperfusion decreased mortality over $21 \mathrm{~d}$, with marked improvement during the first $4 \mathrm{~d}$. Additionally, we are the first to report that bryostatin-1 administration improved recovery from ischemic brain injury and functional outcome up to $21 \mathrm{~d}$ after MCAO in aged animals. Use of bryostatin-1 attenuated both necrotic and apoptotic cell death. The reduced degree of atrophy observed in aged rats treated with bryostatin-1 at $21 \mathrm{~d}$ suggests the potential for both decreased inflammation and cellular death following MCAO and TPA reperfusion. Effects measured in this study were associated with changes in expression and localization of PKC isozymes in neurons and endothelial cells within the peri-infarct region.

Regulation of PKC activity is the primary reported mechanism of action for bryostatin- 1 . Inhibition of PKC isozymes blocked the synaptogenic and cognitive-enhancing effects of bryostatin on normal animals ${ }^{394}$. Therefore, it is plausible that pathological mechanisms initiated following ischemic brain injury involve PKC-mediated pathways ${ }^{33,322}$. Results from this study clearly show increased $\varepsilon P K C$ expression in endothelial cells and a modest decrease in aPKC expression in neurons of bryostatin-1 treated rats at $24 \mathrm{~h}$ following MCAO. The roles of PKC activity following ischemic stroke and reperfusion are complex and poorly defined. What is known is that PKC activity mediates protective and damaging signaling cascades during the injury and reparative phases of ischemic stroke 362, 490; suggesting that timing, localization, and the PKC isozyme activated play critical, and sometimes contrasting, roles in injury response and progression of neuronal 
damage and regeneration following stroke ${ }^{375,394}$. Previous studies report that increased \&PKC activity initiates proliferative and cell survival signaling pathways following ischemic stroke and reperfusion ${ }^{321}, 363,396$, while increased aPKC activity exacerbates neuronal damage following post-stroke brain injury through initiation of anti-proliferative and pro-apoptotic pathways ${ }^{330}$; thus, while still inconclusive, our results suggest that changes in PKC activity in endothelial cells and neurons may play a vital role in the acute neuroprotective actions of bryostatin-1. It should be noted that while bryostatin initially activates both PKC $\alpha$ and $\varepsilon$, it only causes prolonged binding of $\varepsilon P K C$ to the critically required anchoring protein, RACK 1 , that is required for prolonged activation (unpublished data, Sun, Nelson, and Alkon).

Of particular interest, especially during the acute phase of stroke injury, is the role different PKC isozymes play in alterations of endothelial cell function during pathophysiological conditions. Increased PKC activity has been shown to affect structural and functional integrity of the BBB following ischemic brain injury ${ }^{382}$ with $\alpha P K C$ and $\beta P K C$ increasing $B B B$ opening through alterations in tight junction protein localization and $\varepsilon P K C$ enhancing BBB integrity by upregulating the expression of claudin $5^{345,384,491}$. Our study showed that $\alpha \mathrm{PKC}$ and $\varepsilon P K C$ were localized to both neurons and cerebral endothelial cells. No colocalization of aPKC or $\varepsilon P K C$ was observed with GFAPpositive astrocytes; however, that does not mean that changes in PKC activity do not have a significant influence on astrocyte reactivity as demonstrated by our study, which showed a reduction in astrogliosis and tissue damage in bryostatin- 1 treated rats. In fact, in relation to chronic brain response following ischemic injury, attenuation of glial response may end up being the most significant finding of this study. Gaining a better understanding of the critical mediators and timing of this response will be a primary focus of follow-up studies.

In conclusion, administration of bryostatin-1 following MCAO and TPA reperfusion has neuroprotective effects on the magnitude of ischemic brain injury. A notable finding in this study was the marked improvement in survival observed in bryostatin-treated rats, especially during the first week following ischemic insult. While mortality increased in the bryostatin-1 treated group during the $21 \mathrm{~d}$, it must be emphasized that PKC activator treatment, depending on dose and duration, causes three sequential consequences: activation, downregulation (and thus inhibition), and de novo protein synthesis. Thus, careful selection of treatment dosing and duration has a profound impact on PKCmediated phosphorylation of down-stream substrates and associated therapeutic benefits ${ }^{492}$. Bryostatin-1 treated rats displayed reduced ischemic brain injury following MCAO that is characterized by increased survival, reduced infarct volume, decreased atrophy, and improved neurological function.

\subsection{ACKNOWLEDGEMENTS}

This study was supported by a grant from the National Institute of Neurological Diseases and Stroke [RO1 NS061954] to J.D.H. 


\section{FIGURES}

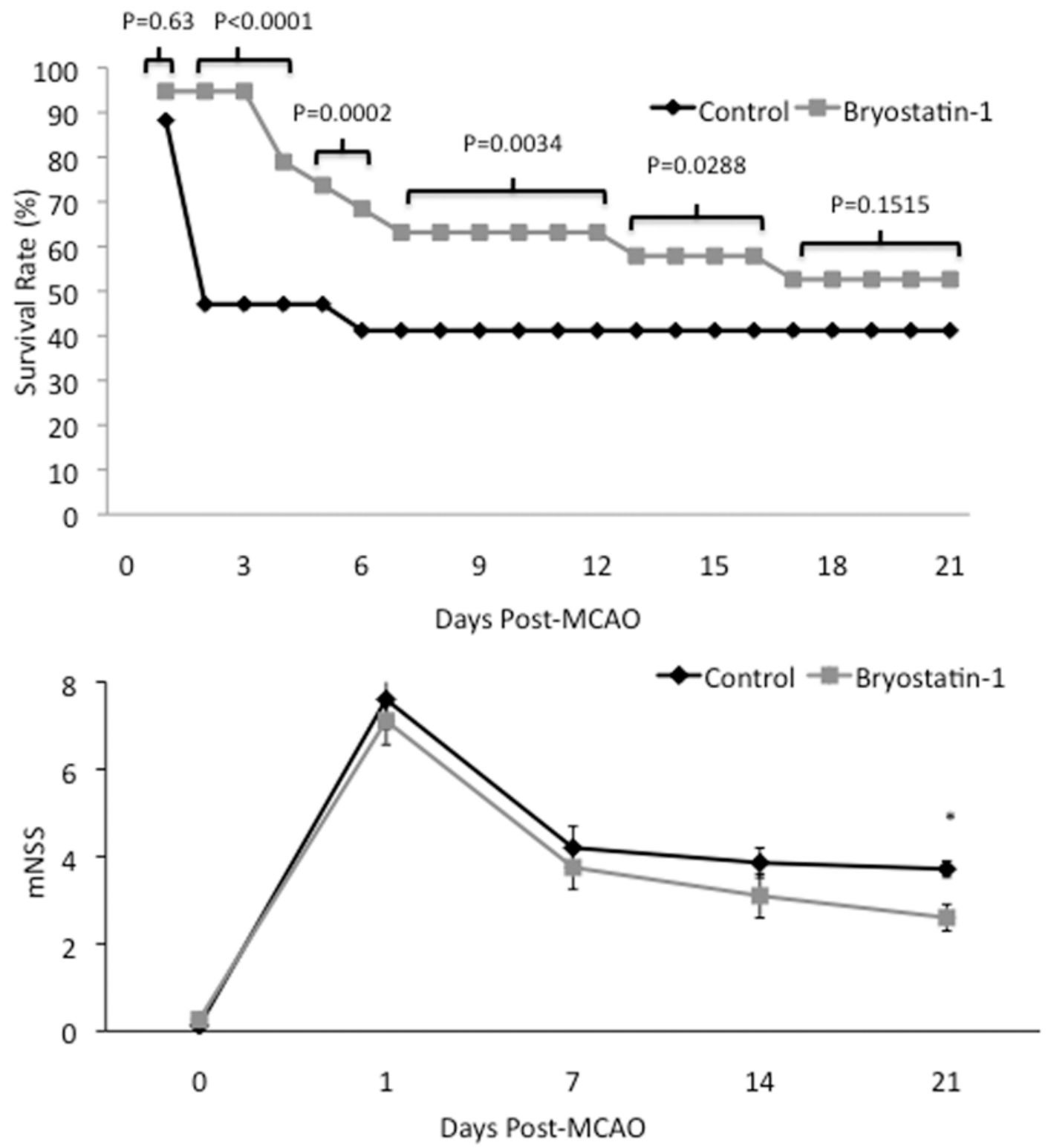

Figure 2.1. A.) Administration of bryostatin-1 significantly increased the survival rate of aged rats from 2 to $17 \mathrm{~d}$ following MCAO and tPA reperfusion. A total of 7 out of 17 saline and 10 out of 19 bryostatin- 1 treated rats survived to $21 \mathrm{~d}$ after MCAO. Fisher's exact test with two-tailed calculation of $P$ value was performed to determine statistical significance in mortality rate $\left({ }^{*} \mathrm{p}<0.001 ;{ }^{\#} \mathrm{P}<0.01 ;{ }^{\dagger} \mathrm{P}<0.05\right)$. B.) Neurological function was also significantly improved in bryostatin-1 treated aged rats at $21 \mathrm{~d}$ after MCAO as compared to saline-treated rats. No difference in neurological score between saline and bryostatin-1 treated rats was measured at 1, 7, and $14 \mathrm{~d}$ after MCAO $(n=7$ saline and 10 bryostatin-1 treated rats). Data presented as mean \pm SEM, * $p<0.05$. 


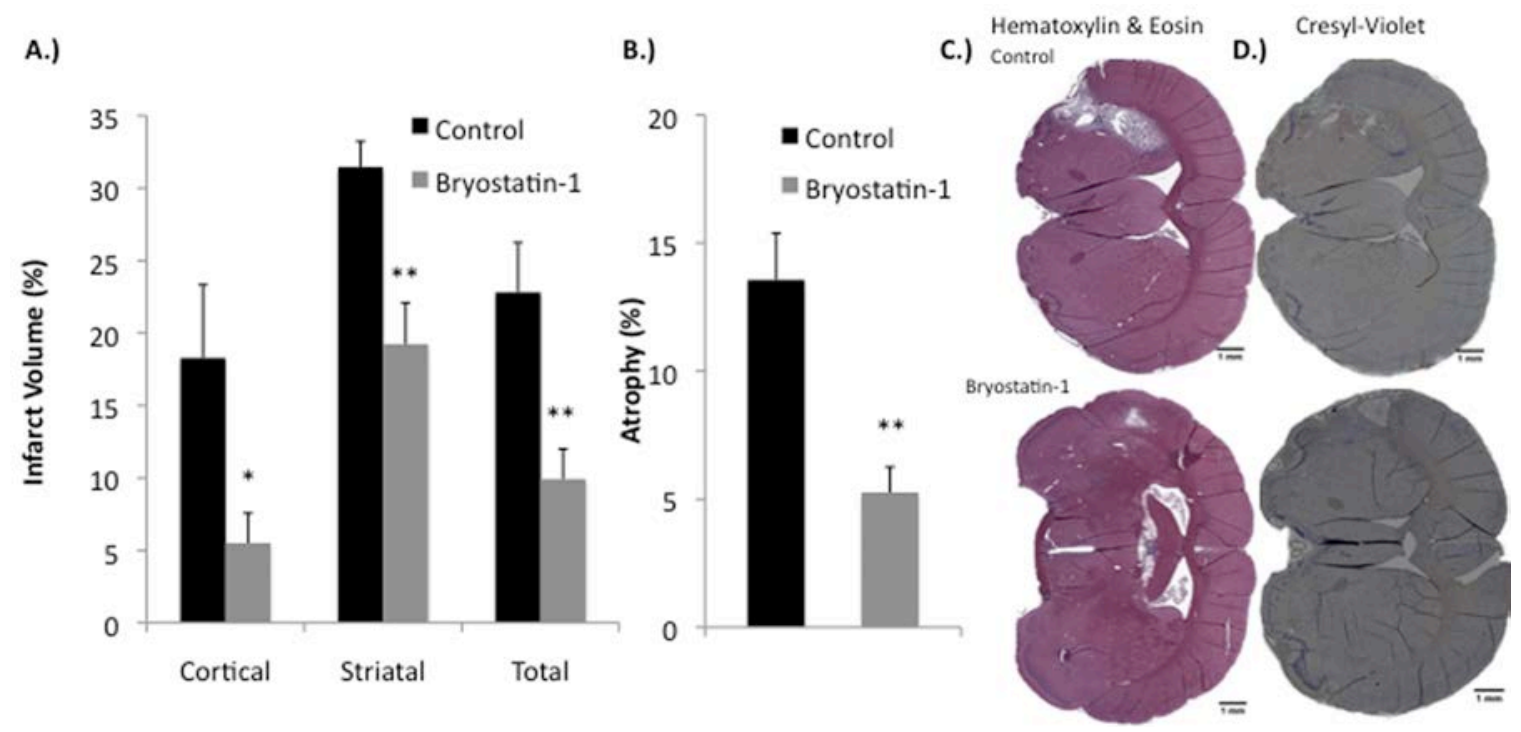

Figure 2.2. A.) Repeated administration of bryostatin-1 decreased infarct volume in the cortex $(p=0.02)$, striatum $(p=0.01)$, and total cerebral hemisphere $(p=0.001)$ of aged rats at $21 \mathrm{~d}$ following MCAO ( $\mathrm{n}=7$ saline and 10 bryostatin-1 rats). B.) Bryostatin-1 significantly reduced cerebral atrophy in the ischemic hemisphere as compared to saline-treated rats at $21 \mathrm{~d}$ after MCAO ( $\mathrm{n}=7$ saline and 10 bryostatin-1 rats). Data presented as mean \pm SEM, * $p<0.05$. C.) Representative photomicrographs of hematoxylin and eosin stained brains in control and bryostatin-1 treated animals, respectively. D.) Representative photomicrographs of cresyl-violet stained brains in control and bryostatin-1 treated animals, respectively. 


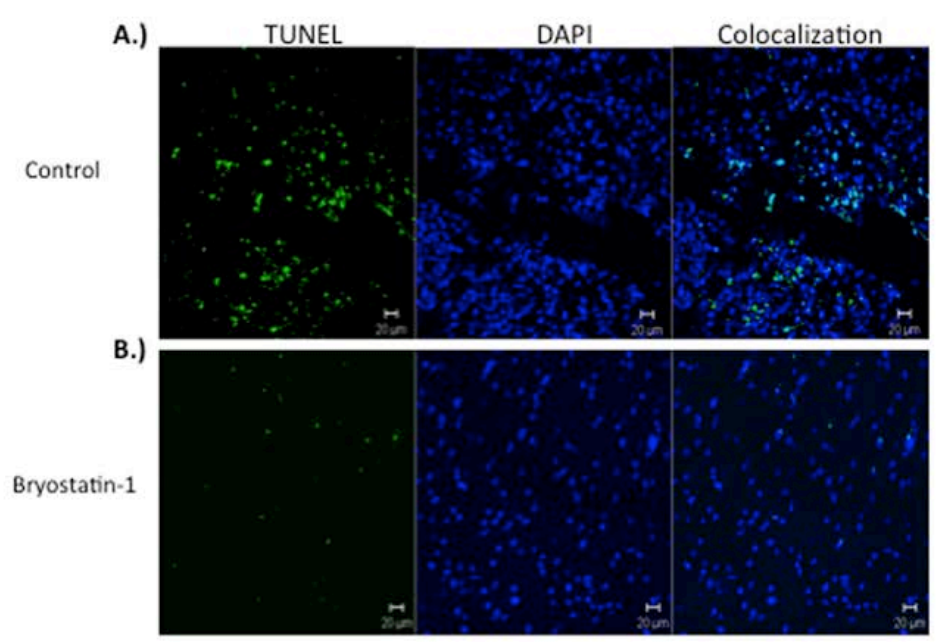

C.)

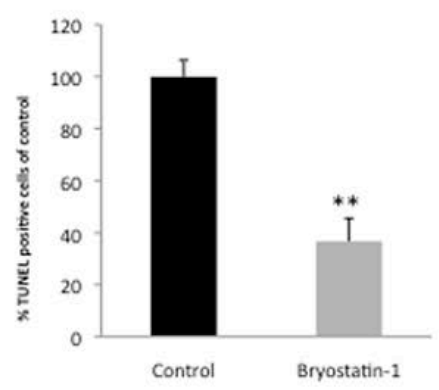

Figure 2.3. Representative photomicrographs showing TUNEL-positive cells (green) in the peri-infarct region at 21d after MCAO in saline group (A) and bryostatin-1 treated (B) aged rats at $21 \mathrm{~d}$ after MCAO. (C) A $84 \pm 15 \%$ decrease in TUNEL positive cells were observed at $21 \mathrm{~d}$ following MCAO in rats treated with bryostatin-1 as compared to salinetreated rats. Data expressed as mean \pm SEM. * denotes statistical significance at $p<0.05$. Cell nuclei were stained using DAPI ( $n=7$ saline and 10 bryostatin- 1 rats). 
A.)

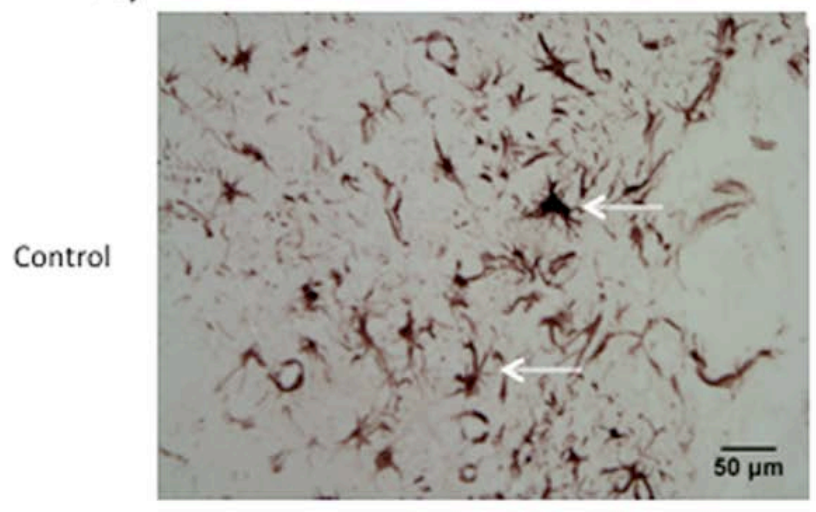

C.)

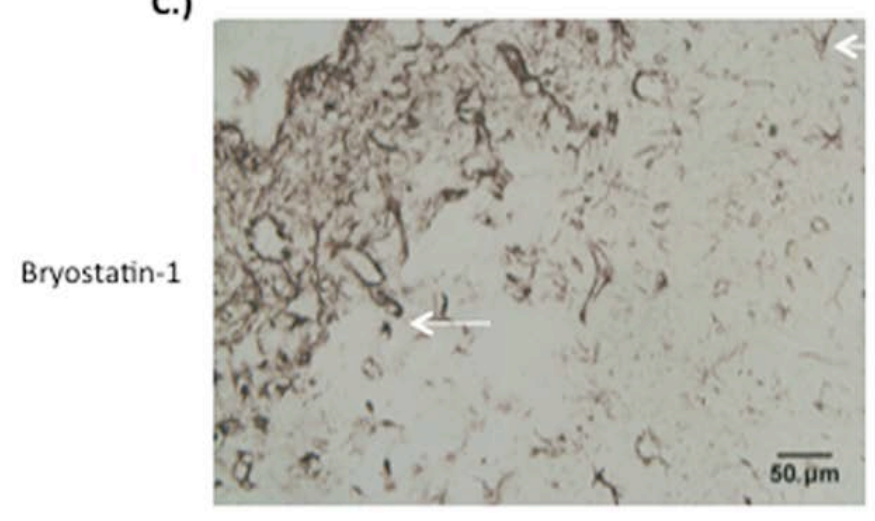

B.)

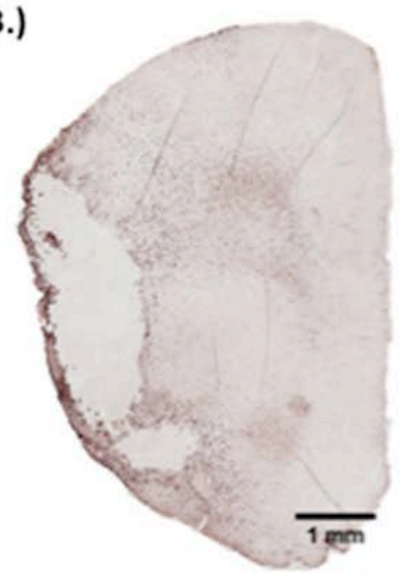

D.)

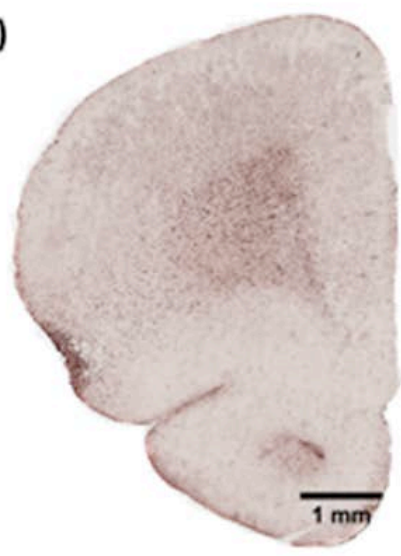

Figure 2.4. Representative photomicrographs of GFAP-staining positive cells in saline $(A \& C)$ and bryostatin-1 (B \& D) treated aged rats at $21 \mathrm{~d}$ following MCAO. Arrows (white) point to difference in astrocyte morphology observed in saline and bryostatin treated brain at $21 \mathrm{~d}$ following MCAO ( $\mathrm{n}=7$ saline and 10 bryostatin-1 rats). 


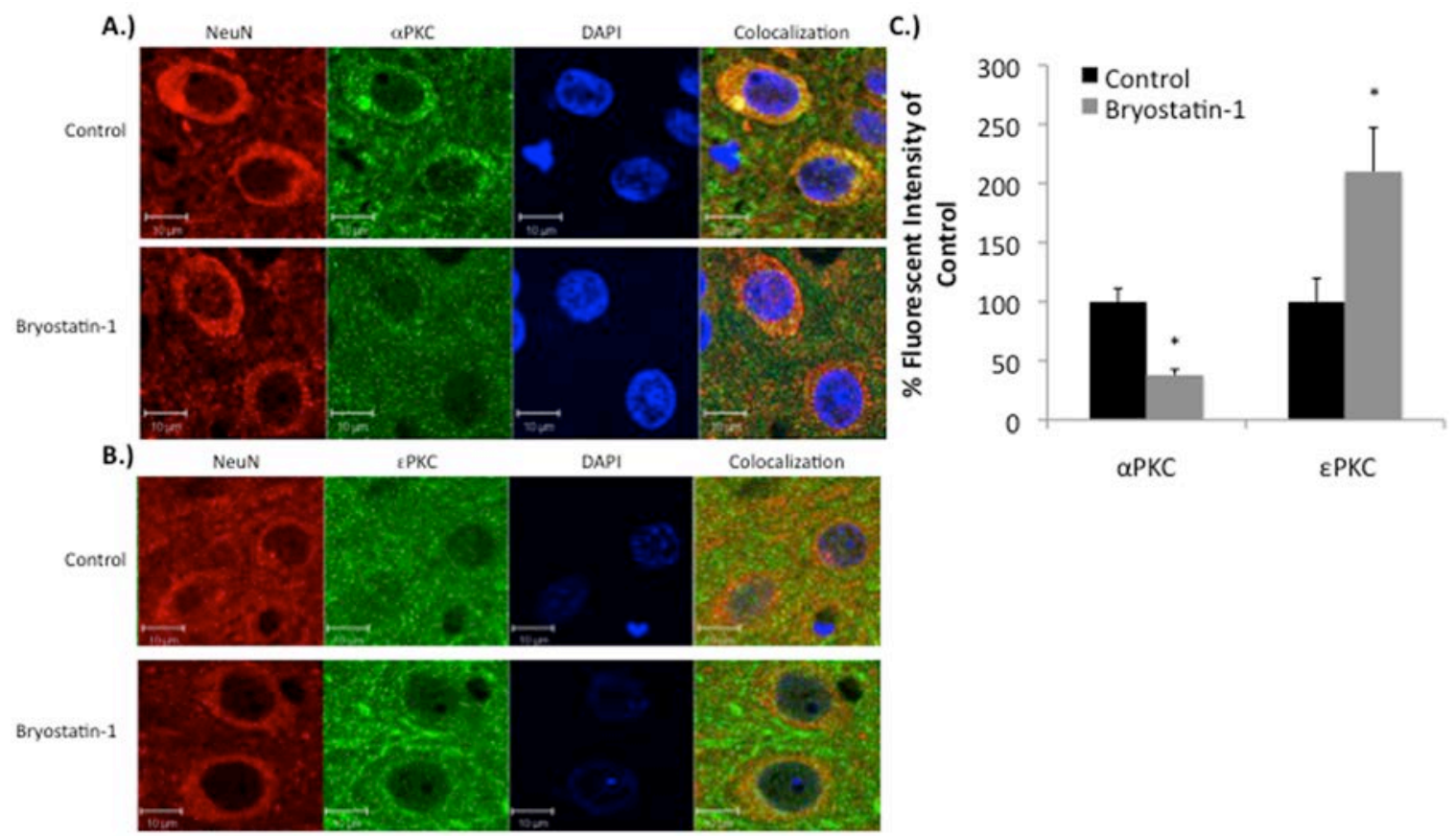

Figure 2.5. Representative images of tri-color confocal immunofluorescent microscopy demonstrating colocalization of NeuN with aPKC (A) and $\varepsilon P K C(B)$ in the penumbra of the infarcted hemisphere of saline and bryostatin-1 treated aged rats at $24 \mathrm{~h}$ after MCAO. (C) In neurons, a 68\% decrease in aPKC expression and a $202 \%$ increase in $\varepsilon P K C$ expression were measured at $24 \mathrm{~h}$ following MCAO in bryostatin-1 treated rats as compared to saline-treated rats. Data expressed as mean \pm SEM. * denotes statistical significance at $p<0.05$. Cell nuclei were stained using DAPI ( $n=4$ rats per group). 


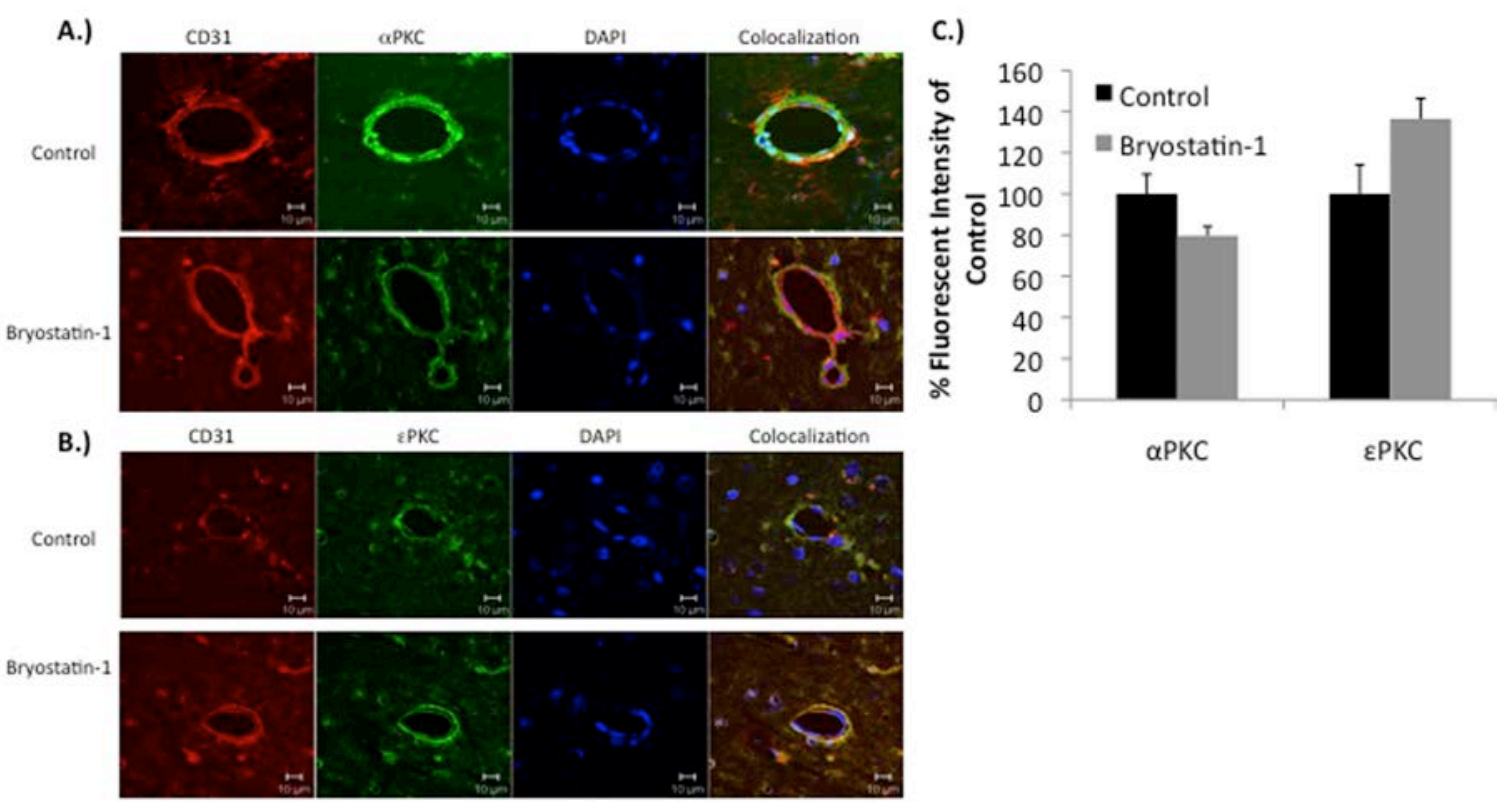

Figure 2.6. Representative images of tri-color confocal immunofluorescent microscopy demonstrating colocalization of CD31 (endothelial cells) with aPKC (A) and عPKC (B) in the penumbra of the infarcted hemisphere of saline and bryostatin-1 treated aged rats at $24 \mathrm{~h}$ after MCAO. (C) In endothelial cells, no statistical difference $(p<0.05)$ in $\alpha \mathrm{PKC}$ and $\varepsilon P K C$ expression were observed at $24 \mathrm{~h}$ following MCAO between bryostatin-1 and saline treated rats. Data expressed as mean \pm SEM. * denotes statistical significance at $p<0.05$. Cell nuclei were stained using DAPI ( $n=4$ rats per group). 


\title{
Chapter Three
}

Currently, tissue plasminogen activator (tPA) remains the only FDA-approved pharmacologic for the treatment of ischemic stroke. Administration of tPA is hampered by an extensive list of contraindications and narrow therapeutic window resulting in less than $5 \%$ of patients receiving tPA. Consequently, there is an immense need for pharmacological agents that can improve the safety to tPA, allowing administration in previously contraindicated patients and those presenting outside the classically defined therapeutic window. One potential candidate based on prior work in other disease and animal models is apyrase. This study examines the effect of apyrase on outcome following ischemic stroke and tPA administration at 6 hours post-stroke.

Combination Treatment of r-tPA and Apyrase Reduces Mortality Rate and Hemorrhage Transformation in Acute Ischemic Stroke in Aged Female Rats

Zhenjun Tan, Xinlan Li, Ryan C. Turner, Aric F. Logsdon, Brandon P. Lucke-Wold, Kenneth H. DiPasquale, Ridong Chen, Jason D. Huber, Charles L. Rosen

This work is currently in preparation for publication submission.

\begin{abstract}
Thrombolytic therapy utilizing the only FDA-approved pharmacologic agent for ischemic stroke treatment, recombinant tissue plasminogen activator ( $r$-tPA), has a narrow time window for administration and is therefore limited to use in a select few patients. Furthermore, r-tPA has direct and deleterious effects on the ischemic neurovascular unit that can substantially increase the risk of intracerebral hemorrhage and intrinsic toxicity. These therapeutic shortcomings necessitate additional investigation of agents that can extend the windows for safe use of thrombolytic therapy. In this study, combination of $r$ tPA and APT 102, a novel form of the naturally occurring human apyrase/ADPase, was investigated in a clinically relevant embolic ischemic stroke model in aged female rats to evaluate whether the combination can safely extend the therapeutic window of r-tPA administration. Results of our study showed that co- administration of APT 102 with rtPA significantly reduced mortality rate and decreased cortical and total hemispheric stroke volumes. Similarly, co-administration produces significant improvement in neurological deficit scores obtained using the modified Neurological Severity Scale (mNSS). Decreased cortical, striatal and total hemorrhage were also observed in APT 102 plus r-tPA groups as determined by hemoglobin extravasation. These data suggest that APT 102 can extend the therapeutic window and prevent intracerebral hemorrhage following r-tPA treatment in aged female rats. APT 102 combined with r-tPA may have potential utility in treating acute ischemia stroke in clinical setting. Prior work indicates this beneficial effect may be achieved through modulation of extracellular ATP and ADP, substances involved in platelet aggregation and endothelial activation, by APT 102.
\end{abstract}

\section{Keywords}

Acute ischemia stroke; APT 102/ apyrase; hemorrhage transformation; infarction size; recombinant tissue plasminogen activator 


\subsection{INTRODUCTION}

Stroke remains a leading cause of death worldwide, consequently a tremendous clinical need for improved acute ischemic stroke therapy exists. Despite clinical trials on numerous drug candidates, pharmacological options for acute ischemic stroke are limited to thrombolytic therapy 493,494 . Unfortunately, thrombolytic therapy is indicated in a small subset of patients due to a narrow therapeutic window and an extensive list of contraindications. Therefore, there is an urgent need for adjunct therapy in conjunction with r-tPA administration that would reduce intracerebral hemorrhage, improve general clinical outcomes, and expand the time window of eligibility for r-tPA.

Reasons for the failed translation of other therapeutics from bench-to-bedside are presently unclear but notable contrasts exist between preclinical models of ischemic stroke and the clinical population and may account for failed translation. Specifically, stroke is a disease of the elderly with approximately $72 \%$ of stroke patients are over the age of $65{ }^{495}$. The Stroke Therapy Academic Industry Roundtable (STAIR) committee recommends that following evaluation of proposed therapeutics in young, healthy male animals, further studies should be performed in aged female animals and animals with comorbid conditions 47,496 . Therefore, we used a model of embolic stroke in aged female rats to investigate whether aging alters efficacy and side effects of r-tPA in a combination treatment paradigm with the belief that doing so may significantly contribute to the successful translation of therapeutics from animal models to stroke patients.

In this study, combination of r-tPA and APT 102, a recombinant form of human apyrase, was investigated in a clinically relevant embolic stroke model in aged female rats to evaluate whether the combination can reduce cerebral infarct volume, improve neurological deficit score, and prevent r-tPA-induced cerebral hemorrhage. Previous studies have shown that APT 102 alone or combination with r-tPA can prevent ischemiareperfusion injury in lung infarction 497, 498, myocardial infarction 499, 500 and ischemic stroke in young male rats ${ }^{501-503}$ making APT 102 a potentially promising therapeutic for ischemic stroke. Our general goal was to determine whether APT 102 will improve outcome of stroke, efficacy and safety of $r$-tPA, and extend the therapeutic window of $r$ tPA and the therapeutics can be translated from bench to bedside.

\subsection{MATERIALS AND METHODS}

\subsubsection{Animals, Surgical Procedures for MCAO and Drug Treatment}

Seventy-two female Sprague-Dawley rats (18-20 months old) were purchased from Harlan Laboratories ( Indianapolis, IN) and housed under 12-h:12-h light-dark conditions with food and water ad libitum. All work involving rats was approved by the West Virginia University Animal Care and Use Committee. Rats were randomly divided into three treatment groups. Saline group $(\mathrm{N}=23)$ : TBS(Tris Buffered Saline) as an i.v. bolus at $6 \mathrm{~h}$ after MCAO; r-tPA group ( $\mathrm{N}=30): 5 \mathrm{mg} / \mathrm{kg}$ of $\mathrm{r}$-tPA in TBS as an i.v. bolus at $6 \mathrm{~h}$ after MCAO; r-tPA+ APT 102 group ( $\mathrm{N}=19): 5 \mathrm{mg} / \mathrm{kg}$ of tPA plus $2.25 \mathrm{mg} / \mathrm{kg}$ of APT 102 in TBS as an i.v. bolus at $6 \mathrm{~h}$ after MCAO.

Rats underwent a reversible embolic MCAO as previously described and modified ${ }^{10,84}$, 504 . Briefly, rats were anesthetized with $2 \%$ isoflurane in a mixture of $30 \%$ oxygen and $70 \%$ nitrous oxide. A servo-controlled homeothermic heating blanket, utilizing a rectal thermometer, was used to maintain body temperature at $37^{\circ} \mathrm{C}$. All surgical procedures were performed with the aid of a Leica ${ }^{\mathrm{TM}}$ Surgical Microscope. Relative regional cerebral blood flow was monitored with a laser Doppler probe. The modified PE 50 microcatheter 
was inserted into the external carotid artery stump and advanced into the MCA. Placement in the proximal MCA was verified by a sudden decrease in cerebral blood flow as measured by laser Doppler. Microcatheter was withdrawn $\sim 1 \mathrm{~mm}$, allowing cerebral blood flow to return to baseline, and then the $25 \mathrm{~mm}$ thoroughly washed fibrinrich clot was injected. Successful MCA occlusion was confirmed by a drop in perfusion greater than $80 \%$ of initial baseline in continuous laser Doppler monitoring. Recanalization was noted if cerebral blood flow increased to $75 \%$ of baseline. Rats not achieving these standards were excluded from the study.

\subsubsection{Neurological Functional Assessments}

Neurological functional assessments were measured at post-MCAO 24 hours. The neurological assessments were made by an investigator blinded to the treatment group. The modified Neurological Severity Scores (mNSS) evaluated the motor, sensory, balance and reflex measures, with scores ranging from 1 to 17, with higher scores indicating greater neurological injury ${ }^{505}$.

\subsubsection{TTC staining and Determination of Infarction Volume}

At $24 \mathrm{hr}$ following MCAO, rats were sacrificed and infarct size and stroke volume were evaluated by 2,3,5-triphenyltetrazolium chloride (TTC; $2 \%$ ) staining of $2 \mathrm{~mm}$ coronal brain slices for $15 \mathrm{~min}$ at $37^{\circ} \mathrm{C}$. Hemispheres were scanned on a flatbed scanner and analyzed using Image $\mathrm{J}$ software. On each slice, the nonstained area (ischemic brain) was marked, and the infarct volume was calculated according to the slice thickness of 2 $\mathrm{mm}$ per slice. The corrected infarction volume (CIV) was given by the following equation: $\mathrm{CIV}=(\mathrm{CHV}-[\mathrm{IHV}-\mathrm{IHI}]) \times \mathrm{d}$, where $\mathrm{CHV}$ was the area of the contralateral cortical or striatal volume in $\mathrm{mm} 2$, IHV was the area of the ipsilateral cortical or striatal volume in $\mathrm{mm} 2$, $\mathrm{IHI}$ was the infarct cortical or striatal area in $\mathrm{mm} 2$, and $\mathrm{d}$ was the thickness of the slice (2 $\mathrm{mm}$ ). Edema index was calculated as follows: Edema index = ([volume of ipsilateral hemisphere-volume of contralateral hemisphere]/volume of contralateral hemisphere) $\mathrm{x} 100 \%{ }^{16}$.

\subsubsection{Spectrophotometric Assay of Hemoglobin}

Hemorrhagic transformation was quantified with spectrophotometric assay of brain hemoglobin content. At 24 hours after MCAO, the animals were perfused transcardially with $0.1 \mathrm{~mol} / \mathrm{L}$ Phosphate-buffered saline under anesthetization of ketamine and xylazine until the outflow fluid from the right atrium was colorless. MCA area in left and right hemisphere were dissected and put in $0.1 \mathrm{~mol} / \mathrm{L}$ phosphate-buffered saline. Brain tissue was then homogenized in $0.1 \mathrm{~mol} / \mathrm{L}$ phosphate-buffered saline. After 30 -minute centrifugation $(13000 \mathrm{~g}), 50 \mathrm{\mu l}$ samples (supernatant) were transferred into wells and $200 \mu \mathrm{l}$ reagent was added. After 15 minutes, optical density was measured at 400nm with a spectrophotometer. The hemoglobin content was expressed as $\mathrm{mg} / \mathrm{dl}$ tissues ${ }^{506}$.

\subsubsection{Statistical Analysis}

Data are presented as mean \pm SEM. Physiologic parameters and functional data were compared using two-way analysis of variance (ANVOA) and comparisons between groups determined using Turkey's post-hoc analysis. Level of significance was set at $p<0.05$. 


\subsection{RESULTS}

3.3.1. APT 102 and r-tPA in Combination Significantly Decreased Mortality Rate at $24 \mathrm{~h}$ Following MCAO

Mortality rate was evaluated at $24 \mathrm{~h}$ following MCAO. APT 102 and r-tPA in combination significantly reduced the mortality rate from $46.67 \%$ ( $n=30, r$-tPA group) to $15.79 \%$ ( $n=19$, r-tPA+ APT 102 group). The mortality rate of saline group was $34.78 \%(n=23$, saline group) (Figure 3.1). Animals dying prior to $24 \mathrm{~h}$ were autopsied. Typical findings were larger ipsilateral hemispheric infarctions, extensive brain edema and macroscopic hemorrhage. Animals expiring prior to $24 \mathrm{~h}$ were not included in the following histological analysis.

\subsubsection{APT 102 and r-tPA in Combination Reduced Cortical and Total Hemispheric but Not Striatal Infarction Size at $24 \mathrm{~h}$ Following MCAO}

Stroke volume of the infarct cortex, striatum, and total cerebral hemisphere were measured at $24 \mathrm{~h}$ following MCAO. Cortical infarct volume was reduced significantly by APT $102 / r$-tPA combination treatment compared with r-tPA alone $(29.15 \pm 4.48 \%$ versus $46.87 \pm 4.72 \%, p<0.05)$. Infarct volume of total cerebral hemisphere was also significantly reduced by APT 102/r-tPA combination treatment compared with r-tPA alone $(33.18 \pm 3.52 \%$ versus $48.15 \pm 3.23 \%, p<0.05)$. Striatal infarct volume was not significantly different in APT 102/r-tPA combination treatment and r-tPA alone $(39.74 \pm 3.07 \%$ versus $49.90 \pm 2.19 \%, p>0.05)$. The infarction volume of cortex, striatum, total cerebral hemisphere from saline group was $44.20 \pm 4.92 \%, 53.74 \pm 3.98 \%, 47.17 \pm 3.71 \%$ respectively (Figure 3.2).

\subsubsection{APT 102 and r-tPA in Combination Improved Neurological Function at $24 \mathrm{~h}$ Following MCAO}

Before MCAO, neurological score was normal (score=0) in all animals. Neurological function (mNSS) was assessed at $24 \mathrm{~h}$ following MCAO. APT 102 and r-tPA in combination significantly improved the neurological score compared with r-tPA alone $(6.78 \pm 0.82$ versus $8.93 \pm 0.61, p<0.05)$. There was no significant difference of neurological score between the saline group $(7.73 \pm 0.49)$ and $r$-tPA group, saline group and r-tPA +APT 102 group (Figure 3.3).

3.3.4. APT 102 and r-tPA in Combination Significantly Decreased Hemorrhage Transformation in Cortex, Striatum and Total Hemisphere at 24 Following MCAO

Hemoglobin levels were measured by hemoglobin assay in cortex, striatum and total hemisphere at $24 \mathrm{~h}$ following MCAO. APT 102 and r-tPA combination significantly decreased hemoglobin levels in cortex $(1.394 \pm 0.084$ versus $1.879 \pm 0.144 \mathrm{mg} / \mathrm{dl}, \mathrm{p}<0.05)$, striatum $(1.368 \pm 0.101$ versus $2.045 \pm 0.289 \mathrm{mg} / \mathrm{dl}, \mathrm{p}<0.05)$, and total hemisphere $(1.37 \pm 0.080$ versus $1.946 \pm 0.200 \mathrm{mg} / \mathrm{dl}, \mathrm{p}<0.05)$ compared with $\mathrm{r}$-tPA group. The hemoglobin levels of cortex, striatum and total hemisphere from saline group were $1.850 \pm 0.142 \mathrm{mg} / \mathrm{dl}, 1.662 \pm 0.098 \mathrm{mg} / \mathrm{dl}, 1.759 \pm 0.098 \mathrm{mg} / \mathrm{dl}$ respectively (Figure 3.4 ).

3.3.5 APT 102 and r-tPA in Combination Failed to Affect Edema Formation at $24 \mathrm{~h}$ Following MCAO

Edema formation (brain swelling) was measured at $24 \mathrm{~h}$ following MCAO. There was no significant difference between saline, r-tPA and r-tPA +APT 102 group (28.43 $\pm 1.54 \%$ versus $25.70 \pm 2.83 \%$ versus $22.35 \pm 2.29 \%, P>0.05$ ) on edema formation (Figure 3.5 ). 


\subsection{DISCUSSION}

To our knowledge, this is the first report of the evaluation of a soluble apyrase in a reproducible and clinically relevant embolic stroke model of aged female rats. This study showed that co- administration of APT 102 with r-tPA significantly reduced mortality rate and improved neurological functional outcome with r-tPA at $6 \mathrm{~h}$ following MCAO. Our findings also demonstrated that administration of APT 102 significantly improved cortical and total infarct volume and decreased hemorrhagic transformation.

Consistent with our previous studies $10,84,504$, higher mortality rates and large infarct volumes were observed in aged female rats at 24h following MCAO and $6 \mathrm{~h} \mathrm{r}$-tPA reperfusion. As a thrombolytic agent, r-tPA has direct and deleterious effects on the neurovascular unit and brain parenchyma that substantially increase death rate, increase infarct volume and cause severe hemorrhage when administered intravenously following MCAO in a delayed fashion. Recent studies show ${ }^{507-509}$ that aged rats are more severely impaired by stroke than are young rats, and show diminished functional recovery. Clinically, aging affects spontaneous recovery of stroke patients and is a risk for fragility of the blood brain barrier and hemorrhagic transformation after thrombolytic therapy 47 .

Vascular endothelium and blood platelets maintain vascular integrity and promote primary and secondary hemostasis following interruption of vessel continuity. Cerebral artery occlusion and reperfusion associated with endothelial damage and activation of platelets 498,510,511. ATP and ADP are released in high concentrations by activated endothelial cells and platelets following aggregation on endothelium. In turn, ATP and ADP further increase additional platelet aggregation, leading to microthrombus formation and the eventual worsening of endothelial injury. Platelet and fibrin deposition downstream of an occlusive lesion contribute significantly to the postischemic hypoperfusion and tissue injury complicating stroke 499, 501, 512-515.

The beneficial effects of co-administration of APT 102 with r-tPA at 6h following MCAO may likely be explained by the effect of apyrase. APT 102, a novel form of the naturally occurring human apyrase/ADPase (CD 39, recombinant soluble human CD 39), can promote the breakdown of extracellular ATP and ADP by catalyzing the removal of the gamma phosphate from ATP and the beta phosphate from ADP 514, 516-519. APT 102 does not act on the platelet per se but exclusively on prothrombolic mediators (ATP and ADP). Moreover, APT 102 prevents hemorrhagic transformation by inhibiting ATP-induced endothelial cell activation and neutrophil infiltration and re-sensitizing platelets ${ }^{499-501,510,}$ 518,520 .

Furthermore, the effects of exogenously administered apyrase are consistent with prior work in cd 39-/- mice demonstrating increased cerebral infarction volumes compared with control cd 39+/+ mice following stroke. Data also showed that endogenous CD 39 is protective in stroke, and that administration of pharmacological doses of sol CD 39 is effective in inhibiting thrombosis and tissue injury in strokes ${ }^{513,517}$. Since sol CD39 does not interfere with primary GPIb-mediated platelet adhesion at the site of vessel damage, sol CD 39 administration should not prevent a layer of platelets from forming at the site of injury or interfere with hemostatic mechanisms that prevent intracerebral hemorrhage 501, 510. Compared with aspirin, sol CD39 may induce more potent inhibition of platlet aggregation by blocking ADP-induced platelet recruitment. sol CD 39 is more efficient in inhibiting platelet recruitment than is aspirin via blockade of the arachidonate/ thromboxane pathway. Sol CD39 disaggregates platelets under recruitment, but it does 
not have a deleterious effect on primary hemostasis. The fact may explain how sol CD 39 can inhibit microvascular thrombosis and confer cerebroprotection in ischemic stroke without worsening intracerebral hemorrhage 499, 500, 510, 516 .

In conclusion, combination of r-tPA and APT 102 reduces mortality rate, decreases cerebral infarct volume, improves neurological deficit scores, and prevents r-tPA-induced hemorrhage transformation in our highly clinical relevant ischemic stroke model in aged female rats. APT 102 can extend the therapeutic window and prevent intracerebral hemorrhage of r-tPA treatment by inhibiting platelet aggregation and endothelial cell activation in aged female rats following MCAO. 


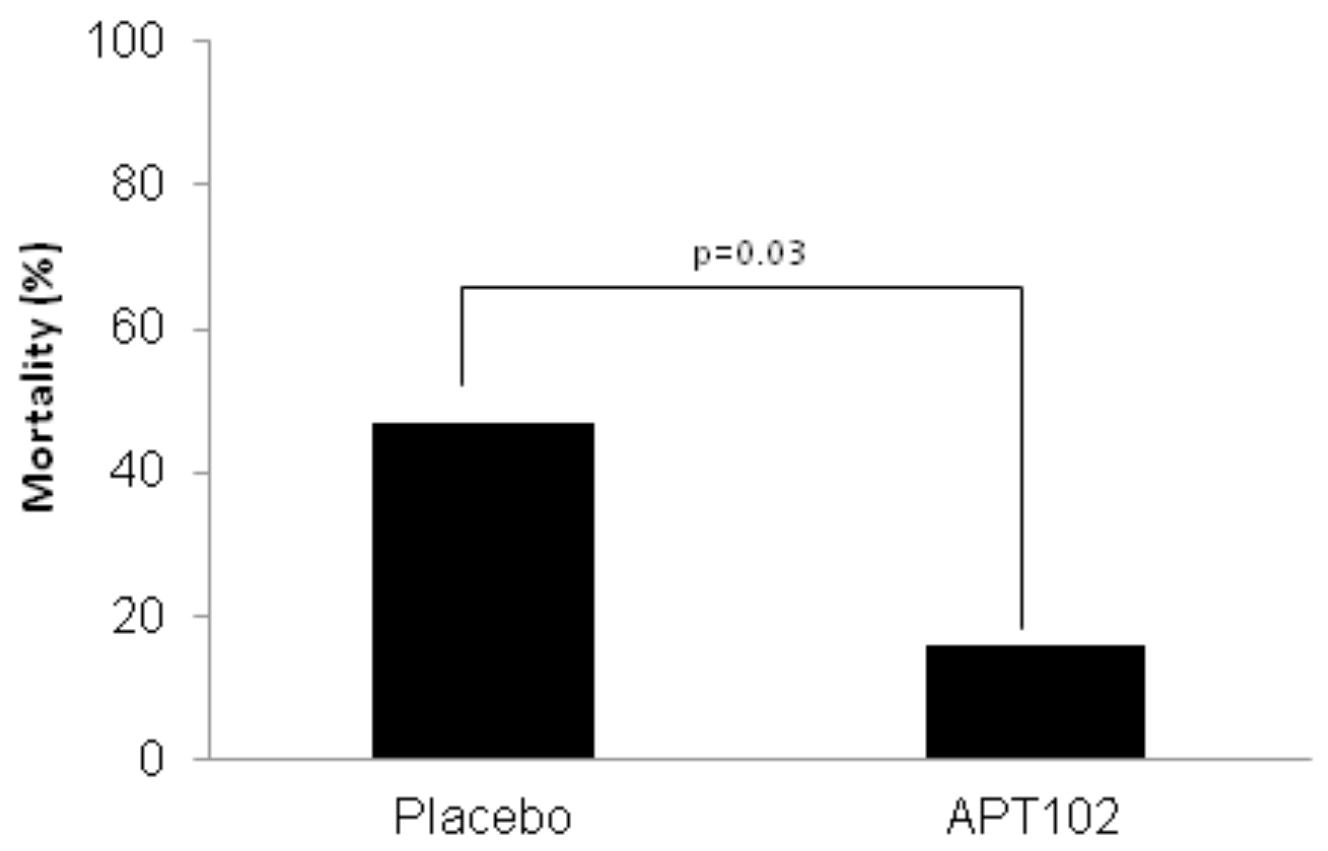

Figure 3.1. Mortality rate was evaluated at $24 \mathrm{~h}$ following MCAO. Results showed a significant decrease $(p<0.05)$ in mortality rate in the $r$-tPA+APT 102 group $(15.79 \%$, $\mathrm{n}=23)$ compared with $\mathrm{r}$-tPA group $(46.67 \%, \mathrm{n}=30)$. The mortality rate of the saline group $(n=23)$ was $34.78 \%$. * represents statistical significance as determined using a $p$-value of $\mathrm{p}<0.05$ 


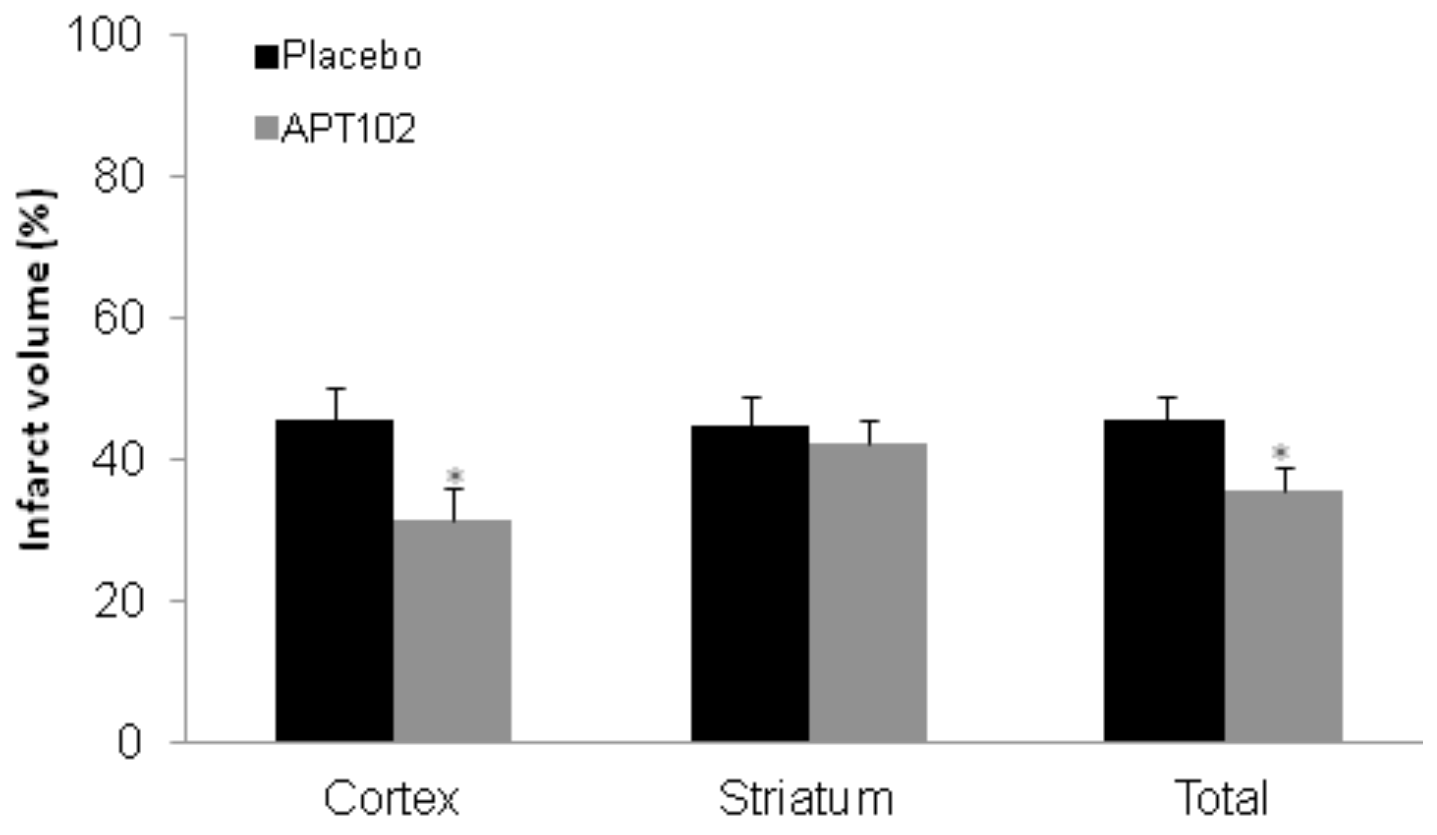

Figure 3.2. Measurement of stroke volume of the infarct cortex, striatum, and total cerebral hemisphere at $24 \mathrm{~h}$ following MCAO. Results showed a significant decrease in cortical stroke volume in the r-tPA+APT 102 group $(29.15 \pm 4.48 \%, n=14)$ compared with the r-tPA group $(46.87 \pm 4.72 \%, n=14, p<0.05)$. No significant difference in striatal stroke volume was observed in r-tPA+APT 102 group $(39.74 \pm 3.07 \%, n=14)$ and the r-tPA group $(49.90 \pm 2.19 \%, n=14, p>0.05)$. Results from the total cerebral hemisphere stroke volume showed a significant $(p<0.05, n=14)$ decrease in the r-tPA+APT 102 group $(33.18 \pm 3.52 \%, n=14)$ as compared to the r-tPA group $(48.15 \pm 3.25 \%, n=14)$. The infarction volume of cortex, striatum, total cerebral hemisphere from the saline group $(n=13)$ was $44.20 \pm 4.92 \%, 53.74 \pm 3.98 \%, 47.17 \pm 3.71 \%$ respectively. * represents statistical significance as determined using a $p$-value of $p<0.05$. 


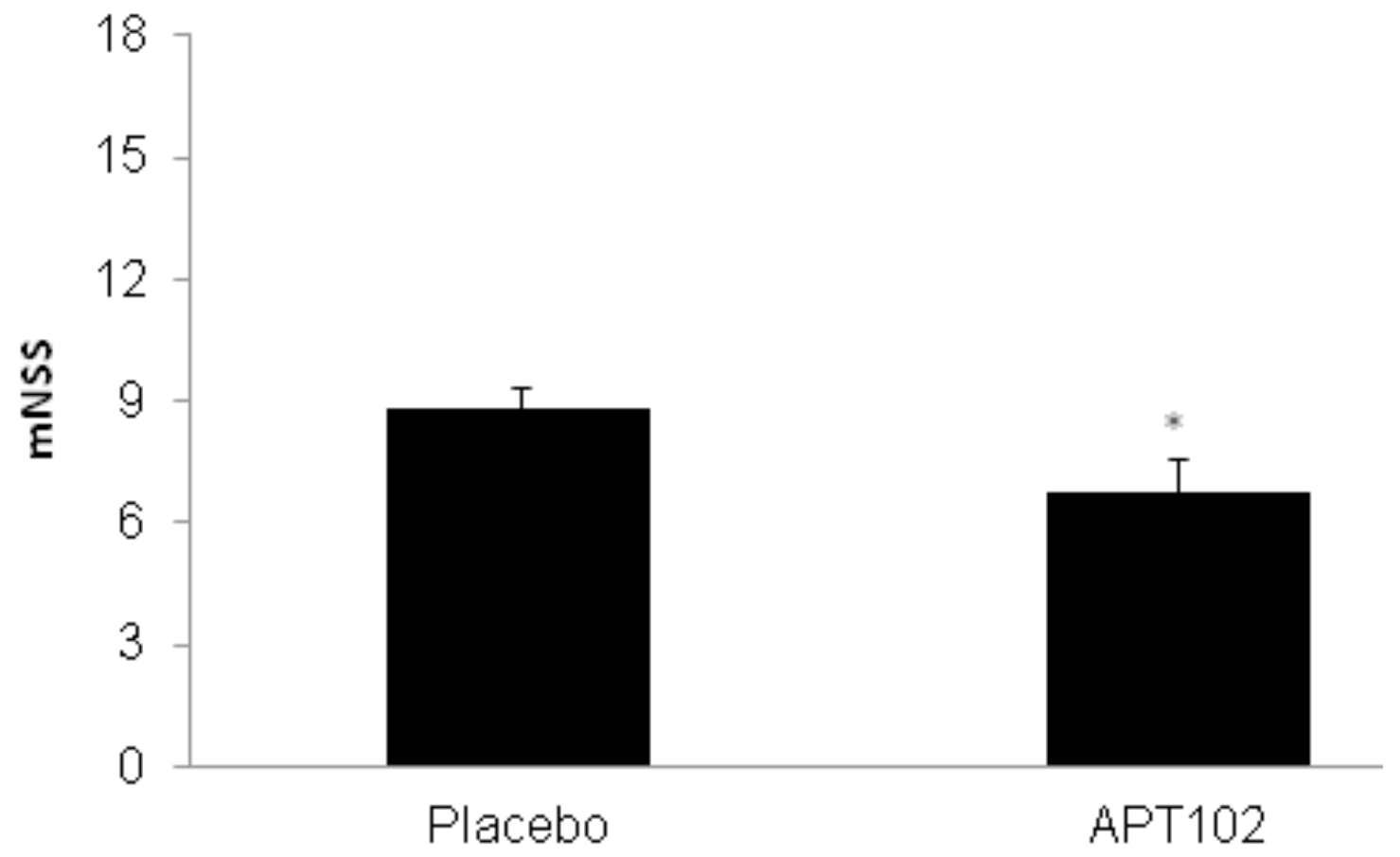

Figure 3.3. Assessment of neurological function using a 17 point $\mathrm{mNSS}$ following MCAO. A significant difference $(p<0.05)$ in mNSS scores were demonstrated between the r-tPA+ APT 102 group (6.78 $\pm 0.82, n=16)$ and the r-tPA group $(8.93 \pm 0.61, n=14)$. The mNSS for the saline group $(n=15)$ was $7.73 \pm 0.49$. * represents statistical significance as determined using a $p$-value of $p<0.05$ 


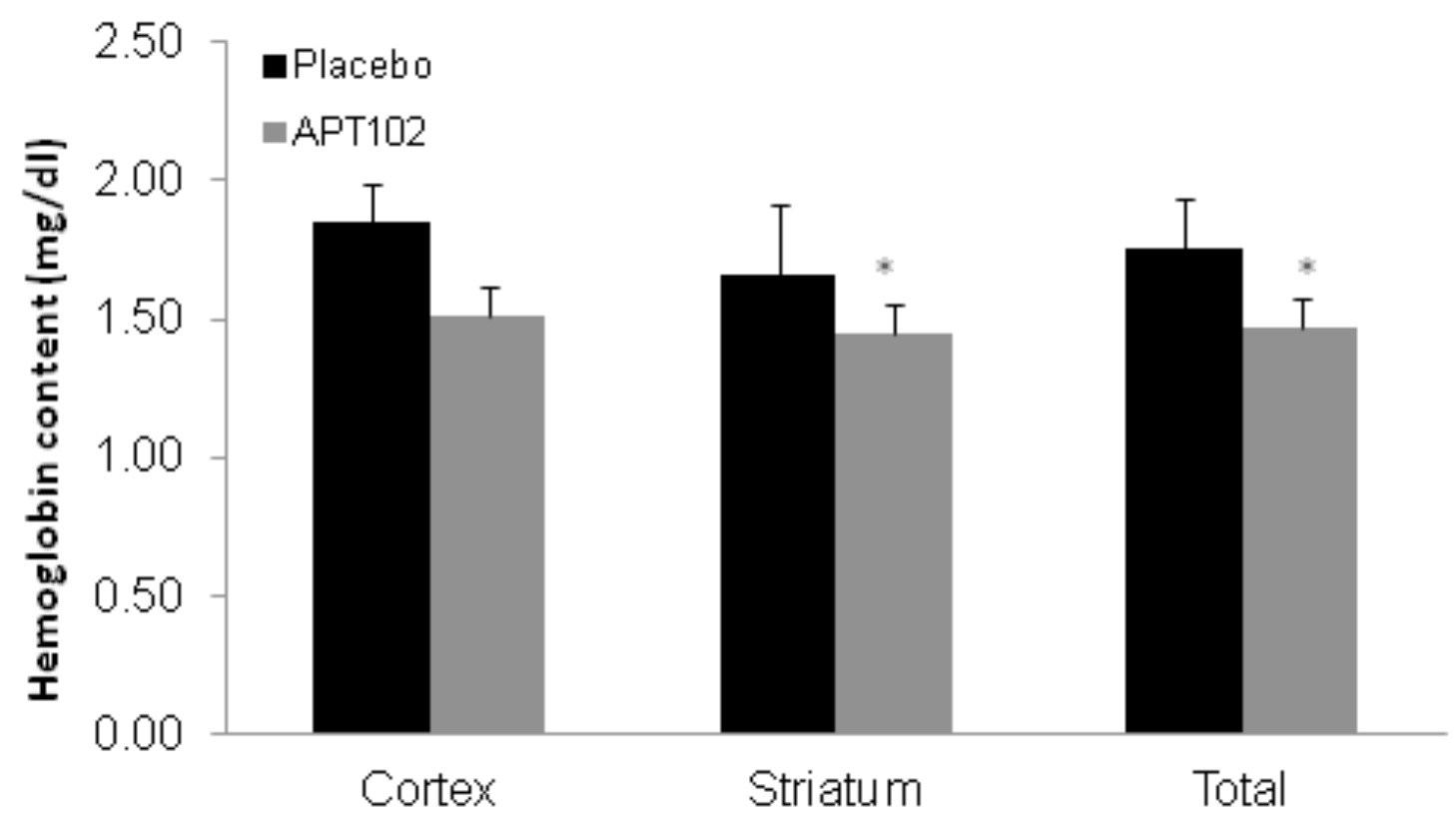

Figure 3.4. Hemoglobin levels were measured in the cortex, striatum and total hemisphere by hemoglobin assays at $24 \mathrm{~h}$ following MCAO. Results showed a significant decrease hemoglobin levels in cortex $(1.39 \pm 0.08$ vs $1.88 \pm 0.14 \mathrm{mg} / \mathrm{dl}$, $p<0.05)$, striatum $(1.37 \pm 0.10$ vs $2.05 \pm 0.29 \mathrm{mg} / \mathrm{dl}, \mathrm{p}<0.05)$, and total hemisphere $(1.37$ \pm 0.08 vs $1.95 \pm 0.20 \mathrm{mg} / \mathrm{dl}, \mathrm{p}<0.05)$ in r-tPA+ APT 102 group $(n=13)$ compared with $r$ tPA group $(n=12)$. The hemoglobin levels of cortex, striatum and total hemisphere from saline group $(\mathrm{n}=15)$ were $1.85 \pm 0.14 \mathrm{mg} / \mathrm{dl}, 1.66 \pm 0.10 \mathrm{mg} / \mathrm{dl}, 1.76 \pm 0.10 \mathrm{mg} / \mathrm{dl}$ respectively. ${ }^{*}$ represents statistical significance as determined using a $p$-value of $p<0.05$ 


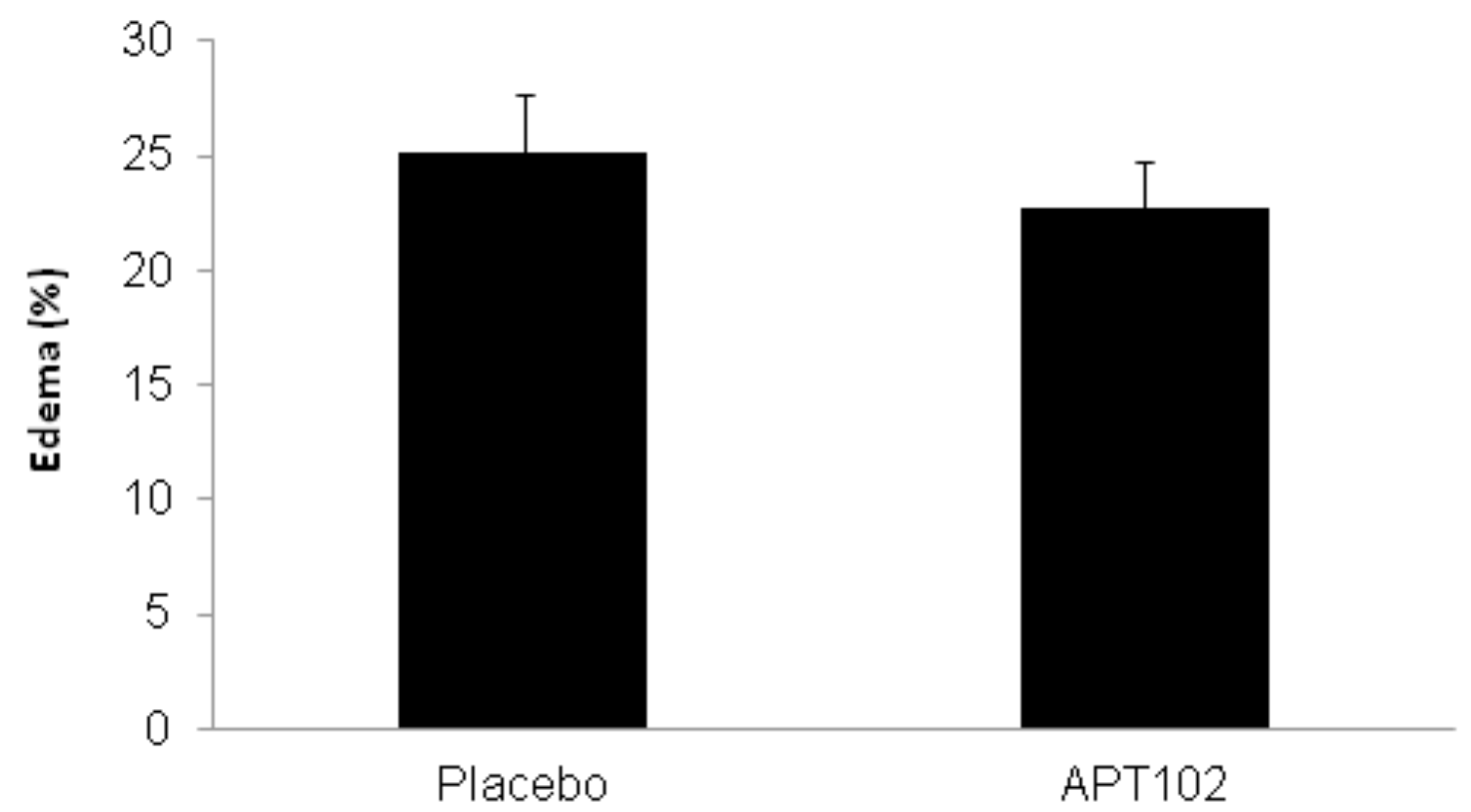

Figure 3.5. Measurement of edema formation in the infarct hemisphere following MCAO. No significant ( $p>0.05$ ) difference was observed between $r$-tPA+APT 102 group (22.35 \pm $2.29 \%, n=14)$ and $r$-tPA group $(25.70 \pm 2.83 \%, n=14)$. Edema index of saline group $(n=13)$ was $28.43 \pm 1.54 \%$. 


\title{
Chapter Four
}

Proposed neuroprotective agents developed using preclinical models of ischemic stroke have failed to translate successfully despite over 100 clinical trials being conducted. Extensive reflection within the stroke community has resulted in the creation of numerous advisory groups that have outlined recommended practices to hopefully increase the likelihood of successful translation from bench-to-bedside. Recurring recommendations include the use of aged animals or those with comorbidities and the implementation of functional assessments as an experimental endpoint in preclinical studies. These suggestions have been made upon the basis that ischemic stroke is a disease of the elderly and clinical endpoints frequently utilize functional outcomes (aka can the patient care for themselves). Functional assessments and the use of aged animals have not been emphasized in preclinical studies and therefore, it remains unclear what the most appropriate functional assessments are and how these may be impacted by age. This chapter explores this issue by comparing performance in numerous assessments across age groups utilized in preclinical ischemic stroke work.

\section{Effect of Aging on Behavioral Assessment Performance: Implications for Clinically Relevant Models of Neurological Disease}

This work was published in Journal of Neurosurgery.

Citation: Turner RC, Seminerio MJ, Naser ZJ, Ford JN, Martin SJ, Matsumoto RR, Rosen CL, Huber JD (2012) Effects of aging on behavioral assessment performance: implications for clinically relevant models of neurological disease. Journal of Neurosurgery 117: 629-637.

\begin{abstract}
The effect of age is often disregarded despite the role of aging in neurologic and neurodegenerative disease development. Functional assessment increases the clinical relevance of animal models of neurologic disease and adds value beyond traditional histologic measures. However, the relationship between age and functional impairment has not been systematically assessed through a battery of functional tests. In the present study, various sensorimotor and behavioral tests were used to evaluate effects of age on functional performance. Aged (18-24 months) Sprague-Dawley rats were found to perform significantly worse on inclined plane test and exhibited alterations in elevated-plus maze and forced swim test compared to young adult animals (3-4 months). Specifically, aged animals exhibited reduced exploration of open arms in elevated-plus maze and higher immobility time in forced swim test. Spatial acquisition and reference memory was diminished in aged animals in comparison to the young adult animals. This work illustrates clear differences between young adult and aged animals, which may have implications in functional assessment for preclinical models of neurologic disease.
\end{abstract}




\subsection{INTRODUCTION}

The assessment of outcome from preclinical neurologic disease models has often emphasized histologic measures and minimized functional and behavioral evaluations 521. Functional assessment following neurologic disease onset and treatment is the primary means of evaluating patients clinically, and therefore, is desirable for translation of preclinical studies to clinical trials ${ }^{522}$. In addition to being clinically relevant, functional assays have the potential to be more sensitive to therapeutic-induced improvements when compared to histologic measures.

One aspect of functional assays that remains poorly understood is the effect of aging on behavior and functional performance. Aging is well documented as a leading risk factor for numerous neurologic diseases ranging from Alzheimer's to stroke and consequently, the use of preclinical models utilizing aged animals has been recognized and recommended $47,523,524$. Preclinical studies utilizing both young and adult animals have illustrated more severe impairment and lessened functional recovery following neurologic injury in comparison to young animals ${ }^{30,33,525-527 .}$

Furthermore, Mehan et al recently demonstrated age-dependent effects on both motor coordination and specific cognitive tasks before and after traumatic brain injury ${ }^{528}$. Similarly, Badan et al documented a $30 \%$ decrease in spontaneous activity in aged animals compared to young animals prior to initiation of neurologic injury ${ }^{30}$. Increased recognition of the utility of using aged animals in preclinical models of disease creates a need for basic understanding of behavioral differences, both before, as well as after, any neurologic injury. This work seeks to elucidate age-dependent effects on behavior and in doing so, establish a foundation for selecting and developing the most appropriate assessments for aged animals. We hope to provide insight into not only the differences in behavior associated with age, but also any similarities that may exist with young adult animals. Lastly, this work will facilitate future work utilizing more clinically relevant preclinical models of neurologic disease.

\subsection{MATERIALS AND METHODS}

\subsubsection{Animals}

Female Sprague-Dawley rats 3-4 months (young adult) and 18-20 months (aged adults) were acquired from Hilltop Animal Laboratory (Scottdale, PA, USA) and housed under 12 $\mathrm{hr}$ light/12 hr dark conditions with food and water available ad libitum. Animals were allowed to acclimate for one week after arrival. All procedures involving rats abided by the policies outlined by the West Virginia University Animal Care and Use Committee. Sprague-Dawley rats were selected based on frequent use in models of neurologic disease, particularly stroke. Clinically, stroke more commonly affects females and therefore, female rats were utilized. Animal weight was recorded using a standard balance prior to conducting assessments.

\subsubsection{Sensorimotor Assessment}

Animals were assessed for sensorimotor ability using standard measures for strength, balance, coordination, and overall locomotor activity. The assessments included gripstrength, inclined plane, rotarod, and locomotor activity. Each of these measures is described in greater detail below. A baseline modified Neurologic Severity Score 
(mNSS), a scoring system widely used in neurologic assessment, particularly for stroke, was utilized ${ }^{529}$.

\subsubsection{Grip-Strength}

A commercially available grip-strength measurement system, the Grip Strength Meter, was acquired from Columbus Instruments (Columbus, $\mathrm{OH}$ ). The grip-strength apparatus consists of a load cell and an interchangeable grip attachment. Two types of grip attachments were utilized in this work - a T bar and a triangle bar. For each attachment, a total of five measures were taken for forelimb grip-strength for each animal and averaged to create a composite for each bar. Animals were grasped by the tail and nape of the neck, forepaws placed on the bar, and pulled in a straight line away from the load cell ${ }^{530}$.

\subsubsection{Inclined Plane}

An inclined plane was constructed similar to that described by Rivlin, et al ${ }^{531}$. The device consists of an $18 \mathrm{~cm} \times 18 \mathrm{~cm}$ platform to which a ridged rubber mat is attached. The platform is affixed to a base via a hinge on one side, allowing rotation from $0-85^{\circ}$. Animals were placed such that their body axis was perpendicular to that of the inclined plane. The maximum incline angle was recorded at which the animal could successfully maintain its position for 5 seconds. During this period, the animal makes corrective adjustments to maintain a normal posture using both fore- and hind-limbs. This process was repeated for a total of five measures per animal, which were then averaged to create a composite value.

\subsubsection{Locomotor Activity}

Locomotor activity was measured using an automated activity monitoring system (San Diego Instruments, San Diego, CA). The animals were given one hour to acclimate to the testing room prior to initiation of testing. Each testing chamber consisted of a plexiglass housing and a $16 \times 16$ photobeam array to detect lateral movements with a separate 8 photobeam array to detect rearing activity. Locomotor activity was quantified over a 30 minute period. Ambulatory, fine, and rearing movements were quantified in order to determine total locomotor activity.

\subsubsection{Rotarod}

The rotarod test requires animals to balance on a rotating drum and is often utilized to assess motor deficits in neurodegenerative disease models in rodents. Two variations to the rotarod test exist - incremental fixed speeds and accelerating ${ }^{532}$. All rotarod studies were conducted with the OMNI-ROTOR by Omnitech, Inc (Columbus, OH).

Training animals consisted of four trials a day over the course of three consecutive days. Animals were placed on the rotarod that was accelerated to a speed of $24 \mathrm{rpm}$ over 20 seconds. The maximum duration spent at this speed was 60 seconds.

For the fixed speed test, animals were placed on the rod and tested at 12, 16, 20, 24, $28,32,36$, and $40 \mathrm{rpm}$ for a maximum of 60 seconds at each speed. The animals were tested a total of three times at each speed with a rest of 30 minutes between each trial. The accelerating rotarod test was set to reach a maximum speed of $50 \mathrm{rpm}$ over a period of 4.5 minutes and was repeated for a total of two trials with a 30 minute rest 
period between trials. The total test duration was 5 minutes per trial. For both the fixed speed and accelerating rotarod tests, the latency to fall was recorded.

\subsubsection{Modified Neurologic Severity Score ( $m N S S)$}

The mNSS, described at length by Watanabe, et al., is a widely used method for quickly assessing severity of injury, particularly in the stroke field ${ }^{529}$. The mNSS (Table 4.1) includes basic measures of sensorimotor performance and reflex integrity. The balance beam used had a square profile with rounded edges and measured $4 \mathrm{~cm} \times 4 \mathrm{~cm}$. The beam was constructed of a synthetic wood alternative.

\subsubsection{Elevated-Plus Maze Assessment}

The elevated-plus maze test was conducted as described by Walf, et al ${ }^{533}$. Time spent in the open arms has been described as correlating inversely with anxiety-like behavior. Animals were placed individually in the center of the maze facing an open arm. ANYMaze Version 4.63 video tracking software (Stoelting Co., Wood Dale, IL) was used to record the number of entries, time, speed, and distance in open versus closed arms. ANY-Maze software settings were as follows: test duration $=300$ seconds and percent body to be considered in arm $=90 \%$.

\subsubsection{Forced Swim Test Assessment}

The forced swim test was performed as described by Porsolt et al ${ }^{534,535}$. Time spent immobile has been identified as correlating with depressive-like activity. Rats were habituated the day prior to testing for a period of seven minutes. On the testing day, rats were acclimated to the testing facility for 60 minutes prior to initiation of experiments. Animals were placed in individual cylinders of water $(20 \mathrm{~cm}$ deep) for a total of seven minutes. The initial two minutes served as an acclimation period and data was not collected from this period. During the remaining five minutes, immobility time was quantified using ANY-Maze Version 4.63 video tracking software (Stoelting Co., Wood Dale, IL). Immobility was defined as no activity other than that required to maintain the animal's head above the surface of the water. In addition to immobility time, speed, distance, and number of immobile episodes were recorded. ANY-Maze software settings were as follows: accustomization period $=120$ seconds, test duration $=300$ seconds, minimum immobility time $=2000$ milliseconds, and immobility sensitivity $=\mathbf{7 5} \%$.

\subsubsection{Spatial Acquisition \& Reference Memory Assessment using the Morris Water Maze}

All Morris water maze studies were conducted in a $182 \mathrm{~cm}$ diameter stainless steel pool with an opaque interior. Columbus Instruments (Columbus, $\mathrm{OH}, \mathrm{USA}$ ) Videomex 4.50 tracking software was utilized for all data acquisition and subsequent analysis. Water was maintained at $19-22{ }^{\circ} \mathrm{C}$ throughout all studies and a platform made of plexiglass, 12 $\mathrm{cm} \times 12 \mathrm{~cm}$ was used. On Days 1-5, four trials were completed per animal from four different positions with the platform being visible on Day 1 and hidden subsequently. Each trial lasted a maximum of two minutes with an inter-trial interval of 15 seconds. Animals not finding the platform within a two minute period were then placed on the platform. The animal remained on the platform for the duration of the inter-trial interval for acquisition to occur. One week after the last acquisition trial, a probe trial was performed in which the platform was removed. For the probe trial, animals were placed in a novel start position facing the tank wall, $180^{\circ}$ from the original platform position to ensure spatial recognition and preference rather than a specific path. For the probe trial, 
the animal was removed after 60 seconds. For the probe trial, the WaterMaze v4.50 was set with a counter $300 \%$ of original platform size. The number of entries, time, and distance in the counter zone was recorded.

\subsubsection{Statistical Analysis}

Data from all experiments was analyzed using GraphPad Prism 4.0 (San Diego, CA). Two-way repeated measures analysis of variance was performed to test for differences between young-adult and aged mice in the water maze-spatial acquisition test (both for latency and distance) and the fixed speed rotarod test. An unpaired $t$ test was used to compare young-adult and aged mice in grip strength, balance beam, locomotor activity, mNSS (placing, tail raise, and reflexes), inclined plane, accelerating rotarod, forced swim test, and elevated plus maze. $\mathrm{P}<0.05$ was considered statistically significant for all data analyzed.

\subsection{RESULTS}

\subsubsection{Sensorimotor}

The effect of aging on strength, balance, and locomotor ability was measured through grip-strength recordings (Figure 4.1a), inclined plane (Figure 4.1b), and overall locomotion (Figure 4.1c). Statistically significant differences were observed when comparing young-adult and aged animals in terms of grip-strength with the $\mathrm{T}$-bar $(\mathrm{t}=2.17$, $\mathrm{p}<0.05)$, grip-strength with the triangle bar $(\mathrm{t}=3.03, \mathrm{p}<0.01)$, and angle of fall on the inclined plane $(t=8.47, p<0.0001)$. No significant difference was seen in total locomotor activity.

The results from two variations of rotarod testing, a fixed speed (Figure 4.2a) and accelerating test (Figure 4.2b), are shown. No significant difference was seen between young-adult and aged animals on either the fixed speed or the accelerating test.

The mNSS, a widely utilized scoring system for stroke, showed that aged animals performed significantly worse than young adults animals on the balance beam component (Figure 4.3; $\mathrm{t}=2.41, \mathrm{p}<0.05$ ). On the other subtests of the mNSS such as placing, raising by the tail, and reflexes, age did not appear to alter ability.

\subsubsection{Elevated Plus Maze}

In the elevated plus maze, young-adult animals spent significantly more time exploring the open arms than did aged animals (Figure 4.4a, $\mathrm{t}=2.39, \mathrm{p}<0.05)$ ). While not reaching statistical significance, there was also a strong trend towards an increased number of entries into the open arm by young animals when compared to the aged (Figure 4.4b). Notably, there was no difference in the average speed between groups in the open arm (Figure 4.4c). In the closed arm, the average speed was different as aged animals failed to explore while young-adult animals moved about with greater frequency (Figure 4.4d, $\mathrm{t}=3.77, \mathrm{p}<0.01)$.

\subsubsection{Forced Swim Test}

In the forced swim test, significant differences were seen in total immobility time (Figure $4.5 a: t=3.83, p<0.05$ ) and the number of immobile episodes (Figure 4.5b:t=3.45, $p<0.05$ ) between the two groups. No significant differences were seen in the total distance traveled (Figure 4.5c), average speed (Figure 4.5d), or the latency to the first immobile episode (Figure 4.5e). 


\subsubsection{Cognitive}

Two-way ANOVA of the spatial acquisition (learning) phase showed differences between young and aged mice in their latency to find the platform (Figure 4.6a: $F(4,88)=17.10$, $\mathrm{p}<0.0005$ ) and distance (Figure 4.6b:F $(4,55)=31.60, p<0.0001)$. Bonferroni post hoc test confirmed a significant difference between latency on Days 3-5 (Figure 4.6a: Day 3, $\mathrm{t}=2.958, \mathrm{p}<0.05$; Day 4, $\mathrm{t}=4.30, \mathrm{p}<0.001$; Day 5, $\mathrm{t}=3.30, \mathrm{p}<0.01)$ and distance on Day 4 (Figure 4.6b: $t=3.32, p<0.01$ ).

In the probe test (reference memory assessment), conducted 7 days after the last day of learning, aged animals exhibited a reduced number of entries (Figure 4.7a: $t=3.132$, $\mathrm{p}<0.01$ ), time spent (Figure 4.7b; $\mathrm{t}=4.93, \mathrm{p}<0.0001$ ), and distance traveled in the counter area (Figure 4.7c; $t=4.88, p<0.0001$ ) compared to the young-adult rats. Aged animals also performed more poorly than the young-adult animals based on the proximity average, a calculation of the average distance from platform and ideal path of travel (Figure 4.7d; $t=2.33, p<0.05$ ).

\subsection{DISCUSSION}

Age is often ignored despite its clinical relevance in many animal models for neurologic or neurodegenerative disease, of which stroke and Alzheimer's Disease are examples. Age is the greatest known risk factor for Alzheimer's Disease ${ }^{524}$ and stroke ${ }^{536}$, yet the use of young-adult animals is pervasive throughout the literature. This clinical reality is in sharp contrast to preclinical studies of stroke, in particular, in which the vast majority of studies utilize young-adult animals. Furthermore, preclinical models of neurologic disease often emphasize histologic measures despite the clinical relevance of functional assessment. The effect of age on common neurologic functional tests was not systematically investigated previously, making the comparison between young and aged models of neurologic disease difficult. This work not only compares the performance of young and aged animals in the widest array of behavioral tests utilized in neurologic disease research but also illustrates fundamental baseline differences, which may provide insight into the most appropriate tasks for functional assessment going forward in aged animal disease models.

This work addresses the effect of age on sensorimotor, anxiety-like behavior, depressive-like behavior, spatial acquisition, and reference memory. Data presented herein illustrate that age does not affect general locomotor activity and basic tests of motor function. In contrast, age appears to adversely affect tasks requiring integration of motor activity and balance as seen in the balance beam component of the mNSS and inclined plane test. In both tests, aged animals performed worse than the young adult animals. In one measure, grip-strength, the aged animals performed better than young adult animals. This is likely not a true reflection of strength but rather a consequence of increased body weight as described by Maurissen, et al ${ }^{537}$. Young-adult animals utilized in this work weighed $256.92 \pm 1.56$ grams while aged animals weighed $391.58 \pm$ 10.53 grams (data reported as mean \pm standard error of the mean).

In the elevated plus maze, aged animals made fewer entries into the open arms and also spent less time in the open arms. This does not appear to be a consequence of impaired motor activity based on sensorimotor results (above) and no difference in average speed in the open arm between young-adult and aged animals. Within the closed arms, aged animals did not explore to the same degree as the young-adult animals, as evidenced by a reduced average speed within the closed arm. 
Changes in immobility time were reported in the forced swim test. Young-adult and aged animals differed significantly in the number of immobile episodes and the total immobility time. Again, this does not appear to be due to fatigue or changes in motor ability as the total distance traveled and average speed was not different between age groups. Similarly, total distance and average speed was not significantly different between aged and young-adult animals, nor was latency to first immobile episode.

Animal age appears to reduce spatial acquisition and reference memory ability as determined with the Morris Water Maze, a well-accepted and utilized cognitive test, in terms of both learning and memory. The Morris Water Maze test revealed significant differences in spatial acquisition between groups, both in terms of latency to find the platform and total distance swam. While Hamm et al showed an age-related impairment in spatial acquisition (learning) ability after traumatic brain injury, differences at baseline were not discussed ${ }^{526}$. Reference memory, as assessed using a probe trial, also appears to be worsened with age. The aged animals made fewer entries, spent less time, and traveled a shorter distance within the counter area. Furthermore, aged animals spent less time exploring near the counter area as determined using the proximity index. Once again, these results cannot be explained based on motor differences as groups exhibited comparable baseline ability on Day 1 of spatial acquisition.

These studies illustrate fundamental differences in animal behavior and function associated with aging. The process of aging, a risk factor for numerous neurologic and neurodegenerative diseases such as stroke, is rarely considered in preclinical models of stroke and therefore, the effect of aging on a variety of behavioral assays utilized in poststroke assessment has been underreported. Due to behavioral differences associated with age, it is clear that animal models seeking to compare young adult and aged animals may need to normalize to baseline ability prior to injury/disease onset rather than simply reporting absolute measurements post-experimental manipulation. Furthermore, the use of aged animal models may necessitate a specifically designed functional assessment for this population. For example, the modified Neurologic Severity Score (mNSS), is widely utilized in post-stroke assessment, yet has never been validated in an aged animal model. In comparing young adult and aged animal performance on the mNSS, striking differences emerge, particularly on the balance beam task. Notably, in a preclinical study of traumatic brain injury by Hamm et al, different sized balance beams were utilized for young versus aged animals to reach a similar level of difficulty after injury ${ }^{526}$. Other subsets of the mNSS such as placing, raising by the tail, and reflexes did not appear to be affected by age.

\subsection{CONCLUSION}

As evident in this work, age affects performance on a variety of behavioral assessments such as the inclined plane, balance beam, elevated-plus maze, forced-swim test, and Morris Water Maze. These differences can not be explained based on motor ability alone as aged animals exhibit similar performance on the rotarod and total locomotor activity assessment. Rather, the data suggest an age-related impairment in the ability to integrate sensorimotor cues such as balance on the inclined plane and balance beam tests. This work may have implications in preclinical models of neurologic disease employing the use of aged animals, such as stroke. Furthermore, the data indicate clear functional differences associated with aging but the biologic causes of these differences remain to be elucidated. 


\section{ACKNOWLEDGEMENTS}

The authors wish to acknowledge the contribution of Samantha J. Martin, James (Neal) Ford, and Matthew Robson for their role in animal testing and preparation. The authors would also like to thank Dr. Diane Miller of the National Institute of Occupational Safety and Health (NIOSH) for supplying the rotarod apparatus. Rolf Hansen also contributed to this work by providing equipment for the elevated plus maze. Furthermore, this work would not have been possible without financial support provided to Dr. Jason Huber (NIH 5RO1NS061954), Ryan Turner (NIH 5T32GM081741), and Mike Seminerio (NIH 5T32GM08174). 


\section{TABLES AND FIGURES}

Table 4.1. Modified Neurologic Severity Score (mNSS). The mNSS utilizes a variety of sensorimotor, balance, and reflex tests to assign a neurologic score. One point is assigned for the inability to perform the tests or for the lack of a tested reflex. Severe injury is defined as $13-18$ points, moderate injury 7-12 points, and mild injury 1-6 points.

Motor Tests

$$
\text { Modified Neurologic Severity Score (mNSS) }
$$

Muscle status - Hemiplegia

Raising by tail (normal $=0$, maximum $=3$ )

1.) Flexion of forelimb

1.) Flexion of hindlimb

1.) Head moving more than 10 to vertical axis within 30 seconds

Placing on floor (normal $=0$, maximum $=3$ )

0.) Normal walk

1.) Inability to walk straight

2.) Circling toward paretic side

3.) Falling down to paretic side

Sensory Tests (maximum of $2-1$ for each test)

1.) Placing (visual and tactile test)

1.) Proprioception (deep sensation)

Beam Balance (normal $=0$, maximum $=6$ )

0.) Balances with steady posture

1.) Grasps side of beam

2.) Hugs the beam and one limb falls down from beam

3.) Hugs the beam and two limbs fall down from beam, or spins on beam

4.) Attempts to balance on beam but falls off ( $>40$ seconds)

5.) Attempts to balance on beam but falls off ( $>20$ seconds)

6.) Falls off without attempting to balance or hang on to beam ( $<20$ seconds)

Reflex absence \& abnormal movements (normal $=0$, maximum $=4$ )

1.) Pinna reflex (head shake when touching auditory meatus)

1.) Corneal reflex (eye blink when touching cornea lightly with cotton)

1.) Startle reflex (a motor response to a brief noise from snapping a clipboard paper)

1.) Seizures, myoclonus, myodystony 
A

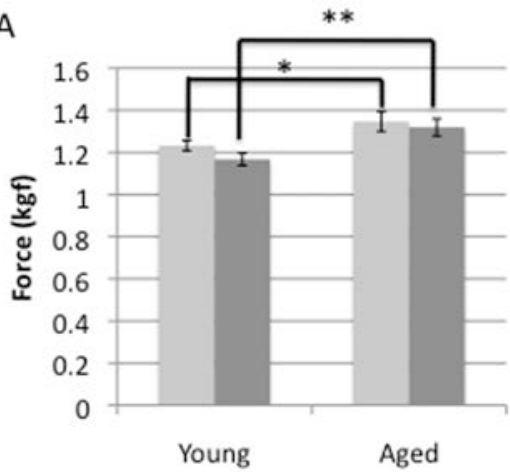

$\mathrm{C}$

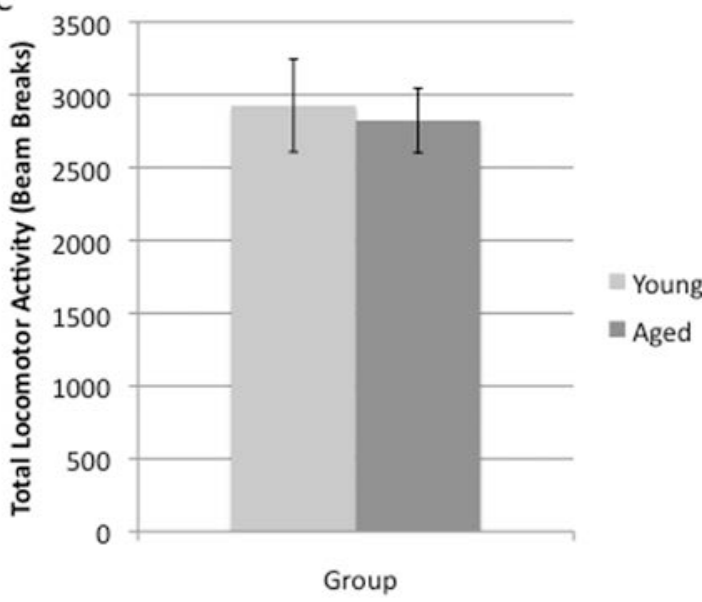

B

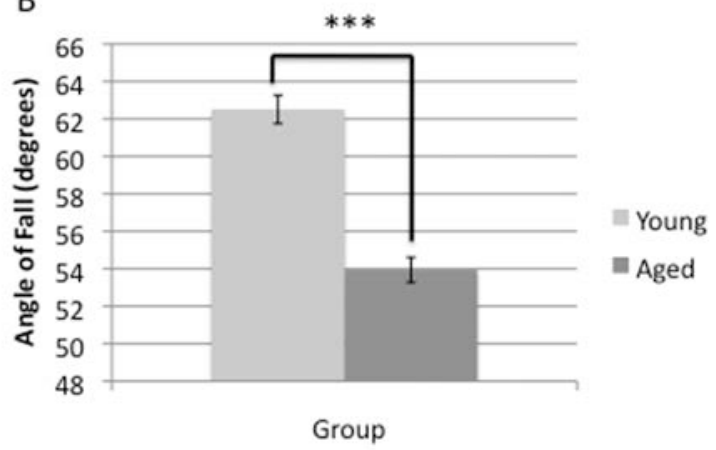

Figure 4.1. The effect of age on sensorimotor assessment with A) Grip Strength Test, B) Inclined Plane Test, and C) Total Locomotor Activity. The grip strength and inclined plane tests were significantly different between young-adult and aged animals $(p<0.05)$ with aged animals exhibiting stronger grip force and young-adult animals performing better on the inclined plane. No difference in total locomotor activity was measured. Statistical significance determined using unpaired t-test. ${ }^{*},{ }^{* *},{ }^{* * *}$ denote statistical significance $(p<0.05, p<0.01$, and $p<0.001)$. 
A

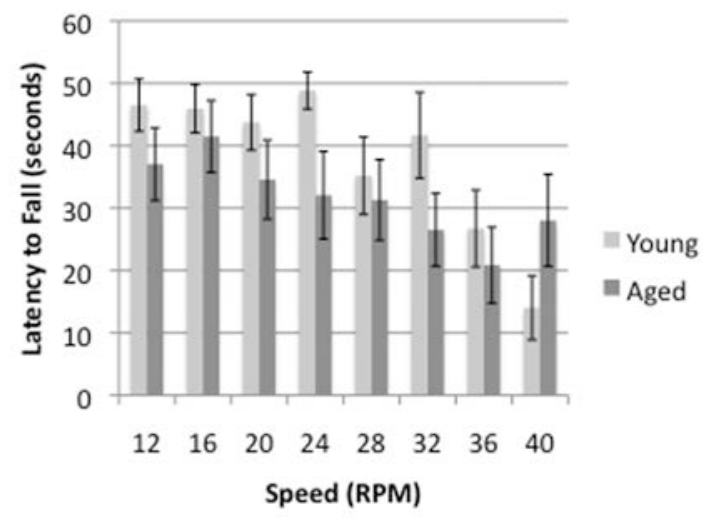

B

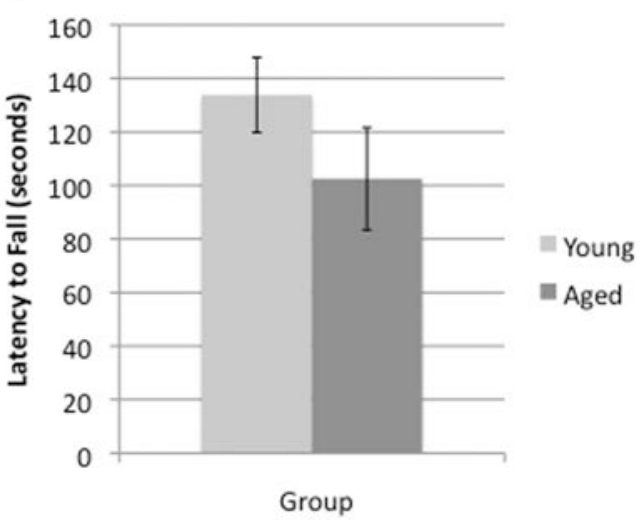

Figure 4.2. Fixed-speed $(A)$ and accelerating $(B)$ rotarod test in young-adult and aged animals. The fixed speed test included trials at 12, 16, 20, 24, 28, 32, 36, and 40 RPM. The accelerating test was conducted over five minutes while reaching a maximum speed of 50 RPM. No statistically significant difference was seen between young-adult and aged animals on either variation of rotarod testing. 


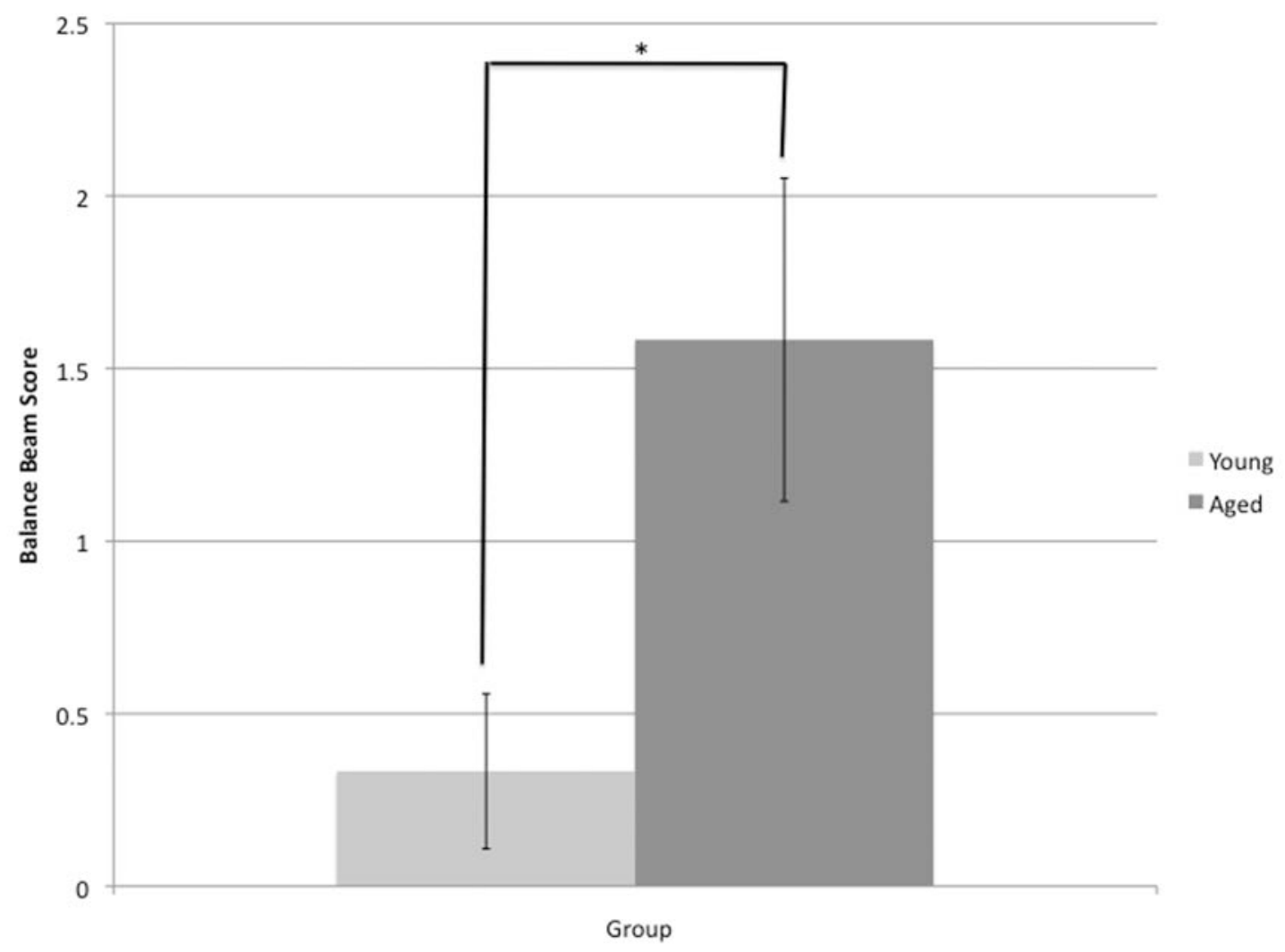

Figure 4.3. Reduced performance on the balance beam subtest (part of $\mathrm{mNSS}$ ) in aged animals. Aged animals were comparable to young-adult animals in all mNSS subtests except for balance beam in which young animals excelled in contrast to aged animals. Statistical significance determined using unpaired t-test. * denotes statistical significance $(p<0.05)$. 
A

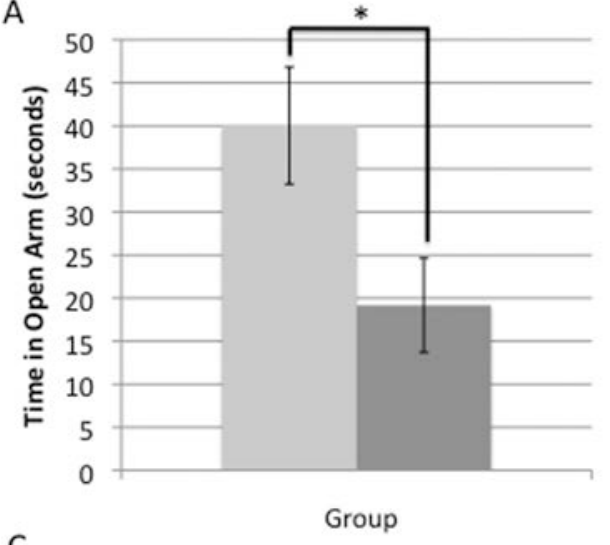

C

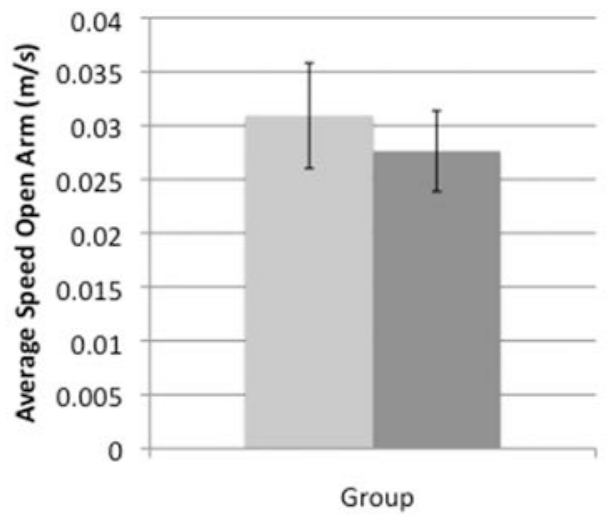

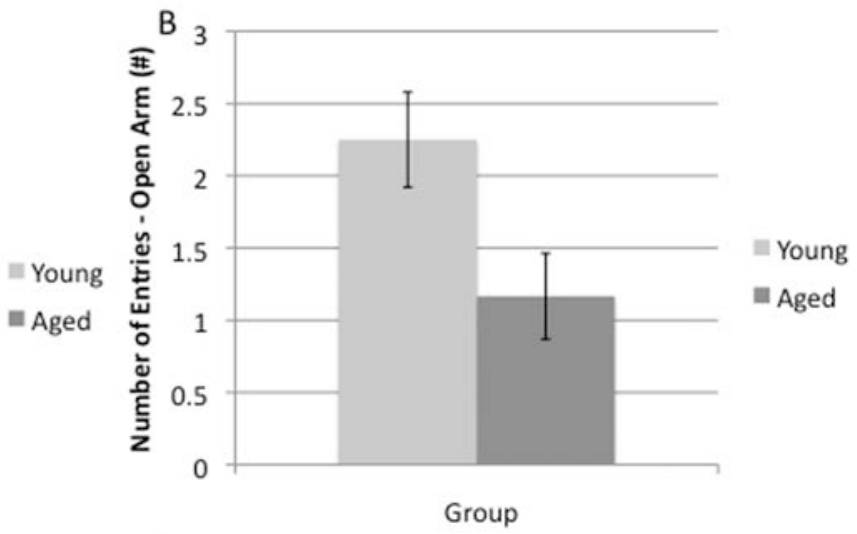

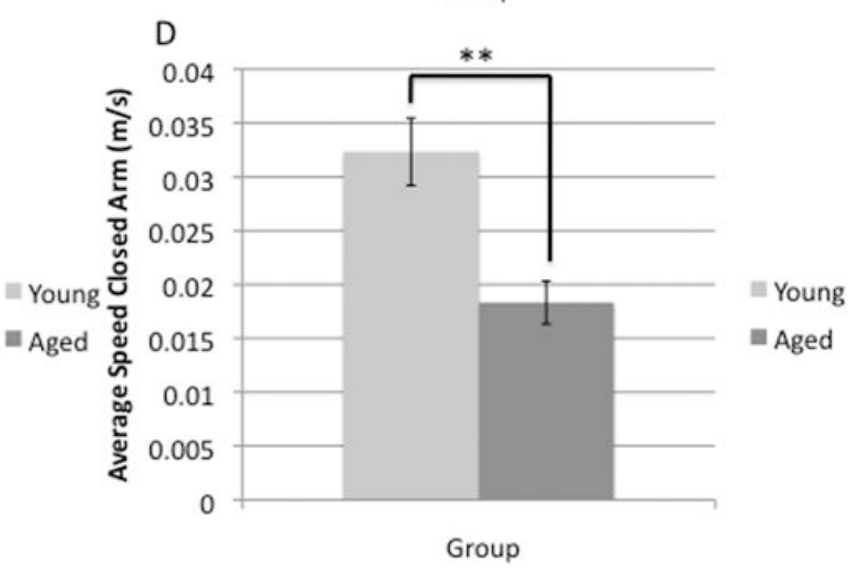

Figure 4.4. Effect of age on behavior using the elevated plus maze assessment. Aged animals made fewer entries into the open arms (A), spent less time in the open arms (B), but did not differ in average speed in the open arms (C). In the closed arms, young animals explored more readily than the aged animals, resulting in a higher average speed within the closed arms (D). Statistical significance determined using unpaired ttest. ${ }^{*},{ }^{* *}$ denote statistical significance $(p<0.05$ and $p<0.01)$, respectively. 

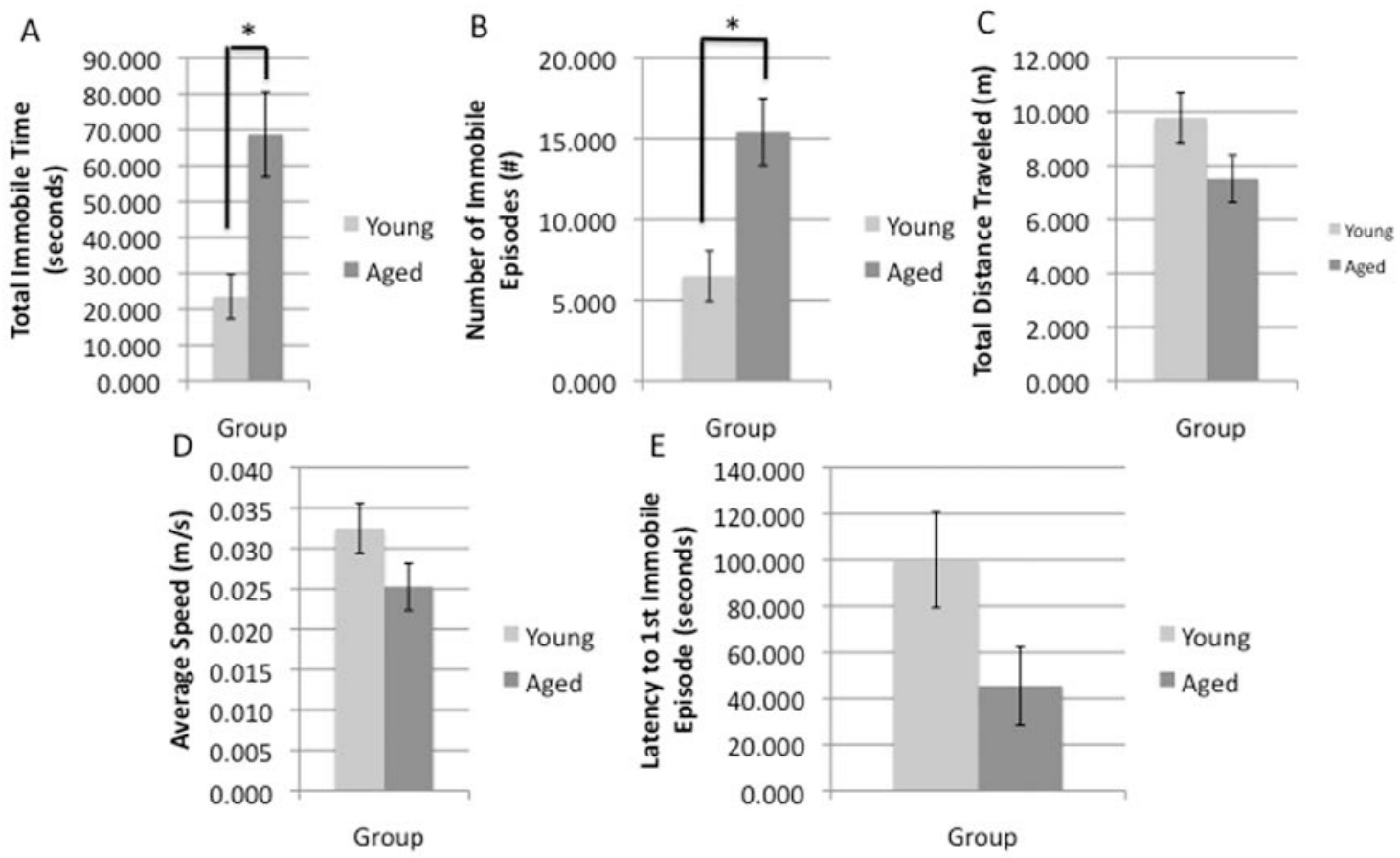

Figure 4.5. Effect of age on total immobility time, immobile episodes, distance traveled, and speed in the forced swim test. Aged animals spent more time immobile (A), had a greater number of immobile episodes (B), but traveled a similar distance $(C)$ at a similar speed (D). There was no statistically significant difference in latency to first immobile episode (E). Statistical significance determined using unpaired t-test. * denotes statistical significance $(p<0.05)$. 

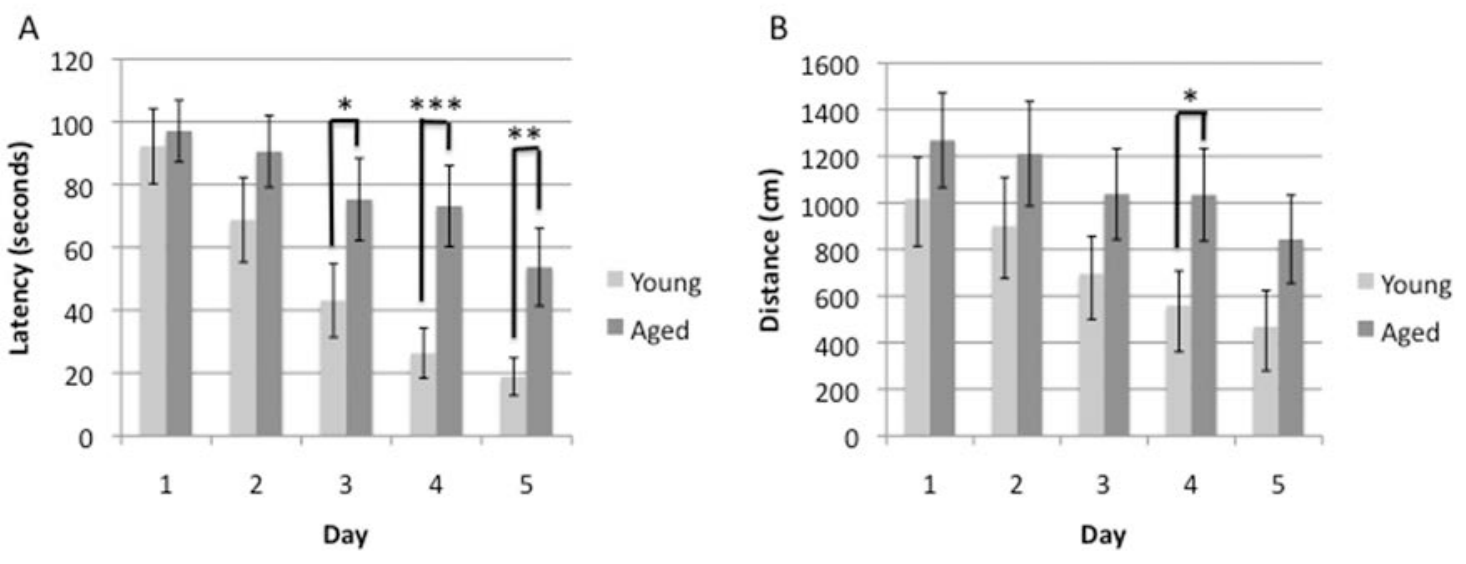

Figure 4.6. The role of age in spatial acquisition determined using the Morris Water Maze. Aged animals exhibited increased latency (A) and distance to platform (B) when compared to young animals at later time-points, despite being nearly identical when first exposed to the maze. Statistical significance determined using two-way ANOVA with Bonferroni post hoc test. ${ }^{*},{ }^{* *},{ }^{* * *}$ denote statistical significance $(p<0.05, p<0.01$, and $\mathrm{p}<0.001)$. 

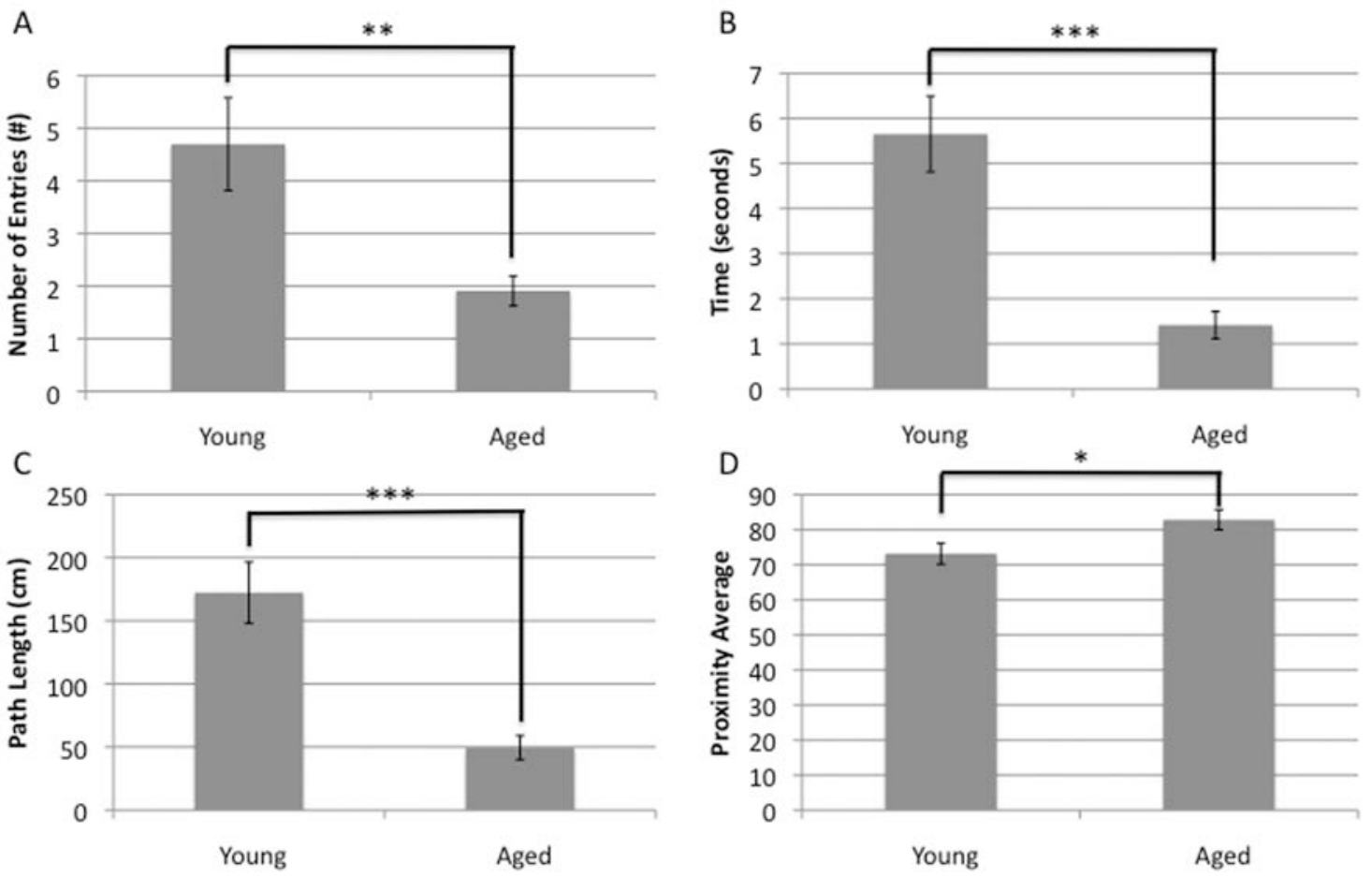

Figure 4.7. Reduction in reference memory in aged animals as measured in the Morris water maze probe test. Aged animals made fewer entries (A), spent less time (B), and traveled less distance $(\mathrm{C})$ in the counter zone compared to young animals. The proximity average (D) was lower amongst young animals, indicating greater reference memory ability. Statistical significance determined using unpaired t-test. ${ }^{*},{ }^{* *},{ }^{* * *}$ denote statistical significance $(p<0.05, p<0.01$, and $p<0.001)$. 


\title{
Chapter Five
}

This work builds upon the previous chapter by applying many of the previously described functional assessments to a clinically relevant model of ischemic stroke at numerous time points post-stroke. By correlating function and infarct volume over time, it becomes clear that some functional assessments, such as the mNSS, correlate more strongly with various infarcted regions, such as the striatum, at different time points. The model of ischemic stroke utilized in this work in aged rodents produces both large cortical and striatal strokes, whereas many studies utilizing young animals produce only striatal strokes due to strong collateral circulation within the cortex. Consequently, we demonstrate the potential utility, of specific functional outcomes as an experimental endpoint in preclinical studies to more closely replicate the clinical scenario.

\section{The Role for Infarct Volume as a Surrogate Measure of Functional Outcome Following Ischemic Stroke in Preclinical Studies}

Ryan C. Turner, Kenneth H. DiPasquale, Aric F. Logsdon, Zhenjun Tan, Rae R. Matsumoto, Jason D. Huber, Charles L. Rosen

This work is currently in preparation for publication submission.

\begin{abstract}
The failed translation of proposed therapeutic agents for ischemic stroke from preclinical to clinical studies has led to increased scrutiny of preclinical studies, namely the model and outcome measures utilized. Preclinical studies routinely use infarct volume as an experimental endpoint or measure in studies employing young-adult, healthy male animals despite the fact that clinically, ischemic stroke is a disease of the elderly, predominantly affecting females, and improvements in functional outcome from pre- to post-intervention remains the most widely utilized assessment. The validity of infarct volume as a surrogate measure for functional outcome remains unclear in clinical studies, as well as preclinical studies, particularly those utilizing a more clinicallyrelevant, aged thromboembolic model. In this work, we addressed the relationship between acute and subchronic functional outcome and infarct volume using a variety of functional assessments ranging from subjective measurements such as the modified Neurologic Severity Score (mNSS), to objective measurements such as grip strength and performance on the inclined plane.
\end{abstract}

\subsection{INTRODUCTION}

Ischemic stroke remains a leading cause of morbidity and mortality worldwide and is associated with significant direct and indirect costs ${ }^{538}$. Present treatment of ischemic stroke is limited to thrombolysis, achieved via either mechanical or pharmacological means, both of which are limited to a small cohort of patients based on time of presentation after stroke onset and an extensive list of contraindications. Consequently, there remains a large unmet need for either alternative treatment options or a means for increasing eligibility for thrombolysis ${ }^{504}$. The development of a successful neuroprotectant, since discovery of the ischemic penumbra by Astrup and colleagues ${ }^{150}$, 539,540 , has been the focus of extensive research in ischemic stroke and produced over 100 candidates progressing to clinical trials based on the work of over 1000 preclinical studies ${ }^{8}$. Unfortunately, none of these proposed neuroprotective agents have translated from bench-to-bedside successfully ${ }^{8}$. 
Reasons for the failed translation remain speculative; however, advisory groups composed of leaders in the field have identified numerous potential reasons for the lack of successful translation 47,135,523,541. These include the animal models and outcome measures utilized. The vast majority of preclinical studies have employed healthy, young-adult male animals, despite the fact that stroke is largely a disease of the elderly and the female gender ${ }^{9}, 523$. The aged brain not only responds to an insult or injury differently, but also exhibits less restorative capacity in comparison to the healthy and young brain 9, 33,51,508,542,543. Additionally, preclinical studies emphasize histological outcome measures, namely infarct volume quantification, whereas the focus of clinical studies is on functional assessments and restoration of physical and cognitive skills ${ }^{523}$, 544 . How infarct volume relates to functional outcome is a subject of controversy with clinical studies demonstrating a moderate correlation (Spearman's rho of $0.43-0.59$ depending on the study) between final infarct volume and function assessed using common scales of neural injury 381,545. Preclinical studies have demonstrated a multitude of findings depending on the model of ischemia, functional assays performed, method of correlation, and time points assessed post-ischemia ${ }^{546-554}$. Variances and discrepancies in these findings are difficult to interpret due to the aforementioned variables as well as the use of generally healthy, young-adult animals in the vast majority of studies, a population that fails to replicate the clinical population afflicted with ischemic stroke ${ }^{10}$.

In this work, we investigate for the first time in an aged thromboembolic model of ischemia and TPA-mediated reperfusion the relationship between infarct volume and infarct location on functional outcome. Specifically, we address the following questions: 1) do acute decrements in functional ability following ischemia correlate with the location or size of the infarct; 2) do chronic deficits in function equate to larger infarct volumes; 3 ) do acute functional deficits correlate with larger infarcts chronically; and 4) does the time point of assessment indicate the inclusion or exclusion of certain functional assessments?

We seek to address these questions in what we believe represents a more clinicallyrelevant preclinical model of ischemic stroke. The use of functional assessments is emphasized in clinical studies and therefore needs to be considered in preclinical work. By incorporating the use of a functional assessment array in a more relevant model of ischemia, we address for the first time using this model the relevance of histological measures as a surrogate measure of outcome. This work illustrates the importance of functional ability as an outcome measure in preclinical ischemic stroke studies on the basis of more closely replicating clinical experience. A range of functional assessments were included in an effort to address multiple components of sensorimotor ability (strength, balance, etc).

\subsection{MATERIALS AND METHODS}

\subsubsection{Chemicals and Animals}

All chemicals used in this study were of pharmaceutical grade and purchased from Sigma Aldrich (St. Louis, MO), unless stated otherwise. Human recombinant TPA was gifted by Genentech (San Francisco, CA). Female Sprague-Dawley rats were acquired from Hilltop Animal Laboratory (Scottsdale, PA). All animals used in this work were obtained between 18-21 months of age from an aging colony established at the supplier. Animals were housed under 12 hour light/12 hour dark conditions with food and water available ad libitum. Animals were acclimated for a minimum of seven days prior to all 
experimental procedures. All experimental protocols were approved by the West Virginia University Animal Care and Use Committee.

\subsubsection{Surgery: Middle Cerebral Artery Occlusion (MCAO)}

Rats were anesthetized using inhaled isoflurane (4\% induction, $2 \%$ maintenance) from Halocarbon (River Edge, NJ) and underwent thromboembolic MCAO as previously described. Reperfusion was achieved through administration of human recombinant tPA $(5 \mathrm{mg} / \mathrm{kg}$ i.v., femoral vein; $30 \%$ bolus and $70 \%$ infused over 30 minutes using a syringe drive). Ischemia was defined as a perfusion drop across the MCA territory of $>80 \%$ as determined by laser Doppler measurements. Successful reperfusion was considered upon restoration of blood flow to $>80 \%$ of baseline values within 30 minutes of tPA administration. The time of tPA administration was varied from 2-6 hours to produce ischemia of a multitude of severities and consequently, infarcts of different sizes. Rats were then divided into acute and chronic assessment groups with the acute group being sacrificed at 24 hours post-MCAO and the chronic group at 21 days postMCAO.

\subsubsection{Behavioral Testing}

All functional testing was performed at baseline to obtain a baseline value for normalization on an animal-by-animal basis, based on previous experience of the authors and the potential for weight and size-related effects ${ }^{538}$. There was no more than 1 week between baseline testing and induction of MCAO. Following MCAO, testing occurred at 24 hours, 72 hours, 7 days, 14 days, and 21 days in the chronic assessment paradigm and only at 24 hours in the acute paradigm, immediately prior to sacrifice.

\subsubsection{Modified Neurological Severity Score (mNSS)}

The mNSS, described previously by Watanabe and colleagues ${ }^{529}$, is a widely used method for assessing the severity of functional deficits after neurological injury in rodents. The test includes measures of sensorimotor performance and basic reflex integrity (Table 5.1). The balance beam used was a synthetic composite material and possessed a square profile with rounded corners and measured $4 \times 4 \mathrm{~cm}$.

\subsubsection{Grip Strength}

The Grip Strength Meter was acquired from Columbus Instruments (Columbus, Ohio). This apparatus is composed of a load cell and interchangeable grip attachment. This work utilized the T-bar attachment. A total of 5 measures were taken for forelimb grip strength for each rat and averaged to produce a composite score at each time point. The rats were grasped by the tail and nape of the neck, forepaws placed onto the bar, and pulled in a straight line away from the load cell by gripping the animal at the base of the tail.

\subsubsection{Activity Monitoring}

Activity was recorded using an automated monitoring system from San Diego Instruments (San Diego, CA). Rats were given 1 hour to acclimate to the testing room prior to initiation of testing. Each chamber for testing consisted of a Plexiglass housing surrounded by a photobeam array $(16 \times 16)$ to detect movements in the $x-y$ plane. A separate 8 photobeam array detected rearing activity. Activity was recorded over a 
period of 30 minutes and included measures of ambulatory, fine, rearing, and total activity in increments of 5 minutes.

\subsubsection{Inclined Plane}

An inclined plane was constructed to model that described by Rivlin and Tator 531 . The device consisted of an $18 \times 18 \mathrm{~cm}$ platform with a textured, rubberized coating. The platform was affixed to a base and hinged on one side, allowing rotation from the horizontal to $85^{\circ}$. Rats were placed on the apparatus such that the main axis of the body was perpendicular to the inclined plane. The maximum incline angle at which the rat could successfully maintain its position for 5 seconds was recorded as the plane was elevated slowly. As the plane was elevated, the rat would make corrective adjustments to maintain a normal posture using both fore- and hindlimbs. Rats were subjected to 5 trials on the inclined plane per assessment period with these measurements being averaged to again produce a composite score.

\subsubsection{Histology}

At the time of sacrifice, animals in the acute group were saline perfused prior to brain extraction and subsequent staining with $2 \%$ triphenyl tetrazolium chloride (TTC) as previously described. The chronic group was perfused with $4 \%$ paraformaldehyde prior to brain extraction and immersion in fixative for a minimum of 24 hours. Brains were then processed using a Tissue-Tek VIP 5 automatic processor and paraffin embedded using a Tissue-Tek TEC 5 embedding console system, both of which were acquired from Sakura Finetek (Torrance, CA). Paraffin-embedded tissues were sectioned at $6 \mu \mathrm{m}$ using a Leica RM2235 microtome (Richmond, VA). Alternating sections were stained for infarct volume assessment using standard protocols for hematoxylin \& eosin (H\&E) and cresyl echt violet. Briefly, sections were deparaffinized using xylene and rehydrated through a series of alcohol immersions progressing from $100 \%$ ethanol to $70 \%$ ethanol. For H\&E, sections were immersed in hematoxylin solution for 2.25 minutes, rinsed in running tap water, and dipped in a $0.25 \%$ acid alcohol solution to differentiate. Slides were again rinsed in running tap water and blued in Scott's Tap Water Substitute for one minute prior to immersion in $95 \%$ ethanol for 30 seconds and subsequent counterstaining with Eosin $\mathrm{Y}$ for 30 seconds. For cresyl echt violet, sections were stained in in cresyl echt violet solution ( $\mathrm{pH} 2.5)$ for 4 minutes prior to dehydration and clearance with xylene. These techniques allow for assessment of infarct volume despite the presence of significant gliosis at chronic time points.

\subsubsection{Infarct Volume Quantification}

For quantification of TTC stained sections, images were acquired using a standard flatbed scanner and quantified using Adobe Photoshop (San Jose, California). For quantification of $\mathrm{H} \& \mathrm{E}$ and cresyl echt violet stained sections, images were acquired using an Olympus AX-70 microscope (Center Valley, Pennsylvania) and quantified using ImageJ (Bethesda, Maryland). All quantification was performed by a blinded observer.

\subsubsection{Statistical Analysis}

All data was analyzed using JMP 10 from the SAS Institute (Cary, NC). Correlations between infarct volume and functional performance were assessed by the Spearman rank-sum coefficient. Differences are considered to be statistically significant at the $\mathrm{P}<0.05$ level. 


\subsection{RESULTS}

5.3.1. Acute Impairments in Functional Performance on the mNSS Following MCAO in Aged Rodents Correlate with Striatal Infarct Volume.

At 24 hours after MCAO, rats underwent functional assessment using the mNSS, gripstrength meter, inclined plane, and recording of spontaneous activity immediately prior to sacrifice and infarct volume determination using TTC staining. A moderate correlation was observed based on Spearman rank-order correlation between total mNSS score and indirect striatal infarct volume $(\rho=0.45, P<0.05)$ as well as the balance beam and raising by the tail subtests of the mNSS $(\rho=0.41, P<0.05 ; \rho=0.36, P<0.05$; respectively). Activity monitoring revealed a significant decline in the number of rearings recorded post-stroke with a moderate correlation between rearing and striatal infarct volume observed $(\rho=0.45, P<0.05)$.

Both total mNSS and subtests of the mNSS universally failed to correlate with cortical and total infarct volumes at 24 hours post-MCAO (Table 5.2). While mNSS failed to correlate with striatal and cortical infarct volume in the acute period, both inclined plane and grip strength indicated a strong trend towards correlation with cortical $(\rho=0.44, P=$ $0.05 ; \rho=-0.41, P=0.07)$ and total $(\rho=0.42, P=0.06 ; \rho=-0.42, P=0.06)$ infarct volumes but not striatal infarcts (Table 5.2).

The relationship between the various functional assessments and the edema index was also determined as at 24 hours post-MCAO, significant edema is present and may influence outcome as evident by the numerous proposed and tested therapeutic agents targeting various elements of ischemic stroke pathophysiology associated with edema development. Significant correlations were observed between total $\mathrm{mNSS}(\rho=0.31, \mathrm{P}<$ $0.05)$ and the inclined plane $(\rho=0.54, P<0.05)$.

\subsubsection{Total mNSS Scores Correlate with Cortical, Striatal, and Total Infarct Volume When Assessed at 21 Days Post-MCAO.}

Functional assessments were performed on Day 21 post-MCAO immediately prior to sacrifice and infarct volume determination using hematoxylin and eosin (H\&E) staining. Moderate to strong correlations were observed between total mNSS and cortical $(\rho=$ $0.56, P<0.05)$, striatal $(\rho=0.62, P<0.05)$, and total $(\rho=0.70, P<0.05)$ infarct

volumes. Subtests such as the balance beam and raising by the tail also correlated with various elements of infarct volume when both function and histology were assessed at 21 days post-MCAO (Table 5.3). Specifically, performance on the balance beam subtest of the mNSS correlated with cortical and total infarct volumes $(\rho=0.75, P<0.05 ; \rho=$ $0.75, P<0.05$; respectively) but not striatal. Raising of the tail, another subtest of the mNSS, was related to striatal $(\rho=0.62, P<0.05)$ and total $(\rho=0.54, P<0.05)$ infarct volumes but not cortical (Table 5.3). No other assessments detected deficits or impairments at Day 21 consistent with infarct volume (Table 5.3). The reasons for this are unclear but likely include the gradual return of function with increased time postMCAO to a level frequently near, or approximating, the baseline.

5.3.3. Acute Deficits Determined Using the mNSS May Predict Cortical, Striatal, and Total Infarct Volumes at 21 Days Post-MCAO in Aged Rodents.

Moderate to strong correlations were observed between total mNSS and cortical $(\rho=$ $0.63, P<0.05)$, striatal $(\rho=0.84, P<0.05)$, and total $(\rho=0.81, P<0.05)$ infarct volumes in animals with functional performance assessed at 24 hours post-MCAO and infarct 
volumes determined histologically at Day 21 post-ischemia. Subtests such as the balance beam and raising by the tail also correlated with striatal $(\rho=0.60, P<0.05 ; \rho=$ $0.72, \mathrm{P}<0.05)$ and total $(\rho=0.59, \mathrm{P}<0.05 ; \rho=0.53, \mathrm{P}<0.05)$ infarct volume but not cortical infarction when function was assessed at 24 hours post-MCAO and histology was assessed at 21 days post-MCAO (Table 5.4). No other assessments correlated with any of the infarct measures (Table 5.4).

\subsection{DISCUSSION}

Failure of successful bench-to-bedside translation of proposed therapeutics for ischemic stroke, namely neuroprotectants, has resulted in an extensive array of recommendations for preclinical studies going forward. Some of the most notable and agreed upon guidelines include the incorporation of functional and histological endpoints, prolonged survival times post-MCAO, and perhaps most importantly, the consideration of more clinically relevant models that address aging and/or comorbidities frequently afflicting patients with ischemic stroke. This work shows for the first time in a thromboembolic model of ischemic stroke using aged animals, the relationship between infarct volume and functional impairment at both acute and chronic time points. Moderate to strong correlations were observed between neurological scoring systems such as the mNSS and infarct volume at numerous time points. Perhaps most notable is the strength and persistence of this correlation between total mNSS score and striatal infarct volumes at both Day 1 and Day 21. This finding is in contrast to comparisons between total mNSS and cortical infarct volumes in which no correlation was observed at 24 hours postMCAO between function and volume, but a moderate to strong correlation was found at 21 days post-MCAO and when using Day 1 function as a predictor of Day 21 infarct volume.

Why cortical infarct volumes do not correlate with function acutely remains unclear but potential reasons include the impact of edema acutely following MCAO and disruption of the functional circuitry as a consequence of striatal damage regardless of cortical integrity. Edema occurs rapidly following ischemia and subsequent blood-brain barrier disruption, likely reaching a maximum within the first five days post-ischemia and has been shown to be associated with various neurological deficits identified in otherwise healthy young-adult, male rats ${ }^{555,556}$. These findings are consistent with work presented here in which edema correlated with the total mNSS score and inclined plane. Perhaps more importantly, the striatum serves as a relay for numerous connections related to motor and sensorimotor function in the rat. The striatum, composed of the caudate nucleus, putamen, and ventral striatum (a combination of the nucleus accumbens and surrounding areas), receives afferent connections from the cortex, substantia nigra, and thalamic nuclei (centromedian and parafascicular). Efferent connections originating in the striatum pass to the thalamus and substantia nigra, in addition to numerous intrastriatal connections (caudate to the putamen, putamen to the globus pallidus, etc). Consequently, any lesion within the striatum can alter function of other brain regions and result in anatomical changes outside of the ischemic territory such as thalamic shrinkage. Therefore, impairments in function are likely regardless of cortical health, diminishing the likelihood of strong volume-function correlations with regards to cortical and total volumes acutely ${ }^{557}$. This is particularly true with regards to the aged rodent model utilized herein as cortical volumes are typically larger in this model due to reduce collateralization with age and therefore, increased tissue susceptibility to ischemia ${ }^{558}$. There is less discrepancy in collateral flow with regards to the striatum between young and aged rodents, resulting in more uniformity when considering striatal infarct volumes. Delayed assessments post-stroke, such as those conducted within this work, generally 
display an improvement or recovery in function, a finding consistent with significant plasticity of the rodent brain.

While the inclusion of functional assessments has been identified as a recommended experimental endpoint in preclinical studies, there is little to no consensus as to the most appropriate assessments. A variety of assays have been used in young-adult models using primarily acute time points and have been shown to correlate with infarct volume but have never been applied to an aged thromboembolic model with long-term assessments. We demonstrate herein the applicability of the mNSS but undoubtedly other tests may want to be explored, particularly those that are sensitive to higher order functions such as cognition and complex sensorimotor behaviors. Importantly, the lack of significant correlation between infarct volume and functional outcome at certain time points with certain tests is not entirely surprising but indicates a need for more sensitive functional measures following MCAO that display persistent deficits, a particular problem in the likely less relevant but far more widely used young-adult models of ischemic stroke. Similarly, the plasticity of the rodent brain that allows for functional recovery may necessitate an array of functional assessments dependent on time post-infarct of interest in order to capture both initial deficits and return of function prior to saturation (generally baseline).

\section{Limitations}

There is an inherent bias within preclinical ischemic stroke work, particulary in aged animals, with regards to long-term survival studies as animals with presumably the largest infarctions expire prior to assessment at delayed time points, thus preventing acquisition of data. Animals that survive for weeks post-MCAO generally have smaller infarcts, as was the case within this work. Specifically, animals surviving to 21 days had a total mNSS score of $6.39 \pm 2.68$ on Day 1 post-MCAO, consistent with a mild to moderate impairment rather than a severe deficit. The lack of rodents exhibiting more severe deficits chronically post-stroke is likely a product of minimal post-operative care relative to the clinical scenario. Patients afflicted with stroke are often monitored continuously both during and after ischemia with extensive critical care, intervention, and rehabilitation support 564 , a striking contrast to preclinical studies in which pain medication and nutritional support are applied but little else. Consequently, severely afflicted patients may survive whereas this is unlikely in animals.

Similarly, the eloquence of human behavior, a vital component of post-stroke assessment in which patients are asked to communicate, both verbally and written, as well as perform simple drawings and motor tasks, allows for the observation of abilities the emphasize primarily motor, sensory, or cognition. In rodents most, if not all, functional assessments require extensive motor ability. As such, when significant tissue damage occurs within the striatum or motor-associated parts of the cortex, assessments may be impaired due to reduced movement despite the fact that sensory and cognitive abilities are in tact.

Without access to small animal imaging, this work required histological quantification of infarct volumes and therefore necessitated animal sacrifice. As such, we were unable to assess how acute infarct volume influences chronic functional assessments. Future studies may want to address this question in a clinically relevant model of ischemia. 


\section{TABLES}

TABLE 1: The mass

\begin{tabular}{|c|c|}
\hline Test & Points: \\
\hline \multicolumn{2}{|l|}{ motoer (musscle status: hemiplegia) } \\
\hline raising by tail & $\begin{array}{l}\text { 0-3 (normal- } \\
\text { maxis) }\end{array}$ \\
\hline fleodion of forelimb & 1 \\
\hline thesdion of himdimb & 1 \\
\hline head mowing $>10^{\prime \prime}$ no vertical axis a allin 30 secs & 1 \\
\hline placing on floor & $\begin{array}{l}\text { 0-3 (noermal- } \\
\text { mans) }\end{array}$ \\
\hline normal walk: & 0 \\
\hline inability to walk straight & 1 \\
\hline cincling toward paretic side & 2 \\
\hline falling down to paretic side & 3 \\
\hline sensory & $\begin{array}{l}\text { mase of } 2 \text { (1) } \\
\text { for each } \\
\text { test) }\end{array}$ \\
\hline placing (Misual \& tactile test & 1 \\
\hline proprioception (deep sensafion) & 1 \\
\hline bearm balance & $\begin{array}{l}\text { 0-6 (nommal- } \\
\text { macis) }\end{array}$ \\
\hline balances wí sleady posture & o \\
\hline graspes side of beam & 1 \\
\hline hugs the beam \& 1 limb talls down trom bearm & 2 \\
\hline $\begin{array}{l}\text { hugs the beam \& } 2 \text { limbs thall down trom bearm, or } \\
\text { spins on beam }\end{array}$ & 3 \\
\hline attermpts to balance on beam bur tralls ort $(>-40$ secs) & 4 \\
\hline attermpts to balance on bearm but falls off ( $>20$ secs) & 5 \\
\hline $\begin{array}{l}\text { falls off wilo athempting to belance or hang on to } \\
\text { bearm }(<20 \text { secs) }\end{array}$ & 6 \\
\hline reflex absence \& abnormal moverments & $\begin{array}{l}\text { 0-4 (nomma!- } \\
\text { max) }\end{array}$ \\
\hline $\begin{array}{l}\text { pinna neflex (head shake when touching auditory } \\
\text { meaturs; }\end{array}$ & 1 \\
\hline $\begin{array}{l}\text { cormeal reflex (eype blink when tovching comea light- } \\
\text { ly wi' cottons }\end{array}$ & 1 \\
\hline $\begin{array}{l}\text { Startie reflex (a motor nesponse to a brief noise firom } \\
\text { snapping a clipboard clipl) }\end{array}$ & 1 \\
\hline selveures, myocionus, myodyelony & 1 \\
\hline
\end{tabular}

- The mNSS utilizes a variety of sensonimotor, balance, and nefler tests to assign a neurologic score. One point is assigned for the inability bo perform the tests of foe the lack of a testled penex. Sevene injury is defined as 13-18 points, moderabe injury 7-12 points, and mild injury 1-6 points.

Table 5.1. Description of the mNSS scoring system for neural injury that incorporates assessments of reflex and sensorimotor abilities. Scores from each subsection are summed to produce a cumulative score representing the overall level of impairment. A score of 1-6 represents mild, 7-12 moderate, and 13-18 severe injury. Rats were assessed at baseline, day 1 , day 3 , day 7 , day 14 , and 


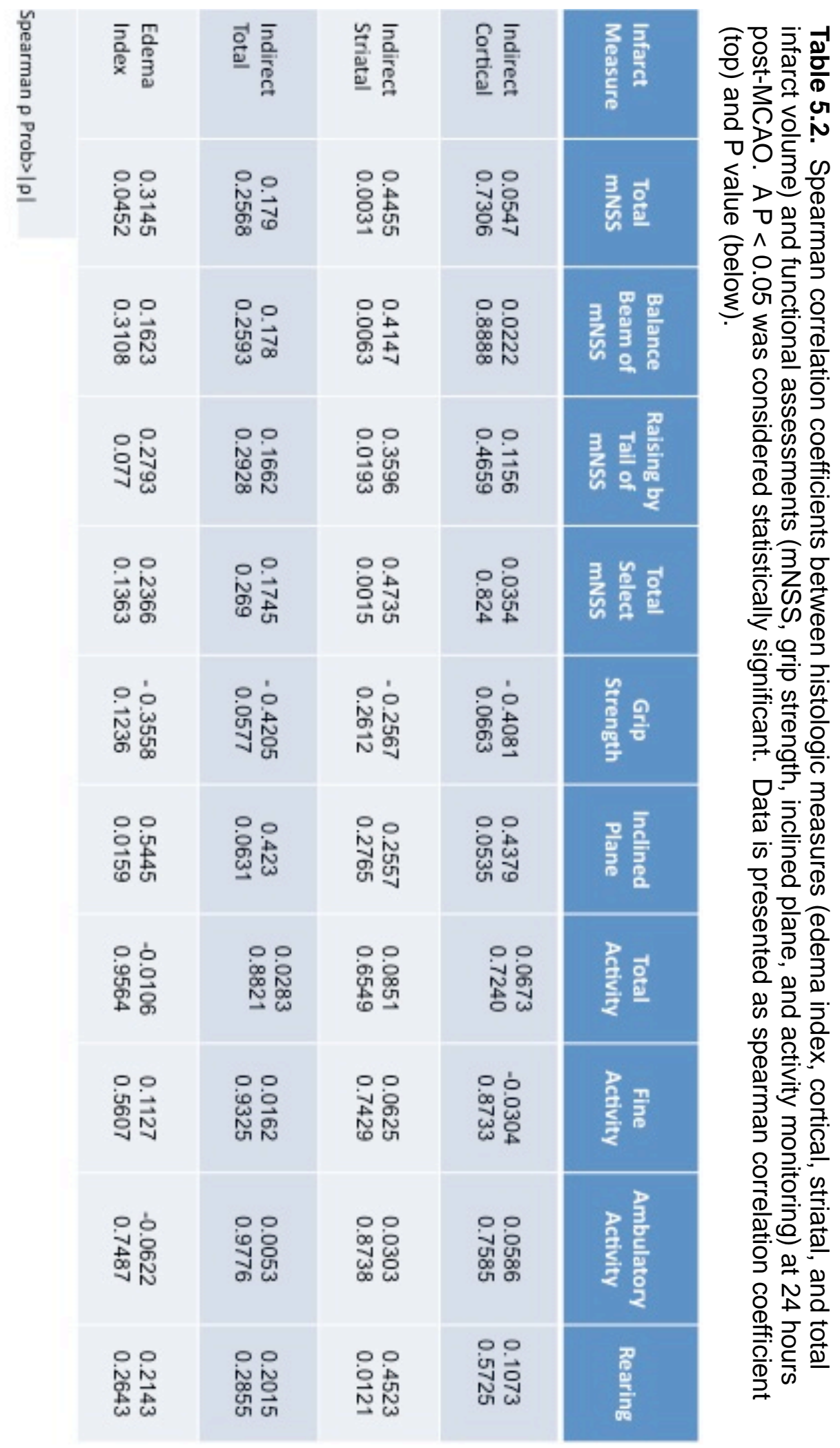




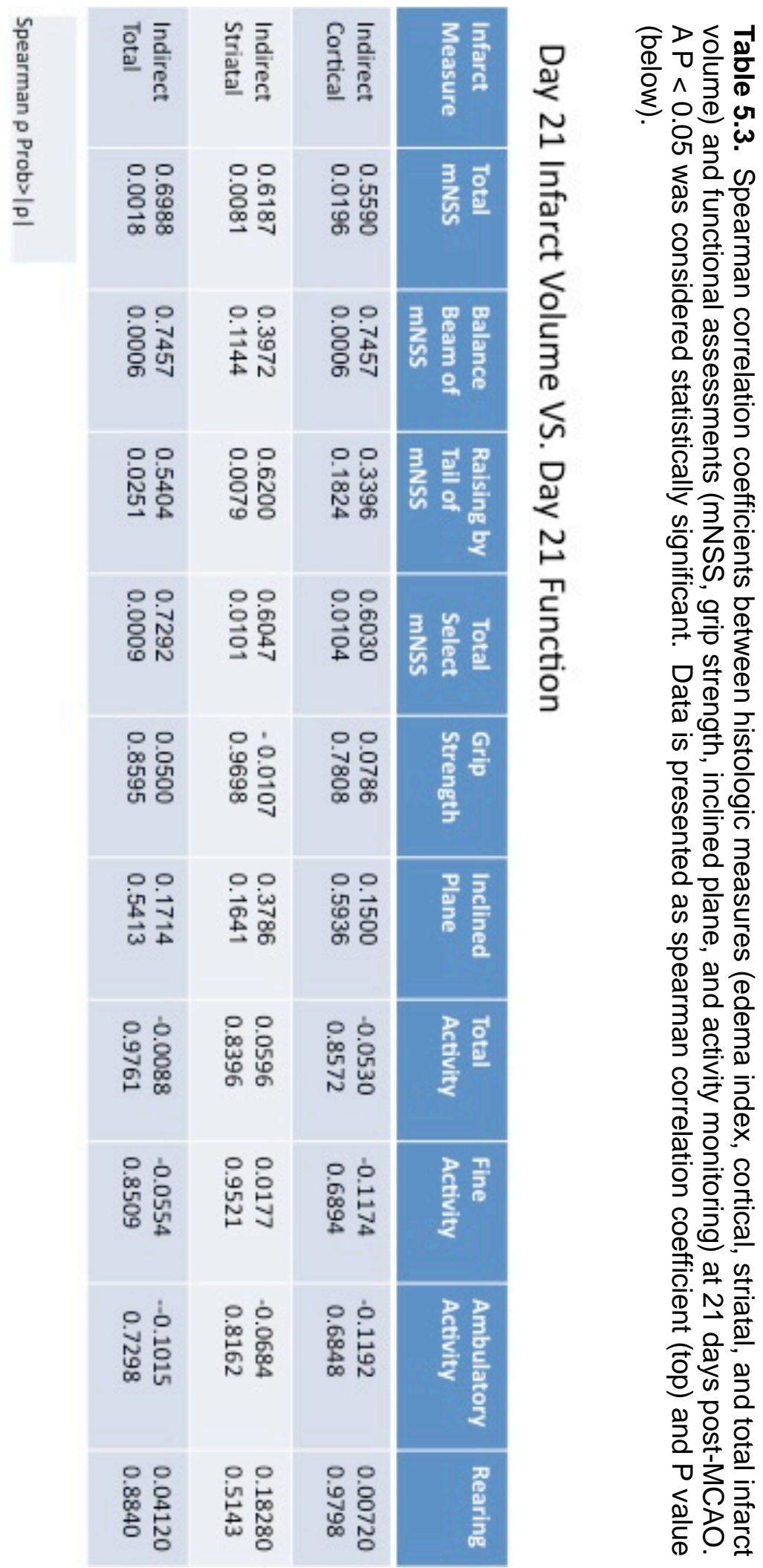




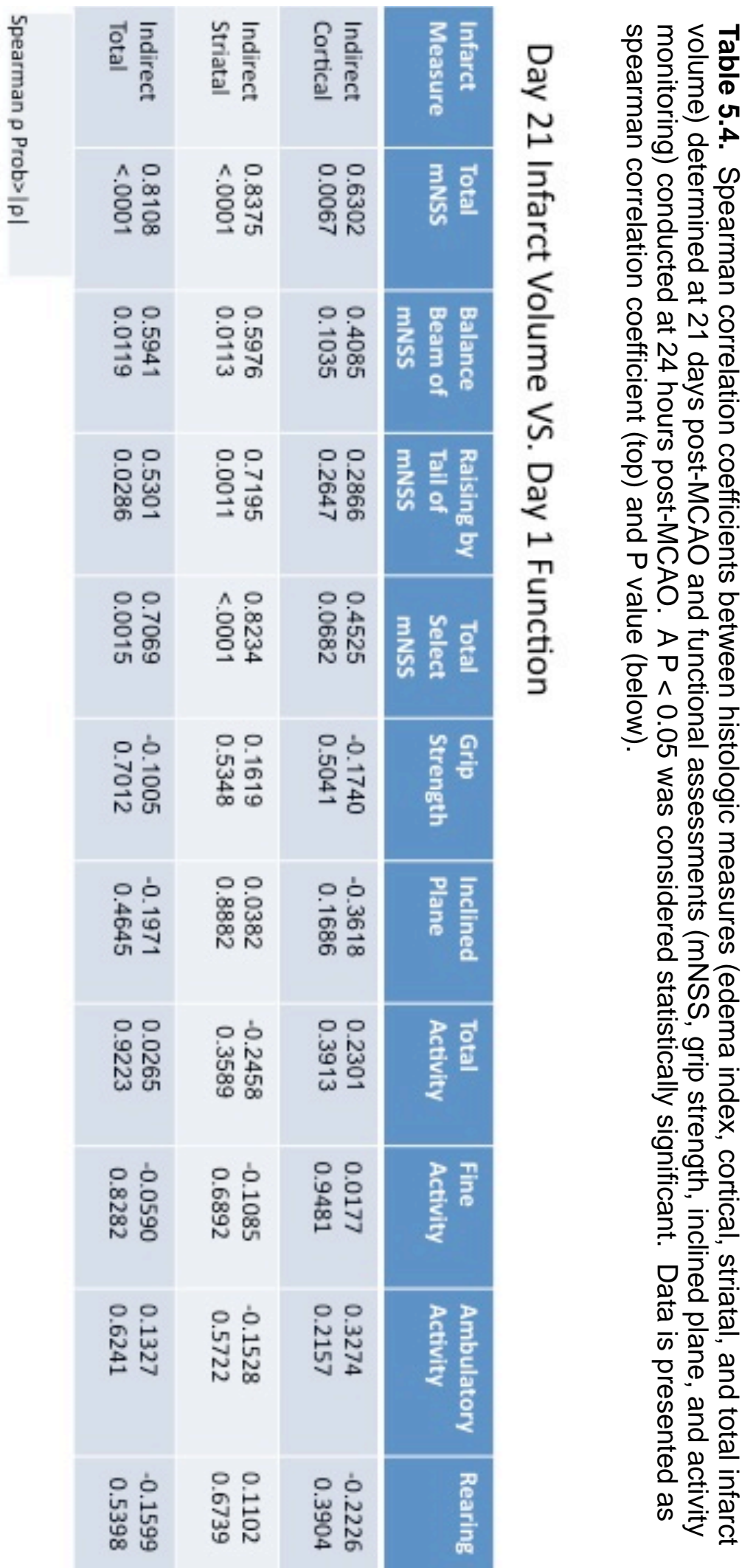




\section{Chapter Six}

Traumatic brain injury, particularly repetitive traumatic brain injury in the form of concussions, has been associated with the development of neurodegenerative disease such as chronic traumatic encephalopathy. This is inherently alarming to those charged with leading or representing certain athletic bodies and hence there are numerous parties attempting to create distance between inherently dangerous activities and health risk. Consequently, alternative explanations have been proposed as precipitating neurologic decline following repetitive trauma, perhaps the most prevalent being a potential role for anabolic steroids. This work investigates this issue by administering steroids in different paradigms, pre- and post-injury, and assessing a well-documented marker of neural and axonal injury, amyloid precursor protein.

\section{Anabolic Steroids and Head Injury}

I This work was published in Neurosurgery.

Citation: Mills JD, Bailes JE, Turner RC, Dodson SC, Sakai J, Maroon JC (2012) Anabolic Steroids and Head Injury. Neurosurgery 70 (1): 205-210.

\section{ABSTRACT}

Background. The suggestion has been made that the neurologic changes seen in the syndrome of chronic traumatic encephalopathy (CTE) may be due to exogenous anabolic steroid use rather than traumatic brain injury (TBI).

Objective. The purpose of this study is to determine if the administration of anabolic steroids alters the pathophysiology of traumatic brain injury.

Methods. Sixty adult male Sprague-Dawley rats were used. A linear acceleration model of traumatic brain injury was used. Experimental groups were as follows: 1) pre-injury anabolic steroids, 2) pre-injury placebo carrier, 3) anabolic steroids without injury, 4) no steroids and no injury, 5) post-injury placebo carrier, 6) post-injury anabolic steroids. Following a 30-day recovery period, rats were sacrificed and brainstem white matter tracts underwent fluorescent immunohistochemical processing and labeling of beta amyloid precursor protein, a marker of axonal injury. Digital imaging and statistical analyses were used to determine if anabolic steroid administration resulted in a significant change in the number of injured axons.

Results. There was no statistically significant difference in number of APP-positive axons by immunohistochemical analysis between the pre-injury anabolic steroid group and the pre-injury placebo carrier group. The same is true when comparing the steroids +no injury with the no steroids+no injury groups and the post-injury steroids with the post-injury placebo carrier groups.

Conclusion. Using a standard acceleration-deceleration laboratory model of mild traumatic brain injury (MTBI), we have shown successful visualization of traumatically injured axons with antibody staining of APP. Our results indicate there is no statistically significant effect of anabolic steroids on the number of APP positive axons in the normal (no linear acceleration injury) rodent corticospinal tract or medial lemniscus, nor is there any statistically significant difference in the number following traumatic brain injury. Using this model and within its limitations, we see no adverse effect or causative role of anabolic steroid administration on the brain following MTBI using APP counts as a marker for anatomical injury. 


\subsection{INTRODUCTION}

While the causal mechanisms of traumatic brain injury (TBI) cross a spectrum from sports-related head impacts to blunt high speed motor vehicle injuries, the underlying pathophysiology remains similar. Autopsy studies from patients with injury classifications ranging from mild traumatic brain injury (MTBI) or concussion to severe traumatic brain injury demonstrate diffuse injury to white matter tracts coursing from the cortex to the brainstem. Initially, traumatic axonal injury (TAI) was thought to involve immediate axonal tearing through the direct action of forces associated with the traumatic insult.

Recent experiments employing anterograde tracers have revealed that TAl is a progressive event involving a focal impairment of axoplasmic transport leading to axonal swelling and ultimate disconnection in the subsequent hours to days . Initial disruption of the axon plasma membrane results in ion channel dysregulation and loss of calcium homeostasis. Thereafter, a series of calcium dependent cascades are activated, resulting in mitochondrial damage and cytochrome c release, activating a caspase-3 mediated apoptotic cascade of proteolytic cleavage of cytoskeletal substrates resulting in the axonal disconnection characteristic of TAI. The extent of TAI is a principal determinant of morbidity and mortality following traumatic brain injury.

To date, no published study has been performed investigating the role of anabolic steroids in the pathophysiology of TBI. The purpose of this study was to determine if chronic administration of anabolic steroids prior to diffuse axonal injury in rats alters the pathophysiology of traumatic brain injury. Furthermore, this study provides insight into the suggestion that anabolic steroid use may be responsible for the pathological alterations seen in Chronic Traumatic Encephalopathy (CTE).

\subsection{MATERIALS AND METHODS}

A total of six experimental groups were utilized in this work. Experimental groups were as follows: 1) pre-injury anabolic steroids, 2) pre-injury placebo carrier, 3) anabolic steroids without injury, 4) no steroids and no injury, 5) post-injury placebo carrier, 6) postinjury anabolic steroids. Following a 30-day recovery period, rats were sacrificed and brainstem white matter tracts underwent fluorescent immunohistochemical processing and labeling of beta amyloid precursor protein, a marker of axonal injury. Digital imaging and statistical analyses were used to determine if anabolic steroid administration resulted in a significant change in the number of injured axons. The experimental groups and methodology is described in greater detail in subsequent sections.

\subsubsection{Pre-Injury Steroid Administration}

Four groups of ten $(n=40)$ adult male Sprague-Dawley rats received anabolic steroids (nandrolone) at a dosage of $15 \mathrm{mg} / \mathrm{kg} /$ week for 12 weeks, or received a carrier placebo. The animals were then subjected to an impact acceleration injury (or sham procedure) resulting in TAI. The four groups therefore were: 1) anabolic steroids with impact injury; 2) anabolic steroids without impact injury; 3) impact injury without steroids; 4) no steroids and no impact injury.

\subsubsection{Post-Injury Steroid Administration}

Two groups of ten $(n=20)$ adult male Sprague-Dawley rats received either nandrolone at a dosage of $15 \mathrm{mg} / \mathrm{kg} /$ week or a carrier placebo for four weeks following an impact acceleration injury. At one month post-injury, rats were sacrificed and following 
transcardial perfusion, their brainstems harvested and sagittal sections were prepared. Immunohistochemical processing was performed to detect abnormal concentration of amyloid precursor protein, the standard marker of TAI. Stereologic microscopic analysis was performed to calculate the density of injured axons within the corticospinal tract and medial lemnsicus.

\subsubsection{Continuous Steroid Infusion}

Subcutaneous continuous osmotic pumps were prefilled with nandrolone or carrier and surgically implanted in rats. Animals received induction anesthesia using a modified medical anesthesia machine. The animals were then shaved and prepared in sterile fashion for surgery, followed by subcutaneous injection of local anesthetic into the planned incision site. A $1 \mathrm{~cm}$ midline incision between the scapulae was made and a subcutaneous pocket was opened using hemostats. The osmotic infusion pump (Azlet; Cupertino, California) was inserted into the pocket and the skin incision was closed. The process was repeated at 6 weeks to obtain 12 weeks of nandrolone or carrier infusion.

\subsubsection{Marmarou Impact Acceleration Injury}

The rats were subjected to an impact acceleration injury (or sham injury procedure) resulting in reproducible MTBI. Rats weighing between 350 and 400 grams received induction anesthesia followed by endotracheal intubation and were maintained on inhaled anesthetic using a modified medical anesthesia machine. The animals were then shaved and prepared in sterile fashion for surgery, followed by subcutaneous injection of local anesthetic into the planned incision site. A $3 \mathrm{~cm}$ midline incision in the scalp was made, periosteal membranes were separated, exposing the bregma and lambda sutures. A metal disk $10 \mathrm{~mm}$ in diameter and $3 \mathrm{~mm}$ thick was attached to the skull with cyanoacrylate and centered between bregma and lambda. The animal was placed prone on a foam bed with the metal disk directly under a plexiglas tube. A 450-gram brass weight was dropped through the tube from a height of 2 meters striking the disk. The animal was then ventilated on $100 \% \mathrm{O} 2$ while the skull was inspected and the incision repaired. When the animal recovered spontaneous respirations, the endotracheal tube was removed and the animal was returned to its cage for postoperative observation. Animals in the sham injury groups underwent an identical procedure with the exception of the weight drop onto the skull. All procedures involving live animals were approved by the Institutional Animal Care and Use Committee of West Virginia University, and were performed according to the principles of the Guide for the Care and Use of Laboratory Animals, published by the Institute of Laboratory Resources, National Research Council (NIH publication 85-23-2985). The animals were housed in the small animal vivarium under veterinary staff supervision. Each group received rat chow ad lib.

\subsubsection{Tissue Processing and Immunohistochemical Analysis}

Following 30 day post-injury survival, animals were euthanized with a lethal dose injection of $0.5 \mathrm{ml}$ Ketamine and $0.5 \mathrm{ml}$ Xylazine. The animals were immediately perfused transcardially with $200 \mathrm{ml}$ cold $0.9 \%$ saline to wash out all blood. This was followed by $4 \%$ paraformaldehyde in Millonigs buffer for 40 minutes. The entire brain, brainstem, and rostral spinal cord were removed and immediately placed in 4\% paraformaldehyde for 24 hours. Following 24 hours fixation, the brain was blocked by cutting the brainstem above the pons, cutting the cerebellar peduncles, and then making sagittal cuts lateral to the pyramids. The resulting tissue containing the corticospinal tracts and the medial lemnisci, areas shown previously to yield traumatically injured axons, was then sagittally cut on a vibratome into 40-micron sections. The tissue then underwent temperature 
controlled microwave antigen retrieval using previously described techniques ${ }^{569}$. The tissue was pre-incubated in a solution containing $10 \%$ normal goat serum (NGS) and $0.2 \%$ Triton $\mathrm{X}$ in PBS for 40 minutes.

The tissue was incubated in polyclonal antibody raised in rabbit against beta amyloid precursor protein (APP) at a dilution of 1:200 in 1\% NGS in PBS overnight. Following incubation in primary antibody, the tissue was washed 3 times in $1 \%$ NGS in PBS, then incubated in a secondary anti-rabbit IgG antibody conjugated with Alexa 488 fluorophore (Molecular Probes; Carlsbad, California) for two hours. The tissue underwent a final wash in $0.1 \mathrm{M}$ phosphate buffer and then was mounted to a glass slide using an anti-fade agent and a coverslip applied. The slides were sealed with acrylic and stored in the dark in a laboratory refrigerator

A stereological method was used to determine an unbiased estimate of the number of APP positive axons per cubic $\mathrm{mm}$ in the corticospinal tract and medial lemniscus. The optical fractionator technique utilitizing Stereolnvestigator 7.0 (MBF Bioscience; Williston, Vermont) and a Nikon Eclipse microscope with 4x and 20x objectives was performed. Sagittal APP stained specimens were examined with low magnification and regions of interest were drawn incorporating the corticospinal tract and medial lemniscus. The software then selected random $50 \mathrm{~mm}^{2}$ counting frames with depth of 15 $\mathrm{mm}$, and APP positive axons were marked. The volume of the ROI was determined using the Cavalieri method, the volume of the sum of the counting frames was calculated, the sum total of injured axons within the counting frames was calculated, and an estimate of the number of APP positive axons per cubic $\mathrm{mm}$ was calculated.

\subsection{RESULTS}

In sham injured animals receiving either nandrolone or carrier, axons throughout the medullary corticospinal tract and medial lemniscus demonstrated a paucity of labeling for APP. These rare labeled axons did not demonstrate vacuolization, swelling, or breakdown; typical characteristics of traumatic axonal injury. In comparison, evaluation of axons from animals 30 days post-injury demonstrated focal labeling of APP within swollen contiguous and terminal axon segments, consistent with previous findings suggestive of impaired axoplasmic transport in traumatic axonal injury. These APP positive injured axons had similar appearance irrespective of the pre-injury treatment with nandrolone or carrier (Figure 6.1).

The stereologic optical fractionator method was utilized to determine an unbiased estimate of the number of APP positive axons per cubic $\mathrm{mm}$ within the corticospinal tract and medial lemnsicus. This demonstrated no statistically significant quantitative difference between the injured animals receiving either carrier or nandrolone; 29,757 \pm 6,185 and $27,301 \pm 3,772$ axons per $\mathrm{mm}^{3}$ respectively, (Figure 6.2). Likewise, there was no significant difference between sham injured animals receiving either nandrolone or carrier; 1,554 \pm 759 and 1,632 \pm 827 axons per $\mathrm{mm}^{3}$ respectively. As expected, a significant difference was observed between the sham injured and injured animals, irregardless of nandrolone or carrier.

To determine if nandrolone would effect injury mechanisms and cellular recovery following injury, the second experimental design was deployed. Again, the stereologic method was utilized to determine an unbiased estimate of the number of APP positive axons per cubic $\mathrm{mm}$. This demonstrated no significant quantitative difference between the injured animals receiving either carrier or nandrolone; 28,136 $\pm 7,160$ and 25,448 \pm 6,955 axons per $\mathrm{mm}^{3}$ respectively, (Figure 6.3). Additionally, there was no significant 
difference between the four groups of injured animals, irrespective of the timing or exposure to nandrolone.

\subsection{DISCUSSION}

This work is necessitated by the fact that the use of anabolic-androgenic steroids (AAS) has increased in recent decades, particularly among athletes seeking a competitive advantage . According to vandenBerg et al., approximately $1.5 \%$ to $2.5 \%$ of US adolescents routinely use anabolic steroids. The rate of use escalates to between $15 \%$ and $30 \%$ amongst community weight trainers. Worse yet is that this issue is far from isolated to adult athletes - $10 \%$ of users are teens according to recent estimates, with the majority being young males . Use among male adolescents reaches $4.5 \%$, with school sponsored sport participants more likely to use anabolic steroids . Not surprisingly, this same group is most likely to suffer TBI.

Sports or recreational activity-associated MTBI is now believed to occur in at least 1.7 million people annually in the United States with some estimating the prevalence as two to three times greater. Additionally, football-specific studies report that $47 \%$ of high school football players sustain a concussion annually. Players suffering a concussion are likely to suffer a second concussion, and multiple concussions are associated with slower recovery of neurologic function . Recent evidence from a survey of retired NFL players indicates that multiple concussions have long term neurologic consequences, including increased risks of clinical depression . The suggestion has been made that the long-term neurologic changes seen in retired NFL players and other competitive athletes is due to the increased prevalence of steroid use, which would appear plausible due to the $9.1 \%$ self-reported use of anabolic steroids during the playing careers of nowretired professional football players.

The syndrome of CTE has been recognized as an important consequence of repetitive MTBI in contact sports ${ }^{420}$, particularly football. It is believed to represent a milder form of exposure to multiple head contacts or violent collisions than is dementia pugilistica which is present in approximately $17 \%$ of retired professional boxers 421,576 . The neurobehavioral syndrome of CTE affects the brain's emotional circuitry, expressed as mood lability, depression, failed business and personal relationships, alcohol and drug abuse, and suicide ${ }^{410,574}$. Using antibody staining for tau protein, the neuropathological picture of CTE has been firmly delineated as tauopathy expressed as neurofibrillary tangles and neuritic plaques ${ }^{423}$. These NFTs and NTs have been shown to be the result of repetitive MTBI in several models, and brain trauma is the only known cause of these typical NFTs and NTs.

The suggestion has been made that the pathologic alterations in CTE could instead be attributed to the interaction of exogenous anabolic steroids, however, to date no experimental paradigm has been carried out to attempt to discern the effects on the mammalian brain of a maintenance administration of testosterone combined with a TBI. Since a controlled clinical trial is unlikely to be performed, we attempted to answer, in a straightforward manner, the question of whether a potentially synergistic effect exists between anabolic steroids and MTBI. Using a standard acceleration-deceleration laboratory model of $\mathrm{MTBI}$, we have shown successful visualization of traumatically injured axons with antibody staining of APP. Our results disclosed no effect on the number of APP positive axons in the rodent corticospinal tract or medial lemniscus, nor was there any difference in the number following traumatic brain injury. 
To the best of our knowledge, the available scientific and medical literature does not contain any studies which incriminate anabolic steroids in causing neuronal damage, loss, or vulnerability following TBI. In conclusion, using this model and within its limitations, we see no adverse effect or causative role of anabolic steroid administration on the brain following MTBI using APP counts as a marker for anatomical injury. To the contrary, our clinical research has shown that the risk of CTE is most likely related to exposure to repetitive $\mathrm{MTBI}$ with a tendency towards a genetic predisposition, specifically the presence of the ApoE3 allele ${ }^{422,423,426 .}$ 


\section{FIGURES}

a

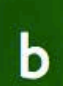

C

Figure 6.1. Micrographs of sagittal sections through rat corticospinal tract following immunohistochemical processing for APP. Injured axons, represented by arrows, had similar appearance in rats receiving either 12 weeks of continuous steroid infusion or carrier infusion. Panel 1a.) steroids and injury, 1b.) no steroids and injury, 1c.) steroids and no injury, 1d.) no steroids and no injury. 


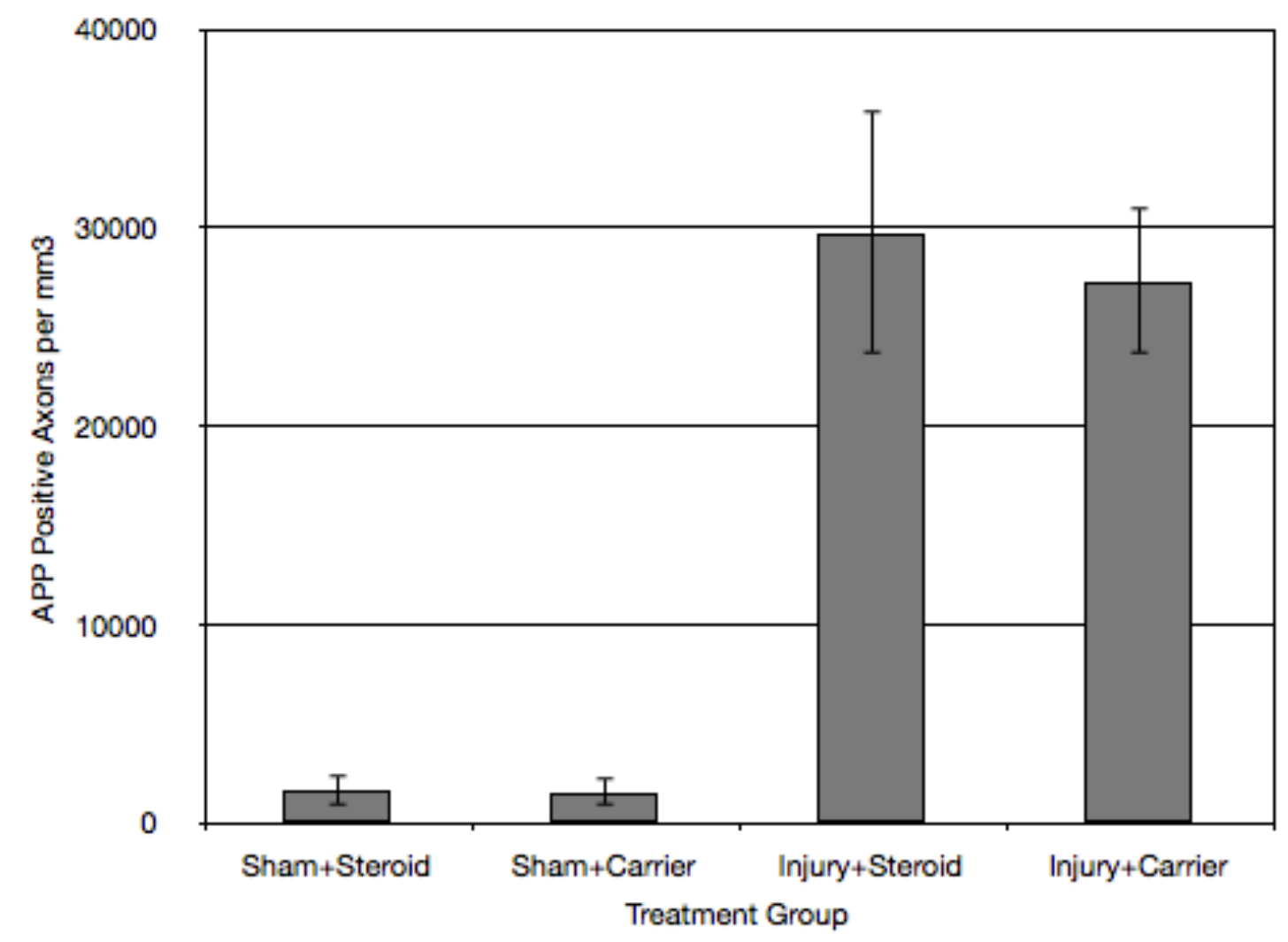

Figure 6.2. Graph demonstrating the density of APP positive axons in corticospinal tracts and medial lemnisci in sham impact injured animals receiving either carrier of nandrolone prior to injury. There was no statistical significance between carrier and steroid-treated groups within any of the respective treatment paradigms $(P>0.05)$. 


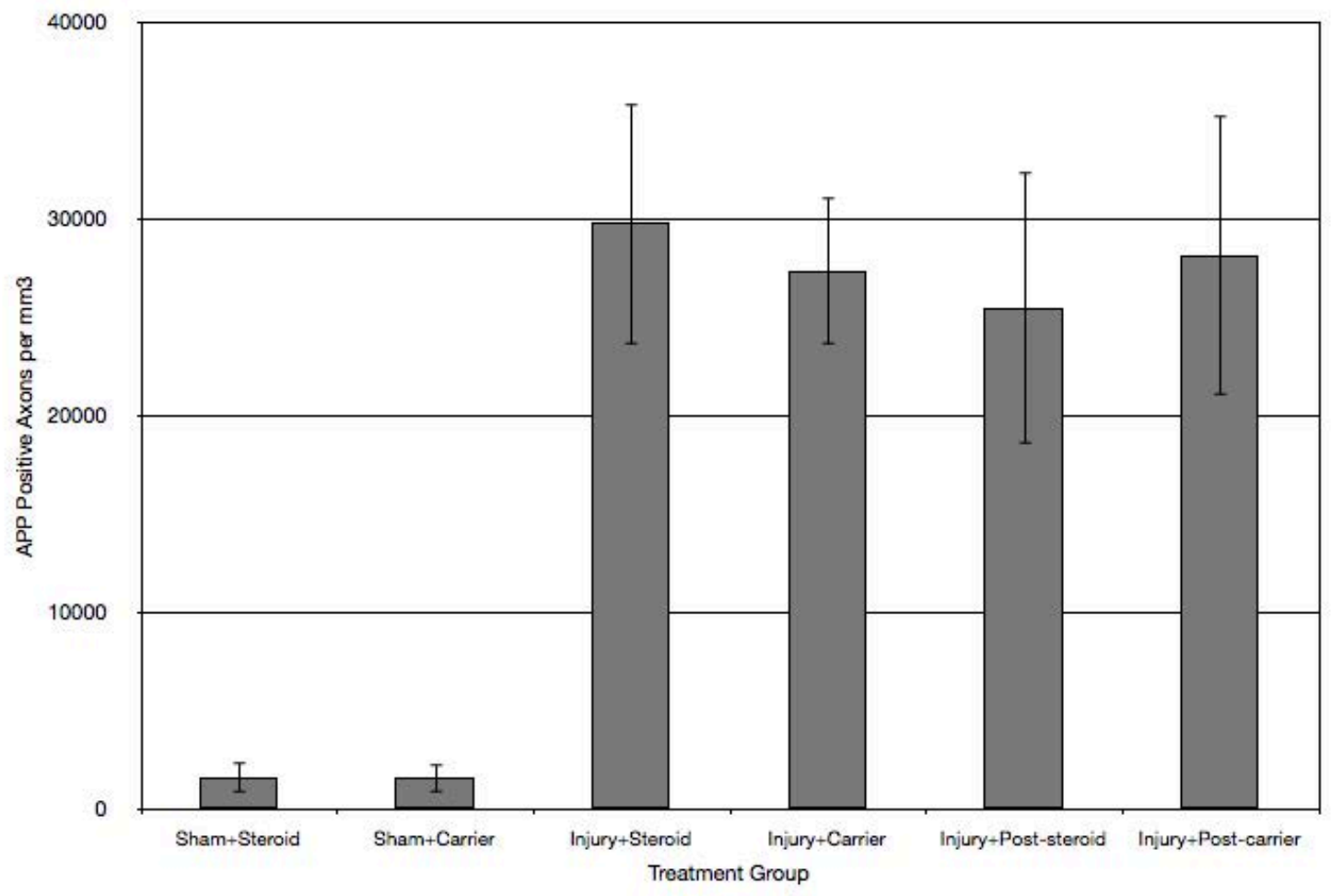

Figure 6.3. Graph demonstrating the density of APP positive axons in corticospinal tracts and medial lemnisci in rats receiving either post-injury steroids or post-injury carrier. There was no statistical significance between carrier and steroid-treated groups within any of the respective treatment paradigms $(P>0.05)$. 


\section{Chapter Seven}

Traumatic brain injury and concussion specifically, remain a major issue on the athletic and battlefield despite significant improvements in technology and materials that have been incorporated in helmets. As such, alternative measures of prevention are desirable. One area of traumatic brain injury that remains uninvestigated, particularly with regards to diffuse axonal injury, is how to restrict brain movement within the cranial vault, thus reducing axonal injury and coup-contrecoup impacts. In this chapter we apply observations from nature, specifically the woodpecker, that may prevent brain movement and neural injury following trauma.

\section{Internal Jugular Vein Compression Mitigates Traumatic Axonal Injury in Rat Model by Reducing Intracranial Slosh Effect}

This work was published in Neurosurgery.

Citation: Smith DW, Bailes JE, Fisher JA, Robles J, Turner RC, Mills JD (2012) Internal Jugular Vein Compression Mitigates Traumatic Axonal Injury in a Rat Model by Reducing the Intracranial Slosh Effect. Neurosurgery 70 (3): 740-746.

\section{ABSTRACT}

Background. Traumatic brain injury (TBI) remains a devastating condition for which traditionally extra-cranial protection has been utilized in the form of helmets, which largely fail to protect against intracranial injury.

Objective. The purpose of this study was to determine if the pathologic outcome after TBI can be improved via slosh mitigation by internal jugular vein (IJV) compression.

Methods. Two groups of 10 adult male Sprague-Dawley rats were subjected to impactacceleration traumatic brain injury. One group underwent IJV compression via application of a collar prior to injury while the second group did not. Intracranial pressure (ICP) and intraocular pressure (IOP) were measured before and after IJV compression to assess collar performance. All rats were sacrificed after a 7-day recovery period, and brainstem white matter tracts underwent fluorescent immunohistochemical processing and labeling of beta amyloid precursor protein (APP), a marker of axonal injury. Digital imaging and statistical analyses were used to determine if IJV compression resulted in a diminished number of injured axons.

Results. IJV compression resulted in an immediate $30 \%$ increase in IOP and ICP. Most notably, IJV compression resulted in greater than $80 \%$ reduction in the number of APPpositive axons as indicated by immunohistochemical analysis.

Conclusion. Using a standard acceleration-deceleration laboratory model of mild TBI, we have shown successful prevention of axonal injury following IJV compression as indicated by immunohistochemical staining of APP. We argue that IJV compression reduces slosh-mediated brain injury by increasing intracranial blood volume, which can be indirectly measured by intracranial and intraocular pressure. 


\subsection{INTRODUCTION}

Traumatic brain injury (TBI) continues to be one of the most common causes of death and morbidity in persons under age 45, even in western societies. A reported 1.7 million people suffer from TBI annually in the United States alone, resulting in an estimated per annum total cost of over $\$ 60$ billion ${ }^{571}$. Historically, prevention of skull and brain injury has focused on the use of helmets as external cranial protection. We believe this approach is fundamentally flawed as irrespective of the benefits helmets have provided for major penetrating brain injuries and skull fractures, both military personnel and athletes are subjected to high velocity, acceleration-deceleration mechanisms which lead to concussive injury ${ }^{577}$. In large part, the human brain's relative freedom of movement within the cranial cavity predisposes to both linear and rotational force vectors, with resultant axonal tearing ${ }^{578}$. We believe that helmets fail to address the linear and rotational forces imparted on the brain following impact and therefore are inadequate for prevention of traumatic axonal injury (TAI) and concussion.

The skull and spinal canal contains only nervous tissue, connective tissue and fat cells and their interstitium, blood, and cerebrospinal fluid (CSF). These fluid contents do not completely fill the rigid container delimited by the skull and bony spinal canal, leaving a 'reserve volume'. The change in volume inside a container for a given change in pressure is termed 'compliance'. Increases in volume of the contents of the skull and bony spinal canal, within the range of reserve volume, occur at low container pressures (due to the high compliance of the system). In the presence of reserve volume, as is seen in a normal physiologic state, acceleration to the skull can result in a differential acceleration between the skull and its contents. As a consequence, the brain and fluids collide with the inside of the skull ${ }^{578}$. Considering the semi-solid properties of the mammalian brain, we will refer to this effect as 'slosh'.

While helmets are effective in preventing the infrequent penetration or fracture of the skull, they have little ability to limit slosh effects. We believe that mitigating slosh by filling the reserve volume (exhausting compliance) will significantly reduce the propensity for differential motion between the skull and its contents. We reasoned that by mitigating slosh, an accelerating force to the skull would tend to move the skull and its contents in unison, preventing collisions amongst intracranial contents and therefore, avoiding brain kinetic and vibrational energy absorption.

In an attempt to mitigate intracranial slosh it is important to recognize that the single intracranial compartment that is most amenable to rapid, reversible change in volume and pressure is the blood. The simplest and most rapid means of increasing the blood compartment is to inhibit its outflow by mechanically obstructing the draining veins in the neck. As such, the aim of this study was to test the hypothesis that reducing intracranial and spinal canal compliance would mitigate brain injury due to acute skull acceleration or deceleration (impact). We used a standardized mild TBI rat model to study the effects of internal jugular vein (IJV) compression on brain injury 562, 579-581.

\subsection{Materials and Methods}

\subsubsection{Animals}

In this work two groups of ten (total of 20) male Sprague-Dawley rats weighing between 350 and 400 grams were used. Animals were acquired from Hilltop Animal Laboratory (Scottdale, PA, USA) and housed under 12 hour light/12 hour dark conditions with rat chow and water available ad libitum. All procedures involving live animals were approved 
by the Institutional Animal Care and Use Committee of West Virginia University, and were performed according to the principles of the Guide for the Care and Use of Laboratory Animals, published by the Institute of Laboratory Resources, National Research Council (National Institutes of Health publication 85-23-2985).

\subsubsection{Marmarou Impact Acceleration Injury Model in Rats}

Anesthesia was induced and maintained with isoflurane using a modified medical anesthesia machine. Body temperature was controlled during the approximately 10 minute procedures using a homeothermic heating blanket with rectal probe, and adequate sedation was confirmed by evaluation of response to heel tendon pinch. The animals were shaved and prepared in sterile fashion for surgery, followed by subcutaneous injection of $1 \%$ lidocaine local anesthetic into the planned incision site. A 3 $\mathrm{cm}$ midline incision in the scalp was made, and periosteal membranes separated, exposing bregma and lambda. A metal disk $10 \mathrm{~mm}$ in diameter and $3 \mathrm{~mm}$ thick was attached to the skull with cyanoacrylate and centered between bregma and lambda. The animal was placed prone on a foam bed with the metal disk directly under a plexiglas tube. A 450-g brass weight was dropped a single time through the tube from a height of $2 \mathrm{~m}$, striking the disk. The animal was then ventilated on $100 \%$ oxygen while the skull was inspected, the disk removed, and the incision repaired. When the animal recovered spontaneous respirations, anesthesia was discontinued and the animal was returned to its cage for post-operative observation . Buprenorphine was used for postoperative analgesia.

\subsubsection{Experimental Protocol}

This work involved two groups, each consisting of 10 animals for a total of 20 animals. Two groups were utilized, a control injury group and an experimental injury group. In the experimental injury group the rats were fitted with a $15 \mathrm{~mm}$ wide collar, with two compressive beads designed to overlay the IJVs and was tightened sufficiently to provide mild compression of the veins without compromising the airway. The collar was then fixed in circumference with a Velcro fastener. The collar was left in position for three minutes prior to administrating experimental brain injury.

\subsubsection{Assessment of Intracranial Reserve Volume}

\subsubsection{Intracranial Pressure (ICP) Measurement}

ICP was measured in five animals using the FOP-MIV pressure sensor (FISO Technologies; Quebec, Canada) as described by Chavko, et al ${ }^{582}$. The head of the rat was shaved and prepped in sterile fashion for surgery. The rat was fixed in a stereotaxic

I apparatus (model 962; Dual Ultra Precise Small Animal Stereotaxic Instrument; $;$ Kopf Instruments, Germany) and a $3 \mathrm{~cm}$ midline incision in the scalp was made. Periosteal membranes were separated, exposing both bregma and lambda. A $2 \mathrm{~mm}$ burr hole was drilled $0.9 \mathrm{~mm}$ caudal from bregma and $1.5 \mathrm{~mm}$ from the midline. The fiberoptic probe was then inserted to a depth of $3 \mathrm{~mm}$ into the cerebral parenchyma.

\subsubsection{Intraocular Pressure (IOP) Measurement}

IOP was measured in all animals using the TonoLab rebound tonometer (Colonial Medical Supply; Franconia, New Hampshire) as described in the literature ${ }^{582-584}$. IOP measurements were taken after induction of anesthesia in all animals and a second time in the experimental group following application of the novel IJV compression device. 
Following application of the IJV compression device in the experimental injury group, IOP readings were taken every 30 seconds while the compression device was in place.

\subsubsection{Tissue Preparation and Immunhistochemical Labeling}

At 7 days post-injury all animals $(n=20)$ were anesthetized and immediately perfused transcardially with $200 \mathrm{ml}$ cold $0.9 \%$ saline to wash out all blood. This was followed by $4 \%$ paraformaldehyde infusion in Millonigs buffer for 40 minutes. The entire brain, brainstem, and rostral spinal cord were removed and immediately placed in $4 \%$ paraformaldehyde for 24 hours. Following 24 hours fixation, the brain was blocked by cutting the brainstem above the pons, cutting the cerebellar peduncles, and then making sagittal cuts lateral to the pyramids. The resulting tissue, containing the corticospinal tracts and the medial lemnisci, areas shown previously to yield traumatically injured axons, was then sagitally cut on a vibratome into 50 micron thick sections. The tissue underwent temperature controlled microwave antigen retrieval using previously described techniques. The tissue was preincubated in a solution containing $10 \%$ normal serum and $0.2 \%$ Triton $X$ in PBS for 40 minutes.

For amyloid precursor protein (APP) labeling, the tissue was incubated in polyclonal antibody raised in rabbit against beta APP (\#51-2700, Zymed, Inc.; San Francisco, California) at a dilution of 1:200 in 1\% NGS in PBS overnight. Following incubation in primary antibody, the tissue was washed 3 times in 1\% NGS in PBS, then incubated in a secondary anti-rabbit IgG antibody conjugated with Alexa 488 fluorophore (A11008, Molecular Probes; Eugene, Oregon), diluted at 1:200 for two hours. The tissue underwent a final wash in $0.1 \mathrm{M}$ phosphate buffer and then was mounted using an antifade agent and cover slipped. The slides were sealed with acrylic and stored in the dark in a laboratory refrigerator ${ }^{569}$.

\subsubsection{Fluorescent Microscopy and Image Analysis}

The tissue was examined and images acquired using a Olympus AX70 fluorescence microscope system (Olympus; Tokyo, Japan). Ten digital images were obtained from the tissue of each animal and images were then randomized. Individual injured axons were independently counted and data was stored in a spreadsheet (Microsoft Corp.,;Redmond, Washington). Differences between group means were determined using paired t-tests and considered significant if the probability value was less than 0.05 .

\subsubsection{Stereological Quantification of Axonal Injury}

A stereological method was used to determine an unbiased estimate of the number of APP positive axons per cubic $\mathrm{mm}$ in the corticospinal tract and medial lemniscus. The optical fractionator technique utilizing a Stereolnvestigator 9.0 (MBF Bioscience, Inc.,;Williston, Vermont) and a Olympus AX70 microscope with 4x and 40x objectives was performed. Sagittal APP stained specimens were examined with low magnification and regions of interest were drawn incorporating the corticospinal tract and medial lemniscus. The software then selected random 50 micron counting frames with depth of 15 microns, and APP positive axons were marked. The volume of the region of interest (ROI) was determined using the Cavalieri method, the volume of the sum of the counting frames was calculated, the sum total of injured axons within the counting frames was calculated, and an estimate of the number of APP positive axons per cubic $\mathrm{mm}$ was calculated. 


\subsection{RESULTS}

\subsubsection{Assessment of Intracranial Reserve Volume}

\subsubsection{Intracranial Pressure (ICP) Measurement}

ICP was assessed both prior to and after application of the IJV compression device. The baseline ICP was $10.23 \pm 1.68 \mathrm{mmHg}$ and was increased to $16.63 \pm 2.00 \mathrm{mmHg}$ following IJV compression (Figure 7.1: $\mathrm{p}<0.01$ ). Notably, this increase of greater than $30 \%$ from baseline occurred within seconds following IJV compression.

\subsubsection{Intraocular Pressure (IOP) Measurement}

IOP measurements were taken both before and after application of the IJV compression device, similar to ICP recordings. The baseline IOP was $11.18 \pm 2.27 \mathrm{mmHg}$ and was elevated to $16.27 \pm 3.20 \mathrm{mmHg}$ following IJV compression (Figure $7.2: \mathrm{p}<0.01$ ). The increase of $31 \%$ seen in IOP following IJV compression is strikingly similar to that seen in ICP following IJV compression, both in magnitude and rapidity of response (Figure 7.3).

\subsubsection{TBI - Impact Acceleration Model}

None of the animals died from the head trauma. Animals tolerated collar application without any observed untoward effects for the duration of the experiment. Specifically, there were no outward or visible signs of discomfort, intolerance, or respiratory difficulty. All recovered without complication and exhibited normal behavioral and feeding habits up until the day of sacrifice. At necropsy, the brains were grossly normal in appearance.

\subsubsection{Stereologic Analysis of APP Positive Axons}

To determine the density of injured axons in the corticospinal tracts and medial lemnisci, the stereological optical fractionator method was used. Compared to the normal anatomy found in previous experiments with sham animals, control animals without the collar demonstrated focal labeling of APP within many swollen contiguous and terminal axon segments, consistent with impaired axoplasmic transport in traumatic axonal injury. Following microscopic digital image acquisition from multiple areas within the corticospinal tract and medial lemnisci from multiple tissue slices, counting of APP positive axons in animals who received the IJV compression collar demonstrated much fewer APP positive axons, at a frequency much more similar to sham animals, compared to those undergoing injury without IJV compression (Figure 7.4). These abnormal axons demonstrated typical morphological characteristics of traumatic injury, primarily swelling and disconnection. By qualitative analysis, the experimental group showed ( $\mathrm{m} \pm \mathrm{sd}$ ) $13,540 \pm 9808$ vs. $77,474 \pm 25,325(\mathrm{p}<0.01)$ APP positive axons $/ \mathrm{mm}^{3}$ in the control group (Figure 7.5).

\subsection{DISCUSSION}

\subsubsection{Internal versus External Brain Protection}

The main finding of this study is that compression of the IJVs for 3 min prior to head trauma, led to physiological alterations in intracranial compliance, as evidenced by modest increases in ICP and IOP, while simultaneously and markedly reducing the pathologic index of primary neuronal injury in a standardized rat model of TBI. We had 
reasoned that the reduction in brain volume compliance would prevent the differential motions between the cranium and the brain that lead to energy absorption and neuronal primary and secondary injuries. These pathological changes include axonal tearing that disrupt axoplasmic transport resulting in axonal swelling and activation of the apoptotic cascades, as evidenced in this model by a statistically significant reduction in APP counts of injured axons.

To date, no effective pharmacological treatment has been discovered for TBI. Although omega-3 fatty acids such as docosahexaenoic acid (DHA), and progesterone ${ }^{581}$, show some promise in animal studies, efficacy in humans has not been proven, emphasizing the importance of prevention in $\mathrm{TBI} 579,580,585$. Heretofore, standard prophylactic measures designed to protect the brain against injury in the case of head trauma have emphasized external cranial prevention through the use of helmets. As effective as they may be in preventing penetrating injuries and fractures, helmets have limitations in their protective abilities, especially for concussion and axonal injury ${ }^{577}$. The explanation may be furthered by an analogy to automobiles: placing additional armor on the outside of the car may not be as effective in preventing passenger injuries during collisions as simply wearing a seat belt, or filling up the passenger compartment with an activated airbag. To counteract this reality, our approach involves increasing resistance to venous return and increasing intracranial blood volume, theoretically preventing or reducing the brain movement inside the skull resulting from impact. The energy imparted to a nonsloshing brain space traverses its contents as a series of elastic collisions between molecules--rather than being absorbed in the form of frictional, acoustic, and kinetic energies associated with relative motion (shear and collision) between compartments. A well known example of elastic collisions is that of Newton's cradle (Figure 7.6) where the energy imparted by striking of the first ball-bearing in a series of contiguous suspended ball-bearings is transmitted through the series of contiguous ball-bearings to the last, without apparent motion of those in between.

In our animal model, applying the collar increased intracranial volume as indicated by an increase in ICP and IOP by $30 \%$ and $31 \%$, respectively. In humans, safe, gentle compression of IJVs dates back to 1918 when Quenkenstedt developed a simple test to prove patency of the spinal column. In this maneuver, a spinal needle is placed in the lumbar subdural space and then the IJVs are compressed causing a rise in spinal pressure. IJV compression may also occur upon placement of tight fitting neck stabilization collars, and have been shown to increase ICP ${ }^{586}$, whereas wearing shirts with tight collars or neckties, has also been shown to increase IOP ${ }^{587}$. Notably, only mild compressive pressure is required to partially occlude the IJVs as they are a low pressure system, often with. As the inflow of cerebral arterial blood continues after partial cerebral venous outlet obstruction, the intracerebral and venous pressure will increase until the jugular venous resistance is overcome or the blood drainage is redirected to other venous channels. In either case there is a reduction in intracranial compliance and a modest increase in ICP.

\subsubsection{Implications to Humans}

Increasing intracranial blood volume to provide brain protection from head trauma and blast injuries, in sports or on the battlefield, could be readily implemented by wearing an elastic collar-type device (Figure 7.7). Mild compression of the IJVs, particularly if applied along some length of the vein, would not pose any danger of airway or carotid compression. Clothing and jewelry, some of which are restrictive in nature and may apply some compression of the neck, are ubiquitously worn without any known adverse effects. 


\subsubsection{Do Analogous Mechanisms Exist in Nature to Limit Brain Trauma?}

In terms of biological-inspired discovery, another species exposed to repeated head trauma is the woodpecker. This bird experiences forces of $1500 \mathrm{G}$ per peck, for 12,000 pecks a day, totaling about 85 million head impacts over its average lifespan ${ }^{588}$. Its protective mechanisms are all reminiscent of preventing slosh. It restricts eye movement by closing its thick inelastic eyelids on impact. A pectin structure within the globe increases pressure and volume and thus prevents relative movement of the vitreous and retina. Its CSF space is remarkably small resulting in low intracranial volume compliance. Finally, woodpeckers have a hyoid and omohyoid structure similar to that in man ${ }^{589}$. Due to the direct proximity of the omohyoid atop the IJVs it is intriguing to speculate that upon contraction of the omohyoid, perhaps with each peck, the IJVs may be partially occluded and intracranial compliance exhausted.

\subsubsection{Limitations}

This was a pilot and proof of concept study which had sufficient power to identify a difference between the treatment and control group in a cohort of 20 animals because of the magnitude of the difference in outcome. This is a standardized measure of axonal injury that may or may not be translatable to other types of trauma and axonal damage in rats, much less other species, including man. Nevertheless, the immunohistochemical technique we employed is specific for axonal damage and results in a reliable range of measured damaged neurons. In addition, the Marmarou model of accelerationdeceleration injury ${ }^{562}$ is an accepted and well-studied methodology by which to quantify the extent of TBI, in which we, and others, have published experience $579,580,585$. A hallmark of this model is the ability to cause diffuse axonal injury as a consequence of not only the initial impact but also the rebound experienced following injury as the animal is placed on a foam pad with known spring constant. An essential component of this is an unimpeded range of motion of the head and neck regions in particular. The IJV compression device utilized in this work (Figure 7), composed primarily of an elastic band with compression beads overlying the IJVs rather than a rigid material, did not impair or alter the biomechanics of the model. The reduction in damaged axons, as evidenced by a marked reduction in APP counts, in the experimental group with the IJV compression device is highly statistically significant $(p<0.01)$.

Additionally, we measured the change in ICP after applying the collar in five rats. There may have been a variation of increase in ICP in the remaining test rats, but if anything, this would be expected to reduce the variability within experimental groups. Our results show that every study rat had a reduction in axonal injury greater than the 95\% confidence interval of the control group. We did not test the protective effect of increasing intracranial volume from blast injury; however, the rationale for its effectiveness is the same.

\subsection{CONCLUSIONS}

We conclude that venous compression in the neck reduced the extent of axonal injury in a standardized mild TBI model in rodents. We suggest that our mechanism restricted brain venous drainage and increased its blood volume, thus shifting intracranial physiology to the steep part of the volume-compliance curve. The lack of compliance inside the confines of the skull and spinal canal prevented slosh energy absorption and the resultant axonal injury by causing them to approach a more elastic collision when the 
skull was struck during the weight drop method. Further research is needed to ascertain whether higher species, including humans, may benefit from slosh mitigation during TBI. 
Figures

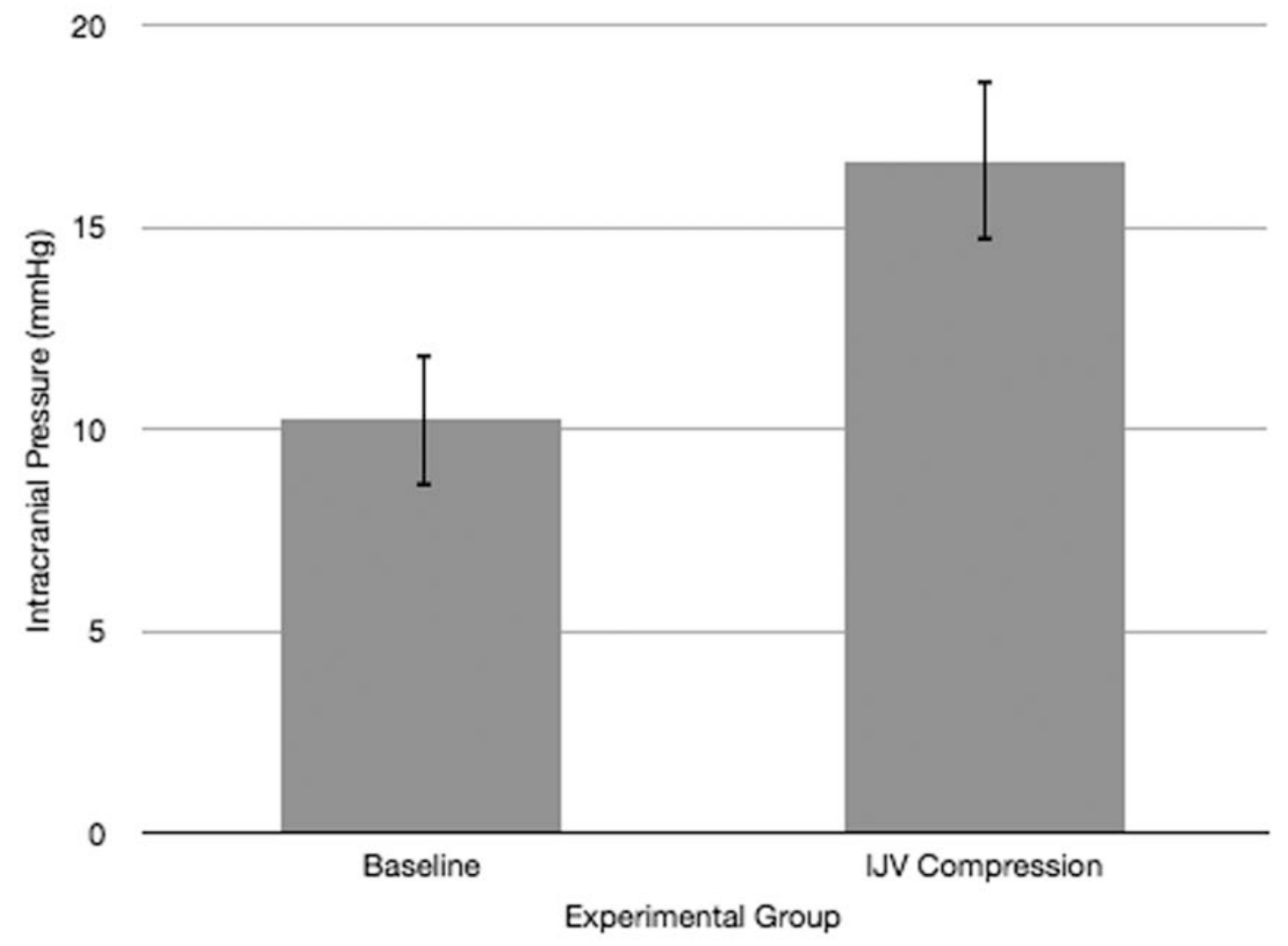

Figure 7.1.: Change in intracranial pressure (ICP) as a consequence of IJV compression, $p$-value $<0.01$. 


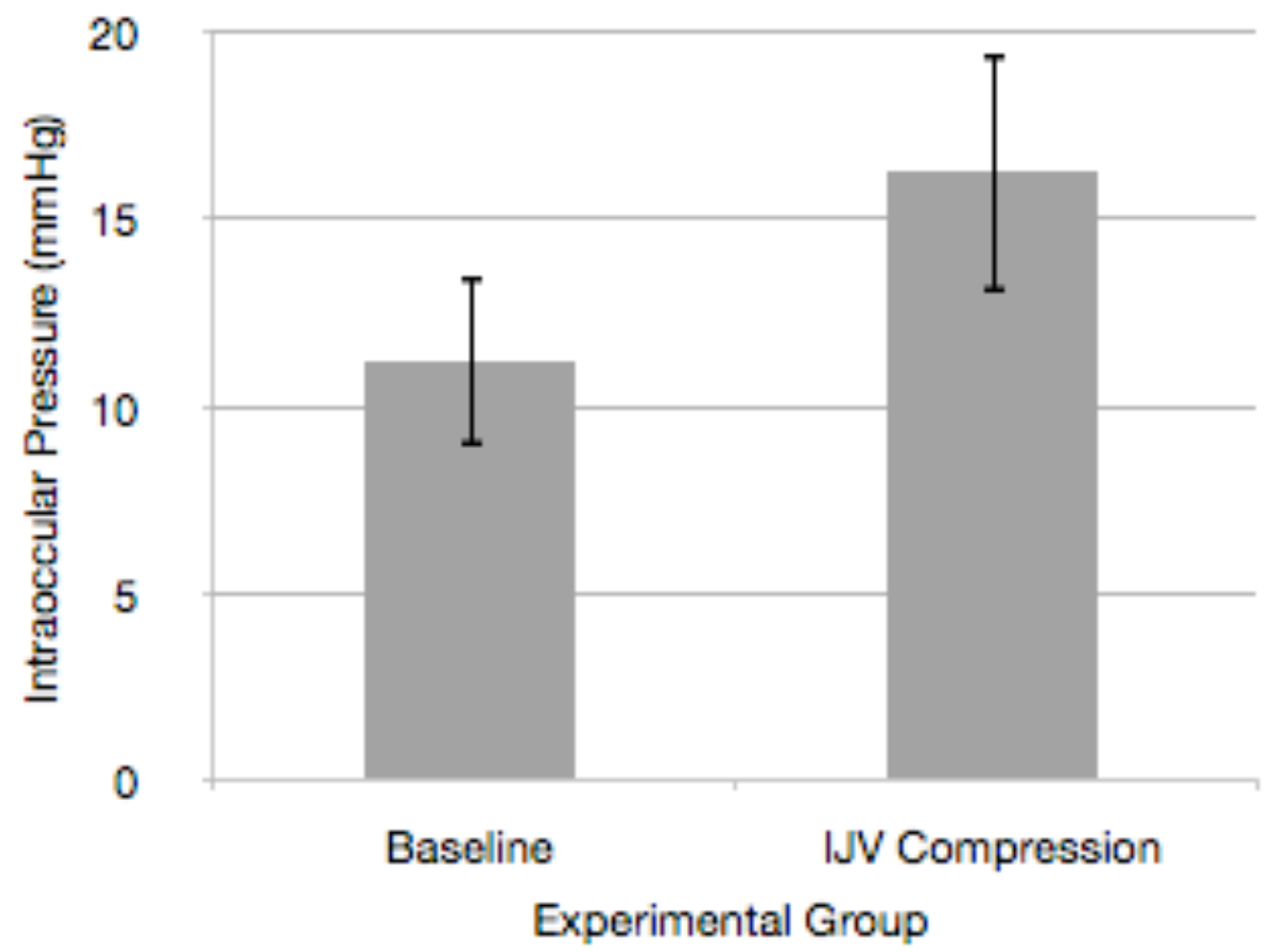

Figure 7.2.: Change in intraocular pressure (IOP) as a consequence of IJV compression, p-value $<0.01$. 


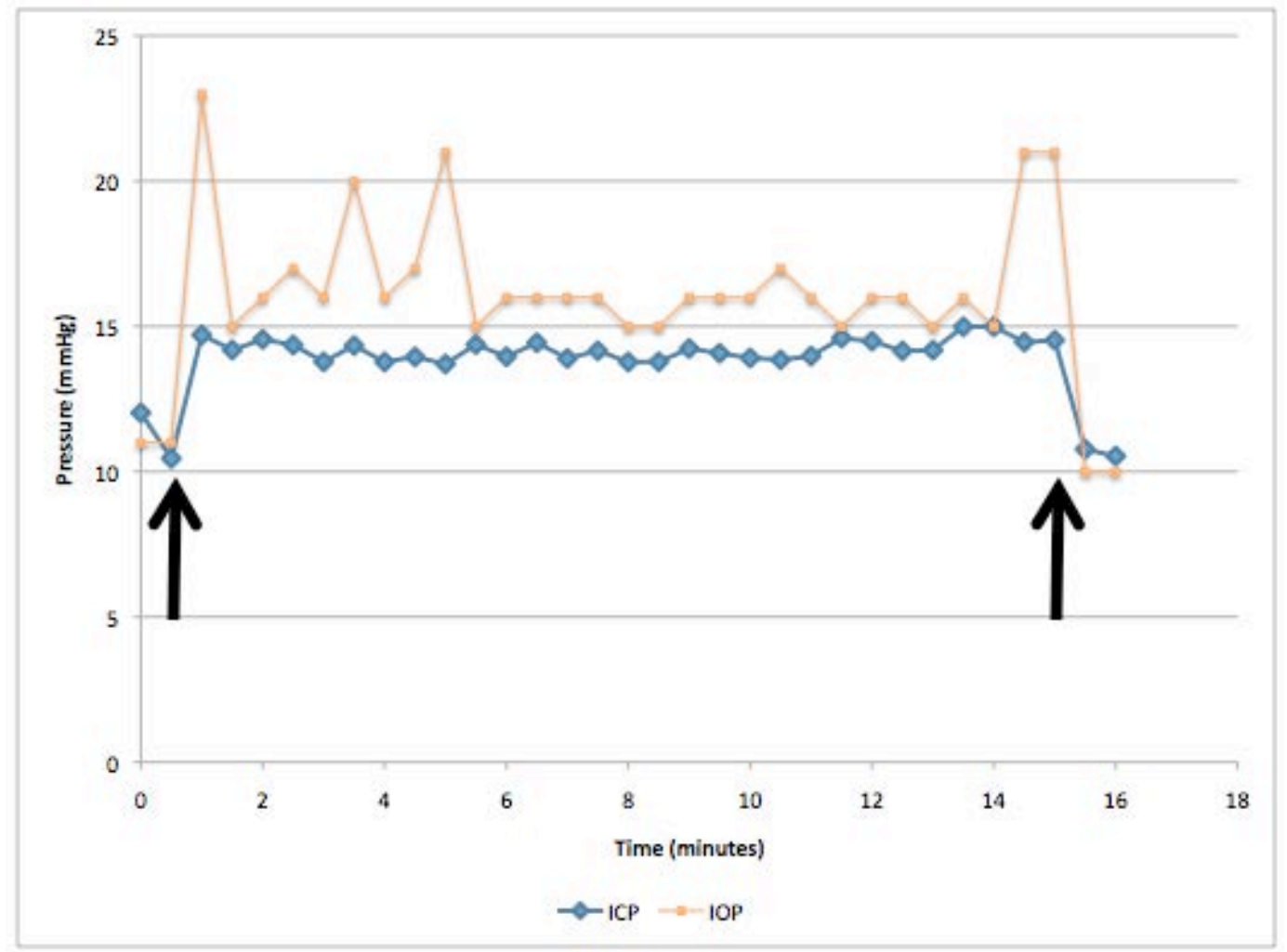

Figure 7.3.: Representative tracing of physiologic changes seen in intracranial pressure (ICP) and intraocular pressure (IOP) over a 15 minute period caused by application (arrow on left) and removal of IJV compression (arrow on right). Of note is the rapid response seen in both ICP and IOP following IJV compression as well as the duration for which these changes are sustained. 


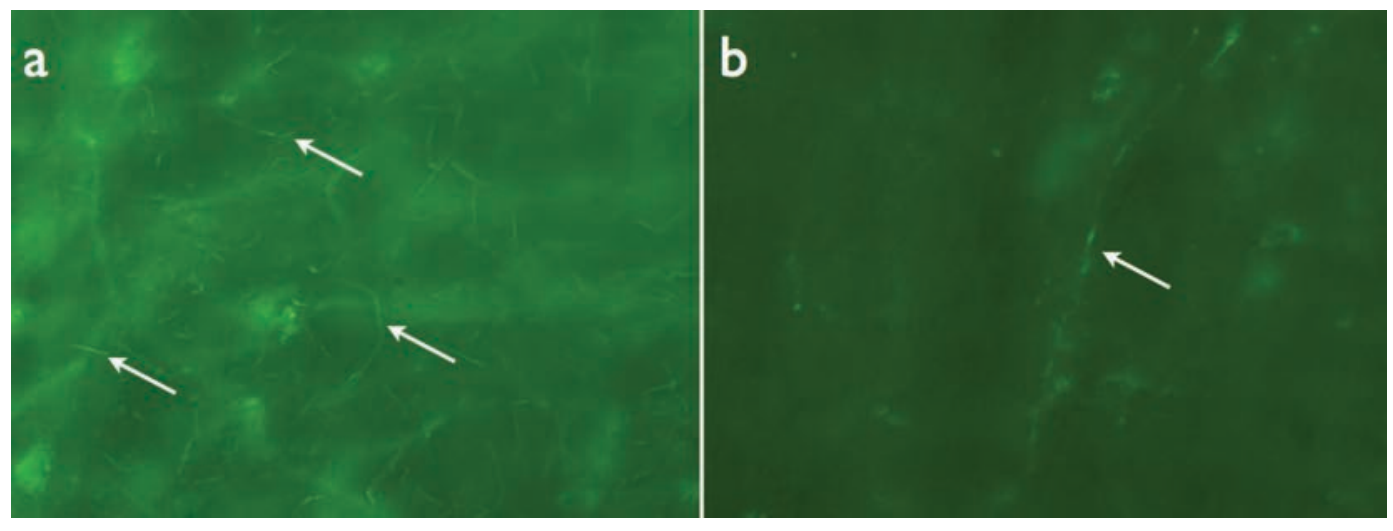

Figure 7.4.: Corticospinal tracts stained for APP post-injury (a) control (b) application of IJV compression device. Arrows represent injured axons. 


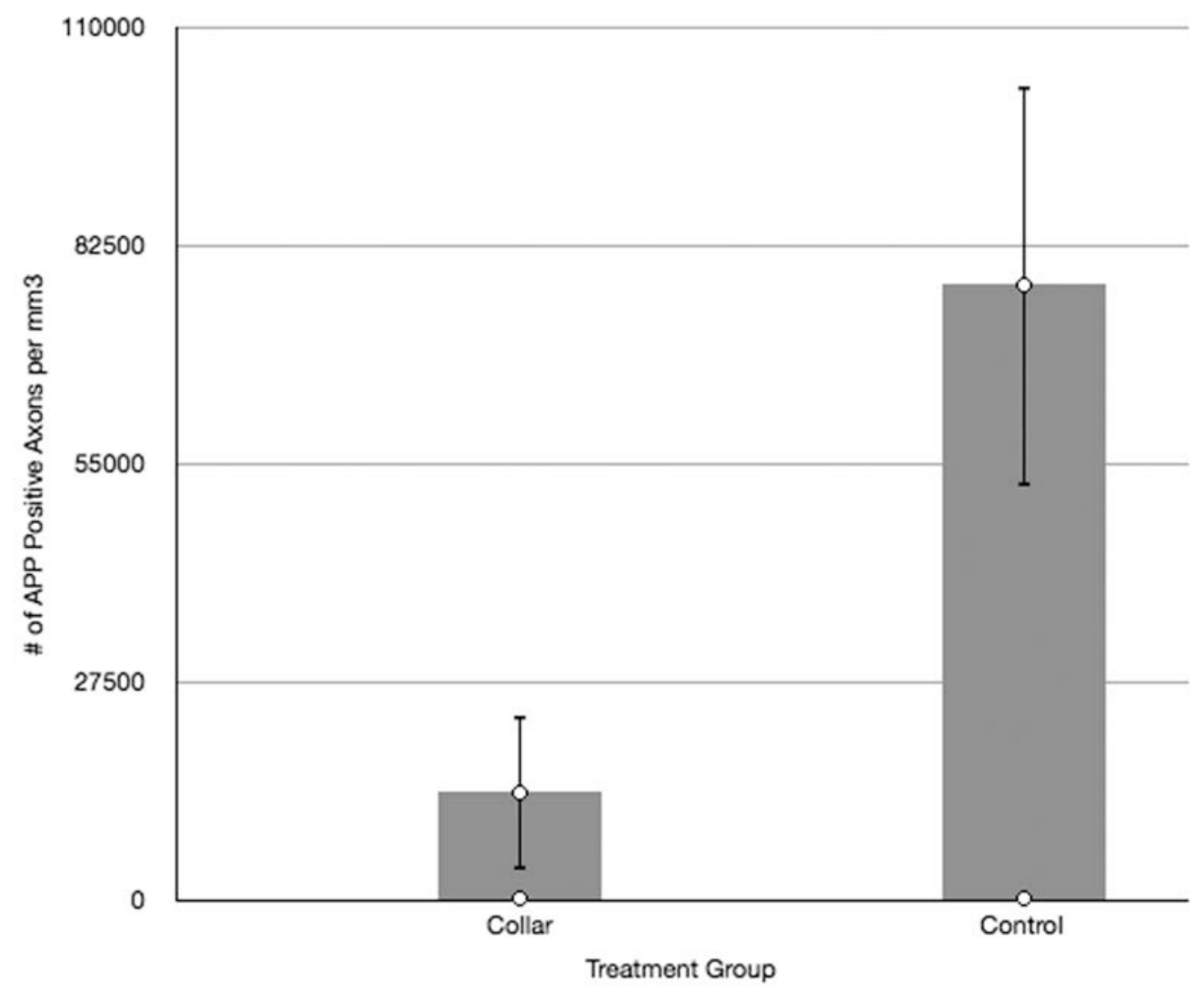

Figure 7.5.: Effect of IJV compression on axonal injury as indicated by APP staining, pvalue $<0.01$. 


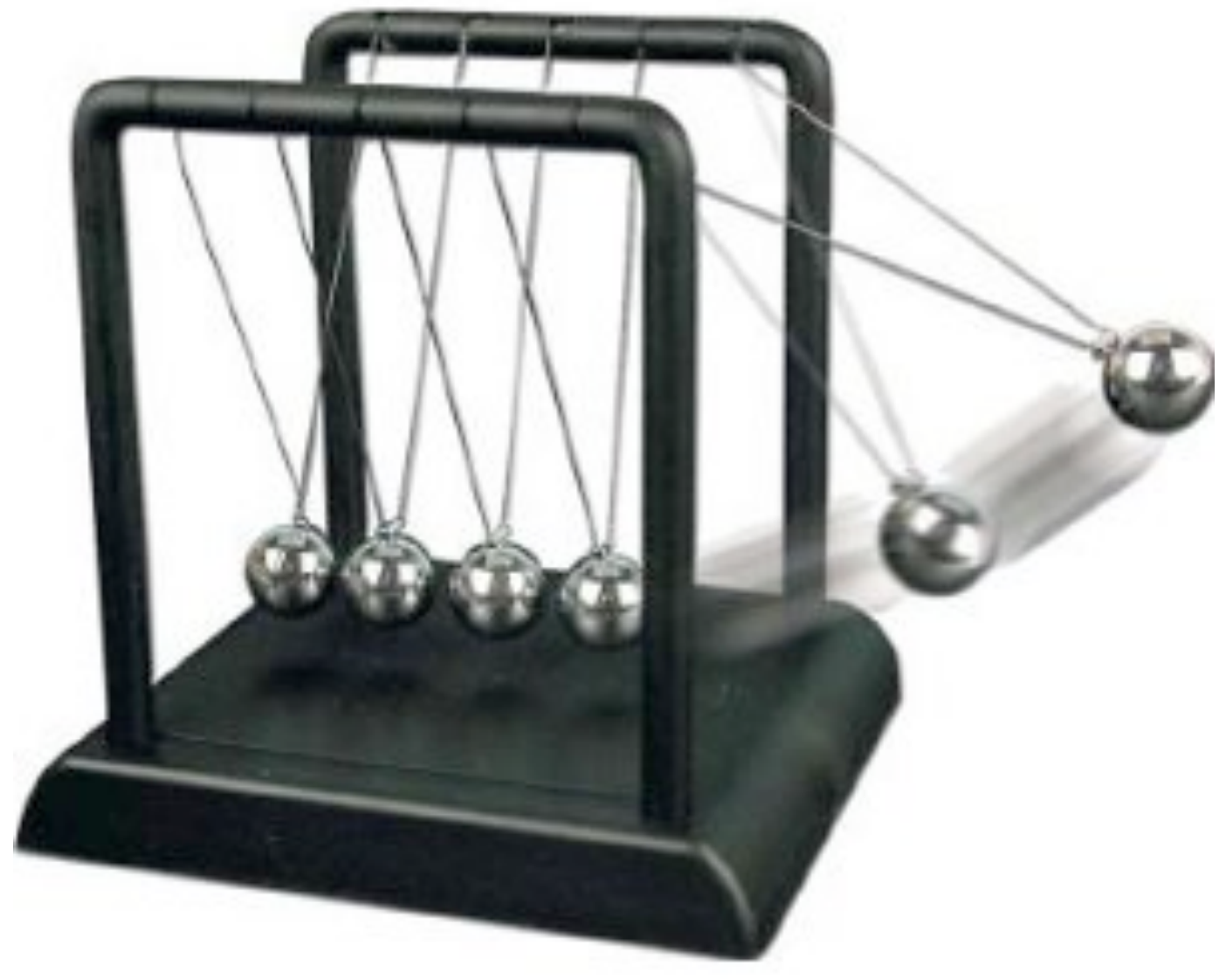

Figure 7.6.: Newton's cradle 

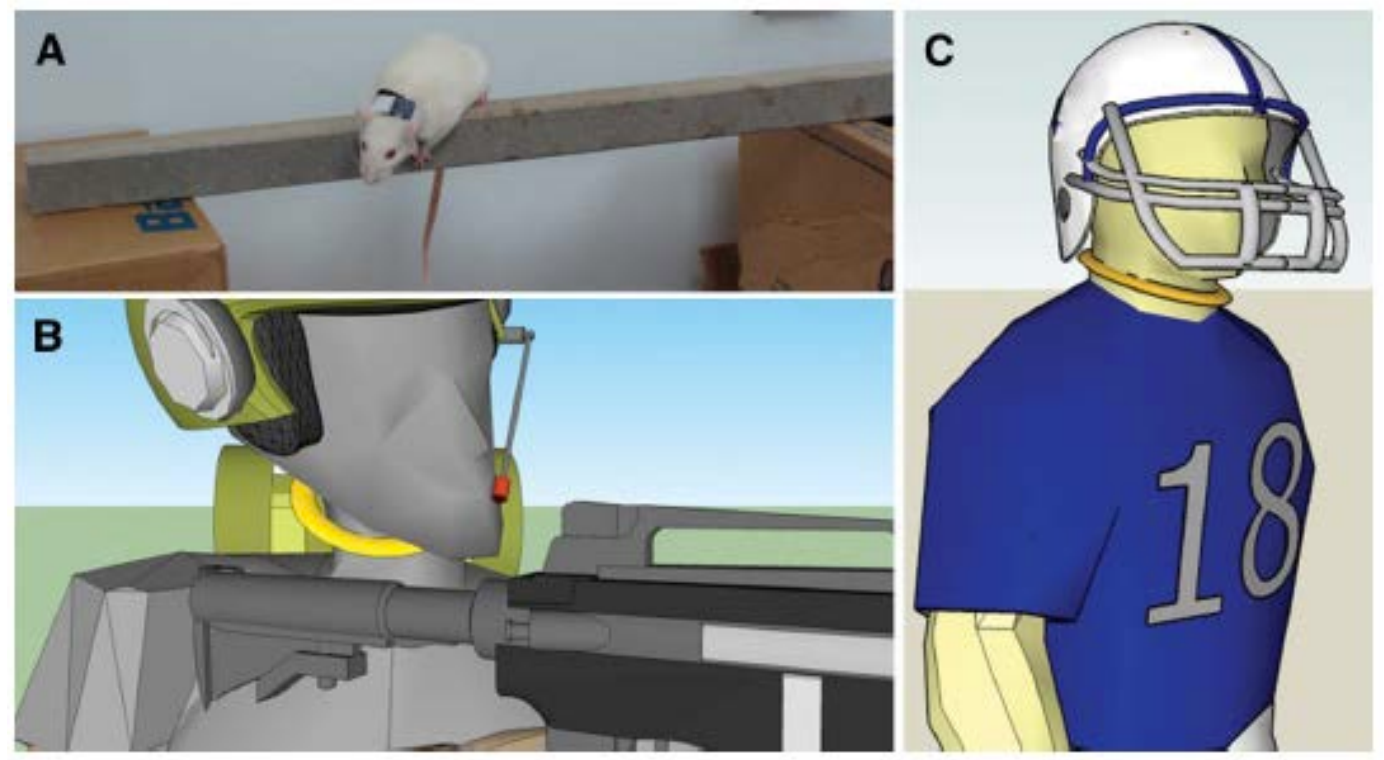

Figure 7.7.: IJV Compression Device (A) Actual device used in this study applied to a rat traversing a blance beam. No observable changes in behavior were noted following collar application with the rat. (B \& C) Artistic rendering of a comparable device applied to a soldier on the battlefield and an athlete on the football field, respectively. 


\section{Chapter Eight}

In this work, we build upon prior observations of reduced axonal injury associated with internal jugular vein compression (Chapter Seven) by exploring the effect on neural degeneration and glial activation. We also explore additional brain regions such as the cortex, striatum, hippocampus, and cerebellum, whereas prior work focused only on the corticospinal tracts.

\section{Effect of Slosh Mitigation on Histologic Markers of Traumatic Brain Injury}

This work was published in Journal of Neurosurgery.

Citation: Turner RC, Naser ZJ, Bailes JE, Smith DW, Fisher JA, Rosen CL (2012) Effect of slosh mitigation on histologic markers of traumatic brain injury. Journal of Neurosurgery 117: 1110-1118.

\section{ABSTRACT \\ Background. Helmets successfully prevent most cranial fracture and skull trauma but TBI and concussion continue to occur with frightening frequency, despite the widespread use of helmets on the athletic and battlefield. Protection against such injury is needed.}

Objective. To determine if slosh mitigation reduces neural degeneration, gliosis, and neuroinflammation.

Methods. Two groups of 10 adult male Sprague-Dawley rats underwent an impactacceleration traumatic brain injury. To one group a collar, inducing internal jugular vein (IJV) compression, was applied prior to injury whereas the second group received no manipulation prior to injury. All rats were sacrificed 7 days post-injury and the brains fixed and paraffin-embedded. Sections were processed and stained for markers of neural degeneration (Fluoro-Jade B), gliosis (Glial Fibrillary Acidic Protein), and neuroinflammation (Ionized Calcium Binding Adapter Molecule 1).

Results. Compared to control animals, animals undergoing IJV compression resulted in a reduction of degenerative neurons by $48.7-59.1 \%$, reactive astrocytes by $36.8-45.7 \%$, and microglial activation by $44.1-65.3 \%$.

Conclusion. We conclude that IJV compression, a form of slosh mitigation, markedly reduces markers of neurologic injury in a common model of TBI. We believe slosh mitigation may have potential for preventing TBI in the clinical population based on these findings and those of previous studies. 


\subsection{INTRODUCTION}

Traumatic brain injury (TBI) remains an immense public health problem and a leading cause of morbidity and mortality in those under 45 years old in the United States and industrialized countries ${ }^{590}$. Treatment options for TBI remain relatively limited in scope, emphasizing control of edema and intracranial pressure (ICP) and subsequent rehabilitation. While numerous therapeutic agents such as progesterone ${ }^{581,591-593}$ and DHA $579,580,585$ have been found to be neuroprotective in preclinical models, and some are presently in clinical trials, few alternatives presently exist for the clinical management of TBI. Furthermore, it is unlikely any treatment aimed at injury reversal will be as effective as injury prevention.

Efforts aimed at preventing TBI have continued to focus on extra-cranial protection in the form of helmets and improved helmet technology such as innovative shapes, shell materials, and padding. Emphasis on helmet technology may not be ideal due to limitations of helmets in preventing the acceleration-deceleration forces causing axonal injury and subsequent concussion. Drawing on lessons from nature, such as the woodpecker, may provide insight into protective mechanisms from repetitive TBI.

The woodpecker anatomy is notable for a multitude of reasons ranging from a small subdural space to a unique tongue structure ${ }^{594-596}$. The tongue is notable in that it extends posteriorly from the base of the mouth, around the neck, over the occiput, and into the right nostril ${ }^{594}$. This arrangement, in which a muscular sling is formed in the neck, has been identified as a possible shock absorber by limiting motion in the neck ${ }^{594}$. In fact, the long tongue and hyoid bone experience significant stress during collision, as evident in biomechanical studies ${ }^{595}$. The hyoid bone has been described by Wang, et al as playing the role of a safety belt for the head of the woodpecker ${ }^{595}$.

While human anatomy clearly differs from that of the woodpecker, some correlates do exist in preventing TBI. For example, professional boxers are capable of sustaining forces of large magnitude when prepared for the impact but suffer deleterious consequences when the blow is unexpected. Much of this has been attributed to a tightening of the neck muscles, one of which is the omohyoid muscle. The omohyoid is unique in that it consists of two muscle bellies joined by a tendon and closely overlies and crosses the internal jugular vein (IJV), a rare anatomic relationship. Notably, contraction of the omohyoid muscle upon yawning results in IJV compression and subsequent changes to intracerebral venous hemodynamics ${ }^{597}$. Therefore, the contraction of the omohyoid muscle, as well as other muscles in the neck, may serve a protective effect in humans through stabilization of the neck, much like the tongue/hyoid bone in the woodpecker. Furthermore, contraction of the omohyoid alters intracerebral hemodynamics, which may serve as a protective mechanism by increasing intracranial volume and reducing intracranial movement, minimizing the collisions experienced by the brain within the skull.

As such, Smith et al postulated that a reduction in intracranial compliance via increased intra-cerebral volume would result in reduced brain injury ${ }^{598}$. This theory, based on the concept of slosh, has been thoroughly investigated in other applications, such as rocket propellants, by groups such as NASA. Ibrahim et al describe slosh as the motion of a given liquids' free surface within a container ${ }^{599}$. Upon colliding with the surface container, energy transfer occurs amongst the involved surfaces ${ }^{599}$. Notably, fluid density must be considered when calculating energy dissipation and motion as it relates to slosh. In the previous work by Smith, et al, intracranial slosh was believed to be 
prevented through IJV compression, thought to result in increased intracranial vascular volume as evident based on correlative physiologic measures such as intracranial and intraocular pressure. Consequently, reduced motion of the cranial contents was believed to occur during trauma, reducing measures of axonal injury by $83 \%{ }^{598}$.

While axonal injury was markedly reduced via internal jugular vein (IJV) compression, presumably due to a reduction in associated slosh mitigation, it was previously unclear to what extent IJV compression alters the response of glial cells to neurologic injury following TBI. Glial cells are likely to influence outcome after TBI significantly as it is well known that astrocytes, oligodendrocytes, and microglia are known to outnumber neurons in humans, and play a clear role in maintaining homeostasis and normal function within the brain 600,601 .

\subsection{MATERIALS AND METHODS}

Tissue samples were obtained from the animals studied by Smith et al. ${ }^{598}$ All procedures involving live animals were approved by the Institutional Animal Care and Use Committee of West Virginia University, and were performed according to the principles of the Guide for the Care and Use of Laboratory Animals, published by the Institute of Laboratory Resources, National Research Council (National Institutes of Health publication 85-23-2985).

\subsubsection{Experimental Protocol}

In brief, twenty male Sprague Dawley rats weighing between 350 and $400 \mathrm{~g}$ were tested. Anesthesia was induced and maintained with isoflurane and temperature regulated with a homeothermic heating blanket. The experimental injury group (10 rats) were fitted with a $15 \mathrm{~mm}$ wide collar, with two compressive beads designed to overlay the IJVs and was tightened sufficiently to produce mild compression of the IJVs without compromising the airway. Increases in intraocular and intracranial pressure confirmed IJV compression 598 . The remaining rats were the control group. The collar was left in position for three minutes prior to administrating experimental brain injury. All animals were subjected to a standardized acceleration-deceleration head injury model in which a 450-gram weight was dropped from a height of 2 meters, striking a brass disk previously surgically affixed to the skull 562,602 .

\subsubsection{Tissue Preparation for Histology and Immunohistochemistry}

At 7 days post-injury all animals $(n=20)$ were anesthetized and immediately perfused transcardially with $200 \mathrm{ml}$ cold $0.9 \%$ saline. This was followed by $4 \%$ paraformaldehyde infusion in Millonigs buffer for 40 minutes. The entire brain, brainstem, and rostral spinal cord were removed and immediately placed in $4 \%$ paraformaldehyde for 24 hours. Following 24 hours fixation, the brain was blocked into $2 \mathrm{~mm}$ thick sections. The first section, from the forebrain, was taken from 1.72-3.72 $\mathrm{mm}$ from bregma. The second section, from the cerebellum, was taken from $9.12-11.12 \mathrm{~mm}$ from bregma. The resulting tissue was then processed using the Tissue Tek-VIP 5 Automatic Tissue Processor (Sakura Finatek; Torrence, California) and embedded into paraffin using a Tissue-Tek Tec 5: Tissue Embedding Console system (Sakura Finatek; Torrence, California). These embedded tissues were then sliced at a thickness of $6 \mu \mathrm{m}$ using a Leica RM2235 microtome (Leica Microscopes; Buffalo Grove, Illinois) and the slices were mounted on glass slides for staining. All slides were heat-fixed and de-paraffinized via a series of xylene and alcohol washes. 


\subsubsection{Cresyl Violet}

For cresyl violet staining, the tissue was incubated in $0.1 \%$ cresyl violet solution for 10 minutes. Following the incubation, the tissue was rinsed in $\mathrm{dH}_{2} \mathrm{O}$ and differentiated in $95 \%$ ethyl alcohol for 30 seconds. Following the differentiation, the tissue was washed 2 times in xylene and then mounted using an antifade agent. The slides were then cover slipped, sealed with acrylic, and stored in the dark in a laboratory refrigerator.

\subsubsection{Hematoxylin \& Eosin (H\&E)}

Tissue was incubated in hematoxylin (Gill's 1X) for 5 minutes prior to rinsing in deionized water until water is clear (approximately 5 minutes). Sections were then dunked 2-3 times in acid alcohol $(1 \% \mathrm{HCl}$ in $70 \% \mathrm{EtOH})$. The sections should be pink following this step. Sections were then rinsed for 5 minutes prior to placement in ammonia water (1 $\mathrm{mL} \mathrm{NH}{ }_{4} \mathrm{OH}$ in $1 \mathrm{~L} \mathrm{H}_{2} \mathrm{O}$ ), resulting in sections darkening significantly. The tissue was then rinsed for 5 minutes in deionized water and then placed in Eosin $Y$ for 1 minute prior to final rinses and cover-slipping.

\subsubsection{Fluoro-Jade B (FJB)}

For FJB labeling, the tissue was incubated in $0.06 \%$ potassium permanganate for 10 minutes after rehydrating with a series of alcohol and deionized water rinses. Slides were washed for 2 minutes in deionized water prior to incubation for 20 minutes in $0.0004 \%$ FJB in $0.1 \%$ acetic acid. Following incubation in the antibody, the tissue was washed 3 times in $\mathrm{dH}_{2} \mathrm{O}$ and then mounted using an antifade agent. The slides were then cover slipped, sealed with acrylic, and stored in the dark in a laboratory refrigerator.

\subsubsection{Glial Fibrillary Acidic Protein (GFAP)}

For GFAP labeling, the tissue was incubated in polyclonal antibody raised in rabbit against anti-cow GFAP (Dako, Glostrup, Denmark) at a dilution of 1:500 in 4\% horse serum in DPBS overnight. Following the incubation in primary antibody, the tissue was washed 3 times in DPBS and then incubated in a secondary Biotinylated anti-rabbit IgG antibody (Vector Laboratories, Burlingame, CA) diluted at 1:10000 in 4\% horse serum in DPBS for 4 hours. Following the incubation in the secondary antibody, the tissue was then incubated in Avidin D-HRP (Vector Laboratories, Burlingame, CA) diluted at 1:1000 in DPB for 1 hour. Following the incubation in the Avidin D-HRP, the tissue was rinsed 3 times in DPBS and then Chromagen solution DAB (Vector Laboratories, Burlingame, CA) for 5 minutes. The tissue was then rinsed 3 times in DPBS and left out to dry overnight. The tissue underwent a final wash in xylene and then was mounted using an antifade agent and cover slipped. The slides were sealed with acrylic and stored in the dark in a laboratory refrigerator.

\subsubsection{Ionized Calcium Binding Adapter Molecule (Iba-1)}

For lba-1 labeling, the tissue was processed identically to GFAP with the following exceptions: the primary antibody was rabbit anti-lba 1 (Wako Chemicals USA,;Richmond, Virginia) at a dilution of 1:500 and the chromagen solution was Nova Red (Vector Laboratories; Burlingame, California).

\subsubsection{Stereological Quantification}

A stereological method was used to determine an unbiased estimate of the number of each staining marker per cubic $\mathrm{mm}$ in the hippocampus, striatum, cortex and cerebellum 
areas. The optical fractionator technique utilizing a Stereolnvestigator 9.0 (MBF Bioscience, Inc.; Williston, Vermont) and a Olympus AX70 microscope with 4x-100x objectives was performed. Specimens were examined with low magnification and regions of interest were drawn. The software then selected random 75 micron counting frames with depth of 6 microns, and the object of interest was marked. The volume of the region of interest $(\mathrm{ROI})$ was determined using the Cavalieri method, the volume of the sum of the counting frames was calculated, the sum total of the object of interest within the counting frames was calculated, and an estimate of the number per cubic $\mathrm{mm}$ was calculated. All microscopy was performed by an observer blinded to the experimental treatment.

\subsubsection{Statistics}

Data from all experiments was analyzed using GraphPad Prism 4.0 (San Diego, California). An unpaired $t$ test was used to compare between control animals and those undergoing IJV compression for FJB-positive neurons, GFAP-positive astrocytes, and Iba-1-positive microglia. $\mathrm{P}<0.05$ was considered statistically significant for all data analyzed.

\subsection{RESULTS}

\subsubsection{Histopathology}

At one week after TBI, no gross or histopathological abnormalities were seen in either experimental group (IJV compression or control). Cresyl violet (Figure 8.1) and hematoxylin and eosin (Figure 8.2) staining revealed no contusions or cortical cell loss underlying the impact site, no hippocampal cell loss, and no noticeable difference in striatal or cerebellum integrity.

\subsubsection{Neural Degeneration}

Fluoro-Jade B, a marker of neural degeneration, was utilized to visualize and quantify degenerating neurons. A difference between control animals and those undergoing IJV compression was grossly apparent in all tissue regions visualized (Figure 8.3) and was confirmed with stereologic quantification (Figure 8.4). Statistical analysis comparing control animals to those undergoing IJV compression showed a significant difference in cortex $(t=8.522, p<0.0001)$, hippocampus $(t=8.835, p<0.0001)$, striatum $(t=9.439$, $\mathrm{p}<0.0001)$, and cerebellum $(\mathrm{t}=6.302, \mathrm{p}<0.0001)$.

\subsubsection{Glial Proliferation}

Astrocyte reactivity has been previously described as both a precursor to neural injury as well as a marker of neural repair. At one week after traumatic brain injury, control animals exhibited increased numbers of reactive astrocytes, at each location assessed using GFAP as a marker, in comparison to animals receiving TBI with IJV compression and slosh mitigation (Figures 8.5-8.6). Statistical analysis comparing control animals to those undergoing IJV compression showed a significant difference in cortex $(\mathrm{t}=8.201$, $\mathrm{p}<0.0001)$, hippocampus $(\mathrm{t}=9.334, \mathrm{p}<0.0001)$, striatum $(\mathrm{t}=8.296, \mathrm{p}<0.0001)$, and cerebellum $(t=8.600, p<0.0001)$.

\subsubsection{Inflammation \& Immune Reactivity}

Microglial activation following traumatic brain injury was assessed using lonized Calcium Binding Adapter Molecule 1 (lba-1) as a marker of microglia. Microglia morphology 
varies between resting and activated microglia, with the later exhibiting hypertrophic, bushy, and ameboid morphology. At one-week post-TBI, slosh mitigation significantly reduced the number of activated microglia at all locations measured (Figures 8.7-8.8). Statistical analysis comparing control animals to those undergoing IJV compression showed a significant difference in cortex $(t=12.60, p<0.0001)$, hippocampus $(t=9.176$, $p<0.0001)$, striatum ( $t=9.133, p<0.0001)$, and cerebellum ( $t=8.979, p<0.0001)$.

\subsection{DISCUSSION}

In this study we confirmed the protective effect of IJV compression, which we believe leads to slosh mitigation ${ }^{598}$, on neuronal damage by using an alternative marker of neural degeneration, Fluoro-Jade $B{ }^{603}$ which has previously been shown to be elevated in numerous TBI models ${ }^{604-608}$. We further corroborated these findings by showing an associated marked reduction in post-traumatic glial activation in the experimental group. Degenerating neurons were visualized by Fluoro-Jade $B$ and were reduced between 48.7-59.1\% depending on location within the brain. Similarly, reactive astrocytes, which were measured using GFAP, were reduced between 36.8-45.7\%. Microglial activation, indicated by lba-1 staining, was reduced $44.1-65.3 \%$. Notably, activated microglia were seen throughout the brain in a diffuse nature, consistent with findings in 'focal' injury models such as 'controlled-cortical impact' ${ }^{606}$.

Astrocytes, the most abundant type of glial cell, are essential in maintaining homeostasis in the healthy brain and also play a critical role in response to injury. Astrocytes have previously been shown to reduce neuronal death in co-culture models of ischemia when compared to neuron-only cultures ${ }^{609}$. The notion of astrocytes facilitating neuronal survival has been furthered by the observation that loss of astrocytes following TBI precedes neuronal degeneration ${ }^{608}$. It is believed that astrogliosis, which is usually initiated shortly after CNS injury, is involved in the process of restoring CNS homeostasis 610. However, in excess, it may result in glial scarring which may impede neural repair and contribute to axonal degeneration ${ }^{611 .}$

Similarly, microglia are capable of secreting both neurotrophic as well as neurodegenerative factors ${ }^{612-614}$. While it is unclear whether or not microglial activation precedes or is a consequence of axonal injury, it is known to occur in both the acute and chronic phases following TBI, and is therefore a suitable marker for neurologic injury ${ }^{613}$.

\subsection{CONCLUSION}

Our findings suggest that IJV compression, and associated slosh mitigation, may be a powerful technique for reducing TBI. This technique reduces not only axonal injury as previously reported, but also the number of degenerating neurons, reactive astrocytes, and activated microglia. While axonal injury is a well-documented pathological finding after TBI, and one for which current preventative techniques are inadequate, injury to neurons and associated axons hardly exists in isolation. Rather than taking a neuroncentered approach to TBI prevention and treatment, it seems likely that the neuron-glial interaction must be considered. Microglia and astrocytes are intimately involved in not only the initial injury process and subsequent inflammation, but also the long-term repair and recovery that occurs. We believe that IJV compression, and presumably slosh mitigation, offers a novel form of TBI prevention and addresses the intracranial environment more effectively than extracranial protective devices such as helmets. IJV compression likely leads to not only increased retention of blood in the cranium but also may alter the density gradients amongst structures, reducing energy displacement in 
brain tissue based on the concept of slosh in fluid dynamics. More research is needed to verify that our initial findings may translate to clinical applications and be of benefit based on various TBI outcome measures used clinically.

\section{ACKNOWLEDGEMENTS}

The authors would like to gratefully acknowledge the support of Ms. Penny Humberson as lab manager and Dr. James O'Callaghan and Dr. Diane Miller of the National Institute of Occupational Safety and Health (NIOSH) for provision of reagents and supplies. The technical support of Ms. Diana Richardson of NIOSH was essential in preparation of the tissues for analysis. We would also like to thank Dr. Karen Martin for technical expertise and the West Virginia University Imaging Facility, which is supported in part by the Mary Babb Randolph Cancer Center and NIH grant P20 RR016440. This work was funded by West Virginia University and the Department of Neurosurgery. Additional financial support was provided by the National Institutes of Health (NIH 5 T32 GM 81741 to Ryan C. Turner). 


\section{Figures}

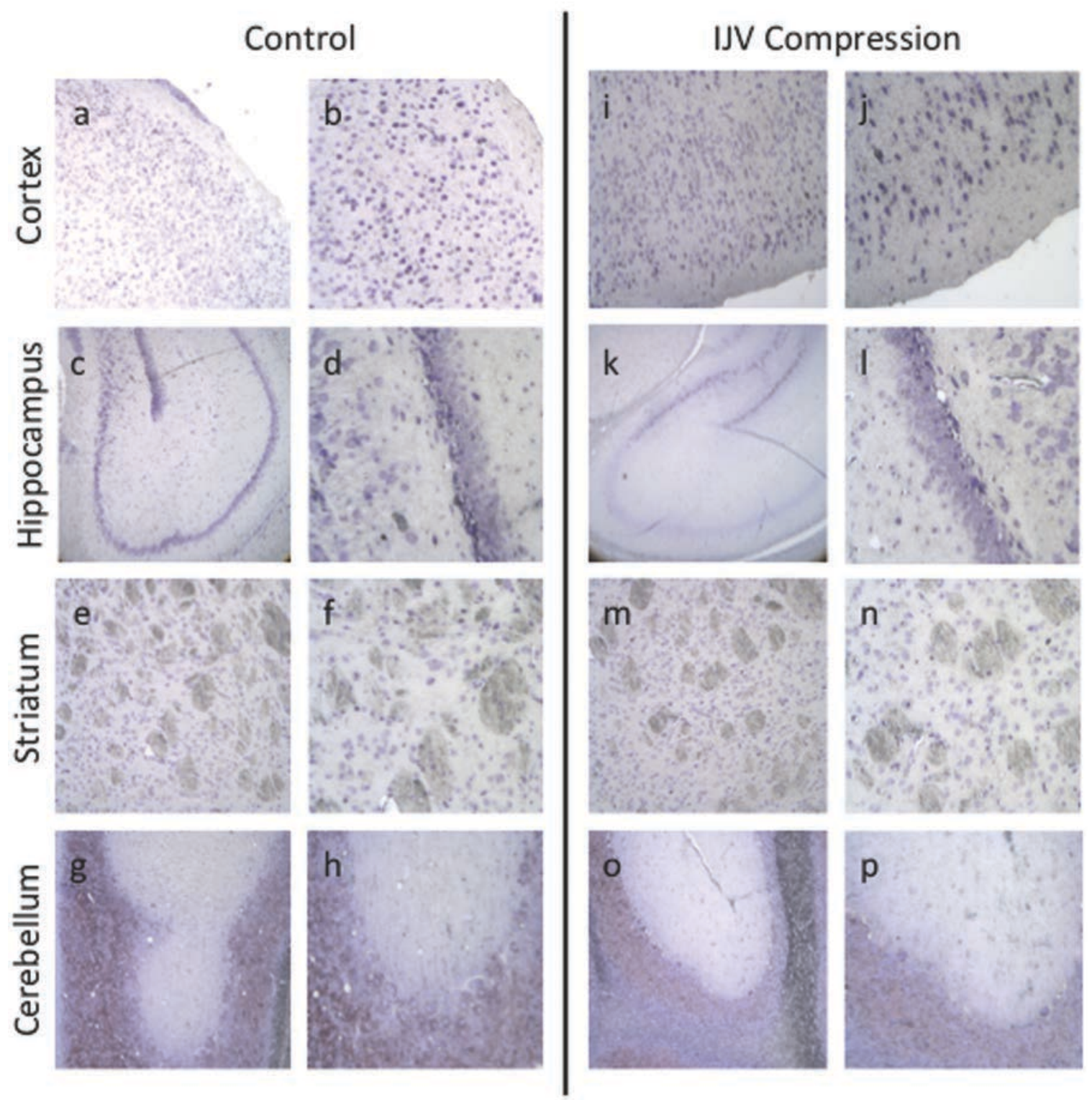

Figure 8.1. Cresyl violet staining illustrated no abnormalities following TBI in control animals (panels a-h) or those undergoing IJV compression (panels $\mathrm{i}-\mathrm{p})$. Low-power view of cortex (a,i), hippocampus $(c, k)$, striatum $(e, m)$, and cerebellum $(g, o)$ in control and IJV compression animals, respectively. High-power view of cortex $(b, j)$, hippocampus $(k, l)$, striatum $(m, n)$, and cerebellum $(o, p)$ in control and IJV compression animals, respectively. 


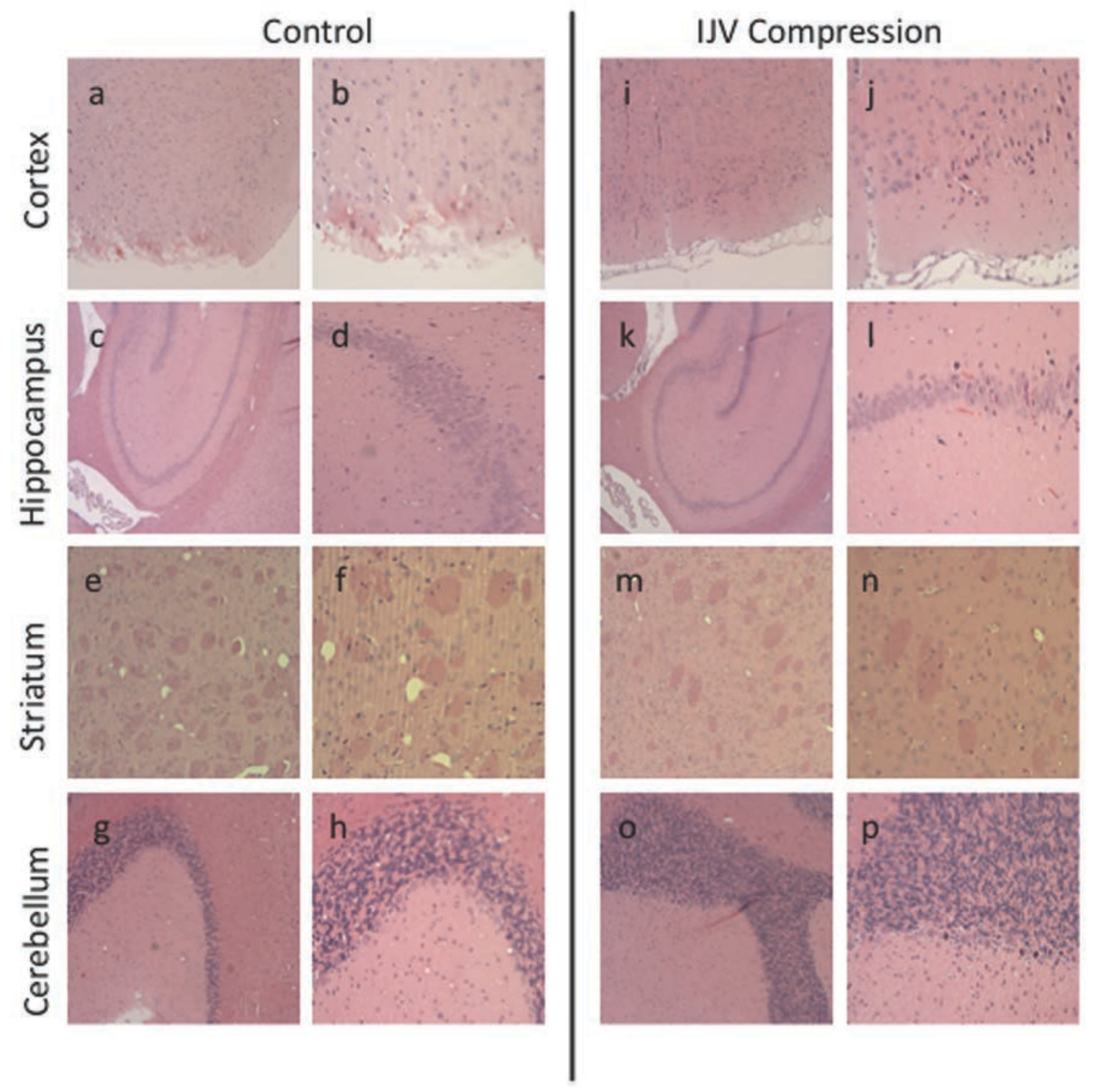

Figure 8.2. Hematoxylin and eosin staining showed no abnormalities following TBI in control animals (panels $a-h)$ or those undergoing IJV compression (panels $i-p)$. Lowpower view of cortex $(a, i)$, hippocampus $(c, k)$, striatum $(e, m)$, and cerebellum $(g, 0)$ in control and IJV compression animals, respectively. High-power view of cortex $(b, j)$, hippocampus $(k, I)$, striatum $(m, n)$, and cerebellum $(0, p)$ in control and IJV compression animals, respectively. 


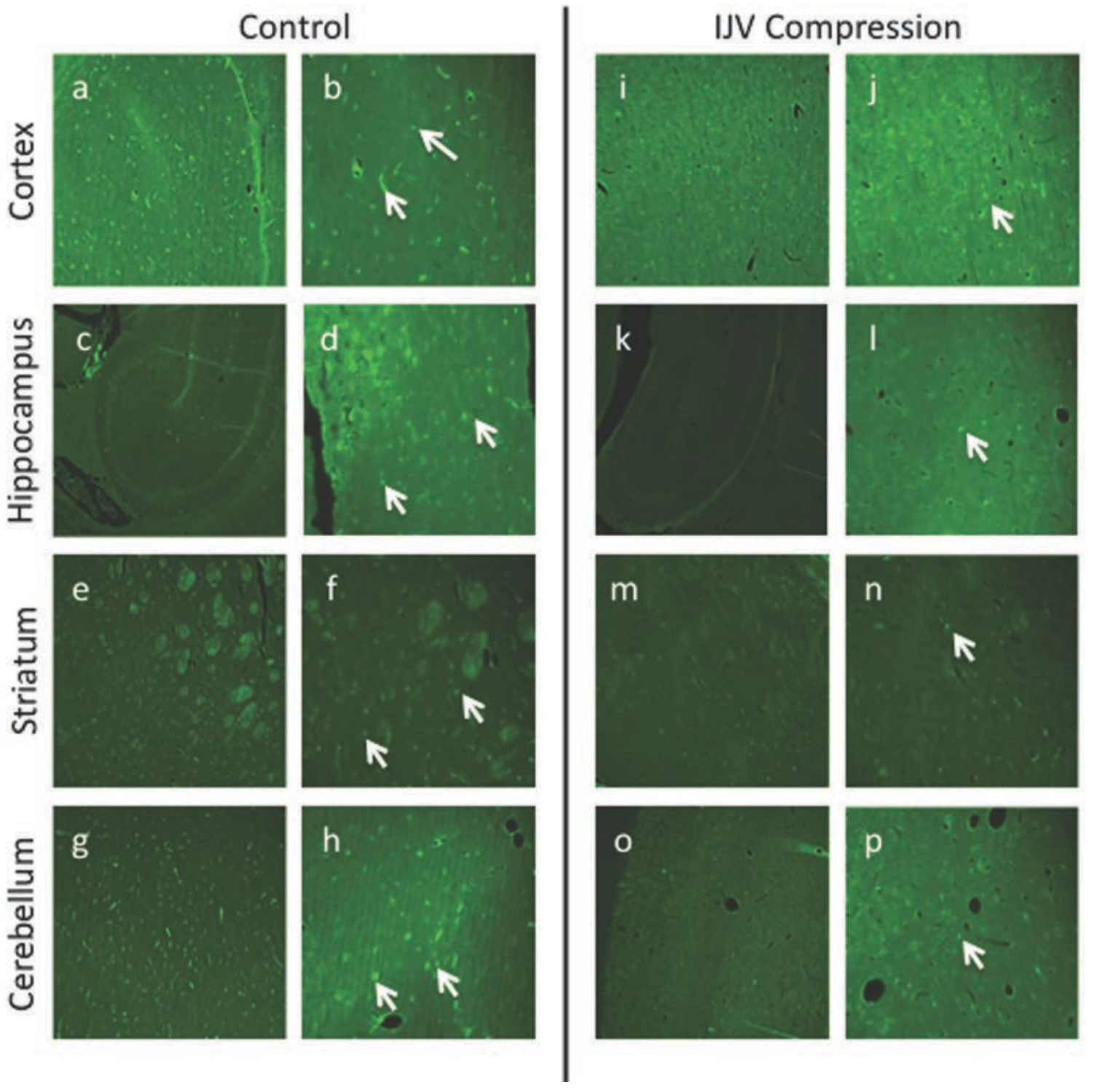

Figure 8.3. Fluoro-Jade B staining showed a significant reduction in degenerating neurons in animals undergoing IJV compression (panels i-p) compared to control animals (panels $a-h)$. Low-power view of cortex $(a, i)$, hippocampus $(c, k)$, striatum $(e, m)$, and cerebellum $(\mathrm{g}, \mathrm{o})$ in control and IJV compression animals, respectively. High-power view of cortex $(b, j)$, hippocampus $(k, I)$, striatum $(m, n)$, and cerebellum $(o, p)$ in control and IJV compression animals, respectively. Arrows represent degenerating neurons. 


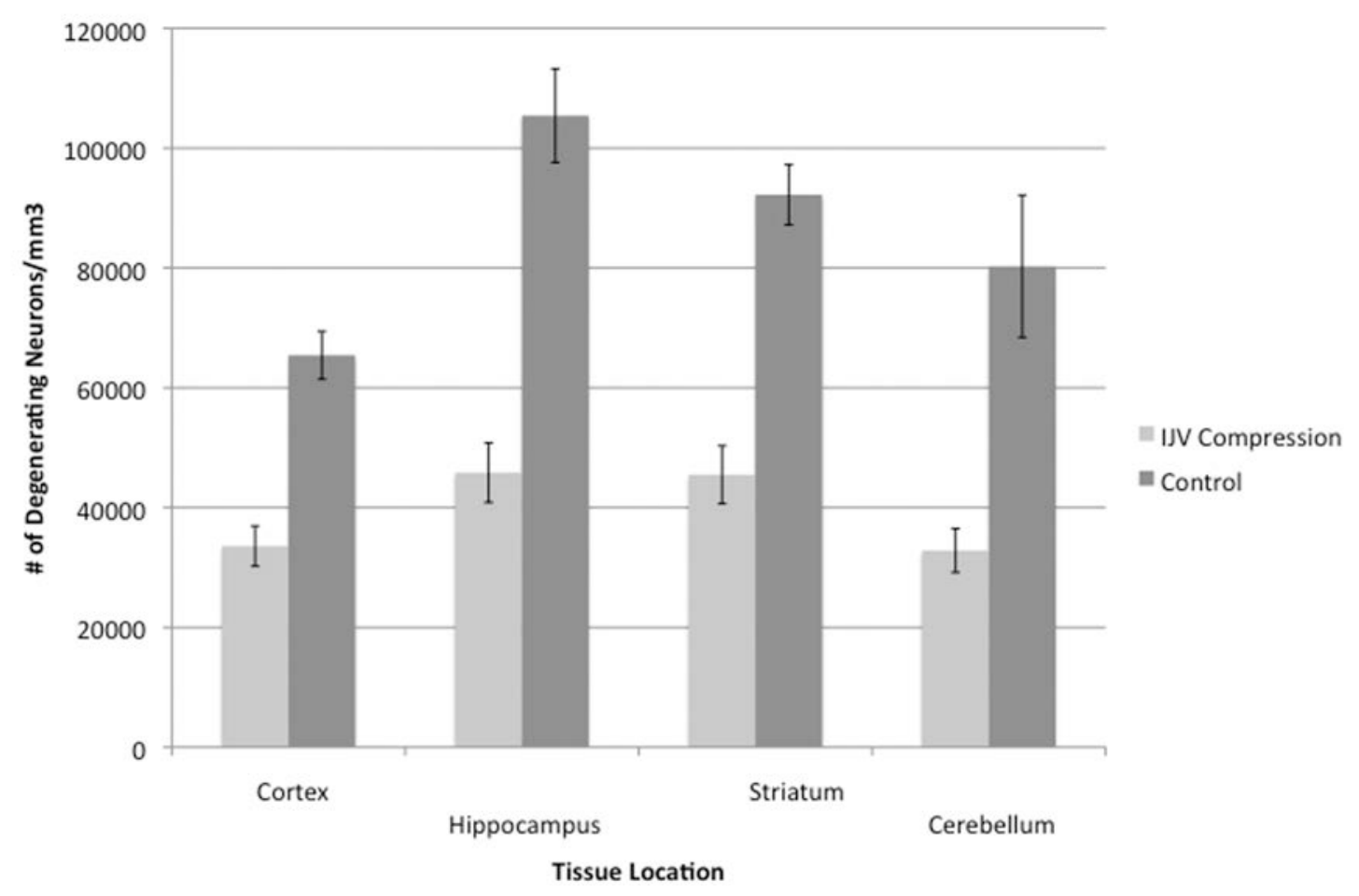

Figure 8.4. Stereologic quantification of degenerating neurons stained with Fluoro-Jade B. A significant reduction in neural degeneration was seen in IJV compression animals at all tissue locations visualized and quantified $(p<0.0001)$. 


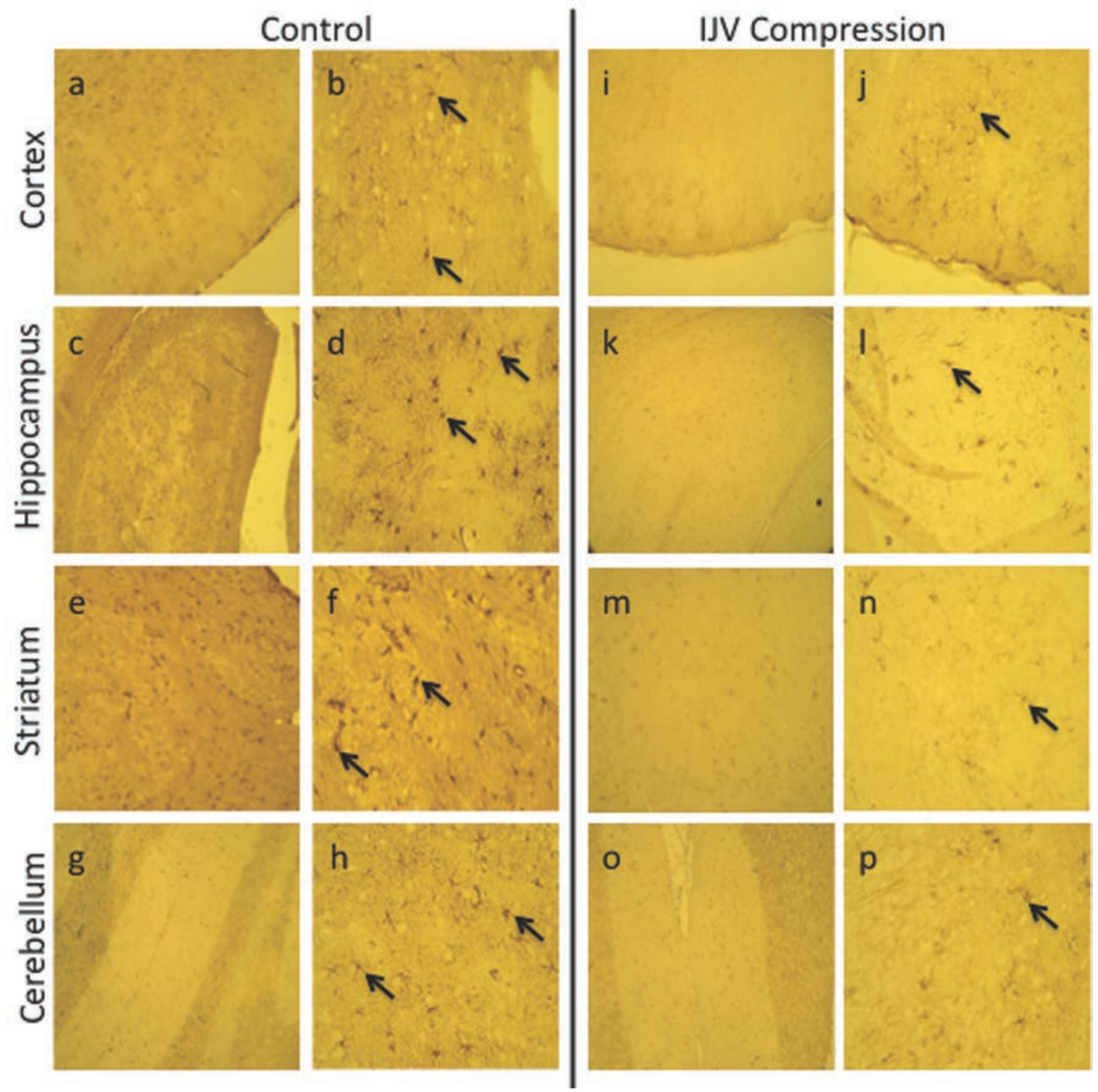

Figure 8.5. GFAP staining showed a significant reduction in reactive astrocytes in animals undergoing IJV compression (panels i-p) compared to control animals (panels ah). Low-power view of cortex $(a, i)$, hippocampus $(c, k)$, striatum $(e, m)$, and cerebellum $(\mathrm{g}, \mathrm{o})$ in control and IJV compression animals, respectively. High-power view of cortex $(\mathrm{b}, \mathrm{j})$, hippocampus $(\mathrm{k}, \mathrm{l})$, striatum $(\mathrm{m}, \mathrm{n})$, and cerebellum $(\mathrm{o}, \mathrm{p})$ in control and IJV compression animals, respectively. Arrows represent reactive astrocytes. 


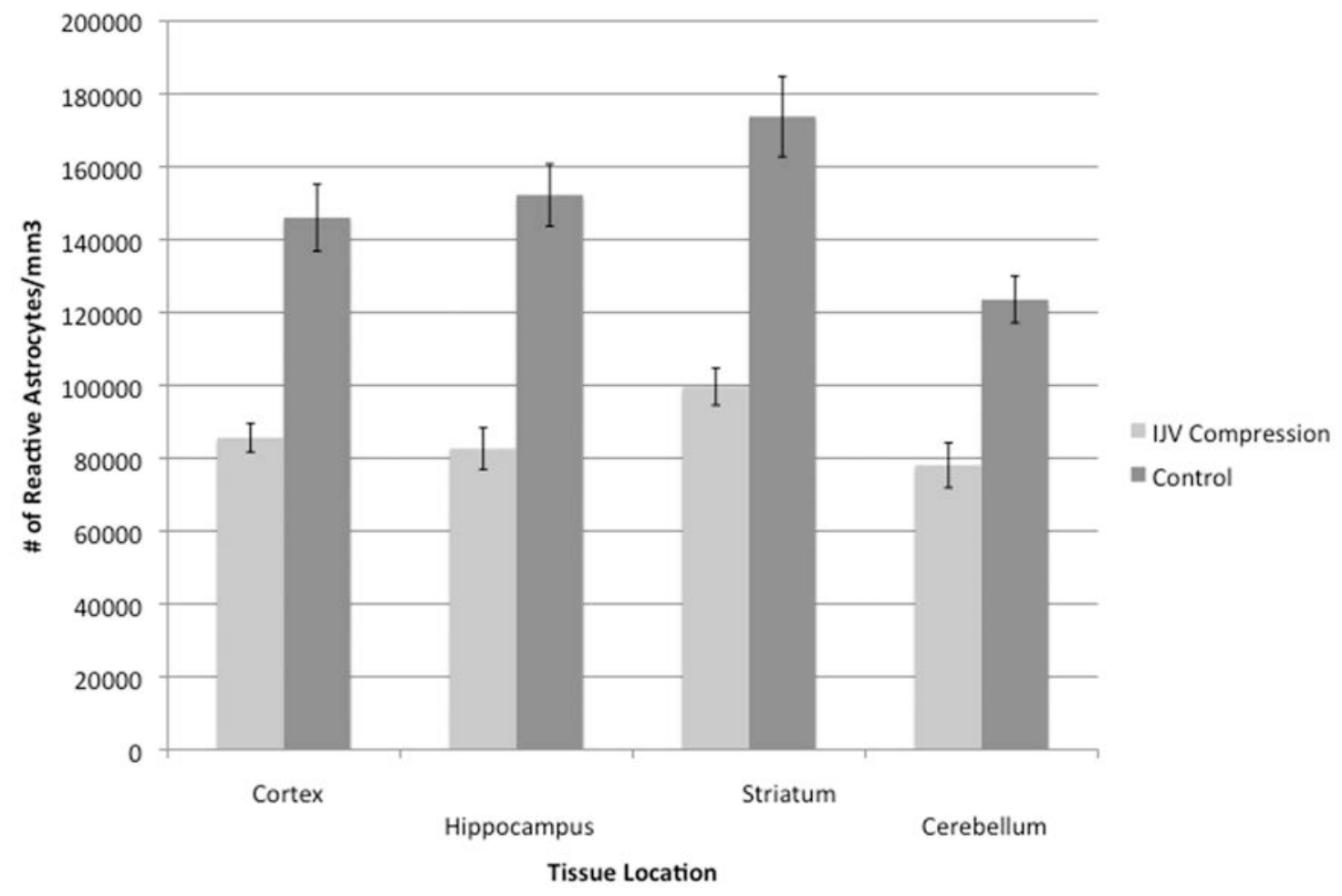

Figure 8.6. Stereologic quantification of reactive astrocytes stained with GFAP. A significant reduction in reactive astrocytes was seen in IJV compression animals at all tissue locations visualized and quantified $(p<0.0001)$. 


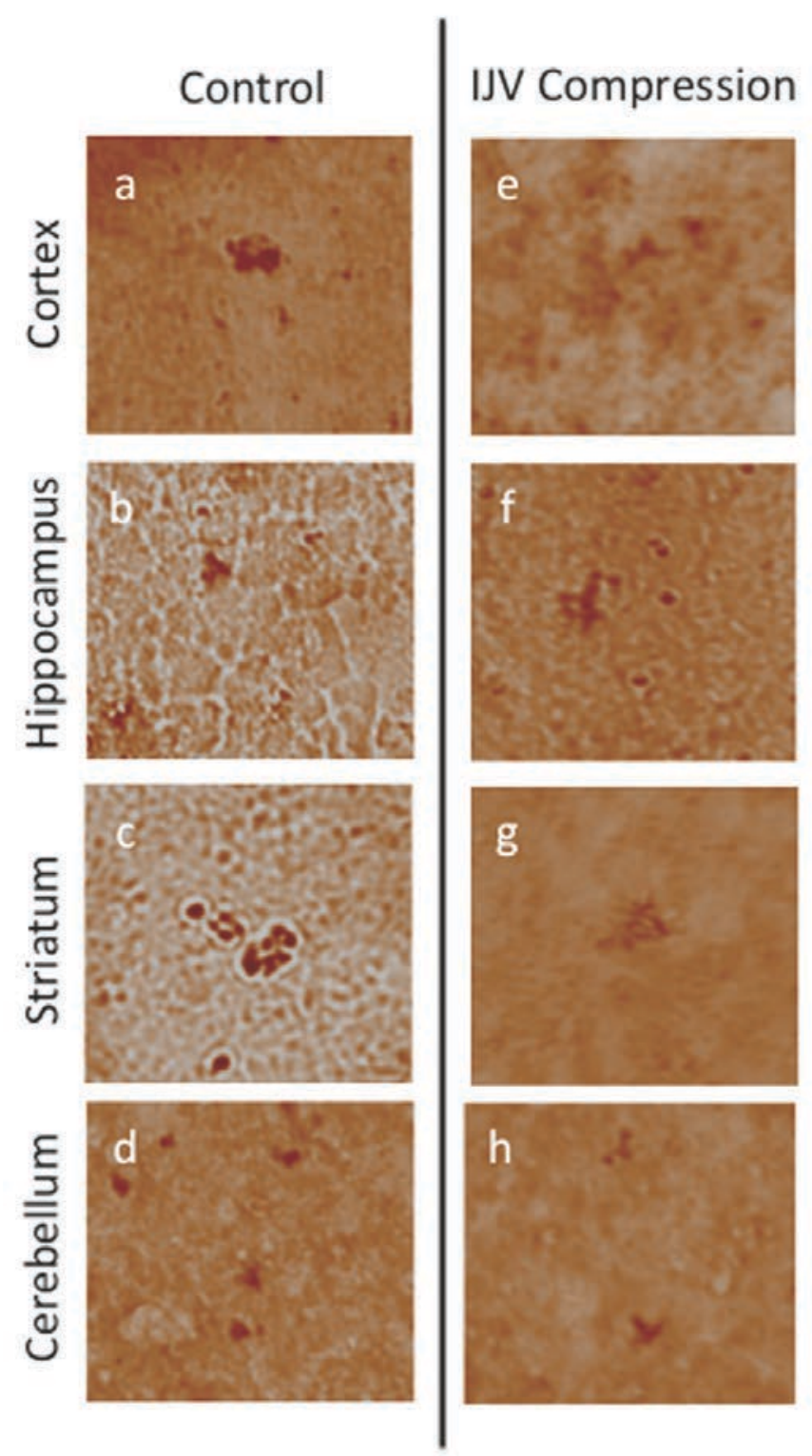

Figure 8.7. $\quad$ lba-1 staining showed a significant reduction in activated microglia in animals undergoing IJV compression (panels e-h) compared to control animals (panels $a-d)$. High-power view of cortex $(a, e)$, hippocampus $(b, f)$, striatum $(c, e)$, and cerebellum $(\mathrm{d}, \mathrm{h})$ in control and IJV compression animals, respectively. 


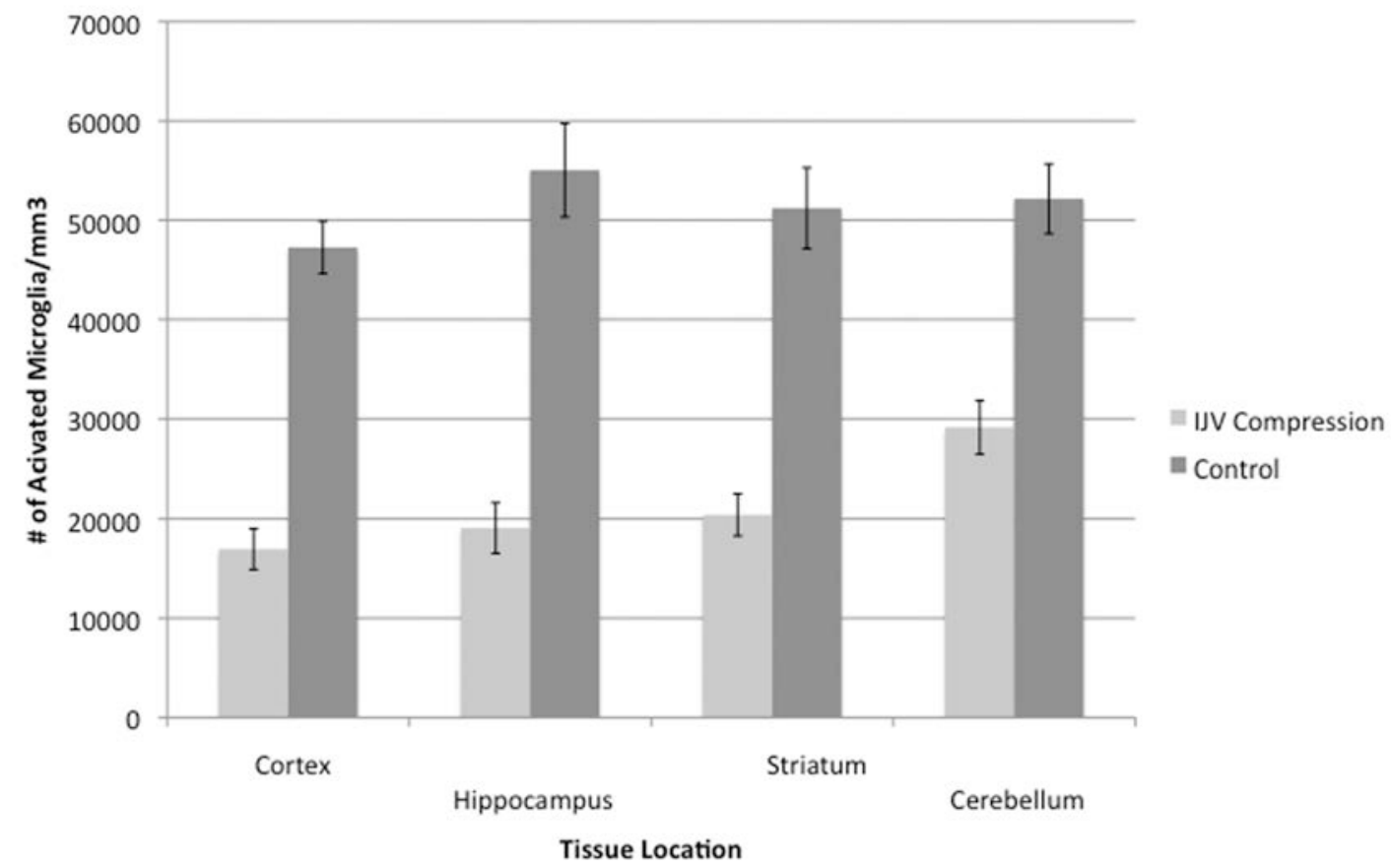

Figure 8.8. Stereologic quantification of activated microglia stained with lba-1. A significant reduction in activated microglia was seen in IJV compression animals at all tissue locations visualized and quantified $(p<0.0001)$. 


\title{
Chapter Nine
}

In addition to diffuse axonal injury following impact with another object, blast-induced neurotrauma has received increased attention due to involvement in military conflicts around the globe. Prior work modeling blast has failed to adequately scale the exposure for rodents based on pulmonary lethality curves ${ }^{406}$. In this work, we present a novel blast model capable of simulating relevant parameters based on scaling concepts, namely overpressure duration. We also show a graded response in many neural injury markers associated with the level of peak overpressure exposure. This work sets the stage for further investigation of blast injury pathophysiology and potential preventative or treatment measures.

\section{Modeling Clinically Relevant Blast Parameters Based on Scaling Principles Produces Functional \& Histological Deficits in Rats}

Ryan C. Turner, Zachary J. Naser, Aric F. Logsdon, Kenneth H. DiPasquale, Garrett J. Jackson, Matthew J. Robson, Robert T. Gettens, Rae R. Matsumoto, Jason D. Huber, Charles L. Rosen

This work is under consideration for publication in Experimental Neurology.

\begin{abstract}
Blast-induced traumatic brain injury represents a leading cause of injury in modern warfare with injury pathogenesis poorly understood. Preclinical models of blast injury remain poorly standardized across laboratories and the clinical relevance is unclear based upon pulmonary injury scaling laws. Models capable of high peak overpressures with short durations may better replicate clinical exposure when scaling principles are considered. In this work, we demonstrate a tabletop shock tube model capable of high peak overpressures of short duration. By varying the thickness of the polyester membrane, peak overpressure can be controlled. We used membranes with a thickness of $0.003,0.005,0.007$, and 0.010 inches to generate peak reflected overpressures of $31.47,50.72,72.05$, and $90.10 \mathrm{PSI}$, respectively. Blast exposure was shown to decrease total activity and produce neural degeneration as indicated by fluoro-jade B staining. Similarly, blast exposure resulted in increased glial activation as indicated by an increase in the number of glial fibrillary acidic protein expressing astrocytes compared to control within the corpus calllosum, the region of greatest apparent injury following blast exposure based on previous reports. Similar findings were observed with regards to activated microglia, some of which displayed phagocytic-like morphology within the corpus callosum following blast exposure, particularly with higher peak overpressures. Furthermore, hematoxylin and eosin staining showed the presence of red blood cells within the parenchyma and red, swollen neurons following blast injury. Exposure to blast with 90.10 PSI peak reflected overpressure resulted in immediate mortality associated with extensive intracranial bleeding. This work demonstrates one of the first examples of blast-induced brain injury in the rodent when exposed to a blast wave scaled from human exposure based on scaling principles derived from pulmonary injury lethality curves.
\end{abstract}




\subsection{INTRODUCTION}

Blast-induced traumatic brain injury has been described as the hallmark injury of modern warfare ${ }^{615,616}$ with $\sim 25,000$ members of the armed services (U.S. and Coalition forces) injured or killed by explosive devices in Iraq and Afghanistan conflicts in the past decade 617. The prevalence of blast exposure is associated with an immense financial and societal burden with estimates of $13-22 \%$ of combat veterans having sustained a traumatic brain injury (TBI) during time in service $406,618,619$. Consequently, there is a clear clinical need for increased understanding of blast injury pathogenesis and the development of improved therapeutics for the treatment, or prevention, of blast-induced brain injury. Increasing efforts to study blast injury has resulted in the propagation of numerous preclinical models of blast injury with the shock tube representing the most widely used model type. Despite the extensive use of shock tube models, model parameters such as size of the driver and driven sections, membrane material and thickness, and gas used seem to vary across laboratories. As such, the shock waves produced vary significantly in peak overpressure and duration.

Recent work by Panzer and colleagues has identified potential discrepancies between many preclinical models and real-world recorded blast parameters with models often exposing small rodents to long duration blasts ${ }^{406}$. Assuming the same scaling principles apply as those discovered with regards to pulmonary blast injury, many current rodent models, in which overpressure exposure durations frequently exceed $4 \mathrm{~ms}$ and in some cases as much as $10 \mathrm{~ms}$ or more, may be subjecting animals to the equivalent of a human exposed to detonation of $27,000-\mathrm{kg}$ of trinitrotoluene (TNT) explosive from over $70 \mathrm{~m}$ away ${ }^{406}$. In other words, many preclinical models may be exposing animals to the equivalent of long-duration nuclear blasts rather than the more common high explosive blasts ${ }^{407}$. Therefore, a preclinical blast model capable of delivering high peak overpressures over a short duration may be of value, particularly should similar scaling laws exist in the brain, as is the case in pulmonary-based work.

In this work, we demonstrate the ability to model blast injury with clinically relevant peak overpressure and duration based on scaling laws from humans to rodents with a novel, tabletop shock tube model. Furthermore, we show the ability to model blast injury of varying severity with different polyethylene terephthalate (PET) membranes that result in reflected peak overpressures ranging from approximately 30-90 PSI while consistently being of short duration $(<3 \mathrm{~ms}$ ). Animals exposed to blast-induced TBI across this range of pressures exhibit alterations in total activity as well as clear histological changes as determined utilizing common markers of neural injury.

\subsection{MATERIALS AND METHODS}

\subsubsection{Animals}

All procedures involving live animals were approved by the Institutional Animal Care and Use Committee of West Virginia University and were performed according to the principles of the Guide for the Care and Use of Laboratory Animals. This worked used thirty-eight 350 gram male Sprague-Dawley rats acquired from Hilltop Lab Animals (Hilltop Lab Animals, Inc.; Scottdale, Pennsylvania). Animals were acclimated for the week prior to experimental use and were housed under 12-hour light/12-hour dark conditions with food and water available ad libitum.

\subsubsection{Design of Blast Model}


A four-piece, machined aluminum shock tube apparatus was constructed and driven using compressed nitrogen gas. The driver and driven sections were separated by clear polyester membranes (Ridout Plastics Co.; San Diego, California) of varying thickness (0.003"-0.010") to achieve a range of peak overpressure exposures. A tapered design of the driver section (Figure 1) was included in an effort to minimize wave reflection and alterations of the blast wave by the expansion wave. This feature is similar in nature to blast tubes utilizing explosives in which the explosive is often detonated in a conical or parabolic-shaped driver section. The driver section was kept short in length in an effort to reduce the amount of gas required for membrane rupture and subsequent blast duration. The overpressure duration and impulse are reduced as described previously due to the shortened driver section ${ }^{407}$. For additional details concerning design of the blast model, please see Appendix.

\subsubsection{Blast Waveform Acquisition \& Analysis}

Shock wave pressures were detected using piezoelectric sensors (PCB Piezotronics; Depew, New York) that were placed in both reflected and incident positions at the exit of the shock tube (Figure 9.1). Data was acquired and processed using the National Instruments input module (NI 9223) connected to the National Instruments receiver USB chassis (CDAQ-9171). Signals were recorded using a custom-designed Labview 12.0 program and the NI data acquisition system (National Instruments; Austin, Texas) with a sampling rate of $500 \mathrm{kHz}$ as described previously ${ }^{620,621 .}$

\subsubsection{Blast Exposure}

Prior to blast exposure, animals were anesthetized by intraperitoneal injection of ketamine (90 mg/kg; Webster Veterinary; Devens, Massachusetts) and xylazine (5 mg/ $\mathrm{kg}$; Webster Veterinary). Animals were oriented with the long axis of the animal perpendicular to the blast front. In other words, the blast was delivered side-on to the head only as the thorax and abdomen were protected using rigid shielding, creating an experimental setup (Figure 9.1) similar to that used by Shridharani and colleagues ${ }^{622}$. With the animal placed directly at the exit of the tube, the effect of expansion waves on the planar conditions of the blast front is minimized as described by Bass and colleagues 407. Animals $(n=38)$ were divided into the following groups: control $(n=10), 0.003$ membrane blast exposure $(n=6), 0.005$ membrane blast exposure $(n=10), 0.007$ membrane blast exposure $(n=9)$, and 0.010 membrane blast exposure $(n=3)$. Immediately following blast exposure, animals were returned to a holding cage equipped with a homeothermic heating blanket equipped with a rectal thermometer to maintain body temperature at $37^{\circ} \mathrm{C}$. Once basic reflexes were restored, animals were returned to the home cage.

\subsubsection{Total Activity Measurements}

Animal activity was measured using an automated activity monitoring system (San Diego Instruments; San Diego, California). Rats were given the hour to acclimate to the testing room prior to initiation of testing. Each testing chamber consisted of a $16 \times 16$ photobeam array surrounding a Plexiglass chamber to record lateral movements. A separate 8 photobeam array to detect rearing activity was located above the $16 \times 16$ array. Activity was quantified over a 30 minute period 24 hours post-blast exposure with total activity, ambulatory, and fine activity reported based on default settings of the data acquisition software (PAS, San Diego Instruments; San Diego, California).

\subsubsection{Histological Preparations}


At 72 hours post-blast exposure, a total of 24 animals (6 control and 6 from each blast exposure group) were anesthetized as described above and perfused transcardially with cold $0.9 \%$ saline followed by $10 \%$ formalin for a total of 10 minutes. The brain was then extracted and placed into fresh $10 \%$ formalin for a minimum of 24 hours. After 24 hours of fixation, the brain was blocked into sections and paraffin embedded as previous described ${ }^{623}$. Briefly, tissues were processed using the Tissue-Tek VIP 5 automatic tissue processor (Sakura Finatek; Tokyo, Japan) and embedded in paraffin using the Tissue-Tek TEC 5 embedding system (Sakura Finatek; Tokyo, Japan). Tissues were sliced using a Leica RM2235 microtome (Leica Microsystems; Wetzlar, Germany) and slices mounted on slides for staining.

Standard protocols were utilized for staining with hematoxylin and eosin (H\&E), fluorojade-B (Millipore; Billerica, Massachusetts), which was used as a marker of neural degeneration, glial fibrillary acidic protein (GFAP) (Dako; Glostrup, Denmark), which was used as a marker of reactive astrocytes, and ionized calcium binding adapter molecule (Wako; Osaka, Japan), which was used as a marker of activated microglia.

Images were acquired from the S1BF region of the cortex, corpus callosum, striatum, hippocampus, and cerebellum. Imaging was performed using a Zeiss Axio Imager 2 (Carl Zeiss AG; Oberkochen, Germany) for all brightfield and fluorescent images shown.

\subsubsection{Histological Quantification}

Stereology and the optical fractionator technique were used to quantify histological results as previously described $598,624,625$. Briefly, a region of interest encompassing the corpus callosum was drawn at low power using an Olympus AX70 microscope (Olympus; Tokyo, Japan) and Stereolnvestigator software (MBF Bioscience; Williston, Vermont). The software then selected random 75- $\mu$ m counting frames with a depth of 6 $\mu \mathrm{m}$, and the object of interest marked by an observed blinded to treatment. The volume of the region of interest previously identified was then determined by the software and the number of cells marked by the observer returned.

\subsubsection{Quantitative Real-Time PCR}

A total of 8 animals were used for quantitative real-time polymerase chain reaction (PCR) and included 4 control animals and 4 animals exposed to blast using the 0.005 membrane. Total RNA was extracted from the brain tissue (cortex, striatum, hippocampus, and cerebellum) of saline-perfused animals using Trizol reagent (Invitrogen; Carlsbad, California) according to the manufacturer's instructions. Sample concentration was determined by spectral absorption and the purity confirmed using a 260/280 ratio of 1.8-2.0. Samples of cDNA were prepared via reverse transcription using commercially available kits (Applied Biosystems; Carlsbad, California). Sample reactions included MultiScribe TM Reverse Transcriptase and random primers, with thermal cycle conditions set as follows: step 1 at $25^{\circ} \mathrm{C}$ for 10 minutes, step 2 at $37^{\circ} \mathrm{C}$ for 120 minutes, step 3 at $85^{\circ} \mathrm{C}$ for 5 seconds, and step 4 at $4^{\circ} \mathrm{C}$ for 10 minutes. For PCR amplification, TaqMan ${ }^{\circledR}$ Universal PCR Master Mix and the following probes were obtained from Applied Biosystems: 18s (Hs99999901_s1) for use as an endogenous control gene, GFAP (Rn00566603_m1), and Iba-1 (Rn00574125_g1). The reaction mixture was prepared based on manufacturer's instructions and the following thermal cycling conditions used: initial holding at $50^{\circ} \mathrm{C}$ for 2 minutes to allow for optimal AmpErase ${ }^{\circledR}$ UNG activity, followed by a first denaturing step at $95^{\circ} \mathrm{C}$ for 10 minutes, then 45 cycles at $95^{\circ} \mathrm{C}$ for 15 seconds, and at $60^{\circ} \mathrm{C}$ for 1 minute. Changes in gene 
expression were determined using the $\Delta \Delta \mathrm{C}_{T}$ method and a threshold value of 0.2 . Threshold cycle values of each gene were normalized to $18 \mathrm{~s}$ rRNA.

\subsubsection{Data Analysis}

Data were analyzed using GraphPad Prism 5.0 (GraphPad Software, Inc.; San Diego, California). A one-way analysis of variance (ANOVA) with post-hoc Tukey's test was used to compare across control and various blast-exposed groups. For gene expression work, a student's t-test was utilized for comparing control and the blast-exposure group (0.005 membrane). $\quad \mathrm{P}<0.05$ was considered statistically significant for all data analyzed.

\subsection{RESULTS}

9.3.1. The Model is Capable of Producing a Characteristic Friedlander-type Blast Across a Range of Peak Overpressures with Short Duration of Exposure.

The ideal blast wave in an open area, also known as the Friedlander curve, consists of a near-instantaneous pressure rise followed by an exponential decay of the overpressure 407. With all membranes tested utilizing the shock tube apparatus (Figure 9.1), ideal blast waves were obtained with a representative curve shown (Figure 9.2a). Peak overpressure increased in a linear fashion with membrane thickness with both reflected $\left(r^{2}=0.92\right)$ and incident $\left(r^{2}=0.93\right)$ sensor orientations allowing for the simulation of multiple peak overpressures in the experiment (Figure 9.2b,c).

9.3.2. High Peak Vverpressure Blast, Even of Short Duration, Is Associated with High Mortality and Extensive Intracranial Bleeds.

While reflected pressures of $31.47 \pm 3.049$ and $50.72 \pm 3.368$ PSI failed to produce any mortality, exposure to $72.05 \pm 1.212 \mathrm{PSI}$ blast was associated with mortality in 3 of 9 experimental animals (33\%) while $90.10 \pm 1.030 \mathrm{PSI}$ blast exposures produced mortality in all 3 animals tested (100\%). Surviving animals failed to exhibit large hematomas at 72 hours post-blast (Figure 9.3a,b) but evidence of microvascular dysfunction and the potential for intraparenchymal bleeds was present (Figure 9.3c,d). Animals exposed to survivable moderate peak overpressures in this work displayed small hematomas immediately following blast exposure (Figure 9.3c), consistent with the work of Reneer and colleagues ${ }^{626}$. Mortality was associated with the production of intracranial bleeds in the cortex, particularly on the contrecoup side as well as in the brainstem (Figure 9.3e,f). Similar findings have been observed utilizing explosives such as TNT in rat models in which animals placed in close range exhibit increased mortality rates associated with brain contusions, lacerations, and hematomas ${ }^{627}$.

\subsubsection{Blast-induced Brain Injury Results in the Presence of Intraparenchymal Red} Blood Cells, Swollen Neurons, and Activated Microglia.

To further examine the effect of blast exposure on neural injury and microvascular dysfunction, particularly considering the presence of hematomas and parenchymal blood on gross examination, brain sections underwent H\&E staining. The intraparenchymal blood was confirmed with H\&E staining and red blood cells clearly passed into the parenchyma and were no longer confined to the vasculature (Figure 9.4a). Evidence of neural injury and swollen, red axons was apparent in some animals (Figure 9.4b). The presence of red neurons indicated a potential anoxic/ischemic insult ${ }^{628}$, consistent with 
the presence of microvascular dysfunction observed following blast exposure ${ }^{629}$. The presence of activated microglia, capable of releasing an array of inflammatory mediators, was also seen with blast exposure and prompted further investigation of astro- and microgliosis.

\subsubsection{Neural Degeneration Occurs in a Dose-response Manner Consistent with Blast Severity.}

Blast-induced brain injury resulted in increased neural degeneration measured using Fluoro-Jade $B$ staining in the S1BF region of the cortex on both the coup $(F(3,23)=$ 47.03, $\mathrm{P}<0.0001)$ and contrecoup $(F(3,23)=110.7, \mathrm{P}<0.0001)$ sides when analyzed by one-way ANOVA (Figure 9.5a-d). Post hoc tests revealed that blast-induced brain injury produced increases in neural degeneration compared to control at $31.47 \pm 3.049$ $\mathrm{PSI}$ on the contrecoup side $(\mathrm{q}=4.51, \mathrm{P}<0.05), 50.72 \pm 3.368 \mathrm{PSI}$ on both coup $(\mathrm{q}=$ 10.29, $P<0.0001)$ and contrecoup $(q=13.69, P<0.0001)$ sides, and $72.05 \pm 1.212 P S I$ on both coup $(q=15.13, P<0.001)$ and contrecoup $(q=23.76, P<0.0001)$ sides (Figure 9.5e-f). Neural degeneration following blast exposure was also observed within other brain regions including the striatum, hippocampus, cerebellum and regions surrounding the corpus callosum (Appendix).

\subsubsection{Blast Injured Brains Demonstrate Increased Glial Activation with Increasing Peak Over-pressure.}

Reactive astrocytes, identified by GFAP staining, were elevated significantly within the corpus callosum on the coup $(F(3,23)=26.08, P<0.0001)$ and contrecoup $(F(3,23)=$ 8.03, $\mathrm{P}<0.001$ ) sides of the brain following blast-induced neurotrauma, consistent with findings from other blast injury models ${ }^{629}$, when analyzed using one-way ANOVA (Figure 9.6a-d). Post hoc tests showed that blast injury resulted in elevated astrocyte reactivity at $50.72 \pm 3.37 \mathrm{PSI}$ on the contrecoup side $(q=5.13, P<0.01)$ and at $72.05 \pm$ $1.21 \mathrm{PSI}$ on both the coup $(q=11.28, P<0.0001)$ and contrecoup $(q=6.03, P<0.01)$ sides (Figure 9.6e-f). Similar findings were observed concerning microglial activation, identified with lba-1 staining, with increased levels of microglial activation relative to control on both coup $(F(3,23)=83.12, P<0.0001)$ and contrecoup $(F(3,23)=25.37, P<$ 0.0001 ) sides (Figure 9.7a-d). Again, although microglial presence was detected throughout the brain and appeared increased throughout, microglial activation was most prominent throughout the corpus callosum and internal capsule, consistent with other studies ${ }^{628}$. Notably, microglia appeared more rounded or amoeboid, typical of phagocytic microglia, within these white matter tracts consistent with white matter damage observed in human subjects following trauma ${ }^{630}$. Previous reports in swine exposed to blast and assessed primarily at two weeks failed to identify extensive microglial activation, a potential reason for the discrepancy between studies ${ }^{628}$. Post hoc tests revealed increased microglial activation following $31.47 \pm 3.049 \mathrm{PSI}$ blast on the contrecoup side $(q=4.71, P<0.05), 50.72 \pm 3.37$ PSI blast on both coup $(q=8.37$, $P<0.0001)$ and contrecoup $(q=7.85, P<0.0001)$ sides, and $72.05 \pm 1.212$ PSI blast on both coup $(\mathrm{q}=20.49, \mathrm{P}<0.0001)$ and contrecoup $(\mathrm{q}=11.92, \mathrm{P}<0.0001)$ sides as well (Figure 9.7e-f).

I Gene expression of GFAP (Figure 9.8a) was increased at 24 hours following blast exposure in the cortex $(t=2.41, P<0.05)$ but not in the striatum $(t=0.02, P>0.05)$, hippocampus ( $t=1.08, P>0.05)$, or cerebellum $(t=0.63, P>0.05)$. The lack of difference in GFAP gene expression in regions outside of the cortex was in contrast to the visible differences seen in these regions at 72 hours based on 
immunohistochemistry (Appendix). No difference in lba-1 gene expression (Figure 9.8b) was detected in the cortex $(t=0.43, P>0.05)$, striatum $(t=0.90, P>0.05)$, hippocampus ( $\mathrm{t}=0.38, \mathrm{P}>0.05)$, or cerebellum $(\mathrm{t}=0.45, \mathrm{P}>0.05)$, consistent with immunohistochemical findings (Appendix).

\subsubsection{Blast-induced Traumatic Brain Injury Produces Deficits in Total Activity Regardless of Severity.}

Total activity of the experimental subjects was decreased at 24 hours post-blast in all injured groups. The total activity level post-injury did not vary based on injury severity with all injury groups demonstrating similar impairments (Figure 9.9a). The deficits in total activity were due to a decrease in ambulatory activity associated with blast exposure (Figure 9.9b). Blast exposure did not alter measures of fine activity (Figure 9.9c).

\subsection{DISCUSSION}

Efforts to model blast injury have been plagued by the lack of standardization across laboratories and the appropriate scaling of blast parameters to the animal species used based on data obtained from human studies. Preclinical studies are varied with regards to animal placement within or outside of the shock tube, orientation to the blast front, protection of the thorax, and blast parameters, namely peak overpressure and duration of exposure. Studies in soldiers revealed common IED exposures producing blast injury result from detonation of $105-\mathrm{mm}$ and $155-\mathrm{mm}$ artillery rounds at a distance of 5-10 meters ${ }^{406}$. Use of the Conventional Weapons Effects Program (CONWEP) ${ }^{631}$ allows for interpretation of these exposures in relation to blast wave parameters with a peak overpressure of 50-1000 kPA (7.25-145.03 PSI) and an exposure duration of 2-10 ms 406. As such, utilizing these parameters and scaling appropriately to preclinical animal species used may represent a more clinically-relevant blast exposure and allow for improved elucidation of blast-induced injury pathophysiology and evaluation of proposed therapeutics.

The concept of scaling blast injury to animal models is based largely upon previous works assessing pulmonary injury and primary blast survival across animal species. Richmond and colleagues identified, in various species, the effect of blast duration on lethality with longer duration blasts requiring a lower peak overpressure to produce mortality when compared to short duration blasts that required a significantly higher peak overpressure ${ }^{632}$. A clear relationship between mass and blast parameters associated with lethality exists, particularly with regards to the more clinically-relevant short duration blast ${ }^{632}$. In fact, classical work by Bowen and colleagues investigated pulmonary injury in relation to blast exposure and created a model system in which scaling is based upon the height of burst divided by the cubed root of the mass ${ }^{633}$. This has been simplified to an equation utilizing the cubed root of the reference mass (human) divided by the mass of the experimental subject $406,407,622$. The scaling equations developed have displayed significant utility in development of injury risk functions based upon the high level of agreement with pre-existing experimental data concerning blast survival and injury risk. As such, while scaling of blast injury and associated blast parameters based on subject weight has not been related to neural injury ${ }^{634}$, scaling represents a logical step forward in more closely replicating clinically relevant exposures in laboratory models $406,635,636$.

To address the question of how scaling affects blast-induced brain injury a shock tube capable of producing a range of peak overpressures with short durations was designed 
and utilized within this work. Furthermore, the model was designed to simulate openfield blast with the rat placed outside of the shock tube in order to increase clinical relevance. Notably, the model produced clear neuropathological changes indicative of neural degeneration and glial reactivity. These findings were consistent with work by Goldstein and colleagues in which single-blast exposure produced neurodegenerative changes consistent with chronic traumatic encephalopathy (CTE) ${ }^{418}$ as well as other work utilizing scaling principles ${ }^{622}$. Similarly, neuropathological changes were observed throughout the cortex as previously described 418 but were most prominent with the corpus callosum and internal capsule, consistent with previous findings in other blast injury models ${ }^{634}$. It remains unclear why specific brain regions may be more susceptible to blast-induced brain injury but one logical explanation is that of spallation. Spallation refers to the interface-based disruption that occurs between tissues of different densities upon a compression wave in the denser medium reflecting at the interface resulting in displacement and fragmentation of the denser medium into the less dense medium ${ }^{637-639}$. Spalling has been identified as a leading cause of endothelial injury and activation of microglia ${ }^{640}$, consistent with our findings. Similarly, endothelial damage results in a loss of tight junction integrity and consequently the potential for increased blood-brain barrier permeability (BBB) and microvascular injury ${ }^{640}$. Notably, increased BBB permeability has been found as late as one month following blastinduced brain injury in a preclinical rodent model, indicating the presence of lasting microvascular dysfunction ${ }^{641}$. Furthermore, blast-induced BBB disruption has been observed to occur more extensively on the contralateral side, as well as at fluid-tissue interfaces such as that surrounding the lateral ventricles ${ }^{642}$, consistent with the findings reported within this study in which the contralateral side was more severely injured in comparison to the ipsilateral side based on standard markers of neural degeneration, astrocyte reactivity, and microglial activation ${ }^{643}$.

The clear neuroinflammatory response, characterized by a graded glial response based on peak overpressure exposure, observed in this work is consistent with findings by Cernak and colleagues in which mild and moderate blast exposure produced increases in GFAP, as well as ED1, a marker of microglia, at numerous time points ${ }^{644}$. Importantly, activated microglia in other blast exposure studies have been associated with changes in neuron appearance such as darkened dendrites ${ }^{645}$ and in vitro work has demonstrated a mild activation of microglia following overpressure exposure with pulse duration altering microglial gene expression ${ }^{646}$. Interestingly, gene expression studies did not entirely match immunohistochemical studies with regard to GFAP and Iba-1 expression. The reason for this discrepancy is not entirely clear but may be due to consideration of the entirety of brain sections for gene expression studies when immunohistochemistry reveals findings largely located at the interface of regions and the gray-white matter junction. Another possibility explaining the discrepancy between immunohistochemical and gene expression findings is the time of analysis. Only one time point for each assay was included and therefore, changes in gene expression could have occurred rapidly following blast and already declined to baseline by 24 hours.

In addition to the neuropathological findings, the model utilized in this work produced deficits in total activity at 24 hours post-blast, regardless of experimental group. How blast-injury models affect behavioral functions in rodents is poorly understood but of clear interest based on clinical findings associated with blast that include retrograde amnesia, confusion, loss of conscioussness, depression, post-traumatic stress disorder (PTSD), vertigo, balance disorders, anxiety, apathy, and difficulty concentrating and performing executive functions 636,641,647-649. Previous reports have demonstrated 
impairment on the accelerating rotarod following direct cranial blast injury ${ }^{650}$ and on the balance beam following whole body blast exposure ${ }^{620}$ while others have shown that shielding of the torso prevents neural injury and associated behavioral deficits following blast ${ }^{651}$. While the significance of these findings is unclear and how these findings translate from one model to another, let alone to the clinical population, there is a clear need for further studies in standardized, clinically-relevant models.

It is clear that future studies, in addition to understanding the effect of scaled blast exposure on functional ability and behavior-related changes, need to better characterize the inflammatory processes associated with blast exposure and how these relate to neural injury and neurodegeneration. While it is unlikely blast exposure can be completely prevented, particularly in civilians exposed to terrorist-type attacks, it may be possible to improve therapeutic options. The need for improved therapeutics is particularly clear in the military in which evacuation of blast-exposed troops is often delayed by hours to several days due to the nature of conflict. As such, secondary responses within the brain often occur and may represent a potential target as described previously ${ }^{652}$.

\section{ACKNOWLEDGEMENTS}

The authors would like to thank Nicholas St. John for contributing to the design of the blast model, Brandon P. Lucke-Wold for his assistance with preliminary experiments, and Ms. Diana Richardson of the National Institute of Occupational Safety and Health $(\mathrm{NIOSH})$ for assistance in tissue preparation. The authors would also like to thank Mr. James Edward Robson and Mr. Peter Bennett for assistance in model construction. The authors are also grateful for the assistance of Dr. Kymberly Gyure for assistance with neuropathological examination. This work was supported by grant funding provided to Ryan C. Turner (5T32GM08174), Jason D. Huber (5RO1-NS061954), and Rae R. Matsumoto (5RO1-DA013978) by the National Institutes of Health. The West Virginia University Department of Neurosurgery also funded this work. Funding was also provided to Dr. Robert T.T. Gettens by the American Society for Quality, Biomedical Division in the form of the Dr. Richard J. Schlesinger Grant for construction of the blast model. 


\section{FIGURES}

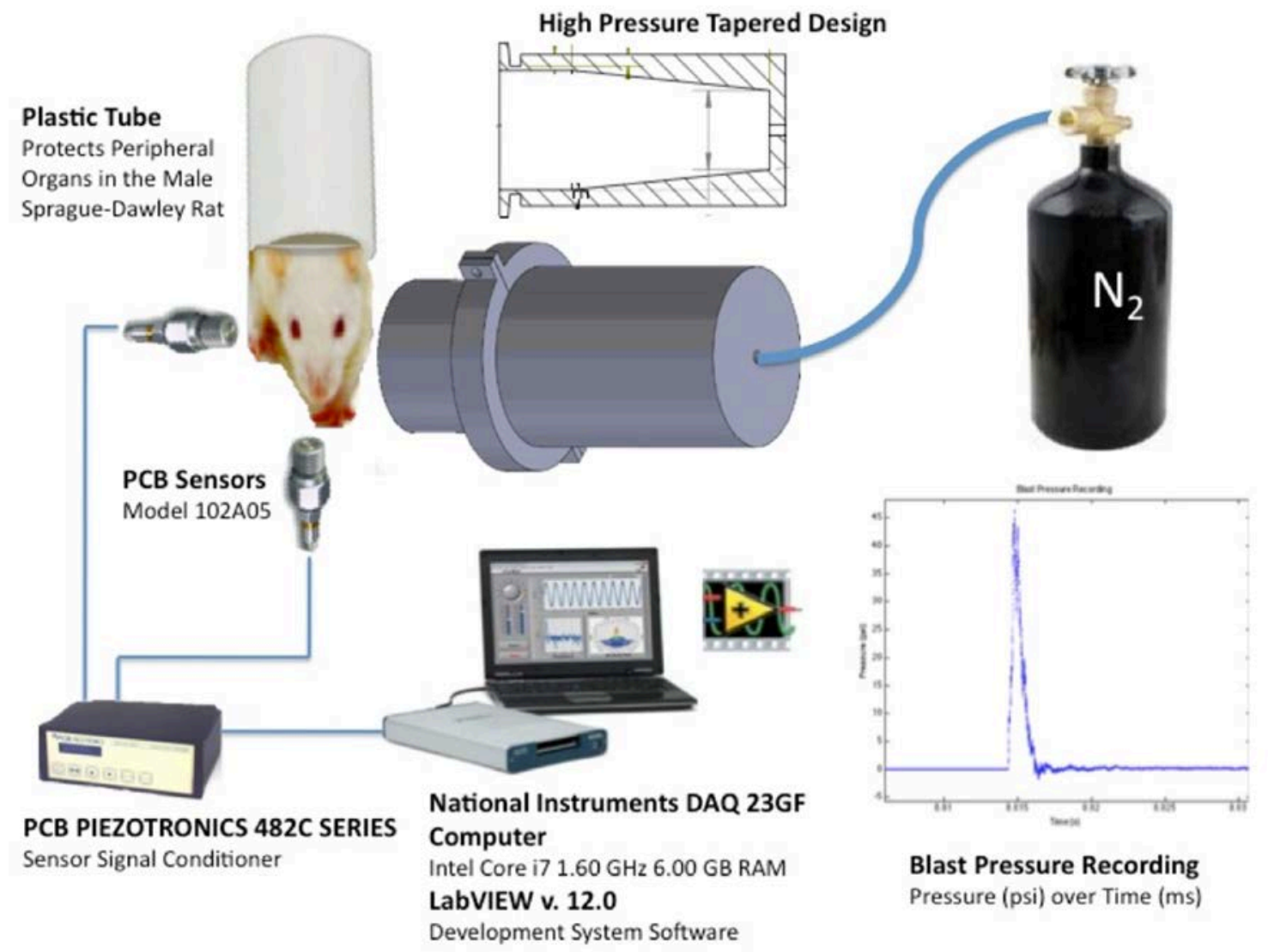

Figure 9.1. Experimental setup utilized in this work demonstrating the novel shock tube apparatus for simulating blast-induced brain injury and data acquisition procedures. The shock tube utilizes a tapered driver section to reduce wave reflection and alterations of the shock wave by the expansion wave. The driver section was kept short to reduce the volume of gas required to produce membrane rupture and therefore, reduced overpressure duration and impulse. The abdomen and thorax of animals was protected using a rigid apparatus. 


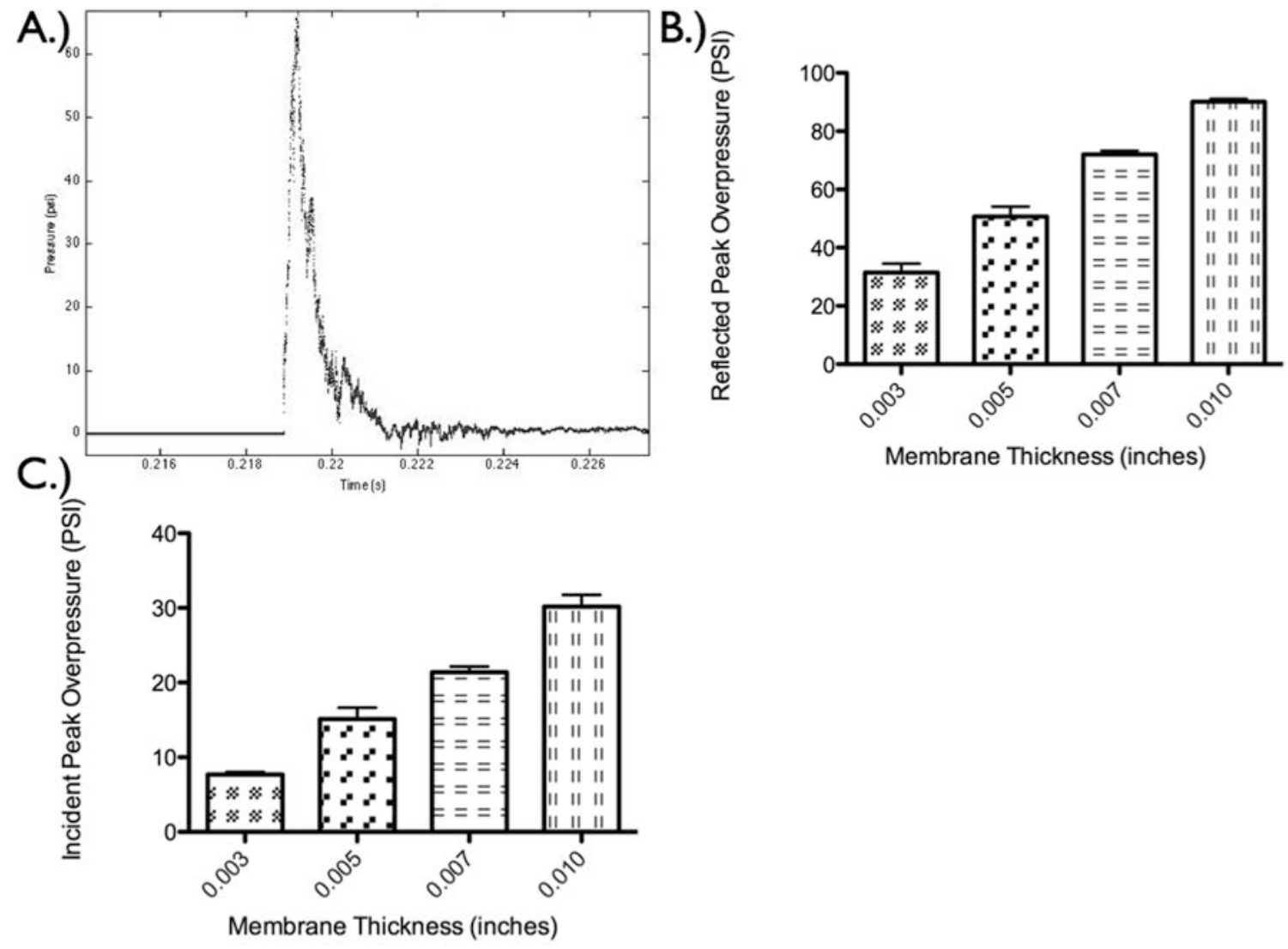

Figure 9.2. A.) Sample shock wave recording (reflected sensor) representing a classical Friedlander wave of short duration. B.) Peak reflected overpressure recorded as a function of membrane thickness. C.) Peak incident overpressure recorded as a function of membrane thickness. 


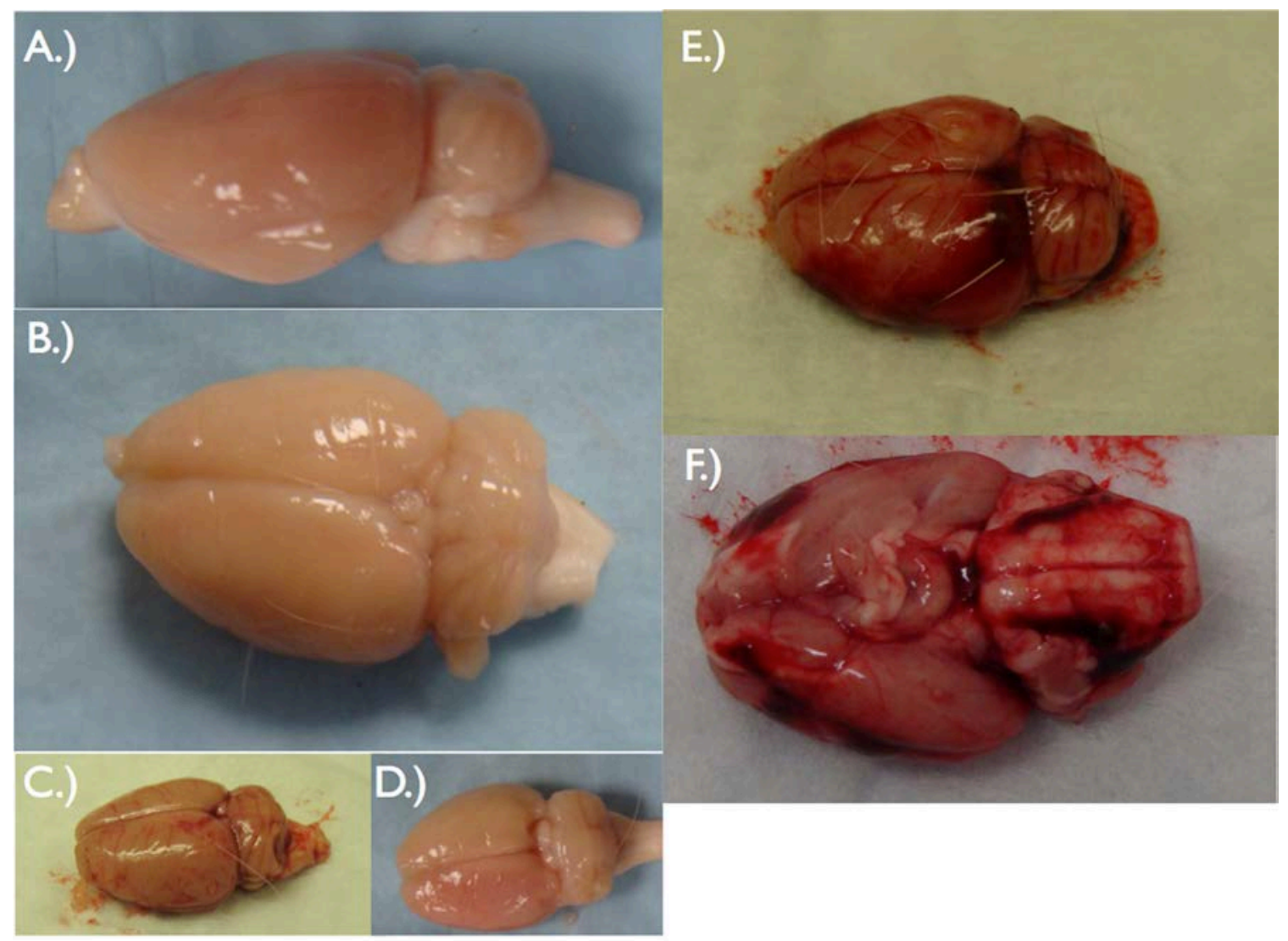

Figure 9.3. Gross examination of single blast-exposed brains. A.) Brain of a perfused control animal, B.) $31.47 \pm 3.049$ PSI (reflected) blast-exposed brain at 72 hours postblast following perfusion demonstrating no apparent hematomas or gross changes, $\mathbf{C}$.) Presence of hematomas and contusion immediately following exposure to $50.72 \pm 3.368$ PSI (reflected) blast on the contrecoup side, D.) At 72 hours post-blast, $72.05 \pm 1.212$ PSI (reflected) blast exposure results in the appearance of parenchymal blood and an overall husky appearance on the contrecoup side, E.) Immediately following $90.10 \pm$ $1.030 \mathrm{PSI}$ (reflected) blast, clear evidence of bleeds on the cortex, and F.) Immediately following $90.10 \pm 1.030$ (reflected) PSI blast, brainstem bleeds were apparent and associated with mortality. 

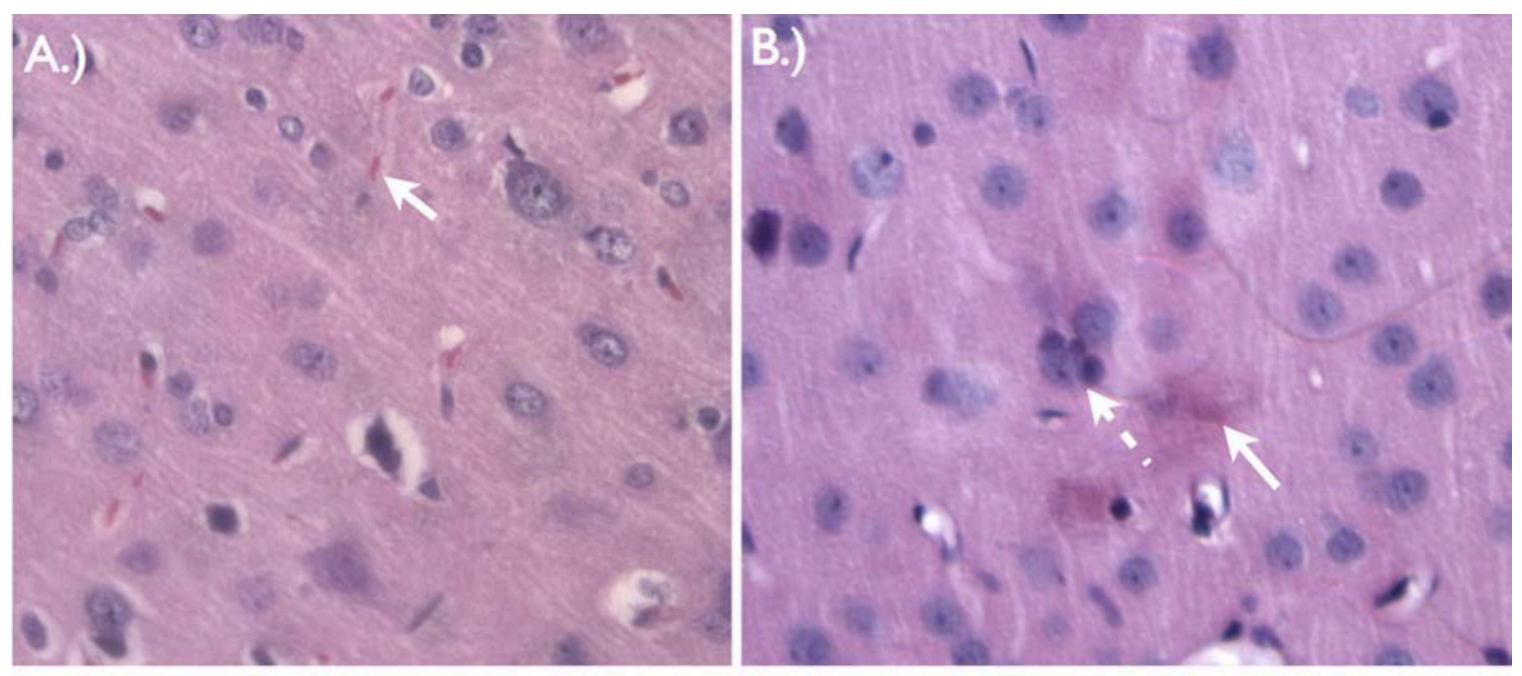

Figure 9.4. Blast exposure, particularly at high peak overpressures, was associated with microvascular damage and neural injury on H\&E staining. A.) Presence of red blood cells within the parenchyma at 72 hours following $72.05 \pm 1.212 \mathrm{PSI}$ (reflected) blast. B.) Swollen, red neuron at 72 hours following $72.05 \pm 1.212$ (reflected) PSI blast. Red neurons typically are associated with anoxic/ischemic injury indicating the presence of secondary damage, likely related to microvascular dysfunction, following blast injury. 

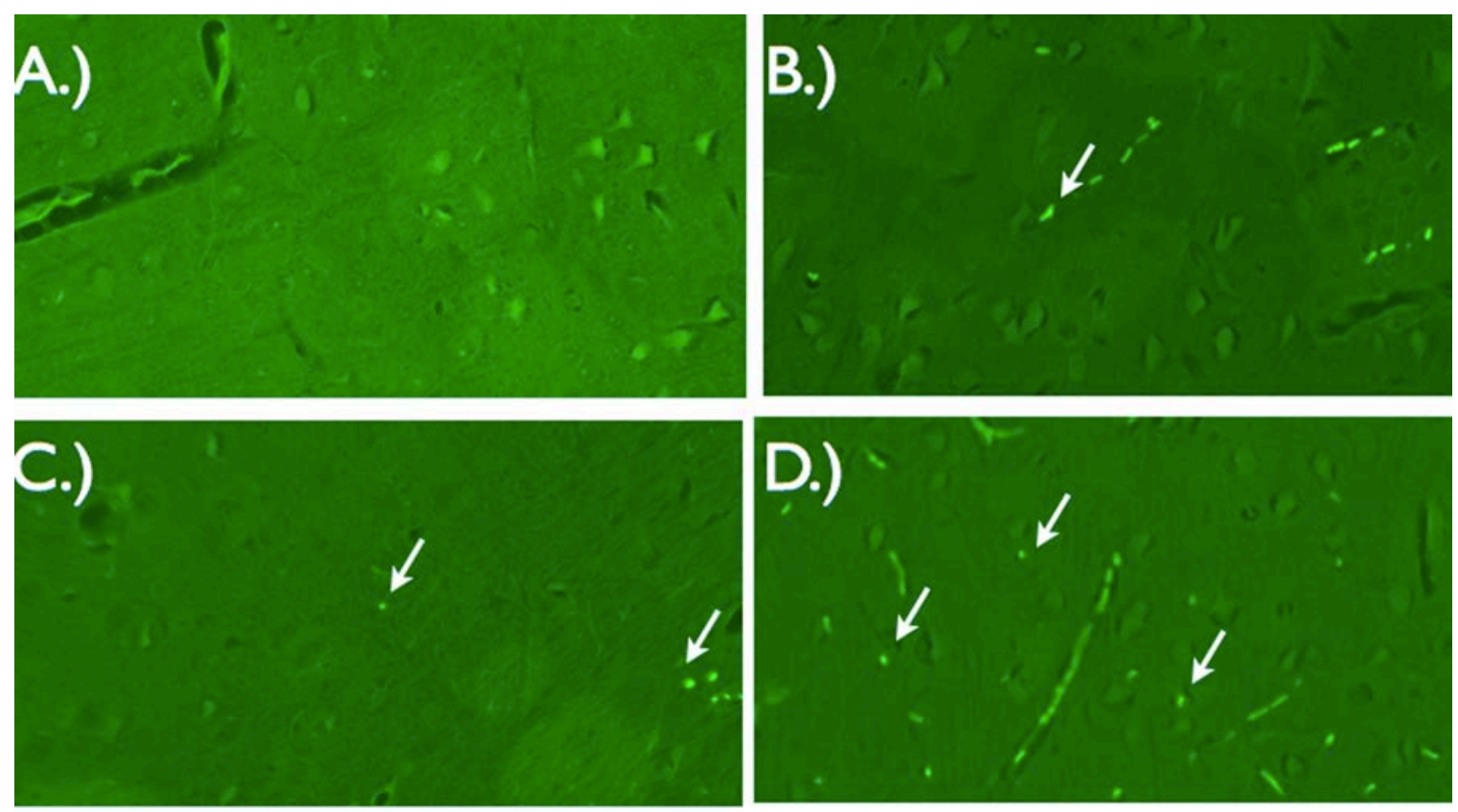

E.)

F.)
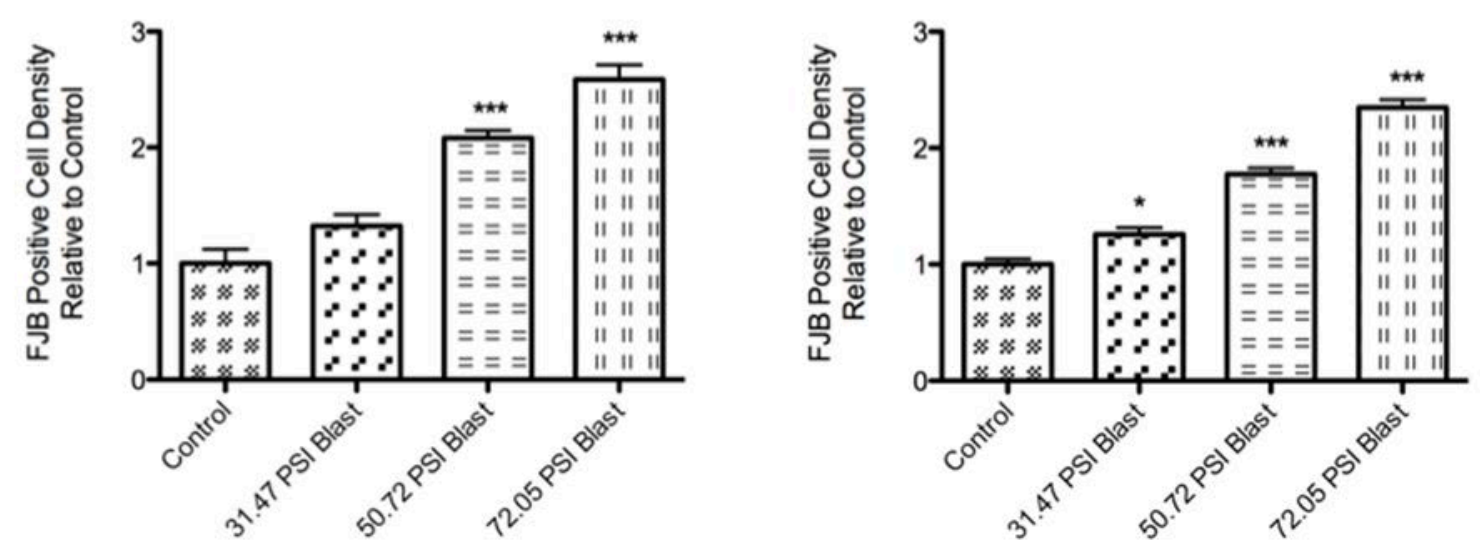

Figure 9.5. Blast exposure produced neural degeneration within the S1BF region of the cortex at 72 hours post-exposure as indicated with fluoro-jade B staining. A.) $31.47 \pm$ 3.049 PSI (reflected) blast on the coup side, B.) $50.72 \pm 3.368$ PSI blast on the coup side, C.) $31.47 \pm 3.049 \mathrm{PSI}$ blast on the contrecoup side, and D.) $72.05 \pm 1.212 \mathrm{PSI}$ blast on the contrecoup side. Quantification of Fluoro-Jade B (FJB) positive neurons using stereology demonstrated a graded response based on peak overpressure exposure in the S1BF region of the cortex on coup $(F(3,23)=47.03, P<0.0001)$ and contrecoup $(F(3,23)=110.70, P<0.0001)$ sides $(\mathbf{E}$ and $\mathbf{F}$, respectively). Post hoc analysis showed that blast exposure resulted in increased neural degeneration relative to control with $31.47 \pm 3.049 \mathrm{PSI}$ on the contrecoup side $(\mathrm{q}=4.51, \mathrm{P}<0.05), 50.72 \pm$ $3.368 \mathrm{PSI}$ on both coup $(\mathrm{q}=10.29, \mathrm{P}<0.0001)$ and contrecoup $(q=13.69, P<0.0001)$ sides, and $72.05 \pm 1.212 \mathrm{PSI}$ on both coup $(\mathrm{q}=15.13, \mathrm{P}<0.001)$ and contrecoup $(\mathrm{q}=$ 23.76, $\mathrm{P}<0.0001$ ) sides. 

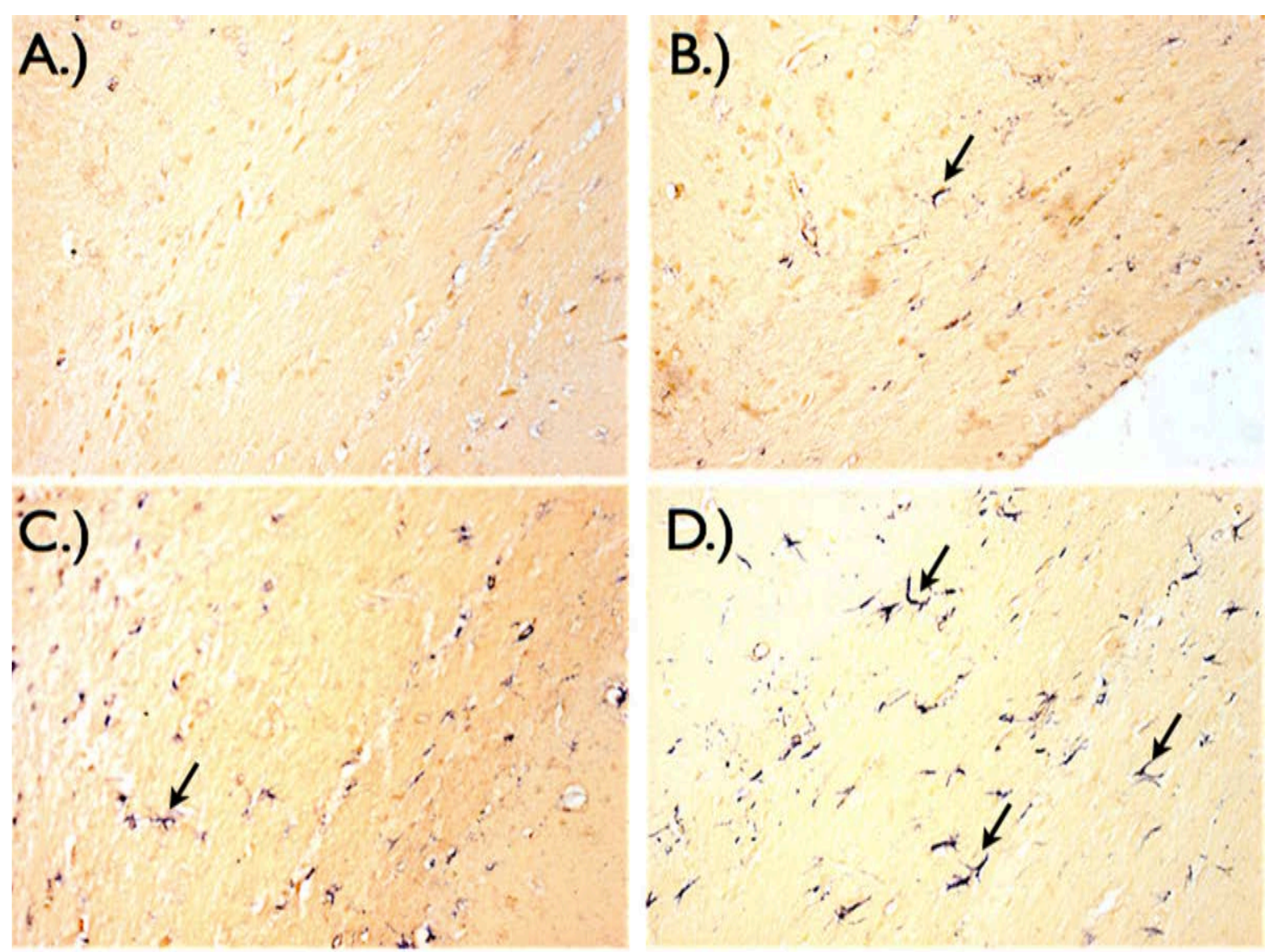

E.)

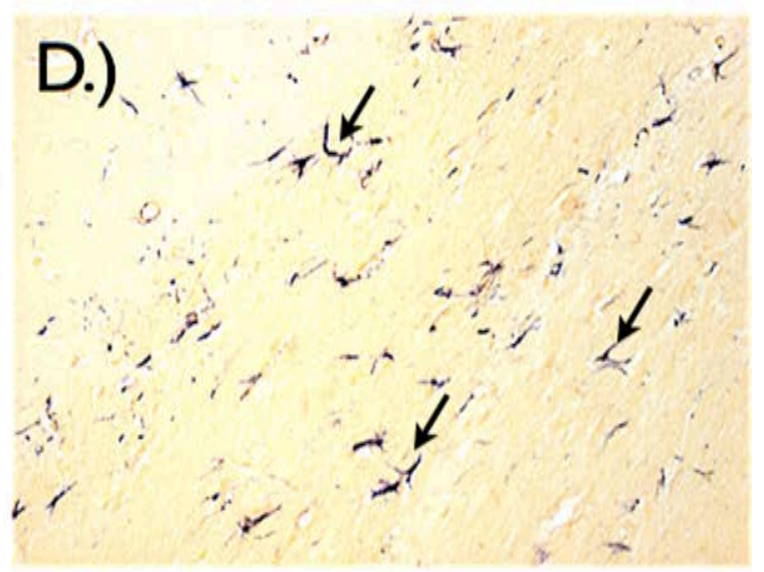

F.)
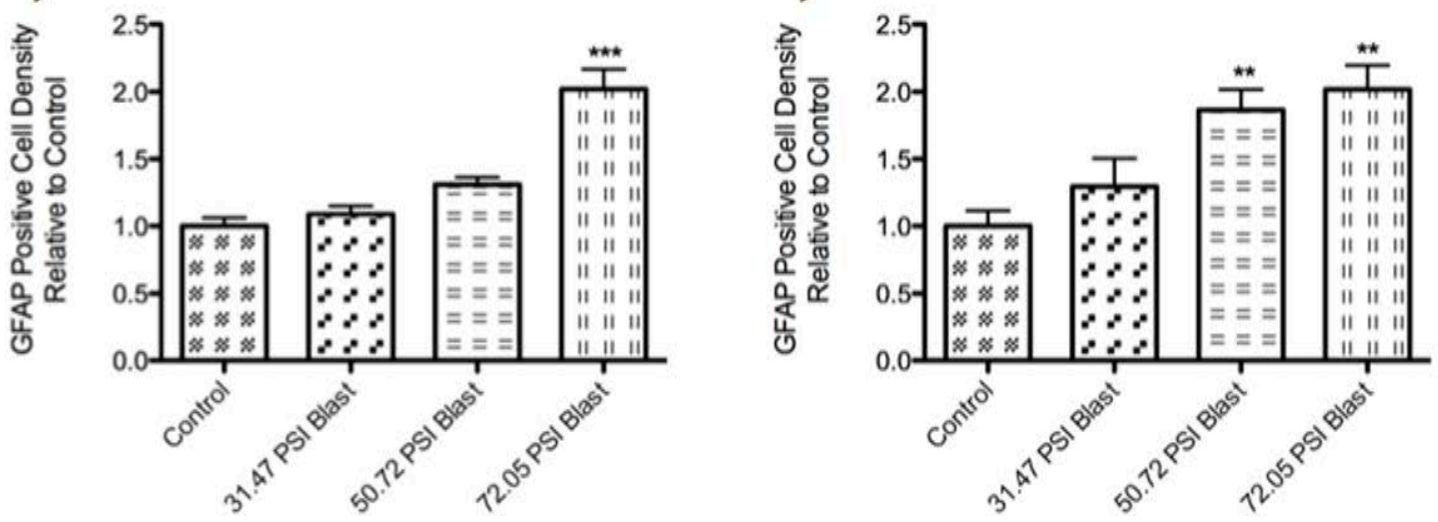

Figure 9.6. Blast exposure produced extensive astrocyte reactivity within the corpus callosum at 72 hours post-exposure as indicated with glial fibrillary acidic protein (GFAP) staining. A.) $31.47 \pm 3.049 \mathrm{PSI}$ blast on the coup side, B.) $72.05 \pm 1.212 \mathrm{PSI}$ blast on the coup side, C.) control (no blast exposure) on the contrecoup side, and D.) $72.05 \pm$ 1.212 PSI blast on the contrecoup side. Quantification of Glial Fibrillary Acidic Protein (GFAP) positive astrocytes using stereology demonstrated a graded response based on peak overpressure exposure in the corpus callosum on the coup $(F(3,23)=26.08$, $\mathrm{P}<0.0001)$ and contrecoup $(\mathrm{F}(3,23)=8.03, \mathrm{P}<0.001)$ sides ( $\boldsymbol{E}$ and $\mathbf{F}$, respectively). Post hoc analysis showed that blast exposure resulted in increased astrocyte reactivity relative to control with $50.72 \pm 3.368 \mathrm{PSI}$ on the contrecoup side $(q=5.13, P<0.01)$ and 
at $72.05 \pm 1.212 \mathrm{PSI}$ on both the coup $(q=11.28, P<0.0001)$ and contrecoup $(q=6.03$, $P<0.01$ ) sides. 

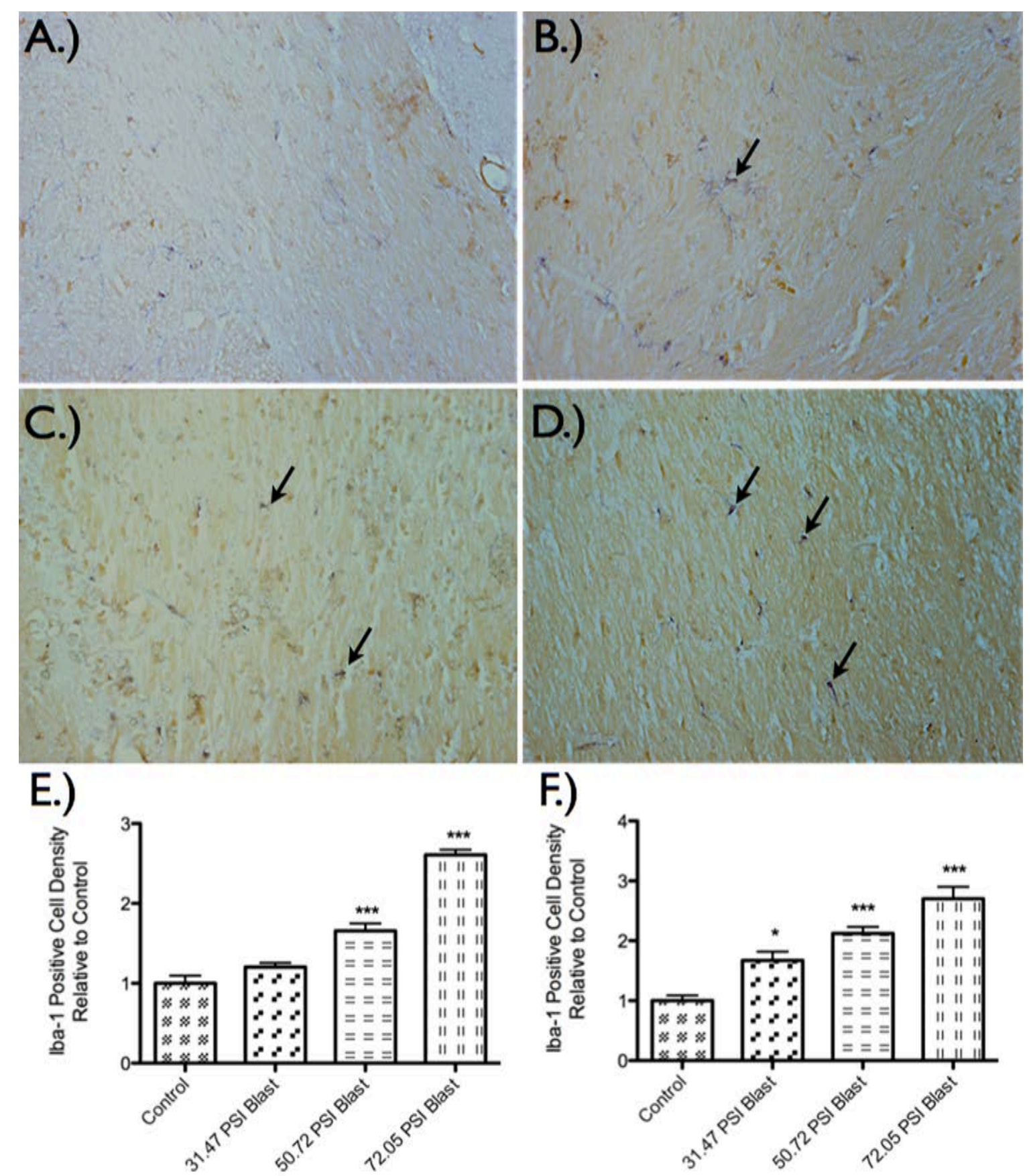

F.)

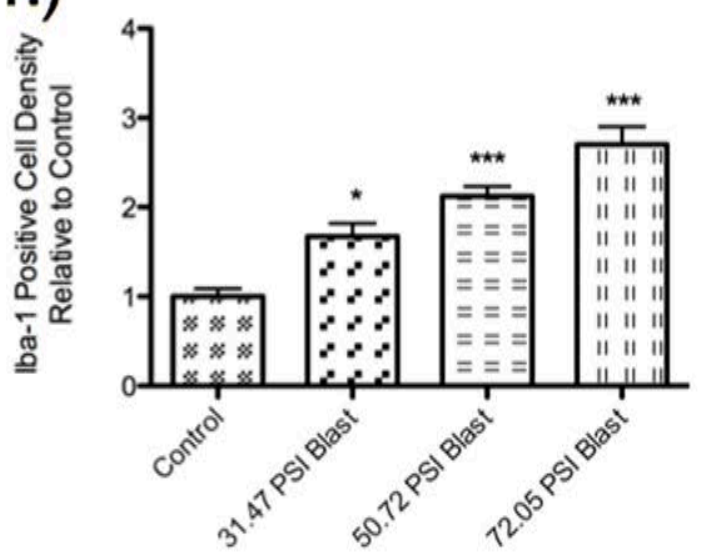

Figure 9.7. Blast exposure produced microglial changes within the corpus callosum at 72 hours post-exposure as indicated with ionized calcium-binding adapter molecule 1 (lba-1) staining. A.) Control (no blast exposure) on the coup side, B.) $50.72 \pm 3.368 \mathrm{PSI}$ blast on the coup side, C.) $31.47 \pm 3.049$ PSI blast on the contrecoup side, and D.) $72.05 \pm 1.212 \mathrm{PSI}$ blast on the contrecoup side. Quantification of Ionized Calciumbinding Adapter Molecule 1 (lba-1) positive microglia using stereology demonstrated a graded response based on peak overpressure exposure in the corpus callosum on the coup $(F(3,23)=83.12, P<0.0001)$ and contrecoup $(F(3,23)=25.37, P<0.0001)$ sides $(E$ and $\mathbf{F}$, respectively). Post hoc analysis showed that blast exposure resulted in increased microglial activation relative to control with $31.47 \pm 3.049 \mathrm{PSI}$ blast on the 
contrecoup side $(\mathrm{q}=4.71, \mathrm{P}<0.05), 49 \mathrm{PSI}$ blast on both coup $(\mathrm{q}=8.37, \mathrm{P}<0.0001)$ and contrecoup $(q=7.85, P<0.0001)$ sides, and $72.05 \pm 1.212$ PSI blast on both coup $(q=20.49, P<0.0001)$ and contrecoup $(q=11.92, P<0.0001)$ sides as well. 

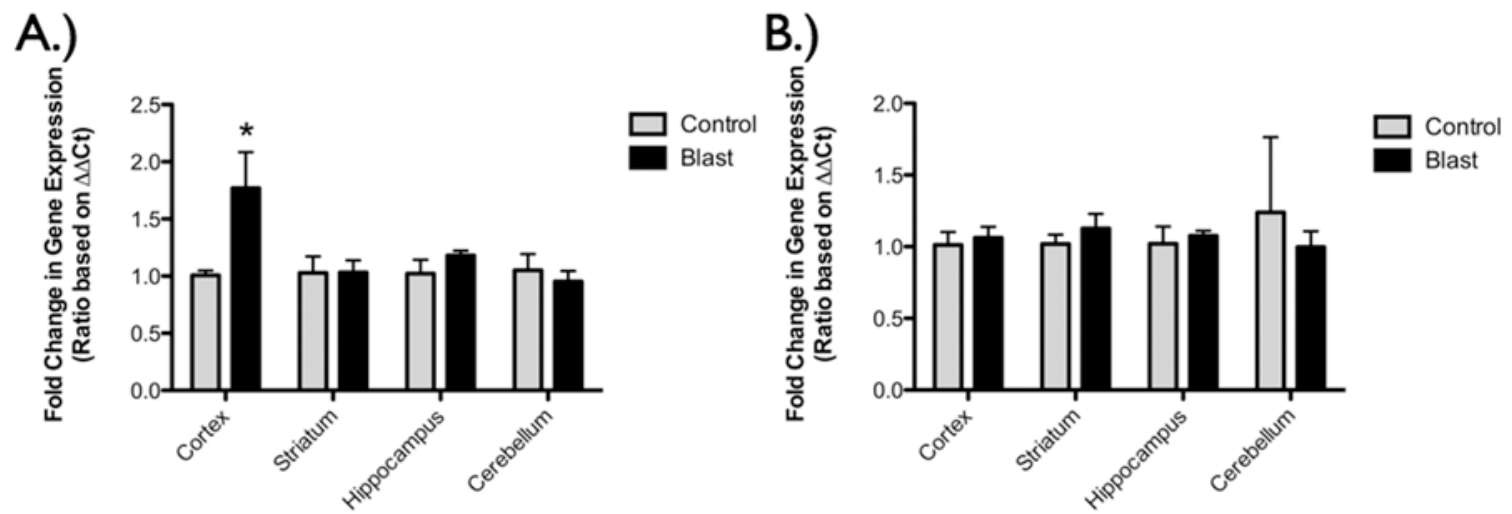

Figure 9.8. Effect of 50.72 PSI blast exposure on gene expression at 24 hours postinjury. A.) Gene expression of glial fibrillary acidic protein (GFAP) was increased within the cortex $(\mathrm{t}=2.412, \mathrm{P}<0.05)$ but not in the striatum, hippocampus, or cerebellum. B.) Gene expression of ionized calcium binding adapter molecule (Iba-1) was not changed within any of the brain regions studied at 24 hours post-blast. 


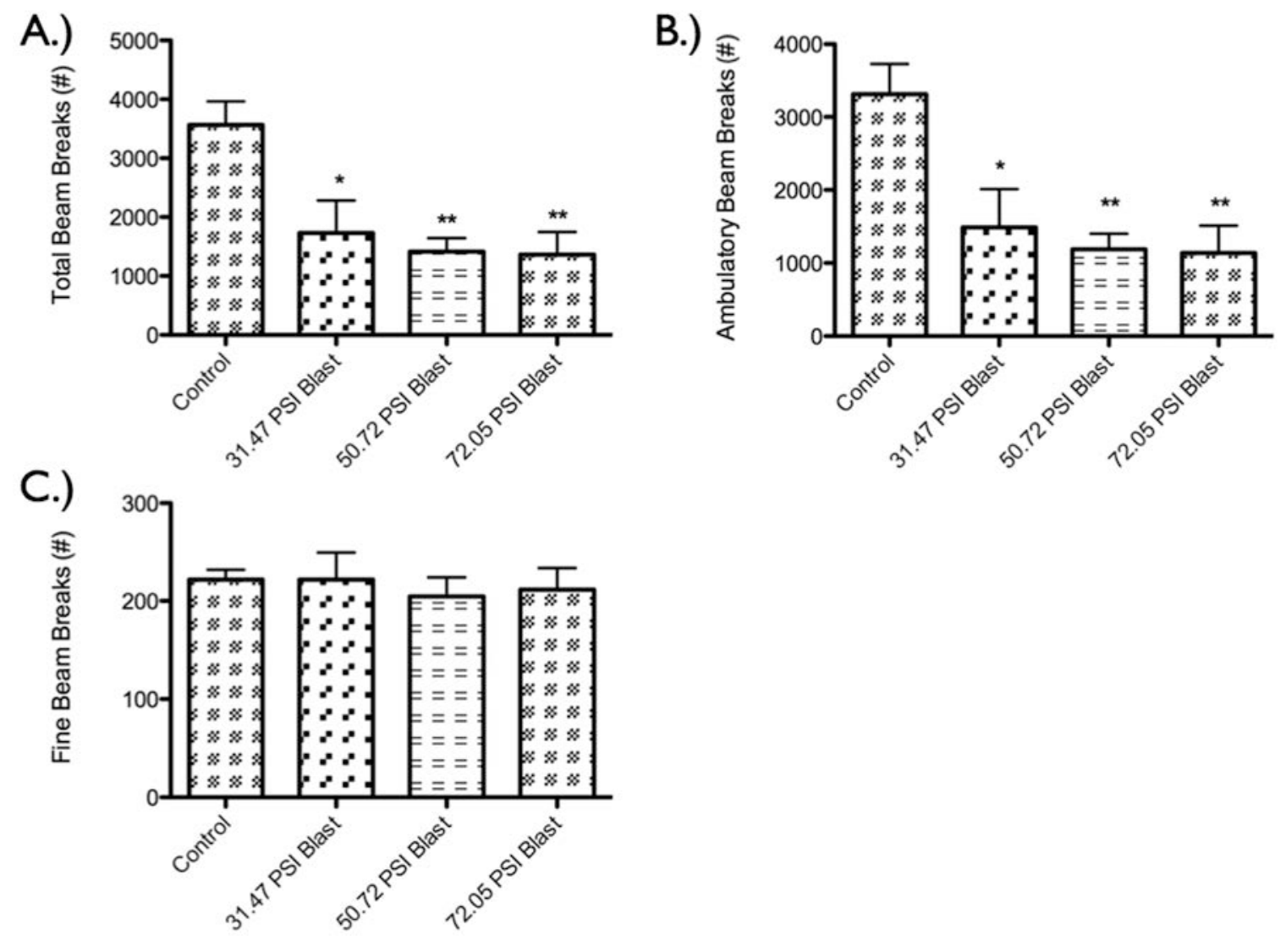

Figure 9.9. Effect of blast exposure on total, ambulatory, and fine activity levels at 24 hours post-injury. A.) Total activity was significantly altered with blast exposure $(F(3,23)$ $=6.55, \mathrm{P}<0.05)$ with post hoc analysis identifying a reduction relative to control with $31.47 \pm 3.049$ PSI blast $(q=4.50, P<0.05), 50.72 \pm 3.368$ PSI blast $(q=5.28, P<0.05)$, and $72.05 \pm 1.212 \mathrm{PSI}$ blast $(\mathrm{q}=5.40, \mathrm{P}<0.05)$. B.) Ambulatory activity was reduced with blast exposure $(\mathrm{F}(3,23)=6.74, \mathrm{P}<0.05)$ with post hoc analysis identifying a reduction relative to control with $31.47 \pm 3.049 \mathrm{PSI}$ blast $(\mathrm{q}=4.59, \mathrm{P}<0.05), 50.72 \pm$ 3.368 PSI blast $(q=5.34, P<0.05)$, and $72.05 \pm 1.212$ PSI blast $(q=5.47, P<0.05)$ exposures. C.) Fine activity failed to demonstrate differences across any group comparisons $(F(3,23)=0.17, P>0.05)$. 


\title{
Chapter Ten
}

As neurotrauma has been associated with long-term neurodegeneration, there is increasing interest in rapid identification of persons sustaining even 'mild' neurotrauma on the athletic or battlefields. The diagnosis of concussion currently relies largely upon self-reported symptoms, as no consistent positive findings are observed with standard medical imaging procedures. As such, numerous tests, both physical and neuropsychological in nature, have been developed in an attempt to assist in concussion diagnosis and return-to-play guidelines. Study of trauma and concussion in preclinical studies has relied largely upon mortality rate and histological markers of damage rather than functional impairment. Therefore, it remains unclear the level or severity of injury truly being studied as impairments are not well-characterized. In this work, we address this issue using a clinically-relevant model of diffuse axonal injury and an array of functional assessments conducted in the acute period, typical of when symptoms associated with concussion present.

\section{Elucidating the Severity of Preclinical Traumatic Brain Injury Models: A Role for Functional Assessment?}

This work is currently in preparation for publication submission.

\begin{abstract}
Concussion remains a symptom-based diagnosis clinically, yet preclinical studies investigating traumatic brain injury emphasize histological endpoints with functional assessments often minimized or ignored all together. Recently, clinical studies have identified the importance of cognitive and neuropsychiatric symptoms, in addition to somatic complaints, following concussion. How these findings may translate to preclinical studies is unclear at present. This work addresses the contrasting endpoints utilized clinically compared to those in preclinical studies and the potential role of functional assessments in a commonly used model of diffuse axonal injury (DAI), the impact-acceleration model. We show, despite the suggestion that this model represents concussive injury, no functional impairments as determined using common measures of motor, sensorimotor, cognitive, and neuropsychiatric function following injury over the course of one week. The lack of functional deficits is in sharp contrast to neuropathologic findings indicating neural degeneration, astrocyte reactivity, and microglial activation. Future studies are needed to identify functional assessments, neurophysiologic techniques, and imaging assessments more apt to distinguish differences following so-called 'mild' traumatic brain injury (TBI) in preclinical models and determine whether these models are truly studying concussive or subconcussive injury. These studies are needed to not only understand mechanisms of injury and production of subsequent deficits, but also for rigorous evaluation of potential therapeutic agents.
\end{abstract}




\subsection{INTRODUCTION}

The diagnosis of concussion remains challenging due to the lack of definitive diagnostic tests such as imaging, neuropsychological assessments, accelerometer recordings ${ }^{653}$, or the identification of biomarkers. While advances have been made in the research environment in many of these areas as evidenced by the advent of diffusion tensor imaging (DTI) for imaging axonal injury 440,654 and the identification of biomarkers such as neuron-specific enolase, $\mathrm{S100B}$, and glial fibrillary acidic protein, clinical diagnosis of concussion continues to rely largely on the self-reported symptoms of the patient 653,655 . Despite this reliance on clinically observed and measured, or self-reported, symptoms for the diagnosis of concussion in humans, preclinical studies modeling mild traumatic brain injury (TBI) or concussion continue to emphasize histologic measures of injury for characterizing model severity, revealing a clear disconnect between preclinical and clinical studies. Consequently, it remains unclear the precise severity of preclinical models and how accurately these models replicate concussive injury in humans. While variations of the fluid percussion injury model, a commonly used preclinical model of TBI suspected to potentially replicate concussion with the use of 'mild' settings, have been characterized to varying degrees using both behavioral and histological endpoints, the same cannot be said of the impact-acceleration model, another widely used preclinical model of TBI and one that has been described as potentially more relevant based on the ability to model closed head injury and acceleration-deceleration biomechanics associated with concussion ${ }^{656,657}$. Furthermore, this model produces significant diffuse axonal injury, a common pathological feature of concussion and one that may correlate to cognitive performance ${ }^{658}$. As the diagnosis of concussion clinically represents a symptom-based diagnosis without the presence of gross anatomical changes, it remains unclear how adequate current preclinical models are at replicating a concussive-like state and associated pathophysiology. This deficiency will likely limit meaningful translation of therapeutics and preventative measures from bench-to-bedside and for these reasons, the suggestion has been made that studies should clearly characterize how concussion is defined within the context of a preclinical model with regards to behavioral deficits produced ${ }^{656}$.

\subsubsection{Clinical Diagnosis of Concussion}

The definition of concussion has evolved over time with recent work and policy statements moving away from previous definitions that required a period of loss of consciousness to one in which concussion is described as a temporary disturbance in function associated with a complex pathophysiological process induced via impact or trauma to the head or body 659,660 . Despite reports indicating just short of four million concussions occurring yearly in the United States, diagnosis relies on self-reported symptoms with no definitive diagnostic test available and specifically, no structural injury detected with standard neuroimaging protocols ${ }^{660}$. Commonly reported symptoms include physical, cognitive, emotional, and sleep-related complaints ranging from headaches to dizziness to difficulty concentrating or confusion to irritability, sadness, and changes in sleep patterns ${ }^{659}$. Other symptoms related to mood disorders, namely anxiety and depression, have also been reported following concussion, but are frequently difficult to distinguish from larger neuropsychiatric disorders and therefore the clinical significance is difficult to interpret ${ }^{661-663}$.

As such, it is clear that the clinical diagnosis of concussion relies on behavioral and functional changes rather than any imaging or histological findings. This is in sharp contrast to preclinical studies in which injury severity is based largely on histological 
findings and mortality rates, while functional outcomes and behavioral analyses, if even included, are not emphasized.

\subsubsection{Preclinical Functional and Behavioral Assessments of TBI}

While proposed diagnostic tools in the clinic for concussive injury have grown increasingly technically advanced, particularly when considering neuroimaging and neuropsychological testing, preclinical assessments used in TBI models have historically been largely simplistic in nature. Frequently measured modalities include sensorimotor assessments, learning and memory, and less frequently, anxiety-like or depressive-like behaviors. Increasing emphasis has been placed in recent years on the incorporation of more advanced tests and those addressing neurobehavioral disorders as these are one of the most prominent long-term consequences of TBI exposure clinically ${ }^{661}$. Motor function can be assessed with activity measurements and open field exploration while balance/coordination has been assayed using the rotarod, inclined plane, beam walk, and balance beam ${ }^{663-673}$. Learning and memory have been evaluated following TBI using various iterations of the Morris water maze $528,658,665,668,673-686$, radial arm maze 665, 670, 672, T-maze 686,687, and avoidance tasks 673, 686, 688, 689. Anxiety-like measures utilized previously include the elevated-plus maze and measures of peripheral versus central exploratory activity within the open field test $658,663,672,685,689-693$. Depressive-like activity has been assessed in the forced swim test and tail suspension test $673,685,686,688$, $689,692,694$. How these various behavioral measures are affected following TBI is dependent on numerous factors including, but not limited to, the severity of injury, location of injury, type of injury (global versus focal, open versus closed), age at time of injury, and the length of recovery period prior to assessment. Characterization of these deficits or changes following injury may allow for better understanding of model severity and relationship to clinical practice rather than the more commonly utilized histological measures that are limited to controlled preclinical studies.

\subsubsection{Role of Aging in Concussive Injury}

Preclinical studies have largely neglected to consider the role of the aging process in the severity of injury sustained and subsequent recovery characteristics. While TBI is generally thought to be a condition associated with the adolescent and young-adult population, the prolonged careers of athletes and military personnel, two populations at high risk for sustaining a concussive injury, alike in recent years necessitates the consideration of age as an experimental variable or paradigm. Previous preclinical work assessing the effect of aging on outcome from TBI, as well as other forms of neurologic injury, has generally revealed a greater initial injury severity and prolonged deficits in comparison to young-adult animals 525,526 . These studies largely utilized models requiring craniotomy 525, 659, 668 rather than simulating true closed-head injury more analogous to that sustained on the athletic or battlefield in the form of a concussion. Consequently, it remains unclear how age alters functional and histological outcomes following diffuse axonal injury.

\subsubsection{Summary}

In this work, we address the discrepancy between clinical diagnosis of concussion and the potential role of functional and behavioral measures in the preclinical study of TBI and more specifically, DAl produced using the impact-acceleration model. We also investigate the effect of aging on the injury process and how this may influence behavioral and functional deficits. We perform a basic characterization of neural injury 
following impact-acceleration injury and relate observed changes to those, or lack thereof, observed with behavioral/functional studies.

\subsection{MATERIALS AND METHODS}

\subsubsection{Animals}

Two age groups, one 3-4 months $(n=16)$ and the other 9-12 months $(n=15)$, of male, Sprague Dawley rats were utilized in this work. All animals utilized in this work were acquired from Hilltop (Hilltop Lab Animals, Inc.; Scottsdale, Pennsylvania) at 3-4 months of age and housed in the West Virginia University vivarium for aging when required. Animals were given standard rat chow and water ad libitum. All work involving live animals was approved by the Institutional Animal Care and Use Committee of West Virginia University and was performed according to the principles of the Guide for the Care and Use of Laboratory Animals, published by the Institute of Laboratory Resources, National Research Council (National Institutes of Health publication 85-23-2985).

\subsubsection{Induction of Traumatic Brain Injury}

Each age cohort of animals was divided into two groups, with animals receiving either a sham surgery or impact-acceleration injury. Anesthesia was induced and maintained using isoflurane at a $4 \%$ and $2 \%$ concentration, respectively. Adequate anesthesia was confirmed by evaluating the response to heel tendon pinch. Body temperature was controlled during the approximately 10-minute procedure with a homeothermic heating blanket and rectal probe. Animals were prepared in sterile fashion and received an impact-acceleration injury as described previously 436,580, 585. Briefly, a $10 \mathrm{~mm}$ diameter and $3 \mathrm{~mm}$ thick stainless steel disk was affixed to the skull between bregma and lambda with cyanoacrylate. The animal was placed in a prone position on a foam bed with the metal disk directly beneath a $2 \mathrm{~m}$ tall plexiglass tube. A 450-gram weight was dropped from the top of the tube, striking the metal disk. The disk was then removed, the skull inspected, and the wound sutured. The animal was then returned to its cage and monitored post-operatively.

\subsubsection{Functional Characterization}

\subsubsection{Locomotor Activity}

Total activity was assessed using an automated activity monitoring system (San Diego Instruments; San Diego, California) that records photobeam breaks in the $x, y$, and $z$ planes. Animals were acclimated to the room for one hour prior to initiation of testing. Each testing chamber consists of a square Plexiglass housing and $16 \times 16$ photobeam array to detect lateral movements. An $8 \times 8$ array, located above the $16 \times 16$ array, detects rearing-associated movements. Activity was quantified over 30 minutes with fine, ambulatory, and rearing photobeam breaks summed to produce the total photobeam breaks.

\subsubsection{Rotarod}

Two versions of rotarod testing were conducted, a fixed speed and an accelerating variant. All studies were conducted using the OMNI-ROTOR (Omnitech Electronics, Inc.; Columbus, Ohio). Rats were trained over a period of five consecutive days with 5 trials per day. Animals were placed on the rotarod and the rotarod was accelerated to a 
maximum of 24 RPM over a period of 20 seconds. Once reaching 24 RPM, the maximum time allowed per trial was 60 seconds.

For the fixed speed testing, animals were tested at speeds of $12,16,20,24,28,32,36$, and 40 RPM for up to 60 seconds. Three trials were conducted at each speed with a rest period of 30 minutes between each trial. For accelerated testing, the rotarod was set to reach a maximum speed of 50 RPM over a period of four minutes with a total test duration of five minutes per trial. The accelerated test was repeated twice for each animal. For both fixed and accelerating tests, the latency to fall was recorded.

\subsubsection{Elevated Plus Maze}

The elevated plus maze, a measure of anxiety-related activity, was conducted as previously described 533,544 . Animals were placed one at a time in the center of the maze, facing an open arm. Data was recorded over a period of five minutes using ANYMaze Version 4.63 video tracking software (Stoelting Co; Wood Dale, Illinois.). Specifically, time spent in open versus closed arms, number of entries into each arm type, speed within given arms, and distance traveled was recorded. A body percentage of $90 \%$ was required to be in a given arm to register a change in arm presence.

\subsubsection{Forced Swim Test}

The forced swim test, described at length by Porsolt et al, has been used as a measure of depressive-like activity 534,535 . Animals were acclimated to the testing room for 60 minutes prior to initiation of testing. On the day before testing, animals were habituated to the water-filled testing cylinder for seven minutes, a length of time consistent with the testing period. During the testing period, animals were placed in the water for a total of sevenminutes, the first two of which served as an acclimation period. Data related to immobility time, speed, distance traveled, and number of immobile episodes was recorded over the subsequent five minutes using ANY-Maze Version 4.63 video tracking software.

\subsubsection{Morris Water Maze}

The Morris water maze was used to assess memory as previously described. Animals were trained prior to induction of injury and tested post-injury for the ability to recall the platform location. All trials were conducted in a 182-cm diameter pool using Videomex 4.50 tracking software (Columbus Instruments, Columbus, Ohio) for data acquisition. The water temperature was maintained at $19-22{ }^{\circ} \mathrm{C}$ and a platform $(12 \mathrm{~cm} \times 12 \mathrm{~cm})$ made of plexiglass was used. Animals were trained on Days 1-6 via a series of four trials per day, each from a different location around the pool, and each with a maximum time of two minutes. Once animals found the platform, 15 seconds was allowed to elapse for spatial acquisition to occur. If animals were unsuccessful in finding the platform after two minutes, they were placed on the platform for 15 seconds. After induction of injury, on Day 1, a one-trial test with the platform in place was utilized to assess memory. Once the platform was discovered, animals were removed immediately to prevent additional learning. On Day 6, a traditional probe trial was performed in which the platform was removed and time spent in each quadrant and the counter zone recorded.

Functional testing was performed beginning 24 hours post-injury over a one-week period. Testing was spread over various days to minimize the level of exertion required by the animal in an effort to reduce the potential for fatigue, consistent with the work of 
Shultz and colleagues ${ }^{658}$. Assays requiring more extensive exertion or those of longer duration were the only task completed on a given day. At 24 hours post-injury, animals underwent activity monitoring and a single trial in the Morris water maze. At 48 hours, the elevated-plus maze and the first rotarod fixed speed test were conducted. At 72 hours, the accelerating rotarod test was conducted. At 96 hours, the forced swim test was performed and at 120 hours, activity monitoring was conducted. The final day prior to sacrifice, Day 6 (144 hours), the probe trial in the water maze was performed along with the second fixed speed rotarod test.

\subsubsection{Histology}

\subsubsection{Tissue Preparation}

At the conclusion of functional testing, seven days after injury, all surviving animals were anesthetized and immediately perfused transcardially with physiologic saline followed by 4\% paraformaldehyde. The entire brain, brainstem, and rostral spinal cord were extracted and placed in 4\% paraformaldehyde for 24 hours. Following fixation, the brain was blocked into $2 \mathrm{~mm}$ thick sections and processed using the Tissue-Tek VIP 5 Automatic Tissue Processor (Sakura Finatek; Torrence, CA). Processed tissues were paraffin-embedded with the Tissue-Tek Tec 5 embedding system (Sakura Finatek,; Torrence, CA) and sliced $(6 \mu \mathrm{m})$ using a Leica RM2235 microtome (Leica Microscopes, Buffalo Grove, IL). Sections were mounted on glass slides and heat-fixed. Immediately prior to staining, tissues were de-paraffinized using a series of xylene and alcohol washes as described previously ${ }^{623}$.

\subsubsection{Fluoro-Jade $B$ (FJB) Staining}

FJB staining was used to identify neural degeneration. For FJB labeling, slides were incubated in $0.06 \%$ potassium permanganate for 10 minutes following rehydration through a series of alcohol and deionized $\left(\mathrm{dH}_{2} \mathrm{O}\right)$ water rinses. After incubation in potassium permanganate, slides were rinsed for 2 minutes in $\mathrm{dH}_{2} \mathrm{O}$ water and then incubated for 20 minutes with FJB in $0.1 \%$ acetic acid. Slides were washed three times in $\mathrm{dH}_{2} \mathrm{O}$.

\subsubsection{Glial Fibrillary Acidic Protein (GFAP) Staining}

Slides were incubated in rabbit anti-cow GFAP antibody (Dako, Glostrup, Denmark) at a dilution of 1:500 in 4\% horse serum in dulbecco's phosphate-buffered saline (DPBS) overnight. Tissues were then washed 3 times in DPBS and incubated in biotinylated anti-rabbit immunoglobulin G (IgG) antibody (Vector Laboratories, Burlingame, CA) diluted at 1:10,000 in 4\% horse serum in DPBS for 4 hours. Following secondary antibody incubation, Avidin D horseradish peroxidase (HRP) (Vector Laboratories, Burlingame, CA) diluted at 1:1,000 in DPBS for 1 hour was applied. 3,3'diaminobenzidine (DAB) chromagen solution (Vector Laboratories, Burlingame, CA) was applied for 5 minutes. Tissues were then rinsed 3 times in DPBS and dried overnight.

\subsubsection{Ionized Calcium Binding Adapter Molecule 1 (IBA-1) Staining}

IBA-1 labeling of activated microglia utilized an identical protocol as that used for GFAP staining with the following exceptions: the primary antibody was rabbit anti-lba 1 (Wako Chemicals USA, Richmond, VA) at a dilution of 1:500 and the chromagen solution was Nova Red (Vector Laboratories, Burlingame, CA). 


\subsubsection{Statistical Analysis}

Data were analyzed using GraphPad Prism version 4.0. One-way analysis of variance (ANOVA) with Tukey's post-hoc tests were performed to determine differences between experimental groups on the accelerating rotarod, elevated plus maze, forced swim test, and Morris water maze. Two-way ANOVA with repeated measures was utilized for fixed speed rotarod testing analysis. A p-value of $<0.05$ was considered statistically significant.

\subsection{RESULTS}

10.3.1. Diffuse Axonal Injury Induced Using the Impact-Acceleration Model Produces Neural Degeneration, Reactive Astrocytes, and Microglial Activation in Both Age Groups.

Fluoro-Jade B staining of brain sections revealed diffuse degeneration of neurons throughout the cortex and striatum as indicated by increased fluorescent labeling (Figure 10.1). While injury induced clear degeneration in both age groups examined in comparison to control (Figure 10.1a,c), young-adult specimens (Figure 10.1b) exhibited qualitatively diminished damage whereas older animals exhibited a qualitatively more severe response and level of damage (Figure 10.1d). A diffuse proliferation and hypertrophy of astrocytes, visualized using GFAP staining, was also seen across tissue regions in young and old groups relative to control (Figure 10.2). Microglial activation was enhanced post-injury in both age groups as indicated by lba-1 staining (Figure 10.3). Microglia in the injured brain exhibit increased ramification of processes as well as overall hypertrophy.

10.3.2. Impact-Acceleration Injury Fails to Produce Deficits in Total Activity and Motor Coordination Across Multiple Time Points in Either Age Group up to One-Week PostInjury.

Analysis using a one-way ANOVA and Tukey's post-hoc of total activity indicated by beam breaks revealed no significant difference between injury and control groups within respective age groups, at both Day 1 (Figure 10.4a) and Day 5 (Figure 10.4b) postinjury, although differences were observed across age groups on Day 1 [Day 1: $F(3,29)$ $=10.26, P<0.05$; Young: $q=2.07, P>0.05$; Old: $q=2.78, P>0.05$ and Day 5: $F(3,29)=$ 2.12, $P>0.05$; Young: $q=0.02$, $P>0.05$; Old: $q=1.118, P>0.05]$. More complex motor function or coordination was assessed using two variations of the rotarod - accelerating (Figure 10.4c) and fixed speed (Figure 10.4d,e). The fixed speed test conducted on Day 2 post-injury demonstrated no significant interaction between speed and experimental group using two-way ANOVA and a Bonferroni post-hoc test when comparing within a respective age group (Table 10.1). When repeated on Day 6, the fixed speed test again failed to demonstrate significant deficits between respective age groups (Table 10.2). The accelerating rotarod test, conducted on Day 3 post-injury, did not reveal any deficits across groups $[F(3,23)=1.165, P>0.05$; Young: $q=0.11, P>0.05$; Old: $q=0.22, P>0.05]$.

10.3.3. Diffuse Axonal Injury Does Not Alter Reference Memory in Both Acute and Subacute Periods as Determined Using the Morris Water Maze.

Acute analysis of reference memory using the Morris water maze at 24 hours post-injury (Figure 10.5) showed no deficits as a consequence of injury in either the young or aged groups $[F(3,27)=3.78, P>0.05$; Young: $q=2.58, P>0.05$; Old: $q=2.62, P>0.05]$. Similar findings to those at 24 hours were observed in a probe trial conducted on Day 6 post- 
injury. Specifically, no difference between the injured and non-injured groups within respective age groups was seen with regards to time spent in the counter region, the region in which the platform previously was placed $[F(3,27)=0.58, P>0.05$; Young: $q=1.61, P>0.05$; Old: $q=0.01, P>0.05]$.

\subsubsection{Measures of Anxiety-like and Depressive-like Behaviors Are Unaltered Following} Injury.

The elevated-plus maze, a commonly utilized measure of anxiety-like behavior, failed to show differences between control and injured animals within respective age groups on Day 2 post-injury (Figure 10.6a,b). Time spent in the open arms $[F(3,29)=5.00, P<0.05$; Young: $q=0.55, P>0.05$; Old: $q=3.00, P>0.05]$ and number of entries into the open arms $[F(3,29)=2.15, P>0.05$; Young: $q=0.05, P>0.05$; Old: $q=1.97, P>0.05]$ were specifically analyzed. The forced swim test, a measure of depressive-like activity, was conducted on Day 4 post-injury. Again, no significant differences were observed between injured and control groups within respective age groups (Figure 10.6c,d). Both total immobility time $[F(3,28)=6.67, P<0.05$; Young: $q=0.33, P>0.05$; Old: $q=3.70, P>0.05]$ and total number of immobile episodes $[F(3,28)=1.02, P>0.05$; Young: $q=0.12$, $P>0.05$; Old: $q=0.28 ; P>0.05]$ were assessed.

10.3.5. Aging Alters Select Behavioral Measures at Baseline and Post-injury in Male Animals.

While no differences were observed in total activity within respective age groups at both 24 hours and Day 4, comparisons across age groups showed reduced activity in the older cohort of animals subjected to injury when compared to both younger control animals $[F(3,29)=10.26, P<0.05 ; q=7.21, P<0.0001]$ and younger injured animals $[F$ $(3,29)=10.26, P<0.05 ; \mathrm{q}=5.95, \mathrm{P}<0.05]$ at 24 hours post-injury (Figure 10.4a). At Day 4 post-injury, no difference in total activity was observed between any groups (Figure 10.4b). Evaluation of fixed-speed rotarod performance revealed a similar phenomena in that at Day 2 post-injury (Figure 10.4c), no difference was observed within respective age groups or between control groups, but older injured animals performed worse than both younger controls (12 RPM: $t=2.73, P>0.05 ; 16$ RPM: $t=1.83, P>0.05 ; 20$ RPM: $\mathrm{t}=2.31, \mathrm{P}>0.05 ; 24$ RPM: $\mathrm{t}=1.41, \mathrm{P}>0.05 ; 28$ RPM: $\mathrm{t}=3.15, \mathrm{P}<0.05 ; 32 \mathrm{RPM}: \mathrm{t}=3.80$, $\mathrm{P}<0.01$; 36 RPM: $\mathrm{t}=3.27, \mathrm{P}<0.05 ; 40 \mathrm{RPM}: \mathrm{t}=2.14, \mathrm{P}>0.05)$ and younger injured animals (12 RPM: $t=2.65, P>0.05 ; 16$ RPM: $t=1.22, P>0.05 ; 20$ RPM: $t=1.63, P>0.05 ; 24$ RPM: $t=1.26, P>0.05$; 28 RPM: $t=3.14, P<0.05 ; 32$ RPM: $t=3.34, P<0.01 ; 36$ RPM: $t=3.29$, $P<0.01$; 40 RPM: $t=2.25, P>0.05)$ at higher speeds. On Day 6, deficits were still observed when contrasting older injured animals with both younger controls (12 RPM: $t=0.34, P>0.05 ; 16$ RPM: $t=0.03, P>0.05 ; 20$ RPM: $t=0.09, P>0.05 ; 24 R P M: t=1.29$, $P>0.05$; 28 RPM: $t=2.47, P>0.05 ; 32$ RPM: $t=2.40, P>0.05 ; 36$ RPM: $t=2.58, P>0.05 ; 40$ $R P M: t=3.41, P<0.01$ ) and younger injured animals (12 RPM: $t=0.12, P>0.05 ; 16$ RPM: $t=0.67, P>0.05 ; 20$ RPM: $t=0.96, P>0.05 ; 24$ RPM: $t=0.63, P>0.05 ; 28 R P M: t=2.26$, $\mathrm{P}>0.05$; 32 RPM: $t=2.97, \mathrm{P}<0.05$; $36 \mathrm{RPM}: \mathrm{t}=2.97, \mathrm{P}<0.05 ; 40$ RPM: $\mathrm{t}=3.12, \mathrm{P}<0.05)$. On the accelerating version of rotarod testing, neither aging nor injury affected the latency to fall $[F(3,23)=1.17, P>0.05]$.

Reference memory at 24 hours was reduced in the older injured group in comparison to the younger injured group as determined by latency to platform measurements using the Morris water maze $[F(3,27)=3.78, P<0.05 ; \mathrm{q}=4.65 ; \mathrm{P}<0.05]$. On Day 6 post-injury, no differences were seen between any of the control or treatment groups with regard to time spent in the counter region, irrespective of age $[F(3,27)=0.58, P>0.05]$. 
Aging appears to alter anxiety-like and depressive-like behaviors as indicated by the elevated-plus maze and forced swim test, respectively. Older control animals spent more time in the open arm of the elevated-plus maze than both younger controls $(q=4.82, P<0.05)$ and younger injured animals $(q=4.81, P<0.05)$. No difference was observed when comparing younger and older injured groups $(q=2.09, P>0.05)$. In the forced swim test, older injured animals spent more time immobile than both younger controls $(q=4.72, P<0.05)$ and younger injured animals $(q=5.79, P<0.05)$.

\subsection{DISCUSSION}

We have demonstrated a lack of functional and behavioral deficits despite evidence of histologic changes utilizing the impact-acceleration model of TBI, and more specifically, diffuse axonal injury (DAI). Preclinical studies of TBI and DAI have consistently emphasized histologic measures for assessing the severity of injury despite the fact that concussion is a clinical diagnosis based on the observation or reporting of somatic as well as cognitive or neuropsychologic function. It would seem logical then that preclinical studies need to incorporate functional assays as not only an experimental endpoint but also to reconcile preclinical models with the severity and clinical relevance of injury being studied. Others have explored this concept, primarily using the fluid percussion model of TBI, and have shown impaired hippocampal spatiotemporal representation in the absence of histological changes ${ }^{687}$. Similarly, a study using lowlevel fluid percussion injury has identified the presence of neuroinflammation after injury despite no difference in axonal injury and no functional impairments ${ }^{695}$.

By observing no effect of injury on an array of behavioral and functional measures following impact-acceleration injury, even though changes in injury markers were observed on post-mortem histopathology, additional questions are raised. One, is the impact-acceleration (Marmarou) model utilized herein truly a model of concussive injury or rather, would it be more appropriately termed a model of subconcussive injury? Second, what preclinical behavioral measures are most appropriate for detecting the subtleties of injury progression and how do these emulate and approximate those used clinically? Third, how does symptom expression relate to neurologic health or neurodegeneration? Four, how are neuropathological findings and functional analysis altered by aging?

\subsubsection{Subconcussive Injury: An Emerging Phenomenon}

Emerging evidence indicates the presence of long-term neurodegeneration upon neuropathological examination of persons with no history of concussion 411, 417 . Importantly, Talavage and colleagues have identified for the first time persons failing to exhibit symptoms associated with concussion but clear deficits on neurocognitive (ImPACT) and neuropsychologic (fMRI) measures ${ }^{448}$. This work, completed in high school football players, indicates changes in neurologic function and health without evidence of concussive injury but rather repetitive subconcussive blows ${ }^{448}$. As these players did not exhibit obvious injury, participation and exposure to further injury continued, consistent with some biomechanical analyses suggesting potential accumulation of injury and altered neurophysiology consistent with the number of subconcussive impacts rather than the shear magnitude 448,449 . The notion of pathological changes occurring in the brain in the absence of clinical presentation of concussion is particularly alarming and indicates the need for further research as does identification of persistent deficits in working memory months after initial TBI 696, 697. 
As clinically measured subconcussive injury likely requires the utilization of increasingly advanced testing modalities such as ImPACT, fMRI, or force accelerometers, translation of this work to preclinical models based on functional assessments will likely necessitate an advanced array of assessment modalities. ImPACT incorporates symptom reporting as well as measurements of verbal and visual memory, processing speed, and reaction time. As such, future preclinical studies may want to incorporate more specific and sensitive studies designed to evaluate similar parameters, when possible, in the rat that may be key in determining concussive versus subconcussive injury. This notion may be true irrespective of preclinical model utilized as our work was consistent with others using the mild fluid percussion injury model in that no functional deficits were observed using locomotor activity, elevated-plus maze, and the forced swim test despite the presence of neuroinflammatory changes ${ }^{695}$. Other measures of behavior, specifically those assessing reaction time and processing or attention deficits, may more closely measure, in preclinical models, the deficits observed in clinic in patients following concussion. While the methodology of these techniques is beyond the scope of this work, various tests assessing these parameters have been extensively reviewed elsewhere 698,699 . The same can be said of more advanced measures of learning and memory such as shuttle avoidance and operant conditioning.

\subsubsection{Symptom Expression as a Correlate of Neuropatholgy}

While the acute effects of clinically defined concussive injury are apparent based upon a combination of self-reported symptoms by the patient and a thorough neurologic assessment by a trained clinician, diagnosis of concussion has historically been viewed as a transient neurologic disturbance with no long-term effects. This view has radically changed in recent years with the emergence of chronic traumatic encephalopathy (CTE), a neurodegenerative condition associated with repetitive head injury - both of the concussive and subconcussive types ${ }^{700}$. CTE is notable in that a history of repetitive head trauma proceeds by years, often decades, development of disease-related symptoms ${ }^{700}$. While the disease pathophysiology remains to be elucidated, it has been hypothesized that the initial brain trauma initiates degenerative processes that emerge slowly over time, hence the existence of a latent period between trauma and symptom emergence ${ }^{432}$. The role the quantity, severity, type, and location of impacts plays in symptom development and severity remain unknown, but it is apparent that more sensitive measures to detect neural damage may be required. In this work, we've shown the lack of acute deficits across a spectrum of functional assays, despite the presence of neuropathological findings. The existence of neural degeneration and glial responses without corresponding symptom expression, albeit using a limited battery of relatively simplistic rodent behavioral tests, indicates the potential for induction of neural damage without clear deficits clinically, at least in the acute period. How deficits develop over time, as well as how neuropathological findings progress, remains to be seen, but may provide further insight into the development of long-term neurodegenerative diseases such as CTE and warrants future study.

Prior work has demonstrated learning impairments utilizing the Morris water maze, a spatial acquisition task, following TBI. These deficits appear largely time and model dependent though as controlled cortical impact has repeatedly been reported to produce acquisition deficits $528,673,674,685$ while fluid percussion injury generally appears to produce impairments only at more moderate and severe levels. In fact, 1.0 atmosphere (atm) fluid percussion injury has been reported to produce histological damage upon microscopic analysis, without any associated water maze deficits ${ }^{668}$. This is in contrast to 1.5 and 1.7-2.0 atm fluid percussion injury producing acquisition and memory-related 
deficits 668,684 and 1.2 atm causing impairment not on original platform trials but in reversal studies ${ }^{658}$. Work using variations of the weight-drop model, some of which produce more linear acceleration while others are rotational in nature, have resulted in reports of similar variability with regards to deficits, with some identifying acquisition deficits at one week post-injury and beyond 675-677,686 whereas others demonstrate deficits only with heavier weights ${ }^{678}$, no deficits in acquisition ${ }^{679}$, or no deficits in memory ${ }^{665}$. Our work is notably different in that we focused on the acute period after injury, based on the period in which patients most commonly report symptoms following concussion, whereas prior studies have generally employed longer periods between injury and functional assessment. Similarly, animals were trained in the acquisition task of the maze prior to injury with memory assessed following injury, hence an evaluation of retrograde amnesia. Retrograde amnesia has been described as likely time-dependent in nature with longer intervals between training and injury allowing for enhanced consolidation and therefore greater likelihood of retention ${ }^{701}$.

Other tests of learning and memory may demonstrate greater sensitivity for detecting deficits or distinguishing between injury severity following neurotrauma. Depending on the location and severity of injury, fluid percussion has been shown to produce deficits in the radial arm maze for as few as 5 days after mild injury, 15 days after moderate injury, and more persistent deficits in others 665,670 . In contrast, a modified weight-drop procedure fails to produce differences in response latency or number of visits to the baited arms or all arms at any time point post-injury, although an increase in working memory errors on Day 3 and reference memory errors at Day 5 post-injury were reported ${ }^{672}$. Non-spatial memory, such as that assessed by the novel object recognition test has successfully demonstrated deficits both acutely and chronically with some ability to distinguish between varying severities or number of impacts in the case of repetitive injury models 673,702 . Passive avoidance and other avoidance tasks have revealed impairments following various forms of injury, namely controlled cortical impact $(\mathrm{CCl})$ and modified weight-drop, at numerous time points, particularly those beyond 1-2 weeks $673,688,689$. Some discrepancy is impairment with one study utilizing a modified weightdrop reporting a lack of deficit at 7,60, and 90 days but diminished memory at 30 days 686. Lastly, other cognitive assessments have also demonstrated utility for distinguishing between sham and injured animals. This includes fear conditioning 663,703 , the swim Tmaze ${ }^{686,687}$, and the dig task ${ }^{686}$. Whether these other techniques for assessing learning and memory would reveal deficits in the acute period using the standard weightdrop model is unclear but may represent more sensitive indices of behavioral assessment going forward.

Furthermore, emerging evidence from clinical studies on concussed patients identifies the potential for neuropsychiatric changes. We investigated anxiety-like and depressivelike behaviors using the elevated plus maze and forced swim test respectively. While we did not identify injury-dependent deficits within respective age groups in the acute period, it is possible the lack of difference between groups is a product of either the time of assessment, model utilized, and/or severity of injury. Previous work has reported contrasting findings with some indicating an increase in anxiety-like behavior 663, 692, 693 whereas others report anxiolytic-like effects of traumatic brain injury 658,685. Notably, multiple studies have identified no difference in anxiety-like behaviors following TBI when assessed within one week of injury $672,690-692$, furthering illustrating the potential timedependency of functional changes following injury. Likewise, injury severity may greatly influence findings as mild modified weight-drop injury has been shown to produce anxiety-like behavior, but the same study failed to demonstrate findings in moderate- and 
severely-injured animals ${ }^{689}$. Depressive-like behavior was reported to increase following modified weight-drop in mice $686,688,689,694$, although this finding was not always consistent over time ${ }^{686}$ and in one instance was detected only on tail suspension but not forced swim test ${ }^{689}$. Interestingly, depressive-like behavior (forced swim test) and anhedonia (sucrose preference test) were not altered following severe fluidpercussion injury (FPI) ${ }^{692}$ while $\mathrm{CCl}$ resulted in depressive-like behavior at both Day 21 and 24 post-injury 673,685 .

More simplistic functional tests, mainly those assessing motor and/or balance, have also been used following injury in preclinical models of TBI. These tests display a range of findings with many failing to report deficits with more mild variations of injury or deficits that resolve within a short period of time post-injury ${ }^{663-668,670-672 . ~ M o r e ~ s e v e r e ~ i n j u r y ~}$ models, such as $\mathrm{CCl}$, have been reported as producing lasting deficits ${ }^{673}$.

The frequent lack of functional deficits observed following injury in preclinical models is perhaps most alarming considering the frequently reported histological damage indicating lasting effects of brain injury, even when injury is below the threshold of detection based on commonly used functional and behavioral assays ${ }^{695}$. Clinical studies have identified similar discrepancies between symptom resolution and metabolic disturbances. Specifically, Vagnozzi and colleagues showed a resolution of symptoms by Day 15 post-concussion in all athletes studied, yet metabolite ratios did not normalize until Day $30{ }^{453}$.

\subsubsection{Role of Aging in Neuropathology \& Functional Deficits Post-TBI}

Aging has been attributed, via clinical experience, with poorer outcomes post-TBI ${ }^{528,704}$, 705. Consequently, there has been an increased emphasis on understanding the effects of aging post-TBI in recent years in an effort to improve the translation of therapeutics from bench-to-bedside. In preclinical studies, aging has been associated with an increase in mortality rate as well as prolonged suppression of reflexes 525, 706. Importantly, aging has also been associated with diminished functional performance both before 528,544, and after, injury 525,528,544,681. While the injury models and functional/ behavioral measures utilized across studies vary, it is clear that older animals exhibit diminished learning and memory, and often complex sensorimotor abilities, in comparison to young-adult animals. In work by Mehan and colleagues, 20 month-old animals performed worse in the Morris water maze and beam walking tasks compared to young-adult animals following laterally administered controlled cortical impact injury ${ }^{528,}$

544 . Use of the impact-acceleration model has produced similar findings in that aged animals, despite being subjected to a less severe injury in terms of energy transferred, exhibited a markedly worse impairment in the water maze at 1 week, 3 weeks, and 5 weeks post-injury in comparison to young-adult animals ${ }^{681}$. Others have identified similar deficits in middle-aged animals compared to young-adult animals with cortical contusion producing more severe deficits in the bilateral tactile adhesive removal test and acquisition, as well as memory, of a reference-based task ${ }^{707}$. Similarly, aging has been associated with larger lesion cavities following $\mathrm{CCl} 707$ and an altered biochemical response as demonstrated by age-dependent changes in protein expression post-injury 528,706 . Why the aged brain appears more susceptible to brain injury remains unclear and necessitates further investigation.

\subsubsection{Future Directions}


Future studies need to elucidate the effect of TBI across a spectrum of time points to address the possibility of delayed effects and the development of deficits over time. While this work demonstrates the lack of detectable functional deficits following a single injury using an array of behavioral measures during the acute and subacute period, chronic deficits that may take longer to develop were not studied. Clinically, changes in affect and cognitive abilities generally arise years after sustaining concussive injury, at least in those cases associated with a diagnosis of CTE 417, 422-424, 708. This reality further indicates the need for long-term preclinical studies to assess not only behavioral changes but also pathological changes.

In addition to the need for longer-term single impact studies, perhaps more importantly, long-term studies incorporating repetitive injury are warranted based on clinical indications and injury paradigms potentially experienced on the athletic or battlefields, frequent locations of concussive injury. Unfortunately, at this point in time preclinical models of disease processes associated with a history of repetitive-concussive injury, such as CTE, have not been established. The lack of representative animal models for these increasingly diagnosed conditions, both in terms of functional/behavioral changes as well as histolgic changes observed on post-mortem analysis, has limited elucidation of disease pathophysiology and therapeutic development.

While numerous models have been utilized to study TBI in rodent models, how these models replicate the clinical scenario, particularly of such a heterogeneous injury as TBI, is unclear. Emerging evidence in preclinical as well as clinical literature indicates a potential role for magnetic resonance spectroscopy (MRS) to gain insight into the biochemistry and pathophysiology of the injury process. Similarly, diffusion tensor imaging (DTI) has been utilized for identification of axonal injury post-TBI. Application of technologies such as these to preclinical models may allow for an enhanced understanding of injury pathophysiology and the severity of injury induced using various models.

\subsubsection{Limitations}

Animals were not assessed until 24 hours post-injury to minimize the potential for anesthetic influences on behavior. By waiting until one day post-injury this issue was avoided. Future studies are likely needed to assess the effect of TBI in this immediate post-injury period. Despite the delay in initial assessments, one would have expected if this was truly a model of concussive injury that some deficits may be detected based on the persistence of symptoms clinically beyond one day in many cases, even though $80-90 \%$ of those afflicted with concussion report resolution of symptoms within 7 days. How the recovery period and resolution of symptoms correlates between clinical and preclinical studies remains to be seen. Furthermore, animals were sacrificed at one week post-injury for neuropathological analysis, preventing the study of potentially late developing deficits weeks or months post-injury.

\section{ACKNOWLEDGEMENTS}

The authors would like to thank Dakota Jackson, Ahmad Hanif, and Emily Reilly for assistance with experimental protocols; Dr. Rae Matsumoto for use of testing equipment; and the West Virginia University Microscopy Imaging Facility for assistance with microscopy. The authors would also like to thank Ms. Diana Richardson of CDC NIOSH for assistance in tissue preparation. This work was supported by grant funding to Ryan 
I Turner (5T32-GM08174) and Jason D. Huber (5RO1-NS061954) from the National Institutes of Health. 


\section{FIGURES}

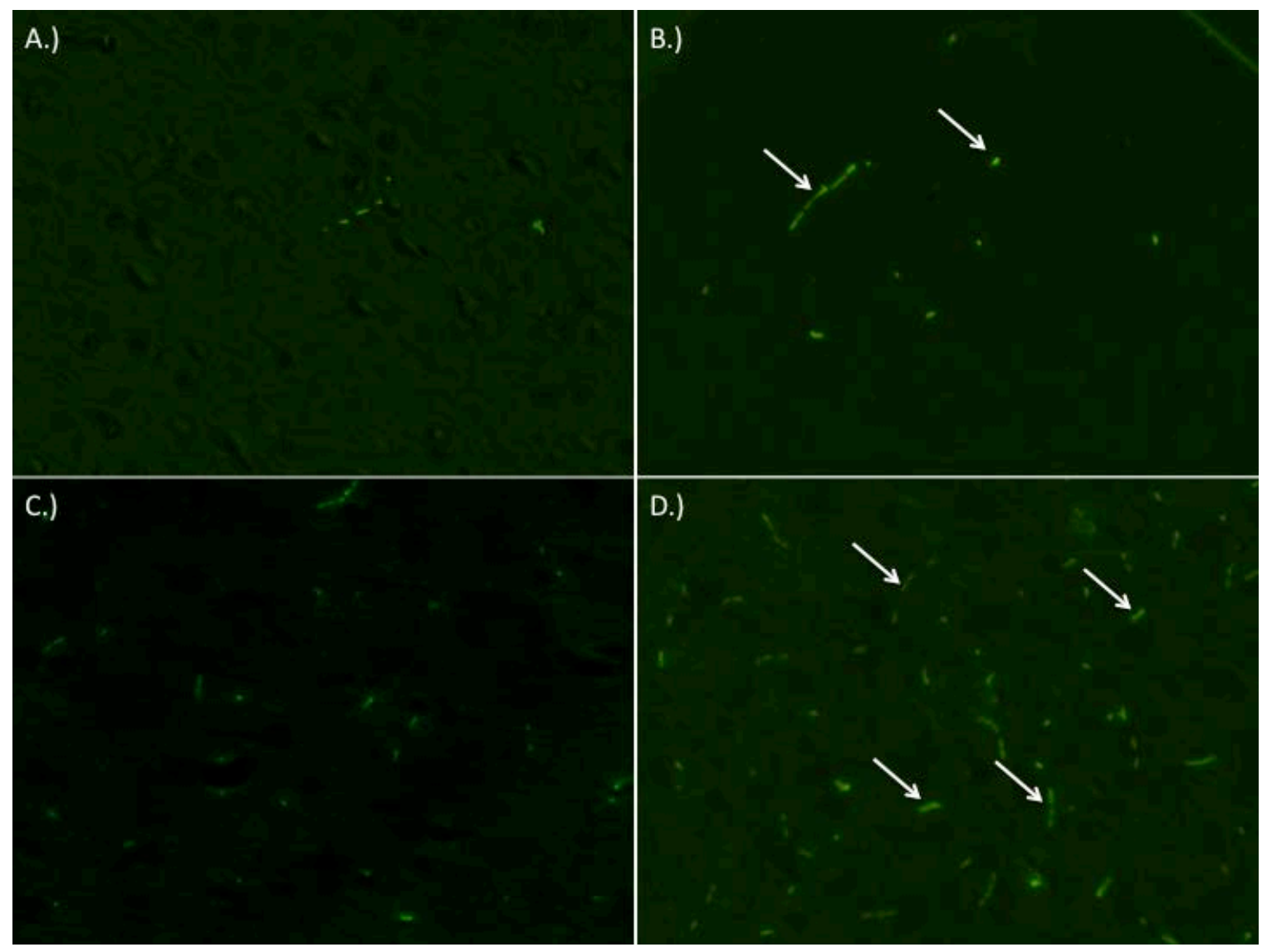

Figure 10.1. Impact-acceleration injury resulted in neural degeneration as evident by Fluoro-Jade B staining in both 3-4 month and 9-12 month-old male rats. Representative sections from sham 3-4 month-old (A), injured 3-4 month-old (B), sham 9-12 month-old (C), and injured 9-12 month-old (D) rats. Arrows indicate degenerating neurons. 


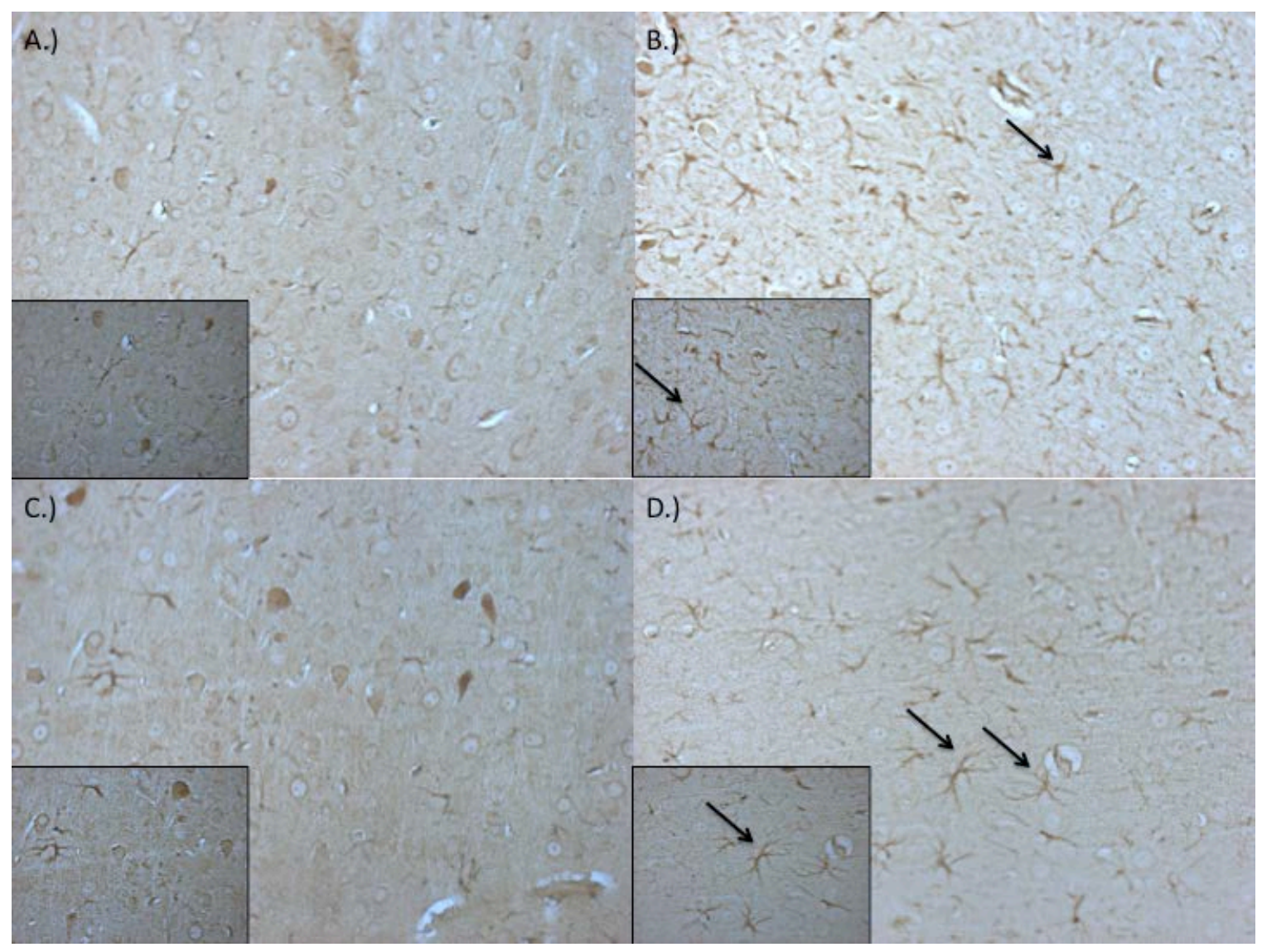

Figure 10.2. Impact-acceleration injury resulted in reactive astrocytes as evident by glial fibrillary acidic protein (GFAP) staining in both 3-4 month and 9-12 month-old male rats. Representative sections from sham 3-4 month-old (A), injured 3-4 month-old (B), sham 9-12 month-old (C), and injured 9-12 month-old (D) rats. Arrows represent reactive astrocytes and insets represent high-powered view of the corresponding panel. 


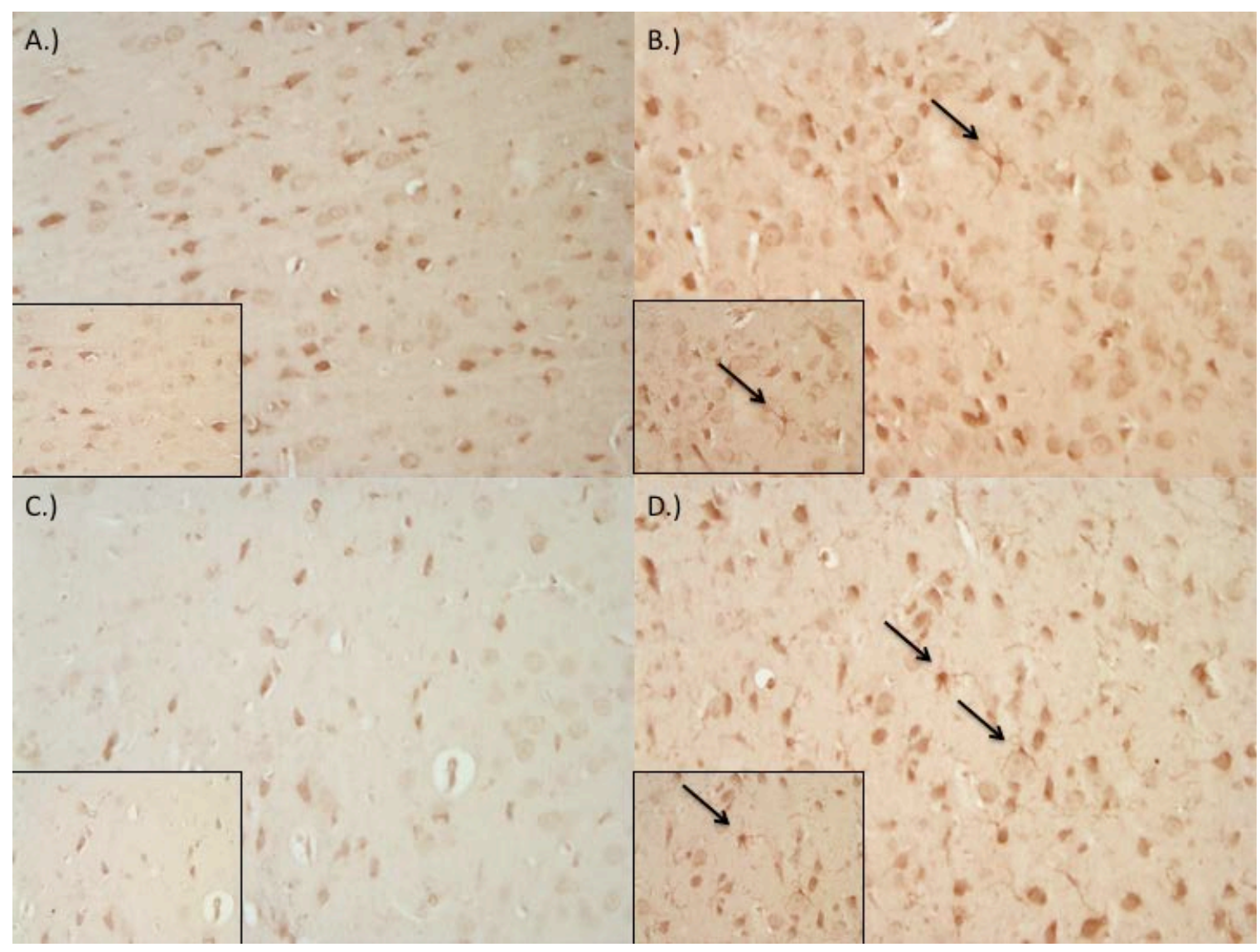

Figure 10.3. Impact-acceleration injury resulted in microglial activation as evident by ionized calcium binding adapter molecule 1 (lba-1) staining in both 3-4 month and 9-12 month-old male rats. Representative sections from sham 3-4 month-old (A), injured 3-4 month-old (B), sham 9-12 month-old (C), and injured 9-12 month-old (D) rats. Arrows represent reactive microglia and insets represent high-powered views of the corresponding panel. 
A.)

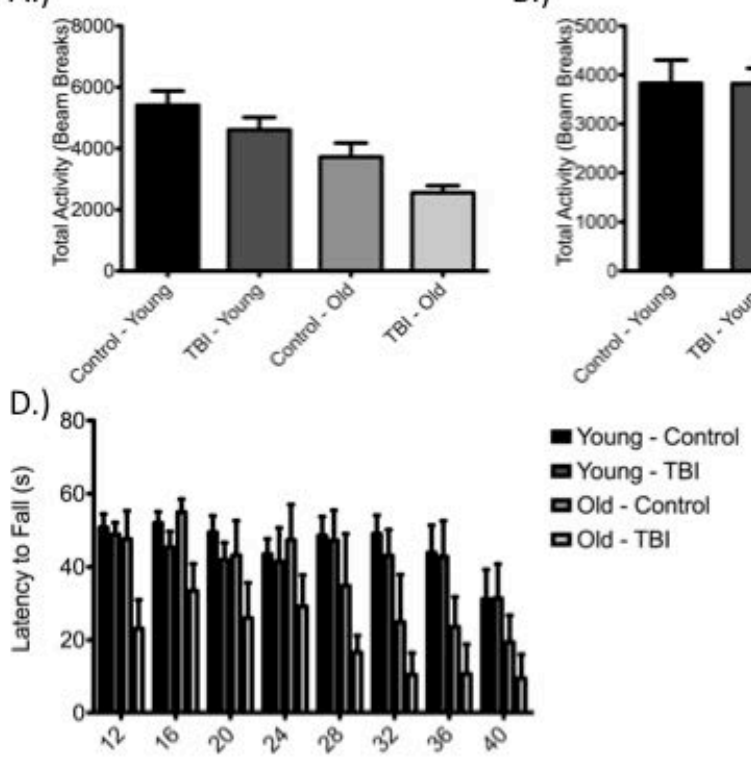

B.)

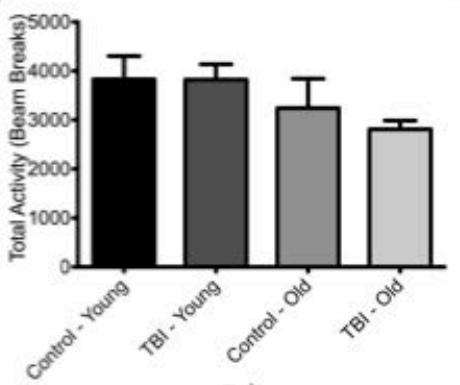

E.)
C.)
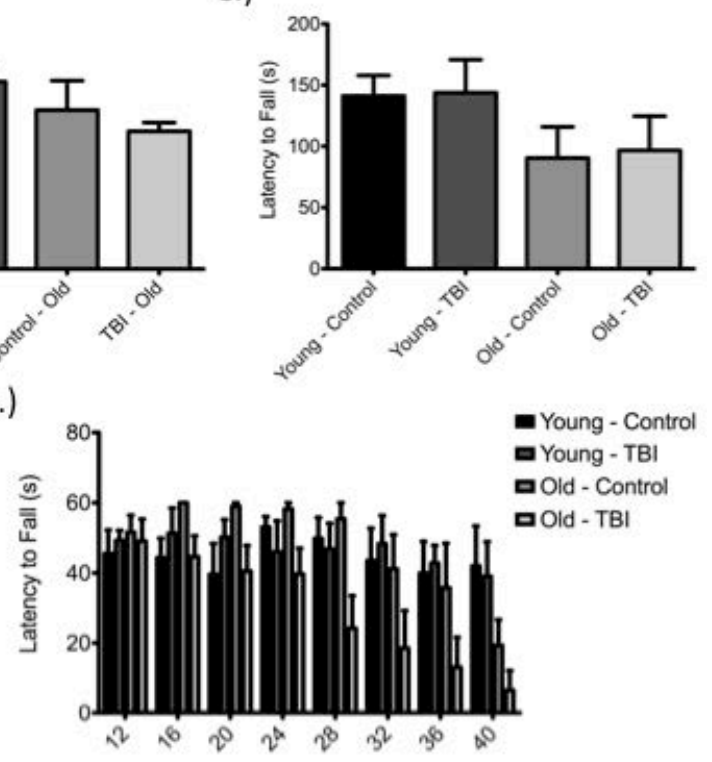

Figure 10.4. Diffuse axonal injury (DAI), produced through impact-acceleration injury, did not result in sensorimotor deficits. Total activity was not altered within respective age groups on Day 1 (A) or Day 5 (B) post-injury. Similar findings were seen in the accelerated rotarod test on Day 3 post-injury $(\mathbf{C})$ and the fixed speed rotarod test conducted on Day 2 (D) and Day 6 (E) post-injury. 
A.)

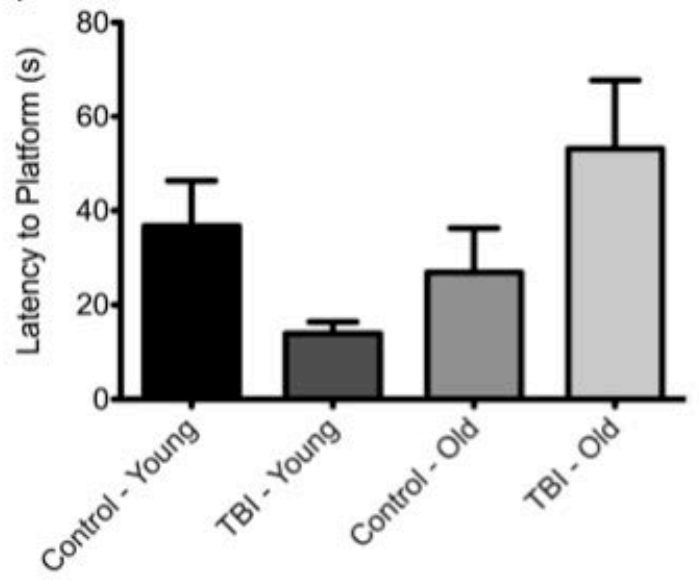

B.)

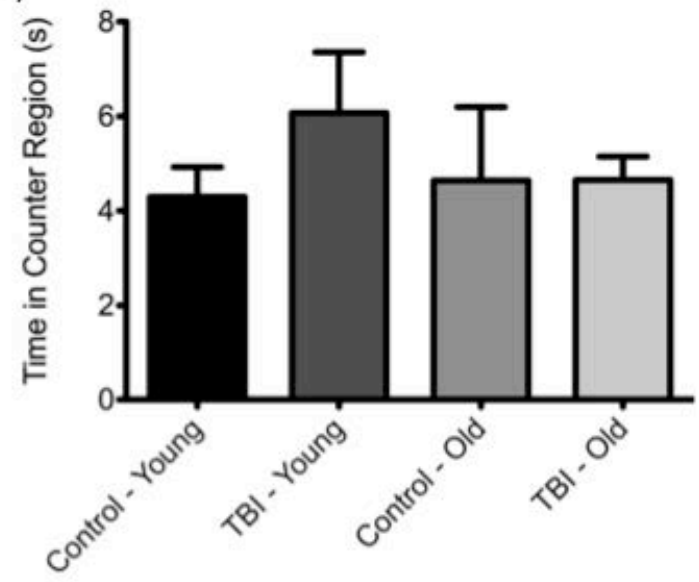

Figure 10.5. Diffuse axonal injury did not produce retrograde amnesia on Day $1(\mathbf{A})$ or Day 6 (B) post-injury as determined using the Morris water maze. 
A.)

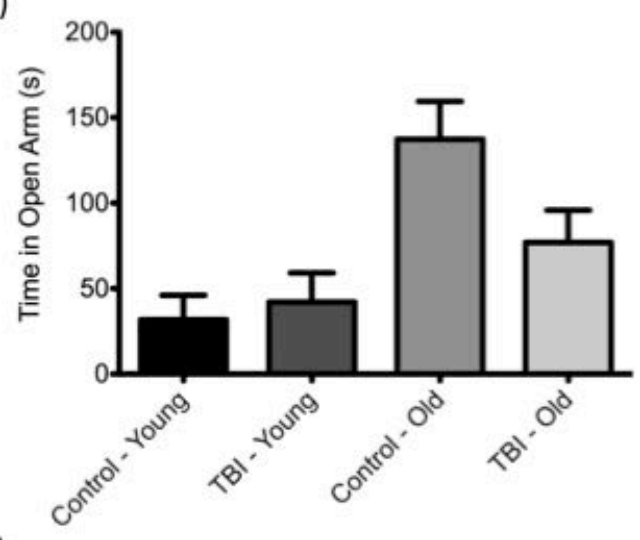

C.)

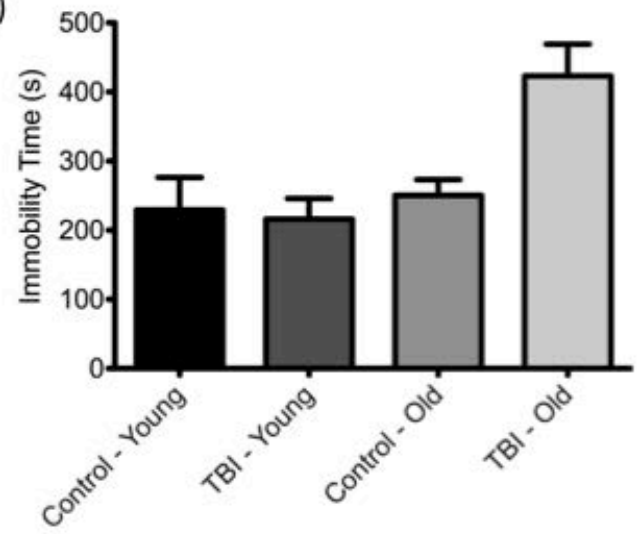

B.)

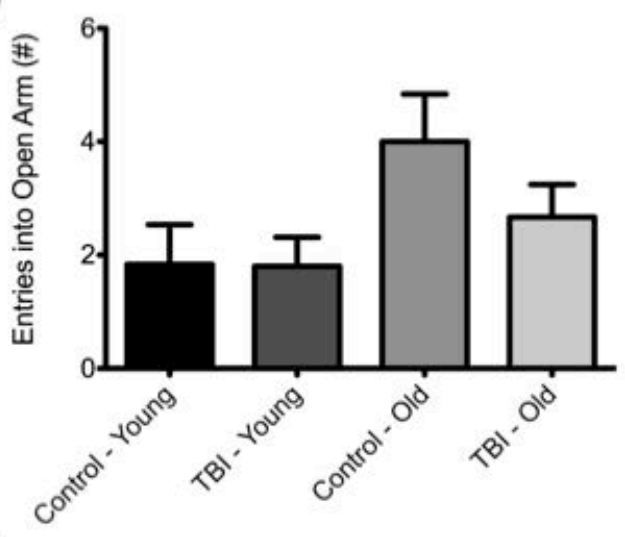

D.)

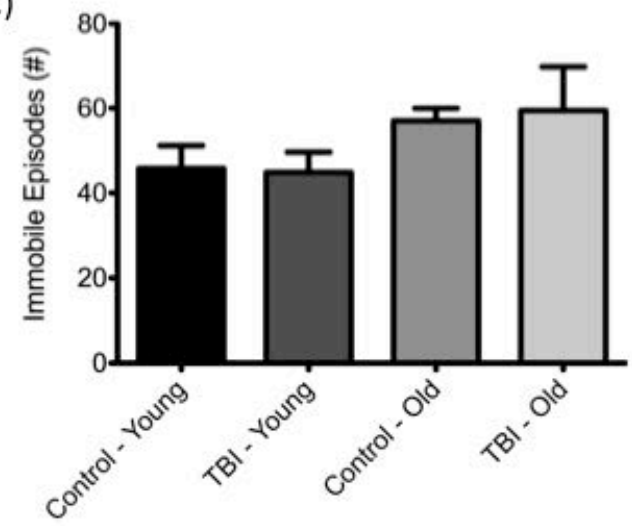

Figure 10.6. Impact-acceleration injury was not associated with changes in anxiety- or depressive-like behavior as determined using the elevated-plus maze and forced swim test, respectively. Both the time spent in open arms $(\mathbf{A})$ and number of entries into open arms (B) were not significantly altered within respective age groups when comparing injured and sham animals. Similar findings were observed in the forced swim test with regards to immobility time (C) and number of immobile episodes (D). 


\section{TABLES}

\begin{tabular}{|c|c|c|c|c|c|c|c|c|c|}
\hline \multicolumn{2}{|c|}{$\begin{array}{l}\text { Experimental } \\
\text { Group }\end{array}$} & \multicolumn{8}{|c|}{ Fixed Speed on Rotarod (RPM) } \\
\hline $\begin{array}{c}\text { Group } \\
\# 1\end{array}$ & $\begin{array}{c}\text { Group } \\
\# 2\end{array}$ & 12 & 16 & 20 & 24 & 28 & 32 & 36 & 40 \\
\hline$Y-I$ & $\mathrm{Y}-\mathrm{S}$ & $\begin{array}{l}t=0.19 \\
P>0.05\end{array}$ & $\begin{array}{l}t=0.66 \\
P>0.05\end{array}$ & $\begin{array}{l}t=0.74 \\
P>0.05\end{array}$ & $\begin{array}{l}t=0.20 \\
P>0.05\end{array}$ & $\begin{array}{l}t=0.13 \\
P>0.05\end{array}$ & $\begin{array}{l}t=0.59 \\
P>0.05\end{array}$ & $\begin{array}{l}t=0.11 \\
P>0.05\end{array}$ & $\begin{array}{l}t=0.02 \\
P>0.05\end{array}$ \\
\hline$Y-1$ & $0-S$ & $\begin{array}{l}t=0.12 \\
P>0.05\end{array}$ & $\begin{array}{l}t=0.85 \\
P>0.05\end{array}$ & $\begin{array}{l}t=0.11 \\
P>0.05\end{array}$ & $\begin{array}{l}t=0.51 \\
P>0.05\end{array}$ & $\begin{array}{l}t=1.09 \\
P>0.05\end{array}$ & $\begin{array}{l}t=1.59 \\
P>0.05\end{array}$ & $\begin{array}{l}t=1.68 \\
P>0.05\end{array}$ & $\begin{array}{l}t=1.05 \\
P>0.05\end{array}$ \\
\hline$Y-1$ & $0-1$ & $\begin{array}{l}t=2.65 \\
P>0.05\end{array}$ & $\begin{array}{l}t=1.22 \\
P>0.05\end{array}$ & $\begin{array}{l}t=1.63 \\
P>0.05\end{array}$ & $\begin{array}{l}t=1.26 \\
P>0.05\end{array}$ & $\begin{array}{l}t=3.14 \\
P<0.05\end{array}$ & $\begin{array}{l}t=3.34 \\
P<0.01\end{array}$ & $\begin{array}{l}t=3.29 \\
P<0.01\end{array}$ & $\begin{array}{l}t=2.25 \\
P>0.05\end{array}$ \\
\hline $\mathbf{Y}-\mathrm{S}$ & $\mathrm{O}-\mathrm{S}$ & $\begin{array}{l}t=0.28 \\
P>0.05\end{array}$ & $\begin{array}{l}t=0.25 \\
P>0.05\end{array}$ & $\begin{array}{l}t=0.54 \\
P>0.05\end{array}$ & $\begin{array}{l}t=0.33 \\
P>0.05\end{array}$ & $\begin{array}{l}t=1.17 \\
P>0.05\end{array}$ & $\begin{array}{l}t=2.05 \\
P>0.05\end{array}$ & $\begin{array}{l}t=1.72 \\
P>0.05\end{array}$ & $\begin{array}{l}t=1.00 \\
P>0.05\end{array}$ \\
\hline $\mathrm{Y}-\mathrm{S}$ & $0-1$ & $\begin{array}{l}t=2.73 \\
P>0.05\end{array}$ & $\begin{array}{l}t=1.83 \\
P>0.05\end{array}$ & $\begin{array}{l}t=2.31 \\
P>0.05\end{array}$ & $\begin{array}{l}t=1.41 \\
P>0.05\end{array}$ & $\begin{array}{l}t=3.15 \\
P<0.05\end{array}$ & $\begin{array}{l}t=3.80 \\
P<0.01\end{array}$ & $\begin{array}{l}t=3.27 \\
P<0.05\end{array}$ & $\begin{array}{l}t=2.14 \\
P>0.05\end{array}$ \\
\hline $0-s$ & $0-1$ & $\begin{array}{l}t=2.14 \\
P>0.05\end{array}$ & $\begin{array}{l}t=1.88 \\
P>0.05\end{array}$ & $\begin{array}{l}t=1.50 \\
P>0.05\end{array}$ & $\begin{array}{l}t=1.58 \\
P>0.05\end{array}$ & $\begin{array}{l}t=1.59 \\
P>0.05\end{array}$ & $\begin{array}{l}t=1.26 \\
P>0.05\end{array}$ & $\begin{array}{l}t=1.13 \\
P>0.05\end{array}$ & $\begin{array}{l}t=0.87 \\
P>0.05\end{array}$ \\
\hline
\end{tabular}

Table 10.1. Fixed speed rotarod testing on Day 2 post-DAl does not reveal deficits between injured and sham animals within respective age groups. Deficits are seen when comparing injured aged animals to younger animals (both control and injured) at higher speeds. 


\begin{tabular}{|c|c|c|c|c|c|c|c|c|c|}
\hline \multicolumn{2}{|c|}{$\begin{array}{l}\text { Experimental } \\
\text { Group }\end{array}$} & \multicolumn{8}{|c|}{ Fixed Speed on Rotarod (RPM) } \\
\hline $\begin{array}{c}\text { Group } \\
\# 1\end{array}$ & $\begin{array}{l}\text { Group } \\
\# 2\end{array}$ & 12 & 16 & 20 & 24 & 28 & 32 & 36 & 40 \\
\hline$Y-1$ & $Y-S$ & $\begin{array}{l}t=0.36 \\
P>0.05\end{array}$ & $\begin{array}{l}t=0.67 \\
P>0.05\end{array}$ & $\begin{array}{l}t=1.01 \\
P>0.05\end{array}$ & $\begin{array}{l}t=0.68 \\
P>0.05\end{array}$ & $\begin{array}{l}t=0.29 \\
P>0.05\end{array}$ & $\begin{array}{l}t=0.46 \\
P>0.05\end{array}$ & $\begin{array}{l}t=0.28 \\
P>0.05\end{array}$ & $\begin{array}{l}t=0.27 \\
P>0.05\end{array}$ \\
\hline$Y-1$ & $0-S$ & $\begin{array}{l}t=0.20 \\
P>0.05\end{array}$ & $\begin{array}{l}t=0.74 \\
P>0.05\end{array}$ & $\begin{array}{l}t=0.76 \\
P>0.05\end{array}$ & $\begin{array}{l}t=1.05 \\
P>0.05\end{array}$ & $\begin{array}{l}t=0.73 \\
P>0.05\end{array}$ & $\begin{array}{l}t=0.61 \\
P>0.05\end{array}$ & $\begin{array}{l}t=0.60 \\
P>0.05\end{array}$ & $\begin{array}{l}t=1.63 \\
P>0.05\end{array}$ \\
\hline$Y-1$ & $0-1$ & $\begin{array}{l}t=0.12 \\
P>0.05\end{array}$ & $\begin{array}{l}t=0.67 \\
P>0.05\end{array}$ & $\begin{array}{l}t=0.96 \\
P>0.05\end{array}$ & $\begin{array}{l}t=0.63 \\
P>0.05\end{array}$ & $\begin{array}{l}t=2.26 \\
P>0.05\end{array}$ & $\begin{array}{l}t=2.97 \\
P<0.05\end{array}$ & $\begin{array}{l}t=2.97 \\
P<0.05\end{array}$ & $\begin{array}{l}t=3.12 \\
P<0.05\end{array}$ \\
\hline $\mathrm{Y}-\mathrm{S}$ & $0-s$ & $\begin{array}{l}t=0.50 \\
P>0.05\end{array}$ & $\begin{array}{l}t=1.30 \\
P>0.05\end{array}$ & $\begin{array}{l}t=1.61 \\
P>0.05\end{array}$ & $\begin{array}{l}t=0.43 \\
P>0.05\end{array}$ & $\begin{array}{l}t=0.46 \\
P>0.05\end{array}$ & $\begin{array}{l}t=0.19 \\
P>0.05\end{array}$ & $\begin{array}{l}t=0.35 \\
P>0.05\end{array}$ & $\begin{array}{l}t=1.88 \\
P>0.05\end{array}$ \\
\hline $\mathrm{Y}-\mathrm{S}$ & $0-1$ & $\begin{array}{l}t=0.34 \\
P>0.05\end{array}$ & $\begin{array}{l}t=0.03 \\
P>0.05\end{array}$ & $\begin{array}{l}t=0.09 \\
P>0.05\end{array}$ & $\begin{array}{l}t=1.29 \\
P>0.05\end{array}$ & $\begin{array}{l}t=2.47 \\
P>0.05\end{array}$ & $\begin{array}{l}t=2.40 \\
P>0.05\end{array}$ & $\begin{array}{l}t=2.58 \\
P>0.05\end{array}$ & $\begin{array}{l}t=3.41 \\
P>0.05\end{array}$ \\
\hline $0-s$ & $0-1$ & $\begin{array}{l}t=0.22 \\
P>0.05\end{array}$ & $\begin{array}{l}t=1.31 \\
P>0.05\end{array}$ & $\begin{array}{l}t=1.57 \\
P>0.05\end{array}$ & $\begin{array}{l}t=1.58 \\
P>0.05\end{array}$ & $\begin{array}{l}t=2.67 \\
P>0.05\end{array}$ & $\begin{array}{l}t=1.93 \\
P>0.05\end{array}$ & $\begin{array}{l}t=1.93 \\
P>0.05\end{array}$ & $\begin{array}{l}t=1.09 \\
P>0.05\end{array}$ \\
\hline
\end{tabular}

Table 10.2. Fixed speed rotarod testing on Day 6 post-DAl does not reveal deficits between injured and sham animals within respective age groups. Deficits are seen when comparing injured aged animals to younger animals (both control and injured) at higher speeds. 


\section{Chapter Eleven}

\subsection{SUMMARY}

In summary, the studies that have been presented herein demonstrate the following:

- Modulating protein kinase c (PKC) via bryostatin-1 administration reduces mortality, infarct volume, and improves functional outcome following stroke.

- APT102 safely extended the therapeutic window for tPA in a clinically relevant animal model of ischemic stroke while significantly reducing the mortality rate.

- Functional endpoints are emphasized in clinical trials yet are often not utilized in preclinical studies. The correlation between functional and histological endpoints is likely age-dependent.

- Traumatic brain injury and axonal injury specifically, resulting from impactacceleration injury, can be reduced through manipulation of intracranial vascular volume.

- Glial activation likely significantly contributes to not only initial injury, but also long term repair and recovery following traumatic brain injury.

- Blast models have frequently failed to consider exposure duration as a clinically relevant parameter and consequently, models have not been scaled appropriately. Scaling to clinically-relevant parameters revealed significant neural injury in a pressure-dependent fashion.

\subsection{FUTURE STUDIES and DIRECTIONS}

\subsubsection{Bryostatin-1 Administration for Neural Injury - Isoform Specificity in Injury?}

While we've shown a clear beneficial role for PKC modulation following ischemic stroke,

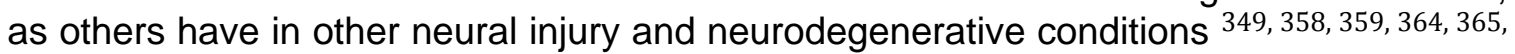
394, 397, 488, 492, it remains unclear the contribution of various PKC isoforms. While bryostatin-1 is believed to be primarily a PKCe activator, other PKC isoforms such as PKC $\alpha$ and PKC $\delta$ are affected by ischemic stroke and may be altered by bryostatin-1 treatment. To address this issue, isoform-specific compounds or conditional knockouts will need to be developed for future studies. Similarly, the precise mechanism via which PKC modulation improves outcome is unclear. Prior work has primarily focused on neuronal effects and largely ignored glial effects, despite the well-documented role of glia in neural injury and degeneration. Furthermore, the role of PKC in specific cell types (neurons, astrocytes, microglia, etc) and across time remains unclear and is of interest considering increased recognition of biphasic signaling events post-injury that may be deleterious initially, but beneficial in recovery and repair or vice-versa. How bryostatin-1 alters outcome in other neural injury conditions, such as blast injury, is another future avenue of investigation.

\subsubsection{APT 102 Extends the Window of Opportunity for tPA Administration but How?}

We've shown a reduced risk of hemorrhagic transformation when IPA is administered at 6 hours post-stroke in combination with APT 102, but the mechanism is not wellelucidated. While others have shown effects on prothrombotic mediators, such as extracellular ATP and ADP ${ }^{709}$, it remains unclear how APT 102 may promote maintenance or restoration of endothelial integrity through direct or indirect effects on platelets and other mediators of thrombosis.

\subsubsection{Preventing Traumatic Brain Injury: Alternative Mechanisms?}


Biologically-inspired discovery led to the revelation of internal jugular vein compression as a preventative measure for diffuse axonal injury through a reduction in not only axonal injury, but also glial activation. Other animals, besides the woodpecker cited as the prime example within this work, experience significant impacts in nature with no apparent deleterious effects, raising the possibility of alternative protective mechanisms existing in nature that could potentially be applied to humans. Such species include big horn sheep and diving gannets, both of which possess respiratory tracts with extensive dead space and exhibit increased levels of arterial $\mathrm{pCO}_{2}$. This altered respiratory gas composition, due to the well-documented effects of increased $\mathrm{pCO}_{2}$, may lead to increased intracranial vascular volume and diminished brain movement within the cranial vault upon impact. Therefore, future studies may administer exogenous $\mathrm{CO}_{2}$ prior to trauma to elucidate the role of increased $\mathrm{pCO}_{2}$ in preventing traumatic brain injury. Other mechanisms exist for increasing intracranial blood flow as well by increasing $\mathrm{pCO}_{2}$ such as pharmacological manipulation with acetazolamide, a carbonic anhydrase inhibitor.

Another potential prevention mechanism lies in the red blood cell and hemoglobin molecule. Traumatic brain injury, particularly diffuse axonal injury associated with closed head injury and concussion, has been notoriously difficult to image regardless of modality utilized. Recent work has shown the potential utility of diffusion tensor imaging (DTI) and tractography for detection of diffuse axonal injury ${ }^{710}$. This detection is based primarily on the detection of iron, the majority of which is found in the heme molecule within hemoglobin. Interestingly, DTI is particularly sensitive for the detection of methemoglobin, the form of hemoglobin incapable of transporting oxygen. Methemoglobin is elevated following trauma according to numerous reports, particularly those associated with blast exposure, in both preclinical and clinical studies indicating a potential role in injury pathogenesis, particularly with regards to free radical production. The conversion of hemoglobin to methemoglobin is notable in that it can be reversed by the enzyme $\mathrm{NADH}$ methemoglobin reductase ${ }^{711}$, an enzyme that can be modulated through administration of methylene blue, which acts as an exogenous electron acceptor for the enzyme.

\subsubsection{Elucidating Blast Injury Pathophysiology}

Development of a clinically relevant blast model, as described herein, will allow for the investigation of neurotrauma pathophysiology and perhaps of most interest and relevance to the general public, elucidation of the role of trauma in neurodegenerative disease development risk with a particular interest in chronic traumatic encephalopathy (CTE). As CTE is characterized by the presence of neurofibrillary tangles, hyperphosphorylated tau, and glial changes, these pathological features represent logical starting points for study and the evaluation of potential therapeutics 700 . Emerging evidence from clinical and preclinical studies alike has implicated endoplasmic reticulum stress in the hyperphosphorylation of tau ${ }^{712-715}$, a precursor to tangle formation. Specifically, components of the PERK-mediated ER stress pathway have been shown to colocalize with pretangle neurons at autopsy in patients afflicted with Alzheimer's disease and other tauopathies 713,715 . While colocalization does not indicate causation, the proximity does indicate a potential role for PERK-mediated ER stress in neuropathological changes, whether direct or indirect in nature. How PERKmediated ER stress is involved in neurotrauma, and the potential for long-term neurodegenerative disease development, remains unclear due to lack of investigation. Studies in other neural injury models, particularly ischemic stroke ${ }^{716}$, indicate a role for PERK-mediated ER stress in pathology. Therefore, PERK-mediated ER stress represents a novel and potentially promising therapeutic target for neural injury and blast 
injury in particular. Numerous pharmacologic modulators exist, such as tunicamycin , thapsigargin, and salubrinal, and have been used extensively in the literature offering further promise for future investigation.

Reconciling Preclinical and Clinical Studies: Integrating Functional and Behavioral Measures with Traditional Histological Studies

Attempts to improve preclinical studies of neural injury, whether it be stroke or neurotrauma, have identified numerous shortcomings in preclinical studies and mechanisms to address these issues. Two of the main weaknesses of preclinical studies include failure to consider age and the use of histological endpoints despite the importance of functional assessments clinically. We've presented in this work the effect of age on behavior and the correlation between infarct volume and functional performance in a clinically relevant model of ischemic stroke using aged female rodents. This correlation is particularly strong when using striatal infarct volumes and noticeably weaker when using cortical volumes. It remains to be seen whether this phenomenon holds up in the clinical population as expected, considering the role of the striatum in signal integration and transmission from various brain regions. 


\section{REFERENCES}

1. Lo EH, Dalkara T, Moskowitz MA. Mechanisms, challenges and opportunities in stroke. Nature Reviews Neuroscience. May 2003;4(5):399-415.

2. Candelario-Jalil E. Injury and repair mechanisms in ischemic stroke: considerations for the development of novel neurotherapeutics. Curr Opin Investig Drugs. Jul 2009;10(7):644-654.

3. Jordan J, Segura T, Brea D, Galindo MF, Castillo J. Inflammation as therapeutic objective in stroke. Curr Pharm Des. 2008;14(33):3549-3564.

4. Hinzman JM, Thomas TC, Burmeister JJ, et al. Diffuse brain injury elevates tonic glutamate levels and potassium-evoked glutamate release in discrete brain regions at two days post-injury: an enzyme-based microelectrode array study. J Neurotrauma. May 2010;27(5):889-899.

5. Xiong Y, Mahmood A, Chopp M. Animal models of traumatic brain injury. Nat Rev Neurosci. Feb 2013;14(2):128-142.

6. Lin Y, Wen L. Inflammatory response following diffuse axonal injury. Int $J$ Med Sci. 2013;10(5):515-521.

7. Ziebell JM, Morganti-Kossmann MC. Involvement of pro- and anti-inflammatory cytokines and chemokines in the pathophysiology of traumatic brain injury. Neurotherapeutics. Jan 2010;7(1):22-30.

8. O'Collins VE, Macleod MR, Donnan GA, Horky LL, van der Worp BH, Howells DW. 1,026 experimental treatments in acute stroke. Ann Neurol. Mar 2006;59(3): 467-477.

9. Rosen CL, Dinapoli VA, Nagamine T, Crocco T. Influence of age on stroke outcome following transient focal ischemia. J Neurosurg. Oct 2005;103(4):687-694.

10. Dinapoli VA, Rosen CL, Nagamine T, Crocco T. Selective MCA occlusion: a precise embolic stroke model. J Neurosci Methods. Jun 30 2006;154(1-2):233-238.

11. Woodruff TM, Thundyil J, Tang SC, Sobey CG, Taylor SM, Arumugam TV. Pathophysiology, treatment, and animal and cellular models of human ischemic stroke. Mol Neurodegener. 2011;6(1):11.

12. Roof RL, Schielke GP, Ren X, Hall ED. A comparison of long-term functional outcome after 2 middle cerebral artery occlusion models in rats. Stroke. Nov 2001;32 (11):2648-2657.

13. Chen J, Li Y, Wang L, et al. Therapeutic benefit of intravenous administration of bone marrow stromal cells after cerebral ischemia in rats. Stroke. Apr 2001;32(4): 1005-1011.

14. Boltze J, Kowalski I, Forschler A, et al. The stairway: a novel behavioral test detecting sensomotoric stroke deficits in rats. Artif Organs. Oct 2006;30(10):756-763.

15. DeVries AC, Nelson RJ, Traystman RJ, Hurn PD. Cognitive and behavioral assessment in experimental stroke research: will it prove useful? Neurosci Biobehav Rev. Jun 2001;25(4):325-342.

16. Yang Y, Shuaib A, Li Q. Quantification of infarct size on focal cerebral ischemia model of rats using a simple and economical method. $J$ Neurosci Methods. Oct 1 1998;84(1-2):9-16.

17. Thore CR, Anstrom JA, Moody DM, Challa VR, Marion MC, Brown WR. Morphometric analysis of arteriolar tortuosity in human cerebral white matter of preterm, young, and aged subjects. J Neuropathol Exp Neurol. May 2007;66(5):337-345.

18. Jucker M, Battig K, Meier-Ruge W. Effects of aging and vincamine derivatives on pericapillary microenvironment: stereological characterization of the cerebral capillary network. Neurobiol Aging. Jan-Feb 1990;11(1):39-46.

19. Shao $\mathrm{WH}$, Li C, Chen L, et al. Stereological investigation of age-related changes of the capillaries in white matter. Anat Rec (Hoboken). Aug 2010;293(8):1400-1407. 
20. Anstrom JA, Brown WR, Moody DM, Thore CR, Challa VR, Block SM. Anatomical analysis of the developing cerebral vasculature in premature neonates: absence of precapillary arteriole-to-venous shunts. Pediatr Res. Oct 2002;52(4):554-560.

21. Chen JJ, Rosas HD, Salat DH. Age-associated reductions in cerebral blood flow are independent from regional atrophy. Neuroimage. Mar 15 2011;55(2):468-478.

22. Brown WR, Thore CR. Review: cerebral microvascular pathology in ageing and neurodegeneration. Neuropathol Appl Neurobiol. Feb 2011;37(1):56-74.

23. Drake $\mathrm{CT}$, ladecola $\mathrm{C}$. The role of neuronal signaling in controlling cerebral blood flow. Brain Lang. Aug 2007;102(2):141-152.

24. Takano T, Tian GF, Peng W, et al. Astrocyte-mediated control of cerebral blood flow. Nat Neurosci. Feb 2006;9(2):260-267.

25. Faraci FM, Heistad DD. Regulation of the cerebral circulation: role of endothelium and potassium channels. Physiol Rev. Jan 1998;78(1):53-97.

26. Russo T, Felzani G, Marini C. Stroke in the Very Old: A Systematic Review of Studies on Incidence, Outcome, and Resource Use. Journal of Aging Research. $2011 ; 2011$.

27. Toescu EC. Normal brain ageing: models and mechanisms. Philos Trans $R$ Soc Lond B Biol Sci. Dec 29 2005;360(1464):2347-2354.

28. Buchhold B, Mogoanta L, Suofu Y, et al. Environmental enrichment improves functional and neuropathological indices following stroke in young and aged rats. Restor Neurol Neurosci. 2007;25(5-6):467-484.

29. Buga AM, Vintilescu R, Pop OT, Popa-Wagner A. Brain Aging and Regeneration after Injuries: an Organismal approach. Aging Dis. Feb 2011;2(1):64-79.

30. Badan I, Buchhold B, Hamm A, et al. Accelerated glial reactivity to stroke in aged rats correlates with reduced functional recovery. J Cereb Blood Flow Metab. Jul 2003;23 (7):845-854.

31. Zhao CS, Puurunen K, Schallert T, Sivenius J, Jolkkonen J. Behavioral and histological effects of chronic antipsychotic and antidepressant drug treatment in aged rats with focal ischemic brain injury. Behav Brain Res. Mar 30 2005;158(2):211-220.

32. Di Napoli M, Shah IM. Neuroinflammation and cerebrovascular disease in old age: a translational medicine perspective. J Aging Res. 2011;2011:857484.

33. Popa-Wagner A, Buga AM, Kokaia Z. Perturbed cellular response to brain injury during aging. Ageing Res Rev. Jan 2011;10(1):71-79.

34. Buga AM, Sascau M, Pisoschi C, Herndon JG, Kessler C, Popa-Wagner A. The genomic response of the ipsilateral and contralateral cortex to stroke in aged rats. $J$ Cell Mol Med. Dec 2008;12(6B):2731-2753.

35. van Uden IW, van Norden AG, de Laat KF, et al. Depressive Symptoms and Amygdala Volume in Elderly with Cerebral Small Vessel Disease: The RUN DMC Study. J Aging Res. 2011;2011:647869.

36. Teggi R, Fabiano B, Recanati P, Limardo P, Bussi M. Case reports on two patients with episodic vertigo, fluctuating hearing loss and migraine responding to prophylactic drugs for migraine. Meniere's disease or migraine-associated vertigo? Acta Otorhinolaryngol Ital. Aug 2010;30(4):217.

37. Hachinski VC, Lassen NA, Marshall J. Multi-infarct dementia. A cause of mental deterioration in the elderly. Lancet. Jul 27 1974;2(7874):207-210.

38. Enciu AM, Constantinescu SN, Popescu LM, Muresanu DF, Popescu BO. Neurobiology of vascular dementia. J Aging Res. 2011;2011:401604.

39. Thome J, Coogan AN, Woods AG, Darie CC, Hassler F. CLOCK Genes and Circadian Rhythmicity in Alzheimer Disease. J Aging Res. 2011;2011:383091.

40. Donnan GA, Baron JC, Ma H, Davis SM. Penumbral selection of patients for trials of acute stroke therapy. Lancet Neurol. Mar 2009;8(3):261-269.

41. Floel A, Warnecke T, Duning T, et al. Granulocyte-colony stimulating factor (GCSF) in stroke patients with concomitant vascular disease--a randomized controlled trial. PLoS One. 2011;6(5):e19767. 
42. Montaner J, Alvarez-Sabin J, Molina C, et al. Matrix metalloproteinase expression after human cardioembolic stroke: temporal profile and relation to neurological impairment. Stroke. Aug 2001;32(8):1759-1766.

43. Odden MC, Coxson PG, Moran A, Lightwood JM, Goldman L, Bibbins-Domingo $\mathrm{K}$. The impact of the aging population on coronary heart disease in the United States. Am J Med. Sep 2011;124(9):827-833 e825.

44. Taussky P, Tawk RG, Daugherty WP, Hanel RA. Medical therapy for ischemic stroke: review of intravenous and intra-arterial treatment options. World Neurosurg. Dec 2011;76(6 Suppl):S9-15.

45. Dronne MA, Grenier E, Chapuisat G, Hommel M, Boissel JP. A modelling approach to explore some hypotheses of the failure of neuroprotective trials in ischemic stroke patients. Prog Biophys Mol Biol. May 2008;97(1):60-78.

46. Schmidt S, Bruehl C, Frahm C, Redecker C, Witte OW. Age dependence of excitatory-inhibitory balance following stroke. Neurobiol Aging. Jul 2012;33(7): 1356-1363.

47. Fisher M, Feuerstein G, Howells DW, et al. Update of the stroke therapy academic industry roundtable preclinical recommendations. Stroke. Jun 2009;40(6): 2244-2250.

48. Shah IM, Ghosh SK, Collier A. Stroke presentation in Type 2 diabetes and the metabolic syndrome. Diabetes Res Clin Pract. Jan 2008;79(1):e1-4.

49. Liu F, Yuan R, Benashski SE, McCullough LD. Changes in experimental stroke outcome across the life span. J Cereb Blood Flow Metab. Apr 2009;29(4):792-802.

50. Cotrina ML, Nedergaard M. Astrocytes in the aging brain. J Neurosci Res. Jan 1 2002;67(1):1-10.

51. Dinapoli VA, Benkovic SA, Li X, et al. Age exaggerates proinflammatory cytokine signaling and truncates signal transducers and activators of transcription 3 signaling following ischemic stroke in the rat. Neuroscience. Oct 13 2010;170(2):633-644.

52. Guevara R, Gianotti M, Oliver J, Roca P. Age and sex-related changes in rat brain mitochondrial oxidative status. Exp Gerontol. Aug 122011.

53. Nakanishi $\mathrm{H}, \mathrm{Wu} \mathrm{Z}$. Microglia-aging: roles of microglial lysosome- and mitochondria-derived reactive oxygen species in brain aging. Behav Brain Res. Jul 19 2009;201(1):1-7.

54. Nakase T, Maeda T, Yoshida Y, Nagata K. Ischemia alters the expression of connexins in the aged human brain. J Biomed Biotechnol. 2009;2009:147946.

55. Popa-Wagner A, Carmichael ST, Kokaia Z, Kessler C, Walker LC. The response of the aged brain to stroke: too much, too soon? Curr Neurovasc Res. Aug 2007;4(3): 216-227.

56. Tripathy D, Yin X, Sanchez A, Luo J, Martinez J, Grammas P. Cerebrovascular expression of proteins related to inflammation, oxidative stress and neurotoxicity is altered with aging. J Neuroinflammation. 2010;7:63.

57. Tirilazad mesylate in acute ischemic stroke: A systematic review. Tirilazad International Steering Committee. Stroke. Sep 2000;31(9):2257-2265.

58. Savitz SI. A critical appraisal of the NXY-059 neuroprotection studies for acute stroke: a need for more rigorous testing of neuroprotective agents in animal models of stroke. Exp Neurol. May 2007;205(1):20-25.

59. Kelly KA, Li X, Tan Z, VanGilder RL, Rosen CL, Huber JD. NOX2 inhibition with apocynin worsens stroke outcome in aged rats. Brain Res. Oct 6 2009;1292:165-172.

60. Franceschi $\mathrm{C}$, Bonafe $\mathrm{M}$, Valensin S, et al. Inflamm-aging. An evolutionary perspective on immunosenescence. Ann N Y Acad Sci. Jun 2000;908:244-254.

61. Hein AM, O'Banion MK. Neuroinflammation and cognitive dysfunction in chronic disease and aging. J Neuroimmune Pharmacol. Mar 2012;7(1):3-6.

62. Pizza V, Agresta A, D'Acunto CW, Festa M, Capasso A. Neuroinflamm-aging and neurodegenerative diseases: an overview. CNS Neurol Disord Drug Targets. Aug 2011;10(5):621-634. 
63. von Bernhardi R, Tichauer JE, Eugenin J. Aging-dependent changes of microglial cells and their relevance for neurodegenerative disorders. J Neurochem. Mar 2010;112 (5):1099-1114.

64. Rosano C, Marsland AL, Gianaros PJ. Maintaining brain health by monitoring inflammatory processes: a mechanism to promote successful aging. Aging Dis. Feb 2012;3(1):16-33.

65. Bitto A, Sell C, Crowe E, et al. Stress-induced senescence in human and rodent astrocytes. Exp Cell Res. Oct 15 2010;316(17):2961-2968.

66. Salminen A, Ojala J, Kaarniranta K, Haapasalo A, Hiltunen M, Soininen H. Astrocytes in the aging brain express characteristics of senescence-associated secretory phenotype. Eur J Neurosci. Jul 2011;34(1):3-11.

67. Csiszar A, Ungvari Z, Koller A, Edwards JG, Kaley G. Aging-induced proinflammatory shift in cytokine expression profile in coronary arteries. FASEB J. Jun 2003;17(9):1183-1185.

68. Brown DR. Role of microglia in age-related changes to the nervous system. ScientificWorldJournal. 2009;9:1061-1071.

69. Luo XG, Ding JQ, Chen SD. Microglia in the aging brain: relevance to neurodegeneration. Mol Neurodegener. 2010;5:12.

70. Njie EG, Boelen E, Stassen FR, Steinbusch HW, Borchelt DR, Streit WJ. Ex vivo cultures of microglia from young and aged rodent brain reveal age-related changes in microglial function. Neurobiol Aging. Jan 2012;33(1):195 e191-112.

71. Baker DJ, Wijshake T, Tchkonia T, et al. Clearance of p16Ink4a-positive senescent cells delays ageing-associated disorders. Nature. Nov 10 2011;479(7372): 232-236.

72. Harry GJ, Kraft AD. Microglia in the developing brain: a potential target with lifetime effects. Neurotoxicology. Mar 2012;33(2):191-206.

73. Hart AD, Wyttenbach A, Perry VH, Teeling JL. Age related changes in microglial phenotype vary between CNS regions: grey versus white matter differences. Brain Behav Immun. Jul 2012;26(5):754-765.

74. Murphy KJ, Miller AM, Thelma R, Cowley F, Cox FF, Lynch MA. The age- and amyloid-beta-related increases in Nogo B contribute to microglial activation. Neurochem Int. Feb 2011;58(2):161-168.

75. Jurgens HA, Johnson RW. Dysregulated neuronal-microglial cross-talk during aging, stress and inflammation. Exp Neurol. Jan 2012;233(1):40-48.

76. Cox FF, Carney D, Miller AM, Lynch MA. CD200 fusion protein decreases microglial activation in the hippocampus of aged rats. Brain Behav Immun. Jul 2012;26 (5):789-796.

77. Gemma C, Bachstetter AD, Bickford PC. Neuron-Microglia Dialogue and Hippocampal Neurogenesis in the Aged Brain. Aging Dis. Dec 1 2010;1(3):232-244.

78. Kim HJ, Kim JY, Sun W. Age-dependent changes in the subcallosal zone neurogenesis of mice. Neurochem Int. Nov 2012;61(6):879-884.

79. McGinn MJ, Colello RJ, Sun D. Age-related proteomic changes in the subventricular zone and their association with neural stem/progenitor cell proliferation. $J$ Neurosci Res. Jun 2012;90(6):1159-1168.

80. Morrison JH, Baxter MG. The ageing cortical synapse: hallmarks and implications for cognitive decline. Nat Rev Neurosci. Apr 2012;13(4):240-250.

81. Chadwick W, Martin B, Chapter MC, et al. GIT2 acts as a potential keystone protein in functional hypothalamic networks associated with age-related phenotypic changes in rats. PLoS One. 2012;7(5):e36975.

82. Dotson V, Horak K, Alwardt C, Larson DF. Relationship of aging and cardiac IL-10. J Extra Corpor Technol. Jun 2004;36(2):197-201.

83. Meador BM, Krzyszton CP, Johnson RW, Huey KA. Effects of IL-10 and age on IL-6, IL-1beta, and TNF-alpha responses in mouse skeletal and cardiac muscle to an acute inflammatory insult. J Appl Physiol. Apr 2008;104(4):991-997. 
84. DiNapoli VA, Huber JD, Houser K, Li X, Rosen CL. Early disruptions of the bloodbrain barrier may contribute to exacerbated neuronal damage and prolonged functional recovery following stroke in aged rats. Neurobiol Aging. May 2008;29(5):753-764.

85. Lee P, Kim J, Williams R, et al. Effects of aging on blood brain barrier and matrix metalloproteases following controlled cortical impact in mice. Exp Neurol. Mar 2012;234 (1):50-61.

86. Itoh $\mathrm{T}$, Imano $\mathrm{M}$, Nishida $\mathrm{S}$, et al. Increased apoptotic neuronal cell death and cognitive impairment at early phase after traumatic brain injury in aged rats. Brain Struct Funct. Jan 2013;218(1):209-220.

87. Popa-Wagner A, Stocker K, Balseanu AT, et al. Effects of granulocyte-colony stimulating factor after stroke in aged rats. Stroke. May 2010;41(5):1027-1031.

88. Caballero B, Coto-Montes A. An insight into the role of autophagy in cell responses in the aging and neurodegenerative brain. Histol Histopathol. Mar 2012;27(3): 263-275.

89. Liu X, Wu Z, Hayashi Y, Nakanishi H. Age-dependent neuroinflammatory responses and deficits in long-term potentiation in the hippocampus during systemic inflammation. Neuroscience. Aug 2 2012;216:133-142.

90. Bruce-Keller AJ, White CL, Gupta S, et al. NOX activity in brain aging: exacerbation by high fat diet. Free Radic Biol Med. Jul 1 2010;49(1):22-30.

91. Knecht S, Ellger T, Levine JA. Obesity in neurobiology. Prog Neurobiol. Jan 2008;84(1):85-103.

92. Katsiki N, Ntaios G, Vemmos K. Stroke, obesity and gender: a review of the literature. Maturitas. Jul 2011;69(3):239-243.

93. Savopoulos C, Michalakis K, Apostolopoulou M, Miras A, Hatzitolios A. Adipokines and stroke: a review of the literature. Maturitas. Dec 2011;70(4):322-327.

94. Hishinuma A, Majima M, Kurabayashi $\mathrm{H}$. Insulin resistance in patients with stroke is related to visceral fat obesity and adipocytokines. J Stroke Cerebrovasc Dis. Jul-Aug $2008 ; 17(4): 175-180$.

95. Jackson RS, Black JH, 3rd, Lum YW, et al. Class I obesity is paradoxically associated with decreased risk of postoperative stroke after carotid endarterectomy. $J$ Vasc Surg. May 2012;55(5):1306-1312.

96. Kastorini CM, Panagiotakos DB. The obesity paradox: methodological considerations based on epidemiological and clinical evidence--new insights. Maturitas. Jul 2012;72(3):220-224.

97. Pan W, Kastin AJ. Tumor necrosis factor and stroke: role of the blood-brain barrier. Prog Neurobiol. Dec 2007;83(6):363-374.

98. Orosco M, Rouch C, Meile MJ, Nicolaidis S. Spontaneous feeding-related monoamine changes in rostromedial hypothalamus of the obese Zucker rat: a microdialysis study. Physiol Behav. Jun 1995;57(6):1103-1106.

99. Subramanian R, MacLeod KM. Age-dependent changes in blood pressure and arterial reactivity in obese Zucker rats. Eur J Pharmacol. Sep 12 2003;477(2):143-152.

100. Langdon KD, Clarke J, Corbett D. Long-term exposure to high fat diet is bad for your brain: exacerbation of focal ischemic brain injury. Neuroscience. May 19 2011;182:82-87.

101. Amantea D, Corasaniti MT, Mercuri NB, Bernardi G, Bagetta G. Brain regional and cellular localization of gelatinase activity in rat that have undergone transient middle cerebral artery occlusion. Neuroscience. Mar 3 2008;152(1):8-17.

102. Deutsch C, Portik-Dobos V, Smith AD, Ergul A, Dorrance AM. Diet-induced obesity causes cerebral vessel remodeling and increases the damage caused by ischemic stroke. Microvasc Res. Jun 2009;78(1):100-106.

103. Berg GD, Wadhwa S. Diabetes disease management in a community-based setting. Manag Care. Jun 2002;11(6):42, 45-50. 
104. Wild S, Roglic G, Green A, Sicree R, King H. Global prevalence of diabetes: estimates for the year 2000 and projections for 2030. Diabetes Care. May 2004;27(5): 1047-1053.

105. Mahler RJ, Adler ML. Clinical review 102: Type 2 diabetes mellitus: update on diagnosis, pathophysiology, and treatment. J Clin Endocrinol Metab. Apr 1999;84(4): 1165-1171.

106. Ripsin CM, Kang H, Urban RJ. Management of blood glucose in type 2 diabetes mellitus. Am Fam Physician. Jan 1 2009;79(1):29-36.

107. Herder C, Roden M. Genetics of type 2 diabetes: pathophysiologic and clinical relevance. Eur J Clin Invest. Jun 2011;41(6):679-692.

108. Medici F, Hawa M, lanari A, Pyke DA, Leslie RD. Concordance rate for type II diabetes mellitus in monozygotic twins: actuarial analysis. Diabetologia. Feb 1999;42(2): 146-150.

109. Pickup JC. Inflammation and activated innate immunity in the pathogenesis of type 2 diabetes. Diabetes Care. Mar 2004;27(3):813-823.

110. Creely SJ, McTernan PG, Kusminski CM, et al. Lipopolysaccharide activates an innate immune system response in human adipose tissue in obesity and type 2 diabetes. Am J Physiol Endocrinol Metab. Mar 2007;292(3):E740-747.

111. Lee M, Saver JL, Hong KS, Song S, Chang KH, Ovbiagele B. Effect of prediabetes on future risk of stroke: meta-analysis. BMJ. 2012;344:e3564.

112. Mukherjee D. Peripheral and cerebrovascular atherosclerotic disease in diabetes mellitus. Best Pract Res Clin Endocrinol Metab. Jun 2009;23(3):335-345.

113. Hotamisligil GS, Arner P, Caro JF, Atkinson RL, Spiegelman BM. Increased adipose tissue expression of tumor necrosis factor-alpha in human obesity and insulin resistance. J Clin Invest. May 1995;95(5):2409-2415.

114. Rees DA, Alcolado JC. Animal models of diabetes mellitus. Diabet Med. Apr 2005;22(4):359-370.

115. Srinivasan K, Ramarao P. Animal models in type 2 diabetes research: an overview. Indian J Med Res. Mar 2007;125(3):451-472.

116. Walder KR, Fahey RP, Morton GJ, Zimmet PZ, Collier GR. Characterization of obesity phenotypes in Psammomys obesus (Israeli sand rats). Int J Exp Diabetes Res. 2000;1(3):177-184.

117. Axen KV, Li X, Fung K, Sclafani A. The VMH-dietary obese rat: a new model of non-insulin-dependent diabetes mellitus. Am J Physiol. Mar 1994;266(3 Pt 2):R921-928.

118. Fan X, Qiu J, Yu Z, et al. A rat model of studying tissue-type plasminogen activator thrombolysis in ischemic stroke with diabetes. Stroke. Feb 2012;43(2):567-570.

119. Hajjar I, Kotchen TA. Trends in prevalence, awareness, treatment, and control of hypertension in the United States, 1988-2000. JAMA. Jul 9 2003;290(2):199-206.

120. Inoue $\mathrm{R}$, Ohkubo $\mathrm{T}$, Kikuya $\mathrm{M}$, et al. Stroke risk in systolic and combined systolic and diastolic hypertension determined using ambulatory blood pressure. The Ohasama study. Am J Hypertens. Oct 2007;20(10):1125-1131.

121. Hjelstuen A, Anderssen SA, Holme I, Seljeflot I, Klemsdal TO. Markers of inflammation are inversely related to physical activity and fitness in sedentary men with treated hypertension. Am J Hypertens. Jul 2006;19(7):669-675; discussion 676-667.

122. Nilupul Perera M, Ma HK, Arakawa $S$, et al. Inflammation following stroke. J Clin Neurosci. Jan 2006;13(1):1-8.

123. Angeli F, Verdecchia $P$, Reboldi GP, et al. Calcium channel blockade to prevent stroke in hypertension: a meta-analysis of 13 studies with 103,793 subjects. $A m J$ Hypertens. Sep 2004;17(9):817-822.

124. Hisham NF, Bayraktutan U. Epidemiology, Pathophysiology, and Treatment of Hypertension in Ischaemic Stroke Patients. J Stroke Cerebrovasc Dis. Jun 82012.

125. Inoue I, Chen L, Zhou L, Zeng X, Wang H. Reproduction of scalp acupuncture therapy on strokes in the model rats, spontaneous hypertensive rats-stroke prone (SHRSP). Neurosci Lett. Nov 29 2002;333(3):191-194. 
126. Masineni SN, Chander PN, Singh GD, Powers CA, Stier CT, Jr. Male gender and not the severity of hypertension is associated with end-organ damage in aged strokeprone spontaneously hypertensive rats. Am J Hypertens. Jun 2005;18(6):878-884.

127. Shimizu M, Ishikawa J, Yano Y, Hoshide S, Shimada K, Kario K. The relationship between the morning blood pressure surge and low-grade inflammation on silent cerebral infarct and clinical stroke events. Atherosclerosis. Nov 2011;219(1):316-321.

128. Sironi L, Maria Calvio A, Bellosta S, et al. Endogenous proteolytic activity in a rat model of spontaneous cerebral stroke. Brain Res. Jun 6 2003;974(1-2):184-192.

129. Aronow WS, Fleg JL, Pepine CJ, et al. ACCF/AHA 2011 expert consensus document on hypertension in the elderly: a report of the American College of Cardiology Foundation Task Force on Clinical Expert Consensus Documents developed in collaboration with the American Academy of Neurology, American Geriatrics Society, American Society for Preventive Cardiology, American Society of Hypertension, American Society of Nephrology, Association of Black Cardiologists, and European Society of Hypertension. J Am Soc Hypertens. Jul-Aug 2011;5(4):259-352.

130. McCullough LD, Alkayed NJ, Traystman RJ, Williams MJ, Hurn PD. Postischemic estrogen reduces hypoperfusion and secondary ischemia after experimental stroke. Stroke. Mar 2001;32(3):796-802.

131. Toung TJ, Traystman RJ, Hurn PD. Estrogen-mediated neuroprotection after experimental stroke in male rats. Stroke. Aug 1998;29(8):1666-1670.

132. Viscoli CM, Brass LM, Kernan WN, Sarrel PM, Suissa S, Horwitz RI. A clinical trial of estrogen-replacement therapy after ischemic stroke. $N$ Engl $J$ Med. Oct 25 2001;345(17):1243-1249.

133. Braeuninger S, Kleinschnitz C. Rodent models of focal cerebral ischemia: procedural pitfalls and translational problems. Exp Trans/ Stroke Med. 2009;1:8.

134. Durukan A, Strbian D, Tatlisumak T. Rodent models of ischemic stroke: a useful tool for stroke drug development. Curr Pharm Des. 2008;14(4):359-370.

135. Liu S, Zhen G, Meloni BP, Campbell K, Winn HR. Rodent Stroke Model Guidelines for Preclinical Stroke Trials (1st Edition). J Exp Stroke Transl Med. Jan 1 2009;2(2):2-27.

136. Orset C, Haelewyn B, Vivien K, Vivien D, Young A. Rodent Models of Thromboembolic Stroke. In: Dirnagl U, ed. Rodent Models of Stroke. Vol 47: Springer Science; 2010:55-70.

137. Lo EH. Experimental models, neurovascular mechanisms and translational issues in stroke research. Brit J Pharmacol. Mar 2008;153:S396-S405.

138. Lacy CR, Suh DC, Bueno M, Kostis JB. Delay in presentation and evaluation for acute stroke: Stroke Time Registry for Outcomes Knowledge and Epidemiology (S.T.R.O.K.E.). Stroke. Jan 2001;32(1):63-69.

139. Durukan A, Tatlisumak T. Acute ischemic stroke: overview of major experimental rodent models, pathophysiology, and therapy of focal cerebral ischemia. Pharmacol Biochem Behav. May 2007;87(1):179-197.

140. Goodman E, Li C, Tu YK, Ford E, Sun SS, Huang TT. Stability of the factor structure of the metabolic syndrome across pubertal development: confirmatory factor analyse of three alternative models. J Pediatr. Sep 2009;155(3):S5 e1-8.

141. Kawai $K$, Sakairi $T$, Harada $S$, et al. Diet modification and its influence on metabolic and related pathological alterations in the SHR/NDmcr-cp rat, an animal model of the metabolic syndrome. Exp Toxicol Pathol. May 2012;64(4):333-338.

142. Kim SY, Park HJ, Choi JS, et al. Ischemic preconditioning-induced expression of gp130 and STAT3 in astrocytes of the rat hippocampus. Brain Res Mol Brain Res. Oct 22 2004;129(1-2):96-103.

143. Ahonen TM, Saltevo JT, Kautiainen HJ, Kumpusalo EA, Vanhala MJ. The association of adiponectin and low-grade inflammation with the course of metabolic syndrome. Nutr Metab Cardiovasc Dis. Mar 2012;22(3):285-291. 
144. Maritano D, Sugrue ML, Tininini S, et al. The STAT3 isoforms alpha and beta have unique and specific functions. Nat Immunol. Apr 2004;5(4):401-409.

145. Adibhatla RM, Hatcher JF. Secretory phospholipase A2 IIA is up-regulated by TNF-alpha and IL-1alpha/beta after transient focal cerebral ischemia in rat. Brain Res. Feb 23 2007;1134(1):199-205.

146. La Porta CA, Comolli R. Age-dependent modulation of PKC isoforms and NOS activity and expression in rat cortex, striatum, and hippocampus. Exp Gerontol. Nov 1999;34(7):863-874.

147. Sajan MP, Nimal S, Mastorides S, et al. Correction of metabolic abnormalities in a rodent model of obesity, metabolic syndrome, and type 2 diabetes mellitus by inhibitors of hepatic protein kinase C-iota. Metabolism. Apr 2012;61(4):459-469.

148. Sun MK, Alkon DL. Pharmacology of protein kinase C activators: cognitionenhancing and antidementic therapeutics. Pharmacol Ther. Jul 2010;127(1):66-77.

149. Barber PA, Zhang J, Demchuk AM, Hill MD, Buchan AM. Why are stroke patients excluded from TPA therapy? An analysis of patient eligibility. Neurology. Apr 24 2001;56 (8):1015-1020.

150. Astrup J, Symon L, Branston NM, Lassen NA. Cortical evoked potential and extracellular $\mathrm{K}_{+}$and $\mathrm{H}_{+}$at critical levels of brain ischemia. Stroke. Jan-Feb 1977;8(1): $51-57$.

151. Lo EH. A new penumbra: transitioning from injury into repair after stroke. Nat Med. May 2008;14(5):497-500.

152. Heidenreich PA, Trogdon JG, Khavjou OA, et al. Forecasting the future of cardiovascular disease in the United States: a policy statement from the American Heart Association. Circulation. Mar 1 2011;123(8):933-944.

153. Stankowski JN, Gupta R. Therapeutic targets for neuroprotection in acute ischemic stroke: lost in translation? Antioxid Redox Signal. May 15 2011;14(10): 1841-1851.

154. Labiche LA, Grotta JC. Clinical trials for cytoprotection in stroke. NeuroRx. Jan 2004;1(1):46-70.

155. Siesjo BK. Pathophysiology and treatment of focal cerebral ischemia. Part I: Pathophysiology. J Neurosurg. Aug 1992;77(2):169-184.

156. Albers GW, Goldstein LB, Hall D, Lesko LM. Aptiganel hydrochloride in acute ischemic stroke: a randomized controlled trial. JAMA. Dec 5 2001;286(21):2673-2682.

157. Meadows ME, Fisher M, Minematsu K. Delayed Treatment with a Noncompetitive Nmda Antagonist, Cns-1102, Reduces Infarct Size in Rats. Cerebrovasc Dis. Jan-Feb 1994;4(1):26-31.

158. Albers GW, Atkinson RP, Kelley RE, Rosenbaum DM. Safety, tolerability, and pharmacokinetics of the N-methyl-D-aspartate antagonist dextrorphan in patients with acute stroke. Dextrorphan Study Group. Stroke. Feb 1995;26(2):254-258.

159. Du C, Hu R, Hsu CY, Choi DW. Dextrorphan reduces infarct volume, vascular injury, and brain edema after ischemic brain injury. J Neurotrauma. Apr 1996;13(4): 215-222.

160. Graham SH, Chen J, Simon RP. A dose-response study of dextrorphan in permanent focal ischemia. Neurosci Lett. Sep 17 1993;160(1):21-23.

161. Muir KW. Magnesium in stroke treatment. Postgrad Med J. Nov 2002;78(925): 641-645.

162. Saver JL, Kidwell C, Eckstein M, Starkman S. Prehospital neuroprotective therapy for acute stroke: results of the Field Administration of Stroke TherapyMagnesium (FAST-MAG) pilot trial. Stroke. May 2004;35(5):e106-108.

163. Yang Y, Li Q, Ahmad F, Shuaib A. Survival and histological evaluation of therapeutic window of post-ischemia treatment with magnesium sulfate in embolic stroke model of rat. Neurosci Lett. May 12 2000;285(2):119-122.

164. Davis SM, Lees KR, Albers GW, et al. Selfotel in acute ischemic stroke : possible neurotoxic effects of an NMDA antagonist. Stroke. Feb 2000;31(2):347-354. 
165. Madden KP, Clark WM, Zivin JA. Delayed therapy of experimental ischemia with competitive N-methyl-D-aspartate antagonists in rabbits. Stroke. Jul 1993;24(7): 1068-1071.

166. Phase II studies of the glycine antagonist GV150526 in acute stroke : the North American experience. The North American Glycine Antagonist in Neuroprotection (GAIN) Investigators. Stroke. Feb 2000;31(2):358-365.

167. Bordi F, Pietra C, Ziviani L, Reggiani A. The glycine antagonist GV150526 protects somatosensory evoked potentials and reduces the infarct area in the MCAo model of focal ischemia in the rat. Exp Neurol. Jun 1997;145(2 Pt 1):425-433.

168. Lees KR, Asplund K, Carolei A, et al. Glycine antagonist (gavestinel) in neuroprotection (GAIN International) in patients with acute stroke: a randomised controlled trial. GAIN International Investigators. Lancet. Jun 3 2000;355(9219): 1949-1954.

169. De Ryck M, Keersmaekers $R$, Duytschaever $H$, et al. Lubeluzole protects sensorimotor function and reduces infarct size in a photochemical stroke model in rats. $J$ Pharmacol Exp Ther. Nov 1996;279(2):748-758.

170. Diener HC, Cortens M, Ford $G$, et al. Lubeluzole in acute ischemic stroke treatment: A double-blind study with an 8-hour inclusion window comparing a 10-mg daily dose of lubeluzole with placebo. Stroke. Nov 2000;31(11):2543-2551.

171. Weiser T. AMPA receptor antagonists for the treatment of stroke. Curr Drug Targets CNS Neurol Disord. Apr 2005;4(2):153-159.

172. Suzuki M, Sasamata M, Miyata K. Neuroprotective effects of YM872 coadministered with t-PA in a rat embolic stroke model. Brain Res. Jan 3 2003;959(1): 169-172.

173. Lyden $\mathrm{P}$, Shuaib A, Ng K, et al. Clomethiazole Acute Stroke Study in ischemic stroke (CLASS-I): final results. Stroke. Jan 2002;33(1):122-128.

174. Marshall JW, Cross AJ, Ridley RM. Functional benefit from clomethiazole treatment after focal cerebral ischemia in a nonhuman primate species. Exp Neurol. Mar 1999;156(1):121-129.

175. Shuaib A, ljaz S, Kanthan R. Clomethiazole protects the brain in transient forebrain ischemia when used up to $4 \mathrm{~h}$ after the insult. Neurosci Lett. Sep 8 1995;197 (2):109-112.

176. Mauler F, Horvath E. Neuroprotective efficacy of repinotan $\mathrm{HCl}$, a $5-\mathrm{HT} 1 \mathrm{~A}$ receptor agonist, in animal models of stroke and traumatic brain injury. $J$ Cereb Blood Flow Metab. Apr 2005;25(4):451-459.

177. Semkova I, Wolz P, Krieglstein J. Neuroprotective effect of 5-HT1A receptor agonist, Bay X 3702, demonstrated in vitro and in vivo. Eur J Pharmacol. Oct 23 1998;359(2-3):251-260.

178. Teal P, Davis S, Hacke W, Kaste M, Lyden PD, Fierus M. A randomized, doubleblind, placebo-controlled trial to evaluate the efficacy, safety, tolerability, and pharmacokinetic/pharmacodynamic effects of a targeted exposure of intravenous repinotan in patients with acute ischemic stroke: modified Randomized Exposure Controlled Trial (mRECT). Stroke. Nov 2009;40(11):3518-3525.

179. Cheney JA, Weisser JD, Bareyre FM, et al. The maxi-K channel opener BMS-204352 attenuates regional cerebral edema and neurologic motor impairment after experimental brain injury. J Cereb Blood Flow Metab. Apr 2001;21(4):396-403.

180. Jensen BS. BMS-204352: a potassium channel opener developed for the treatment of stroke. CNS Drug Rev. Winter 2002;8(4):353-360.

181. Mizoguchi $H$, Leitermann RJ, Narita M, Nagase H, Suzuki T, Tseng LF. Regiondependent G-protein activation by kappa-opioid receptor agonists in the mouse brain. Neurosci Lett. Feb 12 2004;356(2):145-147.

182. Clark WM, Raps EC, Tong DC, Kelly RE. Cervene (Nalmefene) in acute ischemic stroke : final results of a phase III efficacy study. The Cervene Stroke Study Investigators. Stroke. Jun 2000;31(6):1234-1239. 
183. Faden Al, Shirane R, Chang LH, James TL, Lemke M, Weinstein PR. Opiatereceptor antagonist improves metabolic recovery and limits neurochemical alterations associated with reperfusion after global brain ischemia in rats. $J$ Pharmacol Exp Ther. Nov 1990;255(2):451-458.

184. Hossmann KA. Pathophysiological basis of translational stroke research. Folia Neuropathol. 2009;47(3):213-227.

185. Kristian T, Siesjo BK. Calcium in ischemic cell death. Stroke. Mar 1998;29(3): 705-718.

186. Nakamura H, Strong AJ, Dohmen C, et al. Spreading depolarizations cycle around and enlarge focal ischaemic brain lesions. Brain. Jul 2010;133(Pt 7):1994-2006.

187. Hossmann KA. Pathophysiology and therapy of experimental stroke. Cell Mol Neurobiol. Oct-Nov 2006;26(7-8):1057-1083.

188. Kunz A, Dirnagl U, Mergenthaler P. Acute pathophysiological processes after ischaemic and traumatic brain injury. Best Pract Res Clin Anaesthesiol. Dec 2010;24(4): 495-509.

189. Starkov AA, Chinopoulos C, Fiskum G. Mitochondrial calcium and oxidative stress as mediators of ischemic brain injury. Cell Calcium. Sep-Oct 2004;36(3-4): 257-264.

190. Smith WS. Pathophysiology of focal cerebral ischemia: a therapeutic perspective. $J$ Vasc Interv Radiol. Jan 2004;15(1 Pt 2):S3-12.

191. Horn J, de Haan RJ, Vermeulen M, Limburg M. Very Early Nimodipine Use in Stroke (VENUS): a randomized, double-blind, placebo-controlled trial. Stroke. Feb 2001;32(2):461-465.

192. Fogelholm R, Erila T, Palomaki H, Murros K, Kaste M. Effect of nimodipine on final infarct volume after acute ischemic stroke. Cerebrovasc Dis. May-Jun 2000;10(3): 189-193.

193. Roda JM, Carceller F, Diez-Tejedor E, Avendano C. Reduction of infarct size by intra-arterial nimodipine administered at reperfusion in a rat model of partially reversible brain focal ischemia. Stroke. Oct 1995;26(10):1888-1892.

194. Vibulsresth S, Dietrich WD, Busto R, Ginsberg MD. Failure of nimodipine to prevent ischemic neuronal damage in rats. Stroke. Jan-Feb 1987;18(1):210-216.

195. Hellstrom HO, Wanhainen A, Valtysson J, Persson L, Hillered L. Effect of tirilazad mesylate given after permanent middle cerebral artery occlusion in rat. Acta Neurochir (Wien). 1994;129(3-4):188-192.

196. Meden P, Overgaard K, Pedersen H, Boysen G. Effect of Early Treatment with Tirilazad (U74006F) Combined with Delayed Thrombolytic Therapy in Rat Embolic Stroke. Cerebrovasc Dis. 1996;6(3):141-148.

197. Shuaib A, Hussain MS. The past and future of neuroprotection in cerebral ischaemic stroke. Eur Neurol. 2008;59(1-2):4-14.

198. Diener HC, Lees KR, Lyden P, et al. NXY-059 for the treatment of acute stroke: pooled analysis of the SAINT I and II Trials. Stroke. Jun 2008;39(6):1751-1758.

199. Shuaib A, Lees KR, Lyden P, et al. NXY-059 for the treatment of acute ischemic stroke. N Engl J Med. Aug 9 2007;357(6):562-571.

200. Broughton BR, Reutens DC, Sobey CG. Apoptotic mechanisms after cerebral ischemia. Stroke. May 2009;40(5):e331-339.

201. MacManus JP, Buchan AM. Apoptosis after experimental stroke: fact or fashion? $J$ Neurotrauma. Oct 2000;17(10):899-914.

202. Zipfel GJ, Babcock DJ, Lee JM, Choi DW. Neuronal apoptosis after CNS injury: the roles of glutamate and calcium. J Neurotrauma. Oct 2000;17(10):857-869.

203. Bredesen DE. Apoptosis: overview and signal transduction pathways. $J$ Neurotrauma. Oct 2000;17(10):801-810.

204. Wang X, Han W, Du X, et al. Neuroprotective effect of Bax-inhibiting peptide on neonatal brain injury. Stroke. Sep 2010;41(9):2050-2055. 
205. Nuttall ME, Lee D, McLaughlin B, Erhardt JA. Selective inhibitors of apoptotic caspases: implications for novel therapeutic strategies. Drug Discov Today. Jan 12001;6 (2):85-91.

206. Robertson GS, Crocker SJ, Nicholson DW, Schulz JB. Neuroprotection by the inhibition of apoptosis. Brain Pathol. Apr 2000;10(2):283-292.

207. Joly LM, Mucignat V, Mariani J, Plotkine M, Charriaut-Marlangue C. Caspase inhibition after neonatal ischemia in the rat brain. $J$ Cereb Blood Flow Metab. Jan 2004;24(1):124-131.

208. Johnson EM, Jr., Greenlund LJ, Akins PT, Hsu CY. Neuronal apoptosis: current understanding of molecular mechanisms and potential role in ischemic brain injury. $J$ Neurotrauma. Oct 1995;12(5):843-852.

209. Wang $Q$, Tang $X N$, Yenari MA. The inflammatory response in stroke. $J$ Neuroimmunol. Mar 2007;184(1-2):53-68.

210. Huang J, Upadhyay UM, Tamargo RJ. Inflammation in stroke and focal cerebral ischemia. Surg Neurol. Sep 2006;66(3):232-245.

211. Jin R, Yang G, Li G. Molecular insights and therapeutic targets for blood-brain barrier disruption in ischemic stroke: critical role of matrix metalloproteinases and tissuetype plasminogen activator. Neurobiol Dis. Jun 2010;38(3):376-385.

212. Jin R, Yang G, Li G. Inflammatory mechanisms in ischemic stroke: role of inflammatory cells. J Leukoc Biol. May 2010;87(5):779-789.

213. Rossi DJ, Brady JD, Mohr C. Astrocyte metabolism and signaling during brain ischemia. Nat Neurosci. Nov 2007;10(11):1377-1386.

214. Carmignoto G, Gomez-Gonzalo M. The contribution of astrocyte signalling to neurovascular coupling. Brain Res Rev. May 2010;63(1-2):138-148.

215. Zonta M, Angulo MC, Gobbo $S$, et al. Neuron-to-astrocyte signaling is central to the dynamic control of brain microcirculation. Nat Neurosci. Jan 2003;6(1):43-50.

216. Takano T, Oberheim N, Cotrina ML, Nedergaard M. Astrocytes and ischemic injury. Stroke. Mar 2009;40(3 Suppl):S8-12.

217. Hayakawa $K$, Nakano $T$, Irie $K$, et al. Inhibition of reactive astrocytes with fluorocitrate retards neurovascular remodeling and recovery after focal cerebral ischemia in mice. J Cereb Blood Flow Metab. Apr 2010;30(4):871-882.

218. Pettigrew LC, Kasner SE, Albers GW, et al. Safety and tolerability of arundic acid in acute ischemic stroke. J Neurol Sci. Dec 21 2006;251(1-2):50-56.

219. Sorbera LA, Castaner J, Castaner RM. Arundic Acid. Drugs of the Future. 2004;29(5):441-449.

220. Tateishi N, Mori T, Kagamiishi $\mathrm{Y}$, et al. Astrocytic activation and delayed infarct expansion after permanent focal ischemia in rats. Part II: suppression of astrocytic activation by a novel agent (R)-(-)-2-propyloctanoic acid (ONO-2506) leads to mitigation of delayed infarct expansion and early improvement of neurologic deficits. J Cereb Blood Flow Metab. Jun 2002;22(6):723-734.

221. Use of anti-ICAM-1 therapy in ischemic stroke: results of the Enlimomab Acute Stroke Trial. Neurology. Oct 23 2001;57(8):1428-1434.

222. Becker KJ. Anti-leukocyte antibodies: LeukArrest (Hu23F2G) and Enlimomab (R6.5) in acute stroke. Curr Med Res Opin. 2002;18 Suppl 2:s18-22.

223. Bowes MP, Rothlein R, Fagan SC, Zivin JA. Monoclonal antibodies preventing leukocyte activation reduce experimental neurologic injury and enhance efficacy of thrombolytic therapy. Neurology. Apr 1995;45(4):815-819.

224. Yenari MA, Kunis $D$, Sun $G H$, et al. Hu23F2G, an antibody recognizing the leukocyte CD11/CD18 integrin, reduces injury in a rabbit model of transient focal cerebral ischemia. Exp Neurol. Oct 1998;153(2):223-233.

225. Fagan SC, Cronic LE, Hess DC. Minocycline Development for Acute Ischemic Stroke. Trans/ Stroke Res. Jun 1 2011;2(2):202-208. 
226. Hayakawa K, Mishima K, Nozako M, et al. Delayed treatment with minocycline ameliorates neurologic impairment through activated microglia expressing a highmobility group box1-inhibiting mechanism. Stroke. Mar 2008;39(3):951-958.

227. Lampl Y, Boaz M, Gilad R, et al. Minocycline treatment in acute stroke: an openlabel, evaluator-blinded study. Neurology. Oct 2 2007;69(14):1404-1410.

228. Macleod MR, O'Collins T, Horky LL, Howells DW, Donnan GA. Systematic review and metaanalysis of the efficacy of FK506 in experimental stroke. J Cereb Blood Flow Metab. Jun 2005;25(6):713-721.

229. Maeda M, Furuichi Y, Noto T, Matsuoka N, Mutoh S, Yoneda Y. Tacrolimus (FK506) suppresses rt-PA-induced hemorrhagic transformation in a rat thrombotic ischemia stroke model. Brain Res. Feb 13 2009;1254:99-108.

230. Switzer JA, Hess DC, Ergul A, et al. Matrix metalloproteinase-9 in an exploratory trial of intravenous minocycline for acute ischemic stroke. Stroke. Sep 2011;42(9): 2633-2635.

231. Takamatsu $H$, Tsukada $H$, Noda $A$, et al. FK506 attenuates early ischemic neuronal death in a monkey model of stroke. J Nucl Med. Dec 2001;42(12):1833-1840.

232. Burns TC, Steinberg GK. Stem cells and stroke: opportunities, challenges and strategies. Expert Opin Biol Ther. Apr 2011;11(4):447-461.

233. del Zoppo GJ. The neurovascular unit in the setting of stroke. J Intern Med. Feb 2010;267(2):156-171.

234. Pilegaard K, Ladefoged $O$. Total number of astrocytes in the molecular layer of the dentate gyrus of rats at different ages. Anal Quant Cytol Histol. Aug 1996;18(4): 279-285.

235. Block ML, Zecca L, Hong JS. Microglia-mediated neurotoxicity: uncovering the molecular mechanisms. Nature Reviews Neuroscience. Jan 2007;8(1):57-69.

236. Hayakawa K, Qiu J, Lo EH. Biphasic actions of HMGB1 signaling in inflammation and recovery after stroke. Ann N Y Acad Sci. Oct 2010;1207:50-57.

237. Bauer S, Kerr BJ, Patterson PH. The neuropoietic cytokine family in development, plasticity, disease and injury. Nat Rev Neurosci. Mar 2007;8(3):221-232.

238. Helmy A, Carpenter KL, Menon DK, Pickard JD, Hutchinson PJ. The cytokine response to human traumatic brain injury: temporal profiles and evidence for cerebral parenchymal production. J Cereb Blood Flow Metab. Feb 2011;31(2):658-670.

239. Marz P, Herget T, Lang E, Otten U, Rose-John S. Activation of gp130 by IL-6/ soluble IL-6 receptor induces neuronal differentiation. Eur J Neurosci. Dec 1997;9(12): 2765-2773.

240. Derouet D, Rousseau F, Alfonsi F, et al. Neuropoietin, a new IL-6-related cytokine signaling through the ciliary neurotrophic factor receptor. Proc Natl Acad Sci U S A. Apr 6 2004;101(14):4827-4832.

241. Fann MJ, Patterson $\mathrm{PH}$. Neuropoietic cytokines and activin A differentially regulate the phenotype of cultured sympathetic neurons. Proc Natl Acad Sci U S A. Jan 4 1994;91(1):43-47.

242. Bravo J, Heath JK. Receptor recognition by gp130 cytokines. EMBO J. Jun 1 2000;19(11):2399-2411.

243. Sriram K, O'Callaghan JP. Signaling Mechanisms Underlying Toxicant-Induced Gliosis. In: Aschner M, Costa LG, eds. The Role of Glia in Neurotoxicity. Second ed: CRC Press; 2005:141-171.

244. Ihle JN, Thierfelder W, Teglund S, et al. Signaling by the cytokine receptor superfamily. Ann N Y Acad Sci. Dec 11 1998;865:1-9.

245. Ihle JN. The Janus protein tyrosine kinase family and its role in cytokine signaling. Adv Immunol. 1995;60:1-35.

246. Kisseleva T, Bhattacharya S, Braunstein J, Schindler CW. Signaling through the JAK/STAT pathway, recent advances and future challenges. Gene. Feb 20 2002;285 (1-2):1-24. 
247. Briscoe J, Kohlhuber F, Muller M. JAKs and STATs branch out. Trends Cell Biol. Sep 1996;6(9):336-340.

248. Matsuda T, Feng J, Witthuhn BA, Sekine $Y$, Ihle JN. Determination of the transphosphorylation sites of Jak2 kinase. Biochem Biophys Res Commun. Dec 10 2004;325(2):586-594.

249. Barahmand-Pour F, Meinke A, Groner B, Decker T. Jak2-Stat5 interactions analyzed in yeast. J Biol Chem. May 15 1998;273(20):12567-12575.

250. Gupta S, Yan H, Wong LH, Ralph S, Krolewski J, Schindler C. The SH2 domains of Stat1 and Stat2 mediate multiple interactions in the transduction of IFN-alpha signals. EMBO J. Mar 1 1996;15(5):1075-1084.

251. Bromberg JF, Wrzeszczynska MH, Devgan G, et al. Stat3 as an oncogene. Cell. Aug 6 1999;98(3):295-303.

252. Iwamaru A, Szymanski S, Iwado E, et al. A novel inhibitor of the STAT3 pathway induces apoptosis in malignant glioma cells both in vitro and in vivo. Oncogene. Apr 12 2007;26(17):2435-2444.

253. Tian $Y$, Zhang $W$, Xia D, Modi P, Liang D, Wei M. Postconditioning inhibits myocardial apoptosis during prolonged reperfusion via a JAK2-STAT3-Bcl-2 pathway. $J$ Biomed Sci. 2011;18:53.

254. Takeda K, Noguchi K, Shi W, et al. Targeted disruption of the mouse Stat3 gene leads to early embryonic lethality. Proc Natl Acad Sci U S A. Apr 15 1997;94(8): 3801-3804.

255. Herrmann JE, Imura T, Song $B$, et al. STAT3 is a critical regulator of astrogliosis and scar formation after spinal cord injury. J Neurosci. Jul 9 2008;28(28):7231-7243.

256. Okada S, Nakamura M, Katoh $\mathrm{H}$, et al. Conditional ablation of Stat3 or Socs3 discloses a dual role for reactive astrocytes after spinal cord injury. Nat Med. Jul 2006;12 (7):829-834.

257. Kang SH, Yu MO, Park KJ, Chi SG, Park DH, Chung YG. Activated STAT3 regulates hypoxia-induced angiogenesis and cell migration in human glioblastoma. Neurosurgery. Nov 2010;67(5):1386-1395; discussion 1395.

258. Desbaillets I, Diserens AC, de Tribolet N, Hamou MF, Van Meir EG. Regulation of interleukin-8 expression by reduced oxygen pressure in human glioblastoma. Oncogene. Feb 18 1999;18(7):1447-1456.

259. Liu XH, Kirschenbaum A, Lu M, et al. Prostaglandin E2 induces hypoxia-inducible factor-1alpha stabilization and nuclear localization in a human prostate cancer cell line. $J$ Biol Chem. Dec 20 2002;277(51):50081-50086.

260. Zagzag D, Lukyanov $Y$, Lan L, et al. Hypoxia-inducible factor 1 and VEGF upregulate CXCR4 in glioblastoma: implications for angiogenesis and glioma cell invasion. Lab Invest. Dec 2006;86(12):1221-1232.

261. Seki $Y$, Kai H, Shibata R, et al. Role of the JAK/STAT pathway in rat carotid artery remodeling after vascular injury. Circ Res. Jul 7 2000;87(1):12-18.

262. Manea A, Tanase LI, Raicu M, Simionescu M. Jak/STAT signaling pathway regulates nox1 and nox4-based NADPH oxidase in human aortic smooth muscle cells. Arterioscler Thromb Vasc Biol. Jan 2010;30(1):105-112.

263. Byfield G, Budd S, Hartnett ME. The role of supplemental oxygen and JAK/STAT signaling in intravitreous neovascularization in a ROP rat model. Invest Ophthalmol Vis Sci. Jul 2009;50(7):3360-3365.

264. Jung JE, Kim GS, Chan PH. Neuroprotection by Interleukin-6 Is Mediated by Signal Transducer and Activator of Transcription 3 and Antioxidative Signaling in Ischemic Stroke. Stroke. Sep 222011.

265. Jung JE, Kim GS, Chen $\mathrm{H}$, et al. Reperfusion and neurovascular dysfunction in stroke: from basic mechanisms to potential strategies for neuroprotection. Mol Neurobiol. Jun 2010;41(2-3):172-179. 
266. Jung JE, Kim GS, Narasimhan P, Song YS, Chan PH. Regulation of Mnsuperoxide dismutase activity and neuroprotection by STAT3 in mice after cerebral ischemia. J Neurosci. May 27 2009;29(21):7003-7014.

267. Penkowa M, Giralt M, Carrasco J, Hadberg H, Hidalgo J. Impaired inflammatory response and increased oxidative stress and neurodegeneration after brain injury in interleukin-6-deficient mice. Glia. Dec 2000;32(3):271-285.

268. Tuna M, Yilmaz DM, Erman T, Arslan A, Ozgur H, Polat S. Effect of neutralization of rat interleukin 6 bioactivity on inducible nitric oxide synthase up-regulation and cerebral ischemic damage. Neurol Res. Sep 2009;31(7):714-720.

269. Fagan SC, Waller JL, Nichols FT, et al. Minocycline to improve neurologic outcome in stroke (MINOS): a dose-finding study. Stroke. Oct 2010;41(10):2283-2287.

270. Hussain SF, Kong LY, Jordan J, et al. A novel small molecule inhibitor of signal transducers and activators of transcription 3 reverses immune tolerance in malignant glioma patients. Cancer Res. Oct 15 2007;67(20):9630-9636.

271. Lang R, Patel D, Morris JJ, Rutschman RL, Murray PJ. Shaping gene expression in activated and resting primary macrophages by IL-10. J Immunol. Sep 1 2002;169(5): 2253-2263.

272. Lin T, Bost KL. STAT3 activation in macrophages following infection with Salmonella. Biochem Biophys Res Commun. Sep 3 2004;321(4):828-834.

273. O'Farrell AM, Liu Y, Moore KW, Mui AL. IL-10 inhibits macrophage activation and proliferation by distinct signaling mechanisms: evidence for Stat3-dependent and independent pathways. EMBO J. Feb 16 1998;17(4):1006-1018.

274. Jacoby JJ, Kalinowski A, Liu MG, et al. Cardiomyocyte-restricted knockout of STAT3 results in higher sensitivity to inflammation, cardiac fibrosis, and heart failure with advanced age. Proc Natl Acad Sci U S A. Oct 28 2003;100(22):12929-12934.

275. Wen Z, Zhong Z, Darnell JE, Jr. Maximal activation of transcription by Stat1 and Stat3 requires both tyrosine and serine phosphorylation. Cell. Jul 28 1995;82(2): 241-250.

276. Aggarwal BB, Kunnumakkara $A B$, Harikumar $K B$, et al. Signal transducer and activator of transcription-3, inflammation, and cancer: how intimate is the relationship? Ann N Y Acad Sci. Aug 2009;1171:59-76.

277. Sengupta TK, Talbot ES, Scherle PA, Ivashkiv LB. Rapid inhibition of interleukin-6 signaling and Stat3 activation mediated by mitogen-activated protein kinases. Proc Natl Acad Sci U S A. Sep 15 1998;95(19):11107-11112.

278. Chung J, Uchida E, Grammer TC, Blenis J. STAT3 serine phosphorylation by ERK-dependent and -independent pathways negatively modulates its tyrosine phosphorylation. Mol Cell Biol. Nov 1997;17(11):6508-6516.

279. Yokogami K, Wakisaka S, Avruch J, Reeves SA. Serine phosphorylation and maximal activation of STAT3 during CNTF signaling is mediated by the rapamycin target mTOR. Curr Biol. Jan 13 2000;10(1):47-50.

280. Greengard P. The neurobiology of slow synaptic transmission. Science. Nov 2 2001;294(5544):1024-1030.

281. Rawlings JS, Rosler KM, Harrison DA. The JAK/STAT signaling pathway. J Cell Sci. Mar 15 2004;117(Pt 8):1281-1283.

282. Shuai K, Liu B. Regulation of JAK-STAT signalling in the immune system. Nat Rev Immunol. Nov 2003;3(11):900-911.

283. Starr R, Hilton DJ. Negative regulation of the JAK/STAT pathway. Bioessays. Jan 1999;21(1):47-52.

284. Heinrich PC, Behrmann I, Haan S, Hermanns HM, Muller-Newen G, Schaper F. Principles of interleukin (IL)-6-type cytokine signalling and its regulation. Biochem J. Aug 15 2003;374(Pt 1):1-20.

285. Justicia C, Gabriel C, Planas AM. Activation of the JAK/STAT pathway following transient focal cerebral ischemia: signaling through Jak1 and Stat3 in astrocytes. Glia. May 2000;30(3):253-270. 
286. Wen TC, Peng H, Hata R, Desaki J, Sakanaka M. Induction of phosphorylatedStat3 following focal cerebral ischemia in mice. Neurosci Lett. May 11 2001;303(3): 153-156.

287. Yamashita T, Sawamoto K, Suzuki S, et al. Blockade of interleukin-6 signaling aggravates ischemic cerebral damage in mice: possible involvement of Stat3 activation in the protection of neurons. J Neurochem. Jul 2005;94(2):459-468.

288. Yagi T, Yoshioka H, Wakai T, Kato T, Horikoshi T, Kinouchi H. Activation of signal transducers and activators of transcription 3 in the hippocampal CA1 region in a rat model of global cerebral ischemic preconditioning. Brain Res. Nov 8 2011;1422:39-45.

289. Shakarishvili $R$, Beridze $M$. Proinflammatory reactants as determinants of stroke severity in elderly. Georgian Med News. Sep 2008(162):23-27.

290. Ley EJ, Clond MA, Singer MB, Shouhed D, Salim A. IL6 deficiency affects function after traumatic brain injury. J Surg Res. Oct 2011;170(2):253-256.

291. Oliva AA, Jr., Kang Y, Sanchez-Molano J, Furones C, Atkins CM. STAT3 Signaling after Traumatic Brain Injury. J Neurochem. Dec 62011.

292. Zhao JB, Zhang Y, Li GZ, Su XF, Hang CH. Activation of JAK2/STAT pathway in cerebral cortex after experimental traumatic brain injury of rats. Neurosci Lett. Jul 8 2011;498(2):147-152.

293. Hergenroeder GW, Moore AN, McCoy JP, Jr., et al. Serum IL-6: a candidate biomarker for intracranial pressure elevation following isolated traumatic brain injury. $J$ Neuroinflammation. 2010;7:19.

294. Bluyssen HA, Rastmanesh MM, Tilburgs C, et al. IFN gamma-dependent SOCS3 expression inhibits IL-6-induced STAT3 phosphorylation and differentially affects IL-6 mediated transcriptional responses in endothelial cells. Am J Physiol Cell Physiol. Aug 2010;299(2):C354-362.

295. Cayetanot F, Nygard M, Perret M, Kristensson K, Aujard F. Plasma levels of interferon-gamma correlate with age-related disturbances of circadian rhythms and survival in a non-human primate. Chronobiol Int. Dec 2009;26(8):1587-1601.

296. Liu RY, Snider WD. Different signaling pathways mediate regenerative versus developmental sensory axon growth. J Neurosci. Sep $12001 ; 21(17): R C 164$.

297. Decker T, Kovarik P. Serine phosphorylation of STATs. Oncogene. May 15 2000;19(21):2628-2637.

298. Kovarik P, Mangold M, Ramsauer K, et al. Specificity of signaling by STAT1 depends on $\mathrm{SH} 2$ and C-terminal domains that regulate Ser727 phosphorylation, differentially affecting specific target gene expression. EMBO J. Jan 15 2001;20(1-2): 91-100.

299. O'Shea JJ, Gadina M, Schreiber RD. Cytokine signaling in 2002: new surprises in the Jak/Stat pathway. Cell. Apr 2002;109 Suppl:S121-131.

300. Sriram K, Benkovic SA, Hebert MA, Miller DB, O'Callaghan JP. Induction of gp130-related cytokines and activation of JAK2/STAT3 pathway in astrocytes precedes up-regulation of glial fibrillary acidic protein in the 1-methyl-4-phenyl-1,2,3,6tetrahydropyridine model of neurodegeneration: key signaling pathway for astrogliosis in vivo? J Biol Chem. May 7 2004;279(19):19936-19947.

301. Haroon F, Drogemuller K, Handel U, et al. Gp130-dependent astrocytic survival is critical for the control of autoimmune central nervous system inflammation. J Immunol. Jun $12011 ; 186(11): 6521-6531$.

302. Dziennis S, Alkayed NJ. Role of signal transducer and activator of transcription 3 in neuronal survival and regeneration. Rev Neurosci. 2008;19(4-5):341-361.

303. Nicolas CS, Peineau S, Amici M, et al. The Jak/STAT pathway is involved in synaptic plasticity. Neuron. Jan 26 2012;73(2):374-390.

304. Fukuda S, Abematsu M, Mori H, et al. Potentiation of astrogliogenesis by STAT3mediated activation of bone morphogenetic protein-Smad signaling in neural stem cells. Mol Cell Biol. Jul 2007;27(13):4931-4937. 
305. Tsuda M, Kohro Y, Yano T, et al. JAK-STAT3 pathway regulates spinal astrocyte proliferation and neuropathic pain maintenance in rats. Brain. Apr 2011;134(Pt 4): 1127-1139.

306. Dominguez E, Mauborgne A, Mallet J, Desclaux M, Pohl M. SOCS3-mediated blockade of JAK/STAT3 signaling pathway reveals its major contribution to spinal cord neuroinflammation and mechanical allodynia after peripheral nerve injury. $J$ Neurosci. Apr 21 2010;30(16):5754-5766.

307. Sofroniew MV. Reactive astrocytes in neural repair and protection. Neuroscientist. Oct 2005;11(5):400-407.

308. Girolami EI, Bouhy D, Haber M, Johnson H, David S. Differential expression and potential role of SOCS1 and SOCS3 in Wallerian degeneration in injured peripheral nerve. Exp Neurol. May 2010;223(1):173-182.

309. Beurel E, Jope RS. Differential regulation of STAT family members by glycogen synthase kinase-3. J Biol Chem. Aug 8 2008;283(32):21934-21944.

310. Buller $B$, Liu $X$, Wang $X$, et al. MicroRNA-21 protects neurons from ischemic death. FEBS J. Oct 2010;277(20):4299-4307.

311. Gabriely G, Wurdinger T, Kesari S, et al. MicroRNA 21 promotes glioma invasion by targeting matrix metalloproteinase regulators. Mol Cell Biol. Sep 2008;28(17): 5369-5380.

312. Iliopoulos D, Jaeger SA, Hirsch HA, Bulyk ML, Struhl K. STAT3 activation of miR-21 and miR-181b-1 via PTEN and CYLD are part of the epigenetic switch linking inflammation to cancer. Mol Cell. Aug 27 2010;39(4):493-506.

313. Ziu M, Fletcher L, Rana S, Jimenez DF, Digicaylioglu M. Temporal differences in microRNA expression patterns in astrocytes and neurons after ischemic injury. PLoS One. $2011 ; 6(2): e 14724$.

314. Niemand C, Nimmesgern A, Haan S, et al. Activation of STAT3 by IL-6 and IL-10 in primary human macrophages is differentially modulated by suppressor of cytokine signaling 3. J Immunol. Mar 15 2003;170(6):3263-3272.

315. Beurel E, Jope RS. Lipopolysaccharide-induced interleukin-6 production is controlled by glycogen synthase kinase-3 and STAT3 in the brain. $J$ Neuroinflammation. 2009;6:9.

316. Gorina R, Petegnief V, Chamorro A, Planas AM. AG490 prevents cell death after exposure of rat astrocytes to hydrogen peroxide or proinflammatory cytokines: involvement of the Jak2/STAT pathway. J Neurochem. Feb 2005;92(3):505-518.

317. Planas AM, Gorina R, Chamorro A. Signalling pathways mediating inflammatory responses in brain ischaemia. Biochem Soc Trans. Dec 2006;34(Pt 6):1267-1270.

318. Naka T, Nishimoto N, Kishimoto T. The paradigm of IL-6: from basic science to medicine. Arthritis Res. 2002;4 Suppl 3:S233-242.

319. Satriotomo I, Bowen KK, Vemuganti R. JAK2 and STAT3 activation contributes to neuronal damage following transient focal cerebral ischemia. $J$ Neurochem. Sep 2006;98(5):1353-1368.

320. Yoshimura A, Naka T, Kubo M. SOCS proteins, cytokine signalling and immune regulation. Nat Rev Immunol. Jun 2007;7(6):454-465.

321. Bright R, Mochly-Rosen D. The role of protein kinase $C$ in cerebral ischemic and reperfusion injury. Stroke. Dec 2005;36(12):2781-2790.

322. Selvatici R, Marino S, Piubello $C$, et al. Protein kinase $C$ activity, translocation, and selective isoform subcellular redistribution in the rat cerebral cortex after in vitro ischemia. J Neurosci Res. Jan 1 2003;71(1):64-71.

323. Battaini F. Protein kinase $C$ isoforms as therapeutic targets in nervous system disease states. Pharmacol Res. Nov 2001;44(5):353-361.

324. Li X, Pabla N, Wei Q, et al. PKC-delta promotes renal tubular cell apoptosis associated with proteinuria. J Am Soc Nephrol. Jul 2010;21(7):1115-1124. 
325. Muller $\mathrm{KM}$, Tveteraas $\mathrm{IH}$, Aasrum $\mathrm{M}$, et al. Role of protein kinase $\mathrm{C}$ and epidermal growth factor receptor signalling in growth stimulation by neurotensin in colon carcinoma cells. BMC Cancer. 2011;11:421.

326. Yang K, Taft WC, Dixon CE, Todaro CA, Yu RK, Hayes RL. Alterations of protein kinase $C$ in rat hippocampus following traumatic brain injury. J Neurotrauma. Fall 1993;10(3):287-295.

327. da Rocha AB, Mans DR, Regner A, Schwartsmann G. Targeting protein kinase C: new therapeutic opportunities against high-grade malignant gliomas? Oncologist. 2002;7 (1):17-33.

328. Hussain S, Assender JW, Bond M, Wong LF, Murphy D, Newby AC. Activation of protein kinase Czeta is essential for cytokine-induced metalloproteinase-1, -3 , and -9 secretion from rabbit smooth muscle cells and inhibits proliferation. J Biol Chem. Jul 26 2002;277(30):27345-27352.

329. Bright $R$, Steinberg GK, Mochly-Rosen D. DeltaPKC mediates microcerebrovascular dysfunction in acute ischemia and in chronic hypertensive stress in vivo. Brain Res. May 4 2007;1144:146-155.

330. Shirai Y, Adachi N, Saito N. Protein kinase Cepsilon: function in neurons. FEBS J. Aug 2008;275(16):3988-3994.

331. Schaffel R, Morais JC, Biasoli I, et al. PKC-beta II expression has prognostic impact in nodal diffuse large B-cell lymphoma. Mod Pathol. Mar 2007;20(3):326-330.

332. Gruber T, Hermann-Kleiter N, Pfeifhofer-Obermair C, et al. PKC theta cooperates with PKC alpha in alloimmune responses of T cells in vivo. Mol Immunol. Jun 2009;46 (10):2071-2079.

333. Pascale A, Govoni S, Battaini F. Age-related alteration of PKC, a key enzyme in memory processes: physiological and pathological examples. Mol Neurobiol. Feb 1998;16(1):49-62.

334. Qvit N, Mochly-Rosen D. Highly Specific Modulators of Protein Kinase C Localization: Applications to Heart Failure. Drug Discov Today Dis Mech. Summer 2010;7(2):e87-e93.

335. Tan SL, Parker PJ. Emerging and diverse roles of protein kinase $C$ in immune cell signalling. Biochem J. Dec 15 2003;376(Pt 3):545-552.

336. Corsini E, Battaini $F$, Lucchi $L$, et al. A defective protein kinase $C$ anchoring system underlying age-associated impairment in TNF-alpha production in rat macrophages. J Immunol. Sep 15 1999;163(6):3468-3473.

337. Standaert ML, Avignon A, Arnold T, et al. Insulin translocates PKC-epsilon and phorbol esters induce and persistently translocate PKC-beta 2 in $\mathrm{BC} 3 \mathrm{H}-1$ myocytes. Cell Signal. Jun 1996;8(4):313-316.

338. Lahn M, Paterson BM, Sundell K, Ma D. The role of protein kinase C-alpha (PKC-alpha) in malignancies of the gastrointestinal tract. Eur J Cancer. Jan 2004;40(1): $10-20$

339. Dorn GW, 2nd, Mochly-Rosen D. Intracellular transport mechanisms of signal transducers. Annu Rev Physiol. 2002;64:407-429.

340. Schechtman D, Mochly-Rosen D. Adaptor proteins in protein kinase C-mediated signal transduction. Oncogene. Oct 1 2001;20(44):6339-6347.

341. Duquesnes N, Lezoualc'h F, Crozatier B. PKC-delta and PKC-epsilon: foes of the same family or strangers? J Mol Cell Cardiol. Nov 2011;51(5):665-673.

342. Yang T, Roder KE, Bhat GJ, Thekkumkara TJ, Abbruscato TJ. Protein kinase C family members as a target for regulation of blood-brain barrier $\mathrm{Na}, \mathrm{K}, 2 \mathrm{Cl}$-cotransporter during in vitro stroke conditions and nicotine exposure. Pharm Res. Feb 2006;23(2): 291-302.

343. Wang J, Bright R, Mochly-Rosen D, Giffard RG. Cell-specific role for epsilon- and betal-protein kinase $\mathrm{C}$ isozymes in protecting cortical neurons and astrocytes from ischemia-like injury. Neuropharmacology. Jul 2004;47(1):136-145. 
344. Smeda JS, King S, Harder DR. Cerebrovascular alterations in protein kinase Cmediated constriction in stroke-prone rats. Stroke. Mar 1999;30(3):656-661.

345. Fleegal MA, Hom S, Borg LK, Davis TP. Activation of PKC modulates blood-brain barrier endothelial cell permeability changes induced by hypoxia and posthypoxic reoxygenation. Am J Physiol Heart Circ Physiol. Nov 2005;289(5):H2012-2019.

346. Dave KR, Raval AP, Purroy J, et al. Aberrant deltaPKC activation in the spinal cord of Wobbler mouse: a model of motor neuron disease. Neurobiol Dis. Feb 2005;18 (1):126-133.

347. Latchoumycandane C, Anantharam V, Jin H, Kanthasamy A. Dopaminergic neurotoxicant 6-OHDA induces oxidative damage through proteolytic activation of PKCdelta in cell culture and animal models of Parkinson's disease. Toxicol Appl Pharmacol. Nov 1 2011;256(3):314-323.

348. Nakai M, Tanimukai S, Yagi K, et al. Amyloid beta protein activates PKC-delta and induces translocation of myristoylated alanine-rich $\mathrm{C}$ kinase substrate (MARCKS) in microglia. Neurochem Int. Jun 2001;38(7):593-600.

349. Zohar O, Lavy $\mathrm{R}, \mathrm{Zi}$ X, et al. PKC activator therapeutic for mild traumatic brain injury in mice. Neurobiol Dis. Feb 2011;41(2):329-337.

350. Qi X, Disatnik MH, Shen N, Sobel RA, Mochly-Rosen D. Aberrant mitochondrial fission in neurons induced by protein kinase $C\{$ delta\} under oxidative stress conditions in vivo. Mol Biol Cell. Jan 15 2011;22(2):256-265.

351. Budas GR, Churchill EN, Disatnik MH, Sun L, Mochly-Rosen D. Mitochondrial import of PKCepsilon is mediated by HSP90: a role in cardioprotection from ischaemia and reperfusion injury. Cardiovasc Res. Oct 1 2010;88(1):83-92.

352. Inagaki K, Chen L, Ikeno $\mathrm{F}$, et al. Inhibition of delta-protein kinase $\mathrm{C}$ protects against reperfusion injury of the ischemic heart in vivo. Circulation. Nov 11 2003;108(19): 2304-2307.

353. Chen L, Hahn H, Wu G, et al. Opposing cardioprotective actions and parallel hypertrophic effects of delta PKC and epsilon PKC. Proc Natl Acad Sci U S A. Sep 25 2001;98(20):11114-11119.

354. Churchill EN, Ferreira JC, Brum PC, Szweda LI, Mochly-Rosen D. Ischaemic preconditioning improves proteasomal activity and increases the degradation of deltaPKC during reperfusion. Cardiovasc Res. Jan 15 2010;85(2):385-394.

355. Facchinetti MM, de Boland AR. Effect of ageing on the expression of protein kinase $\mathrm{C}$ and its activation by $1,25(\mathrm{OH}) 2$-vitamin D3 in rat skeletal muscle. Cell Signal. Jan 1999;11(1):39-44.

356. Ferreira JC, Brum PC, Mochly-Rosen D. betalIPKC and epsilonPKC isozymes as potential pharmacological targets in cardiac hypertrophy and heart failure. $\mathrm{J} \mathrm{Mol} \mathrm{Cell}$ Cardiol. Oct 2011;51(4):479-484.

357. Sun MK, Alkon DL. Bryostatin-1: pharmacology and therapeutic potential as a CNS drug. CNS Drug Rev. Spring 2006;12(1):1-8.

358. Hongpaisan J, Sun MK, Alkon DL. PKC epsilon activation prevents synaptic loss, Abeta elevation, and cognitive deficits in Alzheimer's disease transgenic mice. $J$ Neurosci. Jan 12 2011;31(2):630-643.

359. Nelson TJ, Alkon DL. Neuroprotective versus tumorigenic protein kinase C activators. Trends Biochem Sci. Mar 2009;34(3):136-145.

360. Shimohata T, Zhao H, Sung JH, Sun G, Mochly-Rosen D, Steinberg GK. Suppression of deltaPKC activation after focal cerebral ischemia contributes to the protective effect of hypothermia. J Cereb Blood Flow Metab. Aug 2007;27(8):1463-1475.

361. Sieber FE, Traystman RJ, Brown PR, Martin LJ. Protein kinase C expression and activity after global incomplete cerebral ischemia in dogs. Stroke. Jul 1998;29(7): 1445-1452; discussion 1452-1443.

362. Matsumoto S, Shamloo M, Matsumoto E, Isshiki A, Wieloch T. Protein kinase Cgamma and calcium/calmodulin-dependent protein kinase II-alpha are persistently 
translocated to cell membranes of the rat brain during and after middle cerebral artery occlusion. J Cereb Blood Flow Metab. Jan 2004;24(1):54-61.

363. Bright R, Sun GH, Yenari MA, Steinberg GK, Mochly-Rosen D. epsilonPKC confers acute tolerance to cerebral ischemic reperfusion injury. Neurosci Lett. Aug 15 2008;441(1):120-124.

364. Sun MK, Hongpaisan J, Alkon DL. Postischemic PKC activation rescues retrograde and anterograde long-term memory. Proc Natl Acad Sci U S A. Aug 25 2009;106(34):14676-14680.

365. Sun MK, Hongpaisan J, Nelson TJ, Alkon DL. Poststroke neuronal rescue and synaptogenesis mediated in vivo by protein kinase $\mathrm{C}$ in adult brains. Proc Natl Acad Sci U S A. Sep 9 2008;105(36):13620-13625.

366. Li J, Niu C, Han S, et al. Identification of protein kinase $\mathrm{C}$ isoforms involved in cerebral hypoxic preconditioning of mice. Brain Res. Oct 26 2005;1060(1-2):62-72.

367. Bu X, Zhang N, Yang X, et al. Proteomic analysis of cPKCbetall-interacting proteins involved in HPC-induced neuroprotection against cerebral ischemia of mice. $J$ Neurochem. Apr 2011;117(2):346-356.

368. Kregel KC, Zhang HJ. An integrated view of oxidative stress in aging: basic mechanisms, functional effects, and pathological considerations. Am J Physiol Regul Integr Comp Physiol. Jan 2007;292(1):R18-36.

369. Majumder PK, Mishra NC, Sun X, et al. Targeting of protein kinase C delta to mitochondria in the oxidative stress response. Cell Growth Differ. Sep 2001;12(9): 465-470.

370. Kanthasamy AG, Kitazawa M, Kanthasamy A, Anantharam V. Role of proteolytic activation of protein kinase Cdelta in oxidative stress-induced apoptosis. Antioxid Redox Signal. Oct 2003;5(5):609-620.

371. Maher P. How protein kinase $C$ activation protects nerve cells from oxidative stress-induced cell death. J Neurosci. May 1 2001;21(9):2929-2938.

372. Kahles T, Kohnen A, Heumueller S, et al. NADPH oxidase Nox1 contributes to ischemic injury in experimental stroke in mice. Neurobiol Dis. Oct 2010;40(1):185-192.

373. Thamilselvan V, Menon M, Thamilselvan S. Oxalate-induced activation of PKCalpha and -delta regulates NADPH oxidase-mediated oxidative injury in renal tubular epithelial cells. Am J Physiol Renal Physiol. Nov 2009;297(5):F1399-1410.

374. Wei H, Mi X, Ji L, et al. Protein kinase C-delta is involved in induction of NOX1 gene expression by aldosterone in rat vascular smooth muscle cells. Biochemistry (Mosc). Mar 2010;75(3):304-309.

375. Reshef A, Sperling O, Zoref-Shani E. Activation and inhibition of protein kinase $C$ protect rat neuronal cultures against ischemia-reperfusion insult. Neurosci Lett. Nov 28 1997;238(1-2):37-40.

376. Kostyak JC, Hunter JC, Korzick DH. Acute PKCdelta inhibition limits ischaemiareperfusion injury in the aged rat heart: role of GSK-3beta. Cardiovasc Res. May 1 2006;70(2):325-334.

377. Bright R, Raval AP, Dembner JM, et al. Protein kinase $C$ delta mediates cerebral reperfusion injury in vivo. J Neurosci. Aug 4 2004;24(31):6880-6888.

378. Dave KR, Bhattacharya SK, Saul I, et al. Activation of protein kinase C delta following cerebral ischemia leads to release of cytochrome $C$ from the mitochondria via bad pathway. PLoS One. 2011;6(7):e22057.

379. Qi X, Inagaki K, Sobel RA, Mochly-Rosen D. Sustained pharmacological inhibition of deltaPKC protects against hypertensive encephalopathy through prevention of blood-brain barrier breakdown in rats. J Clin Invest. Jan 2008;118(1):173-182.

380. Monti M, Donnini S, Giachetti A, Mochly-Rosen D, Ziche M. deltaPKC inhibition or varepsilonPKC activation repairs endothelial vascular dysfunction by regulating eNOS post-translational modification. J Mol Cell Cardiol. Apr 2010;48(4):746-756. 
381. Yoo J, Nichols A, Song JC, et al. Bryostatin-1 attenuates TNF-induced epithelial barrier dysfunction: role of novel PKC isozymes. Am J Physiol Gastrointest Liver Physiol. Apr 2003;284(4):G703-712.

382. Willis CL, Meske DS, Davis TP. Protein kinase $\mathrm{C}$ activation modulates reversible increase in cortical blood-brain barrier permeability and tight junction protein expression during hypoxia and posthypoxic reoxygenation. J Cereb Blood Flow Metab. Nov 2010;30 (11):1847-1859.

383. Ding Q, Chai H, Mahmood N, Tsao J, Mochly-Rosen D, Zhou W. Matrix metalloproteinases modulated by protein kinase Cepsilon mediate resistin-induced migration of human coronary artery smooth muscle cells. J Vasc Surg. Apr 2011;53(4): 1044-1051.

384. Sonobe $\mathrm{Y}$, Takeuchi $\mathrm{H}$, Kataoka $\mathrm{K}$, et al. Interleukin-25 expressed by brain capillary endothelial cells maintains blood-brain barrier function in a protein kinase Cepsilon-dependent manner. J Biol Chem. Nov 13 2009;284(46):31834-31842.

385. Wang Z, Xue Y, Jiao H, Liu Y, Wang P. Doxycycline-mediated protective effect against focal cerebral ischemia-reperfusion injury through the modulation of tight junctions and PKCdelta signaling in rats. J Mol Neurosci. May 2012;47(1):89-100.

386. Arai K, Lee SR, Lo EH. Essential role for ERK mitogen-activated protein kinase in matrix metalloproteinase-9 regulation in rat cortical astrocytes. Glia. Sep 2003;43(3): 254-264.

387. McLennan SV, Fisher E, Martell SY, et al. Effects of glucose on matrix metalloproteinase and plasmin activities in mesangial cells: possible role in diabetic nephropathy. Kidney Int Suppl. Sep 2000;77:S81-87.

388. Alexander JP, Acott TS. Involvement of protein kinase $C$ in TNFalpha regulation of trabecular matrix metalloproteinases and TIMPs. Invest Ophthalmol Vis Sci. Nov 2001;42(12):2831-2838.

389. Esteve PO, Chicoine E, Robledo $\mathrm{O}$, et al. Protein kinase C-zeta regulates transcription of the matrix metalloproteinase- 9 gene induced by $\mathrm{IL}-1$ and TNF-alpha in glioma cells via NF-kappa B. J Biol Chem. Sep 20 2002;277(38):35150-35155.

390. Sariahmetoglu M, Crawford BD, Leon $\mathrm{H}$, et al. Regulation of matrix metalloproteinase-2 (MMP-2) activity by phosphorylation. FASEB J. Aug 2007;21(10): 2486-2495.

391. Carpenter AC, Alexander JS. Endothelial PKC delta activation attenuates neutrophil transendothelial migration. Inflamm Res. May 2008;57(5):216-229.

392. Kaneda H, Ikeno F, Inagaki K, Mochly-Rosen D. Preserved coronary endothelial function by inhibition of delta protein kinase $\mathrm{C}$ in a porcine acute myocardial infarction model. Int J Cardiol. Apr 3 2009;133(2):256-259.

393. Gonthier B, Koncina E, Satkauskas S, et al. A PKC-dependent recruitment of MMP-2 controls semaphorin-3A growth-promoting effect in cortical dendrites. PLoS One. 2009;4(4):e5099.

394. Hongpaisan J, Alkon DL. A structural basis for enhancement of long-term associative memory in single dendritic spines regulated by PKC. Proc Natl Acad Sci U S A. Dec 4 2007; 104(49):19571-19576.

395. Kim J, Koyanagi T, Mochly-Rosen D. PKCdelta activation mediates angiogenesis via NADPH oxidase activity in PC-3 prostate cancer cells. Prostate. Jun 15 2011;71(9): 946-954.

396. Zhao CT, Li K, Li JT, et al. PKCdelta regulates cortical radial migration by stabilizing the Cdk5 activator p35. Proc Natl Acad Sci U S A. Dec 15 2009;106(50): 21353-21358.

397. Kuzirian AM, Epstein HT, Gagliardi CJ, et al. Bryostatin enhancement of memory in Hermissenda. Biol Bull. Jun 2006;210(3):201-214.

398. Sun MK, Alkon DL. Synergistic effects of chronic bryostatin-1 and alphatocopherol on spatial learning and memory in rats. Eur J Pharmacol. Apr 28 2008;584 $(2-3): 328-337$. 
399. Dempsey EC, Newton AC, Mochly-Rosen D, et al. Protein kinase C isozymes and the regulation of diverse cell responses. Am J Physiol Lung Cell Mol Physiol. Sep 2000;279(3):L429-438.

400. Van der Zee EA, Palm IF, O'Connor M, Maizels ET, Hunzicker-Dunn M, Disterhoft JF. Aging-related alterations in the distribution of $\mathrm{Ca}(2+)$-dependent PKC isoforms in rabbit hippocampus. Hippocampus. 2004;14(7):849-860.

401. Sharma D, Sethi P, Hussain E, Singh R. Curcumin counteracts the aluminiuminduced ageing-related alterations in oxidative stress, $\mathrm{Na}+\mathrm{K}+$ ATPase and protein kinase $C$ in adult and old rat brain regions. Biogerontology. Aug 2009;10(4):489-502.

402. Milner R, Campbell IL. Cytokines regulate microglial adhesion to laminin and astrocyte extracellular matrix via protein kinase C-dependent activation of the alpha6beta1 integrin. J Neurosci. Mar 1 2002;22(5):1562-1572.

403. Ansar S, Edvinsson L. Subtype activation and interaction of protein kinase $C$ and mitogen-activated protein kinase controlling receptor expression in cerebral arteries and microvessels after subarachnoid hemorrhage. Stroke. Jan 2008;39(1):185-190.

404. Meier M, King GL. Protein kinase $\mathrm{C}$ activation and its pharmacological inhibition in vascular disease. Vasc Med. 2000;5(3):173-185.

405. Albert-Weissenberger C, Siren AL. Experimental traumatic brain injury. Exp Trans/ Stroke Med. 2010;2(1):16.

406. Panzer MB, Matthews KA, Yu AW, Morrison B, 3rd, Meaney DF, Bass CR. A Multiscale Approach to Blast Neurotrauma Modeling: Part I - Development of Novel Test Devices for in vivo and in vitro Blast Injury Models. Front Neurol. 2012;3:46.

407. Bass CR, Panzer MB, Rafaels KA, Wood G, Shridharani J, Capehart B. Brain injuries from blast. Ann Biomed Eng. Jan 2012;40(1):185-202.

408. Daneshvar DH, Baugh CM, Nowinski CJ, McKee AC, Stern RA, Cantu RC. Helmets and mouth guards: the role of personal equipment in preventing sport-related concussions. Clin Sports Med. Jan 2011;30(1):145-163, x.

409. Gavett BE, Stern RA, Cantu RC, Nowinski CJ, McKee AC. Mild traumatic brain injury: a risk factor for neurodegeneration. Alzheimers Res Ther. 2010;2(3):18.

410. Guskiewicz KM, Marshall SW, Bailes J, et al. Association between recurrent concussion and late-life cognitive impairment in retired professional football players. Neurosurgery. Oct 2005;57(4):719-726; discussion 719-726.

411. Stern RA, Riley DO, Daneshvar DH, Nowinski CJ, Cantu RC, McKee AC. Longterm consequences of repetitive brain trauma: chronic traumatic encephalopathy. PM $R$. Oct 2011;3(10 Suppl 2):S460-467.

412. Shenton ME, Hamoda HM, Schneiderman JS, et al. A review of magnetic resonance imaging and diffusion tensor imaging findings in mild traumatic brain injury. Brain Imaging Behav. Jun 2012;6(2):137-192.

413. Saulle M, Greenwald BD. Chronic traumatic encephalopathy: a review. Rehabil Res Pract. 2012;2012:816069.

414. Gavett BE, Stern RA, McKee AC. Chronic traumatic encephalopathy: a potential late effect of sport-related concussive and subconcussive head trauma. Clin Sports Med. Jan 2011;30(1):179-188, xi.

415. Omalu B, Hammers JL, Bailes J, et al. Chronic traumatic encephalopathy in an Iraqi war veteran with posttraumatic stress disorder who committed suicide. Neurosurg Focus. Nov 2011;31(5):E3.

416. Gavett BE, Cantu RC, Shenton M, et al. Clinical appraisal of chronic traumatic encephalopathy: current perspectives and future directions. Curr Opin Neurol. Dec $2011 ; 24(6): 525-531$.

417. Baugh CM, Stamm JM, Riley DO, et al. Chronic traumatic encephalopathy: neurodegeneration following repetitive concussive and subconcussive brain trauma. Brain Imaging Behav. 2012;6:244-254. 
418. Goldstein LE, Fisher AM, Tagge CA, et al. Chronic traumatic encephalopathy in blast-exposed military veterans and a blast neurotrauma mouse model. Sci Transl Med. May 16 2012;4(134):134ra160.

419. Lakhan SE, Kirchgessner A. Chronic traumatic encephalopathy: the dangers of getting "dinged". SpringerPlus. 2012;1(2).

420. McKee AC, Cantu RC, Nowinski CJ, et al. Chronic traumatic encephalopathy in athletes: progressive tauopathy after repetitive head injury. J Neuropathol Exp Neurol. Jul 2009;68(7):709-735.

421. Omalu BI, Bailes J, Hammers JL, Fitzsimmons RP. Chronic traumatic encephalopathy, suicides and parasuicides in professional American athletes: the role of the forensic pathologist. Am J Forensic Med Pathol. Jun 2010;31(2):130-132.

422. Omalu BI, DeKosky ST, Hamilton RL, et al. Chronic traumatic encephalopathy in a national football league player: part II. Neurosurgery. Nov 2006;59(5):1086-1092; discussion 1092-1083.

423. Omalu BI, DeKosky ST, Minster RL, Kamboh MI, Hamilton RL, Wecht $\mathrm{CH}$. Chronic traumatic encephalopathy in a National Football League player. Neurosurgery. Jul 2005;57(1):128-134; discussion 128-134.

424. Omalu BI, Fitzsimmons RP, Hammers J, Bailes J. Chronic traumatic encephalopathy in a professional American wrestler. J Forensic Nurs. Fall 2010;6(3): 130-136.

425. Omalu BI, Hamilton RL, Kamboh MI, DeKosky ST, Bailes J. Chronic traumatic encephalopathy (CTE) in a National Football League Player: Case report and emerging medicolegal practice questions. J Forensic Nurs. Spring 2010;6(1):40-46.

426. Omalu B, Bailes J, Hamilton RL, et al. Emerging histomorphologic phenotypes of chronic traumatic encephalopathy in American athletes. Neurosurgery. Jul 2011;69(1): 173-183; discussion 183.

427. Adams CW, Bruton CJ. The cerebral vasculature in dementia pugilistica. J Neurol Neurosurg Psychiatry. May 1989;52(5):600-604.

428. Casson IR, Pellman EJ, Viano DC. Chronic traumatic encephalopathy in a National Football League player. Neurosurgery. May 2006;58(5):E1003; author reply E1003; discussion E1003.

429. Corsellis JA, Bruton CJ, Freeman-Browne D. The aftermath of boxing. Psychol Med. Aug 1973;3(3):270-303.

430. Millspaugh JA. DEMENTIA PUGILISTICA. U.S. Naval Medical Bulletin. 1937;35 (3):297-303.

431. Martland HS. PUNCH DRUNK. JAMA. 1928;91(15):1103-1107.

432. Blaylock RL, Maroon J. Immunoexcitotoxicity as a central mechanism in chronic traumatic encephalopathy-A unifying hypothesis. Surg Neurol Int. 2011;2:107.

433. Costanza A, Weber K, Gandy S, et al. Review: Contact sport-related chronic traumatic encephalopathy in the elderly: clinical expression and structural substrates. Neuropathol Appl Neurobiol. Oct 2011;37(6):570-584.

434. Johnson VE, Stewart W, Smith DH. Axonal pathology in traumatic brain injury. Exp Neurol. Jan 202012.

435. Geddes JF, Vowles GH, Nicoll JA, Revesz T. Neuronal cytoskeletal changes are an early consequence of repetitive head injury. Acta Neuropathol. Aug 1999;98(2): 171-178.

436. Mills JD, Bailes JE, Turner RC, Dodson SC, Sakai J, Maroon JC. Anabolic steroids and head injury. Neurosurgery. Jan 2012;70(1):205-209; discussion 209-210.

437. McCrory P. Sports concussion and the risk of chronic neurological impairment. Clin J Sport Med. Jan 2011;21(1):6-12.

438. Prichep LS, McCrea M, Barr W, Powell M, Chabot RJ. Time Course of Clinical and Electrophysiological Recovery After Sport-Related Concussion. J Head Trauma Rehabil. May 142012. 
439. Signoretti S, Lazzarino G, Tavazzi B, Vagnozzi R. The pathophysiology of concussion. PM R. Oct 2011;3(10 Suppl 2):S359-368.

440. Mac Donald CL, Dikranian K, Song SK, Bayly PV, Holtzman DM, Brody DL. Detection of traumatic axonal injury with diffusion tensor imaging in a mouse model of traumatic brain injury. Exp Neurol. May 2007;205(1):116-131.

441. Shah S, Yallampalli R, Merkley TL, et al. Diffusion tensor imaging and volumetric analysis of the ventral striatum in adults with traumatic brain injury. Brain Inj. 2012;26(3): 201-210.

442. Smits M, Houston GC, Dippel DW, et al. Microstructural brain injury in postconcussion syndrome after minor head injury. Neuroradiology. Aug 2011;53(8):553-563.

443. Maller JJ, Thomson $\mathrm{RH}$, Lewis PM, Rose SE, Pannek K, Fitzgerald PB. Traumatic brain injury, major depression, and diffusion tensor imaging: making connections. Brain Res Rev. Sep 2010;64(1):213-240.

444. Tremblay S, De Beaumont L, Henry LC, et al. Sports Concussions and Aging: A Neuroimaging Investigation. Cereb Cortex. May 102012.

445. Sanchez-Carrion R, Fernandez-Espejo D, Junque $C$, et al. A longitudinal fMRI study of working memory in severe TBI patients with diffuse axonal injury. Neuroimage. Nov 15 2008;43(3):421-429.

446. Rosenbaum SB, Lipton ML. Embracing chaos: the scope and importance of clinical and pathological heterogeneity in mTBI. Brain Imaging Behav. Jun 2012;6(2): 255-282.

447. McDonald BC, Saykin AJ, McAllister TW. Functional MRI of mild traumatic brain injury (mTBI): progress and perspectives from the first decade of studies. Brain Imaging Behav. Jun 2012;6(2):193-207.

448. Talavage TM, Nauman E, Breedlove EL, et al. Functionally-Detected Cognitive Impairment in High School Football Players Without Clinically-Diagnosed Concussion. $J$ Neurotrauma. Oct 12010.

449. Breedlove EL, Robinson M, Talavage TM, et al. Biomechanical correlates of symptomatic and asymptomatic neurophysiological impairment in high school football. $J$ Biomech. Apr 30 2012;45(7):1265-1272.

450. Henninger N, Sicard KM, Li Z, et al. Differential recovery of behavioral status and brain function assessed with functional magnetic resonance imaging after mild traumatic brain injury in the rat. Crit Care Med. Nov 2007;35(11):2607-2614.

451. Tshibanda L, Vanhaudenhuyse A, Galanaud D, Boly M, Laureys S, Puybasset L. Magnetic resonance spectroscopy and diffusion tensor imaging in coma survivors: promises and pitfalls. Prog Brain Res. 2009;177:215-229.

452. Signoretti S, Vagnozzi R, Tavazzi B, Lazzarino G. Biochemical and neurochemical sequelae following mild traumatic brain injury: summary of experimental data and clinical implications. Neurosurg Focus. Nov 2010;29(5):E1.

453. Vagnozzi R, Signoretti S, Cristofori L, et al. Assessment of metabolic brain damage and recovery following mild traumatic brain injury: a multicentre, proton magnetic resonance spectroscopic study in concussed patients. Brain. Nov 2010;133 (11):3232-3242.

454. Henry LC, Tremblay S, Leclerc S, et al. Metabolic changes in concussed American football players during the acute and chronic post-injury phases. BMC Neurol. 2011;11:105.

455. Bigler ED, Maxwell WL. Neuropathology of mild traumatic brain injury: relationship to neuroimaging findings. Brain Imaging Behav. Jun 2012;6(2):108-136.

456. Henry LC, Tremblay S, Boulanger Y, Ellemberg D, Lassonde M. Neurometabolic changes in the acute phase after sports concussions correlate with symptom severity. $J$ Neurotrauma. Jan 2010;27(1):65-76.

457. Kleindienst A, Tolias CM, Corwin FD, et al. Assessment of cerebral S100B levels by proton magnetic resonance spectroscopy after lateral fluid-percussion injury in the rat. J Neurosurg. Jun 2005;102(6):1115-1121. 
458. Lin AP, Liao HJ, Merugumala SK, Prabhu SP, Meehan WP, 3rd, Ross BD. Metabolic imaging of mild traumatic brain injury. Brain Imaging Behav. Jun 2012;6(2): 208-223.

459. Lin A, Ramadan S, Box H, Stanwell P, Stern R. Neurochemical changes in athletes with chronic traumatic encephalopathy. Paper presented at: Radiological Society of North America2010; Chicago, IL.

460. Price $P$, Jones $T$. Can positron emission tomography (PET) be used to detect subclinical response to cancer therapy? The EC PET Oncology Concerted Action and the EORTC PET Study Group. Eur J Cancer. Nov 1995;31A(12):1924-1927.

461. Venneti $S$, Wagner AK, Wang $G$, et al. The high affinity peripheral benzodiazepine receptor ligand DAA1106 binds specifically to microglia in a rat model of traumatic brain injury: implications for PET imaging. Exp Neurol. Sep 2007;207(1): 118-127.

462. Provenzano FA, Jordan B, Tikofsky RS, Saxena C, Van Heertum RL, Ichise M. F-18 FDG PET imaging of chronic traumatic brain injury in boxers: a statistical parametric analysis. Nucl Med Commun. Nov 2010;31(11):952-957.

463. Wagner K. Can Science See Inside An NFL Player's Skull Before It's Too Late? BAD BRAINS 2012; http://deadspin.com/5920006/can-science-see-inside-an-nfl-playersskull-before-its-too-late. Accessed August 20, 2012, 2012.

464. Small GW, Kepe V, Ercoli LM, et al. PET of brain amyloid and tau in mild cognitive impairment. N Engl J Med. Dec 21 2006;355(25):2652-2663.

465. Kemp PM, Houston AS, Macleod MA, Pethybridge RJ. Cerebral perfusion and psychometric testing in military amateur boxers and controls. J Neurol Neurosurg Psychiatry. Oct 1995;59(4):368-374.

466. Shen Y, Kou Z, Kreipke CW, Petrov T, Hu J, Haacke EM. In vivo measurement of tissue damage, oxygen saturation changes and blood flow changes after experimental traumatic brain injury in rats using susceptibility weighted imaging. Magn Reson Imaging. Feb 2007;25(2):219-227.

467. Aiken AH, Gean AD. Imaging of head trauma. Semin Roentgenol. Apr 2010;45 (2):63-79.

468. McCrea RP, Harder SL, Martin M, Buist R, Nichol H. A comparison of rapidscanning $X$-ray fluorescence mapping and magnetic resonance imaging to localize brain iron distribution. Eur J Radiol. Dec 2008;68(3 Suppl):S109-113.

469. Cheng JL, Yang $\mathrm{YJ}$, Li HL, Wang J, Wang MH, Zhang Y. In vivo tracing of superparamagnetic iron oxide-labeled bone marrow mesenchymal stem cells transplanted for traumatic brain injury by susceptibility weighted imaging in a rat model. Chin J Traumatol. Jun 1 2010;13(3):173-177.

470. Zhang J, Zhang Y, Wang J, et al. Characterizing iron deposition in Parkinson's disease using susceptibility-weighted imaging: an in vivo MR study. Brain Res. May 12 2010;1330:124-130.

471. Meoded A, Poretti A, Northington FJ, Tekes A, Intrapiromkul J, Huisman TA. Susceptibility weighted imaging of the neonatal brain. Clin Radiol. Aug 2012;67(8): 793-801.

472. Kou Z, Benson RR, Gattu R, Haacke EM. Susceptibility Weighted Imaging Complements to Diffusion Tensor Imaging in Traumatic Brain Injury. Neuroimage. 2009;47:S68.

473. Ashwal S, Babikian T, Gardner-Nichols J, Freier MC, Tong KA, Holshouser BA. Susceptibility-weighted imaging and proton magnetic resonance spectroscopy in assessment of outcome after pediatric traumatic brain injury. Arch Phys Med Rehabil. Dec 2006;87(12 Suppl 2):S50-58.

474. McKee AC, Gavett BE, Stern RA, et al. TDP-43 proteinopathy and motor neuron disease in chronic traumatic encephalopathy. J Neuropathol Exp Neurol. Sep 2010;69 (9):918-929. 
475. Mondello S, Jeromin A, Buki A, et al. Glial neuronal ratio: a novel index for differentiating injury type in patients with severe traumatic brain injury. J Neurotrauma. Apr 10 2012;29(6):1096-1104.

476. Dohi K, Satoh K, Ohtaki H, et al. Elevated plasma levels of bilirubin in patients with neurotrauma reflect its pathophysiological role in free radical scavenging. In Vivo. Sep-Oct 2005;19(5):855-860.

477. Zetterberg H, Tanriverdi F, Unluhizarci K, Selcuklu A, Kelestimur F, Blennow K. Sustained release of neuron-specific enolase to serum in amateur boxers. Brain Inj. Aug 2009;23(9):723-726.

478. Balakathiresan $N$, Bhomia $M$, Chandran $R$, Chavko M, McCarron RM, Maheshwari RK. MicroRNA let-7i is a promising serum biomarker for blast-induced traumatic brain injury. J Neurotrauma. May 1 2012;29(7):1379-1387.

479. Pasinetti GM, Ho L, Dooley C, Abbi B, Lange G. Select non-coding RNA in blood components provide novel clinically accessible biological surrogates for improved identification of traumatic brain injury in OEF/OIF Veterans. Am $J$ Neurodegener Dis. May 30 2012;1(1):88-98.

480. Neselius S, Brisby $\mathrm{H}$, Theodorsson A, Blennow K, Zetterberg $\mathrm{H}$, Marcusson J. CSF-biomarkers in Olympic boxing: diagnosis and effects of repetitive head trauma. PLoS One. 2012;7(4):e33606.

481. Avsar $\mathrm{T}$, Korkmaz $\mathrm{D}$, Tutuncu $\mathrm{M}$, et al. Protein biomarkers for multiple sclerosis: semi-quantitative analysis of cerebrospinal fluid candidate protein biomarkers in different forms of multiple sclerosis. Mult Scler. Jan 172012.

482. Tortelli R, Ruggieri M, Cortese R, et al. Elevated cerebrospinal fluid neurofilament light levels in patients with amyotrophic lateral sclerosis: a possible marker of disease severity and progression. Eur J Neurol. Jun 112012.

483. Yoshiyama $\mathrm{Y}$, Uryu $\mathrm{K}$, Higuchi $\mathrm{M}$, et al. Enhanced neurofibrillary tangle formation, cerebral atrophy, and cognitive deficits induced by repetitive mild brain injury in a transgenic tauopathy mouse model. J Neurotrauma. Oct 2005;22(10):1134-1141.

484. Tran HT, Sanchez L, Esparza TJ, Brody DL. Distinct temporal and anatomical distributions of amyloid-beta and tau abnormalities following controlled cortical impact in transgenic mice. PLoS One. 2011;6(9):e25475.

485. Hoshino $S$, Tamaoka A, Takahashi M, et al. Emergence of immunoreactivities for phosphorylated tau and amyloid-beta protein in chronic stage of fluid percussion injury in rat brain. Neuroreport. Jun 1 1998;9(8):1879-1883.

486. Katzan IL, Hammer MD, Hixson ED, Furlan AJ, Abou-Chebl A, Nadzam DM. Utilization of intravenous tissue plasminogen activator for acute ischemic stroke. Arch Neurol. Mar 2004;61(3):346-350.

487. Shlosberg D, Benifla M, Kaufer D, Friedman A. Blood-brain barrier breakdown as a therapeutic target in traumatic brain injury. Nat Rev Neurol. Jul 2010;6(7):393-403.

488. Etcheberrigaray $\mathrm{R}$, Tan $\mathrm{M}$, Dewachter I, et al. Therapeutic effects of PKC activators in Alzheimer's disease transgenic mice. Proc Natl Acad Sci U S A. Jul 27 2004;101(30):11141-11146.

489. Quattrone A, Pascale A, Nogues X, et al. Posttranscriptional regulation of gene expression in learning by the neuronal ELAV-like mRNA-stabilizing proteins. Proc Natl Acad Sci U S A. Sep 25 2001;98(20):11668-11673.

490. Skaper SD, Facci L, Strijbos PJ. Neuronal protein kinase signaling cascades and excitotoxic cell death. Ann N Y Acad Sci. Jun 2001;939:11-22.

491. Krizbai I, Szabo G, Deli M, et al. Expression of protein kinase C family members in the cerebral endothelial cells. J Neurochem. Jul 1995;65(1):459-462.

492. Alkon DL, Sun MK, Nelson TJ. PKC signaling deficits: a mechanistic hypothesis for the origins of Alzheimer's disease. Trends Pharmacol Sci. Feb 2007;28(2):51-60.

493. Lees $K R$, Bluhmki $E$, von Kummer $R$, et al. Time to treatment with intravenous alteplase and outcome in stroke: an updated pooled analysis of ECASS, ATLANTIS, NINDS, and EPITHET trials. Lancet. May 15 2010;375(9727):1695-1703. 
494. Lloyd-Jones D, Adams RJ, Brown TM, et al. Heart disease and stroke statistics--2010 update: a report from the American Heart Association. Circulation. Feb 23 2010;121(7):e46-e215.

495. Goldstein LB, Adams R, Alberts MJ, et al. Primary prevention of ischemic stroke: a guideline from the American Heart Association/American Stroke Association Stroke Council: cosponsored by the Atherosclerotic Peripheral Vascular Disease Interdisciplinary Working Group; Cardiovascular Nursing Council; Clinical Cardiology Council; Nutrition, Physical Activity, and Metabolism Council; and the Quality of Care and Outcomes Research Interdisciplinary Working Group. Circulation. Jun 20 2006;113 (24):e873-923.

496. Sacco RL, Adams R, Albers G, et al. Guidelines for prevention of stroke in patients with ischemic stroke or transient ischemic attack: a statement for healthcare professionals from the American Heart Association/American Stroke Association Council on Stroke: co-sponsored by the Council on Cardiovascular Radiology and Intervention: the American Academy of Neurology affirms the value of this guideline. Stroke. Feb 2006;37(2):577-617.

497. Eckle T, Fullbier L, Wehrmann M, et al. Identification of ectonucleotidases CD39 and CD73 in innate protection during acute lung injury. J Immunol. Jun 15 2007;178(12): 8127-8137.

498. Sugimoto S, Lin X, Lai J, et al. Apyrase treatment prevents ischemia-reperfusion injury in rat lung isografts. J Thorac Cardiovasc Surg. Sep 2009;138(3):752-759.

499. Kohler D, Eckle T, Faigle M, et al. CD39/ectonucleoside triphosphate diphosphohydrolase 1 provides myocardial protection during cardiac ischemia/ reperfusion injury. Circulation. Oct 16 2007;116(16):1784-1794.

500. Moeckel D, Baum P, Nguyen A, Jeong S, Chen R, Abendschein D. A Novel Human Apyrase Prevents Reocclusion and Substantially Decreases Infarct Size Without Bleeding Time Prolongation After Coronary Fibrinolysis in Dogs. Paper presented at: Arteriosclerosis, thrombosis and vascular biology 20112011; Orlando, Florida.

501. Belayev L, Khoutorova L, Deisher TA, et al. Neuroprotective effect of SolCD39, a novel platelet aggregation inhibitor, on transient middle cerebral artery occlusion in rats. Stroke. Mar 2003;34(3):758-763.

502. Sun G, Zhao X, Grotta J, Savitz SI, Chen C, Aronowski J. Apyrase, APT102, improves the rt-PA beneficial effect in thromboembolic stroke model. Paper presented at: International Stroke Conference2011; Los Angeles, California.

503. Wang C, Shabanzadeh AP, He C, Pegg CC. APT102 protects the ischemically injured brain in rats. Paper presented at: Neuroscience2010; San Diego, California.

504. Tan Z, Li X, Kelly KA, Rosen CL, Huber JD. Plasminogen activator inhibitor type 1 derived peptide, EEIIMD, diminishes cortical infarct but fails to improve neurological function in aged rats following middle cerebral artery occlusion. Brain Res. Jul 24 2009;1281:84-90.

505. Seyfried D, Han Y, Lu D, Chen J, Bydon A, Chopp M. Improvement in neurological outcome after administration of atorvastatin following experimental intracerebral hemorrhage in rats. J Neurosurg. Jul 2004;101(1):104-107.

506. Christoforidis GA, Slivka A, Mohammad Y, Karakasis C, Avutu B, Yang M. Size matters: hemorrhage volume as an objective measure to define significant intracranial hemorrhage associated with thrombolysis. Stroke. Jun 2007;38(6):1799-1804.

507. Popa-Wagner A, Badan I, Vintilescu R, Walker L, Kessler C. Premature cellular proliferation following cortical infarct in aged rats. Rom J Morphol Embryol. 2006;47(3): 215-228.

508. Popa-Wagner A, Badan I, Walker L, Groppa S, Patrana N, Kessler C. Accelerated infarct development, cytogenesis and apoptosis following transient cerebral ischemia in aged rats. Acta Neuropathol. Mar 2007;113(3):277-293. 
509. Popa-Wagner A, Dinca I, Yalikun S, Walker L, Kroemer H, Kessler C. Accelerated delimitation of the infarct zone by capillary-derived nestin-positive cells in aged rats. Curr Neurovasc Res. Feb 2006;3(1):3-13.

510. Marcus AJ, Broekman MJ, Drosopoulos JH, et al. Role of CD39 (NTPDase-1) in thromboregulation, cerebroprotection, and cardioprotection. Semin Thromb Hemost. Apr 2005;31(2):234-246.

511. Marcus AJ, Broekman MJ, Drosopoulos JH, Pinsky DJ, Islam N, Maliszewsk CR. Inhibition of platelet recruitment by endothelial cell CD39/ecto-ADPase: significance for occlusive vascular diseases. Ital Heart J. Nov 2001;2(11):824-830.

512. Bours MJ, Swennen EL, Di Virgilio F, Cronstein BN, Dagnelie PC. Adenosine 5'triphosphate and adenosine as endogenous signaling molecules in immunity and inflammation. Pharmacol Ther. Nov 2006;112(2):358-404.

513. Dwyer KM, Robson SC, Nandurkar HH, et al. Thromboregulatory manifestations in human CD39 transgenic mice and the implications for thrombotic disease and transplantation. J Clin Invest. May 2004;113(10):1440-1446.

514. Imai M, Takigami K, Guckelberger O, et al. CD39/vascular ATP diphosphohydrolase modulates xenograft survival. Transplant Proc. Aug 2000;32(5):969.

515. Wang TF, Guidotti G. CD39 is an ecto-(Ca2+,Mg2+)-apyrase. J Biol Chem. Apr 26 1996;271(17):9898-9901.

516. Marcus AJ, Safier LB, Hajjar KA, et al. Inhibition of platelet function by an aspirininsensitive endothelial cell ADPase. Thromboregulation by endothelial cells. $J$ Clin Invest. Nov 1991;88(5):1690-1696.

517. Pinsky DJ, Broekman MJ, Peschon JJ, et al. Elucidation of the thromboregulatory role of CD39/ectoapyrase in the ischemic brain. J Clin Invest. Apr 2002;109(8): 1031-1040.

518. Robson S. The therapeutic potential of CD39: interview with Dr Simon Robson by Emma Quigley. Expert Opin Ther Targets. Oct 2006;10(5):649-652.

519. Robson SC, Wu Y, Sun X, Knosalla C, Dwyer K, Enjyoji K. Ectonucleotidases of CD39 family modulate vascular inflammation and thrombosis in transplantation. Semin Thromb Hemost. Apr 2005;31(2):217-233.

520. Guckelberger O, Sun XF, Sevigny J, et al. Beneficial effects of CD39/ectonucleoside triphosphate diphosphohydrolase- 1 in murine intestinal ischemia-reperfusion injury. Thromb Haemost. Mar 2004;91(3):576-586.

521. Hunter AJ, Mackay KB, Rogers DC. To what extent have functional studies of ischaemia in animals been useful in the assessment of potential neuroprotective agents? Trends Pharmacol Sci. Feb 1998;19(2):59-66.

522. Cenci MA, Whishaw IQ, Schallert T. Animal models of neurological deficits: how relevant is the rat? Nat Rev Neurosci. Jul 2002;3(7):574-579.

523. Recommendations for standards regarding preclinical neuroprotective and restorative drug development. Stroke. Dec 1999;30(12):2752-2758.

524. Daviglus ML, Plassman BL, Pirzada A, et al. Risk factors and preventive interventions for Alzheimer disease: state of the science. Arch Neurol. Sep $2011 ; 68(9)$ : 1185-1190.

525. Hamm RJ, Jenkins LW, Lyeth BG, White-Gbadebo DM, Hayes RL. The effect of age on outcome following traumatic brain injury in rats. J Neurosurg. Dec 1991;75(6): 916-921.

526. Hamm RJ, White-Gbadebo DM, Lyeth BG, Jenkins LW, Hayes RL. The effect of age on motor and cognitive deficits after traumatic brain injury in rats. Neurosurgery. Dec 1992;31(6):1072-1077; discussion 1078.

527. Popa-Wagner A, Craitoiu S, Buga AM. Central nervous system aging and regeneration after injuries. A systematic approach. Rom J Morphol Embryol. 2009;50(2): 155-167. 
528. Mehan ND, Strauss KI. Combined age- and trauma-related proteomic changes in rat neocortex: a basis for brain vulnerability. Neurobiol Aging. Sep 2012;33(9): 1857-1873.

529. Watanabe T, Okuda $\mathrm{Y}$, Nonoguchi $\mathrm{N}$, et al. Postischemic intraventricular administration of FGF-2 expressing adenoviral vectors improves neurologic outcome and reduces infarct volume after transient focal cerebral ischemia in rats. J Cereb Blood Flow Metab. Nov 2004;24(11):1205-1213.

530. Pan W, Ding Y, Yu Y, Ohtaki H, Nakamachi T, Kastin AJ. Stroke upregulates TNFalpha transport across the blood-brain barrier. Exp Neurol. Mar 2006;198(1): 222-233.

531. Rivlin AS, Tator $\mathrm{CH}$. Objective clinical assessment of motor function after experimental spinal cord injury in the rat. J Neurosurg. Oct 1977;47(4):577-581.

532. Monville C, Torres EM, Dunnett SB. Comparison of incremental and accelerating protocols of the rotarod test for the assessment of motor deficits in the 6-OHDA model. $J$ Neurosci Methods. Dec 15 2006;158(2):219-223.

533. Walf AA, Frye CA. The use of the elevated plus maze as an assay of anxietyrelated behavior in rodents. Nat Protoc. 2007;2(2):322-328.

534. Porsolt RD, Anton $G$, Blavet $N$, Jalfre $M$. Behavioural despair in rats: a new model sensitive to antidepressant treatments. Eur J Pharmacol. Feb 15 1978;47(4): 379-391.

535. Porsolt RD, Brossard G, Hautbois C, Roux S. Rodent models of depression: forced swimming and tail suspension behavioral despair tests in rats and mice. Curr Protoc Neurosci. May 2001;Chapter 8:Unit 8 10A.

536. Sacco RL. Risk factors, outcomes, and stroke subtypes for ischemic stroke. Neurology. Nov 1997;49(5 Suppl 4):S39-44.

537. Maurissen JP, Marable BR, Andrus AK, Stebbins KE. Factors affecting grip strength testing. Neurotoxicol Teratol. Sep-Oct 2003;25(5):543-553.

538. Turner RC, Seminerio MJ, Naser ZJ, et al. Effects of aging on behavioral assessment performance: implications for clinically relevant models of neurological disease. J Neurosurg. Sep 2012;117(3):629-637.

539. Astrup J. Energy-requiring cell functions in the ischemic brain. Their critical supply and possible inhibition in protective therapy. J Neurosurg. Apr 1982;56(4): 482-497.

540. Astrup J, Siesjo BK, Symon L. Thresholds in cerebral ischemia - the ischemic penumbra. Stroke. Nov-Dec 1981;12(6):723-725.

541. Dirnagl U, Macleod MR. Stroke research at a road block: the streets from adversity should be paved with meta-analysis and good laboratory practice. $\mathrm{Br} J$ Pharmacol. Aug 2009;157(7):1154-1156.

542. Petcu EB, Sfredel V, Platt D, Herndon JG, Kessler C, Popa-Wagner A. Cellular and molecular events underlying the dysregulated response of the aged brain to stroke: a mini-review. Gerontology. 2008;54(1):6-17.

543. Popa-Wagner A, Buga AM, Turner RC, Rosen CL, Toescu E. Cerebrovascular disorders: role of aging. J Aging Res. 2012;2012:128146.

544. Turner RC, Seminerio MJ, Naser ZJ, et al. Effects of aging on behavioral assessment performance: implications for clinically relevant models of neurological disease. J Neurosurg. Jun 292012.

545. Saver JL, Johnston KC, Homer D, et al. Infarct volume as a surrogate or auxiliary outcome measure in ischemic stroke clinical trials. The RANTTAS Investigators. Stroke. Feb 1999;30(2):293-298.

546. Bouet V, Freret $T$, Toutain J, Divoux D, Boulouard M, Schumann-Bard P. Sensorimotor and cognitive deficits after transient middle cerebral artery occlusion in the mouse. Exp Neurol. Feb 2007;203(2):555-567. 
547. Encarnacion A, Horie N, Keren-Gill H, Bliss TM, Steinberg GK, Shamloo M. Long-term behavioral assessment of function in an experimental model for ischemic stroke. J Neurosci Methods. Mar 30 2011;196(2):247-257.

548. Freret T, Chazalviel L, Roussel S, Bernaudin M, Schumann-Bard P, Boulouard M. Long-term functional outcome following transient middle cerebral artery occlusion in the rat: correlation between brain damage and behavioral impairment. Behav Neurosci. Dec 2006;120(6):1285-1298.

549. Haelewyn B, Rouillon C, Risso JJ, Abraini JH. Functional (neurologic) recovery following transient focal cerebral ischemia in the rat requires at least $80 \%$ of ipsilateral cortical and subcortical integrity. Exp Neurol. Sep 2008;213(1):238-240.

550. Lim SH, Lee JS, Lee JI, Im S, Ko YJ, Kim HW. The quantitative assessment of functional impairment and its correlation to infarct volume in rats with transient middle cerebral artery occlusion. Brain Res. Sep 16 2008;1230:303-309.

551. Nedelmann M, Wilhelm-Schwenkmezger T, Alessandri B, et al. Cerebral embolic ischemia in rats: correlation of stroke severity and functional deficit as important outcome parameter. Brain Res. Jan 26 2007;1130(1):188-196.

552. Wahl F, Allix M, Plotkine M, Boulu RG. Neurological and behavioral outcomes of focal cerebral ischemia in rats. Stroke. Feb 1992;23(2):267-272.

553. Yager JY, Wright S, Armstrong EA, Jahraus CM, Saucier DM. The influence of aging on recovery following ischemic brain damage. Behav Brain Res. Oct 16 2006;173 (2):171-180.

554. Zausinger S, Hungerhuber E, Baethmann A, Reulen H, Schmid-Elsaesser R. Neurological impairment in rats after transient middle cerebral artery occlusion: a comparative study under various treatment paradigms. Brain Res. Apr 28 2000;863(1-2): 94-105.

555. Tominaga T, Ohnishi ST. Interrelationship of brain edema, motor deficits, and memory impairment in rats exposed to focal ischemia. Stroke. Apr 1989;20(4):513-518.

556. Yoo AJ, Chaudhry ZA, Nogueira RG, et al. Infarct volume is a pivotal biomarker after intra-arterial stroke therapy. Stroke. May 2012;43(5):1323-1330.

557. Trinh VT, Fahim DK, Shah K, et al. Subcortical injury is an independent predictor of worsening neurological deficits following awake craniotomy procedures. Neurosurgery. Feb 2013;72(2):160-169.

558. Agarwal S, Scoffings DJ, Jones PS, et al. Interaction of age with the ischaemic penumbra, leptomeningeal collateral circulation and haemodynamic variables in acute stroke: a pilot study. J Neurol Neurosurg Psychiatry. Mar 2013;84(3):271-276.

559. Raghupathi R, Graham DI, Mclntosh TK. Apoptosis after traumatic brain injury. $J$ Neurotrauma. Oct 2000;17(10):927-938.

560. Buki A, Okonkwo DO, Wang KK, Povlishock JT. Cytochrome c release and caspase activation in traumatic axonal injury. J Neurosci. Apr 15 2000;20(8):2825-2834.

561. Wang HG, Pathan N, Ethell IM, et al. Ca2+-induced apoptosis through calcineurin dephosphorylation of BAD. Science. Apr 9 1999;284(5412):339-343.

562. Marmarou A, Foda MA, van den Brink W, Campbell J, Kita H, Demetriadou K. A new model of diffuse brain injury in rats. Part I: Pathophysiology and biomechanics. $J$ Neurosurg. Feb 1994;80(2):291-300.

563. Stone JR, Walker SA, Povlishock JT. The visualization of a new class of traumatically injured axons through the use of a modified method of microwave antigen retrieval. Acta Neuropathol. Apr 1999;97(4):335-345.

564. Mills JD, Stone JR, Rubin DG, et al. Illuminating protein interactions in tissue using confocal and two-photon excitation fluorescent resonance energy transfer microscopy. J Biomed Opt. Jul 2003;8(3):347-356.

565. Basaria S, Coviello AD, Travison TG, et al. Adverse events associated with testosterone administration. N Engl J Med. Jul 8 2010;363(2):109-122. 
566. Bhasin S, Storer TW, Berman N, et al. The effects of supraphysiologic doses of testosterone on muscle size and strength in normal men. N Engl J Med. Jul 4 1996;335 (1):1-7.

567. Talih F, Fattal O, Malone D, Jr. Anabolic steroid abuse: psychiatric and physical costs. Cleve Clin J Med. May 2007;74(5):341-344, 346, 349-352.

568. vandenBerg $P$, Neumark-Sztainer D, Cafri G, Wall $M$. Steroid use among adolescents: longitudinal findings from Project EAT. Pediatrics. Mar 2007;119(3): 476-486.

569. Evans NA. Current concepts in anabolic-androgenic steroids. Am J Sports Med. Mar 2004;32(2):534-542.

570. Scott DM, Wagner JC, Barlow TW. Anabolic steroid use among adolescents in Nebraska schools. Am J Health Syst Pharm. Sep 1 1996;53(17):2068-2072.

571. Faul M, Xu L, Wald MM, Coronado VG. Traumatic Brain Injury in the United States: Emergency Department Visits, Hospitalizations and Deaths 2002-2006: Centers for Disease Control and Prevention; 2010.

572. Langburt W, Cohen B, Akhthar N, O'Neill K, Lee JC. Incidence of concussion in high school football players of Ohio and Pennsylvania. J Child Neurol. Feb 2001;16(2): 83-85.

573. Guskiewicz KM, McCrea M, Marshall SW, et al. Cumulative effects associated with recurrent concussion in collegiate football players: the NCAA Concussion Study. JAMA. Nov 19 2003;290(19):2549-2555.

574. Guskiewicz KM, Marshall SW, Bailes J, et al. Recurrent concussion and risk of depression in retired professional football players. Med Sci Sports Exerc. Jun 2007;39 (6):903-909.

575. Horn S, Gregory P, Guskiewicz KM. Self-reported anabolic-androgenic steroids use and musculoskeletal injuries: findings from the center for the study of retired athletes health survey of retired NFL players. Am J Phys Med Rehabil. Mar 2009;88(3):192-200.

576. Rabadi $\mathrm{MH}$, Jordan BD. The cumulative effect of repetitive concussion in sports. Clin J Sport Med. Jul 2001;11(3):194-198.

577. Benson BW, Hamilton GM, Meeuwisse WH, McCrory P, Dvorak J. Is protective equipment useful in preventing concussion? A systematic review of the literature. $\mathrm{Br} J$ Sports Med. May 2009;43 Suppl 1:i56-67.

578. Gurdjian ES. Cerebral contusions: re-evaluation of the mechanism of their development. J Trauma. Jan 1976;16(1):35-51.

579. Bailes JE, Mills JD. Docosahexaenoic acid reduces traumatic axonal injury in a rodent head injury model. J Neurotrauma. Sep 2010;27(9):1617-1624.

580. Mills JD, Hadley K, Bailes JE. Dietary supplementation with the omega-3 fatty acid docosahexaenoic acid in traumatic brain injury. Neurosurgery. Feb 2011;68(2): 474-481; discussion 481.

581. Schumacher M, Guennoun R, Stein DG, De Nicola AF. Progesterone: therapeutic opportunities for neuroprotection and myelin repair. Pharmacol Ther. Oct 2007;116(1): 77-106.

582. Chavko M, Koller WA, Prusaczyk WK, McCarron RM. Measurement of blast wave by a miniature fiber optic pressure transducer in the rat brain. $J$ Neurosci Methods. Jan 30 2007;159(2):277-281.

583. Lee EJ, Park KH, Kim DM, Yoo YC, Kang SH, Kim YJ. Assessing intraocular pressure by rebound tonometer in rats with an air-filled anterior chamber. Jpn $J$ Ophthalmol. Nov-Dec 2008;52(6):500-503.

584. Wang WH, Millar JC, Pang IH, Wax MB, Clark AF. Noninvasive measurement of rodent intraocular pressure with a rebound tonometer. Invest Ophthalmol Vis Sci. Dec 2005;46(12):4617-4621.

585. Mills JD, Bailes JE, Sedney CL, Hutchins H, Sears B. Omega-3 fatty acid supplementation and reduction of traumatic axonal injury in a rodent head injury model. $J$ Neurosurg. Jan 2011;114(1):77-84. 
586. Raphael $\mathrm{JH}$, Chotai $\mathrm{R}$. Effects of the cervical collar on cerebrospinal fluid pressure. Anaesthesia. May 1994;49(5):437-439.

587. Teng C, Gurses-Ozden R, Liebmann JM, Tello C, Ritch R. Effect of a tight necktie on intraocular pressure. Br J Ophthalmol. Aug 2003;87(8):946-948.

588. May PR, Fuster JM, Haber J, Hirschman A. Woodpecker drilling behavior. An endorsement of the rotational theory of impact brain injury. Arch Neurol. Jun 1979;36(6): 370-373.

589. Rai R, Ranade A, Nayak S, Vadgaonkar R, Mangala P, Krishnamurthy A. A study of anatomical variability of the omohyoid muscle and its clinical relevance. Clinics (Sao Paulo). Aug 2008;63(4):521-524.

590. Bruns J, Jr., Hauser WA. The epidemiology of traumatic brain injury: a review. Epilepsia. 2003;44 Suppl 10:2-10.

591. Barha CK, Ishrat T, Epp JR, Galea LA, Stein DG. Progesterone treatment normalizes the levels of cell proliferation and cell death in the dentate gyrus of the hippocampus after traumatic brain injury. Exp Neurol. Sep 2011;231(1):72-81.

592. Shear DA, Galani R, Hoffman SW, Stein DG. Progesterone protects against necrotic damage and behavioral abnormalities caused by traumatic brain injury. Exp Neurol. Nov 2002;178(1):59-67.

593. Stein DG. Progesterone exerts neuroprotective effects after brain injury. Brain Res Rev. Mar 2008;57(2):386-397.

594. May PR, Fuster JM, Newman P, Hirschman A. Woodpeckers and head injury. Lancet. Feb 28 1976;1(7957):454-455.

595. Wang L, Cheung JT, Pu F, Li D, Zhang M, Fan Y. Why do woodpeckers resist head impact injury: a biomechanical investigation. PLoS One. 2011;6(10):e26490.

596. Wygnanski-Jaffe T, Murphy CJ, Smith C, et al. Protective ocular mechanisms in woodpeckers. Eye (Lond). Jan 2007;21(1):83-89.

597. Patra P, Gunness TK, Robert R, et al. Physiologic variations of the internal jugular vein surface, role of the omohyoid muscle, a preliminary echographic study. Surg Radiol Anat. 1988;10(2):107-112.

598. Smith DW, Bailes JE, Fisher JA, Robles J, Turner RC, Mills JD. Internal jugular vein compression mitigates traumatic axonal injury in a rat model by reducing the intracranial slosh effect. Neurosurgery. Mar 2012;70(3):740-746.

599. Ibrahim RD, Pilipchuk VN. Recent advances in liquid sloshing dynamics. Appl Mech Rev. 2001;54(2):133-199.

600. Attwell D, Buchan AM, Charpak S, Lauritzen M, Macvicar BA, Newman EA. Glial and neuronal control of brain blood flow. Nature. Nov 11 2010;468(7321):232-243.

601. Bignami A. Glial cells in the central nervous system1991.

602. Foda MA, Marmarou A. A new model of diffuse brain injury in rats. Part II: Morphological characterization. J Neurosurg. Feb 1994;80(2):301-313.

603. Schmued LC, Hopkins KJ. Fluoro-Jade: novel fluorochromes for detecting toxicant-induced neuronal degeneration. Toxicol Pathol. Jan-Feb 2000;28(1):91-99.

604. Anderson KJ, Miller KM, Fugaccia I, Scheff SW. Regional distribution of fluorojade B staining in the hippocampus following traumatic brain injury. Exp Neurol. May 2005;193(1):125-130.

605. Gao X, Deng-Bryant Y, Cho W, Carrico KM, Hall ED, Chen J. Selective death of newborn neurons in hippocampal dentate gyrus following moderate experimental traumatic brain injury. J Neurosci Res. Aug 1 2008;86(10):2258-2270.

606. Haselkorn ML, Shellington DK, Jackson EK, et al. Adenosine A1 receptor activation as a brake on the microglial response after experimental traumatic brain injury in mice. J Neurotrauma. May 2010;27(5):901-910.

607. Sato $\mathrm{M}$, Chang $\mathrm{E}$, Igarashi $\mathrm{T}$, Noble LJ. Neuronal injury and loss after traumatic brain injury: time course and regional variability. Brain Res. Oct 26 2001;917(1):45-54.

608. Zhao X, Ahram A, Berman RF, Muizelaar JP, Lyeth BG. Early loss of astrocytes after experimental traumatic brain injury. Glia. Nov 2003;44(2):140-152. 
609. Vibulsreth S, Hefti F, Ginsberg MD, Dietrich WD, Busto R. Astrocytes protect cultured neurons from degeneration induced by anoxia. Brain Res. Oct 6 1987;422(2): 303-311.

610. Fitch MT, Doller C, Combs CK, Landreth GE, Silver J. Cellular and molecular mechanisms of glial scarring and progressive cavitation: in vivo and in vitro analysis of inflammation-induced secondary injury after CNS trauma. J Neurosci. Oct 1 1999;19 (19):8182-8198.

611. Ridet JL, Malhotra SK, Privat A, Gage FH. Reactive astrocytes: cellular and molecular cues to biological function. Trends Neurosci. Dec 1997;20(12):570-577.

612. Sandhir R, Onyszchuk G, Berman NE. Exacerbated glial response in the aged mouse hippocampus following controlled cortical impact injury. Exp Neurol. Oct 2008;213(2):372-380.

613. Shitaka Y, Tran HT, Bennett RE, et al. Repetitive closed-skull traumatic brain injury in mice causes persistent multifocal axonal injury and microglial reactivity. $J$ Neuropathol Exp Neurol. Jul 2011;70(7):551-567.

614. Venkatesan C, Chrzaszcz M, Choi N, Wainwright MS. Chronic upregulation of activated microglia immunoreactive for galectin-3/Mac-2 and nerve growth factor following diffuse axonal injury. J Neuroinflammation. 2010;7:32.

615. Elder GA, Cristian A. Blast-related mild traumatic brain injury: mechanisms of injury and impact on clinical care. Mt Sinai J Med. Apr 2009;76(2):111-118.

616. Nakagawa A, Manley GT, Gean AD, et al. Mechanisms of primary blast-induced traumatic brain injury: insights from shock-wave research. J Neurotrauma. Jun 2011;28 (6):1101-1119.

617. Champion HR, Holcomb JB, Young LA. Injuries from explosions: physics, biophysics, pathology, and required research focus. J Trauma. May 2009;66(5): 1468-1477; discussion 1477.

618. Schneiderman AI, Braver ER, Kang HK. Understanding sequelae of injury mechanisms and mild traumatic brain injury incurred during the conflicts in Iraq and Afghanistan: persistent postconcussive symptoms and posttraumatic stress disorder. Am J Epidemiol. Jun 15 2008;167(12):1446-1452.

619. Terrio H, Brenner LA, Ivins BJ, et al. Traumatic brain injury screening: preliminary findings in a US Army Brigade Combat Team. J Head Trauma Rehabil. Jan-Feb 2009;24 (1):14-23.

620. Ahlers ST, Vasserman-Stokes E, Shaughness MC, et al. Assessment of the effects of acute and repeated exposure to blast overpressure in rodents: toward a greater understanding of blast and the potential ramifications for injury in humans exposed to blast. Front Neurol. 2012;3:32.

621. Chavko M, Watanabe T, Adeeb S, Lankasky J, Ahlers ST, McCarron RM. Relationship between orientation to a blast and pressure wave propagation inside the rat brain. J Neurosci Methods. Jan 30 2011;195(1):61-66.

622. Shridharani JK, Wood GW, Panzer MB, et al. Porcine head response to blast. Front Neurol. 2012;3:70.

623. Turner RC, Naser ZJ, Bailes JE, Smith DW, Fisher JA, Rosen CL. Effect of slosh mitigation on histologic markers of traumatic brain injury. J Neurosurg. Sep 212012.

624. Schmitz C, Hof PR. Design-based stereology in neuroscience. Neuroscience. 2005;130(4):813-831.

625. Turner RC, Naser ZJ, Bailes JE, Smith DW, Fisher JA, Rosen CL. Effect of slosh mitigation on histologic markers of traumatic brain injury: laboratory investigation. $J$ Neurosurg. Dec 2012;117(6):1110-1118.

626. Reneer DV, Hisel RD, Hoffman JM, Kryscio RJ, Lusk BT, Geddes JW. A multimode shock tube for investigation of blast-induced traumatic brain injury. J Neurotrauma. Jan 2011;28(1):95-104.

627. Cheng J, Gu J, Ma Y, et al. Development of a rat model for studying blastinduced traumatic brain injury. J Neurol Sci. Jul 15 2010;294(1-2):23-28. 
628. de Lanerolle NC, Bandak F, Kang D, et al. Characteristics of an explosive blastinduced brain injury in an experimental model. J Neuropathol Exp Neurol. Nov 2011;70 (11):1046-1057.

629. Svetlov SI, Prima V, Glushakova O, et al. Neuro-glial and systemic mechanisms of pathological responses in rat models of primary blast overpressure compared to "composite" blast. Front Neurol. 2012;3:15.

630. Johnson VE, Stewart JE, Begbie FD, Trojanowski JQ, Smith DH, Stewart W. Inflammation and white matter degeneration persist for years after a single traumatic brain injury. Brain. Jan 2013;136(Pt 1):28-42.

631. Conventional Weapons Effects Program [computer program]. Vicksburg, MS: United States Army Corps of Engineers; 2004.

632. Richmond DR, Damon EG, Fletcher ER, Bowen IG, White CS. The relationship between selected blast-wave parameters and the response of mammals exposed to air blast. Ann N Y Acad Sci. Oct 28 1968;152(1):103-121.

633. Bowen IG, Fletcher ER, Richmond DR, Hirsch FG, White CS. Biophysical mechanisms and scaling procedures applicable in assessing responses of the thorax energized by air-blast overpressures or by nonpenetrating missiles. Ann N Y Acad Sci. Oct 28 1968;152(1):122-146.

634. Park E, Gottlieb JJ, Cheung B, Shek PN, Baker AJ. A model of low-level primary blast brain trauma results in cytoskeletal proteolysis and chronic functional impairment in the absence of lung barotrauma. J Neurotrauma. Mar 2011;28(3):343-357.

635. Rafaels KA, dale' Bass $\mathrm{CR}$, Panzer MB, et al. Brain injury risk from primary blast. J Trauma Acute Care Surg. Oct 2012;73(4):895-901.

636. Risling $M$, Davidsson J. Experimental animal models for studies on the mechanisms of blast-induced neurotrauma. Front Neurol. 2012;3:30.

637. Cernak I, Noble-Haeusslein LJ. Traumatic brain injury: an overview of pathobiology with emphasis on military populations. J Cereb Blood Flow Metab. Feb 2010;30(2):255-266.

638. Covey DC, Born CT. Blast injuries: mechanics and wounding patterns. J Surg Orthop Adv. Spring 2010;19(1):8-12.

639. Yeh DD, Schecter WP. Primary blast injuries--an updated concise review. World J Surg. May 2012;36(5):966-972.

640. Duckworth JL, Grimes J, Ling GS. Pathophysiology of battlefield associated traumatic brain injury. Pathophysiology. Jun 132012.

641. Rubovitch V, Ten-Bosch $\mathrm{M}$, Zohar O, et al. A mouse model of blast-induced mild traumatic brain injury. Exp Neurol. Dec 2011;232(2):280-289.

642. Readnower RD, Chavko M, Adeeb $S$, et al. Increase in blood-brain barrier permeability, oxidative stress, and activated microglia in a rat model of blast-induced traumatic brain injury. J Neurosci Res. Dec 2010;88(16):3530-3539.

643. Garman RH, Jenkins LW, Switzer RC, 3rd, et al. Blast exposure in rats with body shielding is characterized primarily by diffuse axonal injury. $J$ Neurotrauma. Jun 2011;28 (6):947-959.

644. Cernak I, Merkle AC, Koliatsos VE, et al. The pathobiology of blast injuries and blast-induced neurotrauma as identified using a new experimental model of injury in mice. Neurobiol Dis. Feb 2011;41(2):538-551.

645. Kaur C, Singh J, Lim MK, Ng BL, Yap EP, Ling EA. The response of neurons and microglia to blast injury in the rat brain. Neuropathol Appl Neurobiol. Oct 1995;21(5): 369-377.

646. Kane MJ, Angoa-Perez M, Francescutti DM, et al. Altered gene expression in cultured microglia in response to simulated blast overpressure: possible role of pulse duration. Neurosci Lett. Jul 26 2012;522(1):47-51.

647. Magnuson J, Leonessa F, Ling GS. Neuropathology of explosive blast traumatic brain injury. Curr Neurol Neurosci Rep. Oct 2012;12(5):570-579. 
648. Scheibel RS, Newsome MR, Troyanskaya M, et al. Altered brain activation in military personnel with one or more traumatic brain injuries following blast. $J$ Int Neuropsychol Soc. Jan 2012;18(1):89-100.

649. Taber $\mathrm{KH}$, Warden DL, Hurley RA. Blast-related traumatic brain injury: what is known? J Neuropsychiatry Clin Neurosci. Spring 2006;18(2):141-145.

650. Kuehn R, Simard PF, Driscoll I, et al. Rodent model of direct cranial blast injury. $J$ Neurotrauma. Oct 2011;28(10):2155-2169.

651. Koliatsos VE, Cernak I, Xu L, et al. A mouse model of blast injury to brain: initial pathological, neuropathological, and behavioral characterization. J Neuropathol Exp Neurol. May 2011;70(5):399-416.

652. Dalle Lucca JJ, Chavko M, Dubick MA, et al. Blast-induced moderate neurotrauma (BINT) elicits early complement activation and tumor necrosis factor alpha (TNFalpha) release in a rat brain. $J$ Neurol Sci. Jul 15 2012;318(1-2):146-154.

653. Duhaime AC, Beckwith JG, Maerlender AC, et al. Spectrum of acute clinical characteristics of diagnosed concussions in college athletes wearing instrumented helmets: clinical article. J Neurosurg. Dec 2012;117(6):1092-1099.

654. Mac Donald CL, Johnson AM, Cooper D, et al. Detection of blast-related traumatic brain injury in U.S. military personnel. N Engl J Med. Jun 2 2011;364(22): 2091-2100.

655. Finnoff JT, Jelsing EJ, Smith J. Biomarkers, genetics, and risk factors for concussion. PM R. Oct 2011;3(10 Suppl 2):S452-459.

656. Dashnaw ML, Petraglia AL, Bailes JE. An overview of the basic science of concussion and subconcussion: where we are and where we are going. Neurosurg Focus. Dec 2012;33(6):E5: 1-9.

657. Levin H, Robertson CS. Mild TBI in Translation. J Neurotrauma. Oct 92012.

658. Shultz SR, MacFabe DF, Foley KA, Taylor R, Cain DP. A single mild fluid percussion injury induces short-term behavioral and neuropathological changes in the Long-Evans rat: support for an animal model of concussion. Behav Brain Res. Oct 31 $2011 ; 224(2): 326-335$.

659. Harmon KG, Drezner JA, Gammons M, et al. American Medical Society for Sports Medicine position statement: concussion in sport. Br J Sports Med. Jan 2013;47 (1):15-26.

660. McCullough BJ, Jarvik JG. Diagnosis of concussion: the role of imaging now and in the future. Phys Med Rehabil Clin N Am. Nov 2011;22(4):635-652, viii.

661. Fromm L, Heath DL, Vink R, Nimmo AJ. Magnesium attenuates post-traumatic depression/anxiety following diffuse traumatic brain injury in rats. $J$ Am Coll Nutr. Oct 2004;23(5):529S-533S.

662. Kontos AP, Covassin T, Elbin RJ, Parker T. Depression and neurocognitive performance after concussion among male and female high school and collegiate athletes. Arch Phys Med Rehabil. Oct 2012;93(10):1751-1756.

663. Meyer DL, Davies DR, Barr JL, Manzerra P, Forster GL. Mild traumatic brain injury in the rat alters neuronal number in the limbic system and increases conditioned fear and anxiety-like behaviors. Exp Neurol. Apr 12012.

664. Ding Y, Yao B, Lai Q, McAllister JP. Impaired motor learning and diffuse axonal damage in motor and visual systems of the rat following traumatic brain injury. Neurol Res. Mar-Apr 2001;23(2-3):193-202.

665. Hallam TM, Floyd CL, Folkerts MM, et al. Comparison of behavioral deficits and acute neuronal degeneration in rat lateral fluid percussion and weight-drop brain injury models. J Neurotrauma. May 2004;21(5):521-539.

666. Heath DL, Vink R. Improved motor outcome in response to magnesium therapy received up to 24 hours after traumatic diffuse axonal brain injury in rats. $J$ Neurosurg. Mar 1999;90(3):504-509.

667. Heath DL, Vink R. Magnesium sulphate improves neurologic outcome following severe closed head injury in rats. Neurosci Lett. Jun 13 1997;228(3):175-178. 
668. Hylin MJ, Orsi SA, Zhao J, et al. Behavioral and histopathological alterations resulting from mild fluid percussion injury. J Neurotrauma. Jan 92013.

669. Kane MJ, Angoa-Perez M, Briggs DI, Viano DC, Kreipke CW, Kuhn DM. A mouse model of human repetitive mild traumatic brain injury. J Neurosci Methods. Jan 15 2012;203(1):41-49.

670. Lyeth BG, Jenkins LW, Hamm RJ, et al. Prolonged memory impairment in the absence of hippocampal cell death following traumatic brain injury in the rat. Brain Res. Sep 3 1990;526(2):249-258.

671. Mouzon B, Chaytow $\mathrm{H}$, Crynen $\mathrm{G}$, et al. Repetitive mild traumatic brain injury in a mouse model produces learning and memory deficits accompanied by histological changes. J Neurotrauma. Dec 10 2012;29(18):2761-2773.

672. Rostami E, Davidsson J, Ng KC, et al. A Model for Mild Traumatic Brain Injury that Induces Limited Transient Memory Impairment and Increased Levels of Axon Related Serum Biomarkers. Front Neurol. 2012;3:115.

673. Zhao Z, Loane DJ, Murray MG, 2nd, Stoica BA, Faden Al. Comparing the predictive value of multiple cognitive, affective, and motor tasks after rodent traumatic brain injury. J Neurotrauma. Oct 10 2012;29(15):2475-2489.

674. Adelson PD, Fellows-Mayle W, Kochanek PM, Dixon CE. Morris water maze function and histologic characterization of two age-at-injury experimental models of controlled cortical impact in the immature rat. Childs Nerv Syst. Jan 2013;29(1):43-53.

675. Beaumont A, Marmarou A, Czigner A, et al. The impact-acceleration model of head injury: injury severity predicts motor and cognitive performance after trauma. Neurol Res. Dec 1999;21(8):742-754.

676. Beaumont A, Marmarou C, Marmarou A. The effects of human corticotrophin releasing factor on motor and cognitive deficits after impact acceleration injury. Neurol Res. Oct 2000;22(7):665-673.

677. Berman RF, Verweij BH, Muizelaar JP. Neurobehavioral protection by the neuronal calcium channel blocker ziconotide in a model of traumatic diffuse brain injury in rats. J Neurosurg. Nov 2000;93(5):821-828.

678. Dapul HR, Park J, Zhang J, et al. Concussive Injury before or after Controlled Cortical Impact Exacerbates Histopathology and Functional Outcome in a Mixed Traumatic Brain Injury Model in Mice. J Neurotrauma. Feb 202013.

679. DeFord SM, Wilson MS, Rice AC, et al. Repeated mild brain injuries result in cognitive impairment in B6C3F1 mice. J Neurotrauma. Apr 2002;19(4):427-438.

680. Gurkoff GG, Giza CC, Hovda DA. Lateral fluid percussion injury in the developing rat causes an acute, mild behavioral dysfunction in the absence of significant cell death. Brain Res. Mar 10 2006;1077(1):24-36.

681. Maughan $\mathrm{PH}$, Scholten KJ, Schmidt RH. Recovery of water maze performance in aged versus young rats after brain injury with the impact acceleration model. $J$ Neurotrauma. Dec 2000;17(12):1141-1153.

682. Meehan WP, 3rd, Zhang J, Mannix R, Whalen MJ. Increasing Recovery Time Between Injuries Improves Cognitive Outcome After Repetitive Mild Concussive Brain Injuries in Mice. Neurosurgery. Jun 272012.

683. Schmidt RH, Scholten KJ, Maughan PH. Cognitive impairment and synaptosomal choline uptake in rats following impact acceleration injury. J Neurotrauma. Dec 2000;17 (12):1129-1139.

684. Schmidt $\mathrm{RH}$, Scholten KJ, Maughan PH. Time course for recovery of water maze performance and central cholinergic innervation after fluid percussion injury. $J$ Neurotrauma. Dec 1999;16(12):1139-1147.

685. Washington PM, Forcelli PA, Wilkins T, Zapple DN, Parsadanian M, Burns MP. The Effect of Injury Severity on Behavior: A Phenotypic Study of Cognitive and Emotional Deficits after Mild, Moderate, and Severe Controlled Cortical Impact Injury in Mice. J Neurotrauma. Aug 32012. 
686. Zohar O, Rubovitch V, Milman A, Schreiber S, Pick CG. Behavioral consequences of minimal traumatic brain injury in mice. Acta Neurobiol Exp (Wars). 2011;71(1):36-45.

687. Eakin K, Miller JP. Mild traumatic brain injury is associated with impaired hippocampal spatiotemporal representation in the absence of histological changes. $J$ Neurotrauma. Apr 10 2012;29(6):1180-1187.

688. Milman A, Zohar O, Maayan R, Weizman R, Pick CG. DHEAS repeated treatment improves cognitive and behavioral deficits after mild traumatic brain injury. Eur Neuropsychopharmacol. Mar 2008;18(3):181-187.

689. Schwarzbold ML, Rial D, De Bem T, et al. Effects of traumatic brain injury of different severities on emotional, cognitive, and oxidative stress-related parameters in mice. J Neurotrauma. Oct 2010;27(10):1883-1893.

690. Cutler SM, VanLandingham JW, Murphy AZ, Stein DG. Slow-release and injected progesterone treatments enhance acute recovery after traumatic brain injury. Pharmacol Biochem Behav. Jul 2006;84(3):420-428.

691. Hogg S, Moser PC, Sanger DJ. Mild traumatic lesion of the right parietal cortex of the rat: selective behavioural deficits in the absence of neurological impairment. Behav Brain Res. Jun 1998;93(1-2):143-155.

692. Jones NC, Cardamone L, Williams JP, Salzberg MR, Myers D, O'Brien TJ. Experimental traumatic brain injury induces a pervasive hyperanxious phenotype in rats. $J$ Neurotrauma. Nov 2008;25(11):1367-1374.

693. Liu YR, Cardamone L, Hogan RE, et al. Progressive metabolic and structural cerebral perturbations after traumatic brain injury: an in vivo imaging study in the rat. $J$ Nucl Med. Nov 2010;51(11):1788-1795.

694. Shapira M, Licht A, Milman A, Pick CG, Shohami E, Eldar-Finkelman H. Role of glycogen synthase kinase-3beta in early depressive behavior induced by mild traumatic brain injury. Mol Cell Neurosci. Apr 2007;34(4):571-577.

695. Shultz SR, MacFabe DF, Foley KA, Taylor R, Cain DP. Sub-concussive brain injury in the Long-Evans rat induces acute neuroinflammation in the absence of behavioral impairments. Behav Brain Res. Apr 1 2012;229(1):145-152.

696. Broglio SP, Eckner JT, Paulson HL, Kutcher JS. Cognitive decline and aging: the role of concussive and subconcussive impacts. Exerc Sport Sci Rev. Jul 2012;40(3): 138-144.

697. Gosselin N, Bottari C, Chen JK, et al. Evaluating the cognitive consequences of mild traumatic brain injury and concussion by using electrophysiology. Neurosurg Focus. Dec 2012;33(6):E7: 1-7.

698. Blokland A. Reaction time responding in rats. Neurosci Biobehav Rev. Oct 1998;22(6):847-864.

699. Bushnell PJ, Strupp BJ. Assessing Attention in Rodents. 2009.

700. Turner RC, Lucke-Wold BP, Robson MJ, Omalu BI, Petraglia AL, Bailes JE. Repetitive traumatic brain injury and development of chronic traumatic encephalopathy: a potential role for biomarkers in diagnosis, prognosis, and treatment? Front Neurol. 2012;3:186.

701. Zhou Y, Riccio DC. Concussion-induced retrograde amnesia in rats. Physiol Behav. Jun 1995;57(6):1107-1115.

702. Prins ML, Hales A, Reger M, Giza CC, Hovda DA. Repeat traumatic brain injury in the juvenile rat is associated with increased axonal injury and cognitive impairments. Dev Neurosci. 2010;32(5-6):510-518.

703. Reger ML, Poulos AM, Buen F, Giza CC, Hovda DA, Fanselow MS. Concussive brain injury enhances fear learning and excitatory processes in the amygdala. Biol Psychiatry. Feb 15 2012;71(4):335-343.

704. Anderson J, Sandhir R, Hamilton ES, Berman NE. Impaired expression of neuroprotective molecules in the HIF-1alpha pathway following traumatic brain injury in aged mice. J Neurotrauma. Sep 2009;26(9):1557-1566. 
705. Wali B, Sayeed I, Stein DG. Improved behavioral outcomes after progesterone administration in aged male rats with traumatic brain injury. Restor Neurol Neurosci. $2011 ; 29(1): 61-71$.

706. Timaru-Kast R, Luh C, Gotthardt $P$, et al. Influence of age on brain edema formation, secondary brain damage and inflammatory response after brain trauma in mice. PLoS One. 2012;7(8):e43829.

707. Hoane MR, Lasley LA, Akstulewicz SL. Middle age increases tissue vulnerability and impairs sensorimotor and cognitive recovery following traumatic brain injury in the rat. Behav Brain Res. Aug 12 2004;153(1):189-197.

708. McKee AC, Stein TD, Nowinski CJ, et al. The spectrum of disease in chronic traumatic encephalopathy. Brain. Jan 2013;136(Pt 1):43-64.

709. Perez de Leon AA, Tabachnick WJ. Apyrase activity and adenosine diphosphate induced platelet aggregation inhibition by the salivary gland proteins of Culicoides variipennis, the North American vector of bluetongue viruses. Vet Parasitol. Feb 1996;61 $(3-4): 327-338$.

710. Bennett RE, Mac Donald CL, Brody DL. Diffusion tensor imaging detects axonal injury in a mouse model of repetitive closed-skull traumatic brain injury. Neurosci Lett. Apr 4 2012;513(2):160-165.

711. Jaffe ER. Methemoglobin pathophysiology. Prog Clin Biol Res. 1981;51:133-151.

712. Ho YS, Yang X, Lau JC, et al. Endoplasmic reticulum stress induces tau pathology and forms a vicious cycle: implication in Alzheimer's disease pathogenesis. $J$ Alzheimers Dis. 2012;28(4):839-854.

713. Hoozemans JJ, van Haastert ES, Nijholt DA, Rozemuller AJ, Eikelenboom P, Scheper W. The unfolded protein response is activated in pretangle neurons in Alzheimer's disease hippocampus. Am J Pathol. Apr 2009;174(4):1241-1251.

714. Hoozemans JJ, van Haastert ES, Nijholt DA, Rozemuller AJ, Scheper W. Activation of the unfolded protein response is an early event in Alzheimer's and Parkinson's disease. Neurodegener Dis. 2012;10(1-4):212-215.

715. Nijholt DA, van Haastert ES, Rozemuller AJ, Scheper W, Hoozemans JJ. The unfolded protein response is associated with early tau pathology in the hippocampus of tauopathies. J Pathol. Apr 2012;226(5):693-702.

716. Hayashi T, Saito A, Okuno S, Ferrand-Drake M, Dodd RL, Chan PH. Damage to the endoplasmic reticulum and activation of apoptotic machinery by oxidative stress in ischemic neurons. J Cereb Blood Flow Metab. Jan 2005;25(1):41-53. 


\title{
APPENDIX
}

\section{SUPPLEMENTAL METHODS}

\author{
Design of Blast Model
}

A novel blast apparatus was designed to simulate a multitude of peak overpressures, based on membrane thickness, each with a short duration (less than 3 milliseconds). To achieve blasts of short duration, the driver, or high-pressure section of the model, is notably short in overall length (Supplemental Figure 1). The apparatus was constructed of machined aluminum.

\section{Immunohistochemistry}

Hematoxylin and Eosin (H\&E) Staining. H\&E staining was performed according to manufacturer's instructions (Sigma Aldrich). After paraffin removal and rehydration, sections were immersed in Harris Hematoxylin solution for 2.25 minutes, rinsed in running tap water, and differentiated using a $0.25 \%$ acid alcohol solution. After rinsing in tap water, sections were blued in Scott's Tap Water Substitute for one minute prior to placement in $95 \%$ ethanol for 30 seconds and counterstaining with Eosin $\mathrm{Y}$ solution for 30 seconds. Slides were rinsed and coverslipped.

Fluoro-Jade B Staining (FJB). Following deparaffinization and rehydration, sections were incubated in $0.06 \%$ potassium permanganate for 10 minutes. Slides were then washed for 2 minutes in deionized water prior to a 20 minute incubation in $0.0004 \%$ FJB in $0.1 \%$ acetic acid. After incubation, sections were washed three times in deionized water (5 minutes each wash). Slides were coverslipped, sealed with acrylic, and stored in the dark in a laboratory refrigerator.

Glial Fibrillary Acidic Protein (GFAP) Staining. Following deparaffinization and rehydration, endogenous peroxidases were quenched using a 10\% methanol and $10 \%$ hydrogen peroxide solution in DPBS for 15 minutes. Slides were rinsed in DPBS three times (5 minutes each) and permeabilized for 30 minutes using a $1.8 \%$ L-lysine, $4 \%$ horse serum, and $0.2 \%$ Triton X-100 in DPBS. Sections were then incubated in polyclonal antibody raised in rabbit against anti-cow GFAP at a dilution of 1:500 in $4 \%$ horse serum in DPBS overnight. Next, sections were washed three times for 5 minutes each prior to application of biotinylated anti-rabbit $\lg G(1: 10,000)$ in DPBS with $4 \%$ horse serum for 4 hours. Slides were developed using an alkaline phosphatase and fast blue salt as a chromagen. Slides were rinsed, coverslipped, and sealed.

lonized Calcium Binding Adapter Molecule 1 (Iba-1) Staining. Labeling of microglia using lba-1 followed an identical procedure to that used for GFAP with the exception of the antibodies utilized.

\section{SUPPLEMENTAL RESULTS}

Neural degeneration occurs in a dose-response manner consistent with blast severity. Blast exposure was associated with an increase in neural degeneration within the cortex, consistent with peak overpressure exposure (Figure 5). Similar findings were also seen 
in other brain regions observed such as the region surrounding and including the corpus callosum (Supplemental Figure 2), the striatum (Supplemental Figure 3), hippocampus (Supplemental Figure 4), and cerebellum (Supplemental Figure 5).

Blast injured brains demonstrate increased glial activation with increasing peak overpressure. Increasing severity of blast exposure was associated with an increased level of astrocyte reactivity, particularly within the corpus callosum (Figure 6). Examination of the entirety of forebrain sections (Supplemental Figure 6) revealed astrogliosis in the cortex, striatum, and hippocampus as well. Cortical examination showed intense astrogliosis, particularly with more severe blast exposure, at the interface with white matter tracts such as the corpus callosum (Supplemental Figure 7). Mild astrocyte activation was also observed within the cortical mantle of blast-exposed brains (Supplemental Figure 6 - dashed arrow), a region of interest considering the similarities with regions of vascular dysfunction based on gross imaging post-blast (Figure 3 ). In other brain regions such as the striatum and the hippocampus, similar findings were observed to those in the cortex in that GFAP staining was more intense at the interface of tissue regions and was increased with peak overpressure exposure severity (Supplemental Figures 8 and 9, respectively). Astrocyte reactivity was also seen within the cerebellum with higher power images acquired from the region identified in Supplemental Figure 10. Again, astrocyte reactivity appeared most predominate in fiber tracts within the cerebellum and predominated on the contrecoup side with the intensity of staining consistent with the severity of peak overpressure exposure (Supplemental Figure 11). High power (63X objective) examination of these brains regions revealed both an apparent increase in the number of reactive astrocytes as well as astrocyte hypertrophy characterized by enlarged processes and extensive branching (Supplemental Figure 12). Microglial activation followed similar trends to that of astrocyte reactivity in that there was an increase in condensed, phagocytic-type microglia within the corpus callosum following blast exposure (Figure 7). Microglial activation was not limited to the corpus callosum though as activated microglia were also observed within the cortex (Supplemental Figure 13), striatum (Supplemental Figure 14), hippocampus (Supplemental Figure 15), and cerebellum (Supplemental Figure 16). 


\section{SUPPLEMENTAL FIGURE LEGENDS}

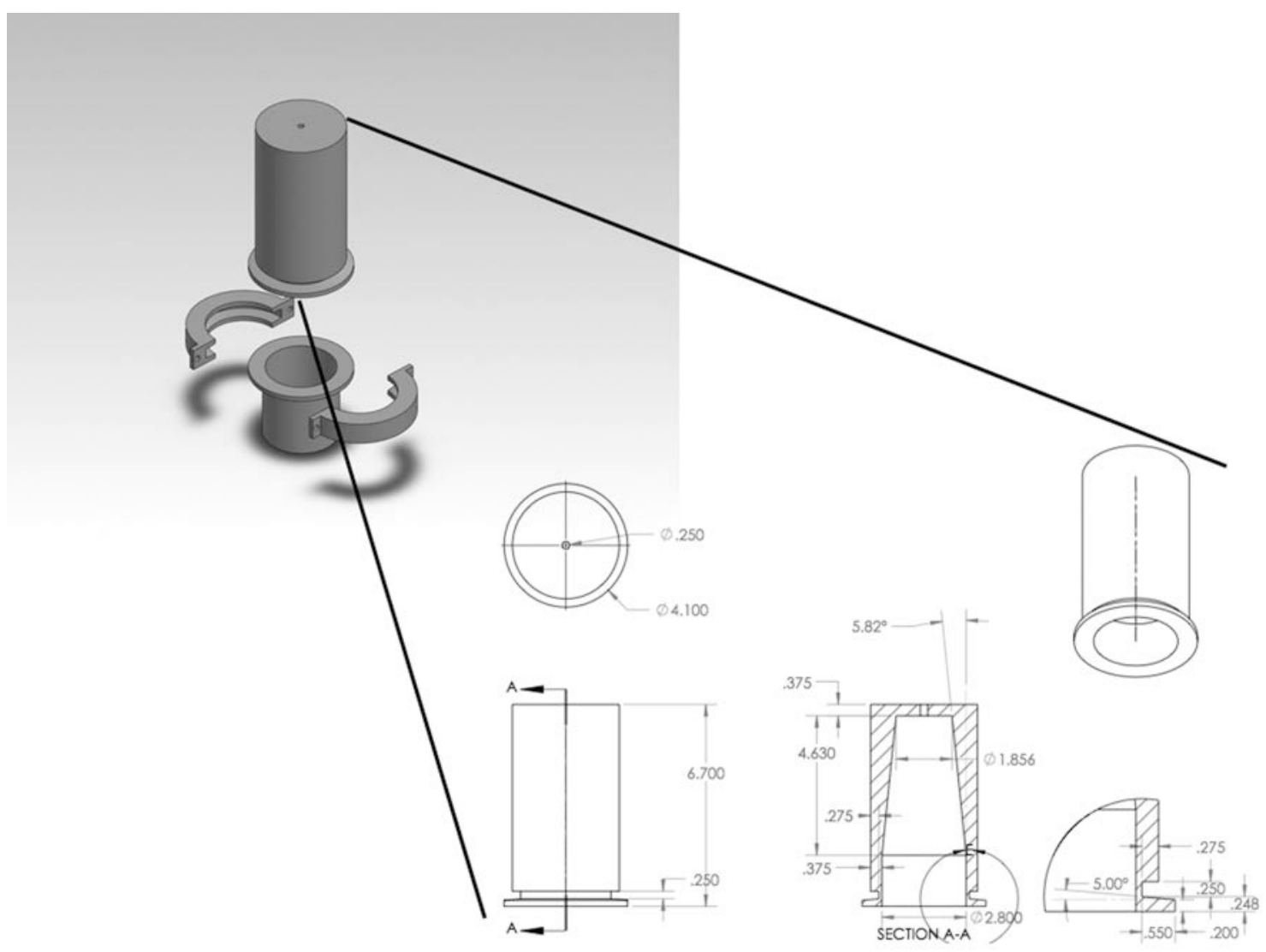

Supplemental Figure 1. An exploded view (not to scale) of the novel blast model utilized within this work with an enlarged engineering sketch shown that demonstrates the tapered design and short driver section. The length of the driver section influences the duration of blast exposure and was kept short deliberately to produce a range of peak overpressures, each with a short duration. Dimensions given are in inches. 


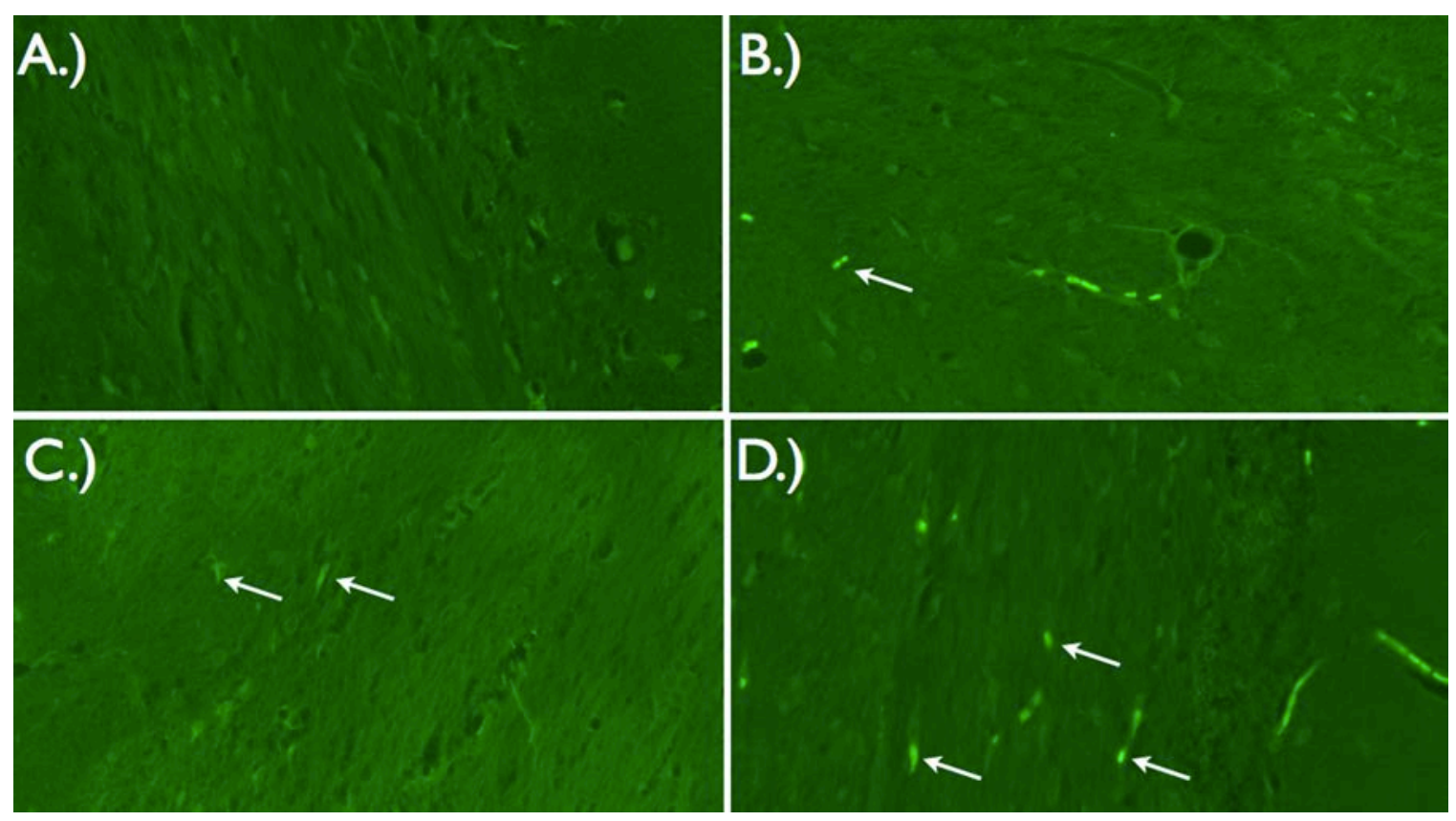

Supplemental Figure 2. Neural degeneration in the area including and directly around the corpus callosum of A.) Control, B.) 31.47 PSI blast, C.) 50.72 PSI blast, and D.) 72.05 PSI blast-exposed animals. Images acquired using 20X objective. 

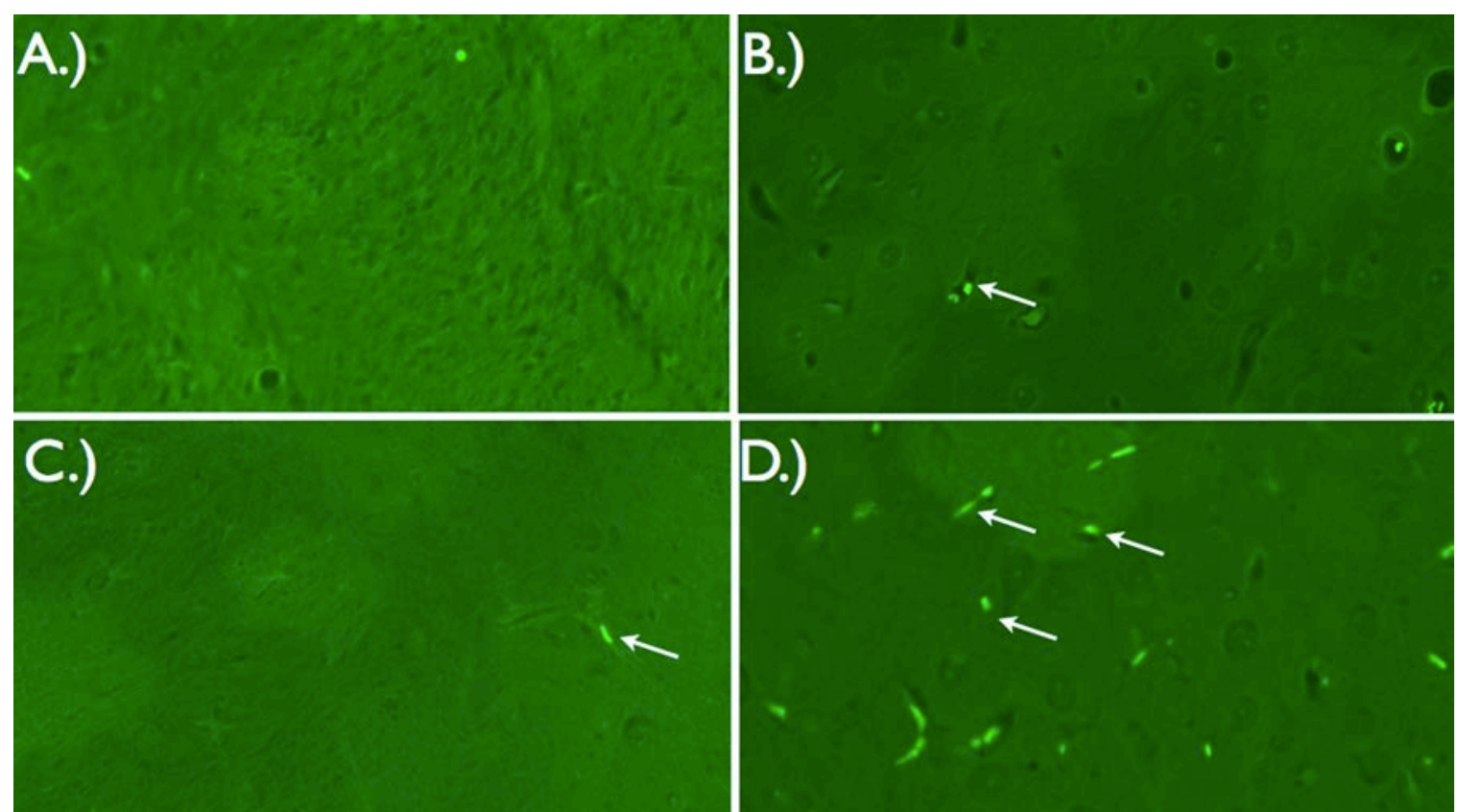

Supplemental Figure 3. Neural degeneration in the striatum of A.) Control, B.) 31.47 PSI blast, C.) 50.72 PSI blast, and D.) 72.05 PSI blast-exposed animals. Images acquired using $20 \mathrm{X}$ objective.FJB Striatum 


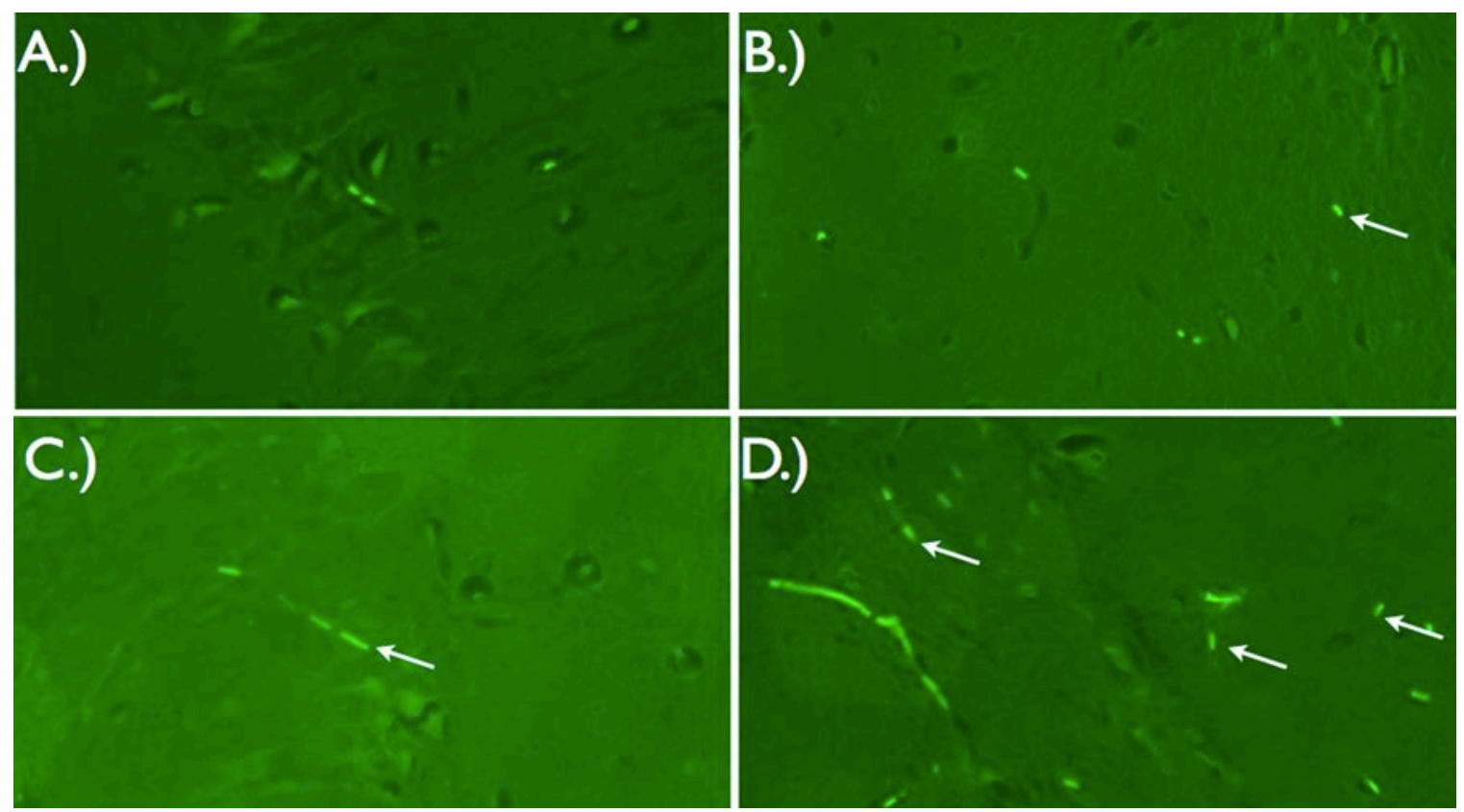

Supplemental Figure 4. Neural degeneration in the hippocampus of A.) Control, B.) 31.47 PSI blast, C.) 50.72 PSI blast, and D.) 72.05 PSI blast-exposed animals. Images acquired using $20 \mathrm{X}$ objective. 


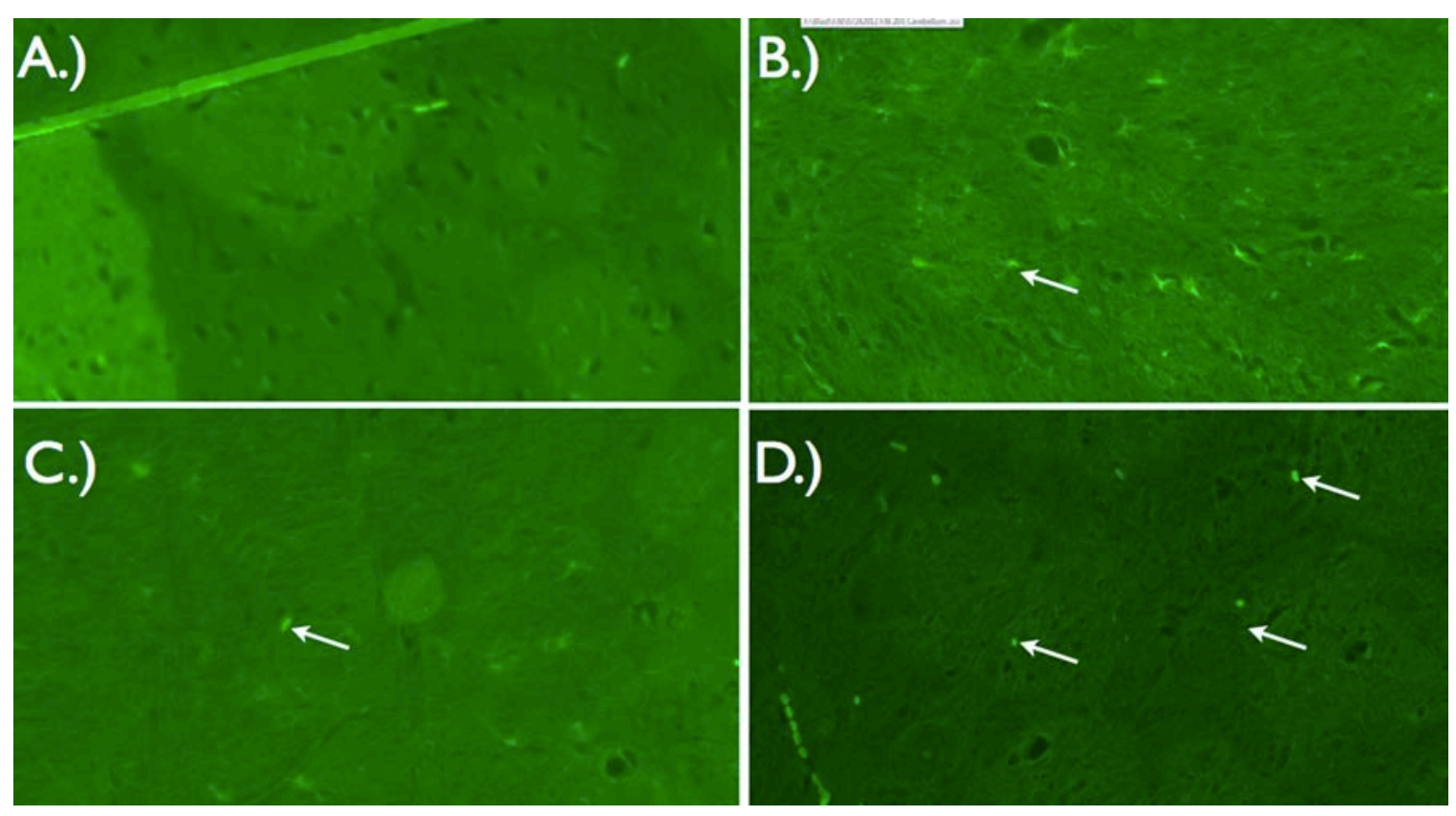

Supplemental Figure 5. Neural degeneration in the cerebellum of A.) Control, B.) 31.47 PSI blast, C.) 50.72 PSI blast, and D.) 72.05 PSI blast-exposed animals. Images acquired using $20 \mathrm{X}$ objective. 


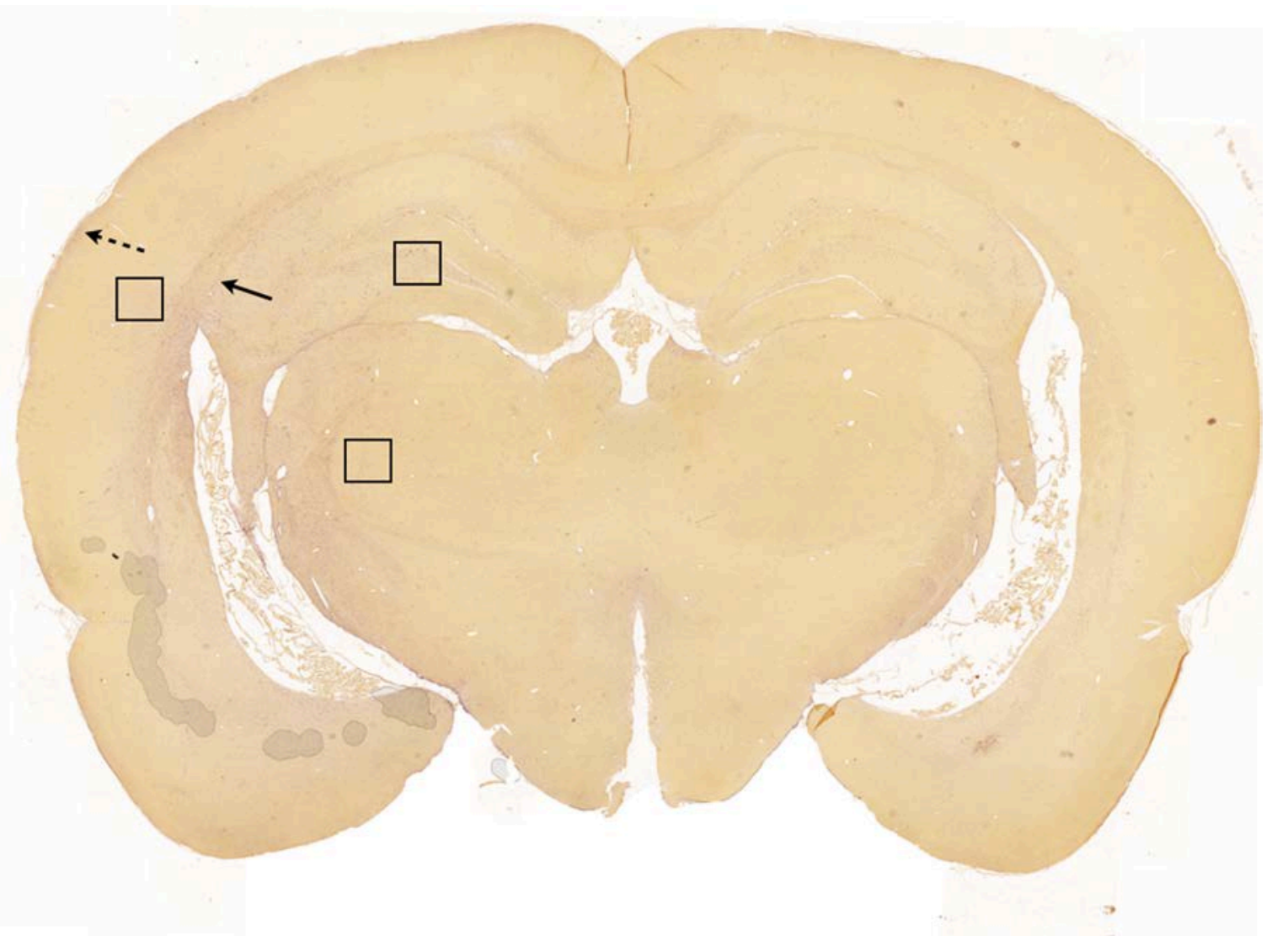

Supplemental Figure 6. Representative image at 72 hours post-injury with 50.72 PSI blast of GFAP staining acquired at low power (10X objective), using image stitching techniques, demonstrating regions from which higher power images were acquired (boxes). While morphological and quantitative studies were conducted using higher power objectives, the extensive GFAP reactivity is visible near the interface of large white matter tracts, such as the corpus callosum, with other brain regions (solid arrow). Some astrocyte reactivity is also apparent within the cortical mantle on the contrecoup side (dashed arrow), a location consistent with the bleeds observed on gross examination in brains exposed to moderate-severe blast (Figure 9.3). 


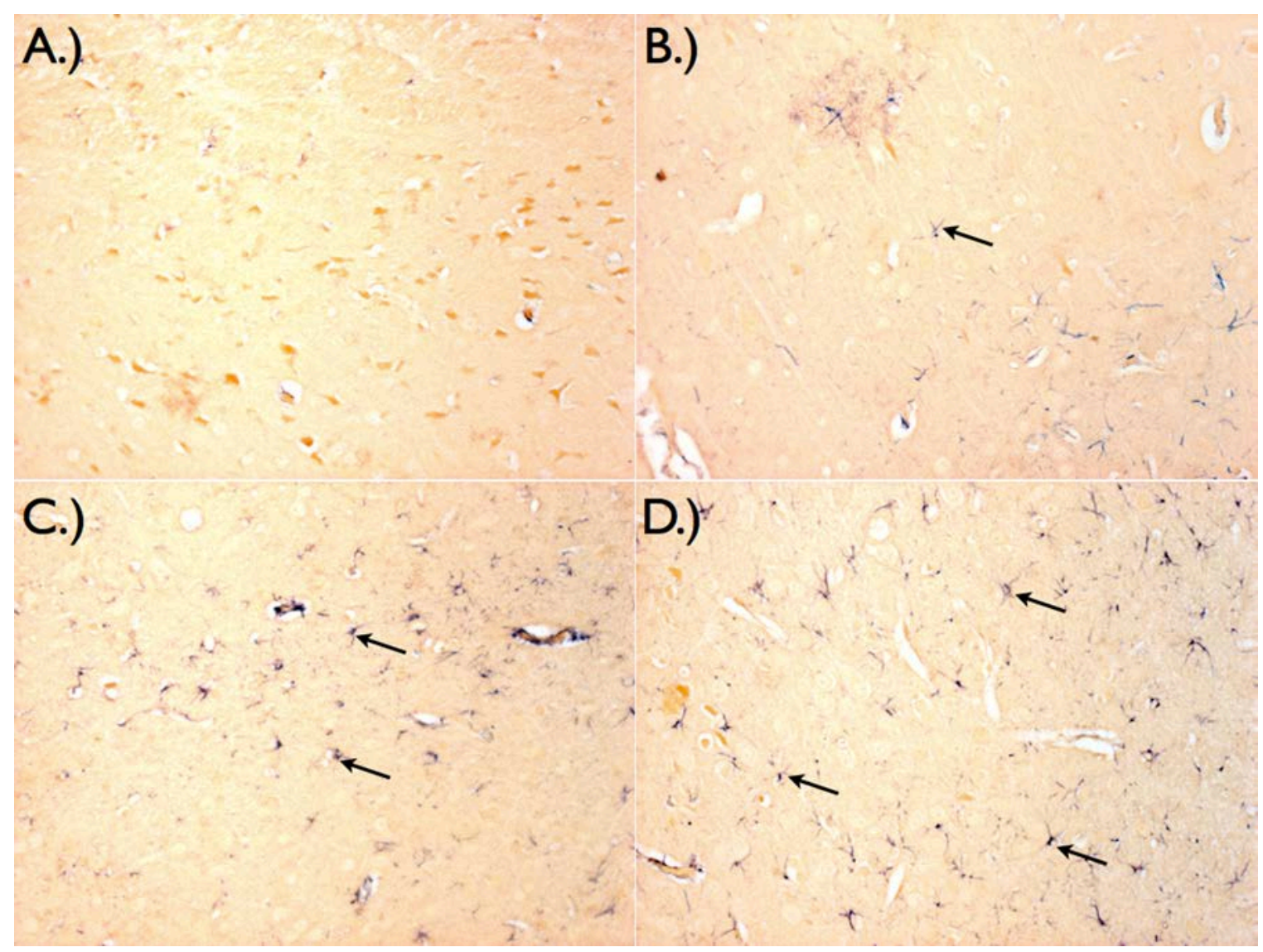

Supplemental Figure 7. Blast exposure was associated with extensive astrocyte reactivity in the cortex, particularly near the interface with long white matter tracts such as the corpus callosum. The level of reactivity appears correlated to peak overpressure exposure, and consequently, blast severity (A. Control, B. 31.47 PSI blast, C. 50.72 PSI blast, and D. 72.05 PSI blast). Similar immunohistochemical findings were observed in other brain regions as well, namely the striatum (Appendix Figure 4), hippocampus (Appendix Figure 5), and cerebellum (Appendix Figures 6 and 7). Images acquired using 20X objective. 


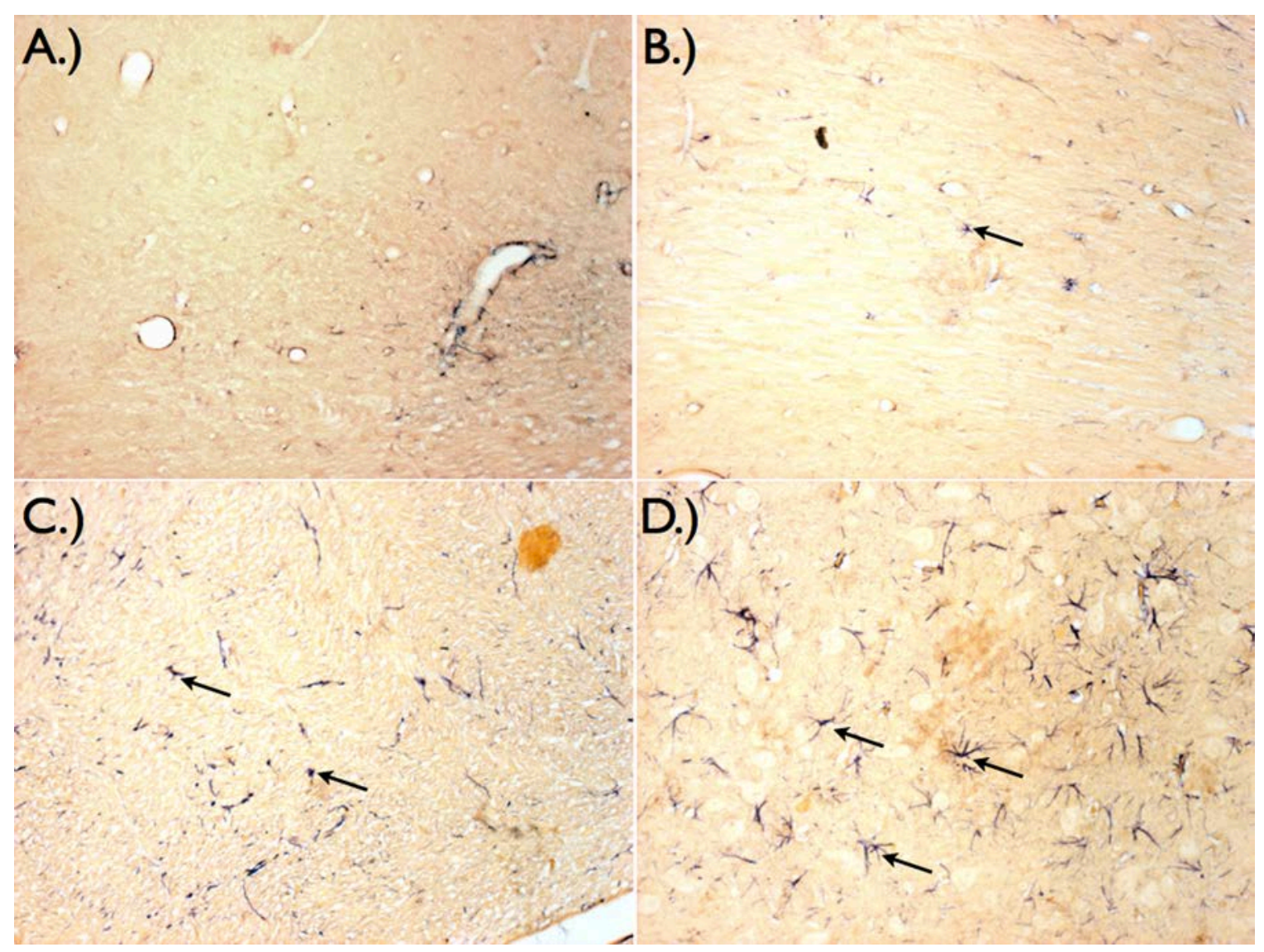

Supplemental Figure 8. Astrocyte reactivity in the striatum of A.) Control, B.) 31.47 PSI blast, C.) 50.72 PSI blast, and D.) 72.05 PSI blast-exposed animals. Images acquired using 20X objective. 


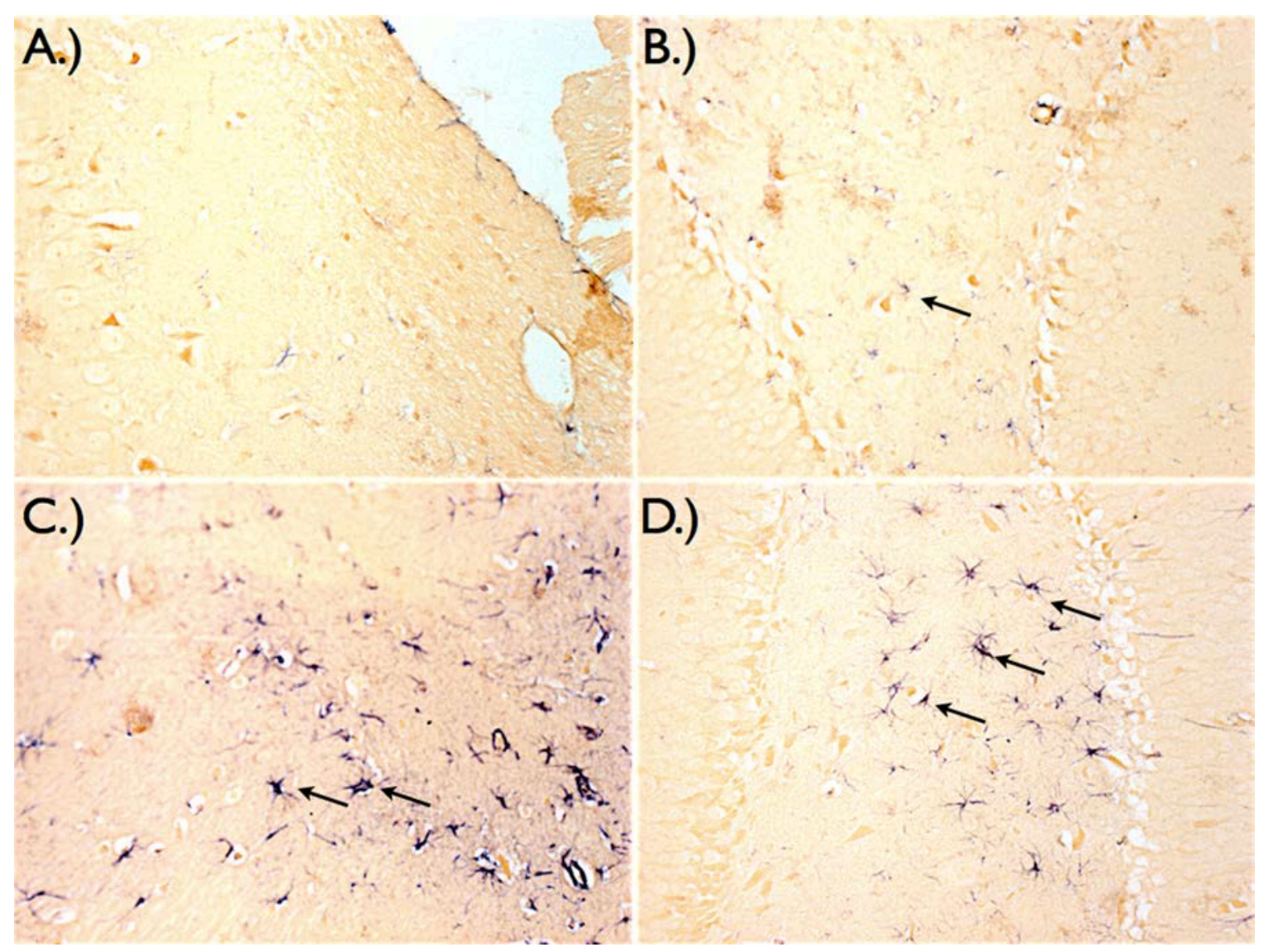

Supplemental Figure 9. Astrocyte reactivity in the hippocampus of A.) control, B.) 31.47 PSI blast, C.) 50.72 PSI blast, and D.) 72.05 PSI blast-exposed animals. Images acquired using $20 \mathrm{X}$ objective. 


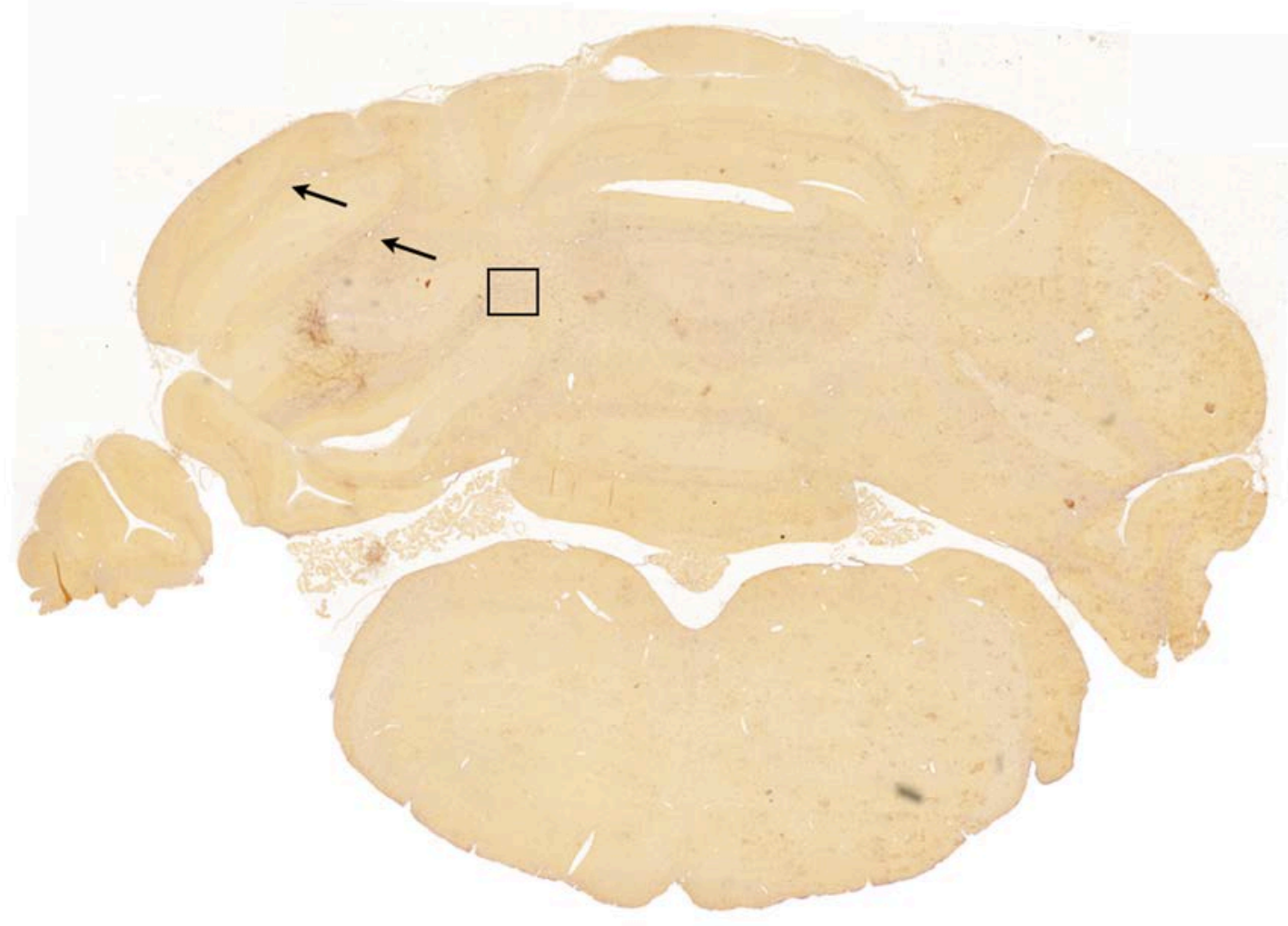

Supplemental Figure 10. Representative image at 72 hours post-injury with 50.72 PSI blast of GFAP staining acquired at low power (10X objective) demonstrating region from which higher power images were acquired (box). Visible astrocyte activation occurred within the white matter in blast-exposed animals. 


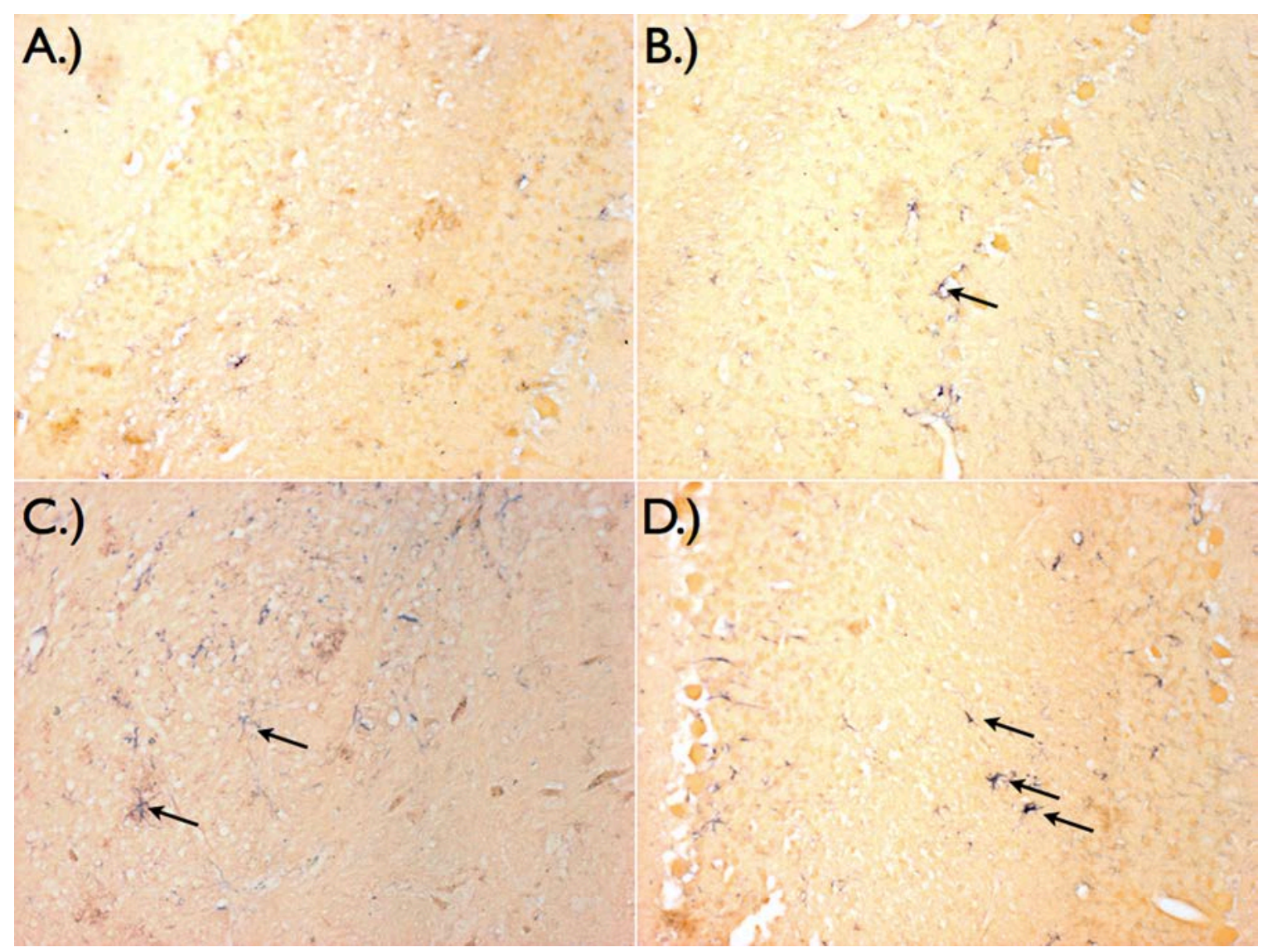

Supplemental Figure 11. Astrocyte reactivity in the cerebellum of A.) Control, B.) 31.47 PSI blast, C.) 50.72 PSI blast, and D.) 72.05 PSI blast-exposed animals. Images acquired using $20 \mathrm{X}$ objective. 

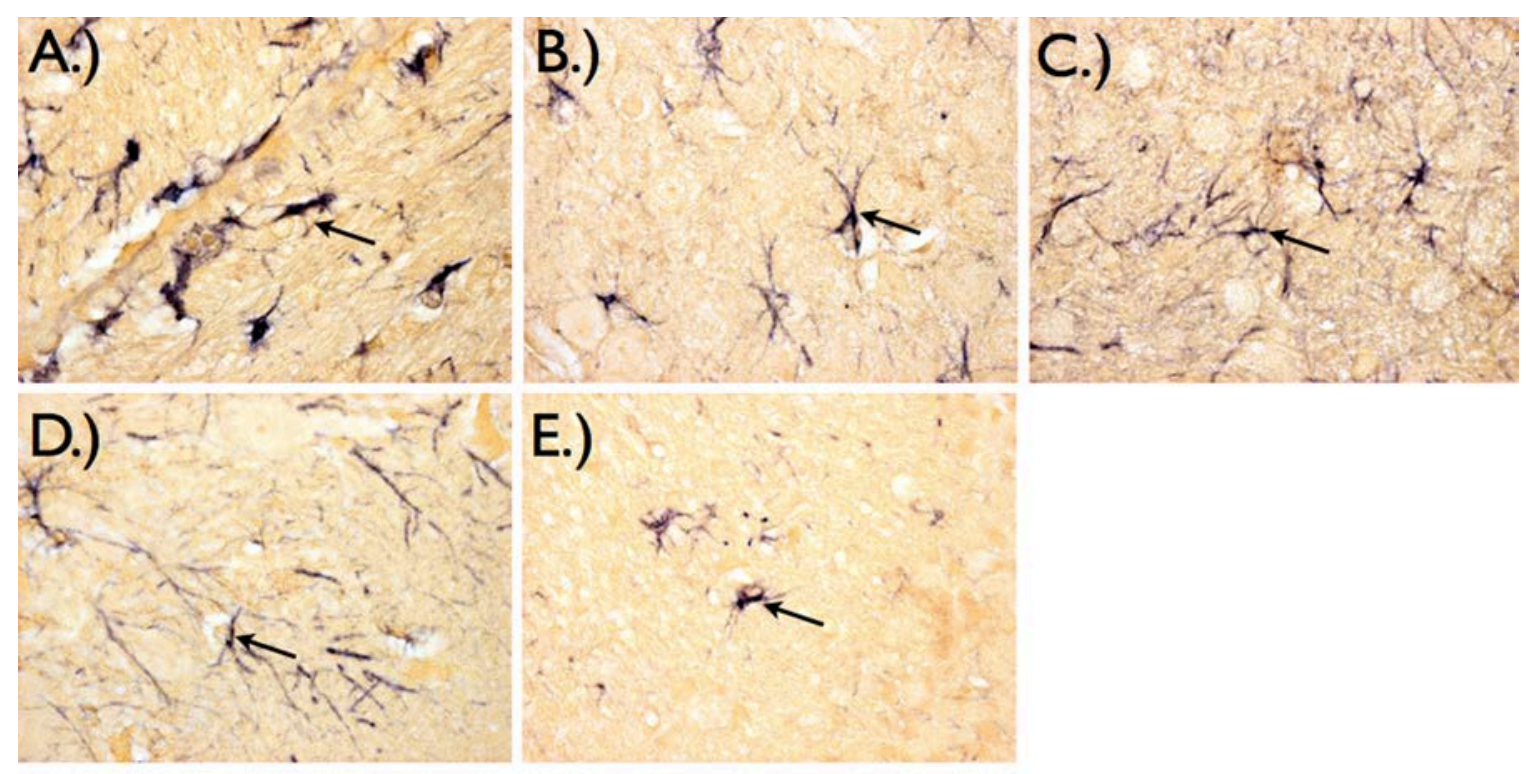

Supplemental Figure 12. High power (63X objective) visualization of astrocyte reactivity in animals exposed to blast reveals both an increase in number of astrocytes as well as significant hypertrophy and branching regardless of brain region but most notable within the corpus callosum. GFAP reactivity at 72 hours post-exposure to 72.05 PSI blast in the A.) corpus callosum, B.) cortex, C.) striatum, D.) hippocampus, and E.) cerebellum. 


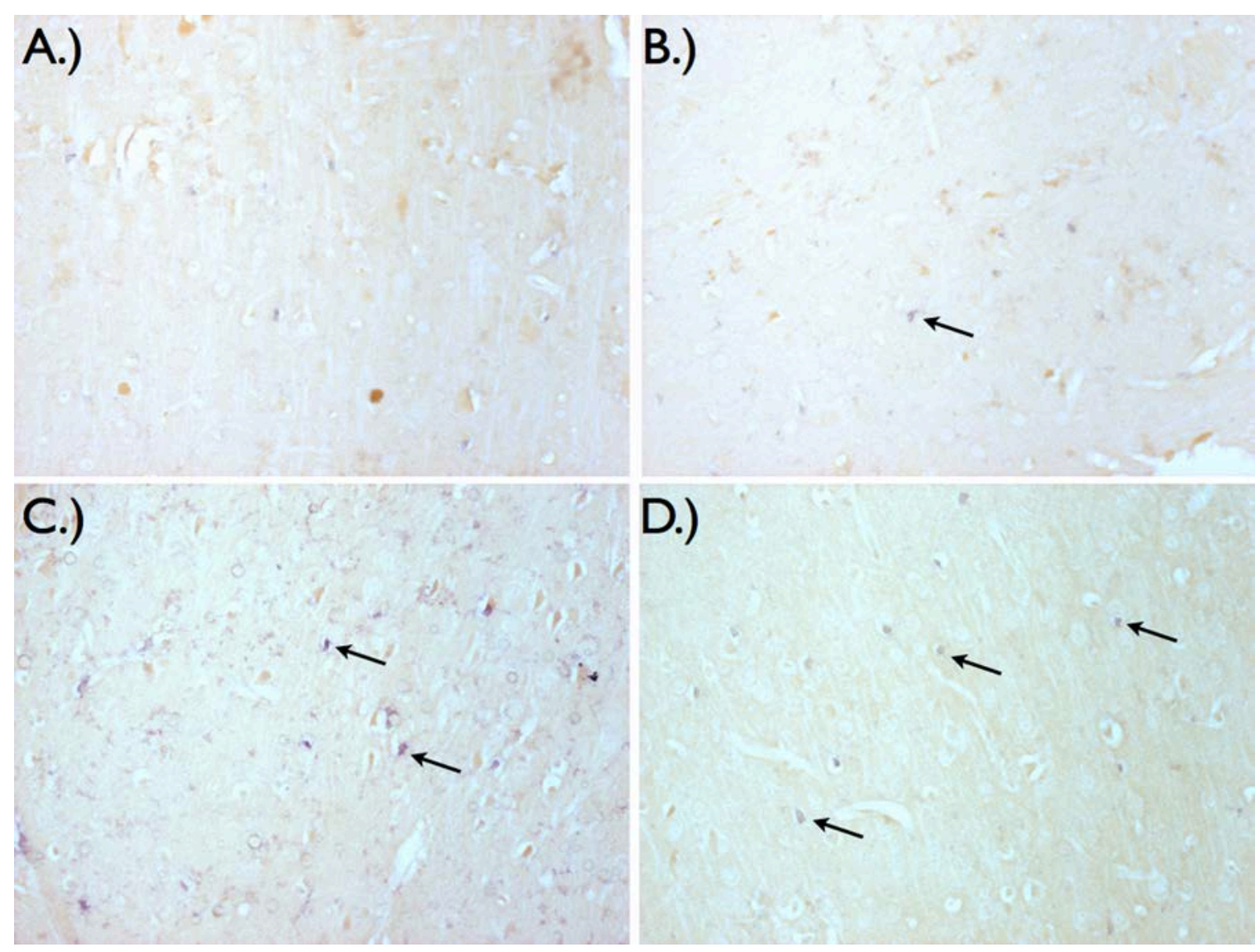

Supplemental Figure 13. Microglial activation in the cortex of A.) Control, B.) 31.47 PSI blast, C.) 50.72 PSI blast, and D.) 72.05 PSI blast-exposed animals. Images acquired using 20X objective. 


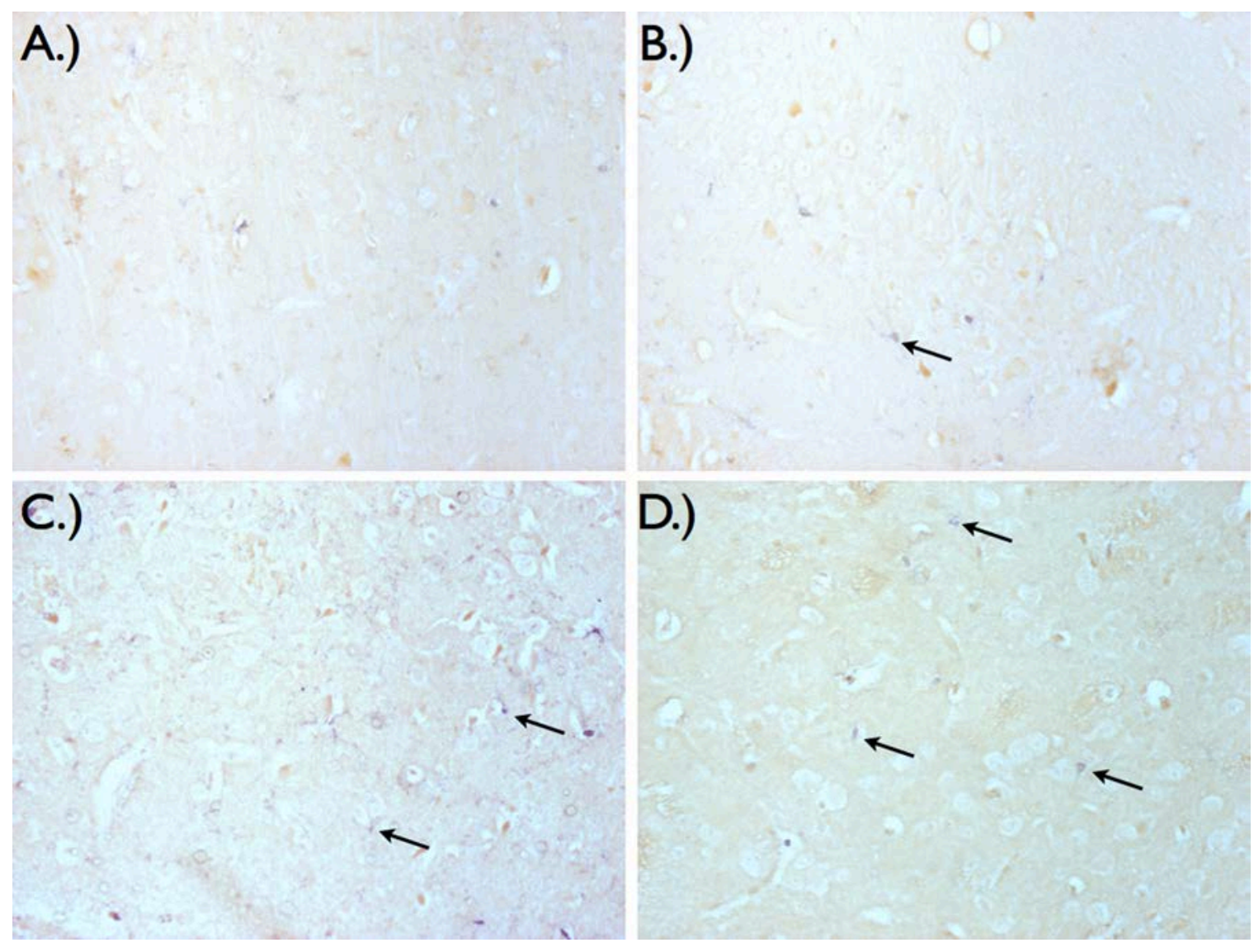

Supplemental Figure 14. Microglial activation in the striatum of A.) Control, B.) 31.47 PSI blast, C.) 50.72 PSI blast, and D.) 72.05 PSI blast-exposed animals. Images acquired using $20 \mathrm{X}$ objective. 


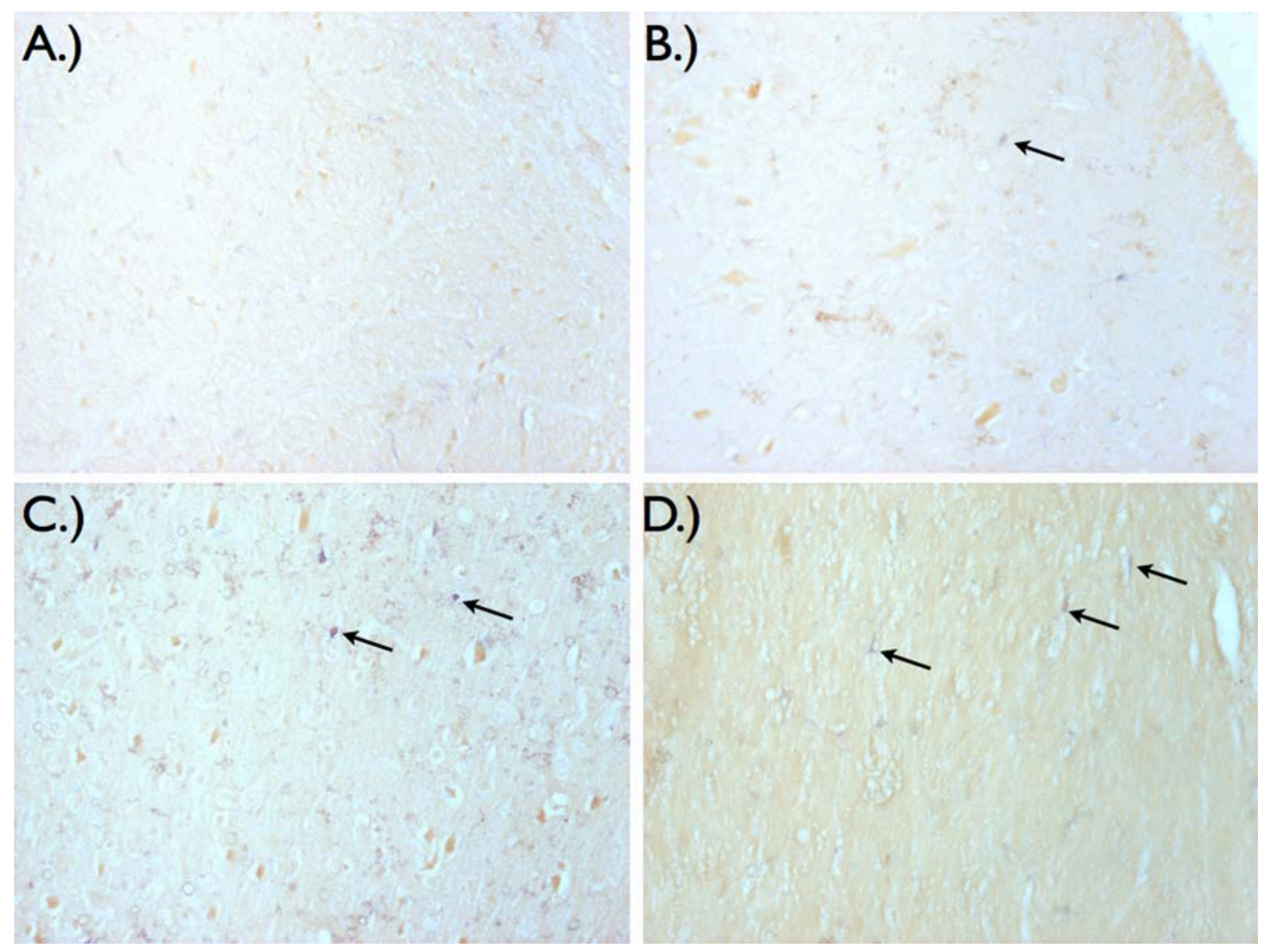

Supplemental Figure 15. Microglial activation in the hippocampus of A.) Control, B.) 31.47 PSI blast, C.) 50.72 PSI blast, and D.) 72.05 PSI blast-exposed animals. Images acquired using $20 \mathrm{X}$ objective. 


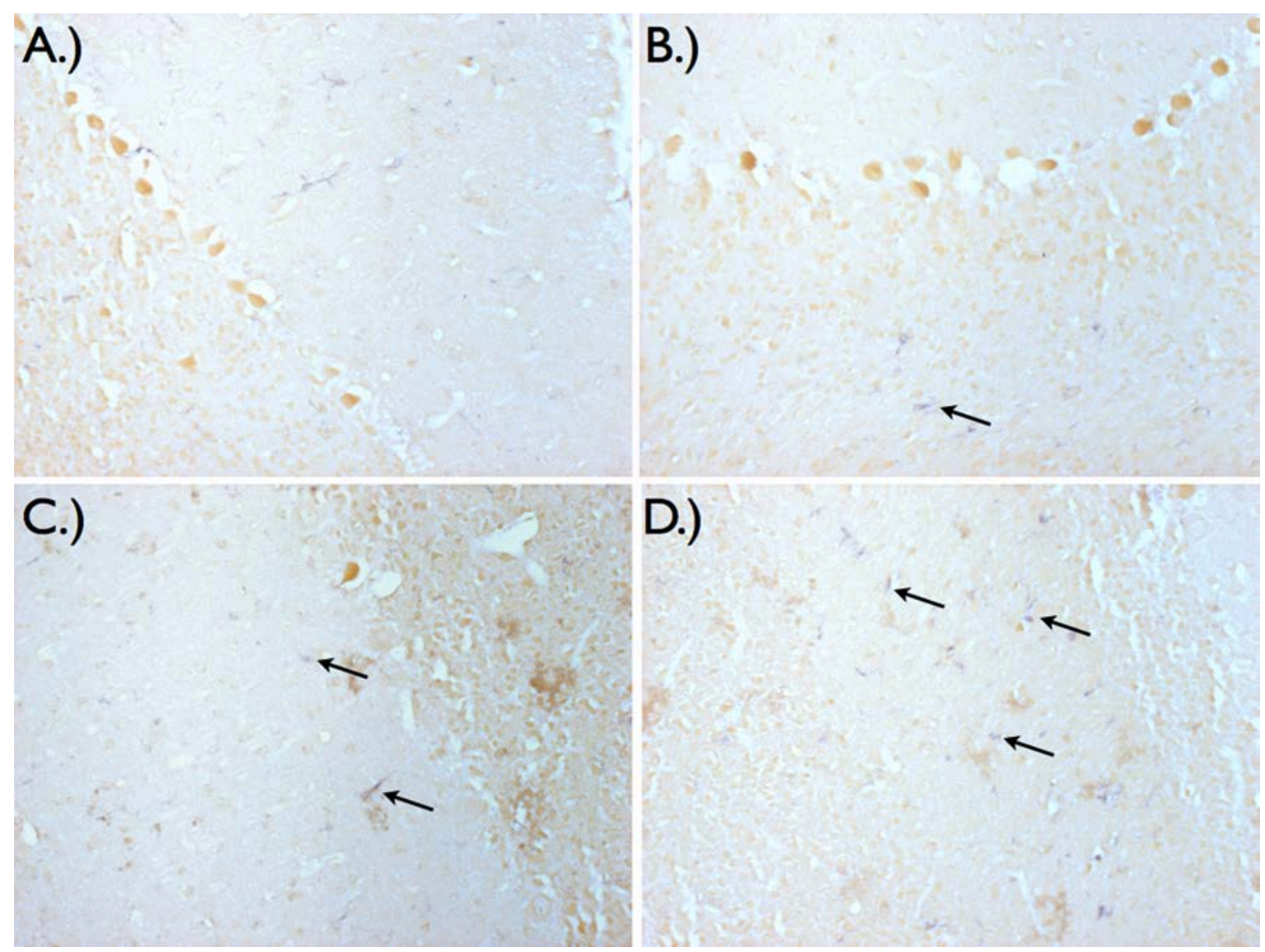

Supplemental Figure 16. Microglial activation in the cerebellum of A.) Control, B.) 31.47 PSI blast, C.) 50.72 PSI blast, and D.) 72.05 PSI blast-exposed animals. Images acquired using $20 \mathrm{X}$ objective. 


\section{CURRICULUM VITAE}

RYAN C. TURNER, B.S.

RESEARCH INTEREST:

PERSONAL

Date of Birth:

Birthplace:

Address:
Ischemic stroke and traumatic brain injury

February 12, 1986

Hoffman Estates, Illinois

Department of Neurosurgery c/o Dr. Charles L. Rosen

West Virginia University School of Medicine Robert C. Byrd Health Sciences Center PO Box 9183

Morgantown, West Virginia 26506

Work: (304) 293-4369

Email: rcturner@hsc.wvu.edu

\section{EDUCATION}

Professional/Graduate:

West Virginia University School of Medicine Morgantown, West Virginia

M.D./Ph.D. Candidate 2008-Present

Undergraduate:

Western New England University

Springfield, Massachusetts

B.S. Biomedical Engineering 2008

Minor: Chemistry

Magna cum Laude

High School:

Yorkville High School

Yorkville, Illinois

\section{ACADEMIC POSITIONS}

M.D./Ph.D. Candidate

Departments of Neurosurgery, Neurobiology and Anatomy

West Virginia University School of Medicine

Morgantown, West Virginia

2008-Present

\section{EXAMINATIONS IN MEDICINE}

MCAT: 29T (Biological: 10, Physical: 10, Verbal: 9, Writing: T)

USMLE Step I: 223/95 


\section{AWARDS}

Presidential Scholar, Western New England University

Springfield, Massachusetts, 2004-2008

Dean's List, Western New England University

Springfield, Massachusetts, 2004-2008

James V. Masi Award, Western New England University

Springfield, Massachusetts, 2005

Skookum Award, Western New England University

Springfield, Massachusetts, 2007

Marston Award, Western New England University

Springfield, Massachusetts, 2008

$2^{\text {nd }}$ Place Presentation, Basic Science Section, Neurosurgical Society of the Virginias, 2011

$2^{\text {nd }}$ Place Presentation, Basic Science Section, Neurosurgical Society of the Virginias, 2012

\section{MEMBERSHIPS}

National Neurotrauma Society 2011-Present American Medical Association 2008-Present

American Physician Scientists Association 2008-Present

Biomedical Engineering Society 2004-2008

\section{PROFESSIONAL SERVICE}

West Virginia University MD/PhD Program Admissions Committee 2010-Present Search Committee for the Assistant Director of HSC Graduate Education 2011 West Virginia University, Health Sciences Center, Graduate Student Organization 2011-2013

West Virginia University, Center for Neuroscience, Seminar Steering Committee 2012 


\section{PROFESSIONAL MEETINGS/WORKSHOPS}

Participant, $31^{\text {st }}$ Annual Northeast Bioengineering Conference, Hoboken, NJ, 2005

Participant, 32nd Annual Northeast Bioengineering Conference, Easton, PA, 2006 Participant, Annual Conference of the Biomedical Engineering Society, Chicago, IL, 2006

Participant, 33 ${ }^{\text {rd }}$ Annual Northeast Bioengineering Conference, Stony Brook, NY, 2007

Participant, Annual Conference of the Biomedical Engineering Society, Los Angeles, CA, 2007

Participant, 34th Annual Northeast Bioengineering Conference, Providence, RI, 2008

APSA Institutional Representative, APSA Annual Meeting, Chicago, IL, 2011

\section{EDITORIAL}

Reviewer, Neurotoxicology and Teratology

Reviewer, British Journal of Medicine and Medical Research

\section{FINANCIAL SUPPORT}

Research Training Program in Behavioral and Biomedical Sciences $\$ 66,448.00$ Behavioral and Biomedical Sciences Predoctoral Trainee NIH Grant: 5T32GM81741

Appointment Effective: July 1, 2011 - June 30, 2013

Behavioral and Biomedical Sciences Training Scholarship January 2011

$\$ 1,000.00$

\section{PUBLICATIONS}

\section{JOURNAL ARTICLES PUBLISHED OR IN PRESS}

1. Tan Z, Turner RC, Leon RL, Li X, Hongpasian J, Zhen W, Alkon D, Rosen CL, Huber JD. Bryostatin-1 mediated protein kinase $C$ activation enhances survival, reduces infarct size, brain atrophy, and neuronal death, and improves function in aged rats following middle cerebral artery occlusion and reperfusion. Stroke. Accepted August 2013.

2. Turner RC, Naser ZJ, Logsdon AF, DiPasquale KH, Jackson GJ, Robson MJ, Gettens RTT, Matsumoto RR, Huber JD, Rosen CL. Modeling Clinically Relevant Blast Parameters based on Scaling Principles Produces Functional and Histological Deficits in Rats. Experimental Neurology. Accepted July 2013.

3. VanGilder RL, Steinhart K, Davidov DM, Huber JD, Turner RC, Wilson KS, Haney E, Davis SM, Chantler PD, Theekie L, Rosen CL, Crocco T, Gutmann L, Barr TL. Systematic Review of Hs-CRP and Long-term Ischemic Stroke Prognosis. Journal of Clinical Neuroscience. Accepted June 21, 2013.

4. Robson MJ, Turner RC, Naser ZJ, McCurdy C, Huber JD, Matsumoto RR. SN79, a sigma receptor ligand, blocks methamphetamine-induced microglial 
activation and cytokine upregulation. Experimental Neurology. Epub ahead of print. DOI: 10.1016/j.expneurol.2013.04.009.

5. Karahalios DG, Mansour NH, Girardot EA, Turner RC. Overcoming Challenges Associated with Upright Imaging of the Cervicothoracic Junction: A Case Report Involving a Novel Repurposing of the O-Arm. International Journal of Medical Robotics and Computer Assisted Surgery. Published online March 14, 2013. Doi://10.1002/rcs.1491.

6. Turner RC, Dodson SC, Rosen CL, Huber JD. The science of cerebral ischemia and the quest for neuroprotection: navigating past failure to future success. A review. May 2013. Journal of Neurosurgery 118(5):1072-1085.

7. Turner RC, Lucke-Wold BP, Lucke-Wold N, Elliott AS, Rosen CL, Huber JD. Neuroprotection for Ischemic Stroke: Moving Past Shortcomings and Identifying Promising Directions. January 2013. International Journal of Molecular Sciences 14(1):1890-1917.

8. Turner RC, Lucke-Wold BP, Robson MJ, Omalu B, Petraglia AL, Bailes JE. Repetitive Traumatic Brain Injury and Development of Chronic Traumatic Encephalopathy: A Potential Role for Biomarkers in Diagnosis, Prognosis, and Treatment? Frontiers in Neurology. Published online January 17, 2013. Doi:// 10.3389/fneur.2012.00186.

9. Lucke-Wold BP, Turner RC, Lucke-Wold N, Rosen CL, Huber JD. Age and the Metabolic Syndrome as Risk Factors for Ischemic Stroke: Improving Preclinical Models of Ischemic Stroke. December 2012. Yale Journal of Biology and Medicine 85:523-539.

10. Turner RC, Naser ZJ, Bailes JE, Smith DW, Fisher JA, Rosen CL. Effect of Slosh Mitigation on Histologic Markers of Traumatic Brain Injury. December 2012. Journal of Neurosurgery 117:1110-1118.

11. Turner RC, Seminerio MJ, Naser ZJ, Ford NJ, Neal SJ, Matsumoto RR, Rosen $\mathrm{CL}$, Huber JD. Effects of aging on behavioral assessment performance: implications for clinically relevant models of neurologic disease. September 2012. Journal of Neurosurgery 117:629-637.

12. Popa-Wagner A, Buga AM, Turner RC, Rosen CL, Toescu E. Cerebrovascular Disorders: Role of Aging. March 2012. Journal of Aging Research. Article ID: 128146. Doi://10.115/2012/128146.

13. Smith DW, Bailes JE, Fisher JA, Robles J, Turner RC, Mills JD. Internal Jugular Compression Mitigates Traumatic Axonal Injury in Rat Model by Reducing Intracranial Slosh Effect. March 2012. Neurosurgery. 70(3):740-746.

14. Mills JD, Bailes JE, Turner RC, Dodson SC, Sakai J, Maroon J. Anabolic Steroids and Head Injury. January 2012. Neurosurgery 70(1):205-210.

15. Turner RC, Dodson SC, Rosen CL. Medical Management of Cerebellar Abscess: A Case Report and Review of the Literature. March/April 2011. West Virginia Medical Journal 107(2):21-23.

\section{JOURNAL ARTICLES SUBMITTED}

1. Barr TL, Huber JD, Turner RC, VanGilder RL, Ding J, Rosen CL, Matarin M, Singleton A, Warach S.A-kinase anchor protein 7 (AKAP7) as a Mediator of Blood Brain Barrier Disruption following Ischemic Stroke. Science Translational Medicine. Submitted August 2013. 
2. Tan Z, Li, X, Turner RC, Logsdon A, DiPasquale K, Lucke-Wold B, Chen R, Huber JD, Rosen CL. Combination treatment of $r$-tPA and apyrase reduces mortality rate and hemorrhagic transformation $6 \mathrm{~h}$ after ischemic stroke in aged female rats. Brain Research. Submitted June 2013.

3. Robson MJ, Turner RC, Naser ZJ, McCurdy CR, O'Callaghan JP, Huber JD, Matsumoto RR. SN79, a sigma receptor antagonist, attenuates methamphetamine-induced astrogliosis through a blockade of OSMR/gp130 signaling and STAT3 phosphorylation. Glia. Submitted June 2013.

4. Lucke-Wold BP, DiPasquale K, Logsdon AF, Nguyen L, Lucke-Wold AN, Turner RC, Huber JD, Rosen CL. Metabolic Syndrome and its Profound Effect on Prevalence of Ischemic Stroke. West Virginia Medical Journal. Submitted January 30, 2013.

5. Matsumoto RR, Seminerio MJ, Turner RC, Robson MJ, Miller DB, O'Callaghan JP. Effects of methamphetamine on body temperature. Pharmacology and Therapeutics. Submitted December 2012. Revisions submitted March 2013.

\section{BOOK CHAPTERS PUBLISHED}

1. Turner RC, Lucke-Wold B, Rosen CL, Huber JD. Modulation of Protein Kinase C Isoforms: A Potential Therapeutic for Neurologic Injury? Ischemic Stroke: Symptoms, Prevention and Recovery. Edited by Lakatos, Virag. Nova Biomedical, New York, NY. Pgs.171-190. ISBN: 978-1-62257-799-6.

2. Turner RC, Lucke-Wold B, Miller DB, O'Callaghan JP, Rosen CL, Huber JD. Neuropoietic cytokines and neural injury: alterations in JAK2/STAT3 signaling associated with aging. Neurological Disorders: New Research. Edited by Chloe E. Thomas and Jayden R. Moore. Nova Biomedical, New York, NY. Pgs. 27-47. ISBN: 978-1-62257-042-3.

3. Turner RC, Elliot AS, Huber JD, Rosen CL. A Clinically Relevant Thromboembolic Stroke Model in the Aged Rat. TRP Channels in Drug Discovery: Volume II. Methods in Pharmacology and Toxicology. Edited by Arpad Szallasi and Tamas Biro. Humana Press, New York, NY. Pgs. 211-229. DOI 10.1007/978-1-62703-095-3_13.

\section{EDITORIAL COMMENTS}

1. Turner RC, Rosen CL. Comment on Zinkel JL - Rat Forebrain Perfusion In Vivo by One Artery Like the Isolated Kidney Model: A Robust Recovery Model Permitting Ischemia Without Anesthesia to Compare Multiple Brain Injury States. April 2013 Neurosurgery 72:662-667.

\section{SELECTED ORAL PRESENTATIONS}

1. R.C. Turner, Z.J. Naser, J.E. Bailes, D.W. Smith, J.A. Fisher, C.L. Rosen. "Effect of Slosh Mitigation on Histologic Markers of Traumatic Brain Injury." Neurosurgical Society of the Virginias Annual Meeting, White Sulphur Springs, WV, January 2012.

2. J.E. Bailes, D.W. Smith, J. Robles, R.C. Turner, Z.J. Naser. "Concussive and Subconcussive Traumatic Brain Injury: Is Limitation Possible Through Slosh 
Mitigation?" Neurosurgical Society of the Virginias Annual Meeting, White Sulphur Springs, WV, January 2011.

3. R.C. Turner, K.A. Kelly, J.D. Huber, C.L. Rosen. "Role of aging and oxidative stress in stroke outcome - interaction of the JAK/STAT signaling pathway and NADPH oxidase." Neurosurgical Society of the Virginias Annual Meeting, White Sulphur Springs, WV, January 2011.

4. K.E. Joyce, R.C. Turner, R.T.T. Gettens. "Design and Validation of an Electrochemical Cell for Characterization of Surface Properties of Alloys." Proceedings of the IEEE EMBS 34th Annual Northeast Bioengineering Conference, Providence, RI, April 2008.

\section{SELECTED POSTER PRESENTATIONS}

1. M.J. Robson, R.C. Turner, Z.J. Naser, J.D. Huber, C.R. McCurdy, R.R. Matsumoto. SN79, a sigma $(\sigma)$ receptor ligand, mitigates methamphetamineinduced astrocyte and microglial activation. Experimental Biology, Boston, MA, April 2013.

2. A.F. Logsdon, R.C. Turner, M.J. Robson, Z.J. Naser, K. DiPasquale, C.L. Rosen, J.D. Huber. Characterization of the PERK-mediated ER stress pathway following traumatic brain injury in male rats: A role in neural injury and degeneration. West Virginia University Van Liere Research Day, Morgantown, WV, March 2013.

3. R.L. VanGilder, S.L. Rellick, J.D. Huber, C.L. Rosen, R.C. Turner. An association between TLR and JAK signaling following cerebral ischemia: A Translational Approach. International Stroke Conference, Honolulu, HI, February 2013.

4. A.S. Elliott, R.C. Turner, J.P. O'Callaghan, C.L. Rosen, J.D. Huber, D.B. Miller. Using RNA-Seq to evaluate the aged female Sprague-Dawley rat cortex transcriptome after repeated bouts of sleep deprivation induced by the gentlehandling method. Society for Neuroscience, New Orleans, LA, October 2012.

5. A. Logsdon, R.C. Turner, Z. Tan, K. DiPasquale, B. Lucke-Wold, C.L. Rosen, J.D. Huber. Alterations to the JAK2/STAT3 signaling pathway may affect ER stress levels in the aged rat stroke model. WVU Center for Neuroscience Annual Retreat, Snowshoe Resort, Snowshoe, WV, July 2012.

6. B.P. Lucke-Wold, R.C. Turner, K. DiPasquale, J.D. Huber, C.L. Rosen. Explosive Exposure: A Blast-Induced Model of Traumatic Brain Injury. WVU Center for Neuroscience Annual Retreat, Snowshoe Resort, Snowshoe, WV, July 2012.

7. K. DiPasquale, R.C. Turner, A. Logsdon, Z. Tan, C.L. Rosen, J.D. Huber. Increasing Clinical Relevance of Preclinical Ischemic Stroke Models: Infarct Volume and Function Correlation. WVU Center for Neuroscience Annual Retreat, Snowshoe Resort, Snowshoe, WV, July 2012.

8. Z. Tan, X. Li, R.C. Turner, A. Logsdon, B. Lucke-Wold, K. DiPasquale, R. Chen, C.L. Rosen, J.D. Huber. Combination treatment of r-tPA and APT 102 reduces cerebral infarct volume, improves neurological deficit score and prevents hemorrhagic transformation in embolic stroke in aged female rats. WVU Center for Neuroscience Annual Retreat, Snowshoe Resort, Snowshoe, WV, July 2012.

9. R.C. Turner*, R.L. VanGilder ${ }^{*}$, S.L. Rellick, T.L. Barr, C.L. Rosen, J.D. Huber. Modulation of Innate-Adaptive Immune System Cross-Talk for Neuroprotection: 
The Role of Lipopolysaccharide in Diffuse Axonal Injury. National Neurotrauma Symposium, Phoenix, AZ, July 2012. ( ${ }^{*}$ denotes co-authorship)

10. R.L. VanGilder ${ }^{\star}$, R.C. Turner*, S.L. Rellick, T.L. Barr, C.L. Rosen, J.D. Huber. The Effects of Lipopolysaccharide Preconditioning on a Diffuse Axonal Injury TBI. National Capital Area TBI Research Symposium. National Institutes of Health. May 2012. ( ${ }^{*}$ denotes co-authorship)

11. N. St. John, R.C. Turner, C.L. Rosen, R.T.T. Gettens. Design of a Blast Wave Characterization Model. American Society for Engineering Education - North East Section, Lowell, MA, April 2012.

12. R.C. Turner, Z.J. Naser, J.E. Bailes, D.W. Smith, J.A. Fisher, C.L. Rosen. Effect of Internal Jugular Vein Compression on Traumatic Brain Injury - A Histologic Approach to Studying Slosh Mitigation. American Association of Neurological Surgeons Annual Meeting, Miami, FL, April 2012.

13. R.C. Turner, Z.J. Naser, J.E. Bailes, D.W. Smith, J.A. Fisher, C.L. Rosen, J.D. Huber. Effect of Slosh Mitigation on Histologic Markers of Traumatic Brain Injury. West Virginia University Van Liere Research Day, Morgantown, WV, March 2012.

14. R.C. Turner, J.D. Mills, J.E. Bailes, S.C. Dodson, J. Sakai, J.C. Maroon. Anabolic Steroids and Traumatic Brain Injury - Do Steroids Play a Role in Chronic Traumatic Encephalopathy? National Neurotrauma Symposium, Hollywood, FL, July 2011.

15. J.E. Bailes, D. Smith, J. Fisher, J. Robles, R.C. Turner, J.D. Mills. Internal Jugular Vein Compression Mitigates Traumatic Axonal Injury in Rat Model by Reducing Intracranial Slosh Effect. National Neurotrauma Symposium, Hollywood, FL, July 2011.

16. J.E. Bailes, D. Smith, J. Fisher, J. Robles, R.C. Turner, J.D. Mills. Internal Jugular Vein Compression Mitigates Traumatic Axonal Injury in Rat Model by Reducing Intracranial Slosh Effect. WVU Center for Neuroscience Annual Retreat Stonewall Jackson Resort, Roanoke, WV, June 2011.

17. R.C. Turner, J.D. Mills, J.E. Bailes, S.C. Dodson, J. Sakai, J.C. Maroon. Effect of Anabolic Steroids on Traumatic Brain Injury. West Virginia University Van Liere Research Day, Morgantown, WV, March 2011.

18. K.E. Joyce, R.C. Turner, R.T.T. Gettens. Design and Validation of an Electrochemical Cell for Surface Characterization of Biomedical Alloys. American Society for Quality (ASQ) 62nd Annual Conference, Houston, TX, May 2008.

\section{REFERENCES}

Julian E. Bailes, M.D.

Chairman, Department of Neurosurgery

NorthShore University Health System

2650 Ridge Avenue

Evanston, Illinois 60201

Work: (847) 570-1456

E-Mail: jbailes@northshore.org

Charles L. Rosen, M.D., Ph.D.

Chairman \& Program Director, Department of Neurosurgery

Director, Cranial Base Surgery and Neurosurgical Research

Professor, Departments of Neurosurgery, Anatomy and Neurobiology 
West Virginia University School of Medicine

P.O. Box 9183, Robert C. Byrd Health Science Center

Morgantown, West Virginia 26506-9183

Work: (304) 293-0424

E-Mail: crosen@hsc.wvu.edu

Jason D. Huber, Ph.D.

Associate Professor, Department of Basic Pharmaceutical Sciences

West Virginia University School of Pharmacy

P.O. Box 9530, Robert C. Byrd Health Science Center

Morgantown, West Virginia 26506-9530

Work: (304) 293-1474

E-Mail: jdhuber@hsc.wvu.edu 